
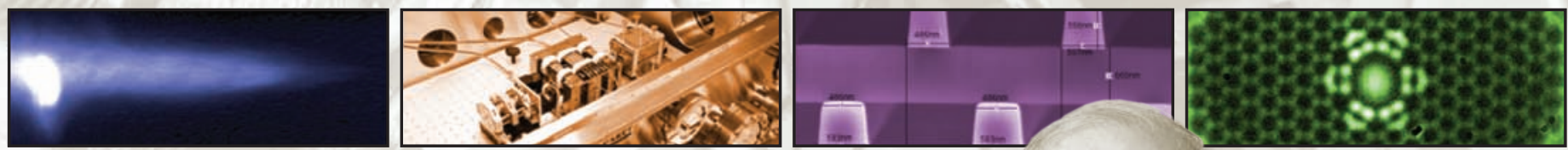

\title{
Proceedings
}

\section{Symposium in Memory of Robert H. Siemann}

July 7th, 2009

SLAC National Accelerator Laboratory

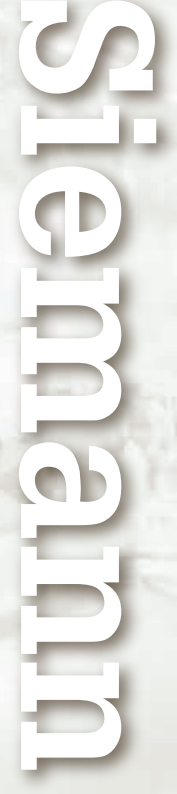




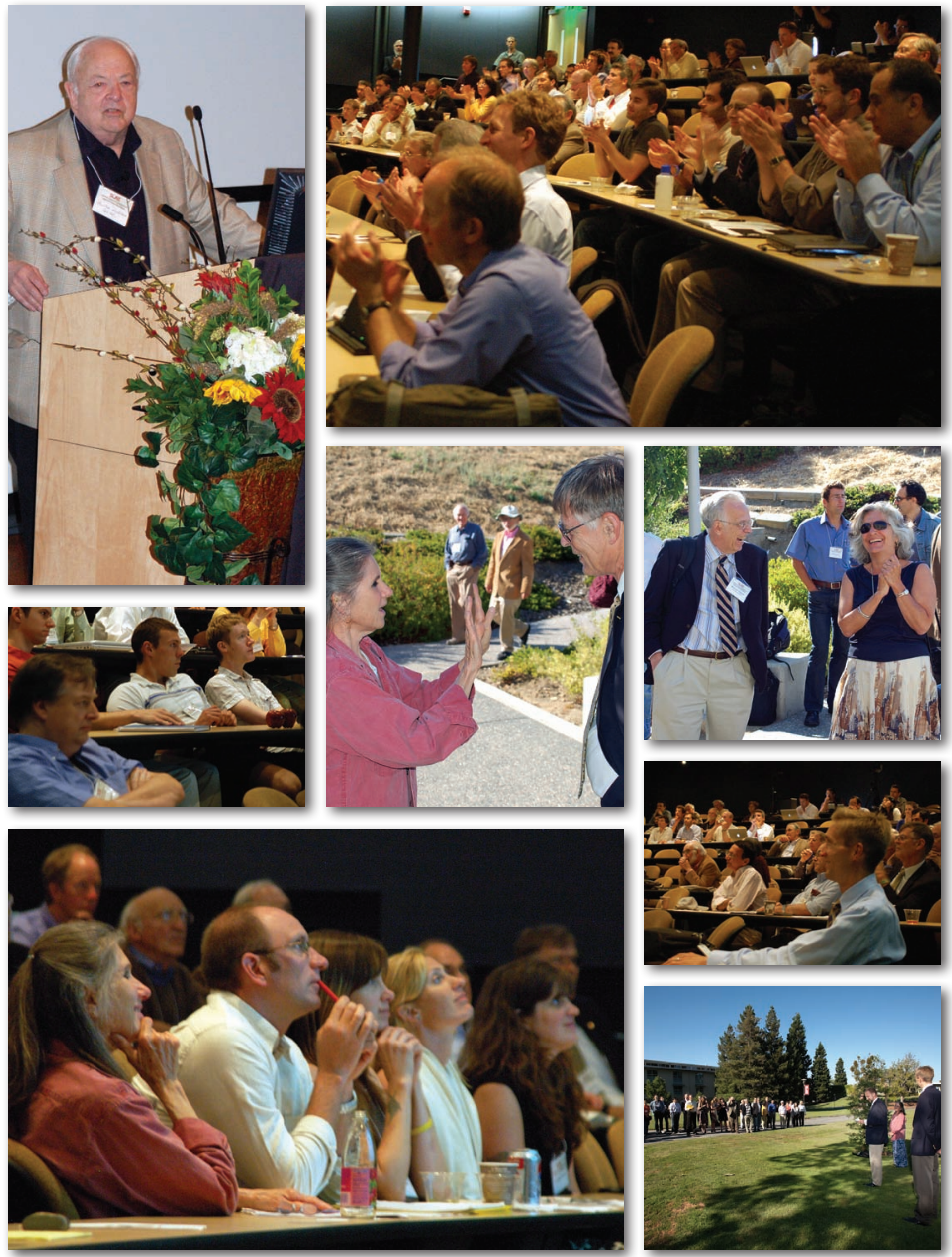




\section{Contents}

Preface

Eric Colby 1

Welcome

Persis Drell.

\section{Early Days}

- Experimental Particle Physics: Bob Siemann and the DECO Experiment Roger Erickson

- Bob's Evolution from Particle Physics to Accelerator Physics

Richard Talman

- Bob as a Mentor at Cornell (and later at Fermilab)

Gerry Jackson

\section{From Cornell to SLAC}

- From SLAC Post-doc to SLAC Professor

Burt Richter 56

- Bob Siemann: SLAC Professor and SLC Physicist

Tor Raubenheimer

\section{Through his student's eyes}

- SLC and the Sawtooth Boris Podobedov

- Reminiscence of Bob Siemann and His Dream on $1 \mathrm{GeV} / \mathrm{m}$

Ping-Jung Chou

- Pulsed Heating

David Pritzkau

\section{Leadership in Accelerator Physics}

- Leadership at Spallation Neutron Source Stuart Henderson

- E-157 Plasma Wakefield Experiment

Tom Katsouleas

- Through his student's eyes: Plasma Accelerators

Chris Barnes

- Laser Accelerators: A Path Toward Coherent Attosecond X-Rays

Bob L. Byer 182

- Through his student's eyes: Laser Accelerators

Ben Cowan 


\section{Legacy, Closing, \& Tree Dedication}

- Bob Siemann and the Community Organizer

Mel Month

- Bob Siemann Brookhaven and the American Physical Society

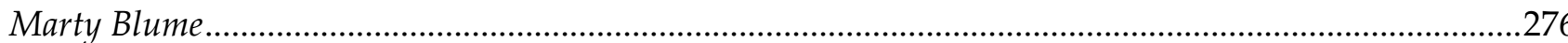

- Remarks from the APS Chair

Swapan Chattopadhyay

- Robert H. Siemann Symposium Closing

Eric Colby

- Tree Dedication

Eric Colby

.303 


\section{Preface}

The Symposium in Memory of Robert H. Siemann was held on July $7^{\text {th }}$, 2009 to honor the life and achievements of one of the pioneers of accelerator physics. Speakers and participants from around the world came together at SLAC to celebrate his contributions to science and to the scientific community.

Speakers from each of the chapters of Bob’s life were asked to provide a broad retrospective of the man and his career. Students provided brief perspectives of specific aspects of his work, and of his role as a mentor. The Siemann family attended and offered personal reminiscences. A redwood tree and memorial plaque were dedicated on the SLAC main Green in his honor. Many who attended the event felt they had learned something they did not know about their longtime colleague.

In the pages that follow, the prepared remarks and slides of speakers have been collected. The richness of the contributions and breadth of topics covered are a reflection of the richness and breadth of Bob's life. The event was video recorded, and may be viewed at the SLAC library or online through the SLAC website.

Persis Drell's sponsorship and Tor Raubenheimer's assistance in arranging the event are both gratefully acknowledged. I am grateful for the help of the Symposium Committee in selecting the program and speakers: Marty Blume, Bob Byer, Alex Chao, Mark Hogan, Gerry Jackson, Chan Joshi, and Maury Tigner. I am especially grateful to the local organizers: Joan Valine and Naomi Nagahashi for flawless logistics, InfoMedia Solutions Team for conference Website application design and support, Nick Arias and Stephanie Santo for help with computer support and the Guestbook, Rob Reape and Communications for A/V support, Ken Soong and Edgar Peralta for photography, Joel England, Johnny Ng, Ian Blumenfeld, and Neil Kirby for setup and preparations, Dieter Walz and Jim Spencer for a fine choice of wines at the reception, and to Sharon West, Terry Anderson, and Robert Noble for help in preparing the Proceedings.

Eric Colby

Symposium Chair

August 2009 


\section{Robert Siemann Symposium Welcome}

7/7/09 -- Persis Drell

Good morning. On behalf of Stanford University and SLAC National Accelerator Laboratory, I would like to welcome you all to the Siemann Symposium in memory of the life of Robert Siemann and his many contributions to accelerator physics and the scientific community. I extend a special welcome to Bob's family; his wife, Hannah, daughters Francesca, Gabriella and Liese and son Evan. You can look forward to speakers who will share reflections from many chapters of Bob's life offering their insights on his scientific and personal legacy. In the late afternoon there will be a dedication of a tree planted in Bob's memory here on the SLAC site, followed by a reception at the Stanford Guest House patio.

Throughout today's talks you will hear two strongly intertwined themes that frame Bob's legacy. You will hear Bob honored for his distinguished work in the area of accelerator science and you will hear about his legacy of teaching and mentoring young scientists.

Bob Siemann completed his bachelor's degree in physics at Brown University in 1964 , and his doctorate in experimental particle physics at Cornell University in 1969. His first association with SLAC was as a research associate from 1969 to 1972. After a year as a physicist at Brookhaven National Laboratory, he joined the faculty of Cornell, where he advanced from assistant professor to full professor. During that period his interests shifted from particle physics to accelerator physics and he made major contributions to the theory and modeling of the beam-beam interaction and beam instabilities, as well as to beam diagnostics. In 1991, already by now a recognized leader in the field of accelerator physics, Burt Richter persuaded Bob to come to SLAC as a Professor. With Bob's arrival, a new Stanford academic program in advanced accelerator physics dawned. A few years after joining Stanford, Bob established a department at SLAC specifically devoted to long-term accelerator research with an emphasis on educating graduate students.

Bob's professional accomplishments in the field of accelerator physics are widely acknowledged and documented as he was named APS and AAAS Fellow and he participated in or led numerous high level advisory committees. He was widely known as a champion of Accelerator Physics as a scientific discipline. He was instrumental in creating the widely respected peer-reviewed journal for accelerator physics, the Physical Review Special Topics - Accelerators and Beams, and served as the first editor of that journal for nine years. This 
publication attracted students and promoted accelerator physics as its own discipline

Bob's dedication as a mentor and advisor is best memorialized in the outstanding students he trained, many of whom you will hear from today. Bob enjoyed working with his students more than anything else, and despite Bob's rigorous and demanding style, his former students universally laud him.

Bob's students appreciated his high level of intellectual honesty and his basic desire to place good science ahead of convenience. Several students have noted that those qualities would lead him to reject 'popular' scientific analysis or conventional solutions, leading to greater depth in understanding of the fundamental accelerator science. Bob taught his students how to ask the right questions, and encouraged them both to create their own ideas and to carry them through to fruition.

Bob's students are a wonderful legacy. More than anyone he championed the importance of educating young accelerator scientists and thanks to Bob and his efforts Stanford University is one of the premier institutions in the country for educating accelerator physicists, probably rivaled only by Cornell, Bob's former home.

The future of the field of particle physics depends critically on innovations in accelerator science, and many other fields, fusion, nuclear physics and photon science, have come to rely on accelerators as their primary tools for discovery. Above all, the future of these fields depends on new generations of accelerator scientists, inspired by mentors like Bob, to make the essential discoveries that we need to advance the frontiers of knowledge.

It is incredibly fitting that in honor of Bob's scientific achieyements and dedication to teaching, that today Stanford University the establishment of two graduate fellowships in Bob Siemann's name. Bob's wife, Hannah Siemann, has very generously given the foundational gift to endow the fellowships to support outstanding physics PhD students who are conducting research at SLAC or in the fields of high energy, particle, or accelerator physics at Stanford.

Dean Richard Saller of Stanford's School of Humanities and Sciences has matched Mrs. Siemann's gift with funds from the William and Flora Hewlett Foundation. Dean Saller stated: "We are all grateful for Mrs. Siemann's generosity in creating these fellowships, which allows us to support talented physics students who - like Robert Siemann - will shape the future of their field"

Siemann's unquestioned commitment to science and the University coupled with his dedication and love of educating young physics students inspired the founding of these fellowships in Bob's memory. I would like to ask all of you, his colleagues and students, who valued him as a friend and as a mentor, who 
enjoyed his company and who now miss him, to strongly consider contributing to this fellowship fund. I personally cannot think of a more fitting tribute to Bob. Bob understood the intimate connection between teaching at advanced levels and research. Accelerator science at SLAC and nationally bears the stamp of Bob's accomplishments and of his character. We are proud to have called him our colleague. With graduate fellowships endowed in his honor, young students will continue to benefit from his legacy.

I hope you enjoy today's Symposium, the science and the personal reflections that you will be hearing.

Thank you and once again...welcome! 


\title{
Experimental Particle Physics: Bob Siemann and the DECO Experiment
}

\author{
Roger Erickson \\ SLAC Linear Accelerator Center \\ Robert H. Siemann Symposium \\ July 7, 2009
}

Thirty five years ago last week, I arrived in Ithaca, hoping to find interesting summer employment before starting graduate school in the fall. I found John DeWire, who was the deputy director of the Laboratory of Nuclear Studies, sitting in his office in Newman Lab that July morning. He looked up, squinting, from his desk and said "Go talk to Bob Siemann. He's the young guy just down the hall."

I walked down the hall as directed and looked into the designated office. Here was a big guy hunched over his desk, writing intensely on a pad of paper. He gripped a pen tightly in his fist as if he were trying to engrave his notes into the desktop.

I introduced myself and said I wanted to work on a high energy physics experiment. Bob dropped the pen, smiled, and launched into a description of a new experiment. This experiment would measure the distributions of all the charged pions and kaons produced by deep-inelastic scattering of electrons off a proton target. The experiment would use a streamer chamber to photograph the tracks of the particles as they curved through a strong magnetic field. The streamer chamber was being built in Hamburg and would be arriving in a few weeks.

Bob explained that we at Cornell had to provide a set of detectors that could unambiguously distinguish which of several charged particles in each event was the electron and provide a trigger pulse to the streamer chamber whenever an electron was scattered at a large angle. To do this, we would need a scintillator hodoscope immediately behind the streamer chamber, followed by a detector that could distinguish electrons from pions.

"Will we use sodium iodide crystals?” I asked. “Too expensive”, he said, dismissing the idea. "Lead glass blocks?" "Nope. We can’t afford that either." "So what else is there?” I asked. He grinned and said, "Look at this.”

In the room across the hall were two rectangular stainless steel boxes, each a little smaller than a kitchen refrigerator. There were fifteen holes on one face of each box to accommodate portholes for photomultiplier tubes. Bob explained that we would fill these boxes with a saturated solution of lead perchlorate in water. This is a crystal clear 
liquid with nearly three times the density of plain water and an index of refraction of 1.6, about the same as flint glass. Electrons will shower in this stuff and produce flashes of Cerenkov light, which can be seen by the phototubes. This was starting to sound interesting.

Bob then said that he wanted the inside walls of the boxes painted with bright white titanium dioxide paint to ensure we would collect as much light as possible. So before lunch of my first day at Cornell, I was painting the interiors of these Cerenkov shower counter tanks.

A few weeks later, the tanks had been moved to the Wilson Synchrotron Lab with their bright white interiors and glass portholes and phototubes installed and were ready to be filled. Lead perchlorate is nasty stuff: it's highly toxic, and it's a strong oxidizing agent. The solution was delivered in small cylindrical barrels, each holding five gallons, and each barrel was covered with warning labels about its toxicity and reactivity. Twenty barrels of this solution were needed to fill each of the two tank detectors.

I looked at the first empty tank and the step ladder and the twenty little barrels lined up on the floor, and realized I had a problem. The filler tube on the top of each tank was nearly six feet above the floor, and each little barrel weighed about 120 pounds. There was no way I would be able to carry these barrels up the ladder.

Bob came to the rescue with a clear plastic hose and a gadget with spinning rollers that could squeeze the hose and force liquid to move through it. But most importantly, he gave me a quick lesson on how to operate the overhead bridge crane and how to use nylon lifting straps in creative ways. By the end of the day, the first tank was filled.

The first tests of the first Cerenkov tank with the real electron beam were disappointing. We couldn't reliably distinguish the signal of a single electron shower from the combined signal of two or more other particles, even if they were widely separated in the tank. When we removed the optical shroud cover from the back and peeked into one of the portholes, we immediately realized what had happened. Instead of the crystal clear liquid, all we could see were millions of time white flecks suspended in a milky white fog.

With fresh resolve, we cleaned the tanks, abandoned the paint idea, and installed shiny stainless steel baffles in the tanks, dividing each tank into fifteen optically isolated compartments, each viewed by one phototube. This arrangement gave us a much cleaner trigger signal, and it allowed us to remove the tubes closest to the beam from the trigger logic, which further reduced the rate of spurious triggers and allowed us to collect more of the interesting higher $\mathrm{Q}^{2}$ events.

During the summer while all this was happening, we started assembling the scintillation counters, using salvaged materials Bob had found in the basement of Newman Lab. When the time came to attach the plastic light pipes and phototubes to the rectangular panels of plastic scintillator, Bob insisted the joints should be glued to ensure optimal 
light transmission. So he led me down to the mysterious subbasement, where there were gallon cans of Hysol epoxy and a triple-beam balance, just like I had used in high school, except this one was all sticky with spilled epoxy.

Under Bob's watchful eye, I carefully weighed out the proper amounts of resin and hardener into a large paper cup. Then, following his instructions, I stirred it very briskly to thoroughly mix the ingredients. With some dismay, I saw that the resulting syrupy mix was nearly opaque. It was full of tiny air bubbles.

"Can we use this stuff like this?” I asked. "Won't the bubbles absorb the light?” He grimaced; "What do you think?" Then with a grin, he said, "Watch this." He set the cup down on an aluminum plate on the table and positioned a glass bell jar over it. The bell jar was connected by a thick flexible hose to a mechanical vacuum pump on the floor. He flicked on a toggle switch, and the pump starting chugging away. Within a few seconds I could see the epoxy start to boil vigorously in the cup. Then quite suddenly, it stopped boiling. Bob turned off the pump, opened the vent valve, and removed the bell jar. The epoxy in the cup was sparkling clear, without a trace of any bubbles.

Bob grinned broadly and said, "Isn't that the cat's meow?" That was the first of many times I heard him use that phrase to express his delight whenever something worked out particularly well.

Bob's familiarity with the epoxy corner in the basement made me think he must have been up to something else involving epoxy. Back in the lab upstairs, I noticed various small saddle-shaped coils of copper wire, some encased in blocks of epoxy. He had been trying to make corrector magnets to fit around the beam pipe of the synchrotron, hoping to improve the beam quality. This was an omen. His interests were already beginning to shift toward the accelerator.

By the time the streamer chamber and magnet arrived from Hamburg, we had an electron beam and a set of trigger counters ready in the $\mathrm{L} 0$ experimental hall. The experiment became known as DECO, a contraction of DESY and Cornell, and it produced a series of new results on quark fragmentation functions, charge retention by forward hadrons, and inclusive rho meson production.

By the end of 1977, the CESR storage ring project was getting started, and DECO had to be dismantled to make way for the CLEO detector. This marked the end of the era of fixed target experiments at the Wilson Synchrotron Lab. By all measures, DECO was a great success. Bob was promoted to Associate Professor, and eventually, I finished a Ph.D. thesis. It was a wonderful time, for which I will always be grateful. 


\title{
Bob's Evolution from Particle Physics to Accelerator Physics
}

\author{
Richard Talman \\ Cornell Laboratory of Elementary-Particle Physics \\ July 7, 2009, Bob Siemann Symposium
}


NUCLEAR INSTRUMENTS AND METHODS II3 (I973) 489-507; (C) NORTH-HOLLAND PUBLISHING CO.

\title{
A POLARIZED PHOTON BEAM PRODUCED BY COHERENT PAIR PRODUCTION IN ORIENTED GRAPHITE*
}

\author{
R. L. EISELE, D. J. SHERDEN, R. H. SIEMANN ${ }^{\dagger}$, C. K. SINCLAIR \\ Stanford Linear Accelerator Center, Stanford University, Stanford, Calif. 94305, U.S.A. \\ D. J. QUINN, J. P. RUTHERFOORD, and M. A. SHUPE \\ Tufts University, Medford, Mass. 02155, U.S.A.
}

Received 18 April 1973

\begin{abstract}
Attenuation by coherent pair production in highly oriented, compression annealed, pyrolytic graphite has been used to polarize a $16-\mathrm{GeV}$ bremsstrahlung beam. The polarizer consists of $61 \mathrm{~cm}$ of graphite crystals, whose reciprocal lattice vectors are oriented at $10.5 \mathrm{mrad}$ to the normal to the beam direction, and can be rotated by $90^{\circ}$ about the beam line to rotate the
\end{abstract}

plane of polarization. A functionally identical assembly of length $30.5 \mathrm{~cm}$ was used as an analyzer to measure the polarization of the beam with the SLAC pair spectrometer. The beam produced intensities greater than $4 \times 10^{8}$ equivalent quanta per beam pulse and had a measured polarization of $0.255 \pm 0.020$. 
PHYSICAL REVIEW D

\title{
Study of charged-pseudoscalar-meson photoproduction from hydrogen and deuterium with 16-GeV linearly polarized photons
}

\author{
D. J. Quinn,* J. P. Rutherfoord, ${ }^{\dagger}$ and M. A. Shupe ${ }^{\ddagger}$ \\ Tufts University, Medford, Massachusetts 02155 \\ D. J. Sherden, R. H. Siemann, ${ }^{\S}$ and C. K. Sinclair \\ Stanford Linear Accelerator Center, Stanford. University, Stanford, California 94305 \\ (Received 23 April 1979)
}

\begin{abstract}
The asymmetries in forward $\pi-N, \pi-\Delta$, and $K^{+}-(\Lambda+\Sigma)$ photoproduction have been measured with a 16$\mathrm{GeV}$ linearly polarized beam. The experimental method and the procedures for extracting cross sections and asymmetries from the data are discussed in detail. Information on the energy and momentum-transfer dependence of cross sections for natural- and unnatural-parity exchange, interference between exchanges of opposite $G$ parity, and vector-meson dominance is obtained and discussed.
\end{abstract}




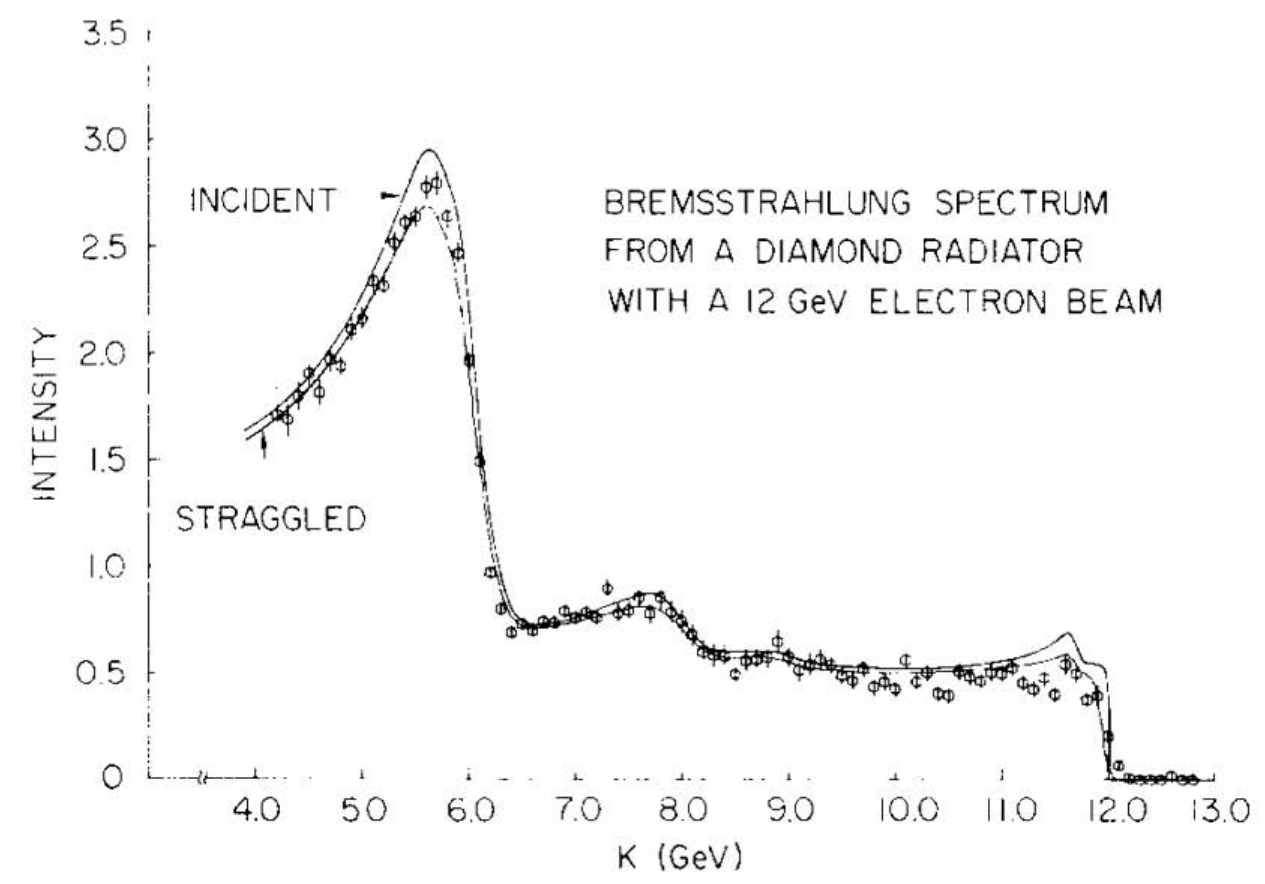

Fig. 1. A measured coherent bremsstrahlung spectrum from a diamond radiator. See ref. 4 for an explanation of the curves. 


\section{Introduction}

It is a measure of success to be recognized unambiguously by first name alone, especially because this honor is bestowed democratically. With a name like Barak or Kobe or Pief it is not so surprising that no ambiguity arises. For that matter, how many people with the name "Persis" do you know. But with a name like "Bob" it is harder to be recognizable by first name alone. When one is reminiscing about old times at Cornell mention of "Bob" gets the response, "Do you mean Bob Wilson or Bob Siemann"?. Certainly both Bobs are warmly remembered at Cornell. 
Bob received his $\mathrm{PhD}$ from Cornell in 1969 measuring wide angle bremsstrahlung with Karl Berkelman. After a stint as Research Associate at SLAC, Bob returned to Cornell in 1973 as assistant (later full) Professor. During the SLAC period Bob was beginning to evolve from elementary particle physicist to accelerator physicist. Perhaps surprisingly (since I was at Cornell) this is the period during which I worked most closely with him, and it is the period to which this remembrance will be limited.

This was a time when particle physicists were still optimistic that sufficiently accurate and detailed measurement of meson scattering and production processes could unlock the secrets of the strong nuclear interaction. Vector mesons had recently been discovered and vector meson dominance was being investigated. More significantly, the quark model had just been introduced, and the organization of mesons and baryons into SU3 families had just been understood. 
I will restrict myself to a solitary thread through Bob's research during this period-Polarization Dependence of Meson Photoproduction. Furthermore I will refer to just the two papers I showed initially. The first paper describes the development of a polarized photon beam at SLAC. It contains no elementary particle results. The second paper, written some years later with very nearly the same authors, summarizes important results obtained with the polarized beam.

Since I wish to concentrate on the instrumentation paper, let me dispense with the second paper right away. You will have to take my word for it that it was an excellent and significant paper reviewing results that could not have been obtained without the beam described in the first paper. Significant comparisons of the data with theoretical models were also given.

I will discuss only the beam line itself. Of course many scientists were involved in the series of advances to be described here. I assume you are more interested in the general flow of the field of research and in the people involved, than in the technical details. I will just drop the names of the leading characters (as it happens, four of them would later become lab directors) along with just enough description of their contributions to support a continuous, somewhat anecdotal, narrative. An analogy to American football may be apt. Moving the ball up the field was the work of many. Bob Siemann carried the ball over the goal line.

As well as honoring Bob I hope also to make physicists jealous of an era in which amazing and varied advances could be made on a time scale of months, not years. 


\section{Preliminary Answers to Some Natural Questions}

Before starting this narrative, let me anticipate some questions that can serve as themes for my talk:

- Why did Bob leave Cornell?

- Why did SLAC recruit Bob?

- Why did Bob convert from particle physics to accelerator physics?

- What did Bob accomplish? 
The first question is easy. Bob thought he could accomplish more at SLAC than at Cornell.

Why SLAC recruited him I can only conjecture, but the accomplishments I intend to describe in this talk surely were important or, I venture to guess, even dominant.

Concerning Bob's evolution in accelerator physics, in spite of my title, in preparing this talk, I have come to realize that, in fact, Bob never really changed in any important sense. Explaining this will be one of the themes of my talk.

Finally the question "What did Bob accomplish?" I will give my own answer to this even though it carries me way beyond the time interval I have been assigned. My answer is that he accomplished two and a half things. To protect myself from the charges of being both too stingy and too flippant, I have to explain my scoring system. I am using the word "accomplish" in the strictest possible sense. Rephrasing the question it becomes "What things would never have happened if Bob had not accomplished them?"

One of these things is obvious - the on-line Phys. Rev. journal. For this Bob gets one point on my accomplishment scale. I leave it to others to say more about this accomplishment. Next, harsh grader that I am, for leading the development of novel acceleration techniques, Bob gets half a point. I am certain Bob would have agreed with this grade, especially after I have explained my grading system. This will be another theme of my talk.

Finally, the actual subject of my talk is justifying the remaining one point that brings Bob's score up to two and a half. 


\section{Early Polarized Photon Measurements}

GeV-scale photons are produced by bremsstrahlung from material targets. In 1956, Uberall showed that, by using an appropriately-oriented single crystal as the target, the radiation would have quite high polarization, especially close to a "resonance edge" in the energy spectrum. I showed this spectrum at the start of the talk. By 1968, workers at Frascati, especially Diambrini, had demonstrated the feasability of this method.

By 1970, Richter and Schwitters (I could just as well have said Burt and Roy) and others (including Bob) had measured polarization dependence of pi+ photoproduction using a beam polarized by the Uberall process. Their method of measurement used a subtraction procedure relying on the edge in the energy spectrum mentioned earlier. Back then the data for this technique was referred to as an "excitation curve". Nowadays it would be referred to as an "inclusive" measurement. This technique was adequate for the early measurements, but one feature was found to be disadvantageous. The edge near which the high polarization exists lies well below the upper end point of the energy spectrum. Photons above the edge gave background counts that tended to swamp the foreground signal. 
By that time rho meson photoproduction from various elements had been measured at Cornell by Mistry, Silverman, me, and others. In 1970, Diambrini spent the year at Cornell, bringing with him the goniometer needed to produce polarized photons from an oriented diamond target. Soon we had measured the polarization dependence of rho photoproduction and found it to be maximal - the rho (vector) mesons "remember" the photon polarization and the plane defined by the pions into which the rho decays remembers the rho polarization.

Diambrini also called our attention to a paper by Cabibbo and others, which described an alternative method for polarizing a photon beam. (Cabibbo has the even greater honor of being identifiable by just his initial - he is the Cabibbo of CKM matrix fame.) For the Cabibbo process the photon beam passes through a thick, properly-oriented single crystal. Photons polarized in one transverse plane are preferentially absorbed by pair production. As with visible light passing through a polaroid film, the emerging radiation is polarized.

Relying on differential absorption, there is an important trade-off between intensity and degree of polarization. But the essential advantage is that the high energy end of the spectrum is polarized in this process. 
During 1970 I was on sabbatic leave in the SLAC group of Dave Ritson (who was himself on leave somewhere else). Bjorn Wiik, Dave Gustavson, and I contemplated the possibility of one-upping the Richter group by using the Cabibbo process to overcome the problems imposed by the Uberall process. For inclusive measurements like this, the low degree of polarization disadvantage is completely out-weighed by the advantage of being able to polarize the high energy end of the spectrum.

But we were not expert in crystallography. We knew that Roy Schwitters had developed an elegant code for calculating the Uberall production process being used in the Richter beam line. From QED, we understood the close connection between bremsstrahlung and electron pair production, and assumed that Roy's code could be adapted for our purposes. We approached Roy about using his code (without, I now confess, explaining how Gustavson intended to modify it) and Roy graciously approved. 
By that time we were confident enough to submit a proposal to use the rho photoproduction process, mentioned earlier, as an analyzer to confirm the applicability of the Cabibbo process and to measure the degree of polarization achievable. McDaniel, the Cornell lab director, had assigned us a few weeks of running time at the Cornell Synchrotron, some months in the future. Such proposals were far less formal in those days than they are today, but it was still a serious commitment. As yet we had no appropriate crystalline target, but we assumed that we could quickly acquire and use a long single crystal of silicon.

Soon, to our horror, with the help of the newly modified code, we realized that the process was not going to work with silicon. The Debye temperature is too low. For that matter, the material proposed by Cabibbo, namely copper, would have failed for the same reason. 
There is nothing like the threat of hanging to concentrate a person's mind. After a few feverish days in the Stanford library I came to realize that carbon was the only practical material for the Cabibbo process. All we needed was a foot-long diamond. But we couldn't see any way of getting one.

Then I considered graphite and, to my amazement, found that carbon exists in crystalline graphite form. Nowadays this form is known as "graphene". Scarcely a week goes by without a seminar on its remarkable properties. At the time, based on its fabrication method, this form of carbon was referred to as "pyrolytic graphite".

A trip to meet Arthur Moore, of Union Carbide, in Cleveland, established that he had some graphene samples. They were shaped like inch-square counter-top tiles. They were a few millimeters thick, which was considerably thicker than the millimeter or so beam width. With care these tiles could be lined up to form the equivalent of a foot-long single crystal.

Unfortunately the tiles were too expensive for us to purchase. Fortunately, through Moore, Union Carbide graciously allowed us to borrow the material. (Later, when Bob Siemann built the serious polarized beam I will get to next, he purchased pyrolytic graphite from the same source - probably including some of the very same tiles.) 
With the crisis averted (and hardly anyone even aware it had ever occurred) we completed planning for the test of the Cabibbo process at Cornell. As I already explained, the azimuthal plane defined by the two pions into which a rho meson decays can be used to infer the polarization of the photon which produced the rho meson. This process can therefore be used as a polarization analyzer.

The Cornell measurements confirmed the (appropriatelyupdated) Cabibbo theory. Though the measurements were not very accurate, they were sufficiently promising to justify proceeding to the rest of the story. 


\section{Development of the Polarized Beam}

Everything up to this point could be called research. So far Bob has been an important actor, but not yet the star. To exploit this research for a usable accelerator facility required development.

Even more than being a futuristic thinker, Bob was an accomplished builder. While the rest of us went on to other things, he did the serious work needed for full implementation of the Cabibbo-polarized beam line. This work is described in the paper I am concentrating on.

If one insists that Bob evolved from particle physicist to accelerator physicist, then this paper may define when the transition occurred. If so then this would be Bob's first accelerator physics paper. 
Many physics and engineering tasks needed to be performed: construction, alignment, cooling, reliable control system, safety assurance, installation of the entire apparatus in a magnetic field to sweep the produced electrons and positrons out of the beam, and so on.

Before the beam line could be used for practical experiments, it was still necessary to determine the optimal crystal orientation, and to accurately measure the resultant degree of polarization. As mentioned already the crystal quality was fairly poor, and the polarization quite low. So empirical measurement was required.

As well as describing the polarization process, the original Cabibbo paper also described how the polarization could be measured, using a second, approximately identical, crystal. For this it was also necessary to measure the attenuation in both polarizer and analyzer in a subsidiary experiment using an electron-positron pair spectrometer.

All this is described in the paper.

As I already mentioned, the results obtained in this beam line are reviewed in the later paper. At the risk of offending other contributors, I think it would be fair to call this the "Bob Siemann beam line". The subsequent polarization measurements could not have been made without this beam line. 
Preliminary results using this beam line were first reported in 1973 at the Bonn Symposium on Electron and Photon Interactions at High Energies, and I had the good fortune to be the rapporteur for that session. Corny though it was, and wishing to explain the method, I asked the Bonn lecture room apparatus attendant whether he had the sort of oven-rack-like wire grid that one uses for lecture demonstration of microwave polarization. The process is the same - one polarization is absorbed, the other is not. The attendant proudly showed me, and allowed me to show, what he said was the very apparatus that Heinrich Hertz had used to demonstate the polarization of electromagnetic waves generated by sparks a century earlier. 


\section{Bob's Skill and Style}

The apparatus paper is a real tour de force of experimental physics. I have already outlined its content. This paper would be ideal for intensive study in a course on experimental physics. You will have to take my word for its excellence in technical content; but I will say a few words about its style.

I cannot be sure what fraction of the paper was in fact written by Bob. But, from my reading the whole paper "sounds like" Bob. To show what I mean let me read out a couple of paragraphs:

"The mounting of the carts was the only serious design flaw of the crystal assembly. Wear in the pivot sockets and at the points where the spring plungers exerted pressure caused the behavior of the pivots to deviate from that of the true pivots. Fortunately the problem was discovered before installation in the beam, and the pivot sockets were replaced with anodized sockets and the pressure points were reinforced with tool steel. Although not a permanent solution, it was an adequate temporary one. A captive pivot is contemplated as a permanent solution."

Rather than suppressing this episode, which many scientists would be tempted to do, after describing the problem in succinct detail, Bob gives both a temporary and a permanent fix; all in five sentences. 
Here is another example:

"During the experiment several of the noncritical functions of the controller failed due to radiation damage or water damage (which occurred as a result of the bursting on several occasions of radiation-damaged water hoses on the sweeping magnet). At several points during the experiment the symmetry measurement was repeated. These measurements showed that the polarization remained constant to within \pm 0.008 , indicating no evidence of deterioration in the polarizing power of the crystals."

I defy anyone to pack more information (even including a numerical error estimate) into a paragraph that would not be out of place a short story.

For Bob paragraphs like these amounted to telling the truth, the whole truth, and nothing but the truth. 


\section{Completing Bob's Accomplishment Score}

To conclude let me complete my tallying up of Bob's accomplishment score.

Success of his polarized beam could only be claimed after it had completed a significant measurement that could not be made any other way. In this he was gloriously successful. So, in my scoring system, Bob gets a full point for this effort. With the point for the online journal this brings his score to 2 .

Much of Bob's subsequent career has been devoted to the far more ambitious task of overcoming the $\mathrm{MeV}$ per meter barrier that prevents one from obtaining truly high energy electrons.

Many people would say that Bob lived long enough to have succeeded in this. But Bob would surely disagree. When he worked on apparatus his definition of success certainly included the requirement that the apparatus make possible physical measurements that would not otherwise be possible. But the advanced acceleration schemes he worked on have not yet met that test. I am certain, that Bob would claim, at most, that the advances have been promising. So I can only award him half a point for this accomplishment.

Finally, what about Bob's evolution from particle physics to accelerator physics? I hope I have convinced you that no such evolution ever needed to occur. From beginning to end Bob was an simply an experimental physicist par excellence. What he enjoyed most, and was best at, was building apparatus that would contribute to the advance of science. 


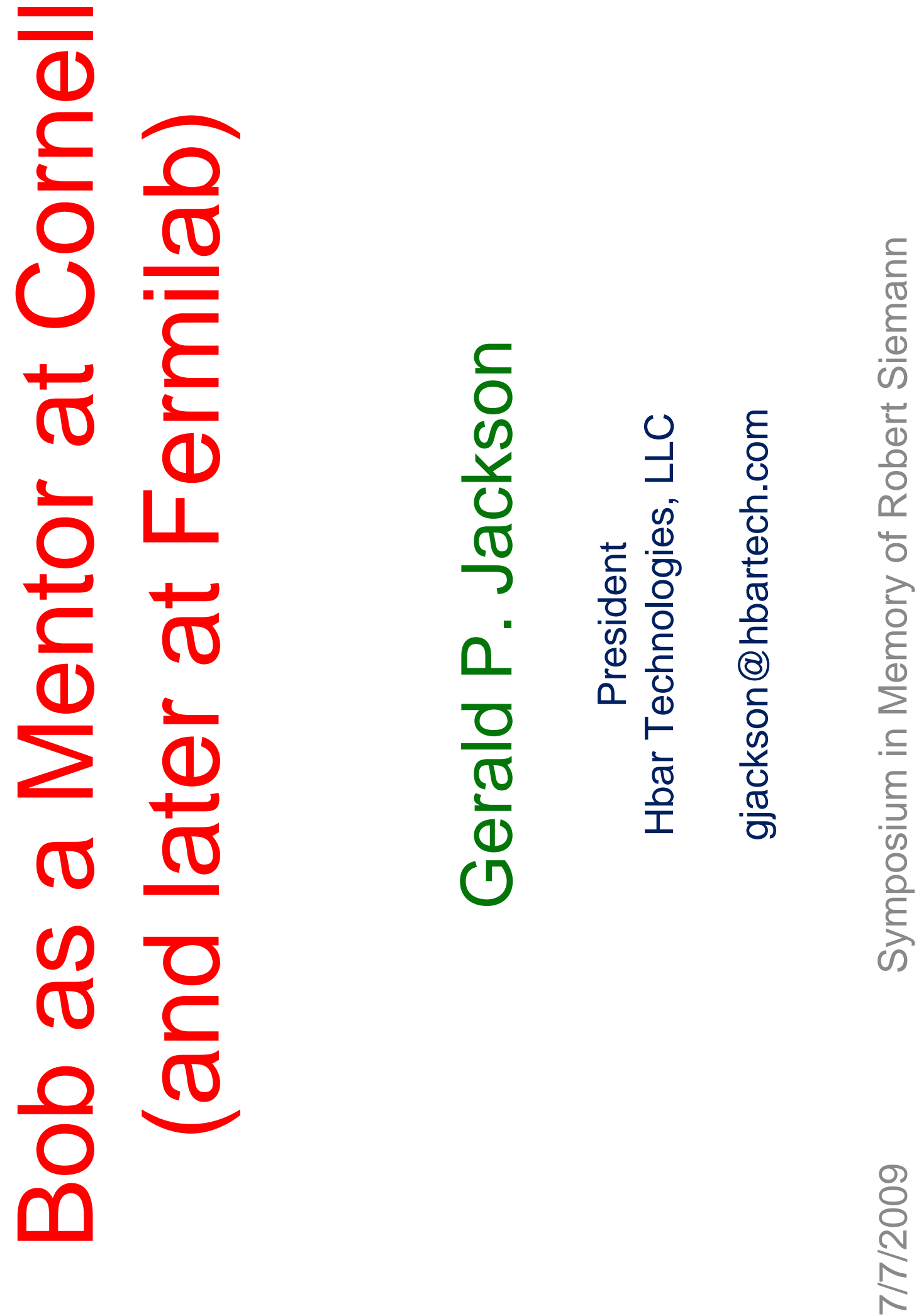




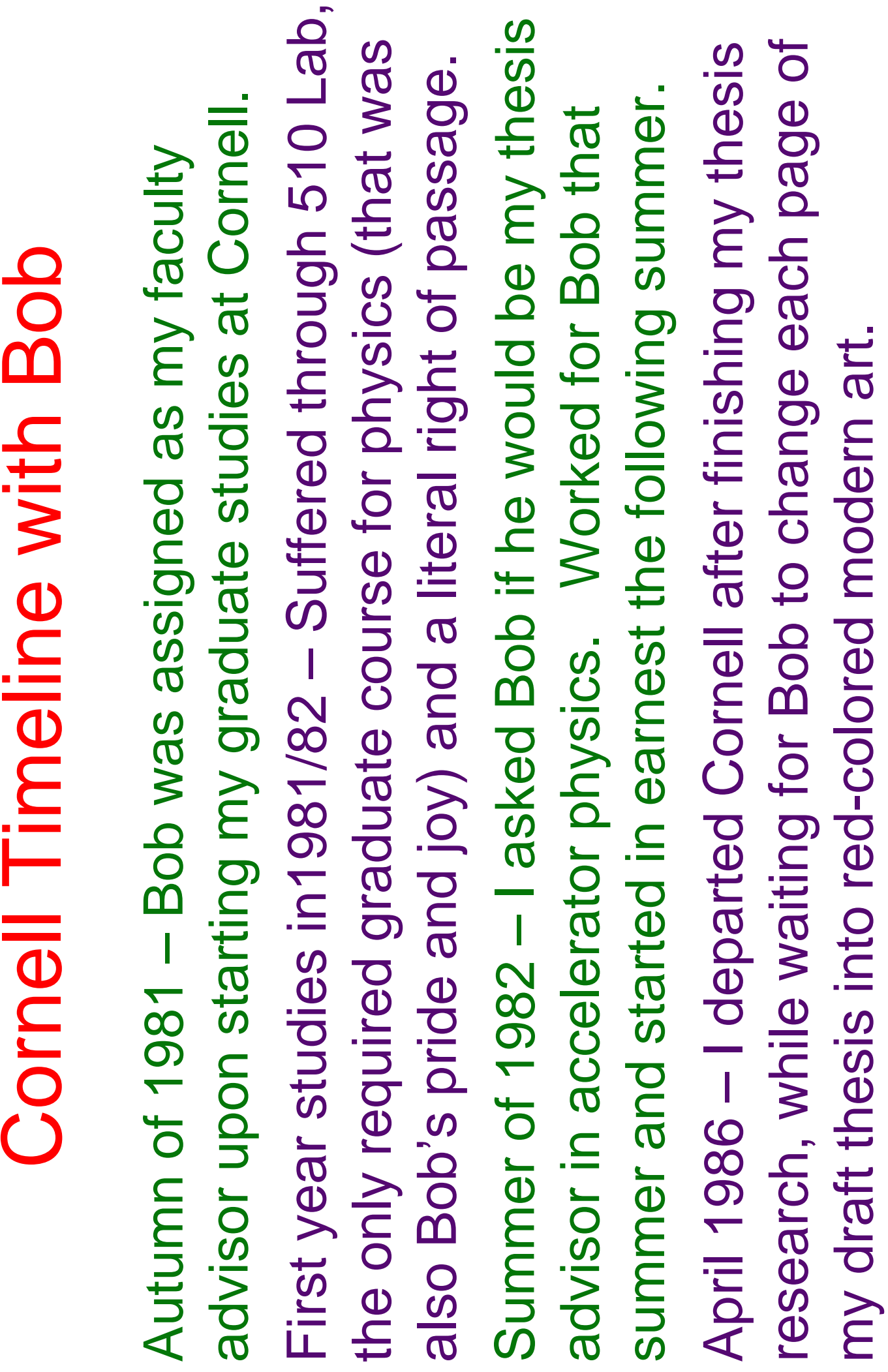




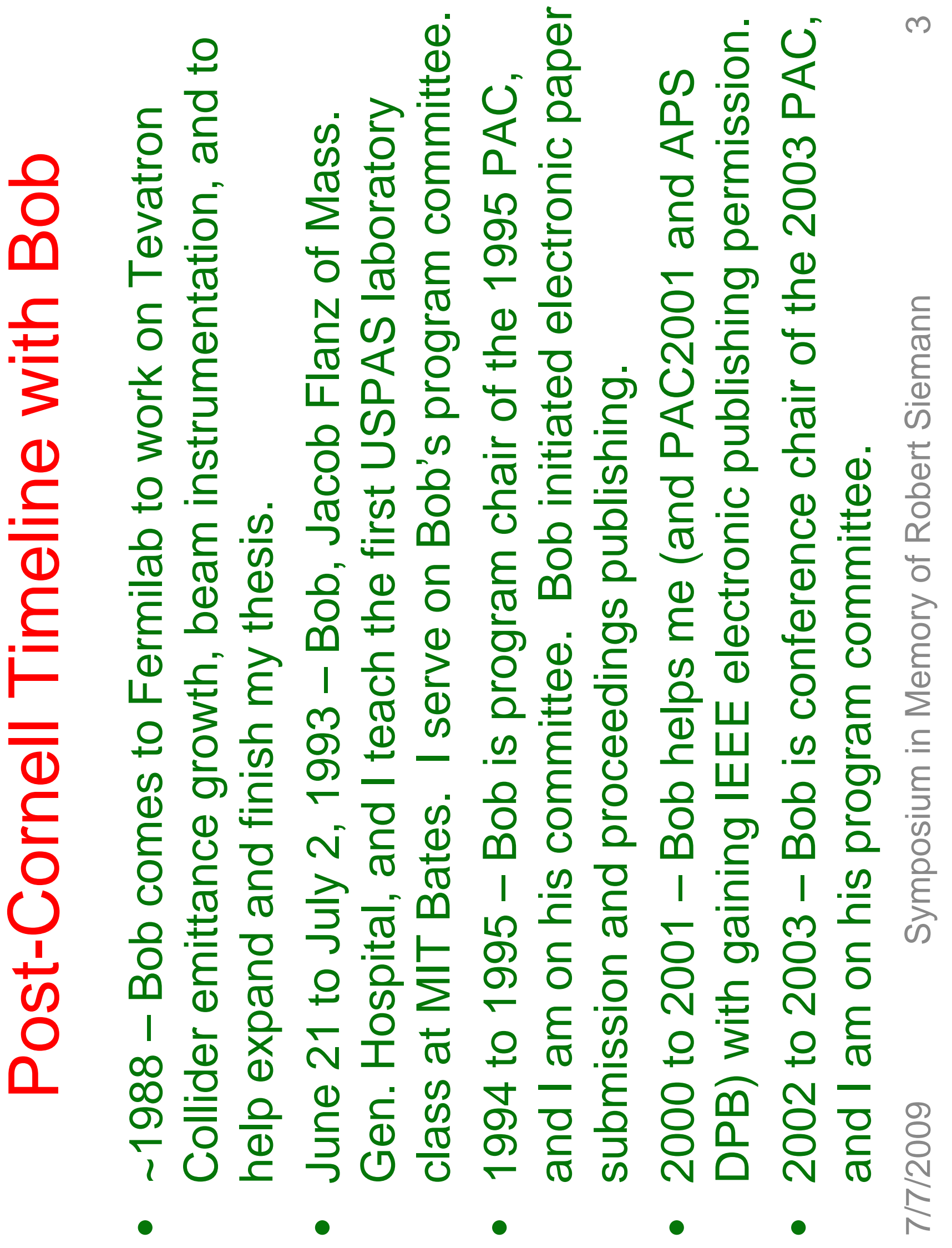


$\overline{1}$

ع

능

$\circlearrowleft$

ㅁํ 음

๑) 임

등

$\bar{\varnothing}$

б $\frac{0}{v}$

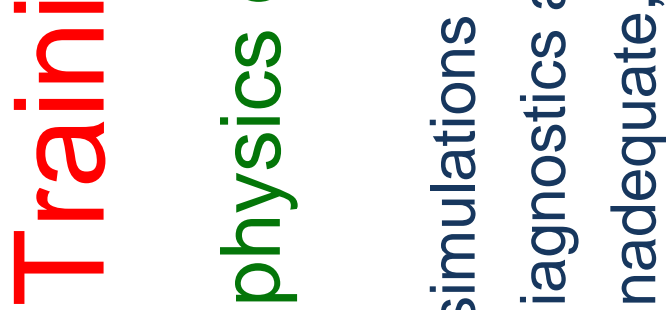

s

$\mathcal{C}$

$\sim \frac{\bar{d}}{\overline{0}}$

¿ $\quad$ U

Q

$\div \quad ㄴ$

O

正

(ర)

U $N$

.$\Xi$ ส

증

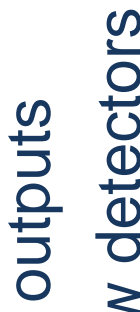

을

$\frac{\pi}{\frac{\pi}{7}} \frac{0}{\pi}$

है

os

$\stackrel{\Phi}{=}$

.

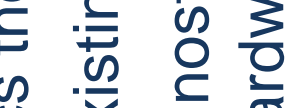

정

业

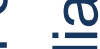

然

त्रे

음

듐

$\Phi$.

$\pm \bar{\omega} \frac{\pi}{\omega} \frac{\bar{D}}{\omega}$

(1)

0

$\longrightarrow$

$\sum_{0}^{0} \sum_{0}^{\infty}$

焉变离焉

Фᄒ

Х

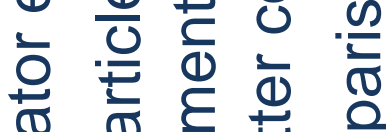

ब

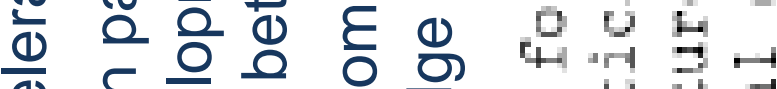

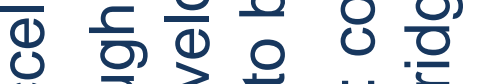

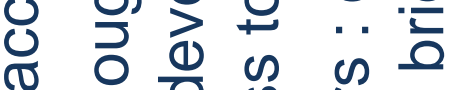

옹 훙

284

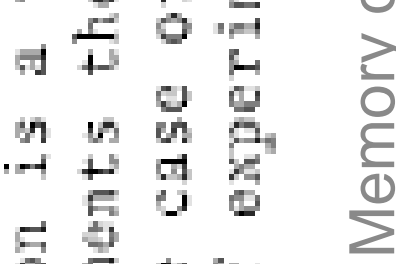

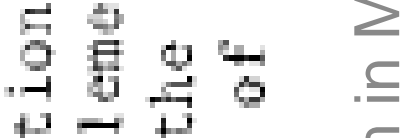

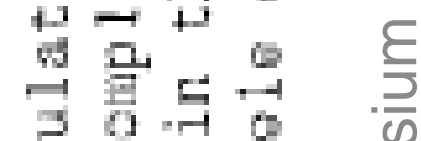

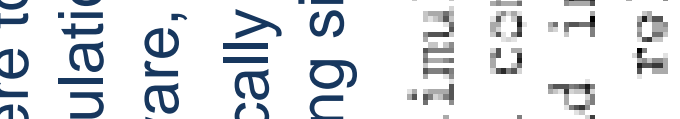

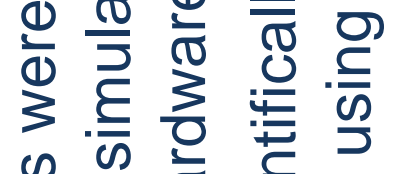

n)

量

更

$4 \frac{1}{3} y$

를

룰용.

에

$j-F$

- $\Rightarrow$ क

o $+c$

न 5

节 


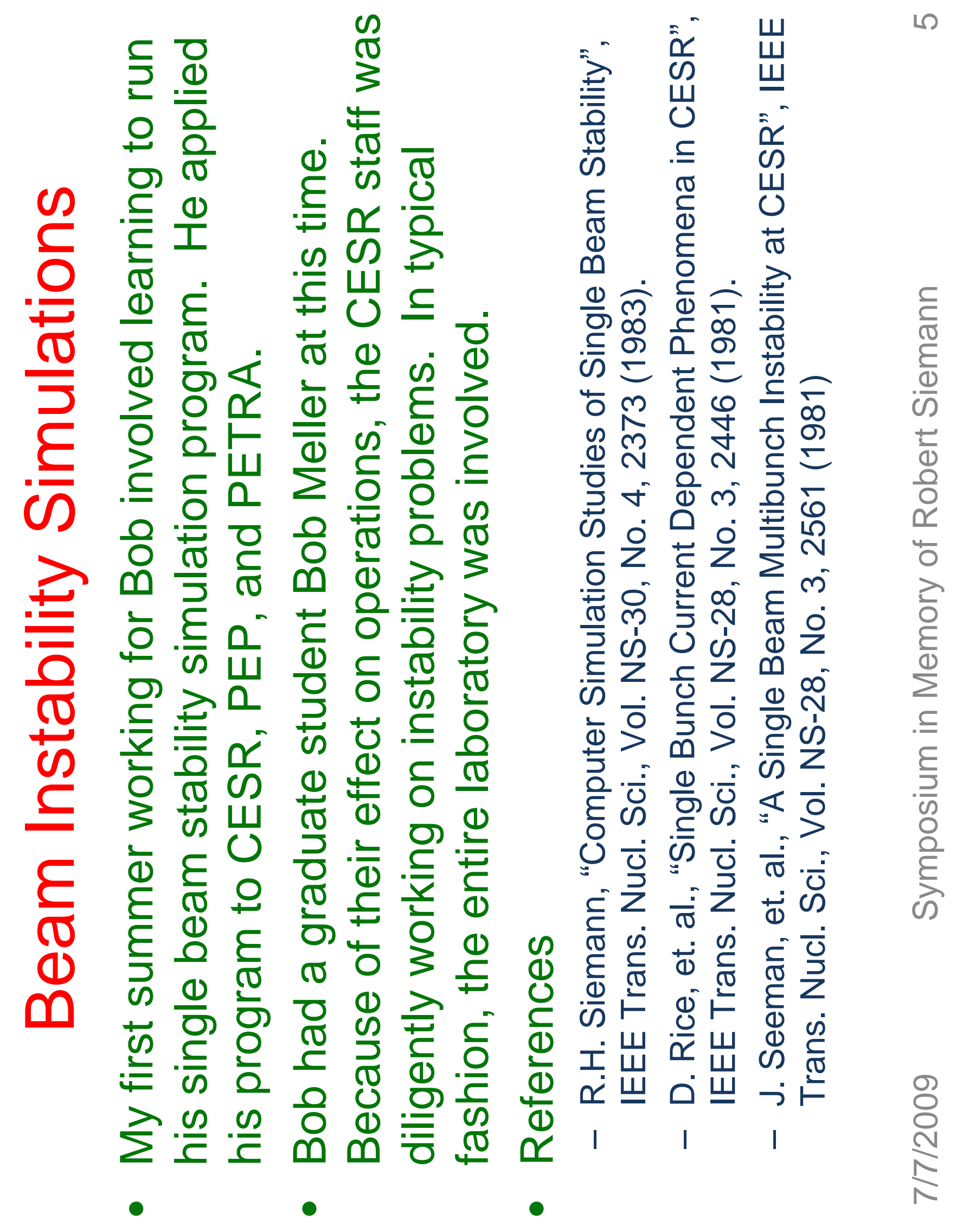



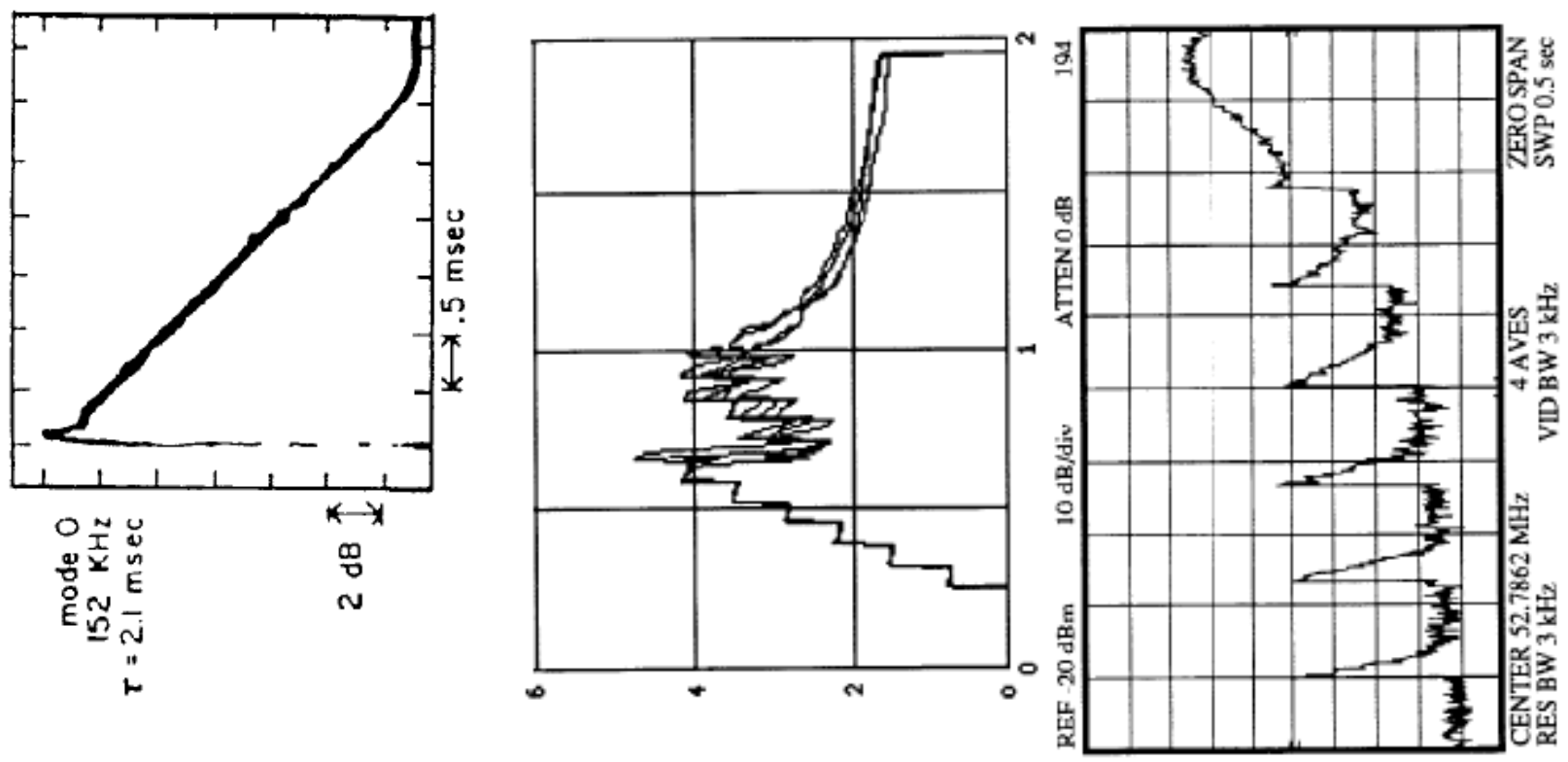

6

(s)

(1)

플

:

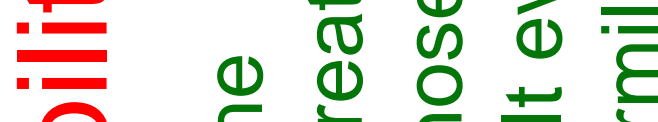

음

(⿻) 은

ज ส

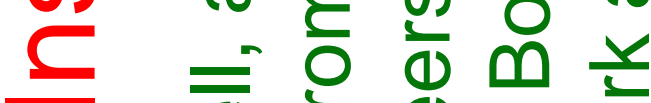

힌 Ф

○ 등

(1) 0 U

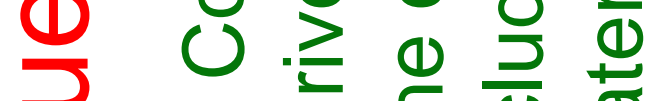

$\sqsupseteq+\overline{0} \cong \overline{0}$

당

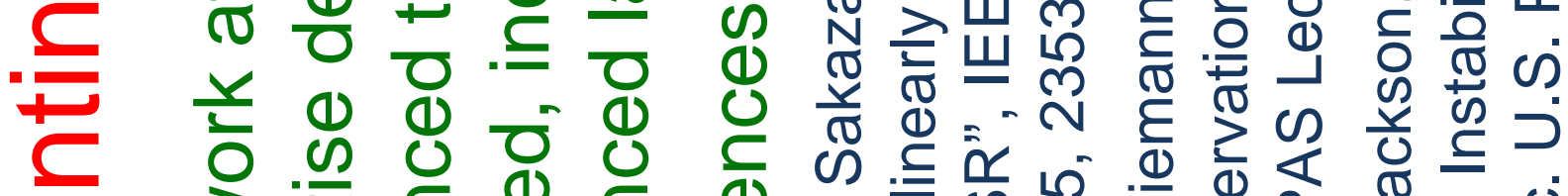

$\cong$ กิ

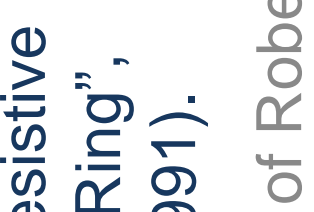

$=$ 舟

$\frac{\frac{5}{5}}{\frac{0}{0}}$

(1)

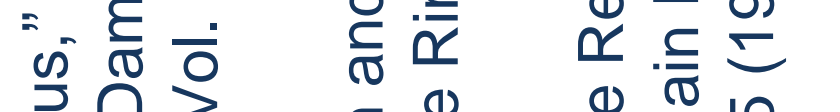
음 हैं

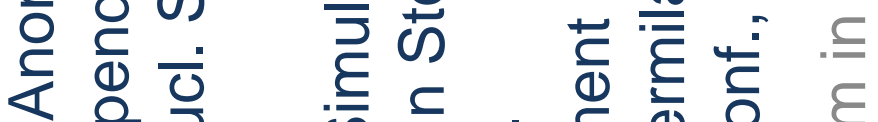

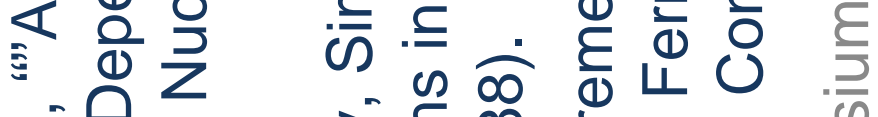
宁 \% ब

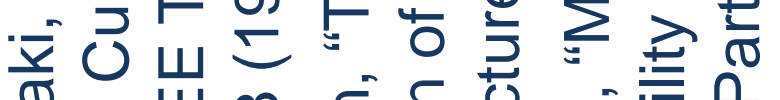

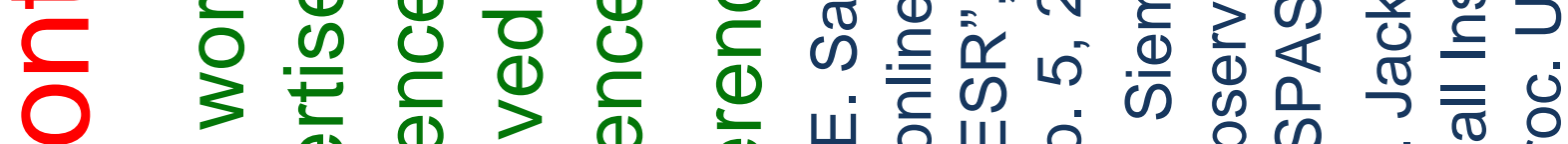

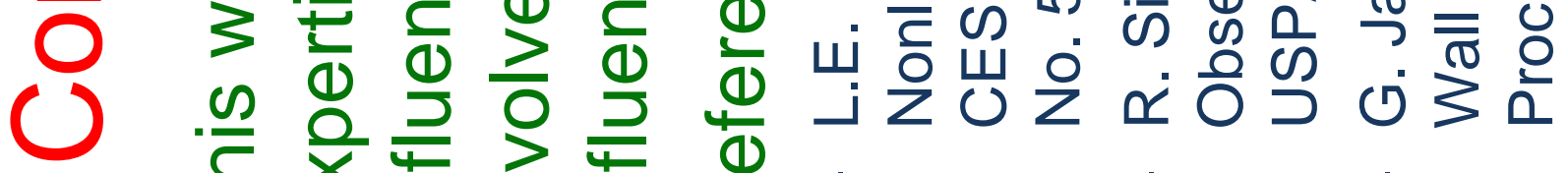

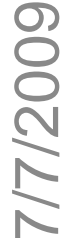




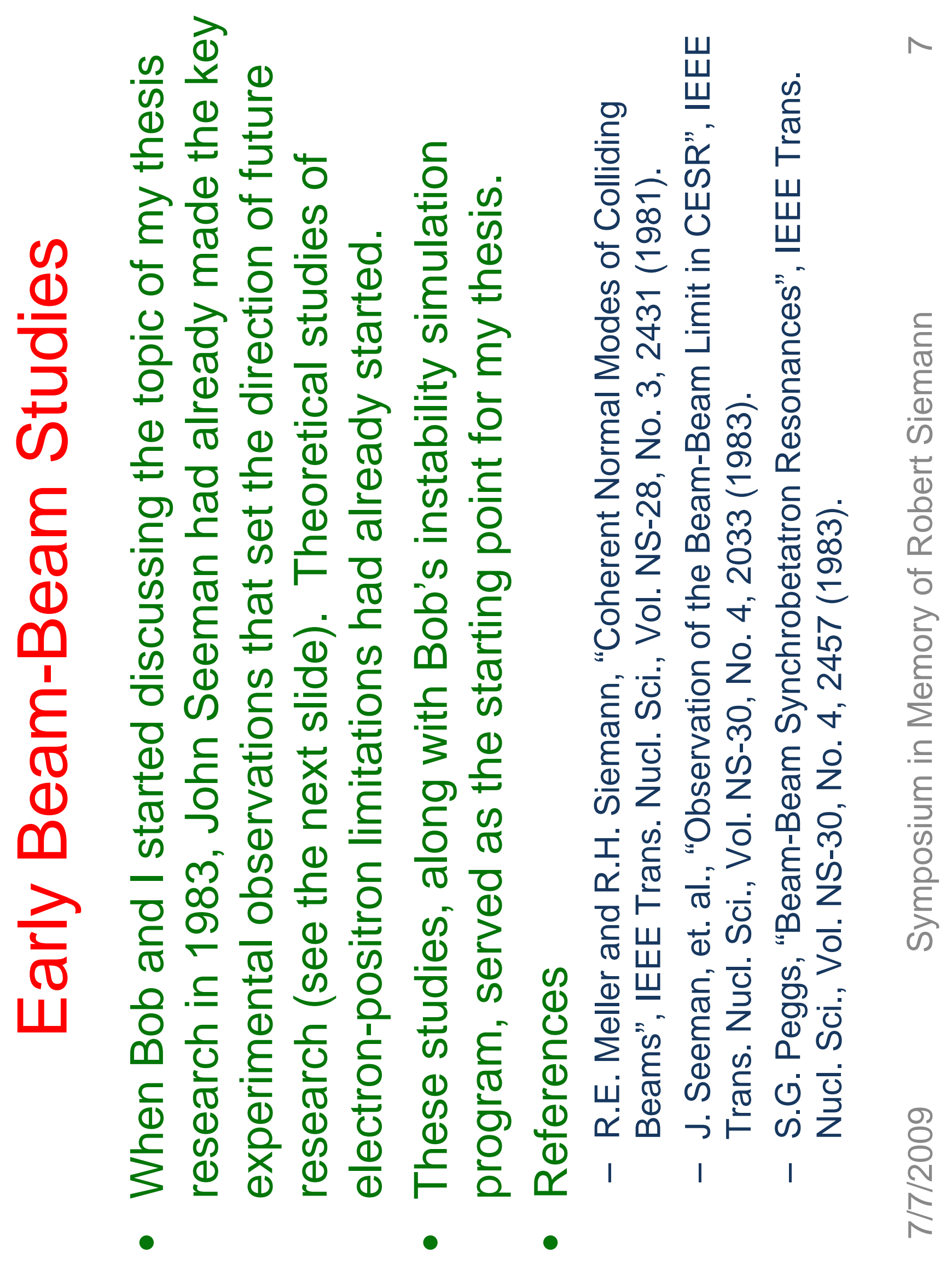




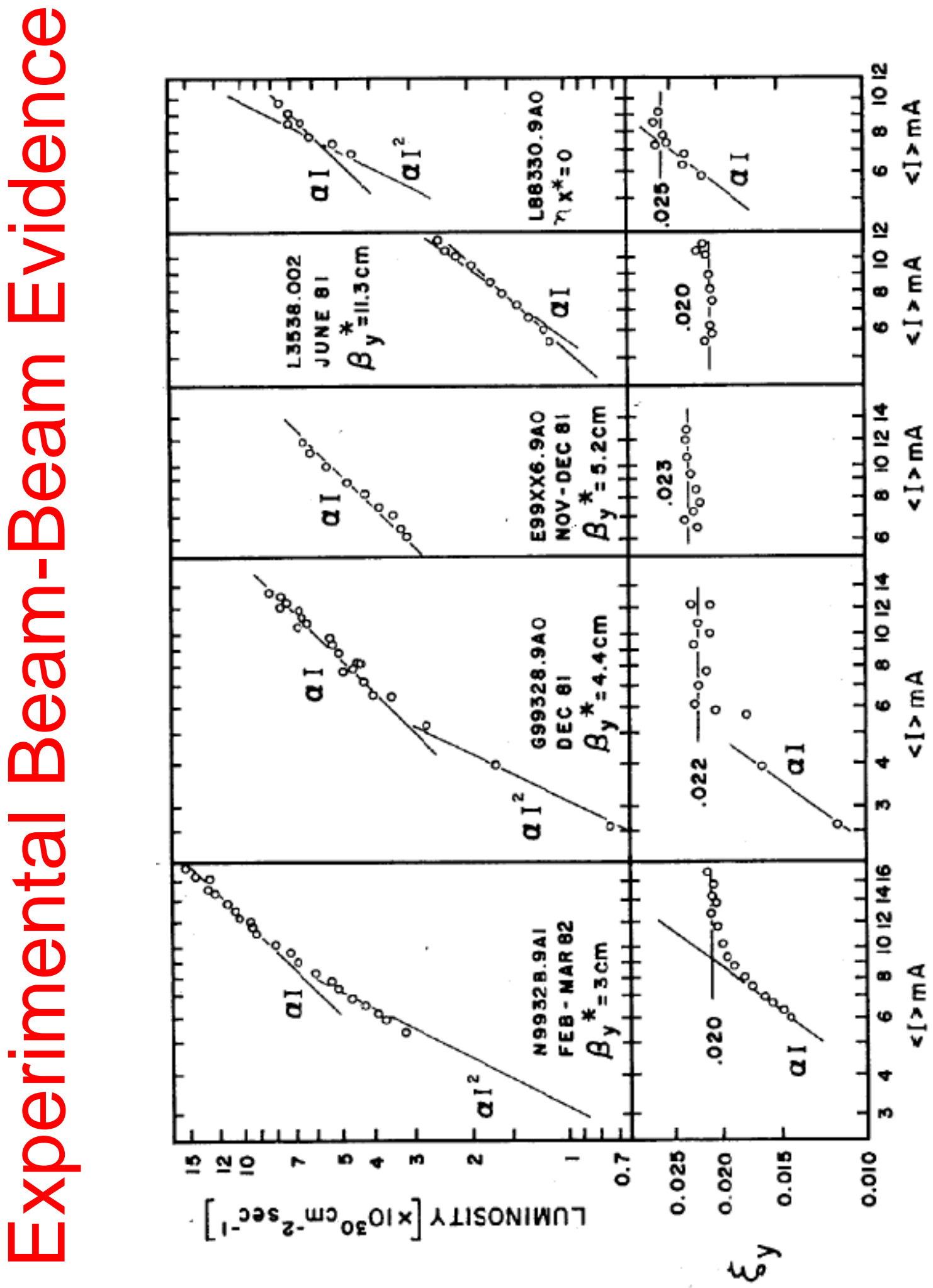




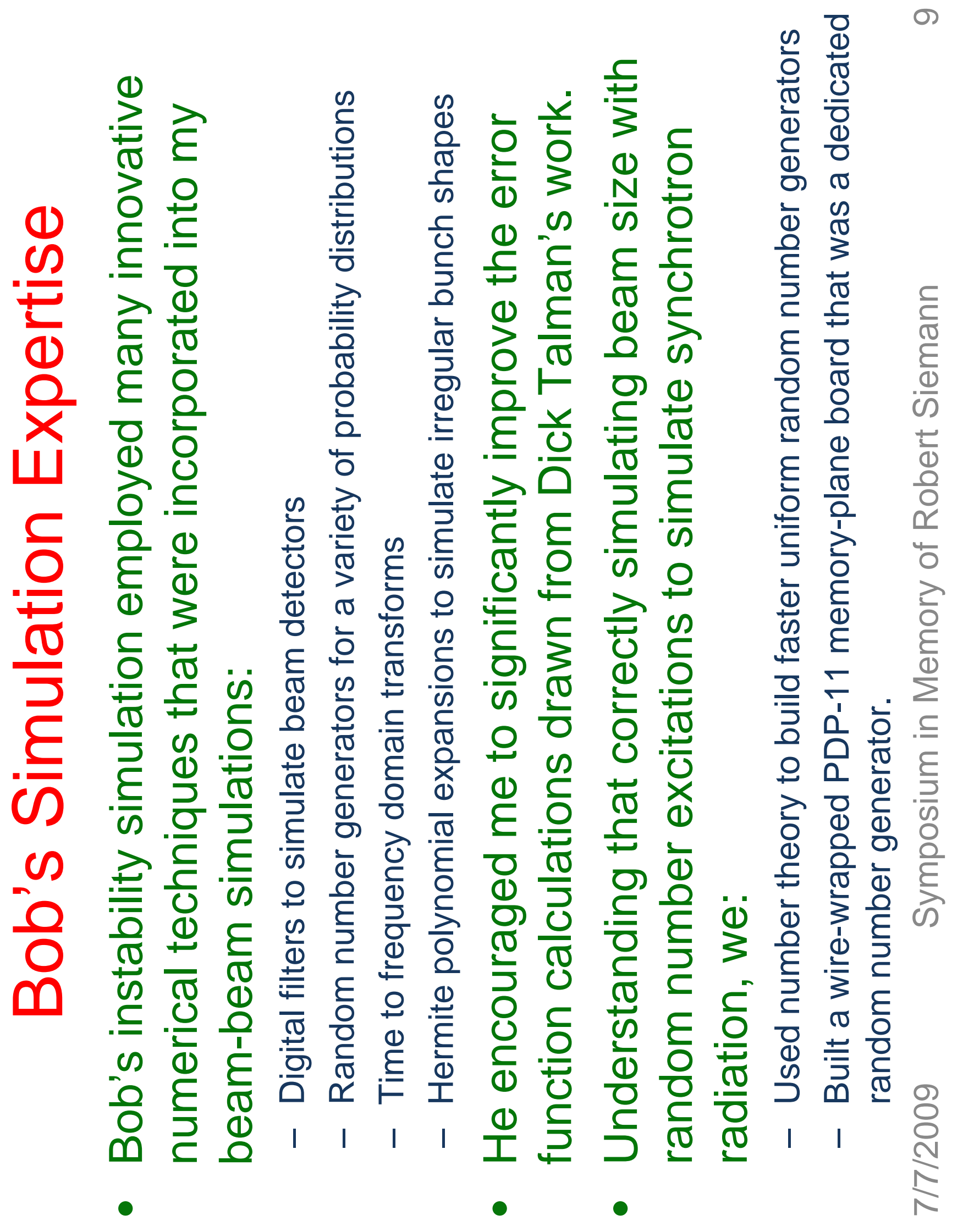



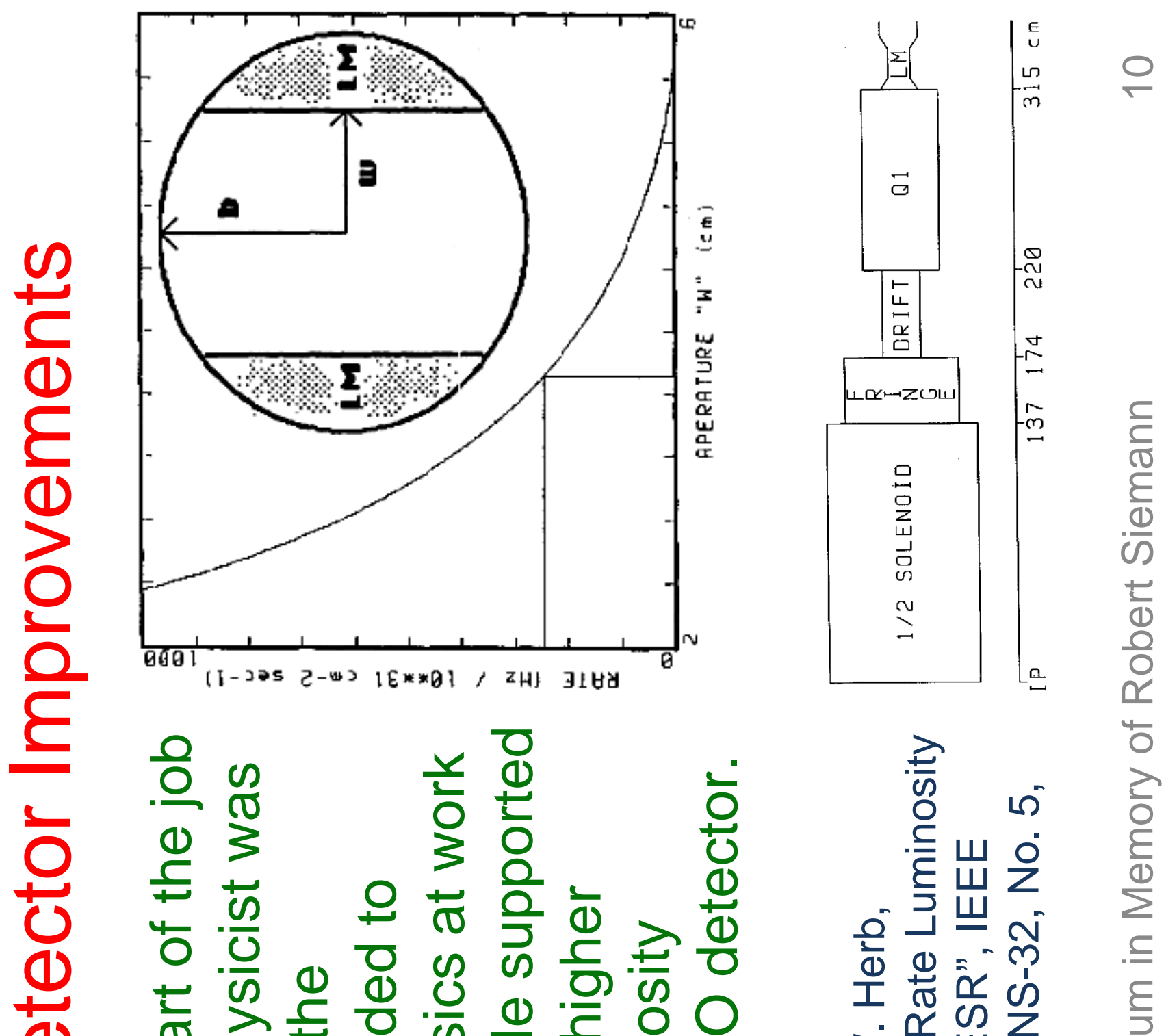

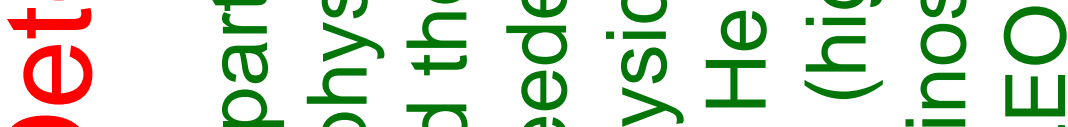

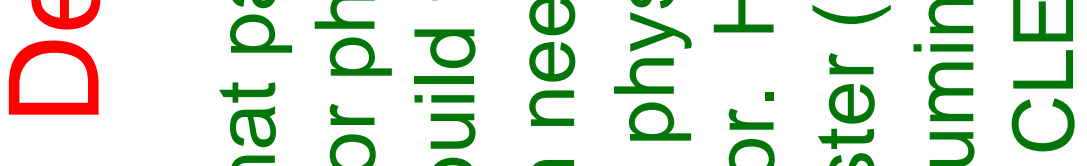

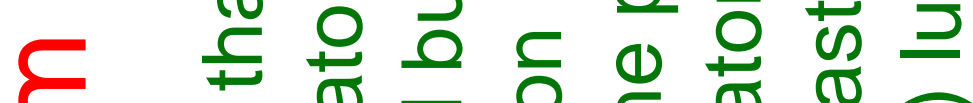

б

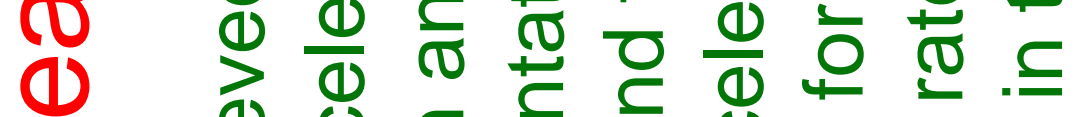

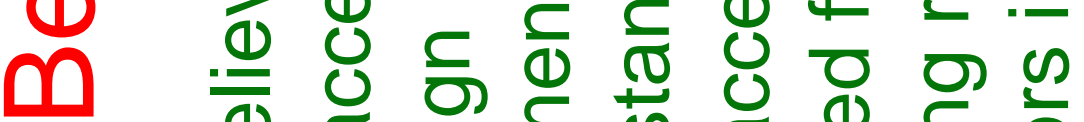

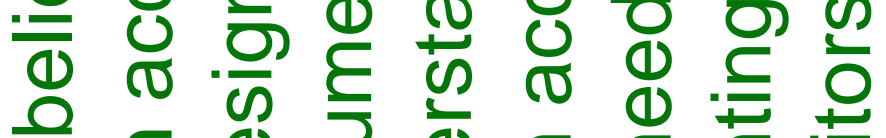

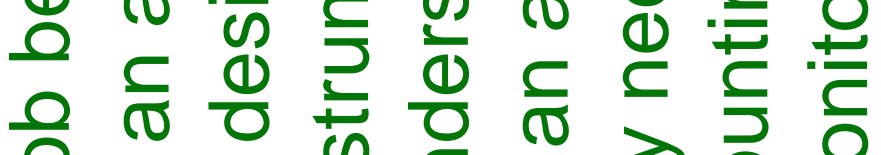

๑

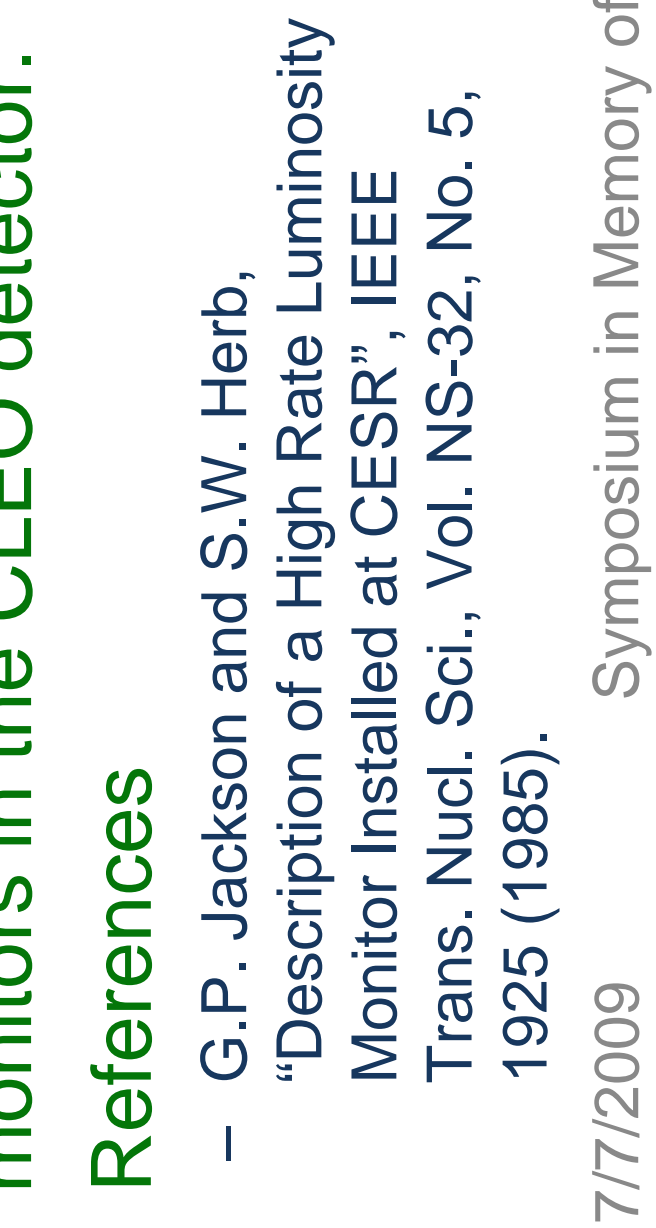




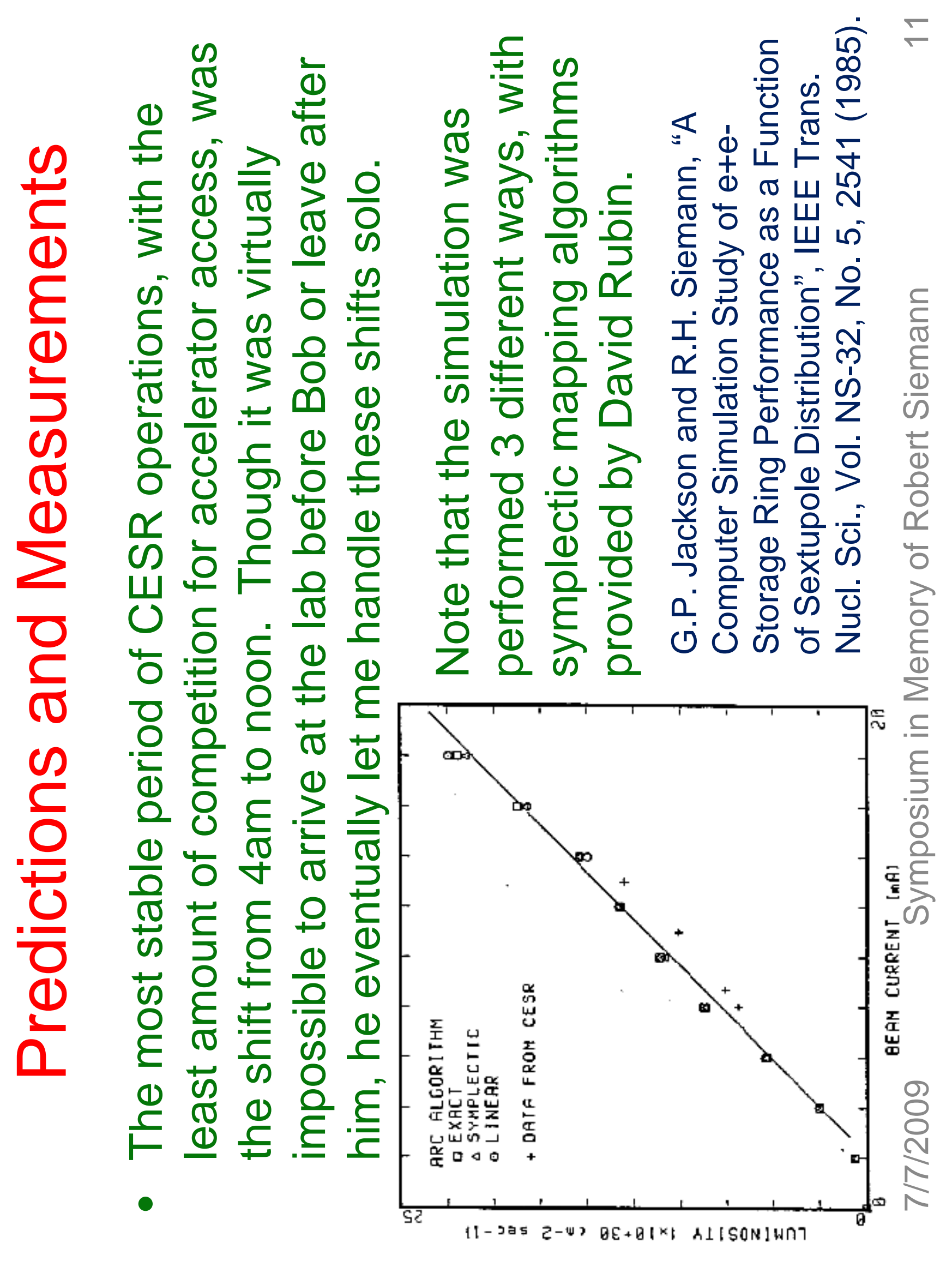




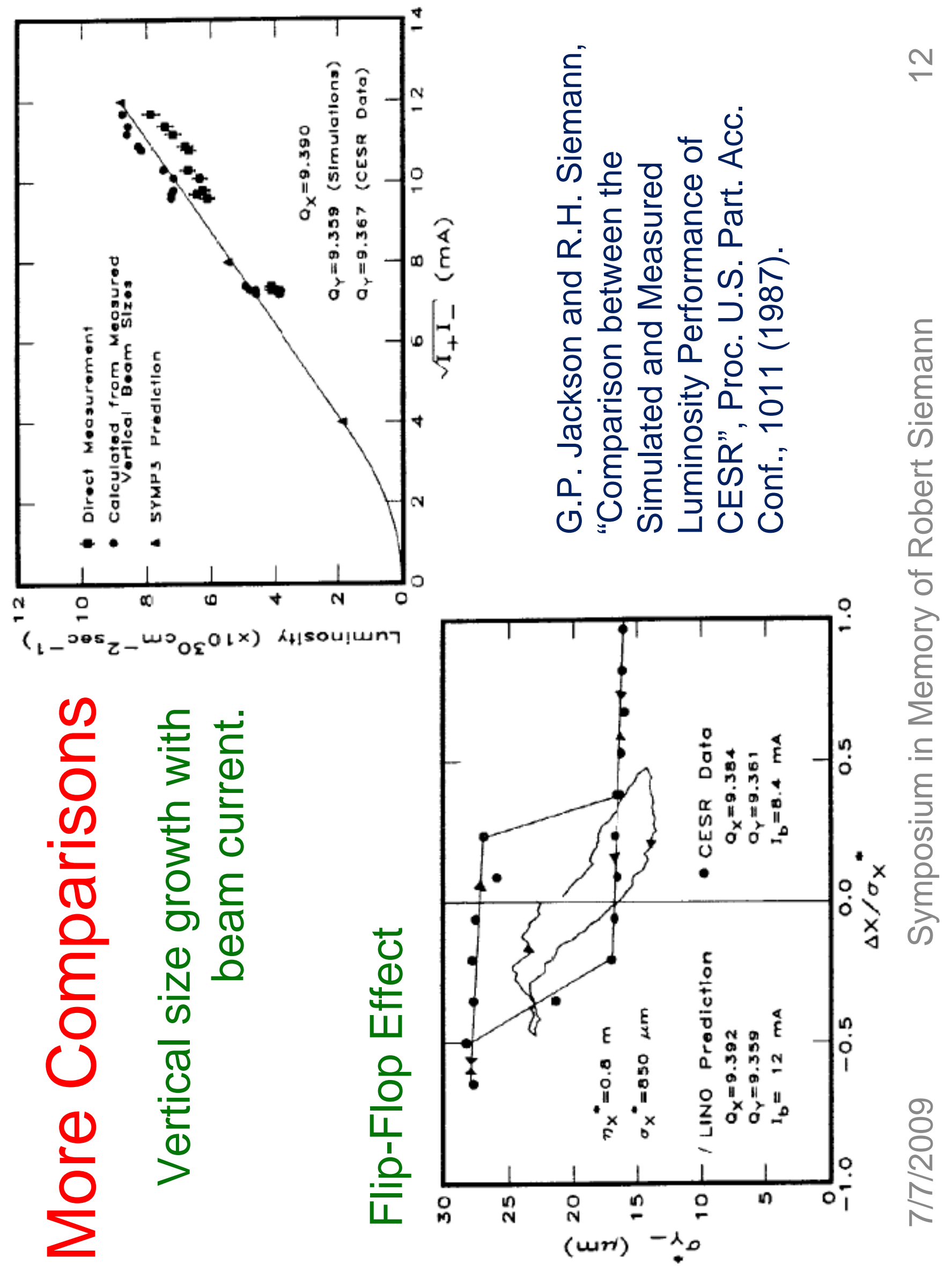




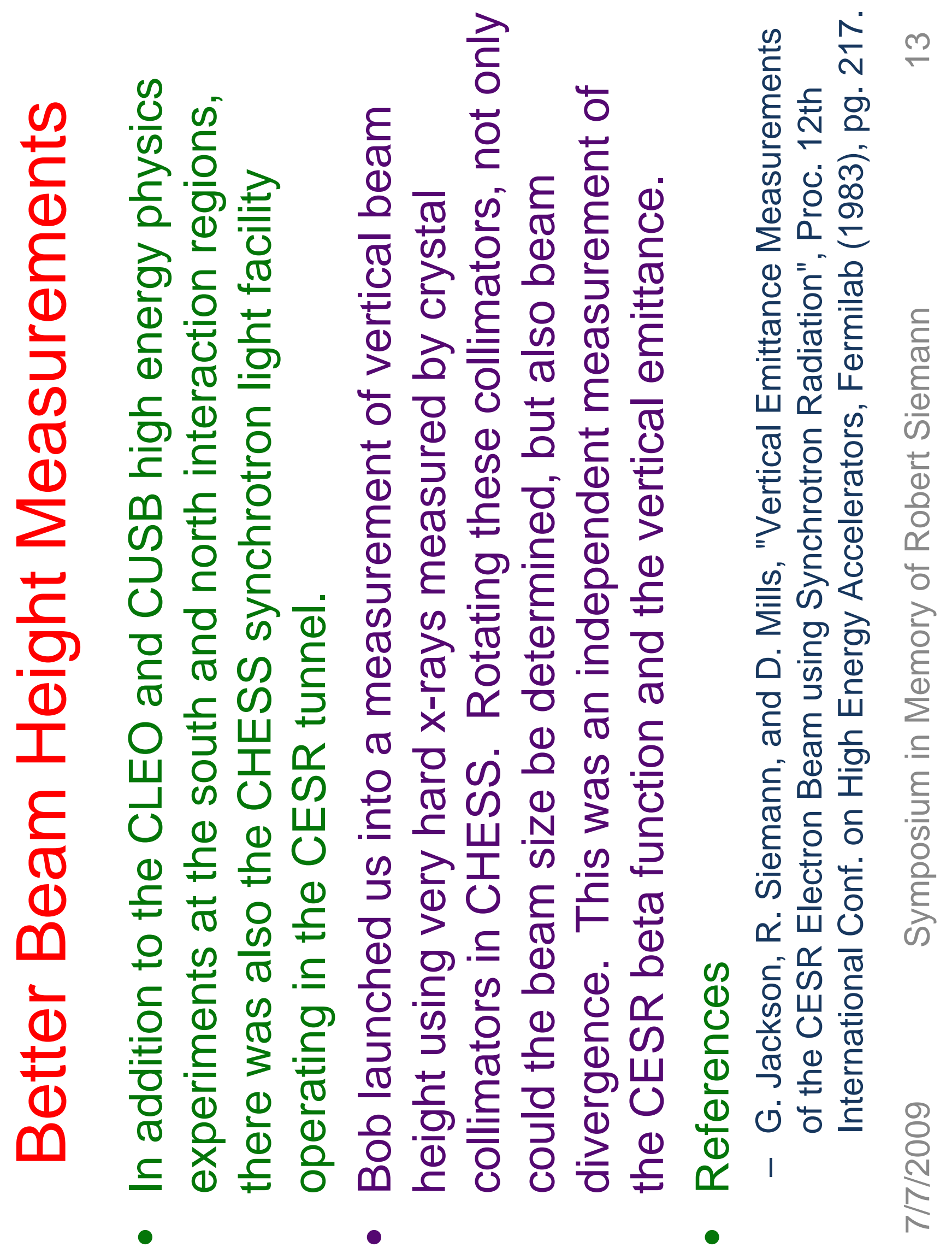




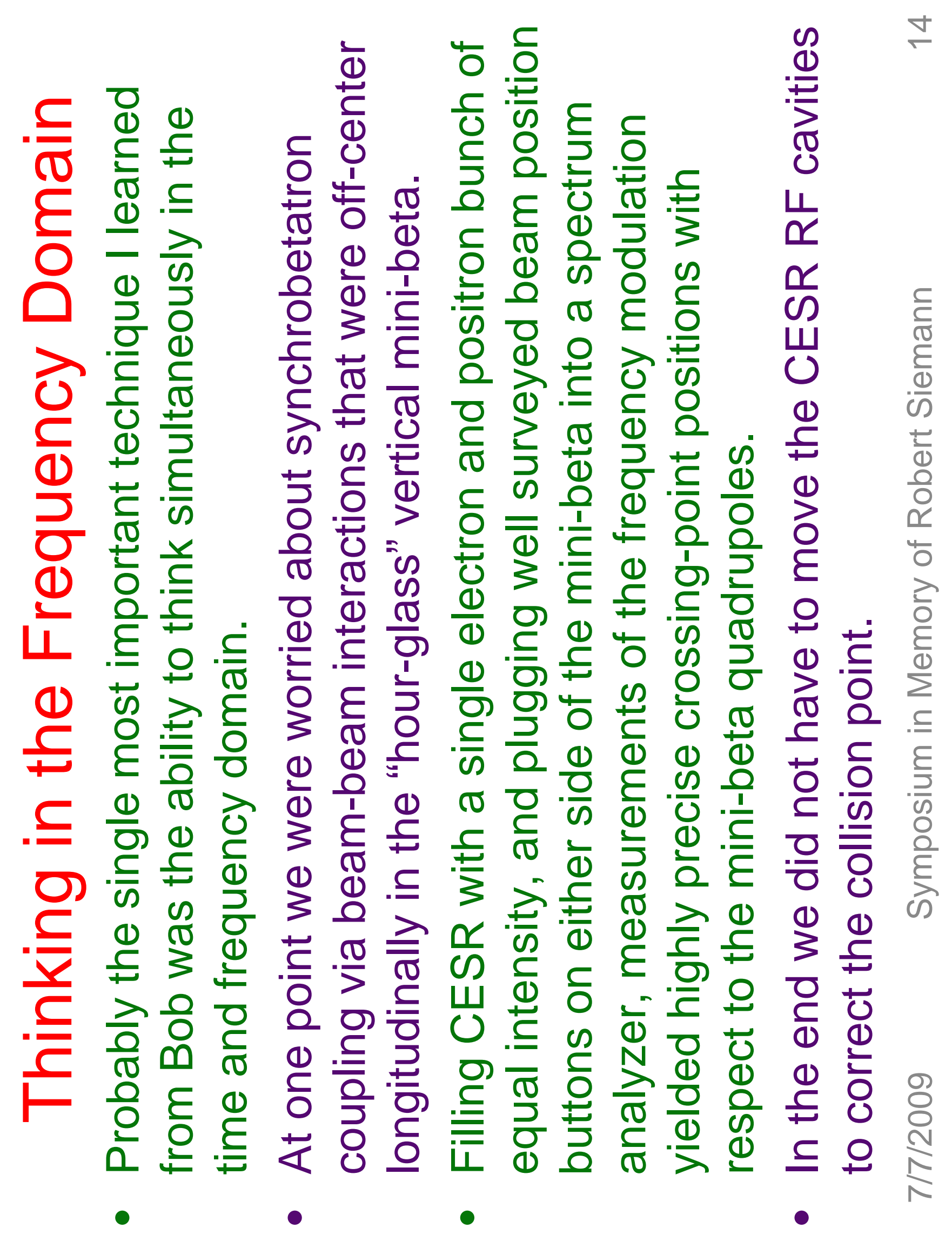




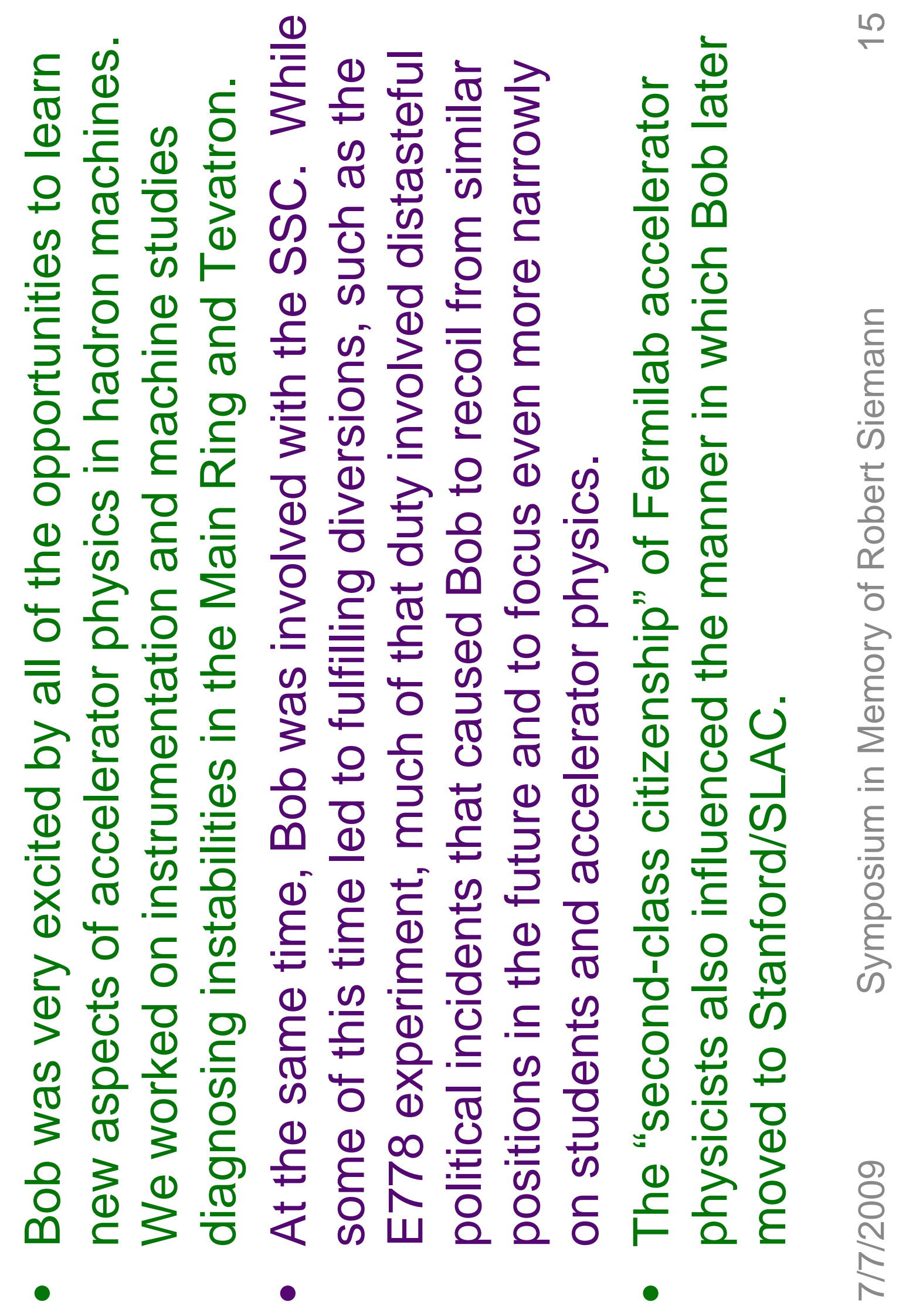



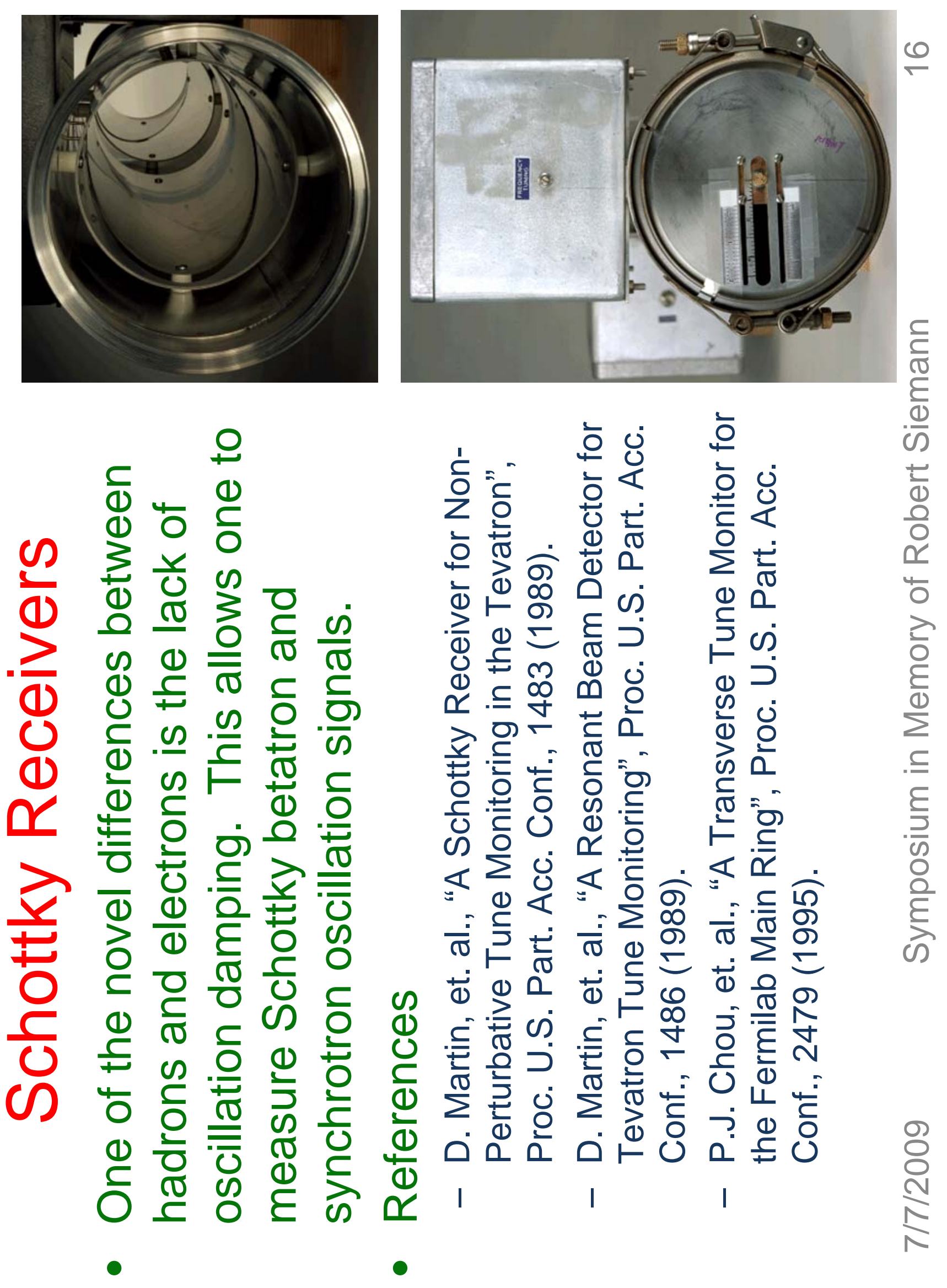


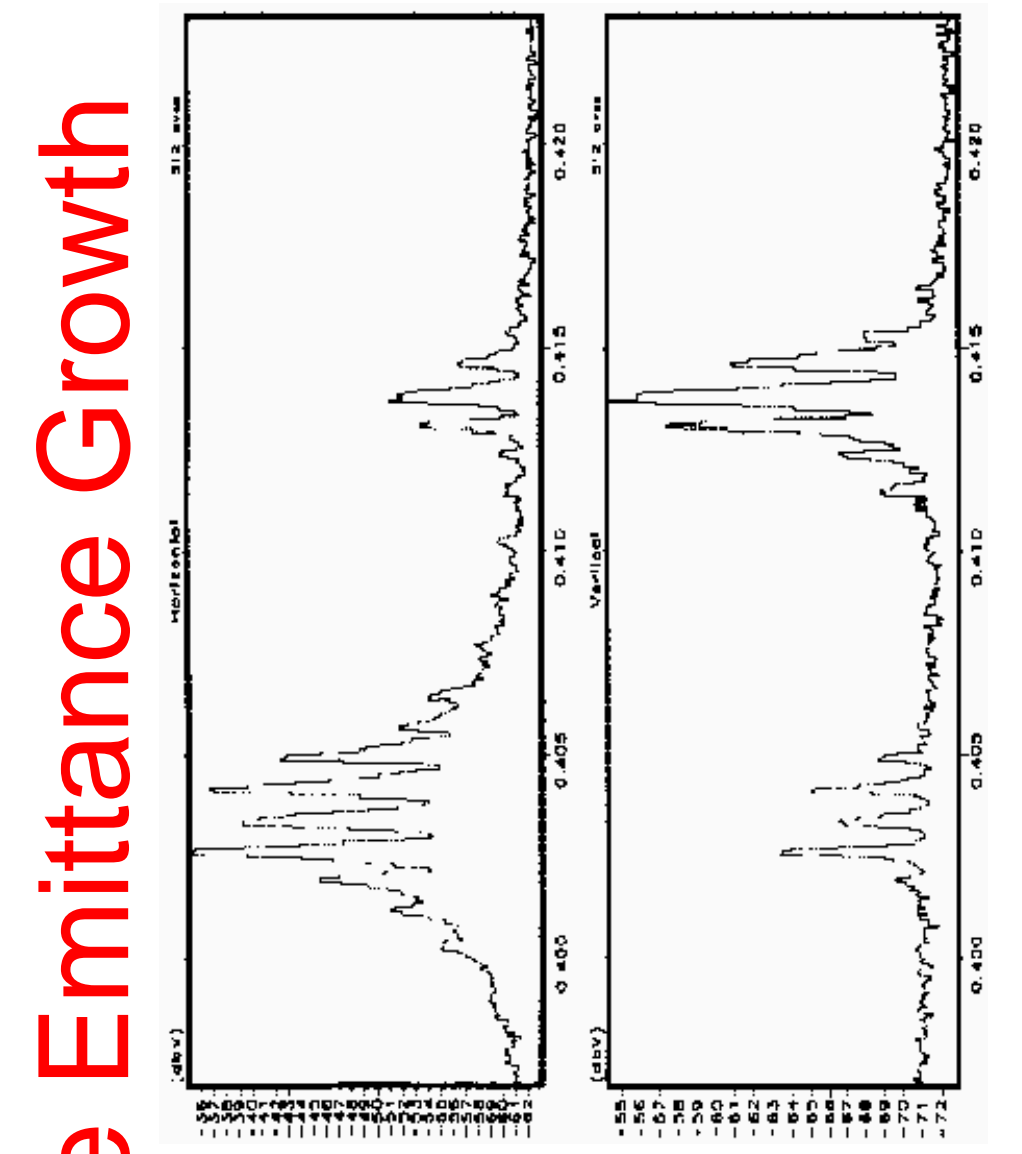

(1)

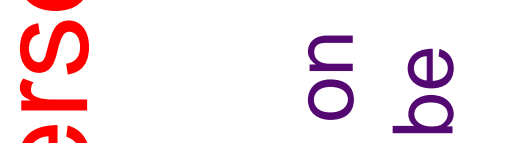

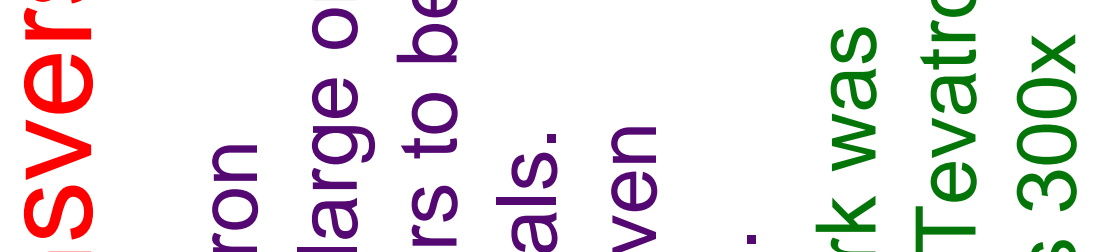

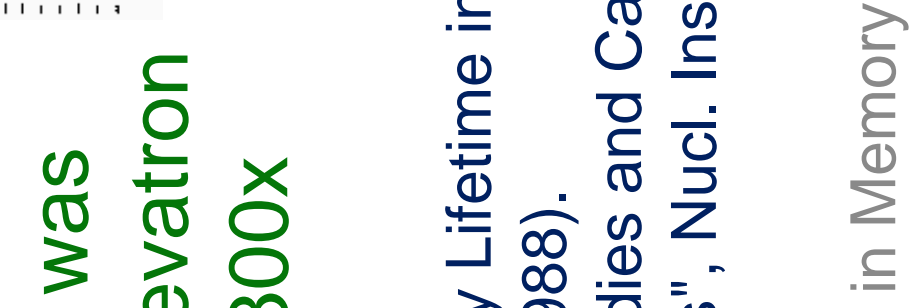

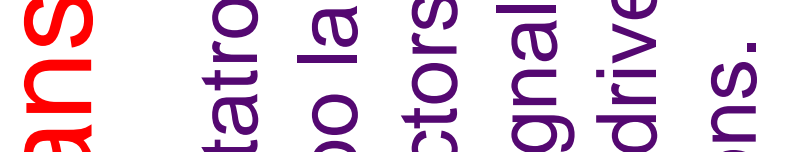

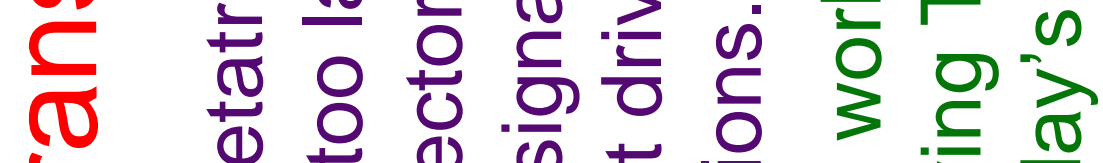

E

ส

응 仓 ঠ

N

(1) ह

‡ एᄄ

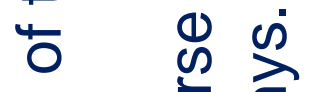

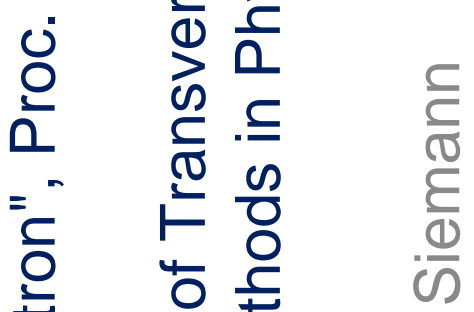

ฮั่

1 음 응 응

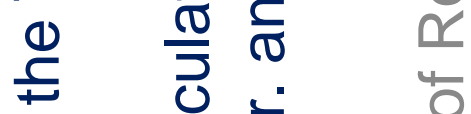

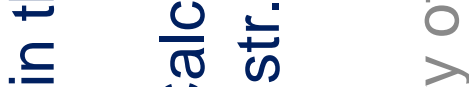

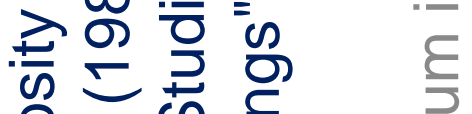

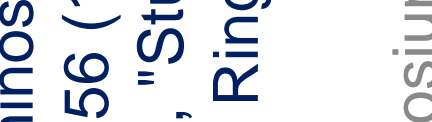

ڤ̊

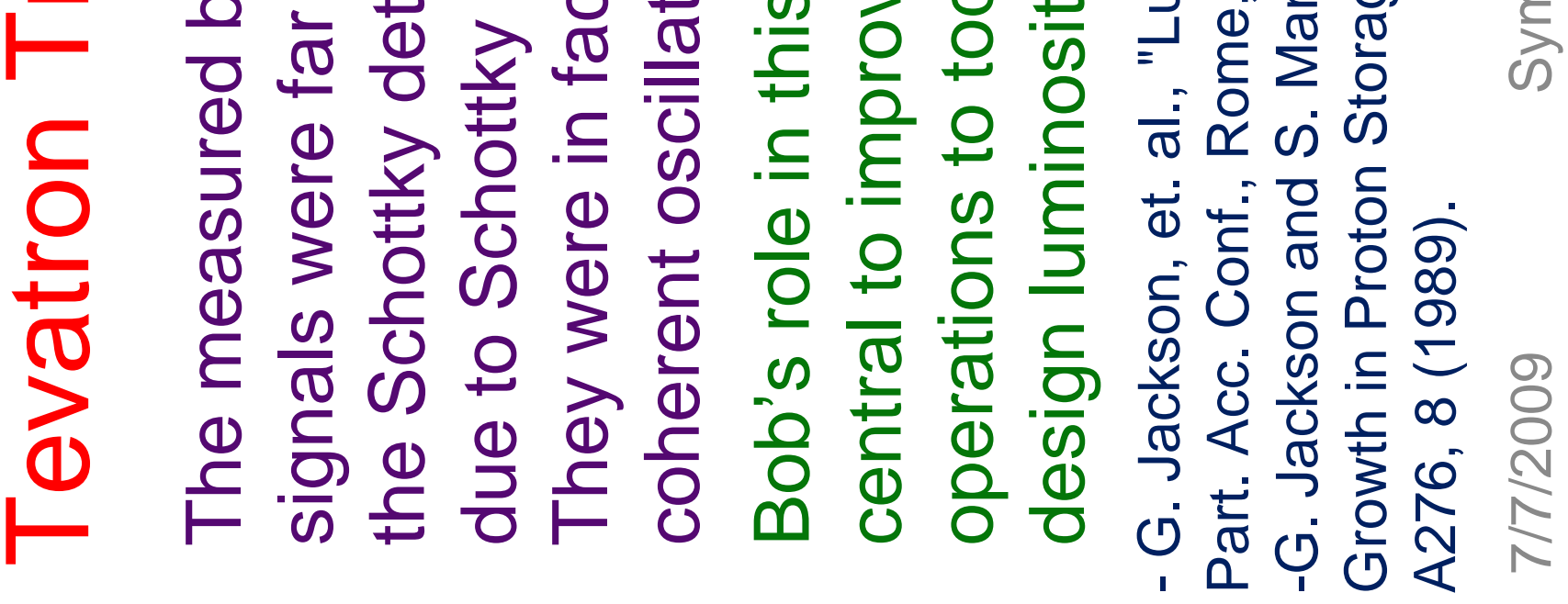




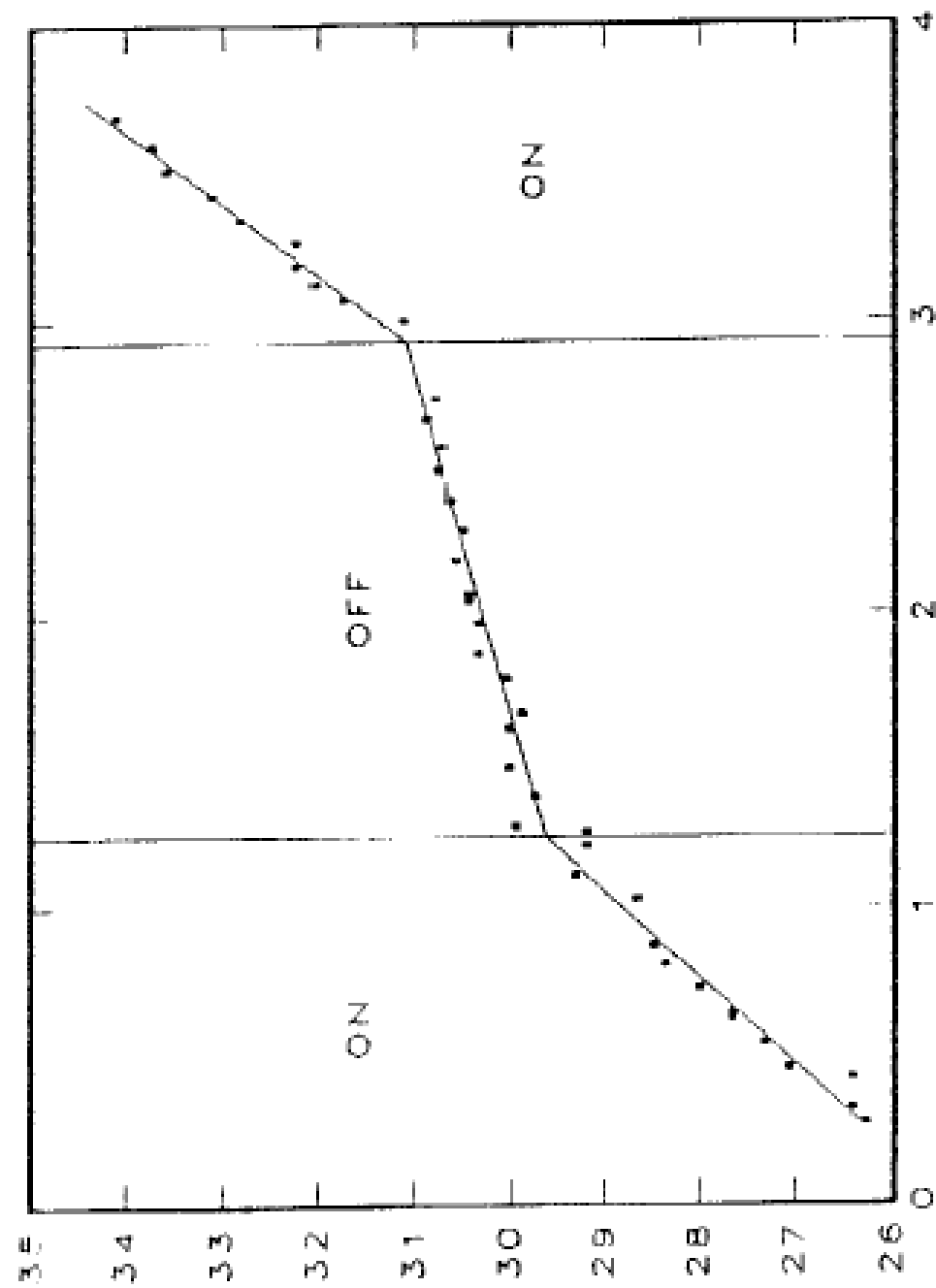

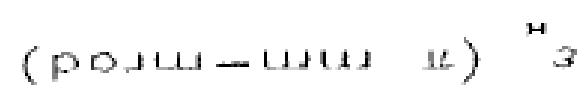

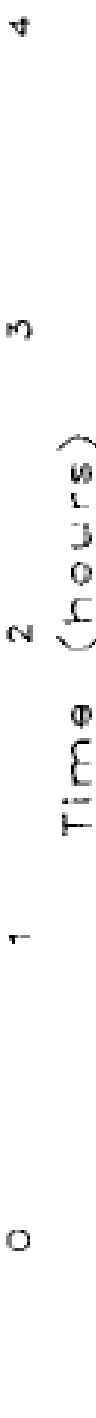

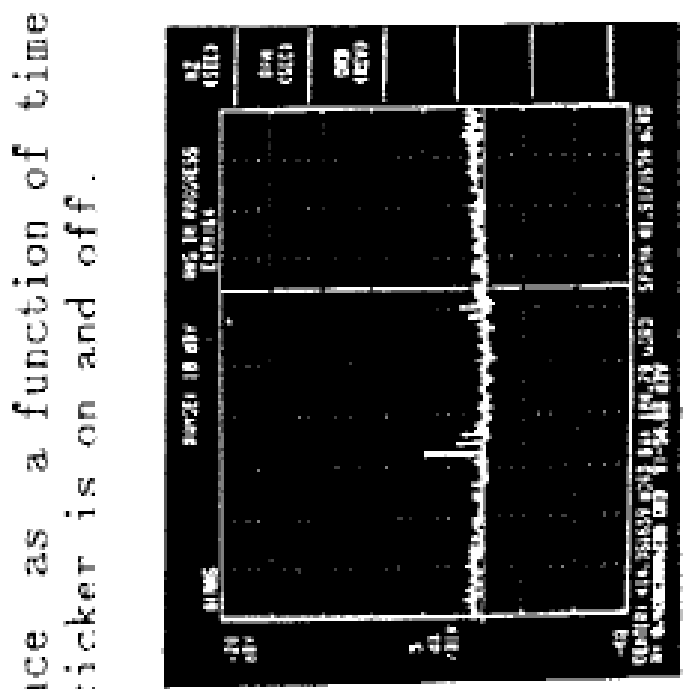

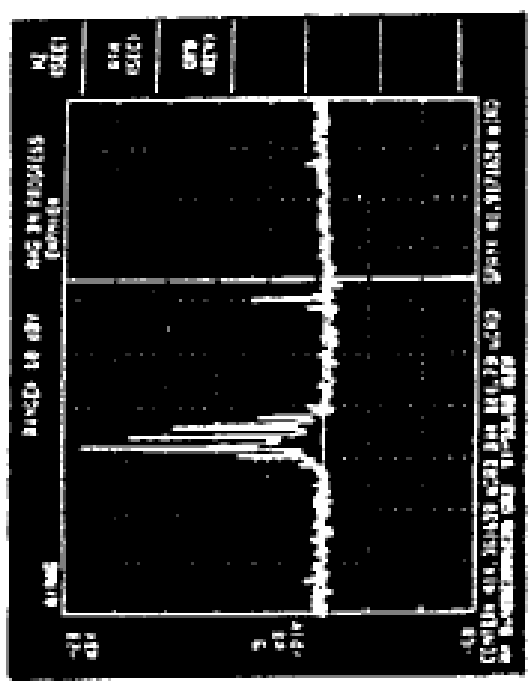

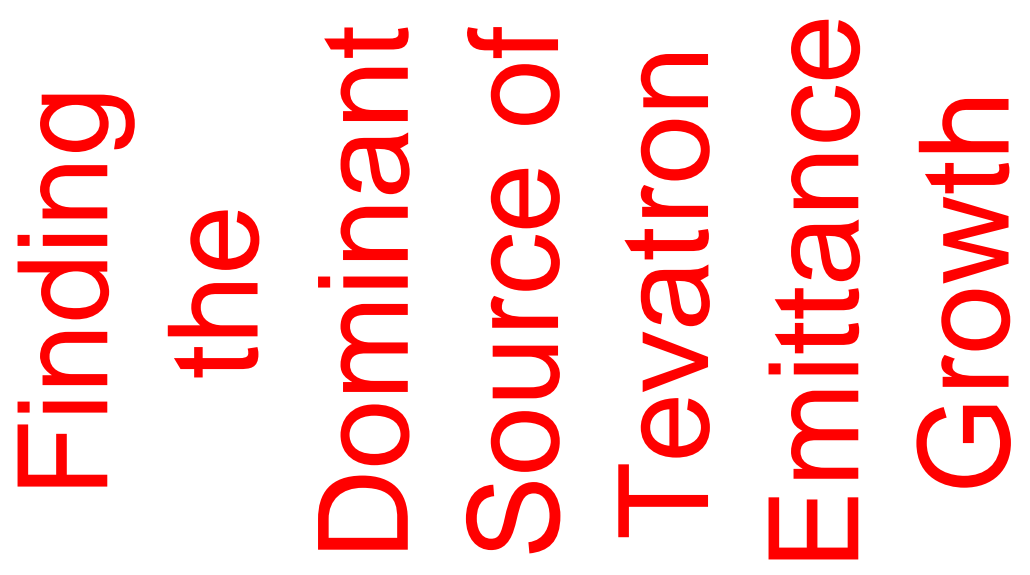




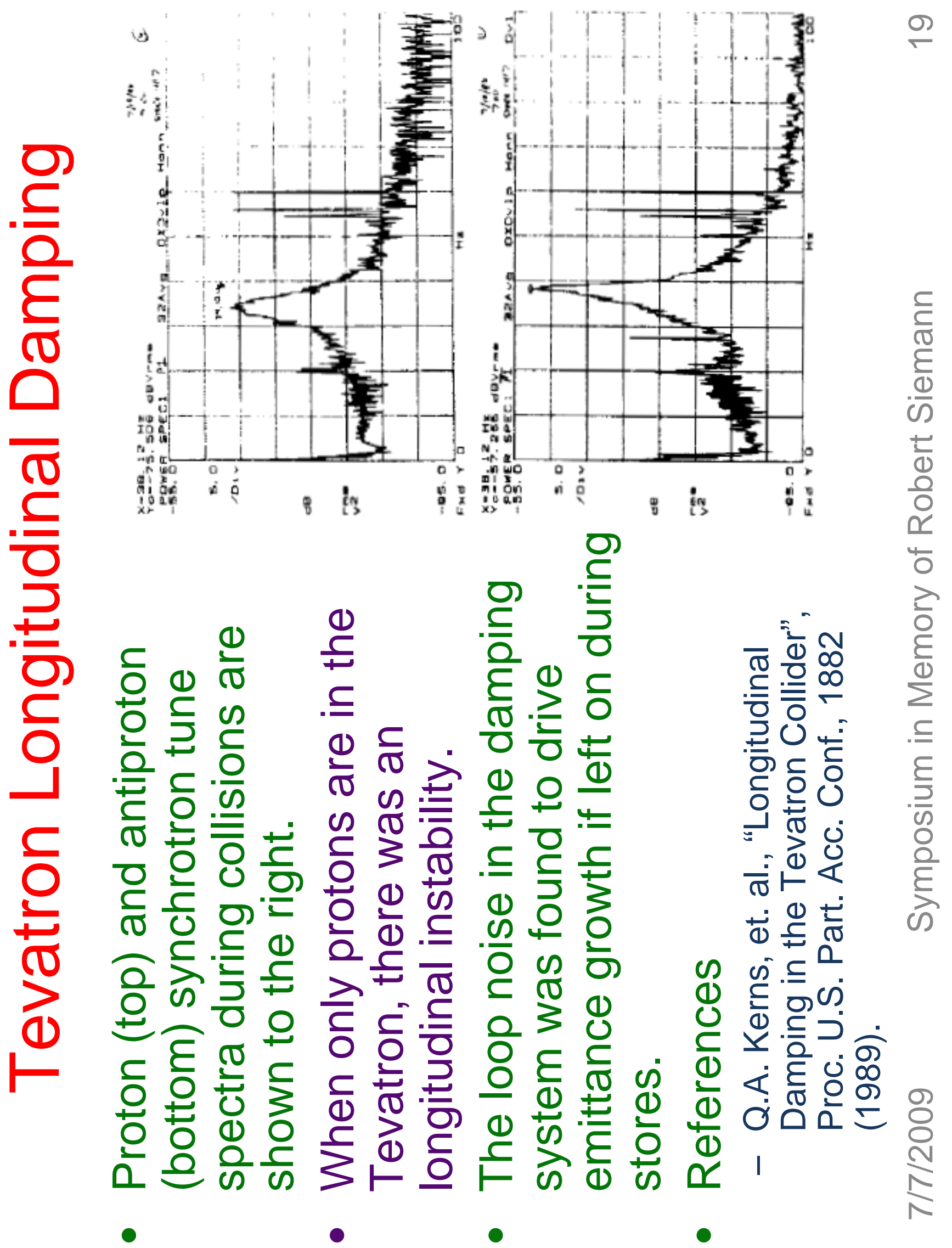




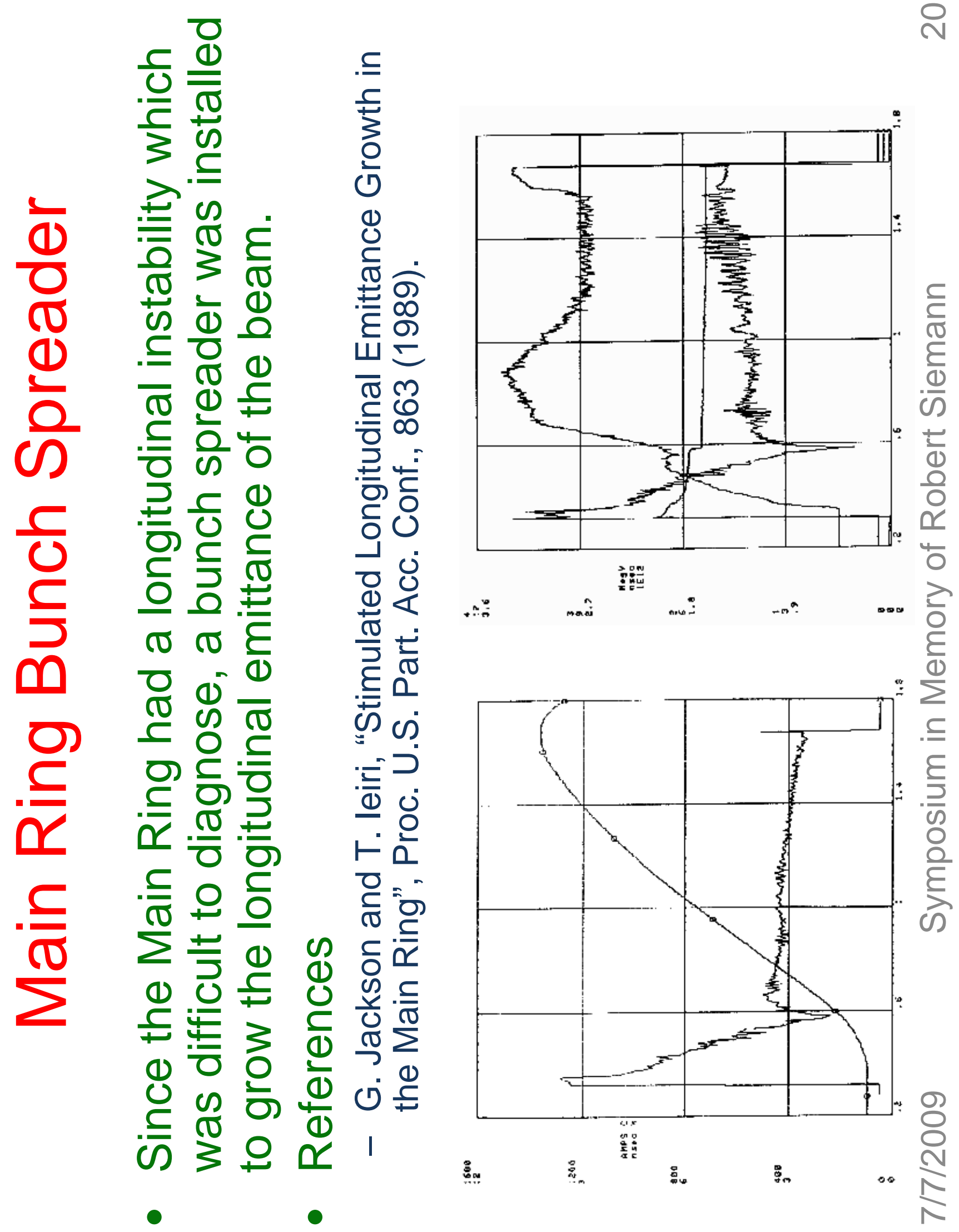


$\div \quad 0 . \bar{n}$

원

-

○. 응 웜

$\sum \stackrel{0}{2} \frac{0}{0}$

등 $\frac{1}{\sigma}$

ठ) ฮั

뒁 $\frac{1}{\sigma}$

(1) 융

-1 凹 ڤ

등 응 응

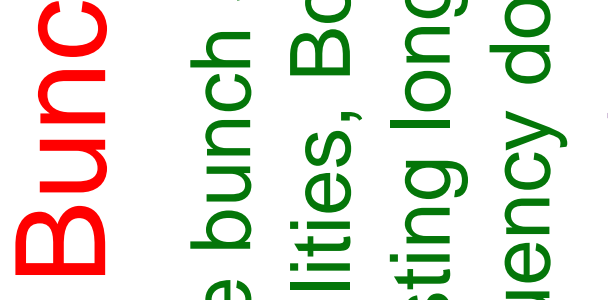

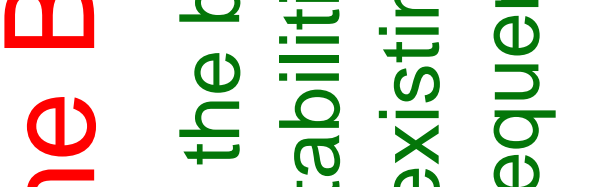

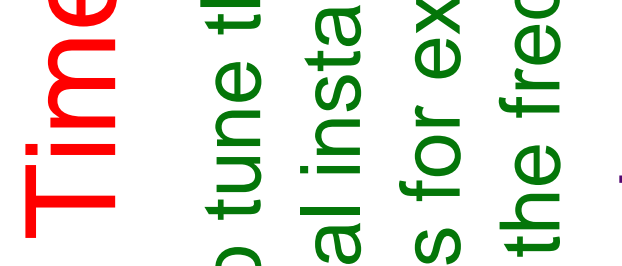

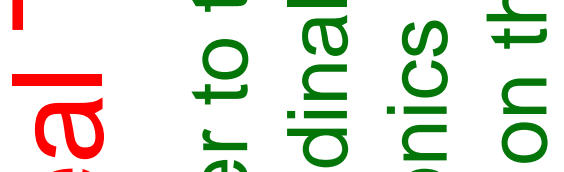

(1) 은 을 을

훙 넝

드 으 엄

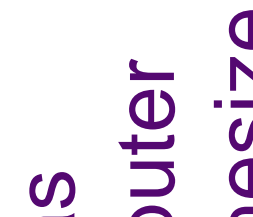

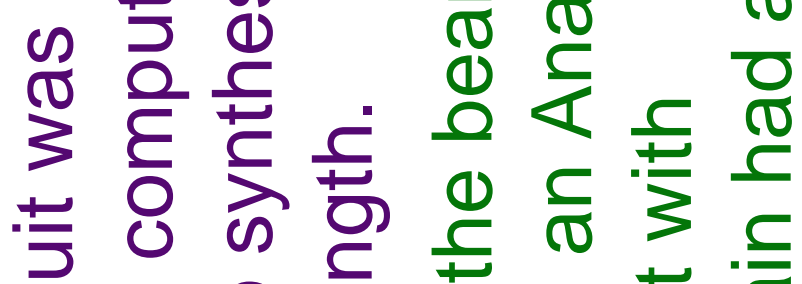

U

言‡

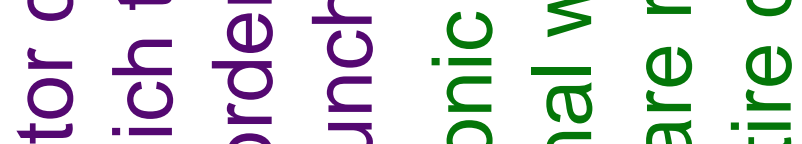

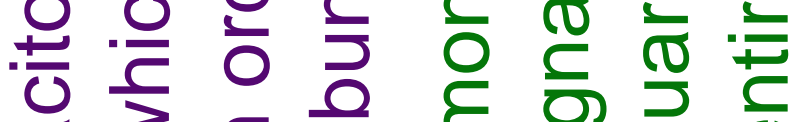

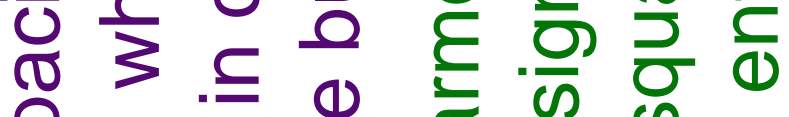

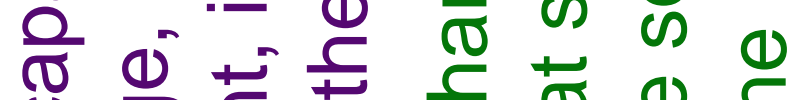

ช

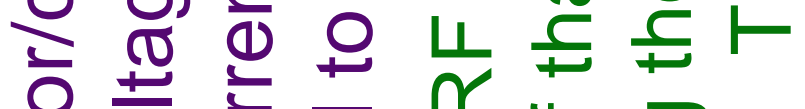

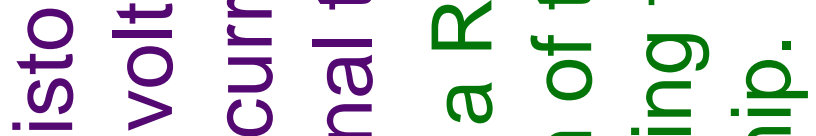

凹

ฮึ

ब ब

ส

응 원 응 등 응

ช

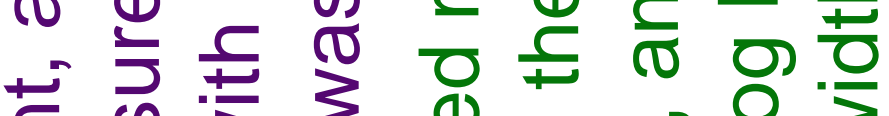

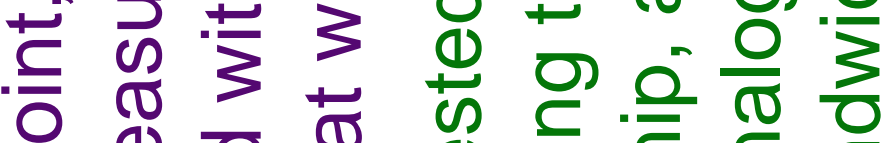

응 0

ल E 巳

(1) $=\frac{2}{2}$

宁依交

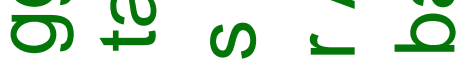

을 즘

D

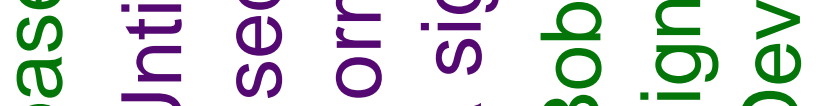

๓ ‘ 


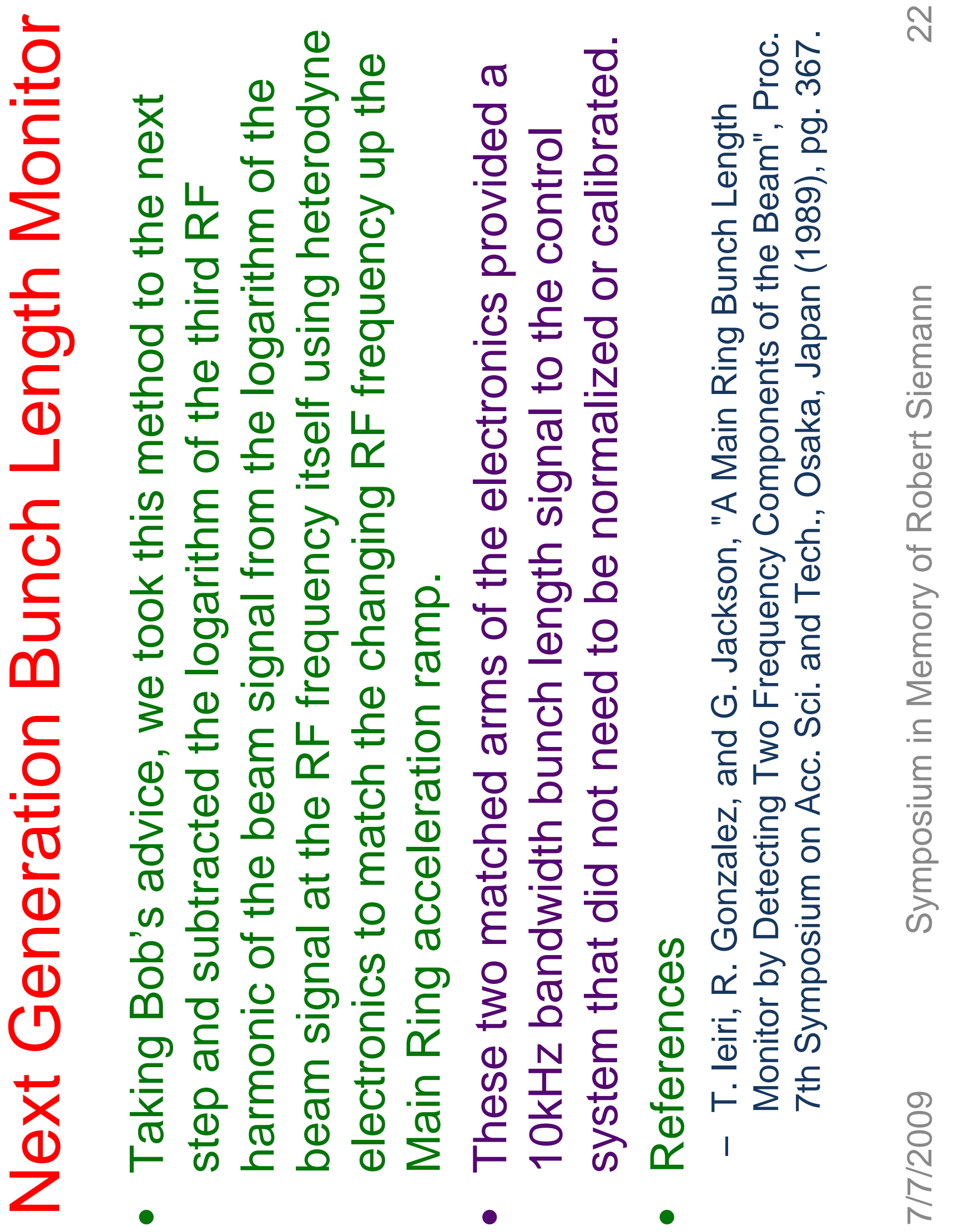


(2) $\frac{0}{0} \frac{0}{0}$ (1)

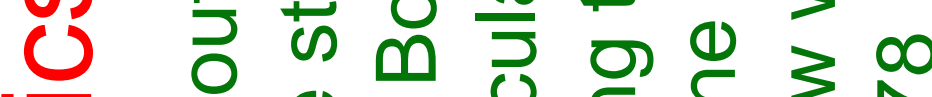

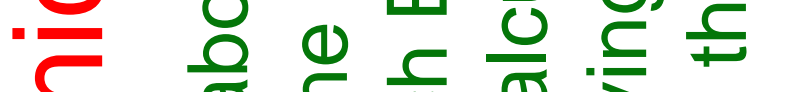

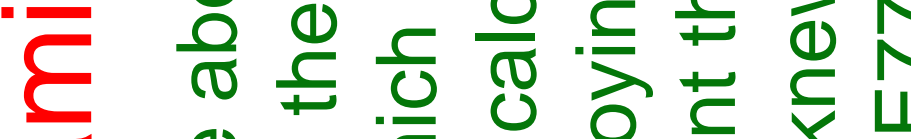

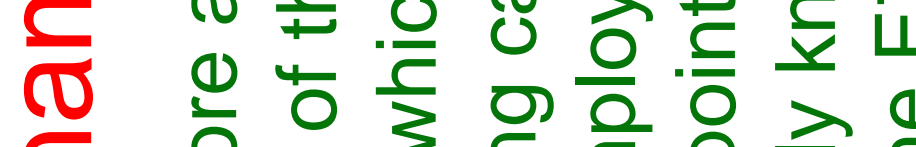

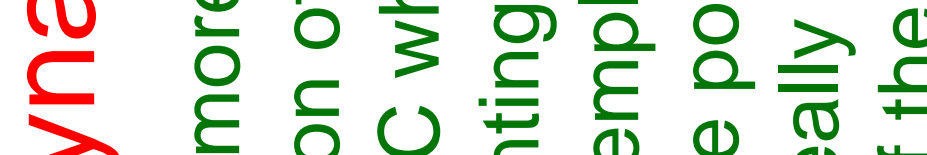

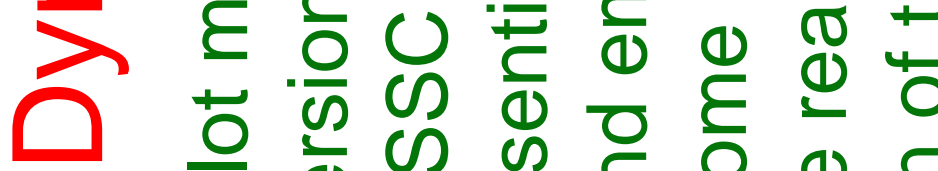

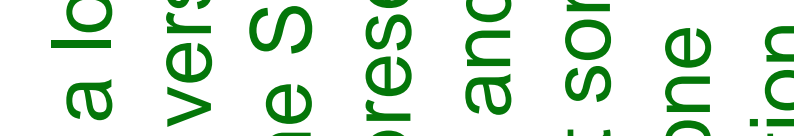

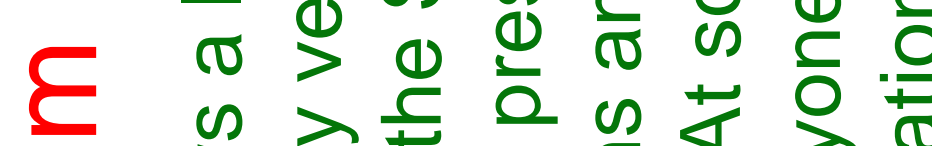

ฮ

Ф)

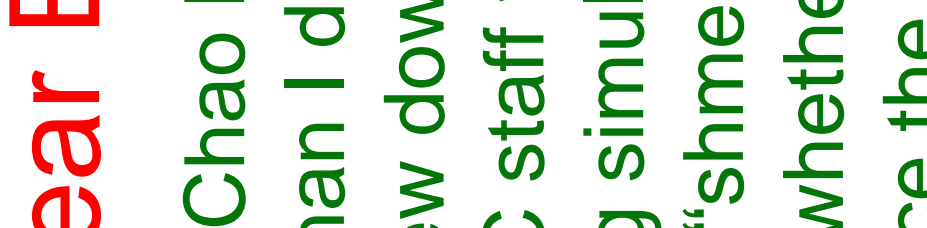

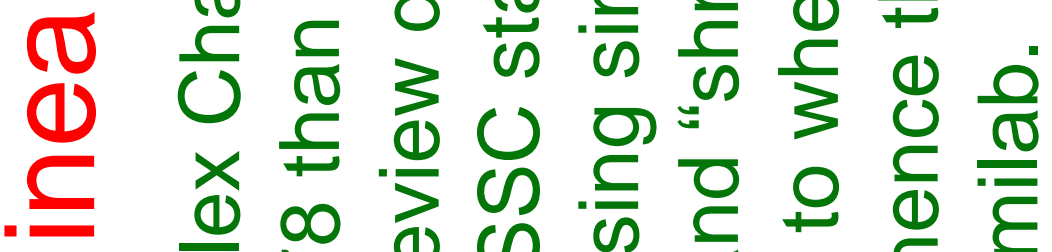

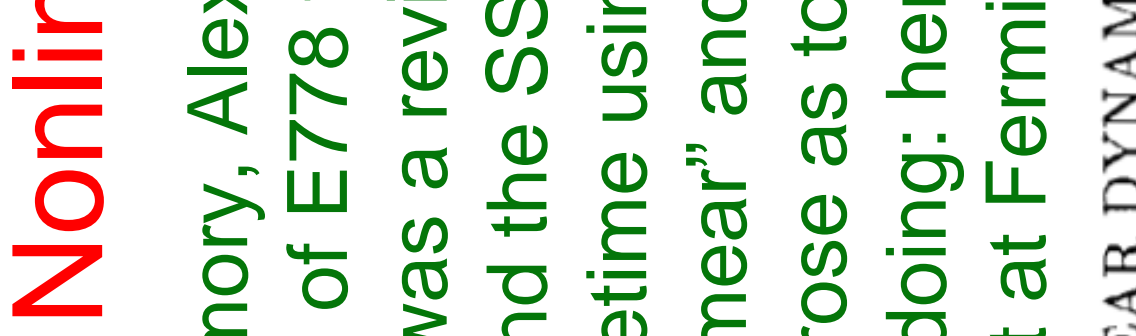

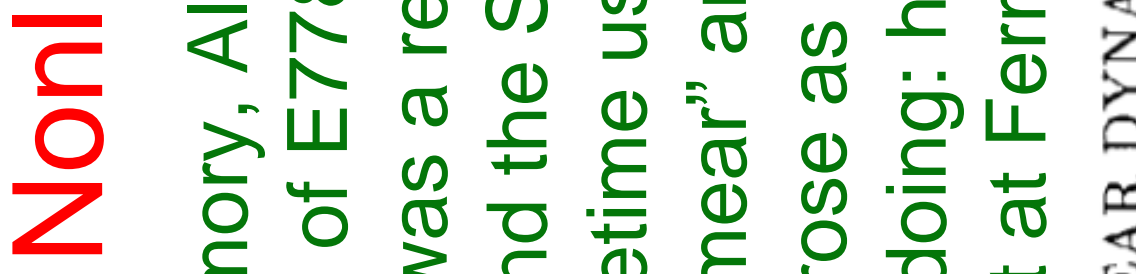

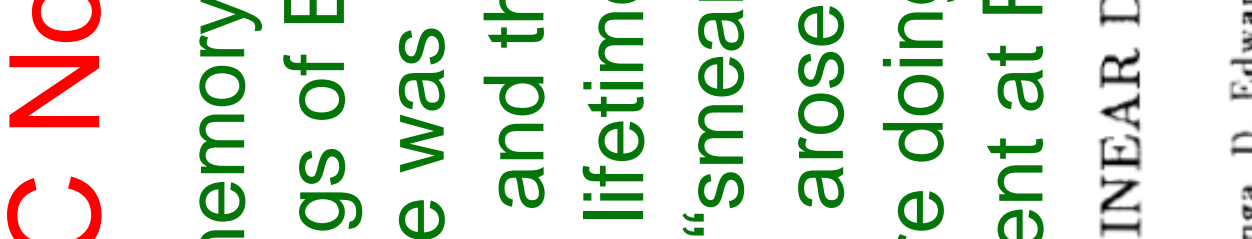

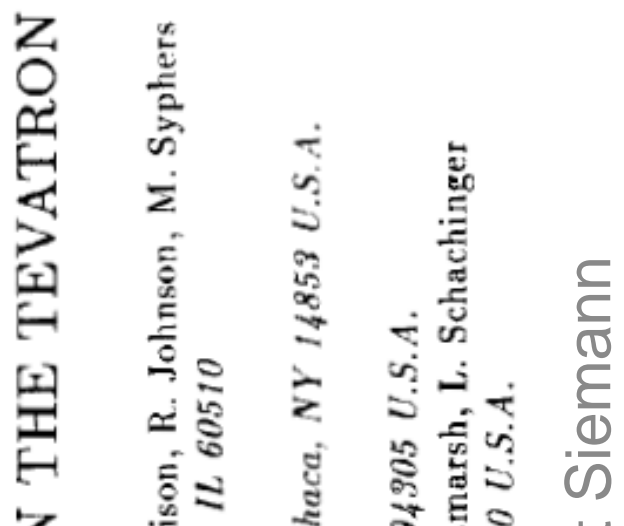

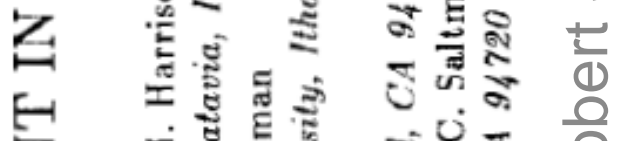

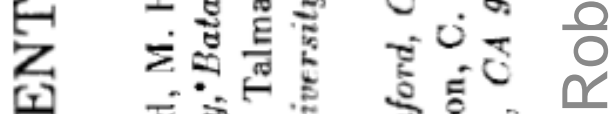

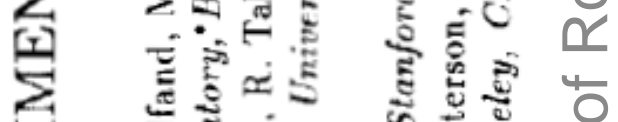

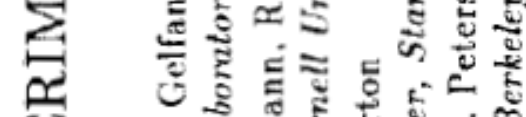

बI 入

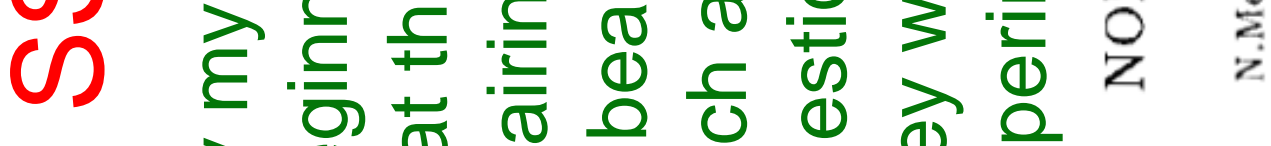




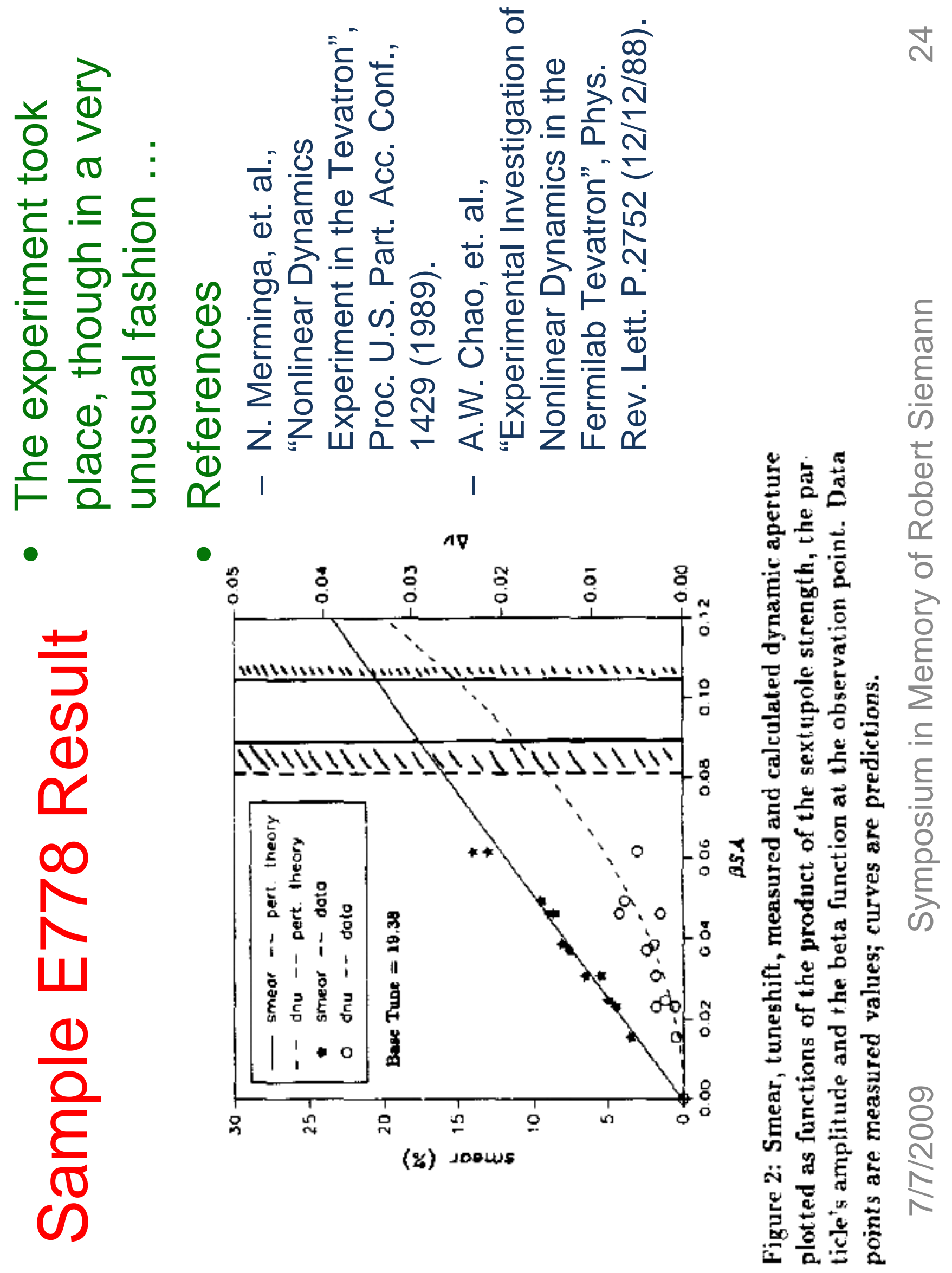




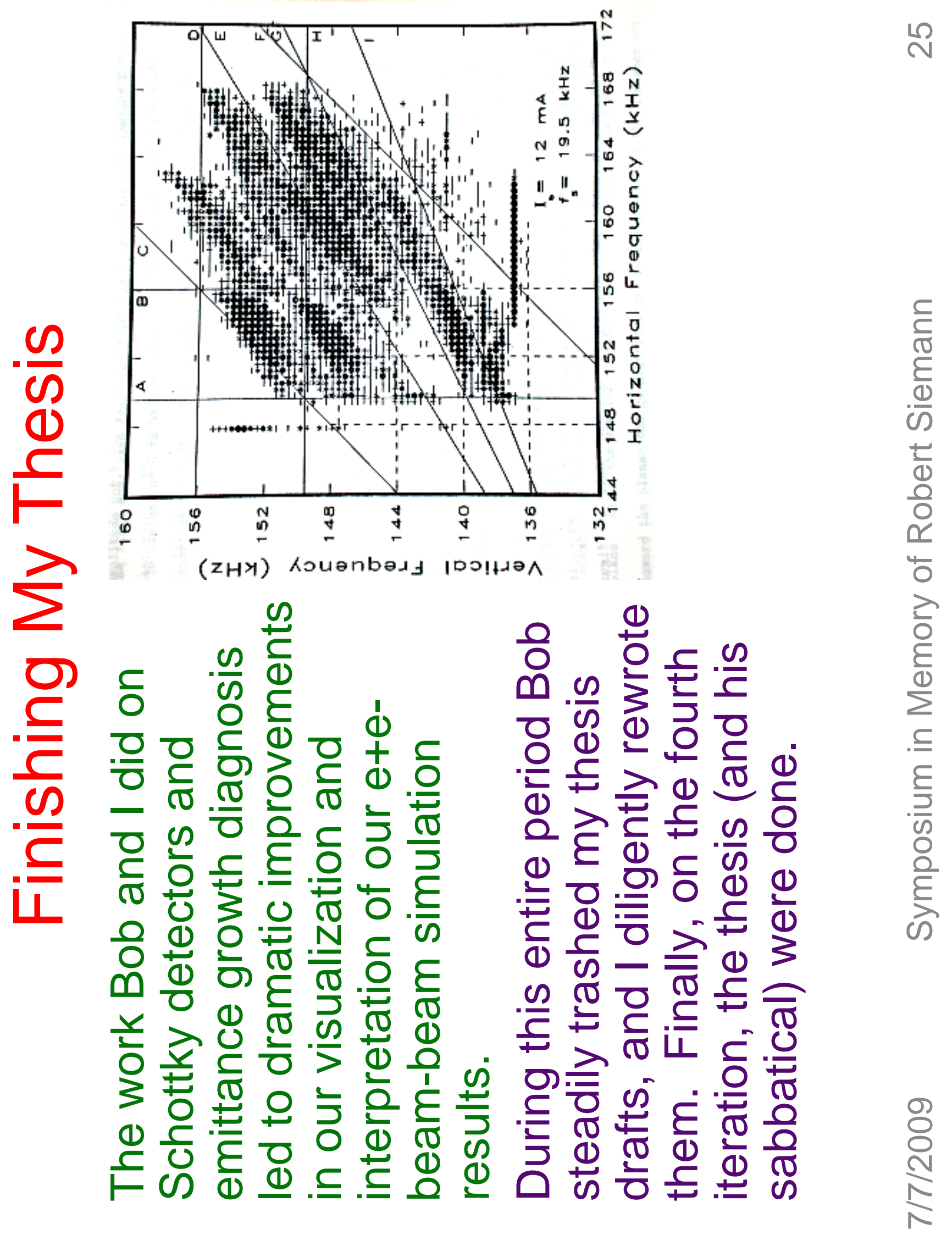




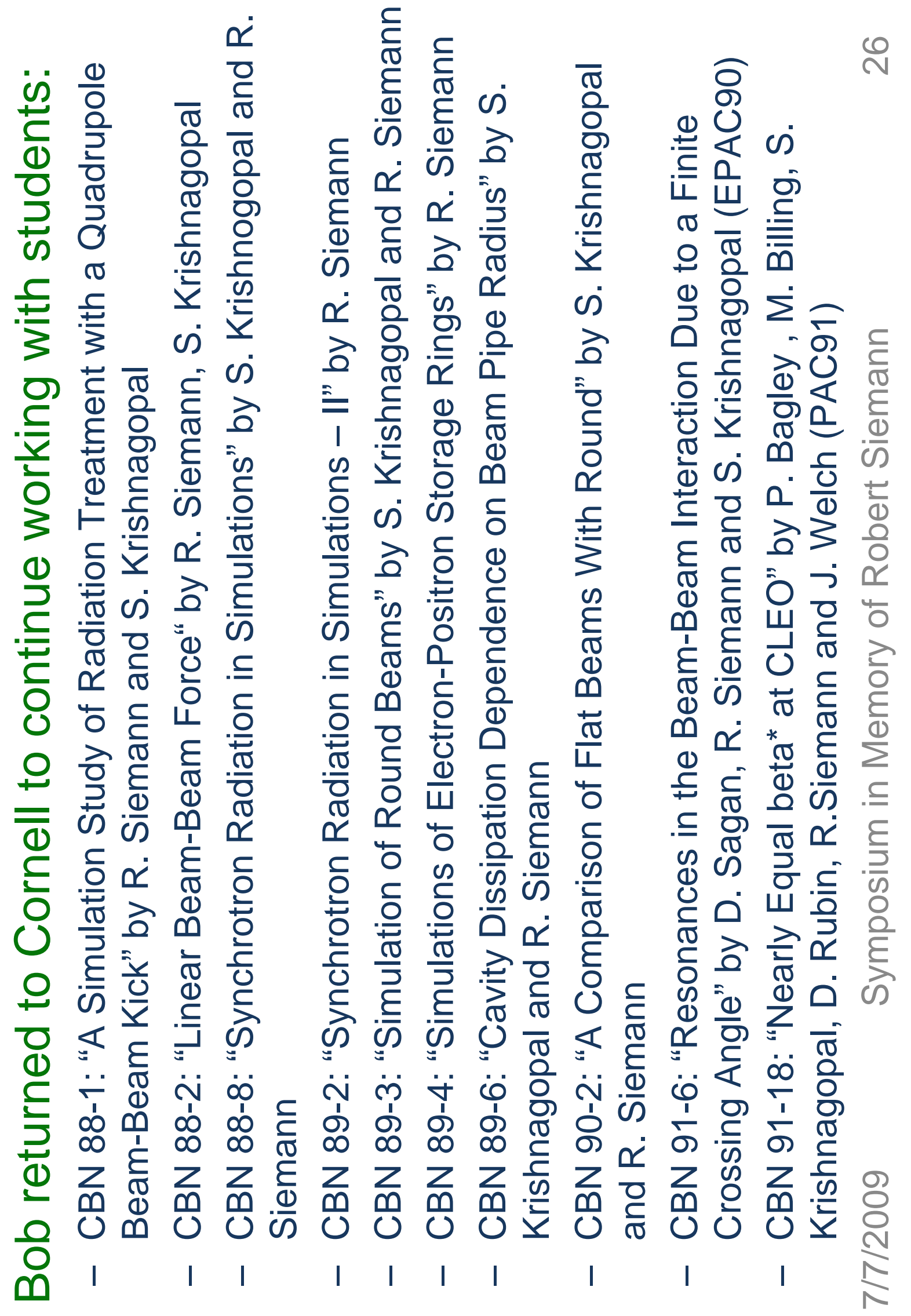



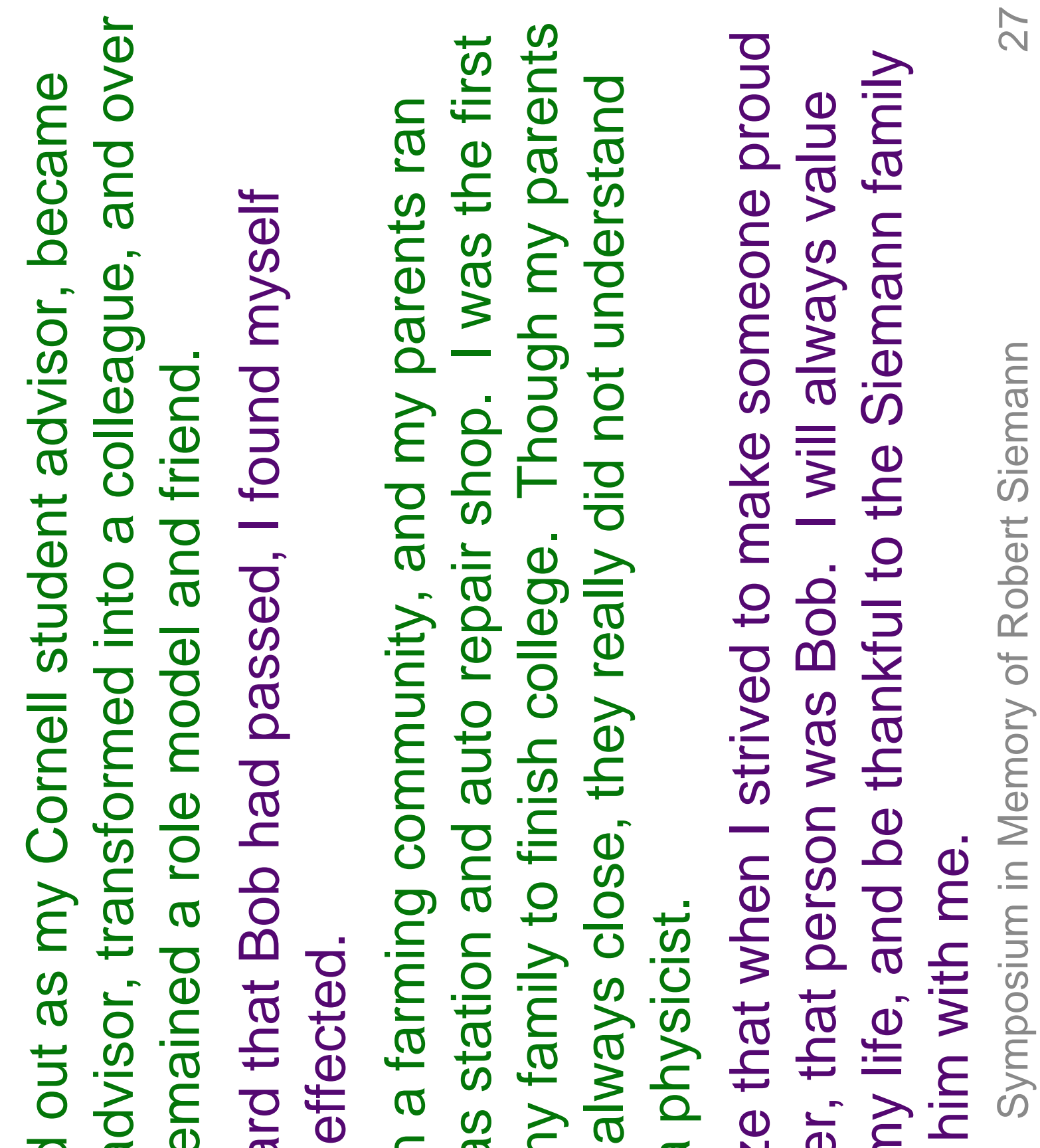

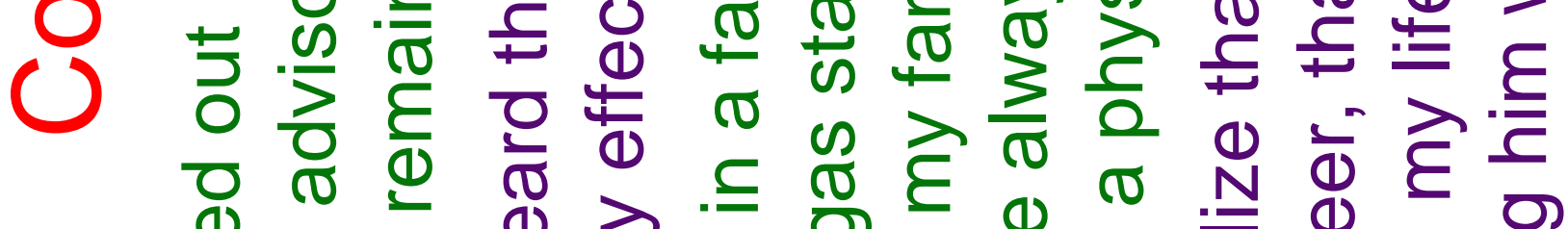

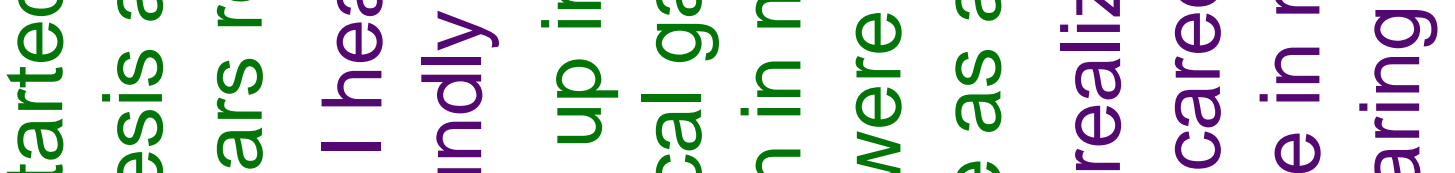

䨔 ब ब

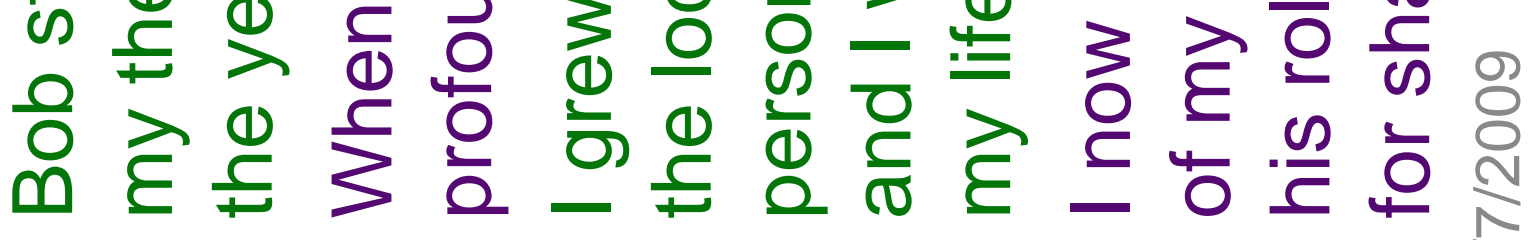
• 


\title{
From SLAC Post-doc to SLAC Professor Robert H. Siemann memorial Symposium
}

\author{
Burton Richter \\ $7 / 8 / 2009$
}

\section{Post-doc Days}

Bob Siemann's association with SLAC began 40 years ago when he accepted a 3-year post-doc job with my group and was with us from 1969 1972. The linac was running. My group, Group C, was doing experiments mostly with photon beams using the spectrometers in End Station A while continuing our efforts to get funding for the colliding beam program. Dick Taylor and the MIT group were doing inelastic electron scattering. Martin Perl was looking for long lived heavy leptons. Joe Ballam and David Leith were getting the bubble chamber grogram together.

It is hard to remember after all this time exactly why I offered Bob a job. He was clearly at least as smart as he was tall, and he was very tall indeed. His $\mathrm{PhD}$ thesis may have has something to do with it because it surely reminded me of my first big experiment as a post-doc a little more than a decade earlier. Bob's thesis was on wide-angle bremstrahlung. My experiment had been on wideangle pair production. We both were testing the limits of QED, and perhaps I suspected a kindred spirit.

The SPEAR storage ring construction project would begin in the fall of 1970 , but when Bob arrived we were not yet sure of funding and physics with it would in any case be too far in the future for a post-doc. Bob had not yet shifted his interests to accelerator physics so did not work on SPEAR design or beam stability issues, but he could not avoid hearing all the talk about it going on in the group and maybe that piqued his interest in accelerators. 
Instead of colliding beams, he became part of many experiments with the SLAC spectrometer facilities, mainly the $20 \mathrm{GeV}$ spectrometer. He had an enormously productive three years at SLAC. There are 14 papers with his name on the author list; some with my group, some Richard Taylor's group, and some with the Tufts group. They included photoproduction experiments including some with polarized targets and beams (Including Roy Schwitters' PhD thesis experiment), electroprodution, deep inelastic scattering, and technology development.

I am particularly fond of one of these experiments; a search for photoproduction of free quarks. The proposal itself had only 2 names on it Robert Siemann and Burton Richter. It was based on a magnetic trick - set a magnetic spectrometer for a momentum beyond the end point of the bremstrahlung spectrum of the beam and no integral charged particle could get through. However, a particle with $1 / 3$ charge would get through if it had a momentum of only one third of the setting. To the surprise of some (Dick Taylor in particular), the program committee was intrigued and the experiment was approved for a month of running time. To no one's very great surprise we did not find any sign of free fractional charged particles - but we had a good time trying.

\section{A Long Interlude}

Bob left SLAC in 1972 and did not return for 18 years, 17 of which were at Cornell. There he did switch his focus from experiments with accelerators to the accelerators themselves. That story has been well told by Dick Talman earlier in this symposium.

I did keep track of Bob's developing career as I did with most of the Group-C graduate students and post-docs, and some of the others young ones that came through SLAC that I thought particularly interesting (Persis Drell was one of those). I wrote many letters for them over the years. 
However, the only thing I did to advance Bob's career at Cornell was indirect. Boyce MacDaniel asked me to tell the NSF why they should fund the CESR machine when the much higher energy PEP would be available. I told the NSF that for studies at the lower energies, a machine optimized for those energies would be much more productive than one optimized for higher energy and then dialed down. CESR was built, the upsilon was discovered at FNAL, and CESR became a physics engine until superseded by the B-factories of KEK and SLAC.

Bob had switched his interests to accelerator physics, and his work contributed to the continuous improvement of the Cornell machine. His increasing prominence in accelerator physics meant that I would see him at conferences and found his work innovative as well as interesting. I particularly liked his papers on the beam - beam interaction.

\section{Return to SLAC}

Here at SLAC, we had finished the SLC, the SLAC Linear Collider, in 1987. It was the first of a new kind of machine. We had foreseen some of its birth problems, but had not clearly foreseen them all. We needed to increase the luminosity of the machine, and one of the arguments going on among the machine experts was about round beams versus flat beams. Bob had written papers about performance in colliding-beam storage rings with round beams versus flat beams which I found particularly interesting.

In colliding beams, the luminosity depends on the particle density in the collision region which is proportional to one over the beam-area; the smaller the beams the better. But, there is a countervailing problem. What is called the tune shift in storage rings or the disruption parameter in linear colliders depends on 
the magnetic field at the surface of the beam. The higher the field the larger the problem and the field depends on the reciprocal of the perimeter of the beam. In a round beam as the area goes down increasing the potential luminosity, the perimeter goes down as well increasing the tune shift or disruption parameter and placing a limit on how small the beam can be. For any beam area, however, I can increase the perimeter by flattening the beam thereby reducing the tune shift.

In going over some old papers for this symposium I found a copy of Gerry Jackson's PhD thesis on work he had done with Bob on the Beam-Beam interaction. SLAC offered Gerry a job, which he turned down for a Wilson Fellowship at FNAL. Maybe I thought if we could not get the student, perhaps we could get the professor. It would have been best, of course, if we could have gotten both.

In any event, we went after Bob. I am not sure if it was the accelerator faculty pushing me or me pushing the faculty. Some informal soundings showed that he was interested in us as well. I can only speculate about why we were attractive at the stage of his career that Bob had reached in the late 1980s. I guess that the reason lay with the directions in which the NSF was willing to move. They were the financial supporters of Cornell, but did not seem interested in advanced accelerator work while Bob was very interested. The attraction of SLAC surely lay in the resources of a larger lab and the directions in which SLAC was moving.

Though it was easy to get Bob Siemann interested in coming back to SLAC, it was not so easy to close the deal and bring him here. The problem was SLAC's academic organization which was much different from the situation at Cornell. Among the DOE labs, SLAC was then and is still the most academically integrated with its contracting university. We have two faculties, high-energy physics and photon science, and the director of the laboratory functions as a kind 
of dean as well as managing the budget and the scientific affairs of the lab. Some of you have had the experience know that dealing with faculty affairs is often more difficult than dealing with the DOE.

When I became director in 1984 the high-energy physics faculty organization was different from what it is now. When the laboratory was first put together by Pief Panofsky, his thinking was that the academic part of the laboratory would just cover high-energy physics. Experimenters and theorists would be regular Stanford University faculty while the accelerator physicists would be research-professors. There was a distinction in title at SLAC, though there was no distinction in practice. But in a university titles do count. Bob was a regular faculty member at Cornell and he made it perfectly clear that if he would not be that at SLAC, he simply wouldn't come. That left the dean, me, with a problem. I had to get SLAC's charter changed because without that charter change, it would not be possible to appoint him as a regular faculty member.

Every department in every university has an area of responsibility and Stanford is no different from the rest. The French Department, for example, can appoint professors to teach French language, French history, and French literature, but it can not appoint a professor to teach physics. The Physics department can appoint people in condensed matter, plasma physics highenergy physics, but not in French. As originally set up, SLAC could only appoint professors in experimental and theoretical physics, but not in accelerator physics. To get Bob we had to get Stanford to amend our academic charter to make accelerator physics a part of our academic mission.

That took a bit of work. A paper was written for the Stanford Provost, who had to be convinced, that laid out the academic side of accelerator physics. Fortunately, there were precedents already at Cornell, the University of Maryland, and U.C.L.A. where accelerator physics was part of the academic curriculum. That helped a lot. The Provost took our paper to his advisory board 
and all came out well. SLAC's charter was amended, accelerator physics was part of SLAC's academic mission, and we now could offer Bob the job that he wanted and could bring him out here. Making the sale, if I can put it that way, was a permanent change. Ever since we brought Bob in, all of our appointments in accelerator physics have been regular academic appointments in both the high-energy physics and the photon science faculties.

Bob brought the family out during and set to work. You will hear about the things that he did in other talks and so I am not going to go into them. I will only say that his appointment that made considerable difference to the laboratory. When he came here, Bob started to work on the linear collider's problems and was a big help in curing some, thus getting its performance improved. Eventually it performed to the point where it could produce physics comparable in importance to that produced by the LEP facility at CERN, thanks both to improved luminosity and to polarized beams.

Bob continued to do some work on colliding beams with linear colliders. He and I produced a design for a $5 \mathrm{TeV}$ center-of-mass machine with a reasonable beam-strahlung parameter. I can't find my copy of our notes and hope they turn up in Bob's papers. Who knows, we might still build it.

Bob's interests evolved toward advanced accelerator systems such as laser and plasma acceleration which became his principle focus. He started a program on wake-field acceleration that has worked very well. His contributions to the development of the kinds of new techniques of acceleration may very well make much higher energy machines more affordable, something badly needed for the future. He was the driver of the effort to set up a facility here to test out advanced accelerator ideas that is finally going to be realized too, unfortunately, not in time for him to use it. 
Bringing Bob here was good for the laboratory as well as the accelerator science field because of the larger scope that he had for his advanced accelerator work. That larger scope came from the DOE's willingness to spend more money looking to the future than the NSF was willing to spend to develop the accelerator technologies to enable that future. It helped us recruit Bob and recruiting Bob was part of our good fortune. 

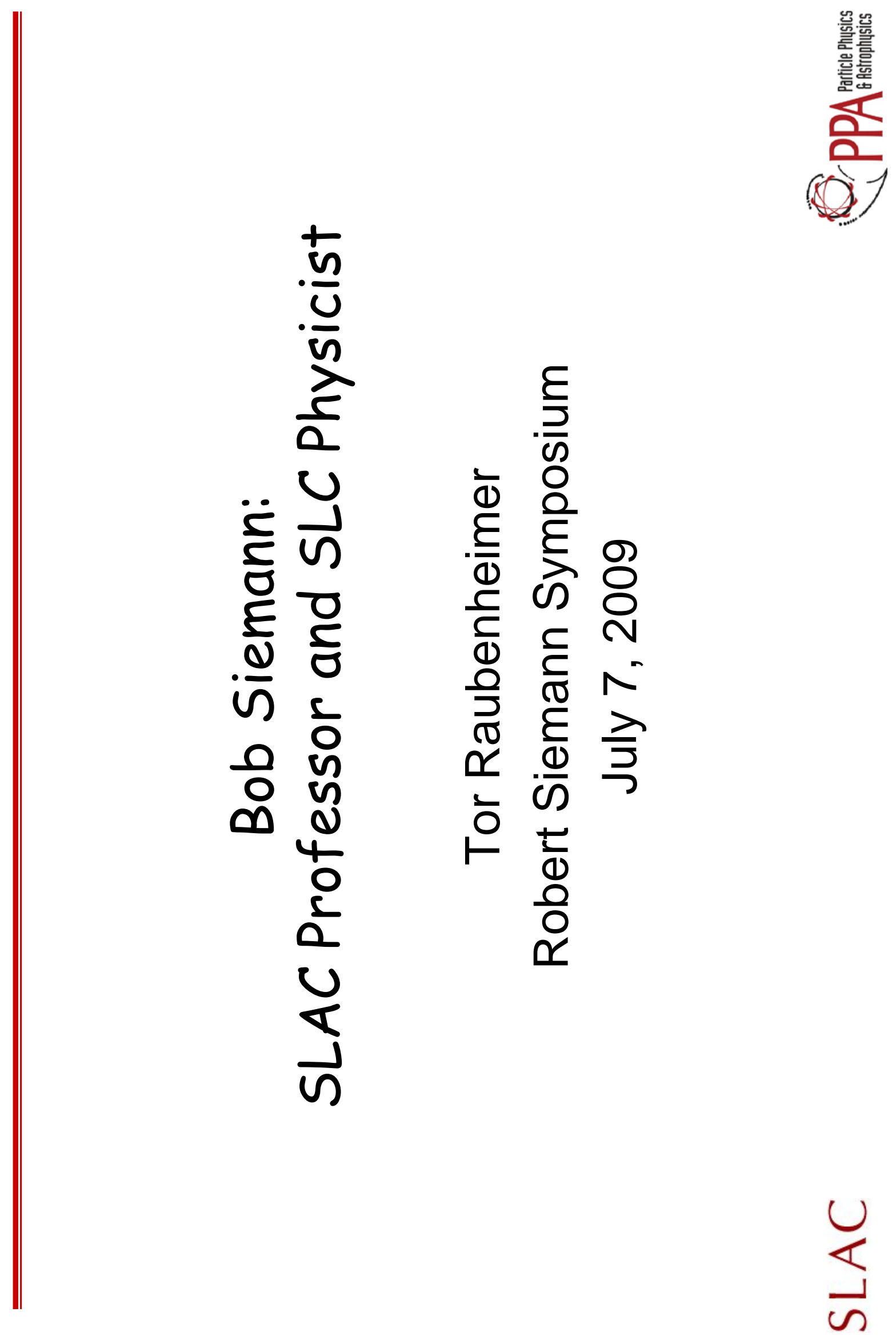


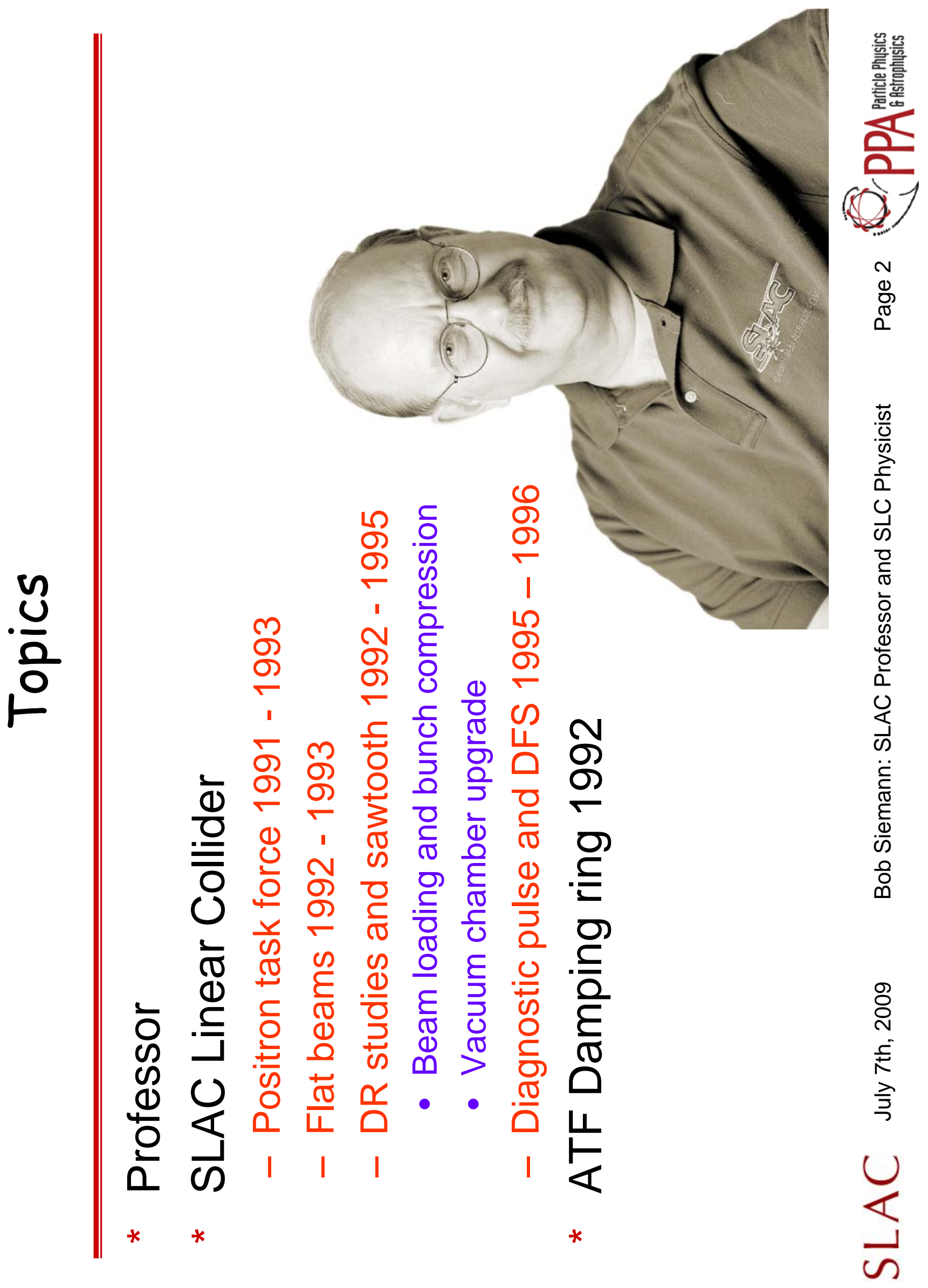




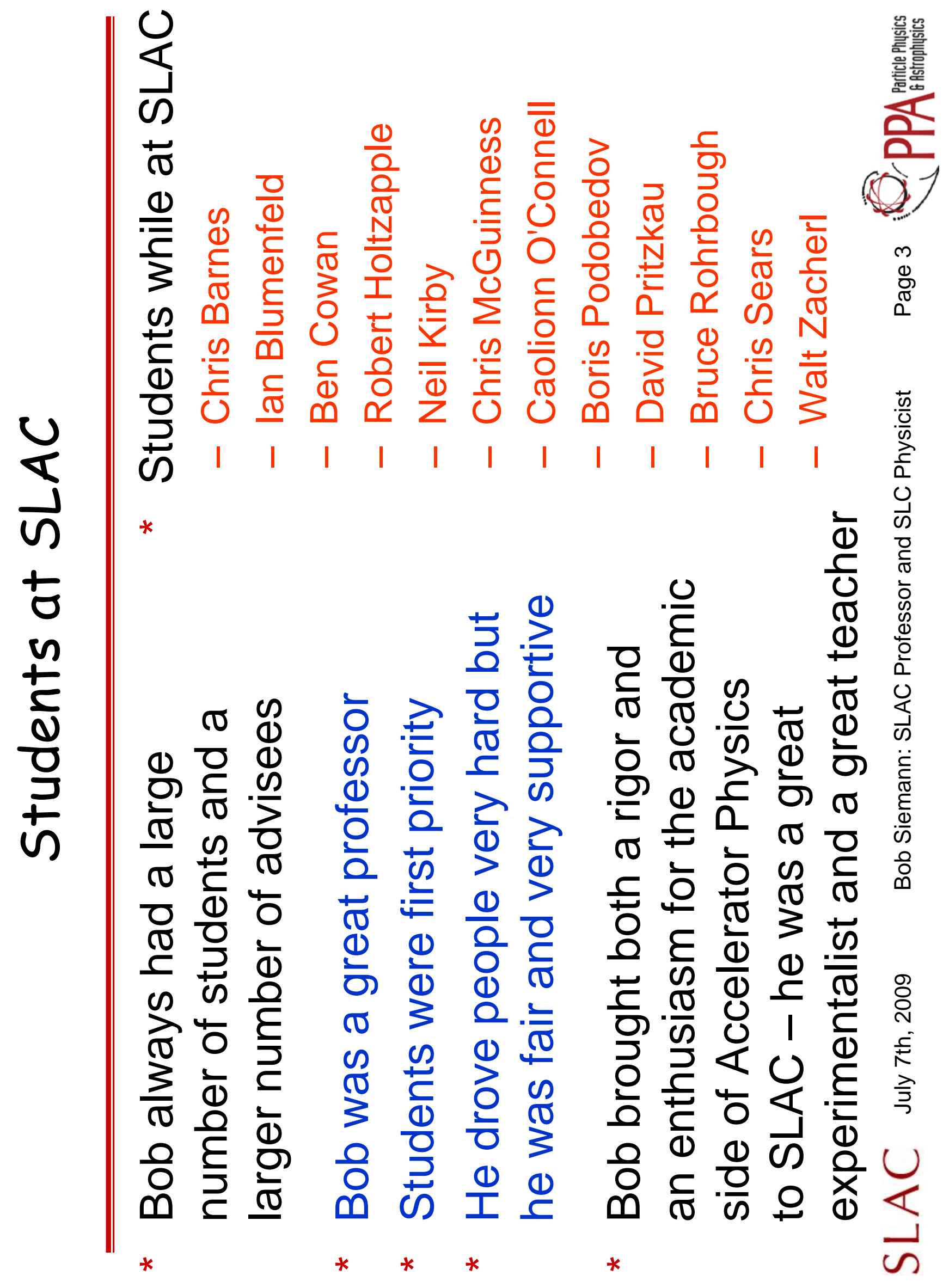




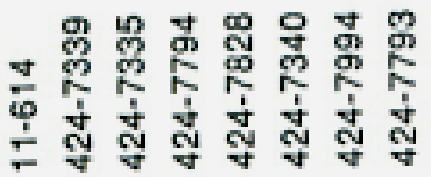

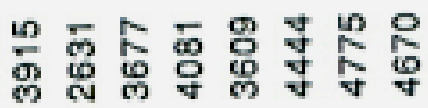

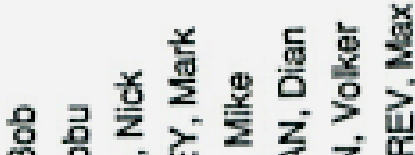

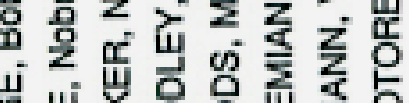

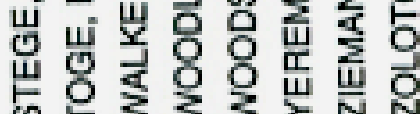

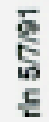

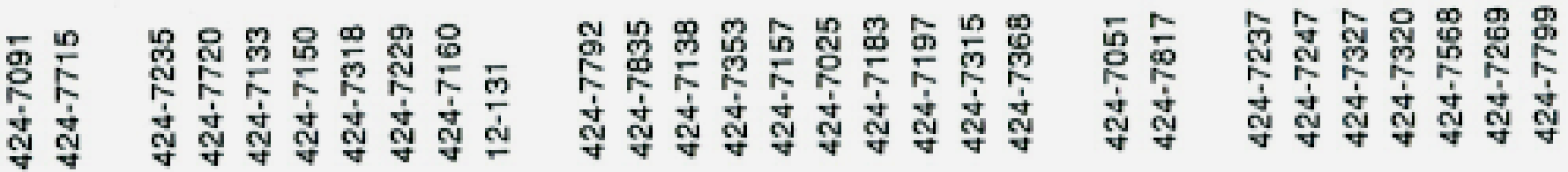

马

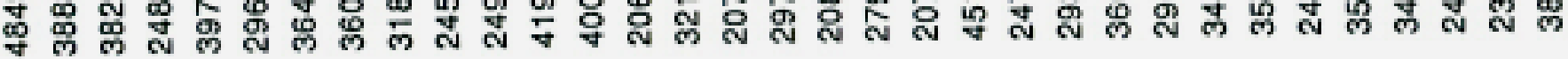

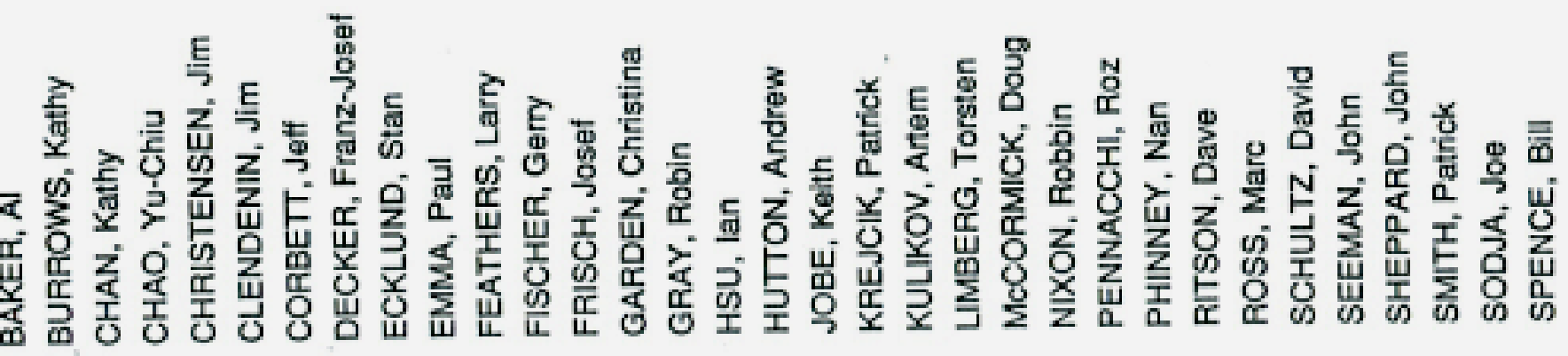

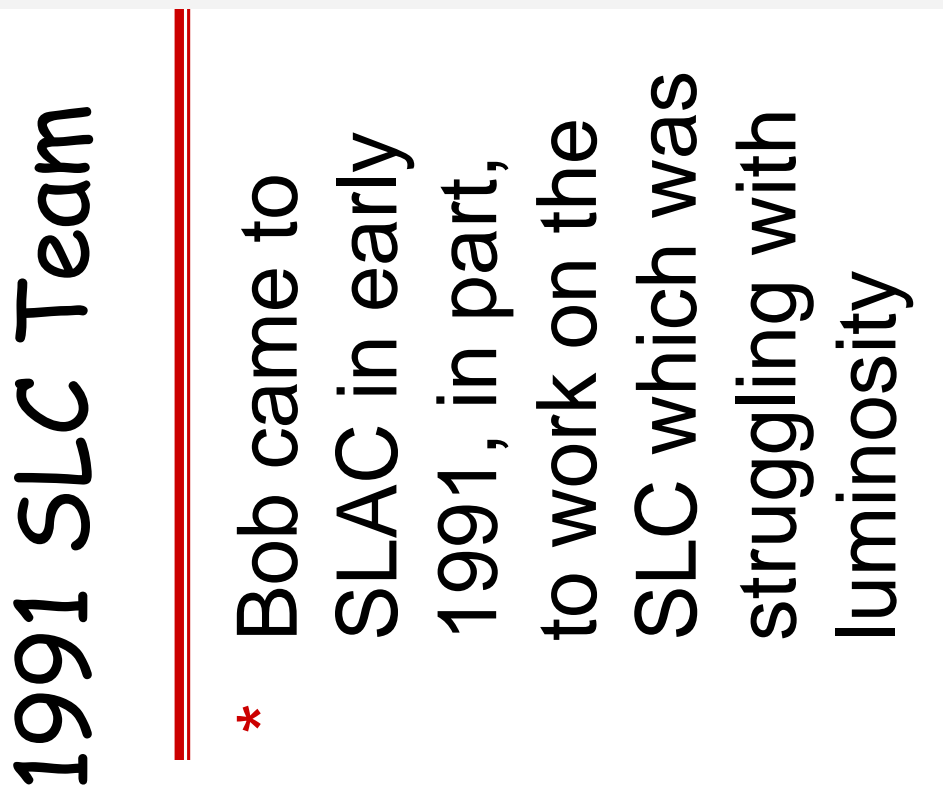

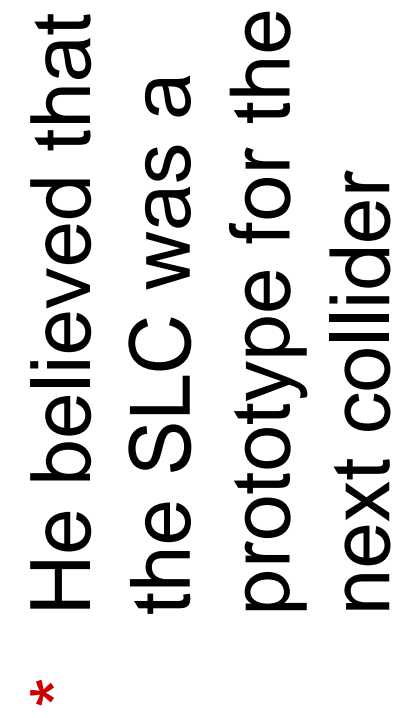

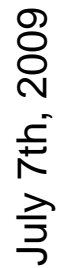

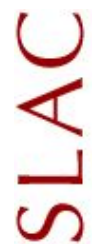




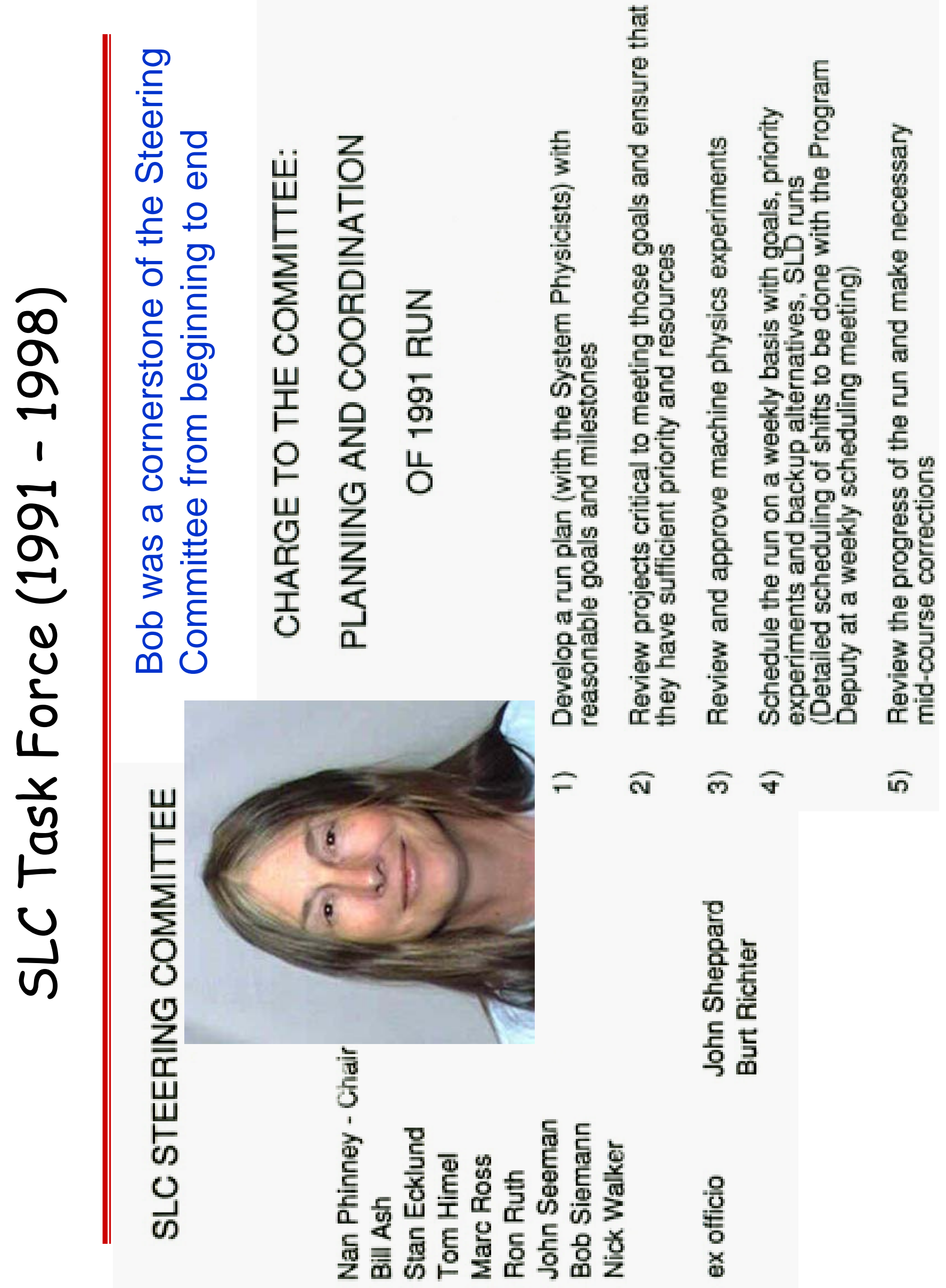




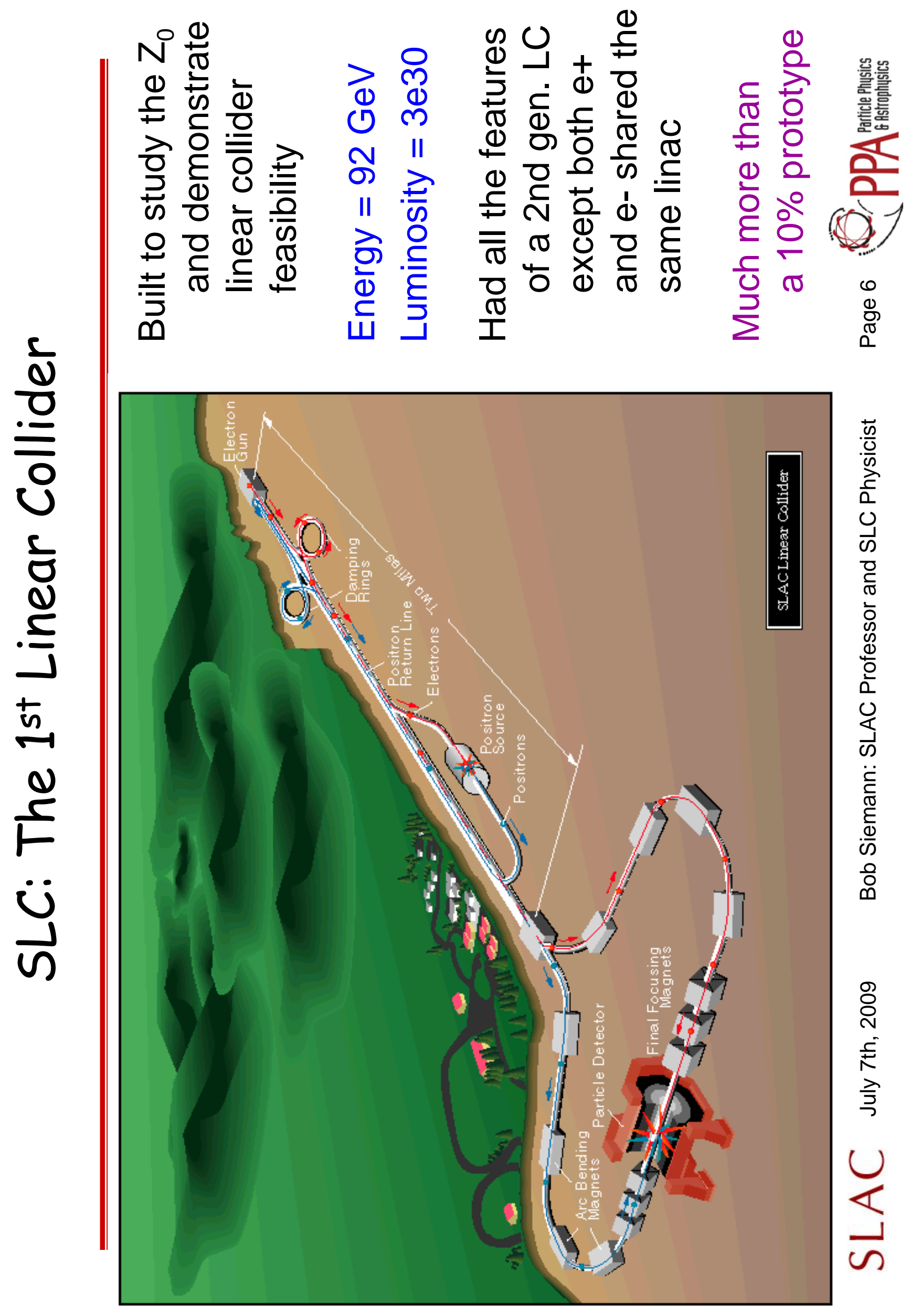




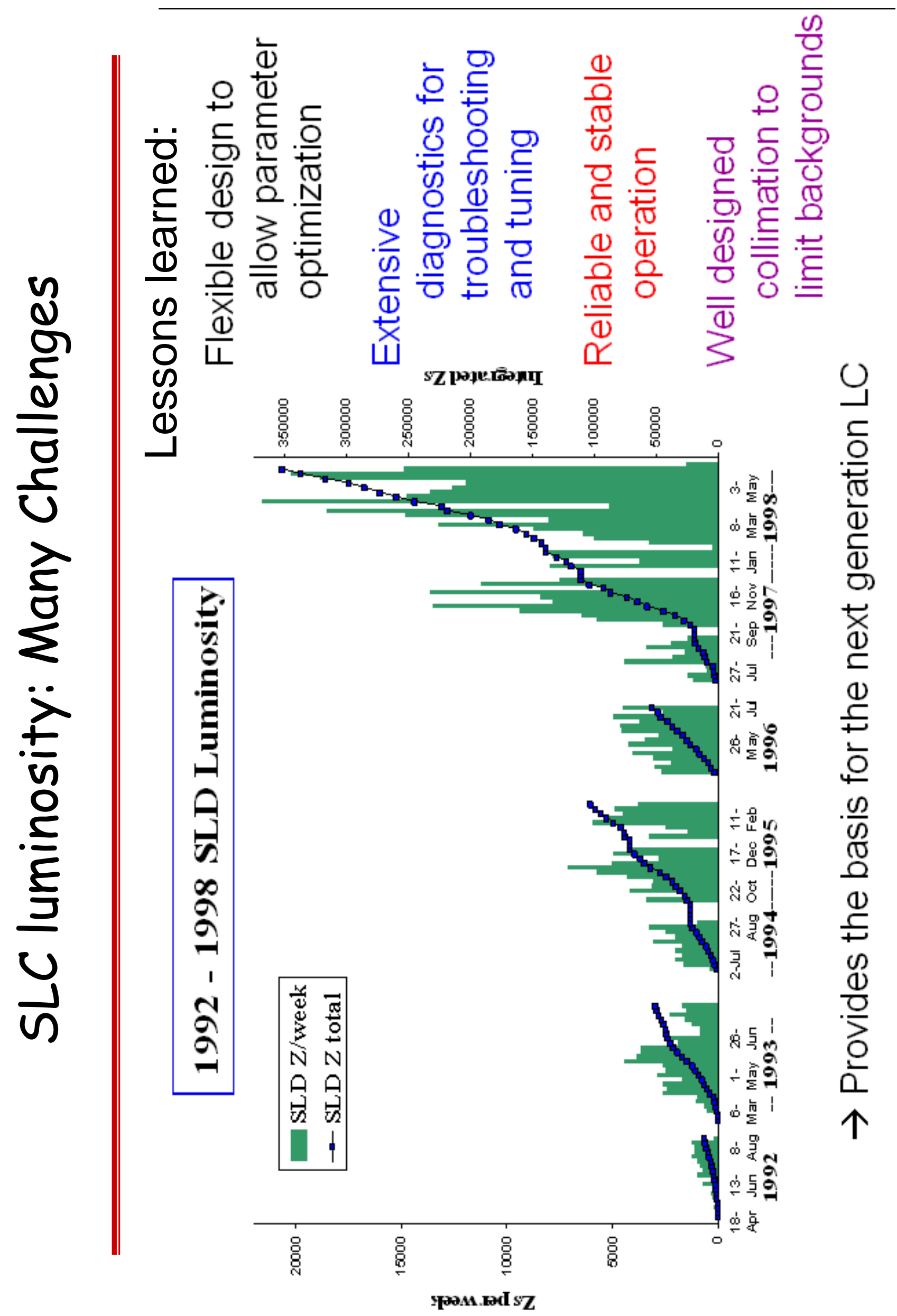



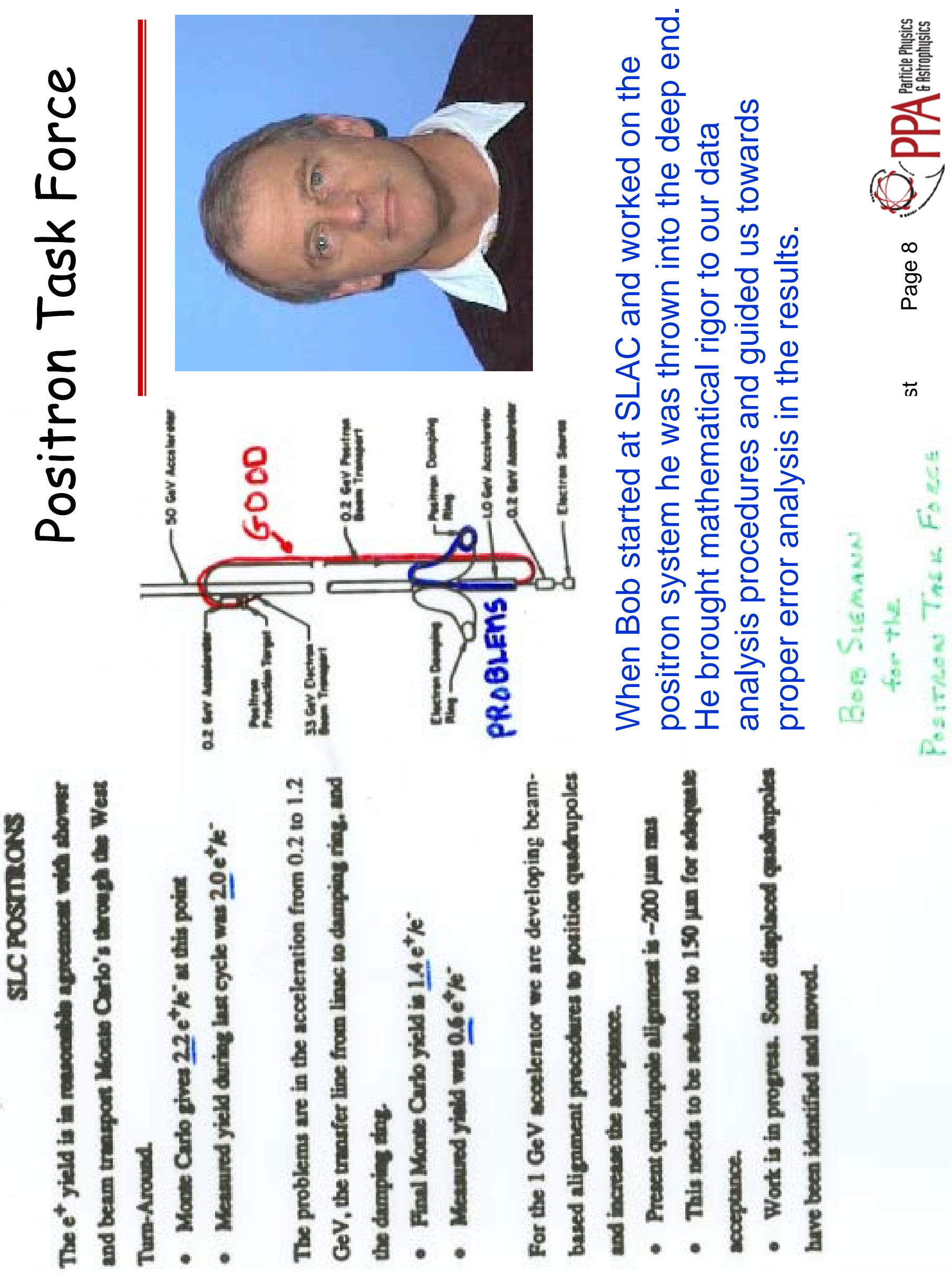

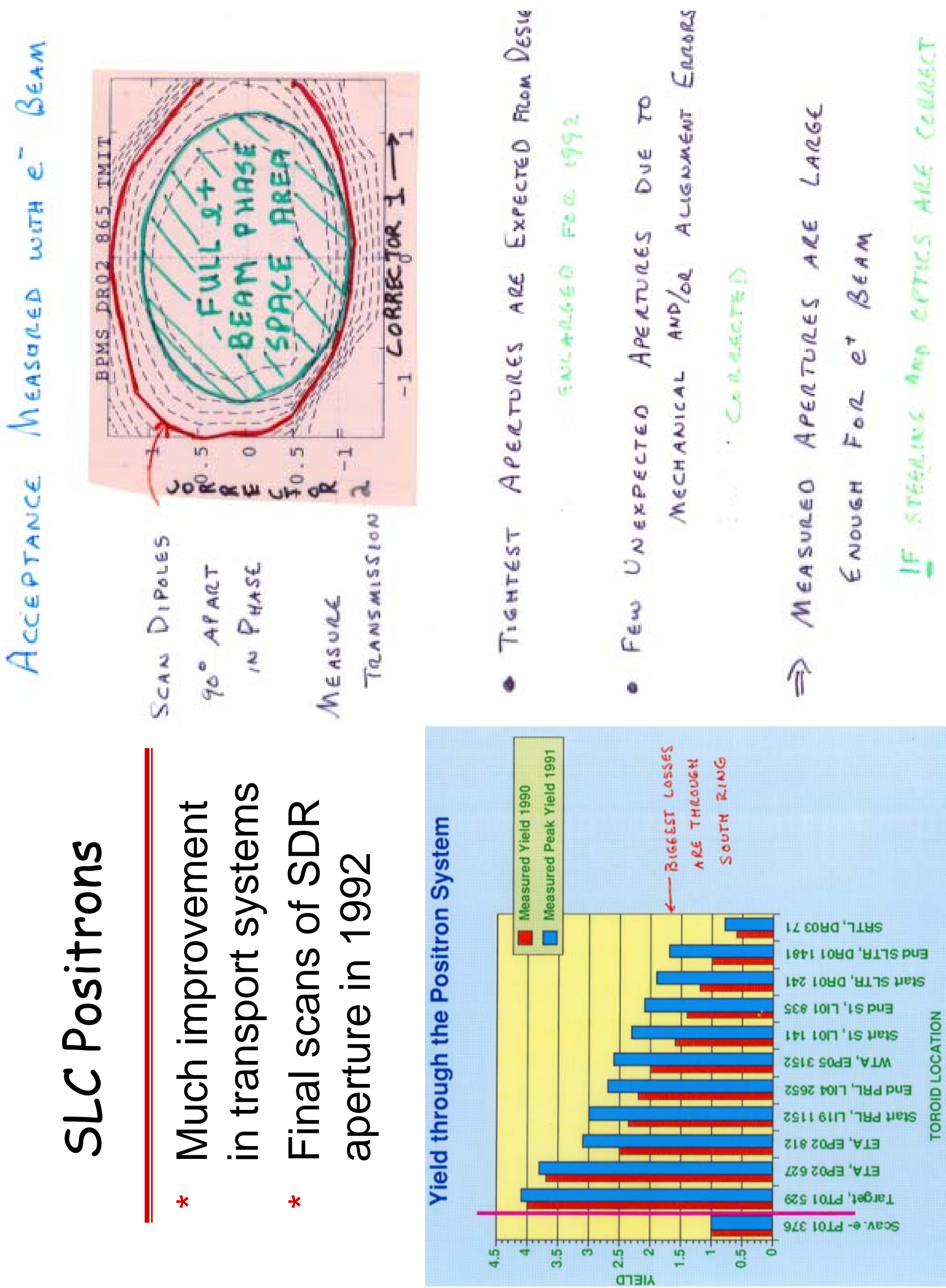


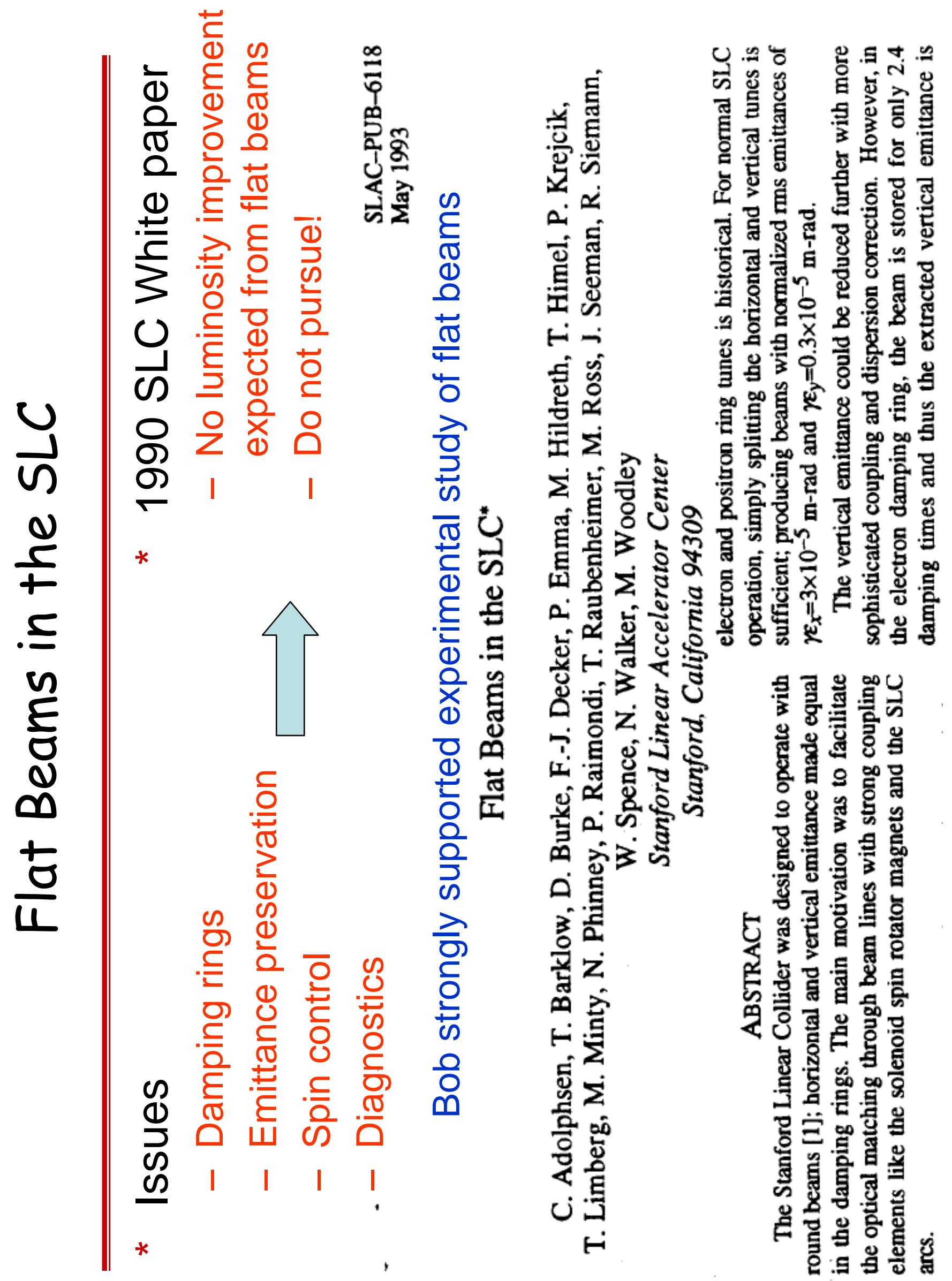



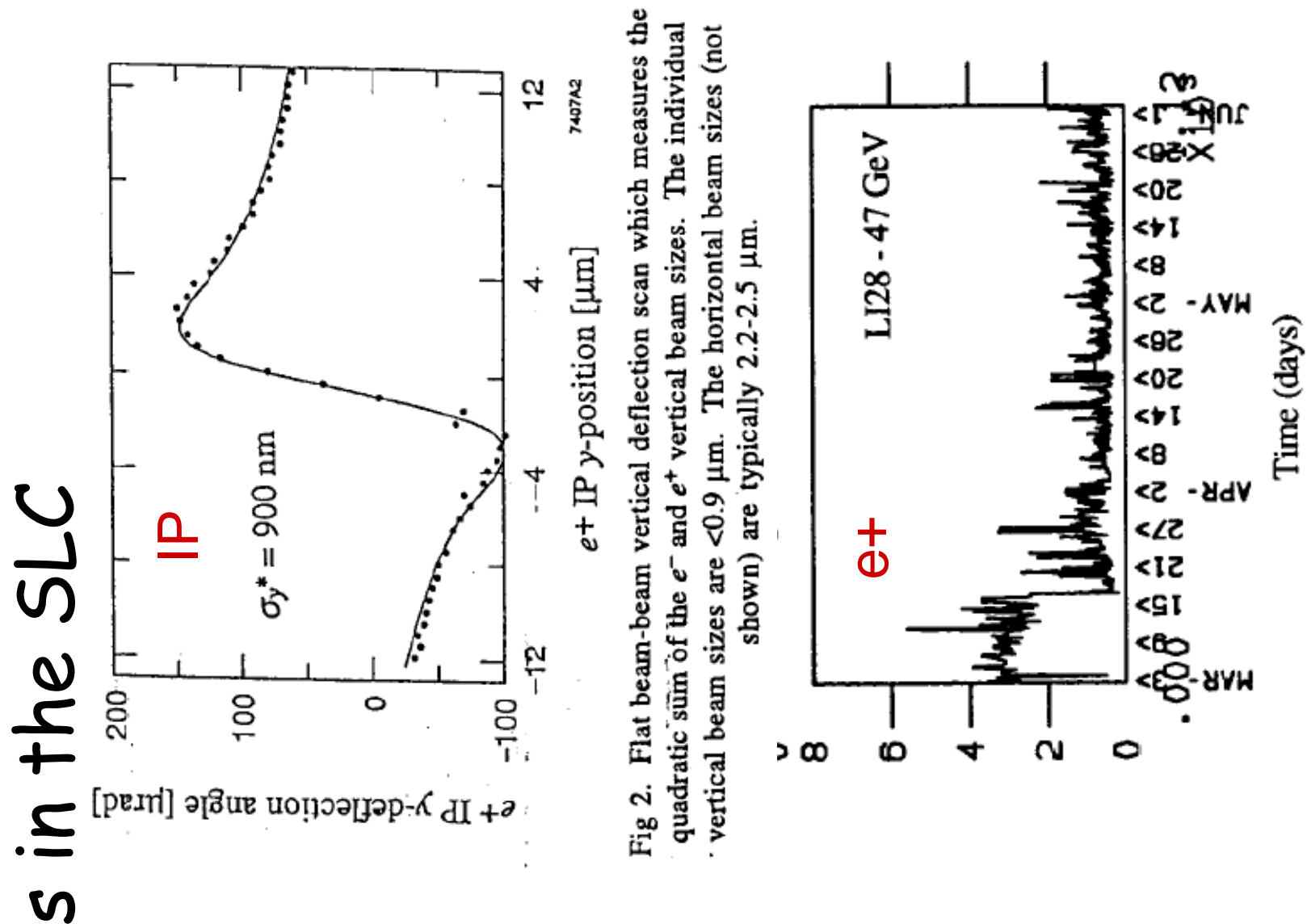

รั 敋 壱怘 is ㅎํ 륭 กิ 品 ๘ 응 은

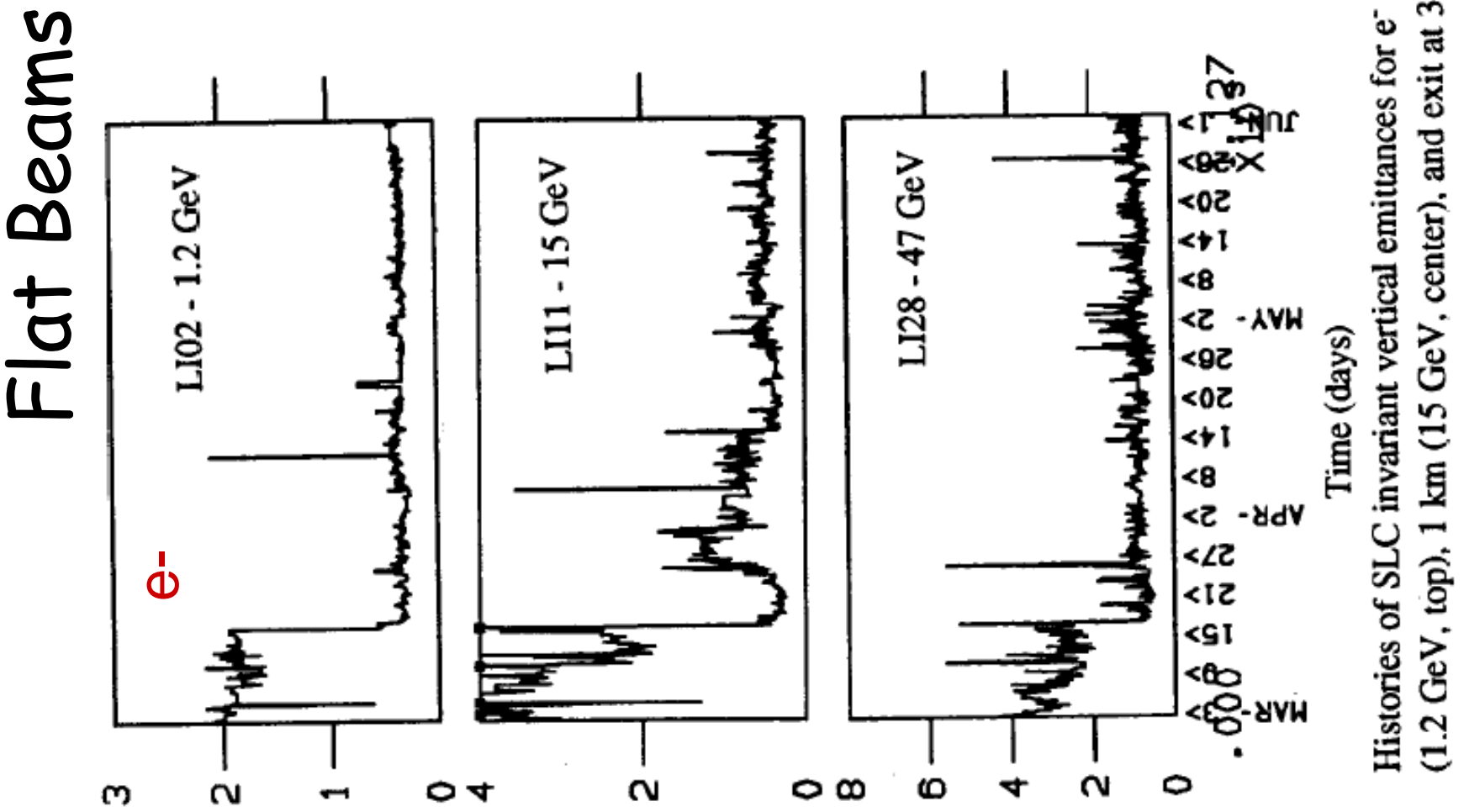

$$
\text { (UI-I s-0I .) } \Lambda_{3} \ell
$$




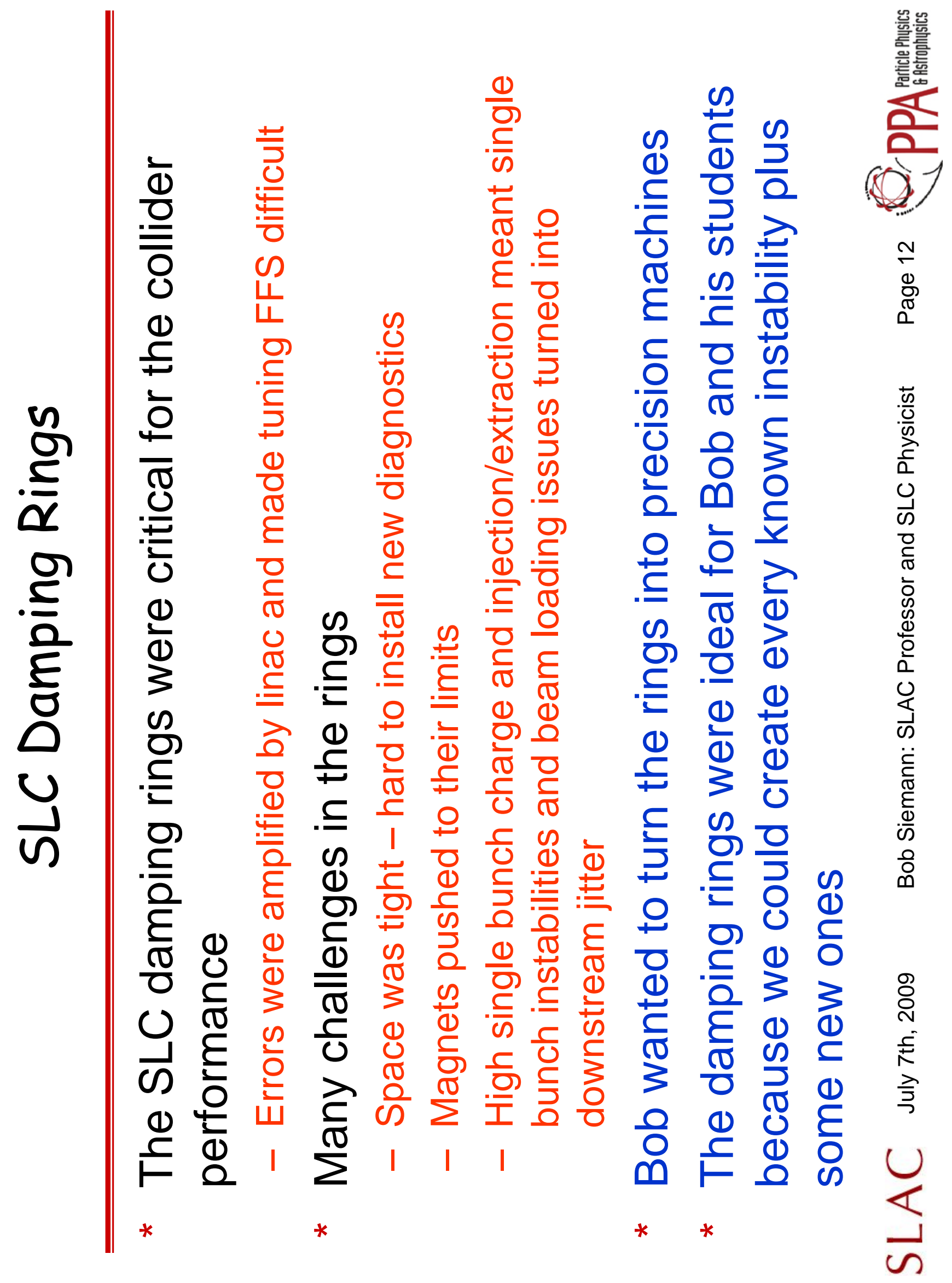




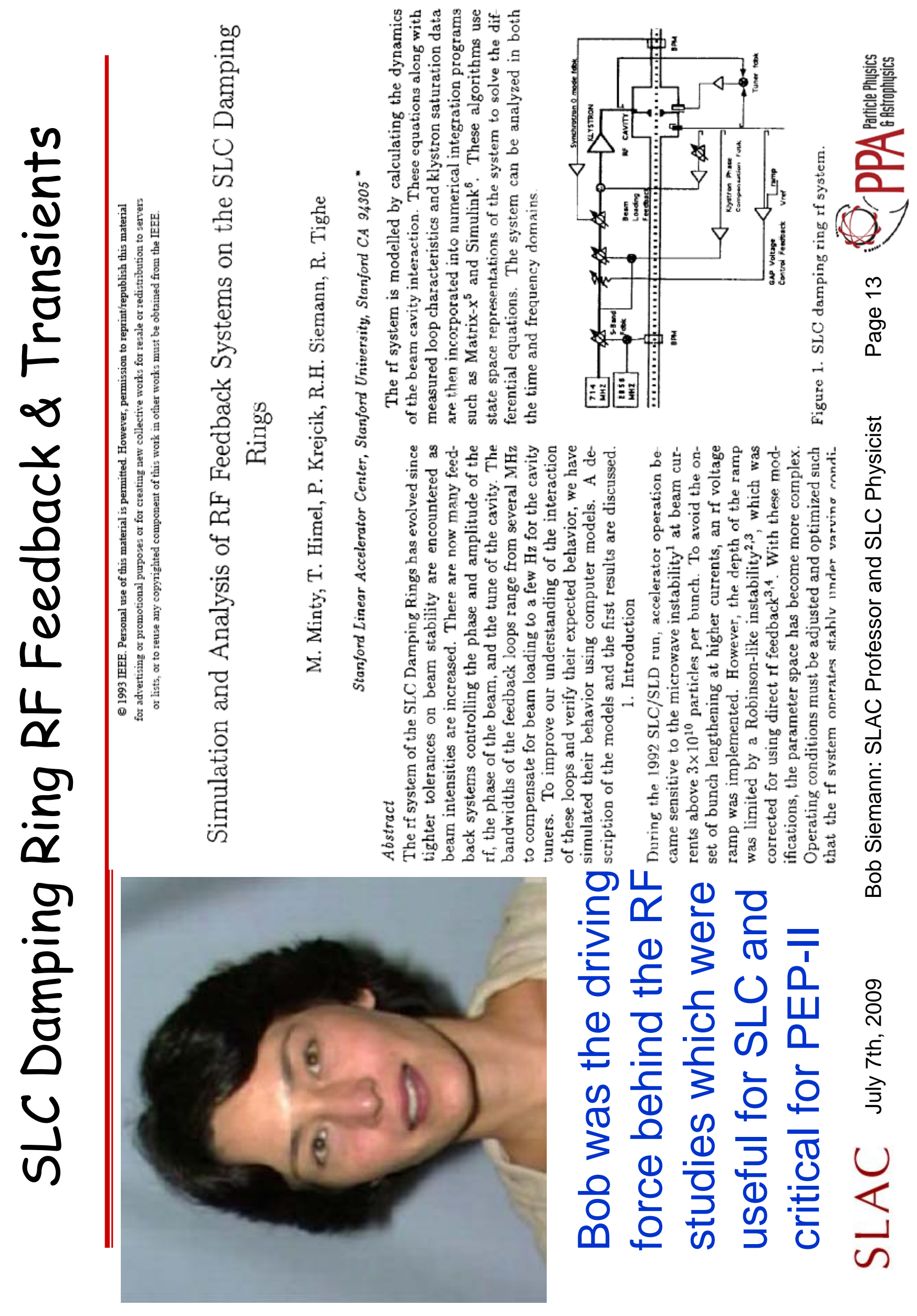




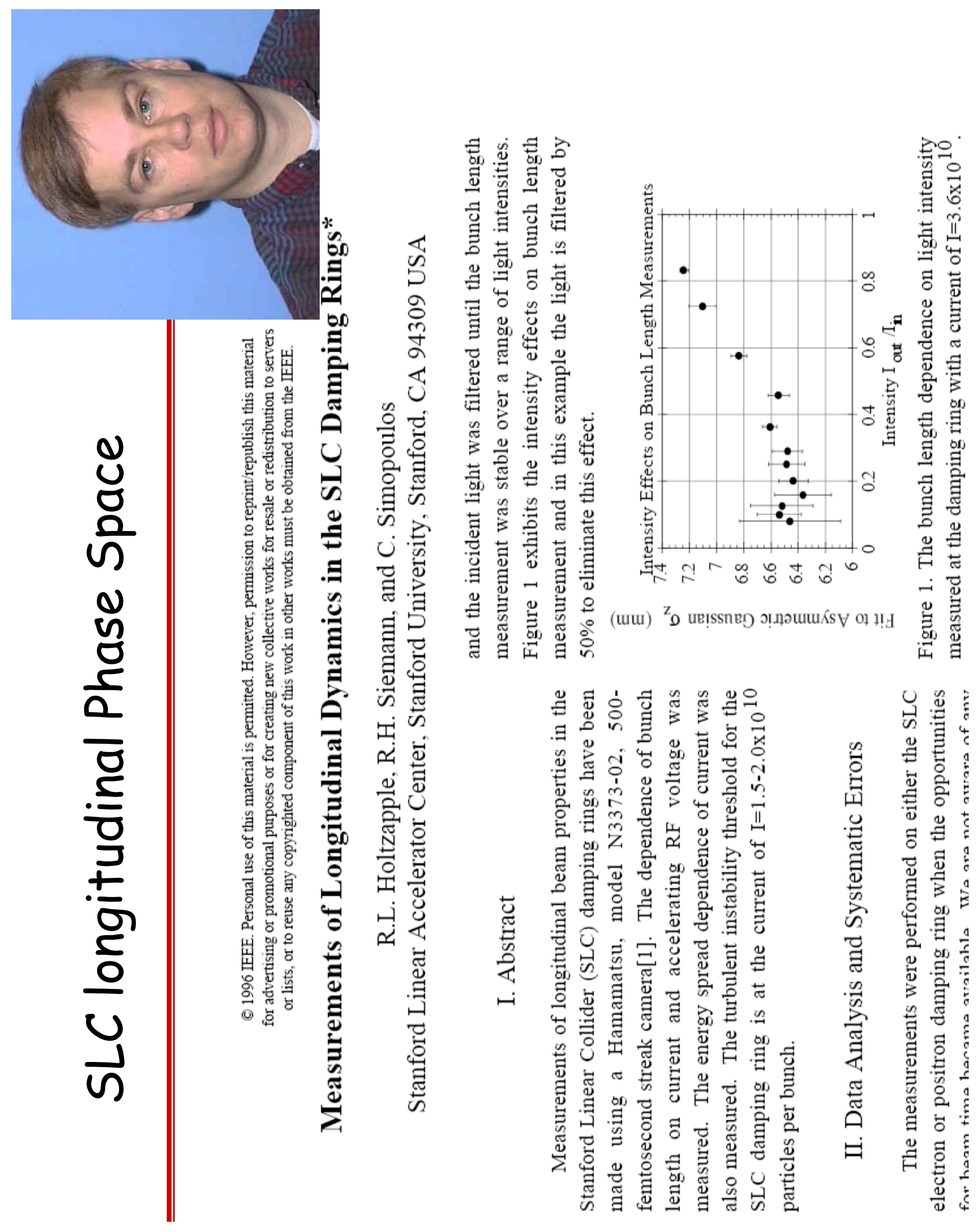




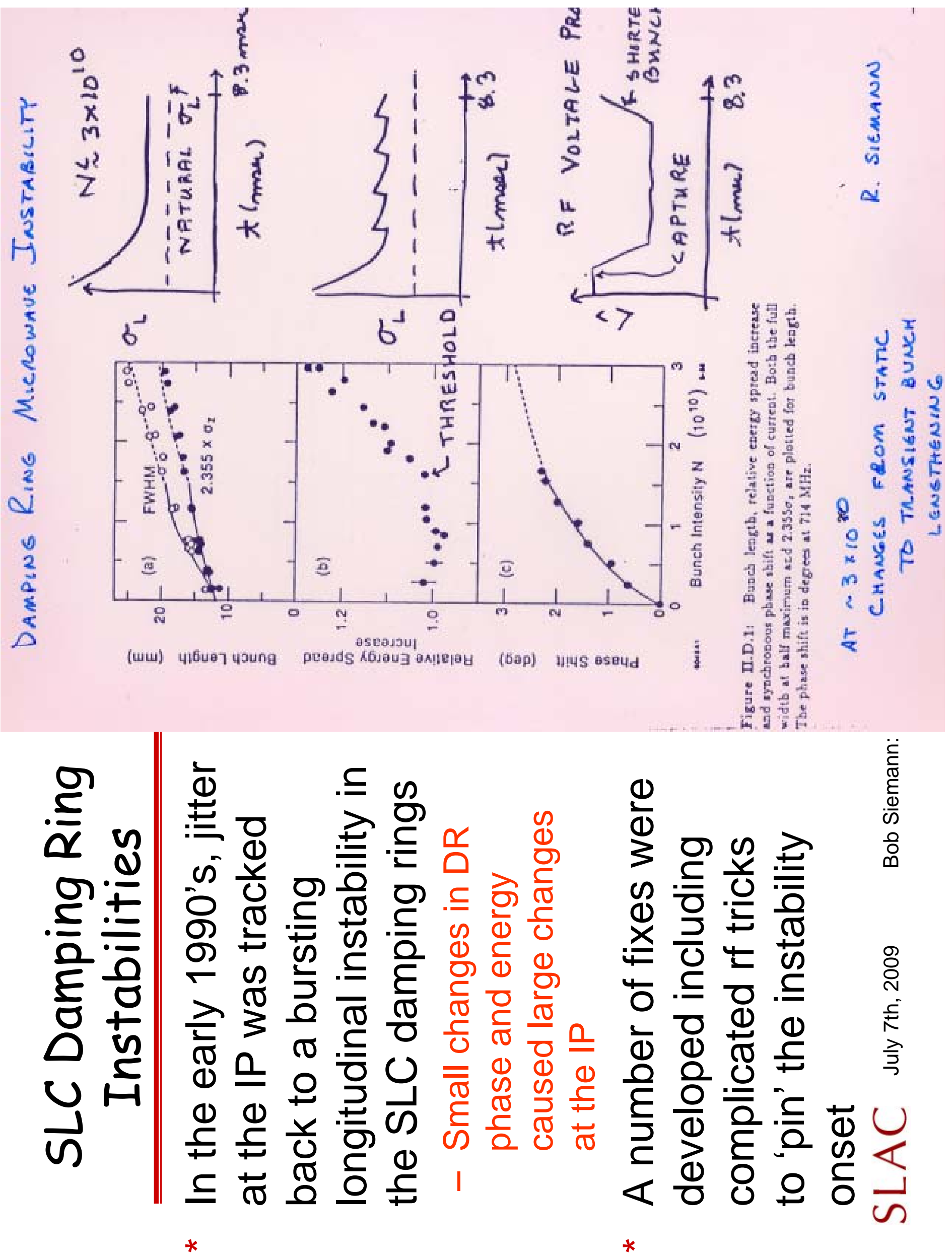




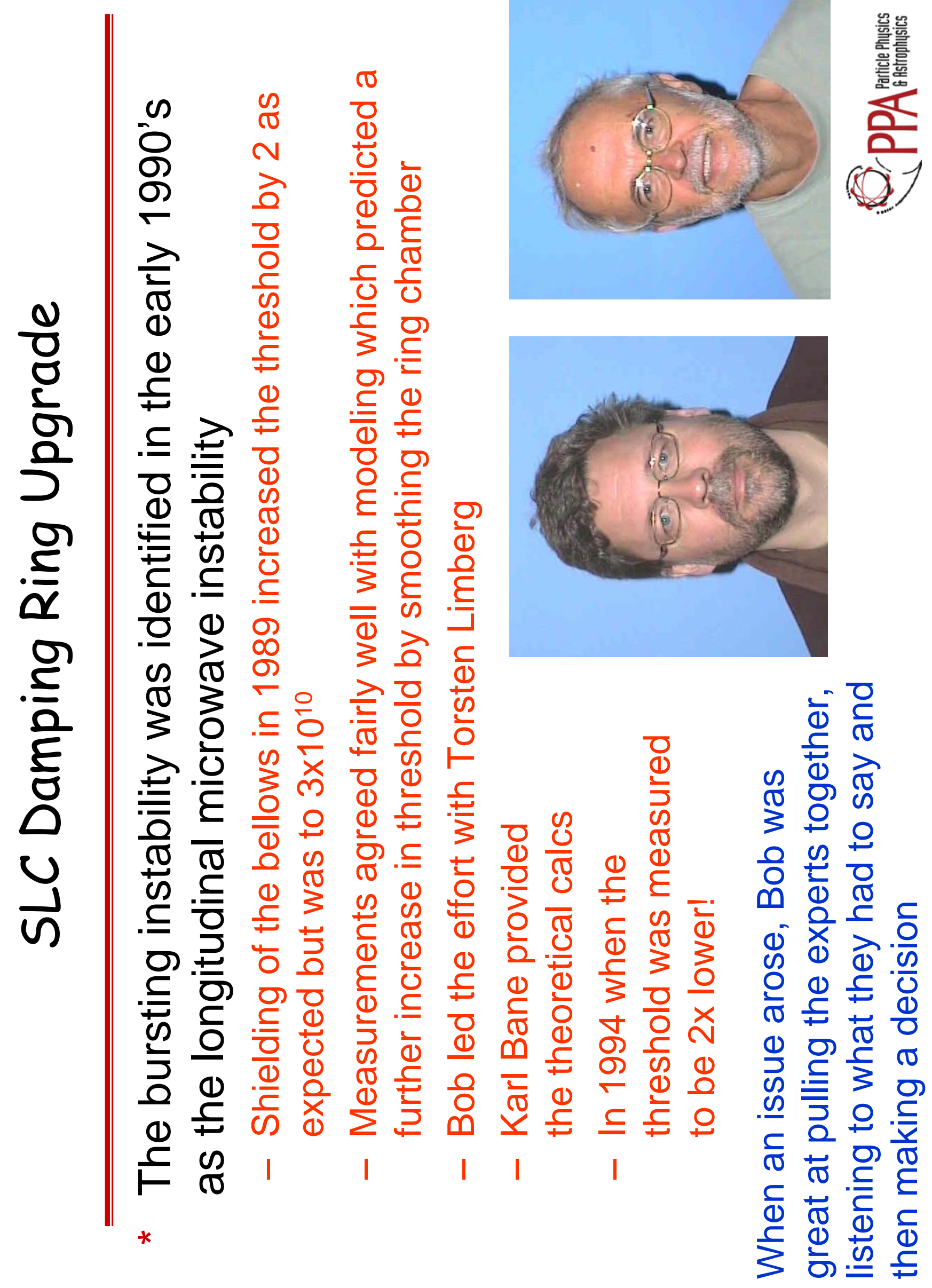




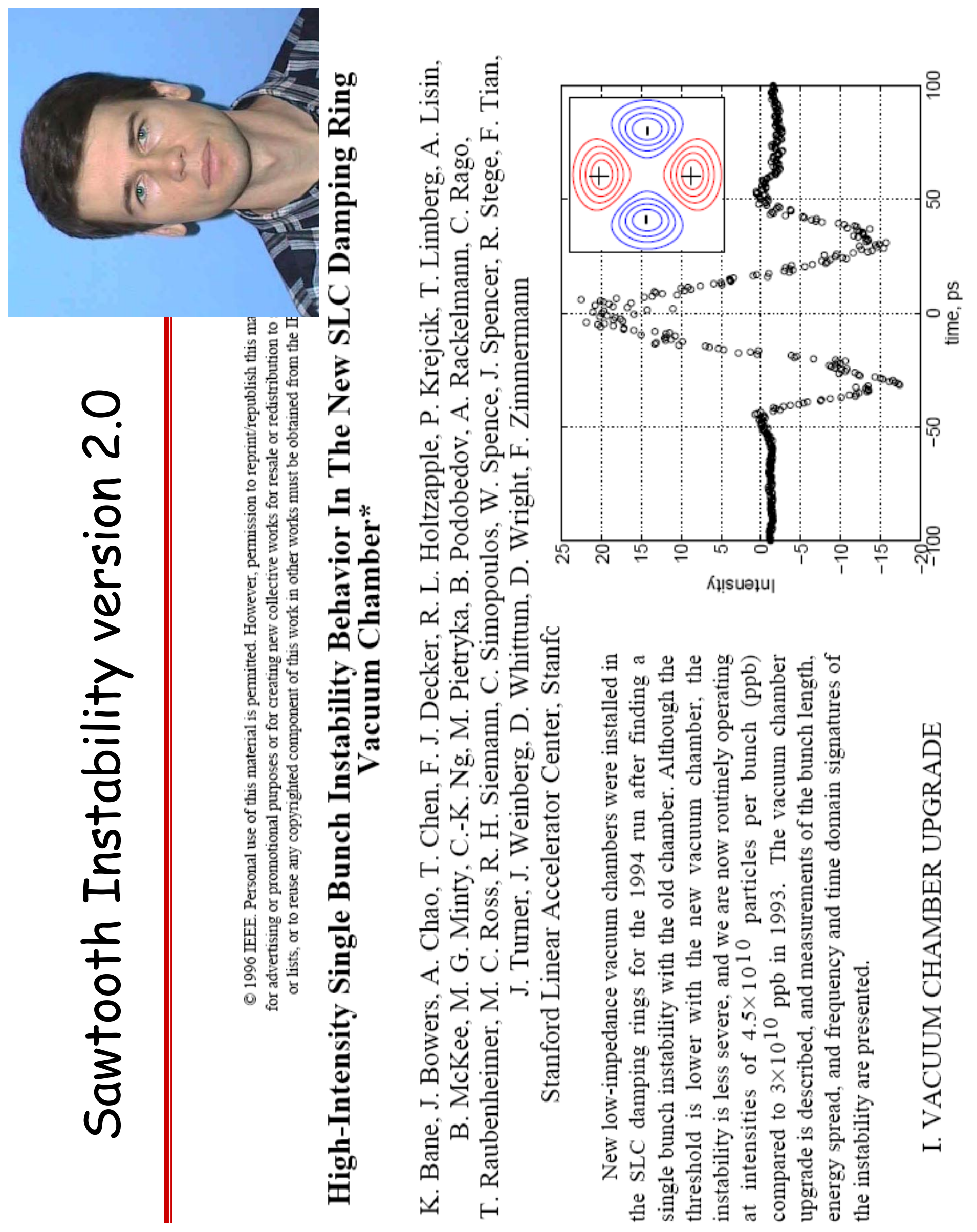



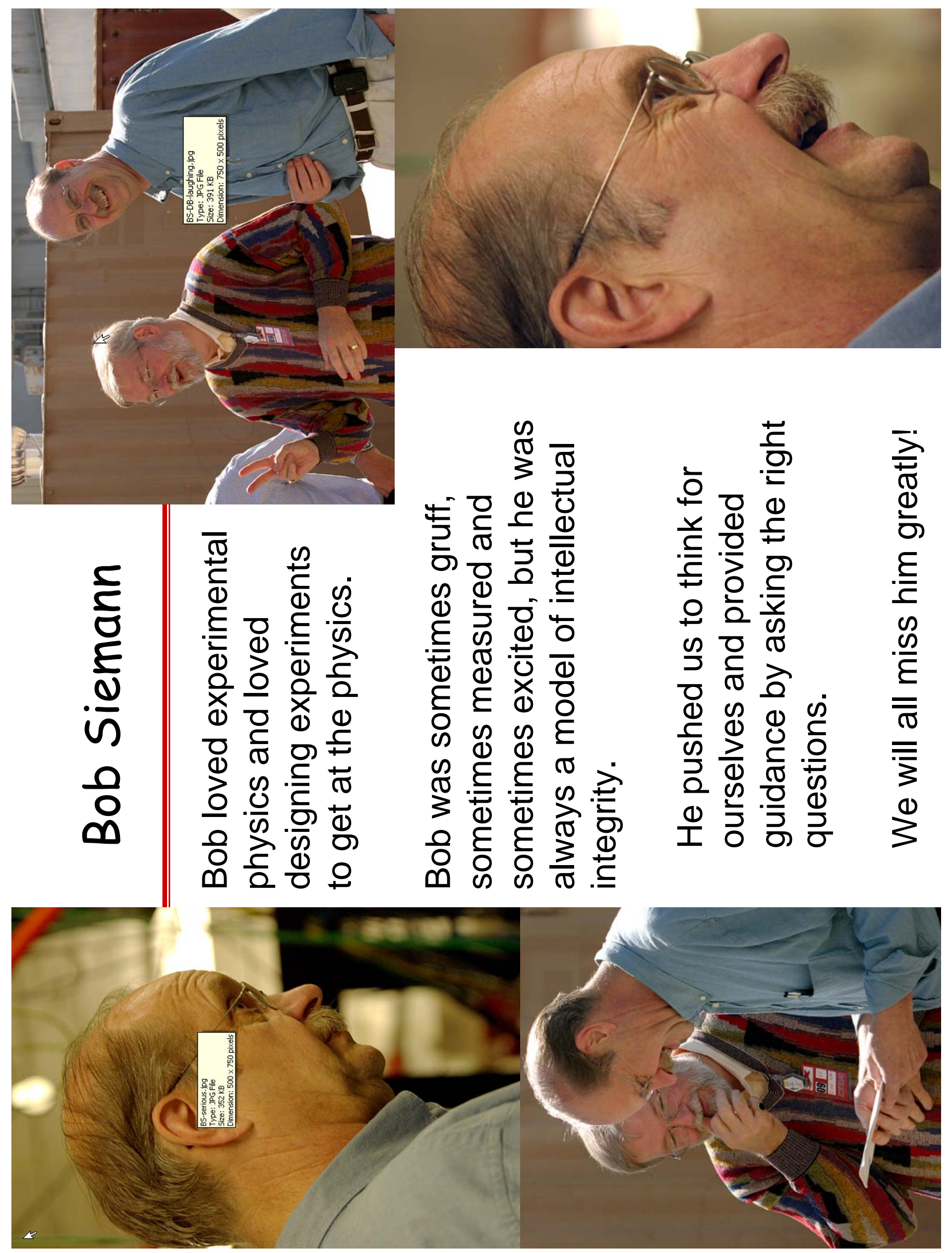

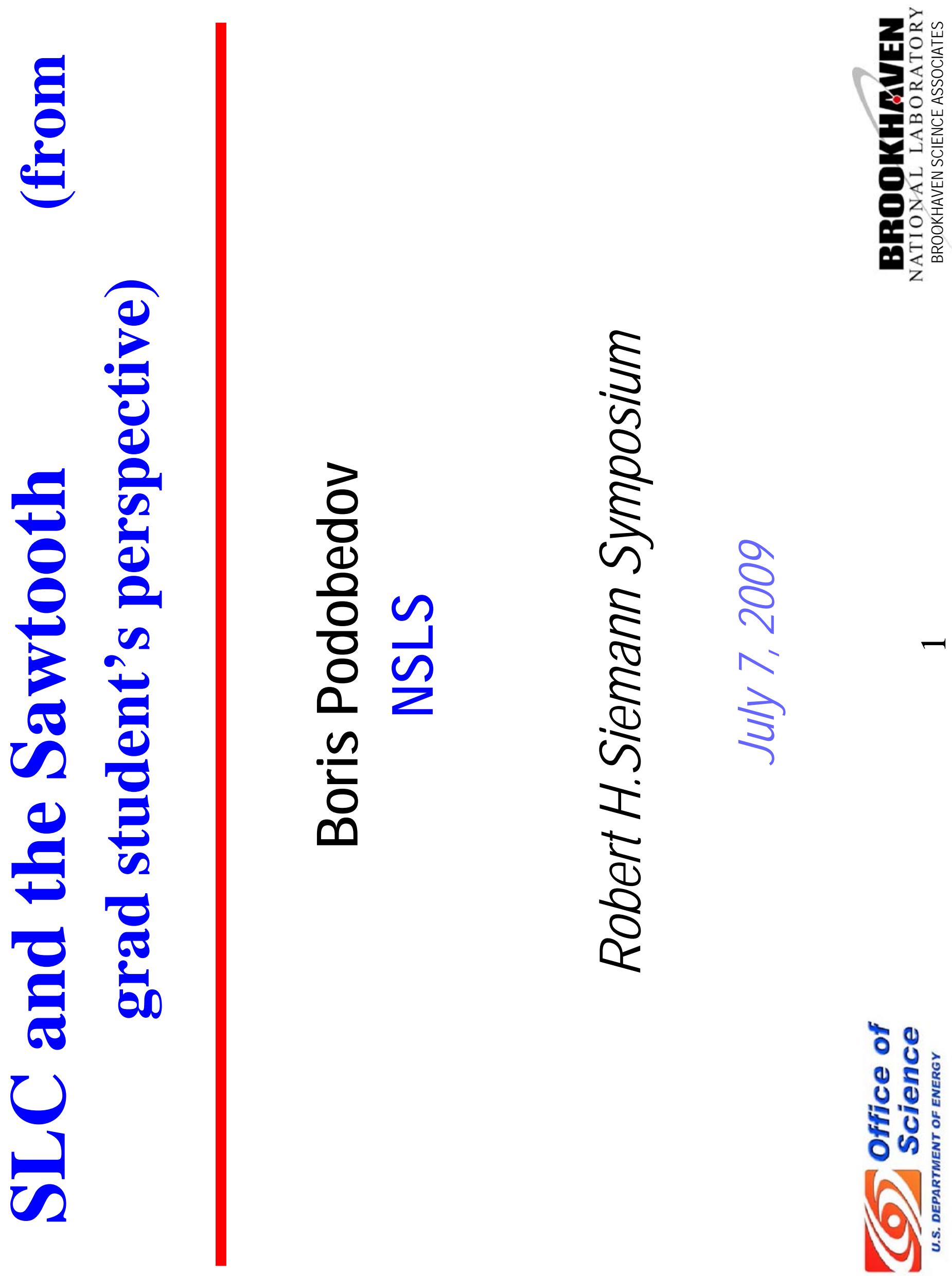


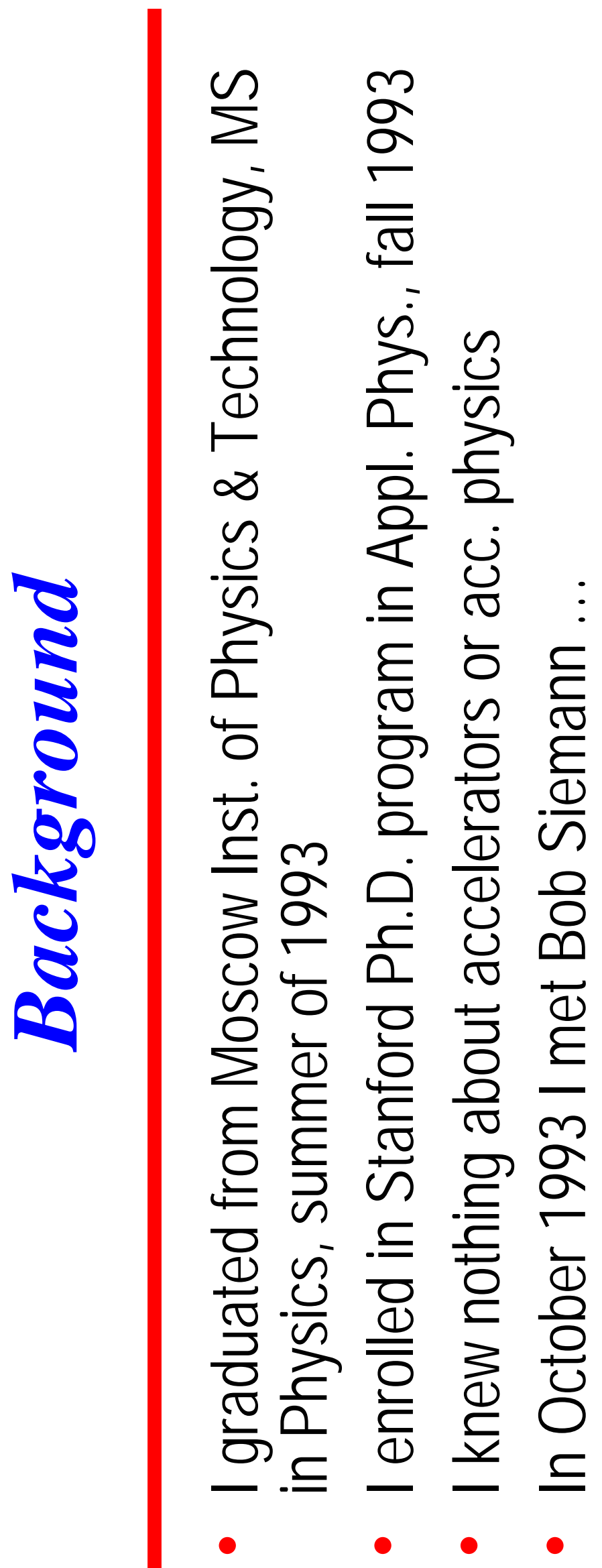




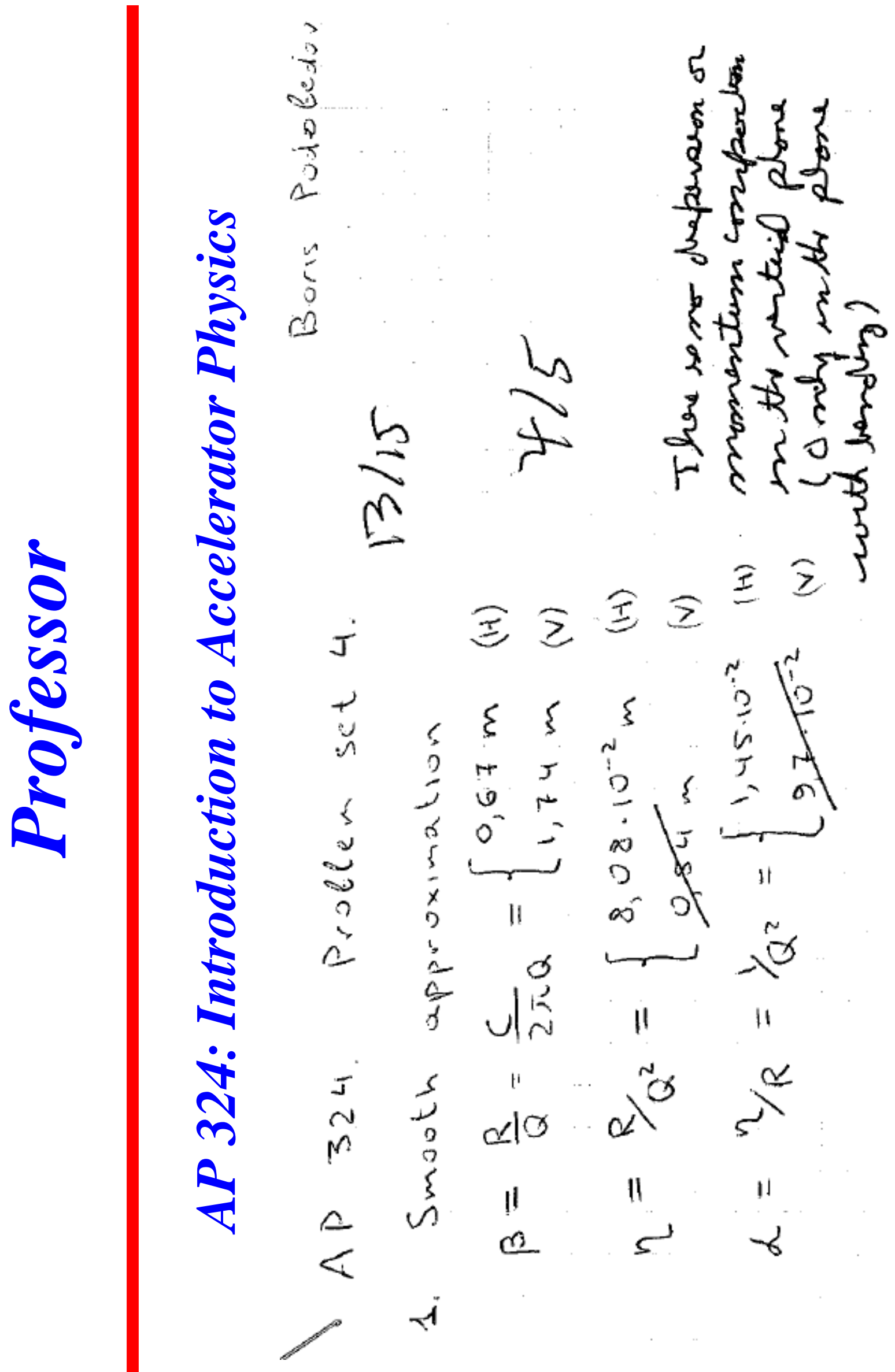


僉
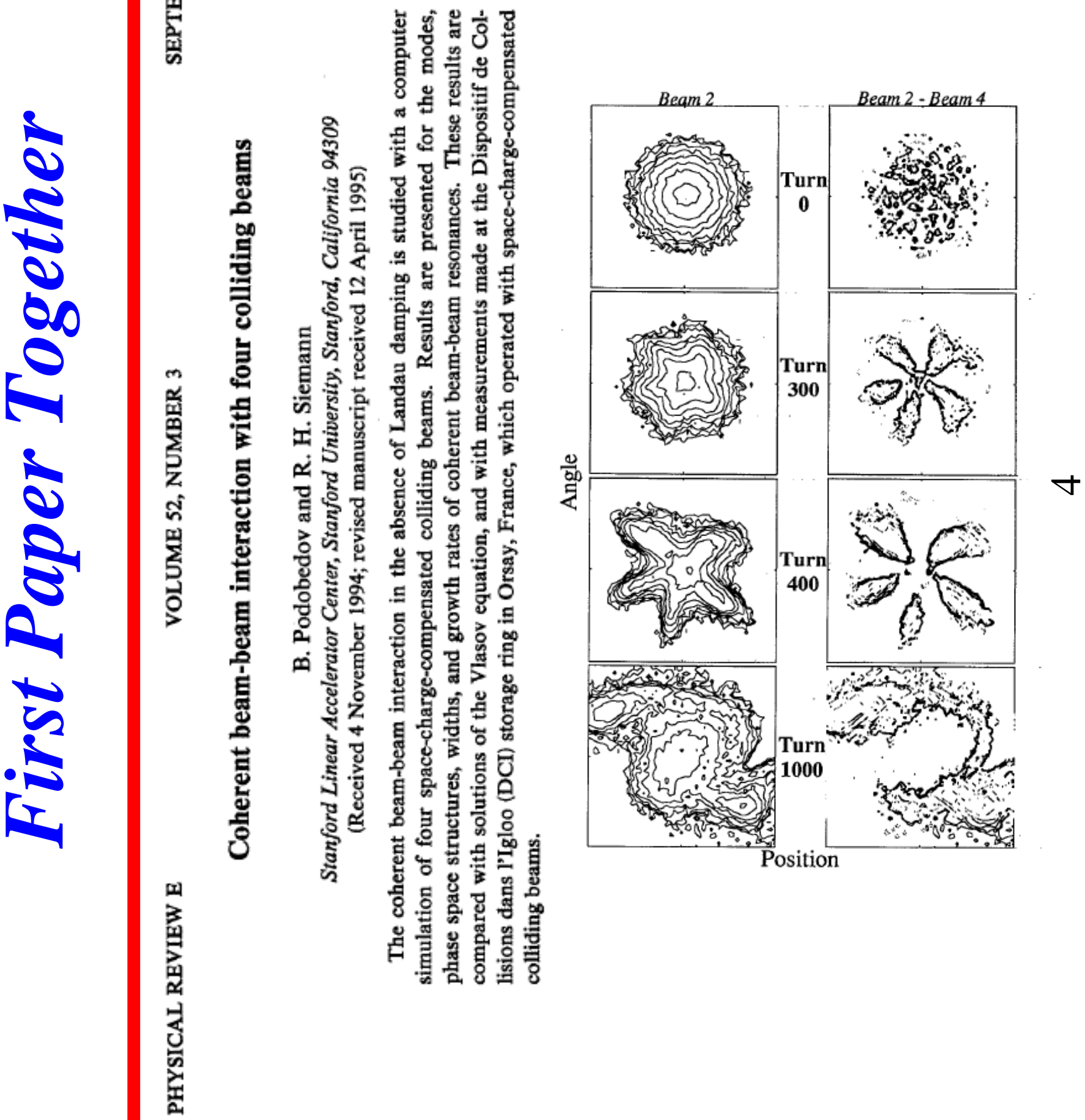

瓷 


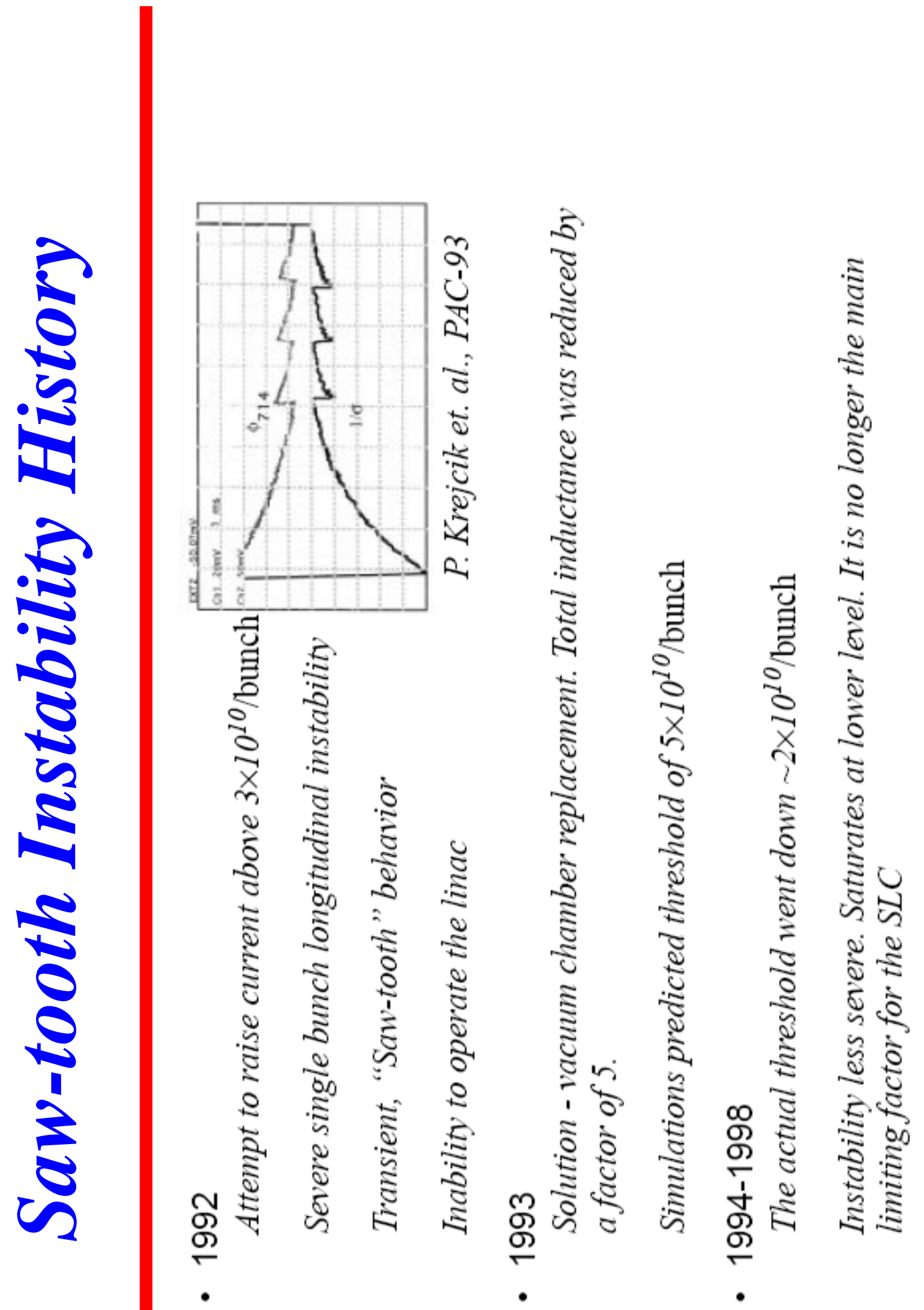

ธ 


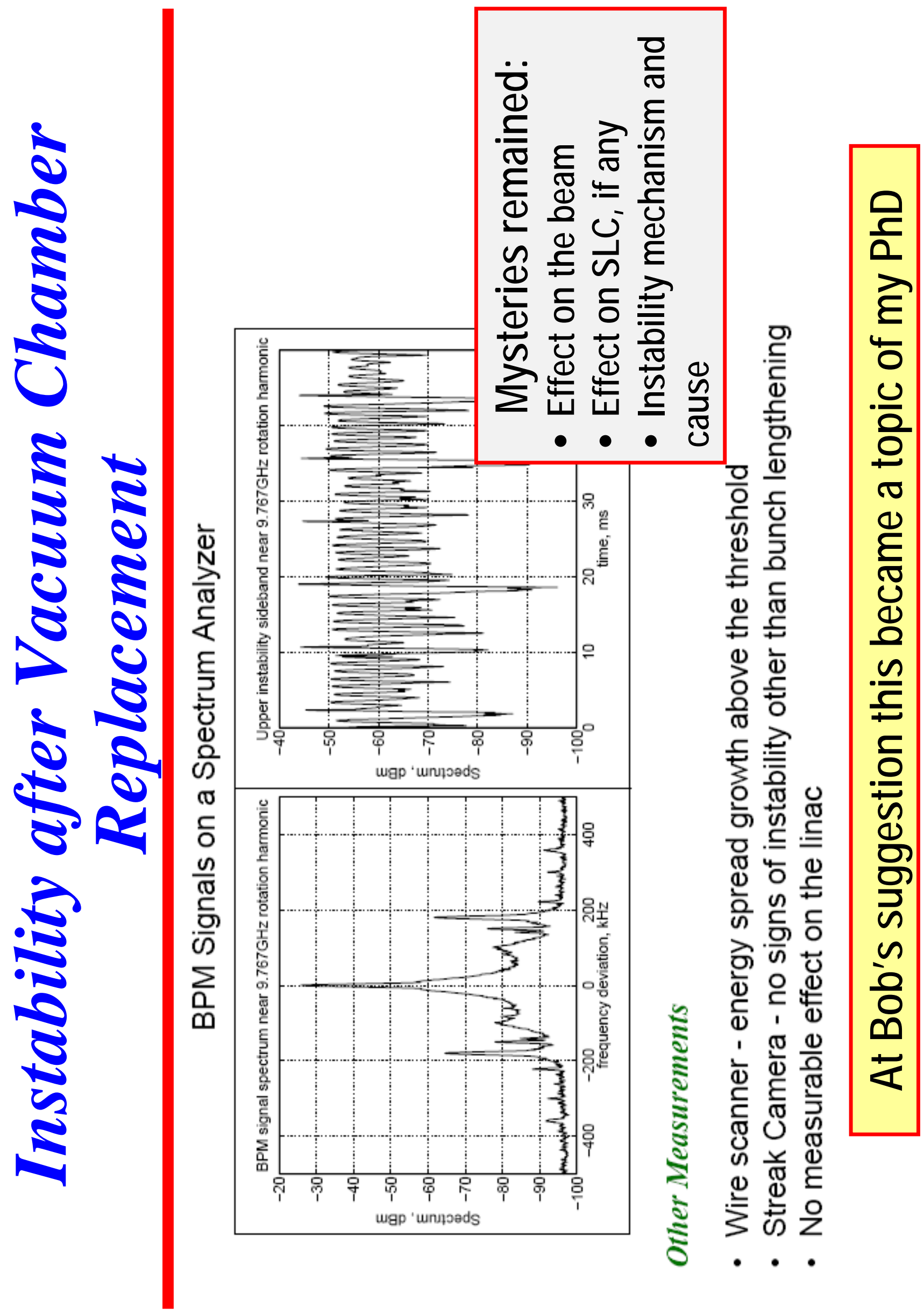




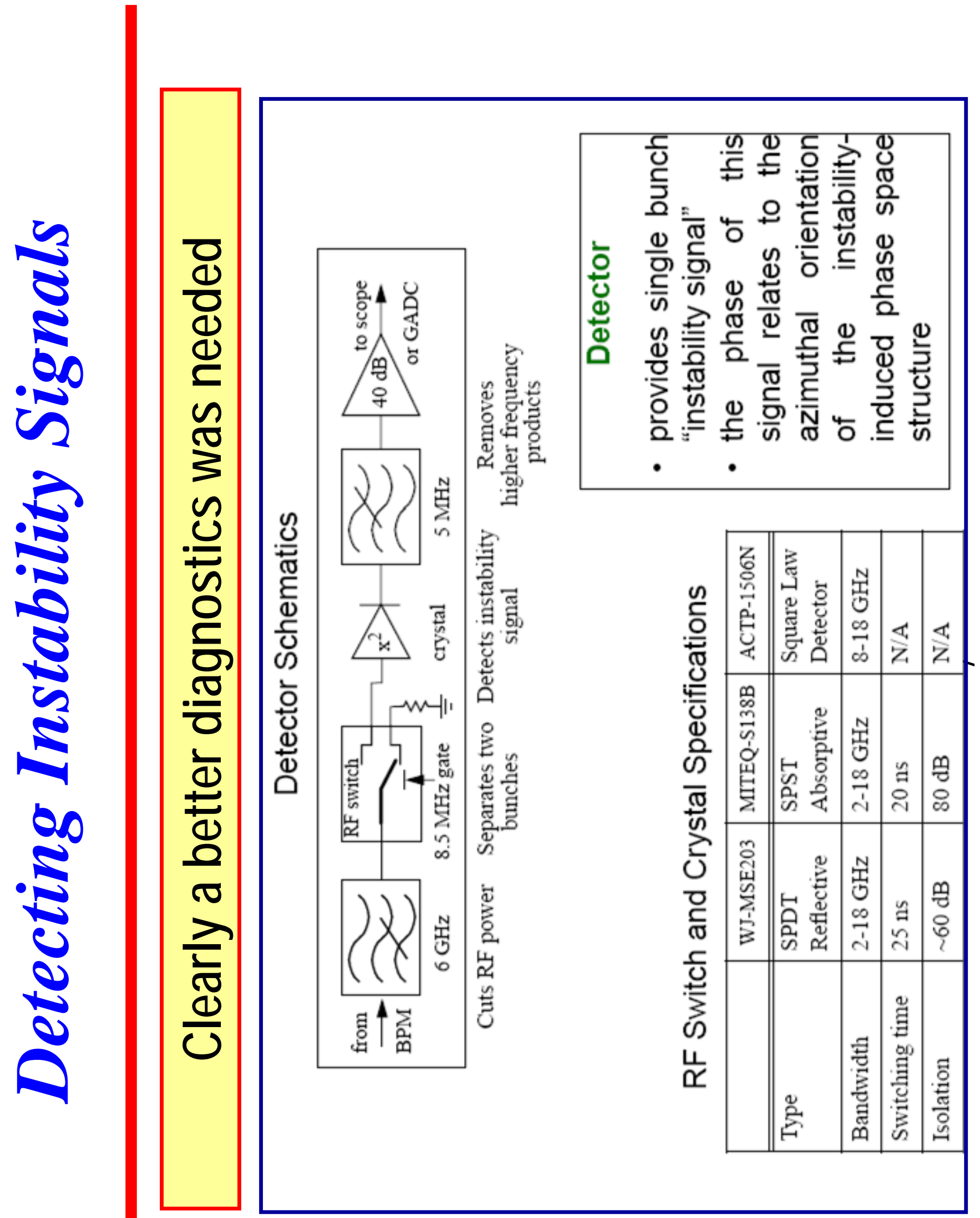




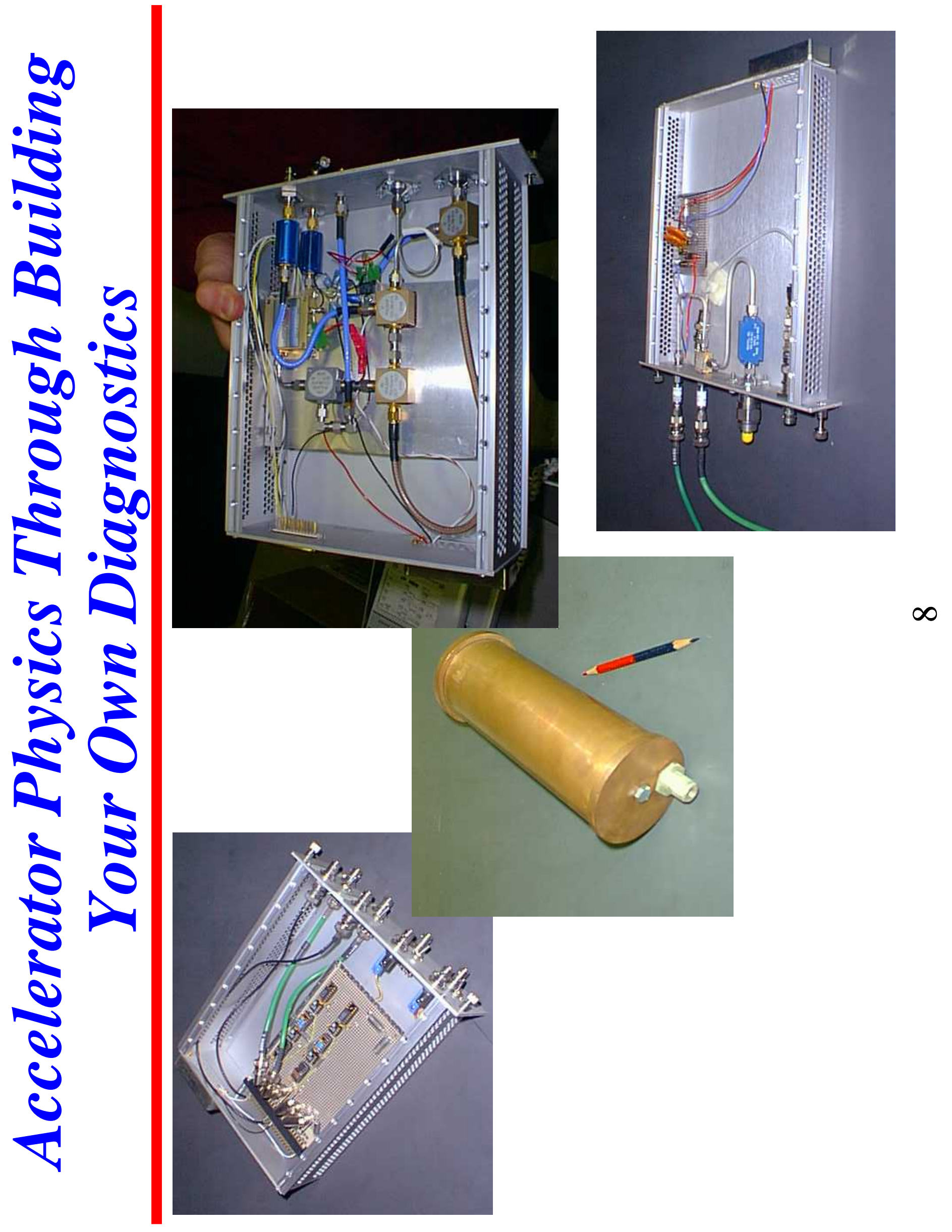




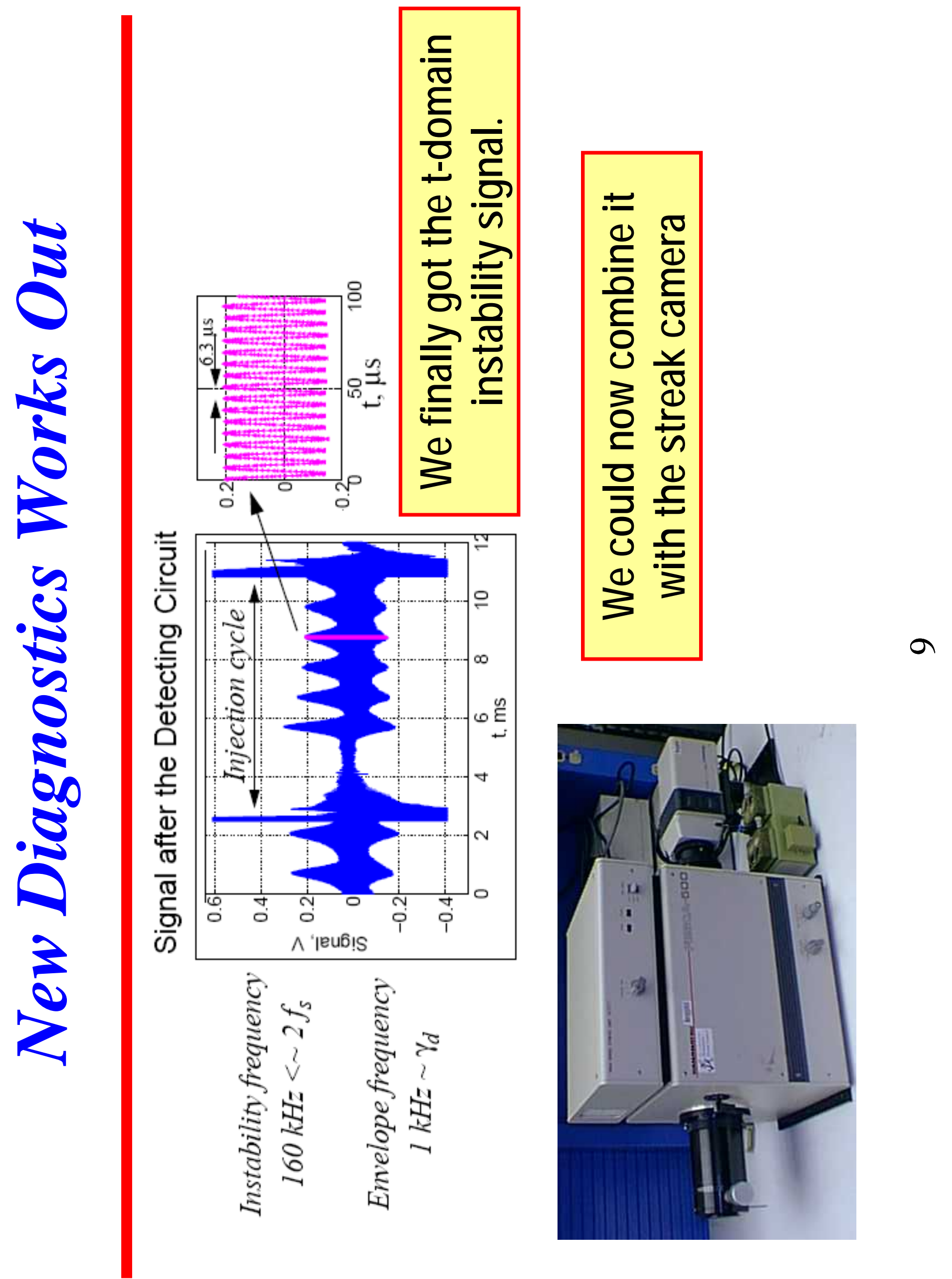




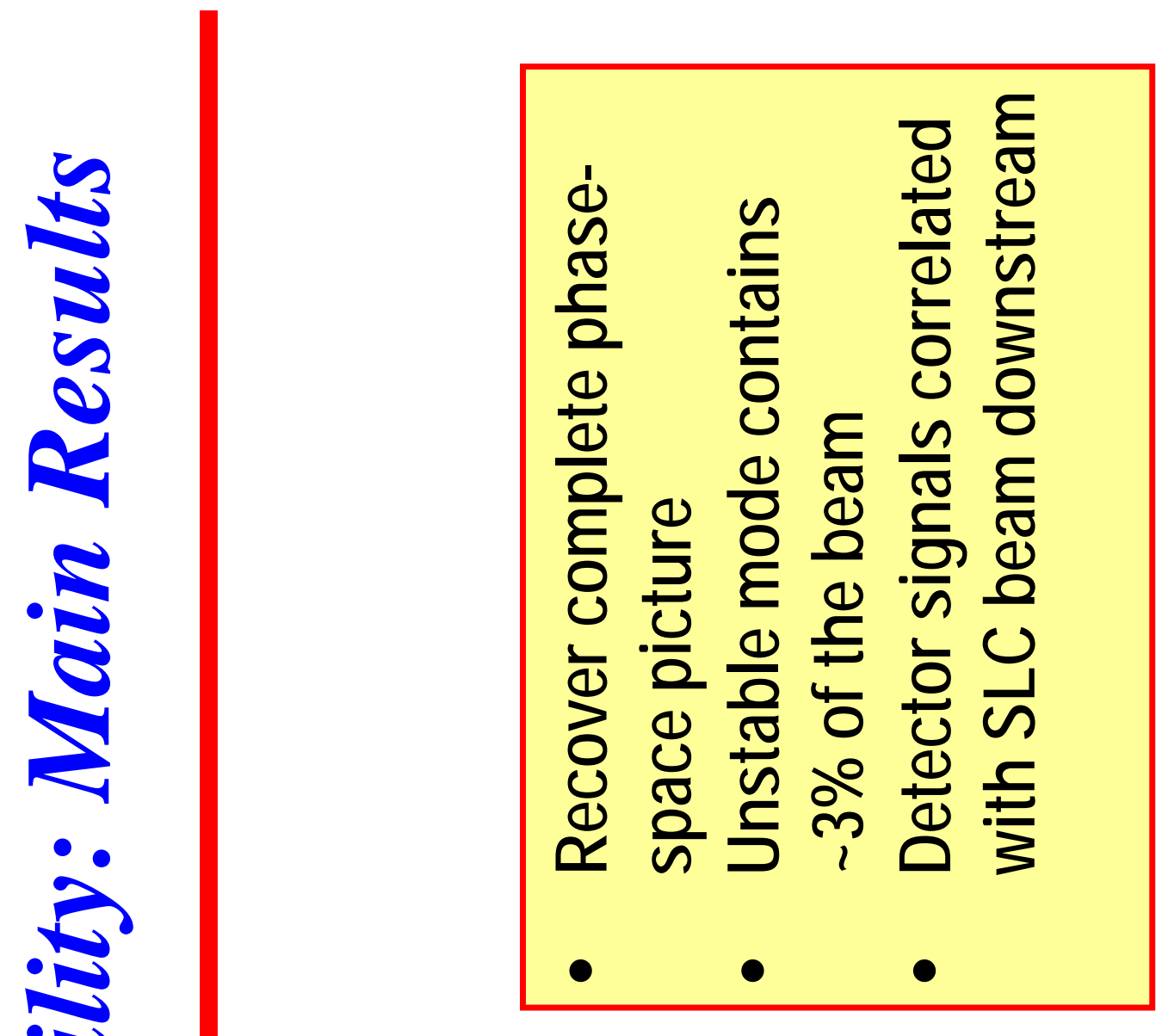

\section{우}
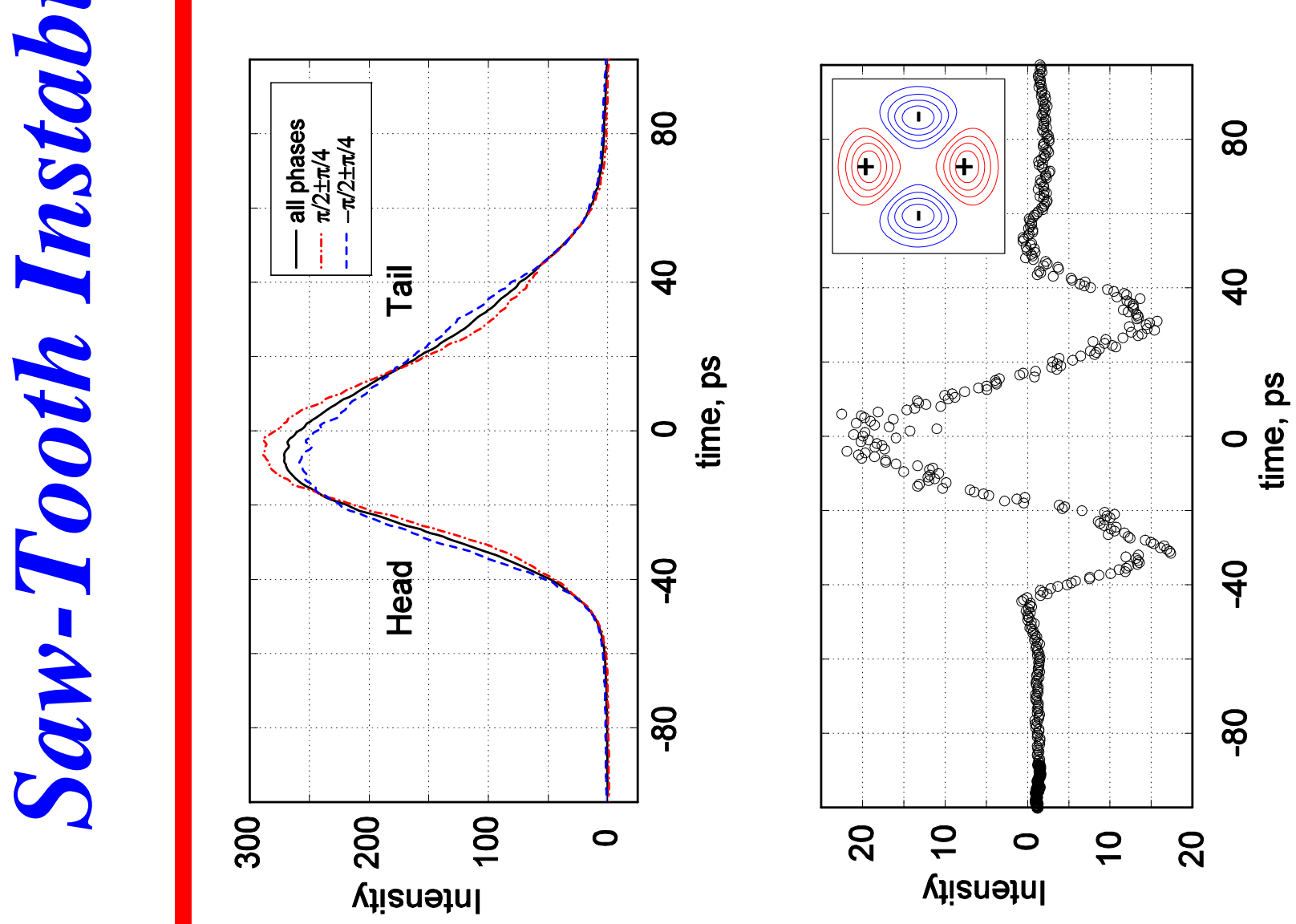


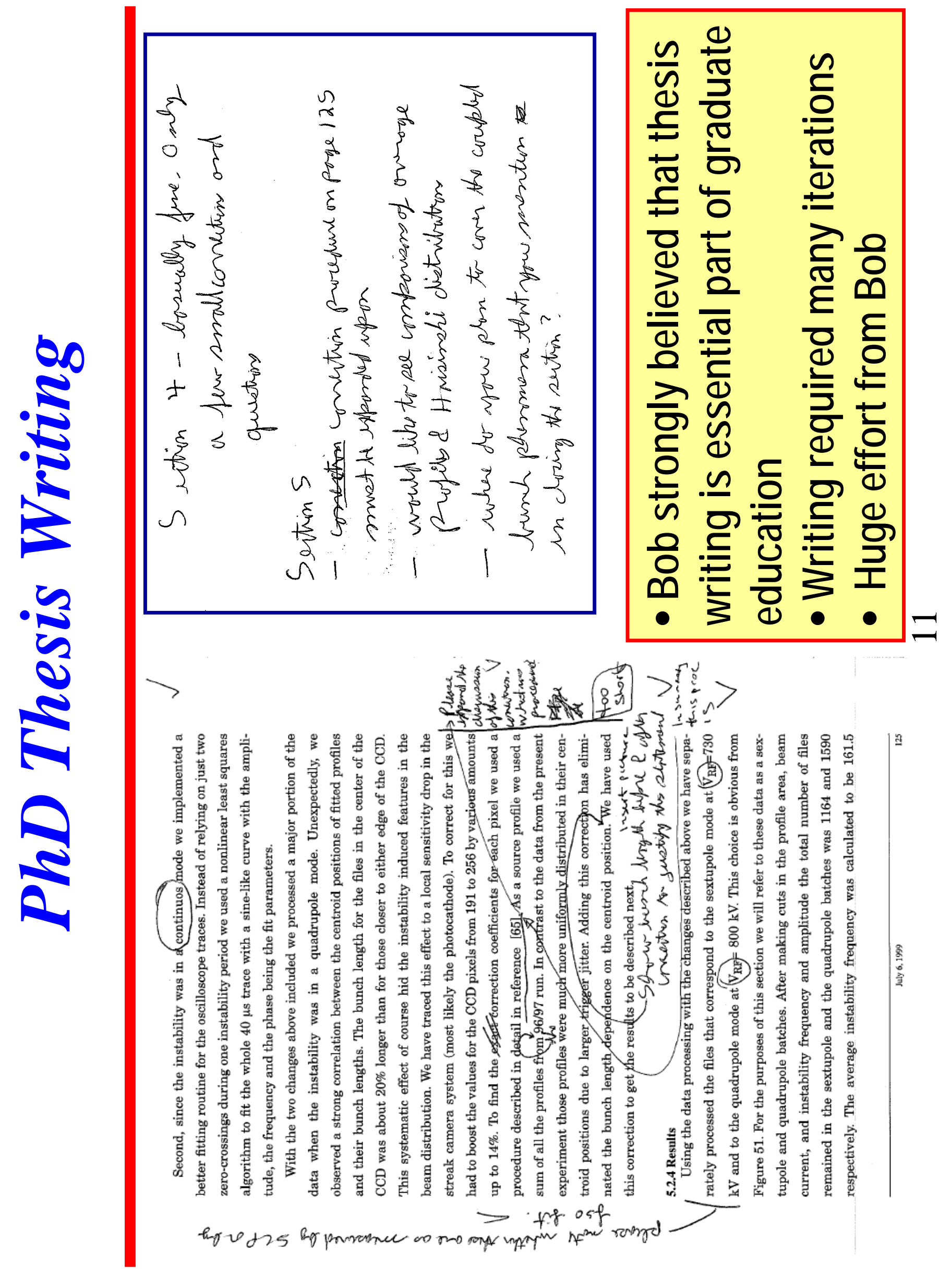




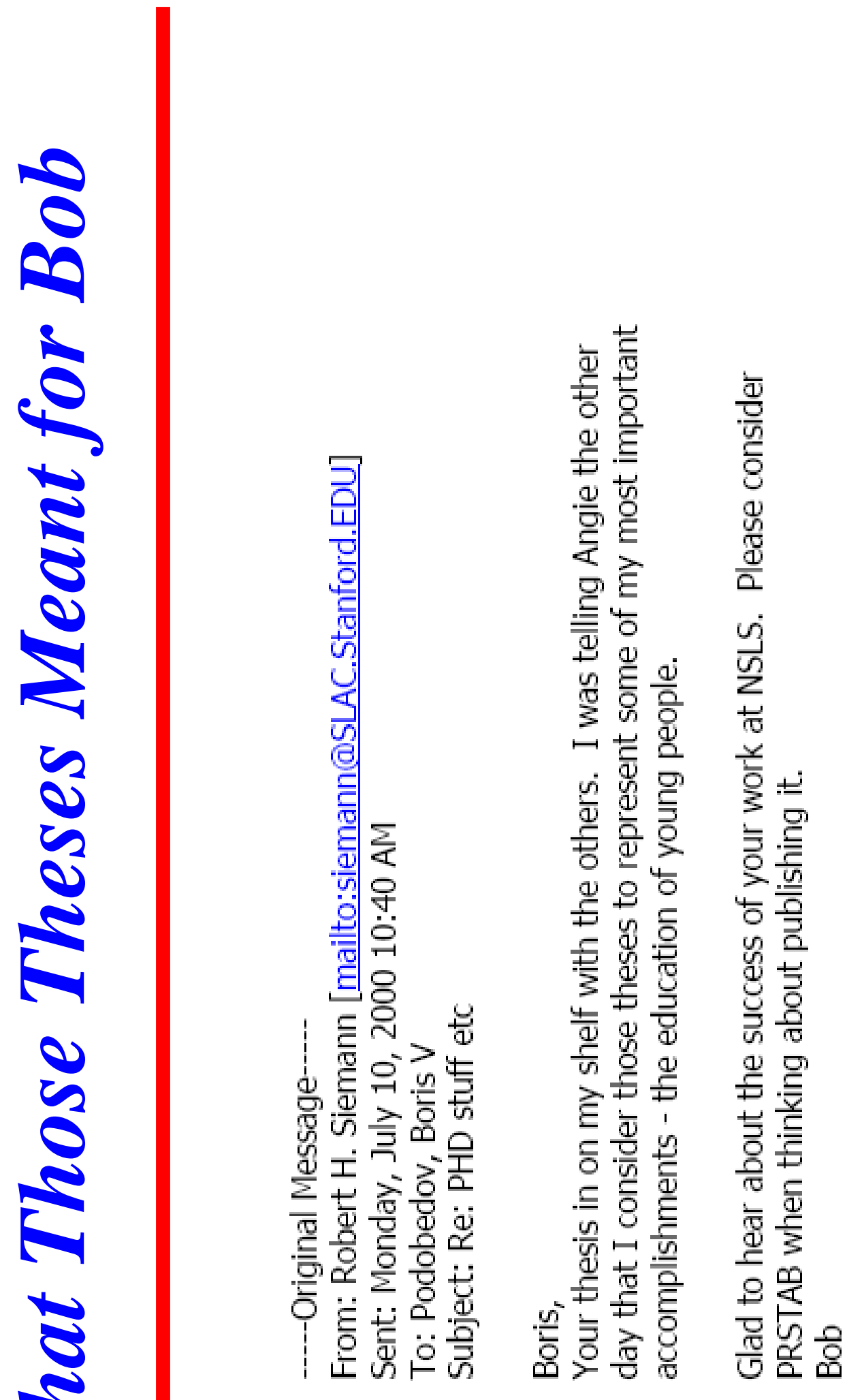




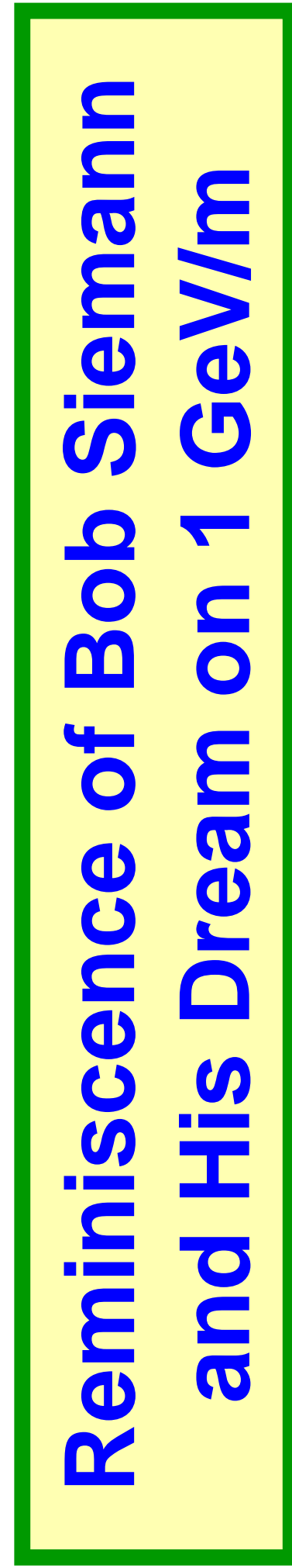

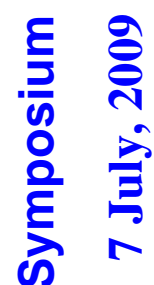

0
2
2
2

宅

(1)

U

II

0

ש

$\rightarrow$ E

ह

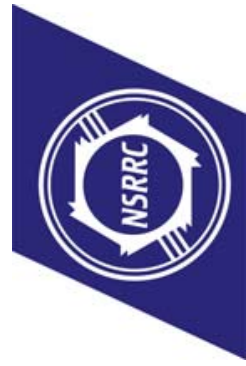




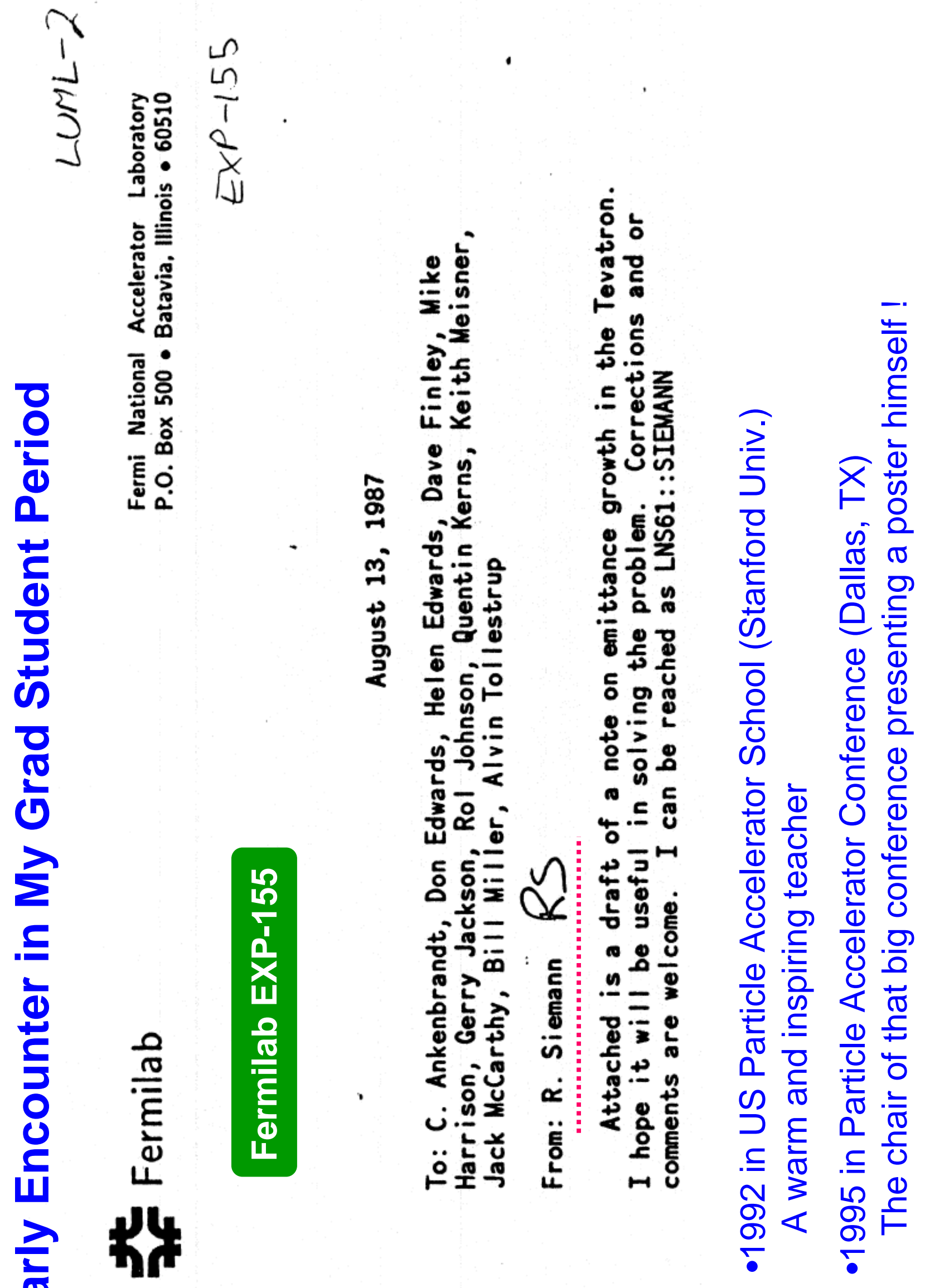

II 


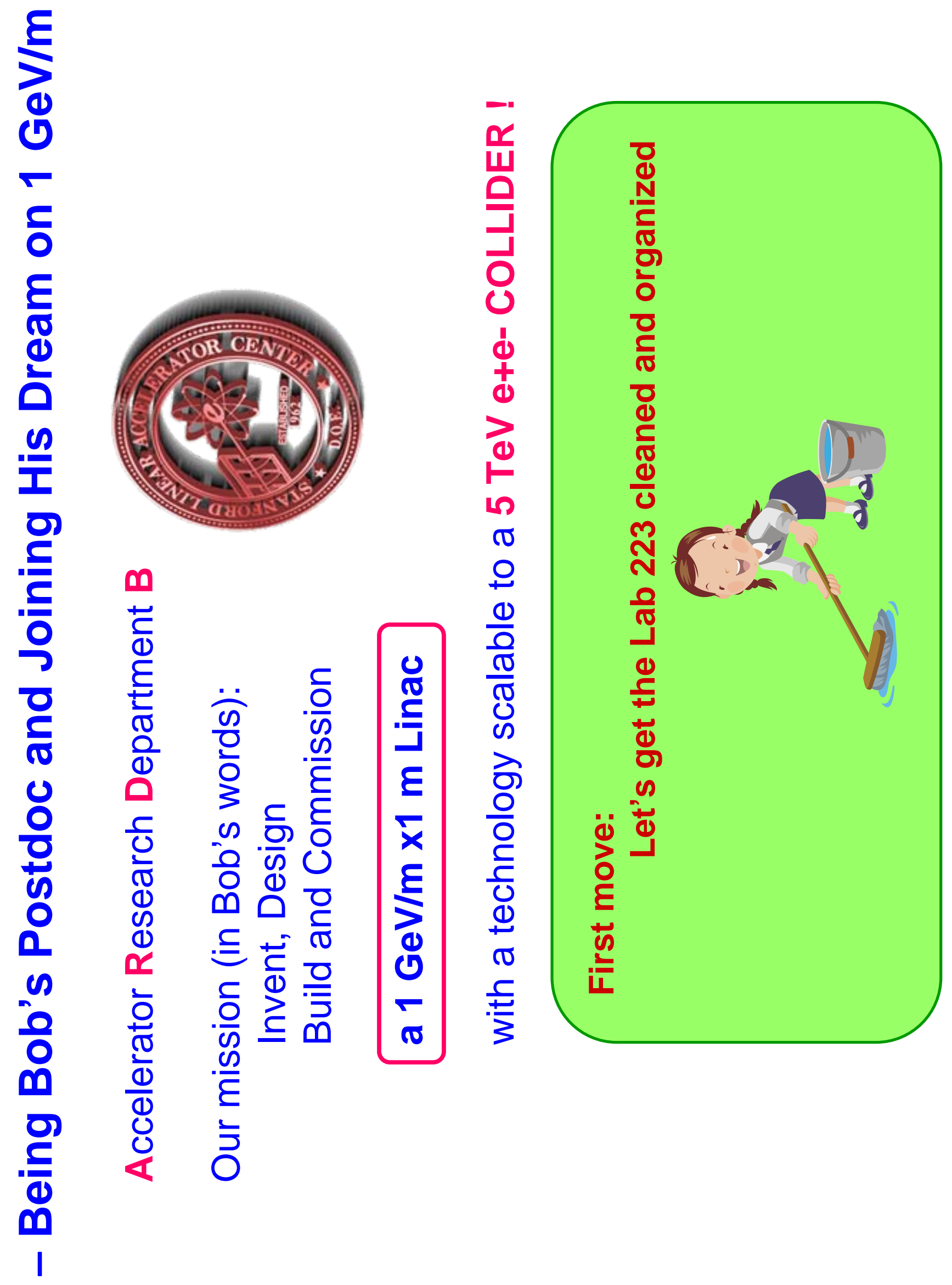




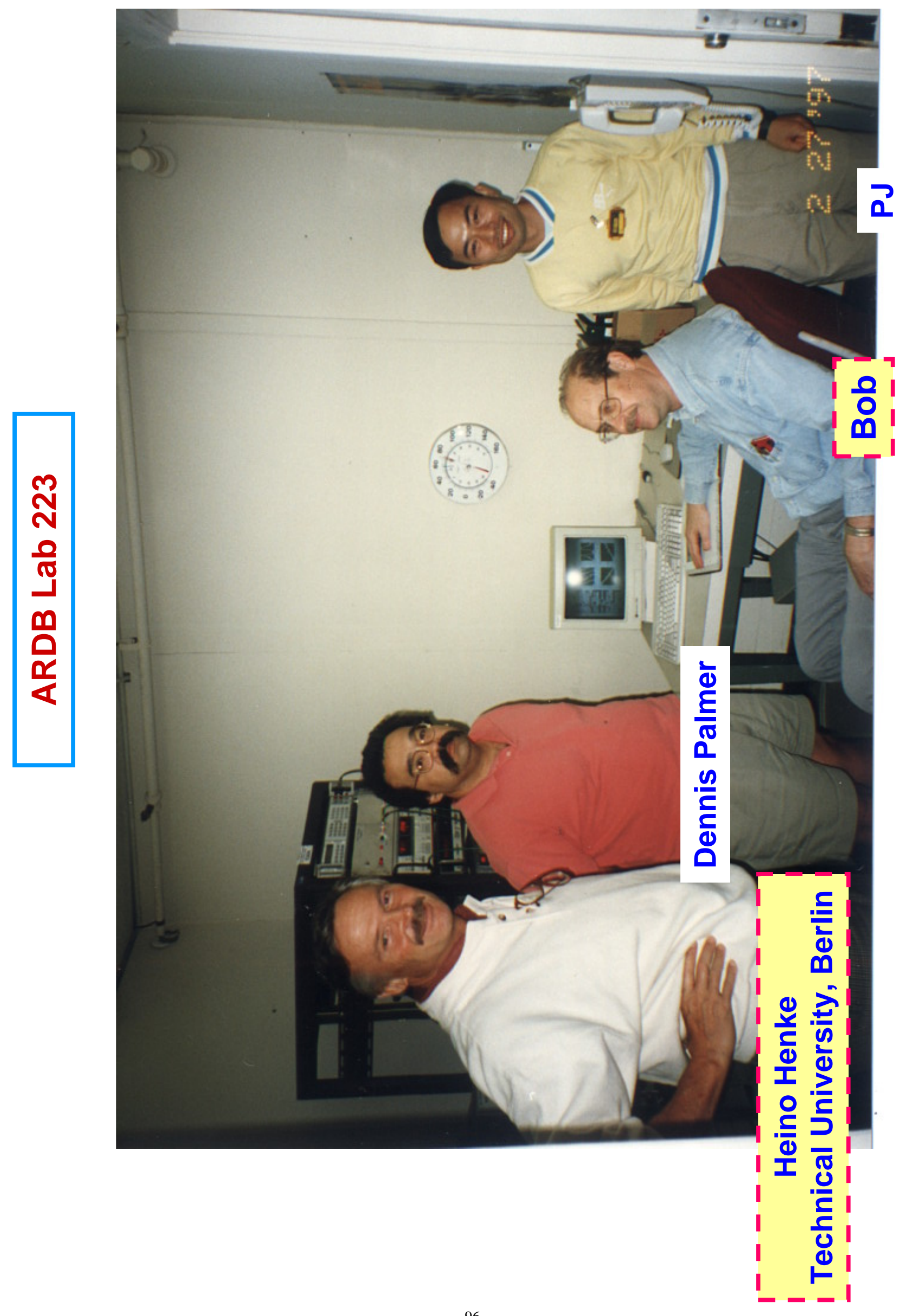




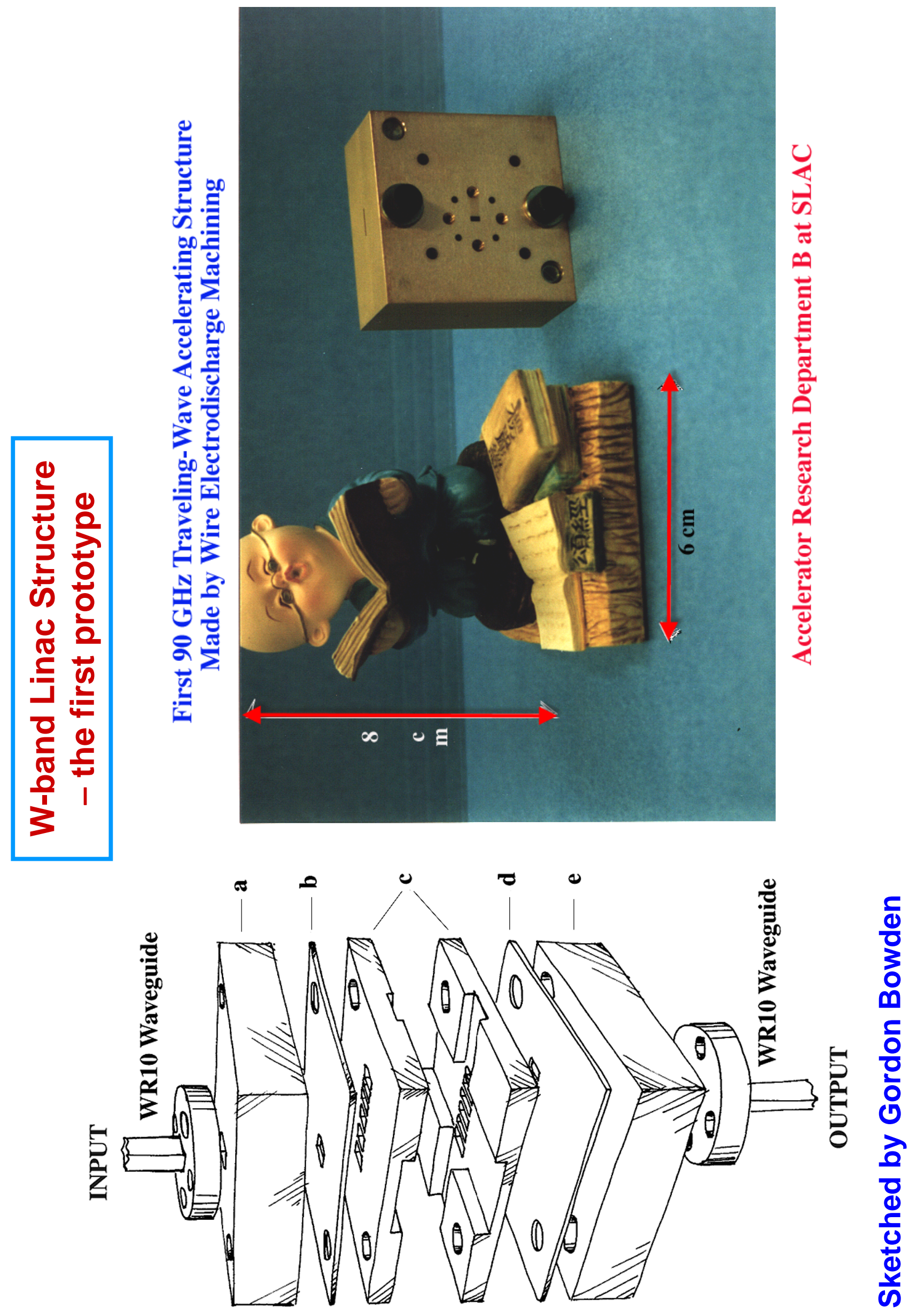



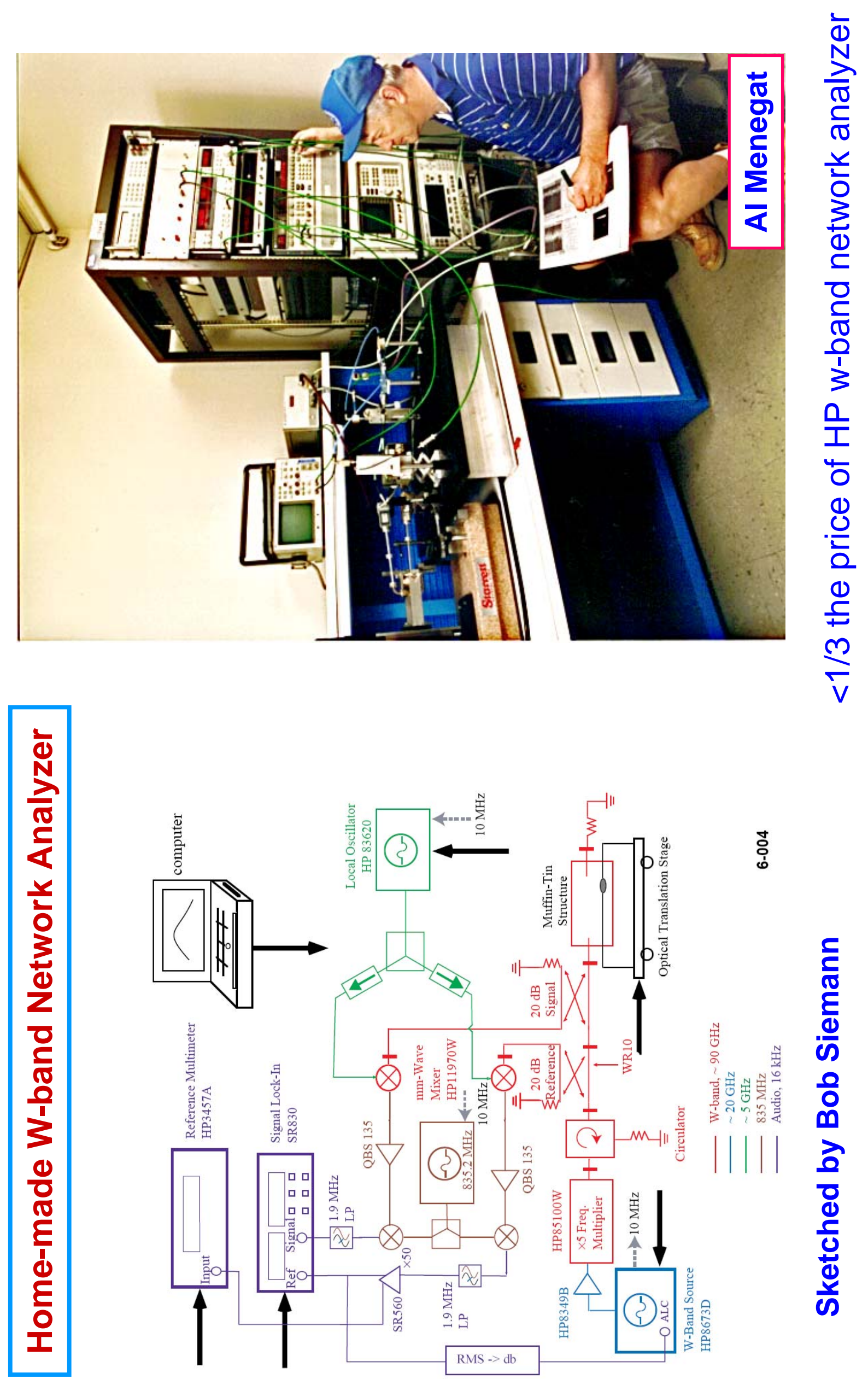

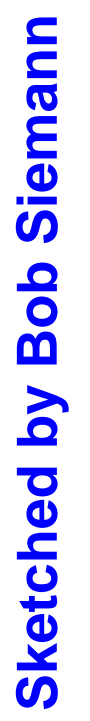




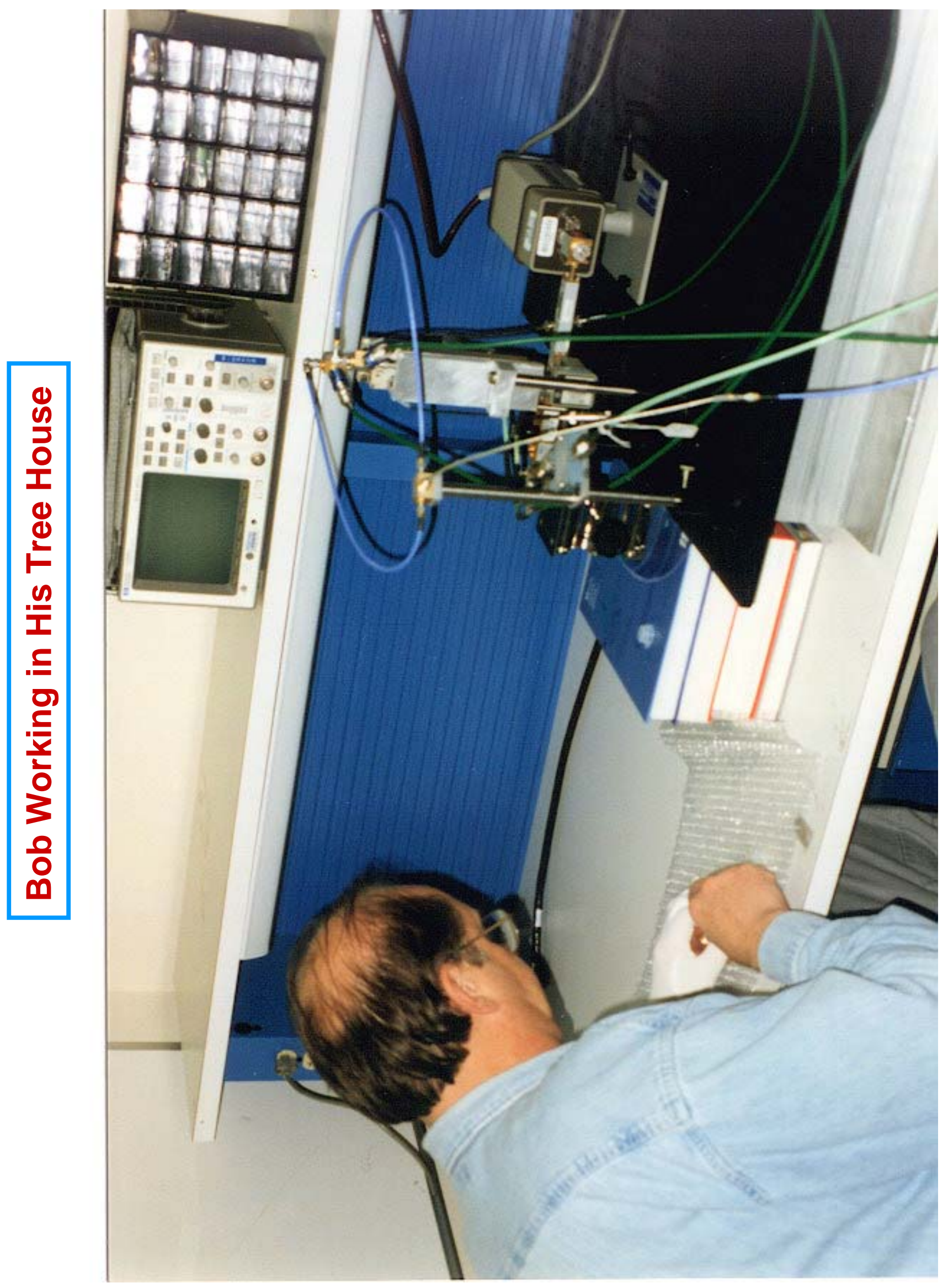




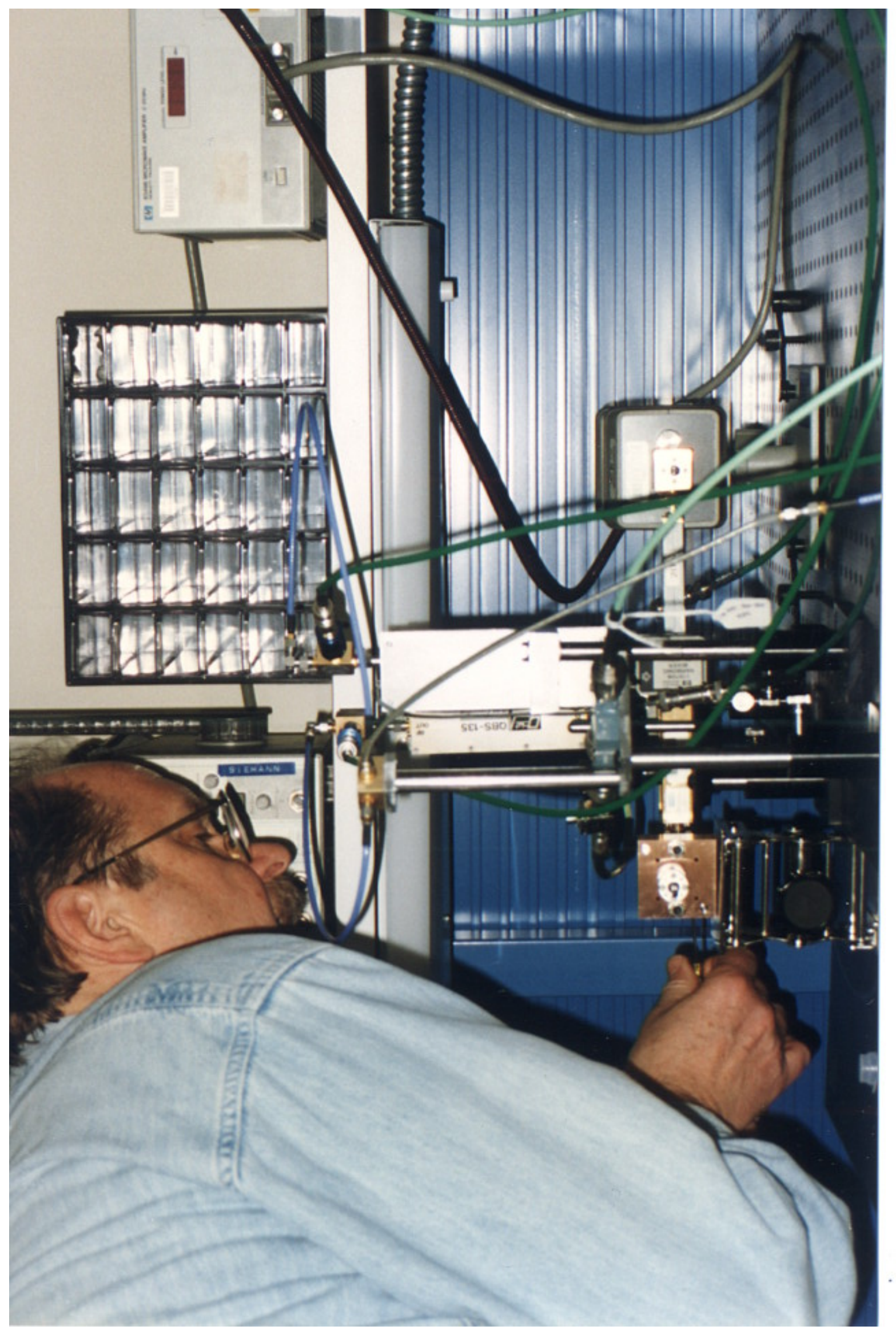




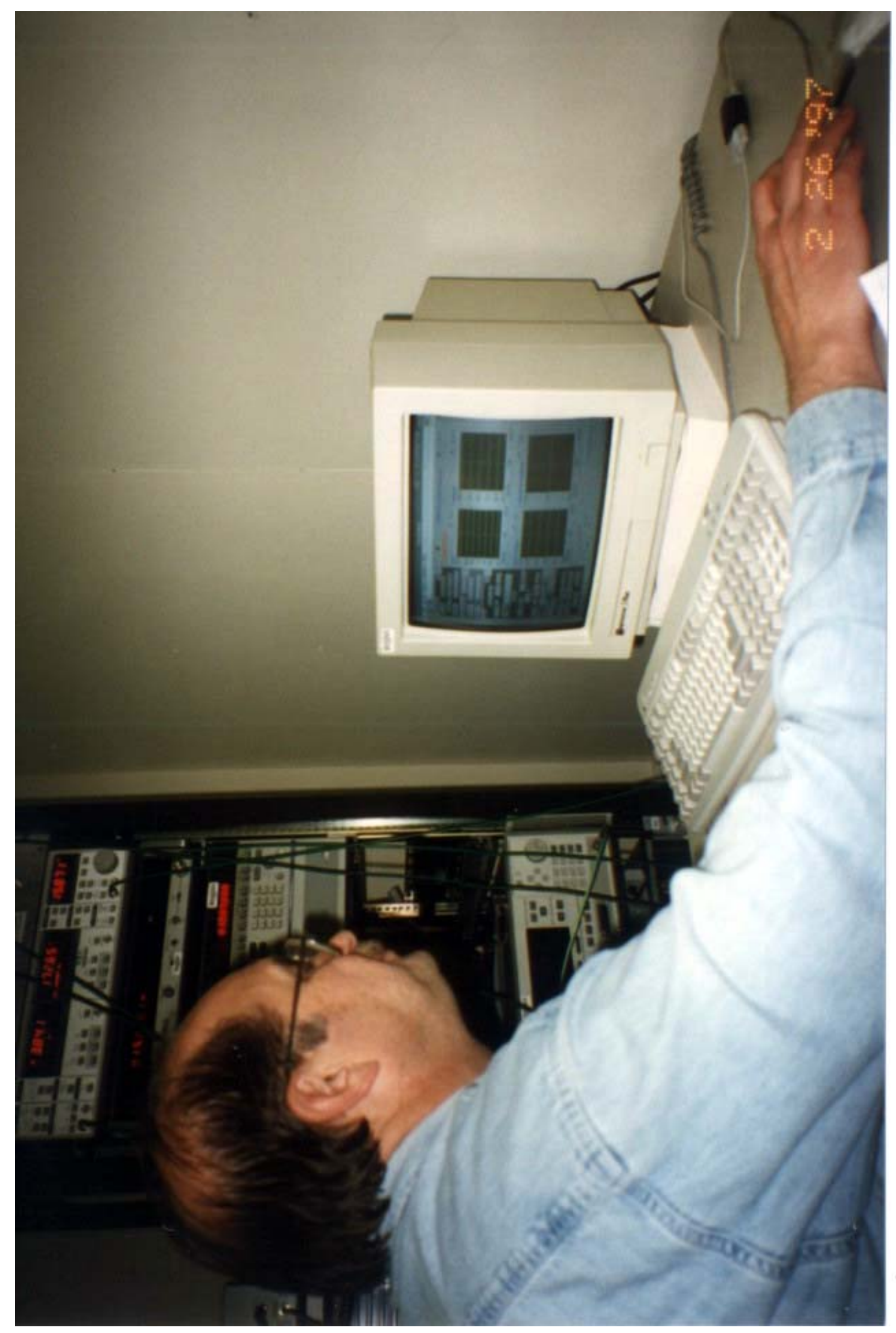



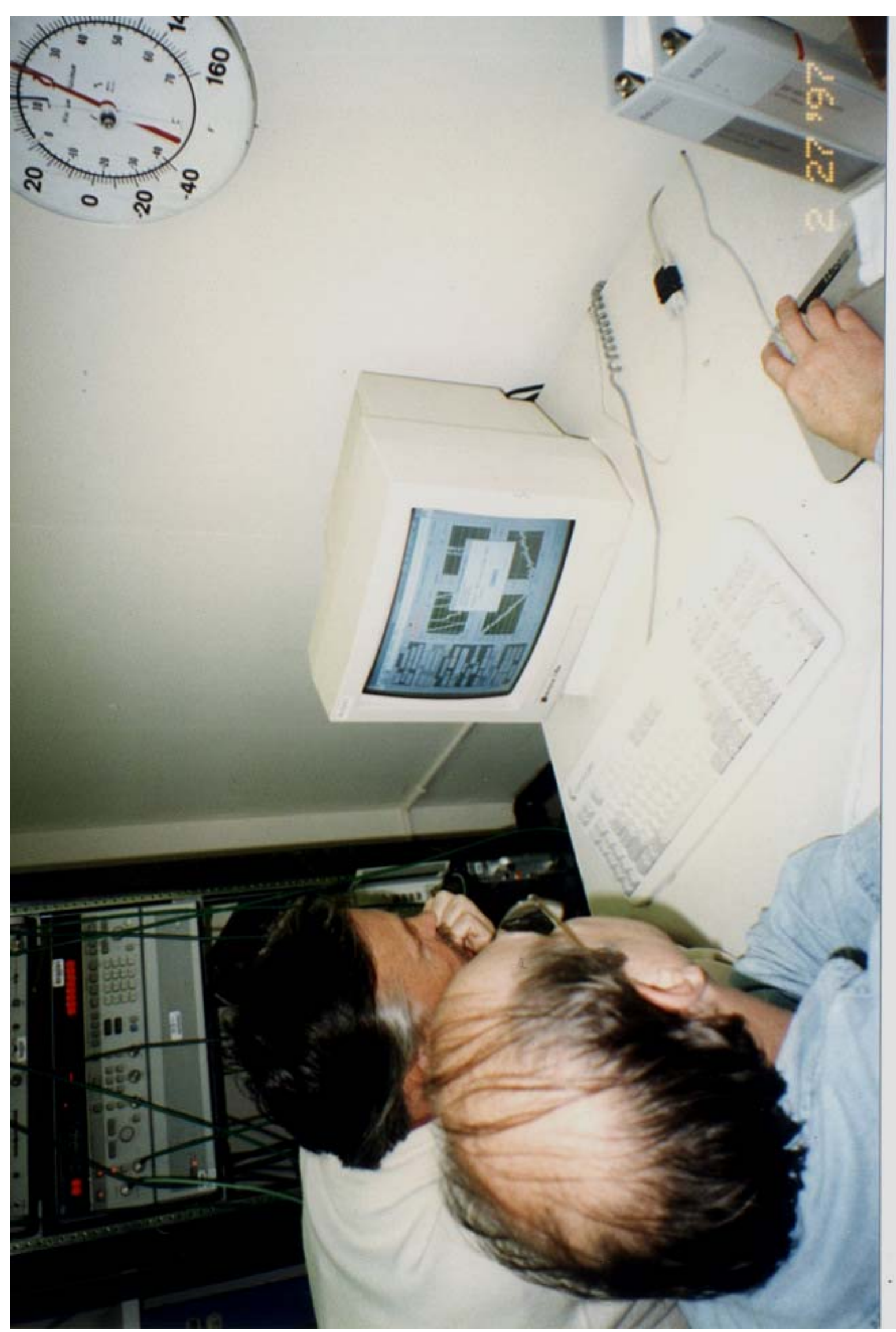


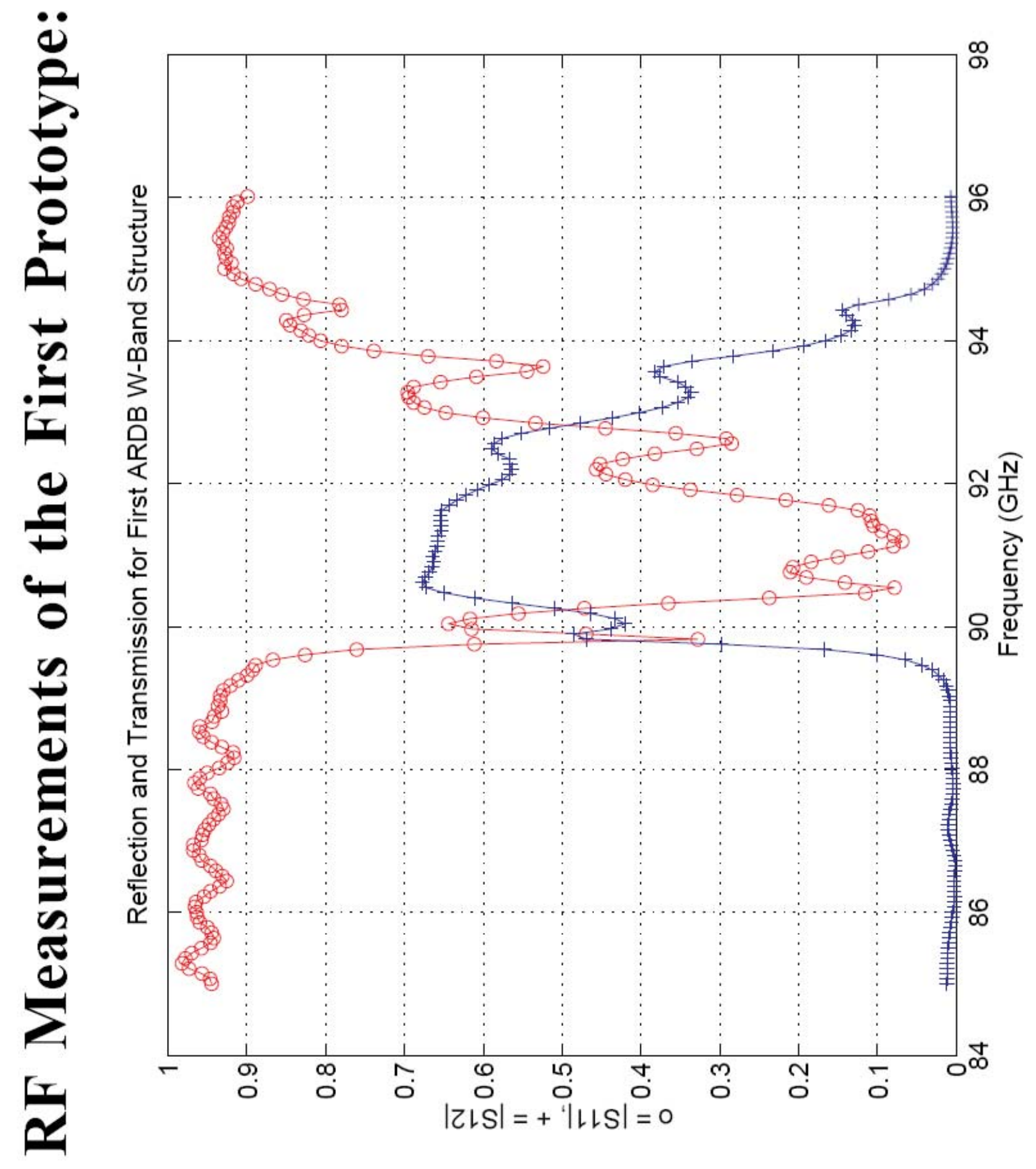




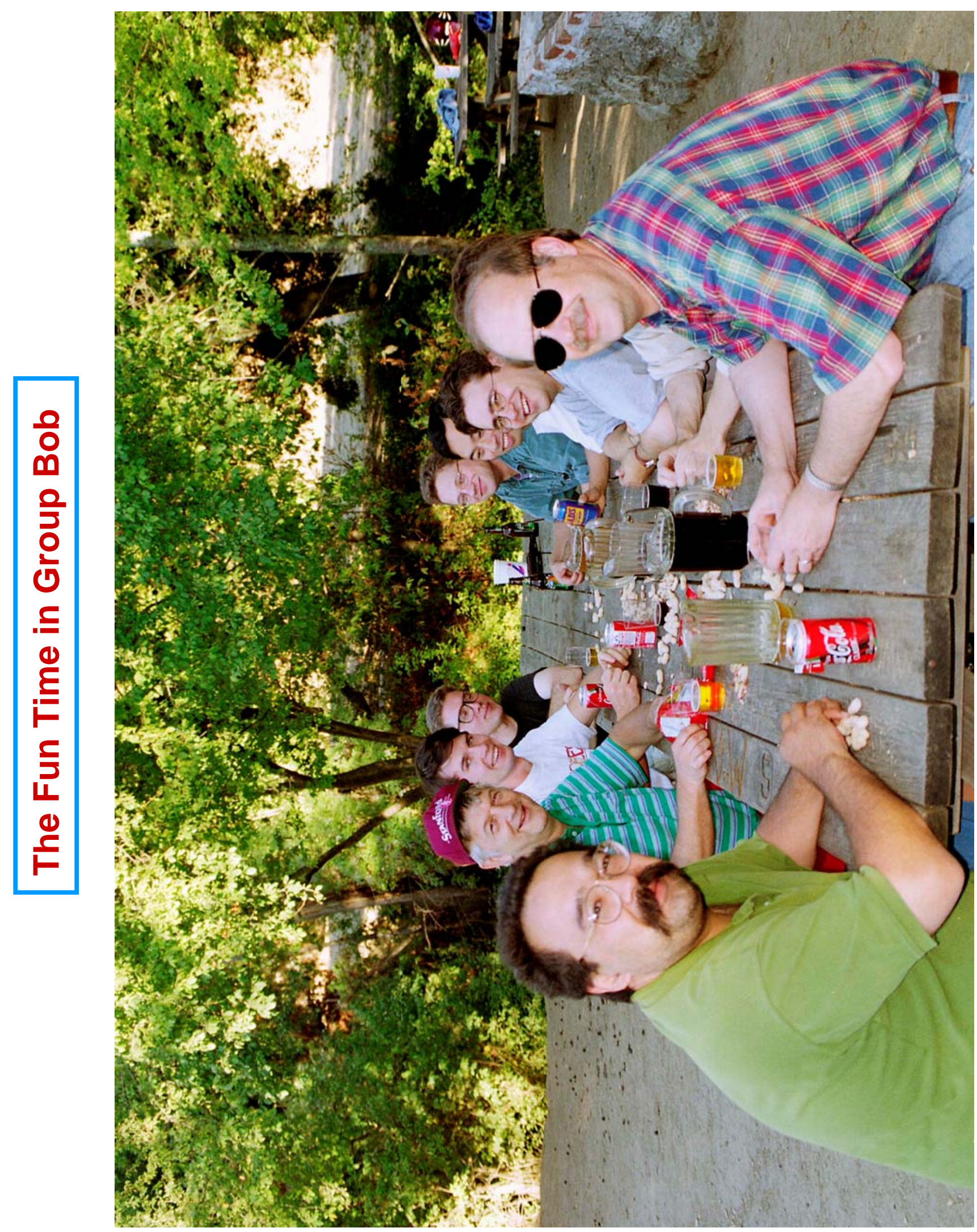




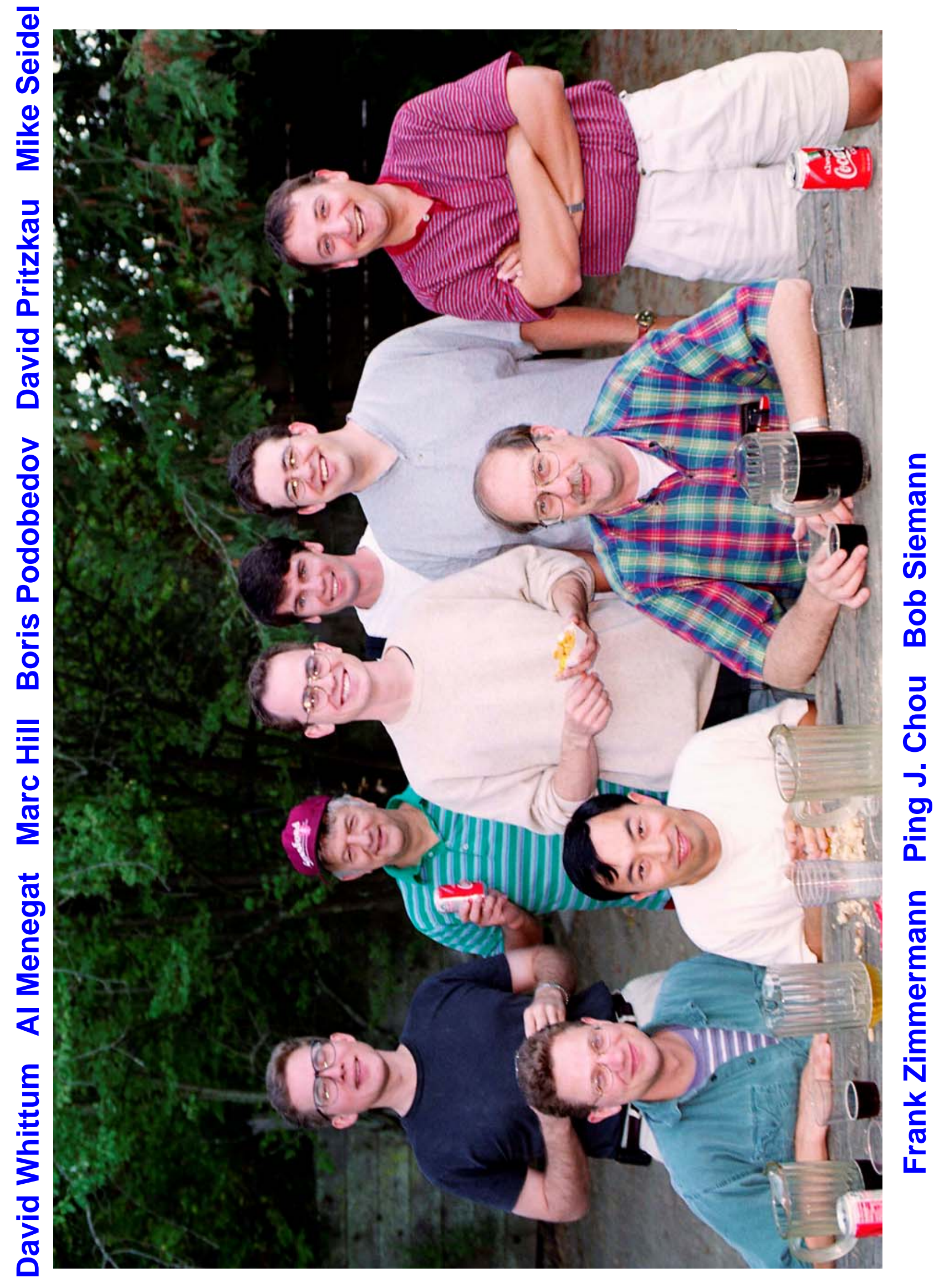




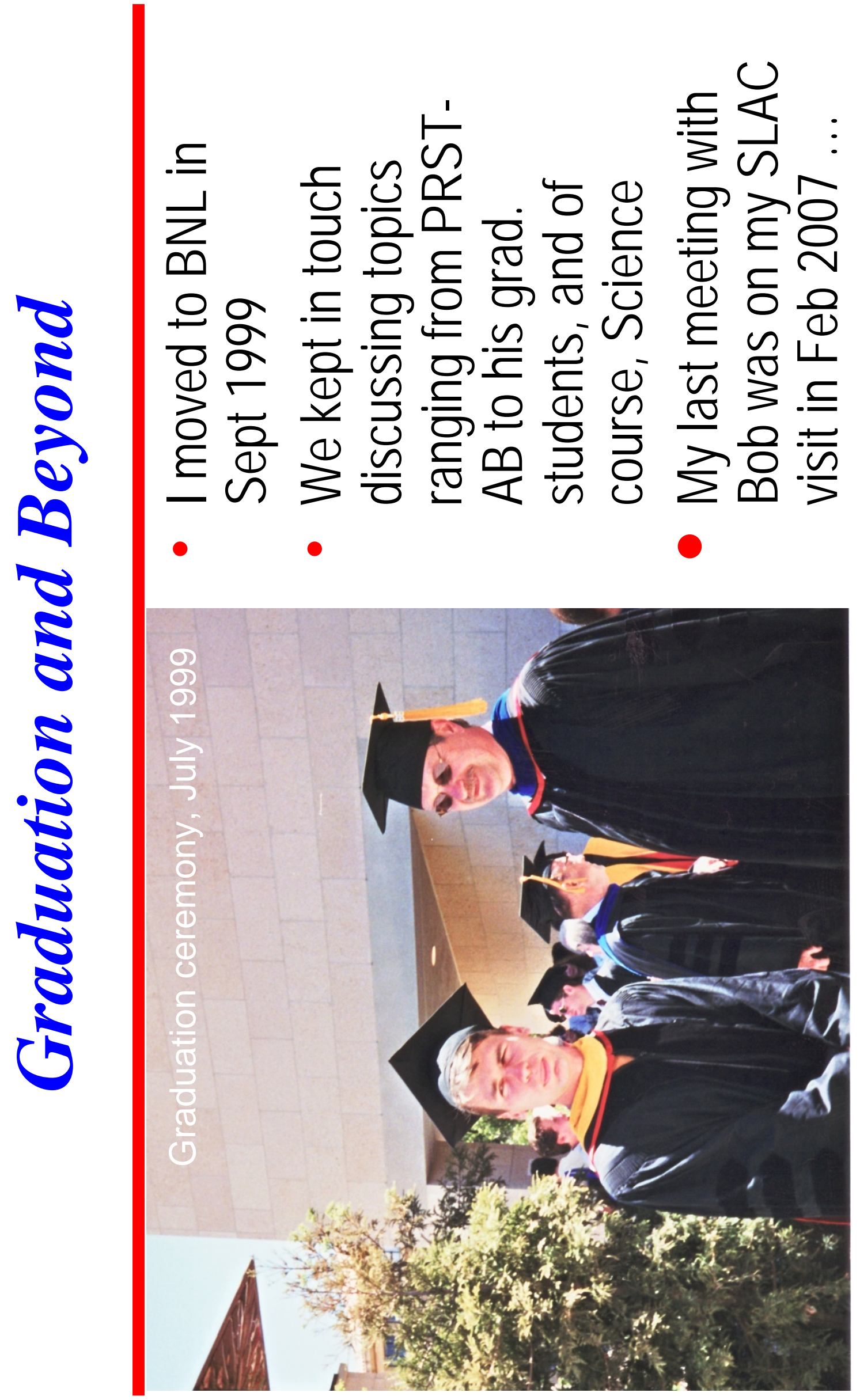


$\frac{0}{6}$
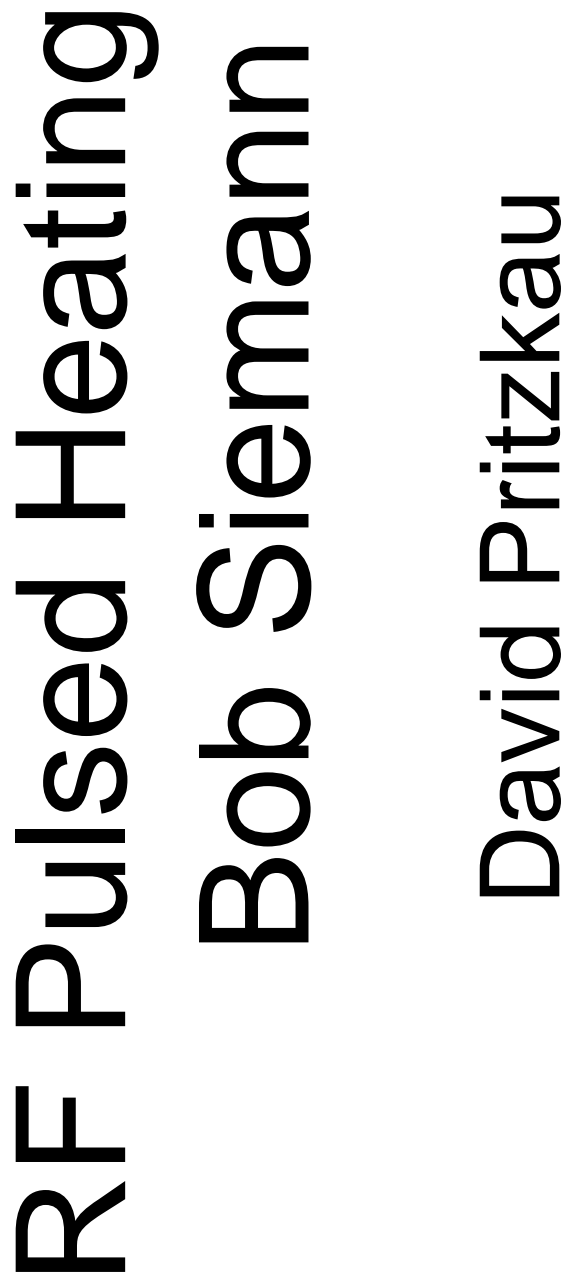


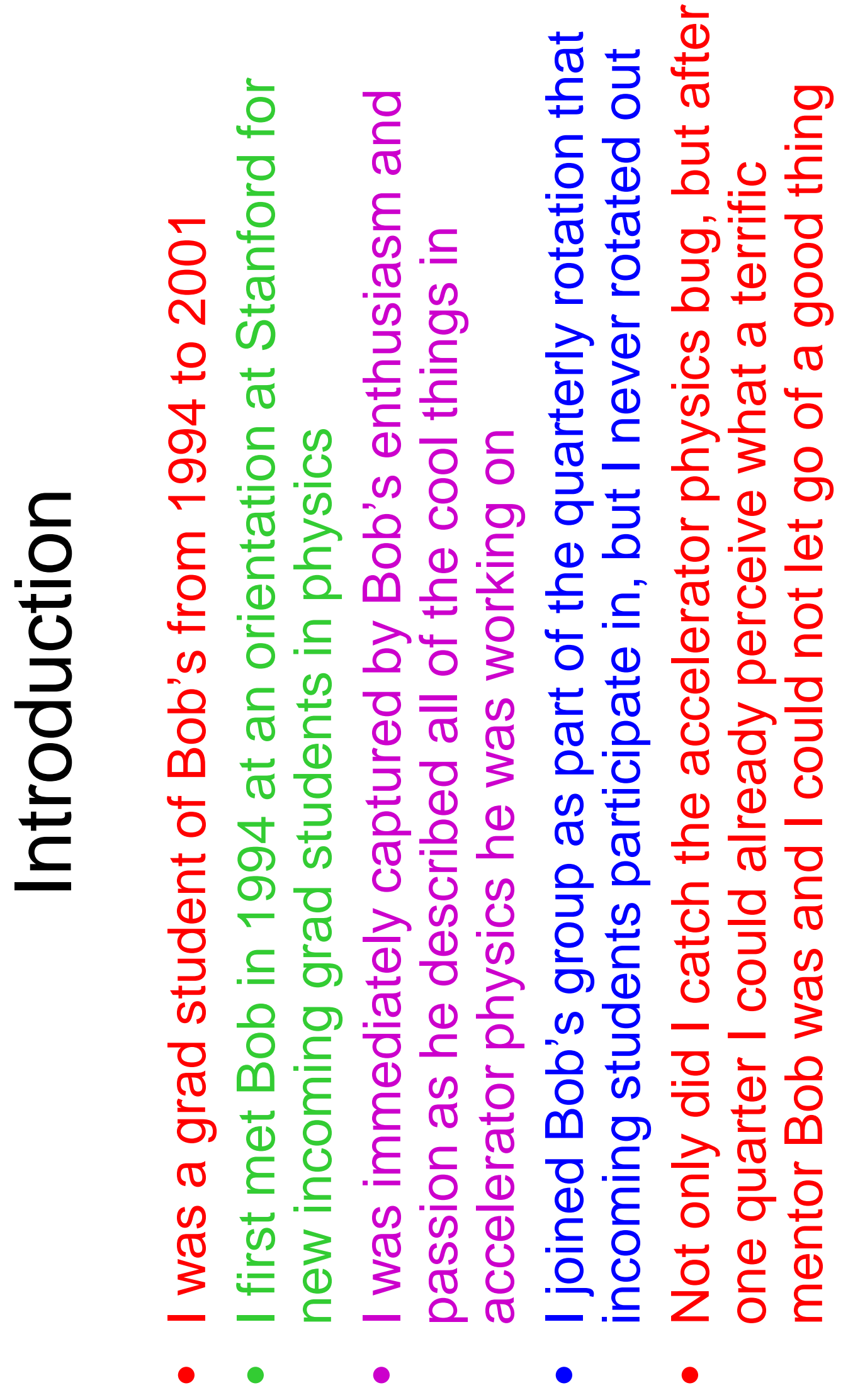




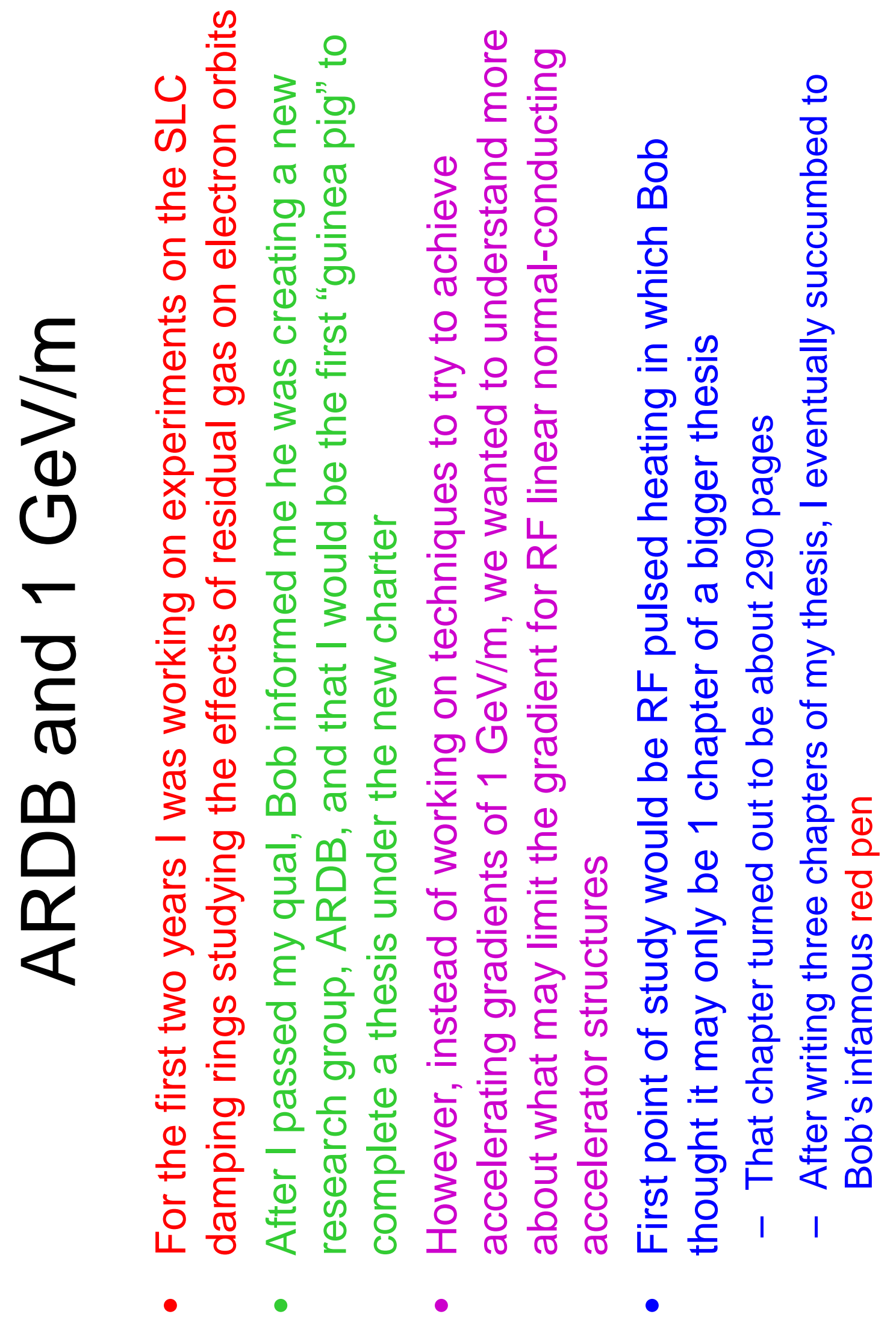




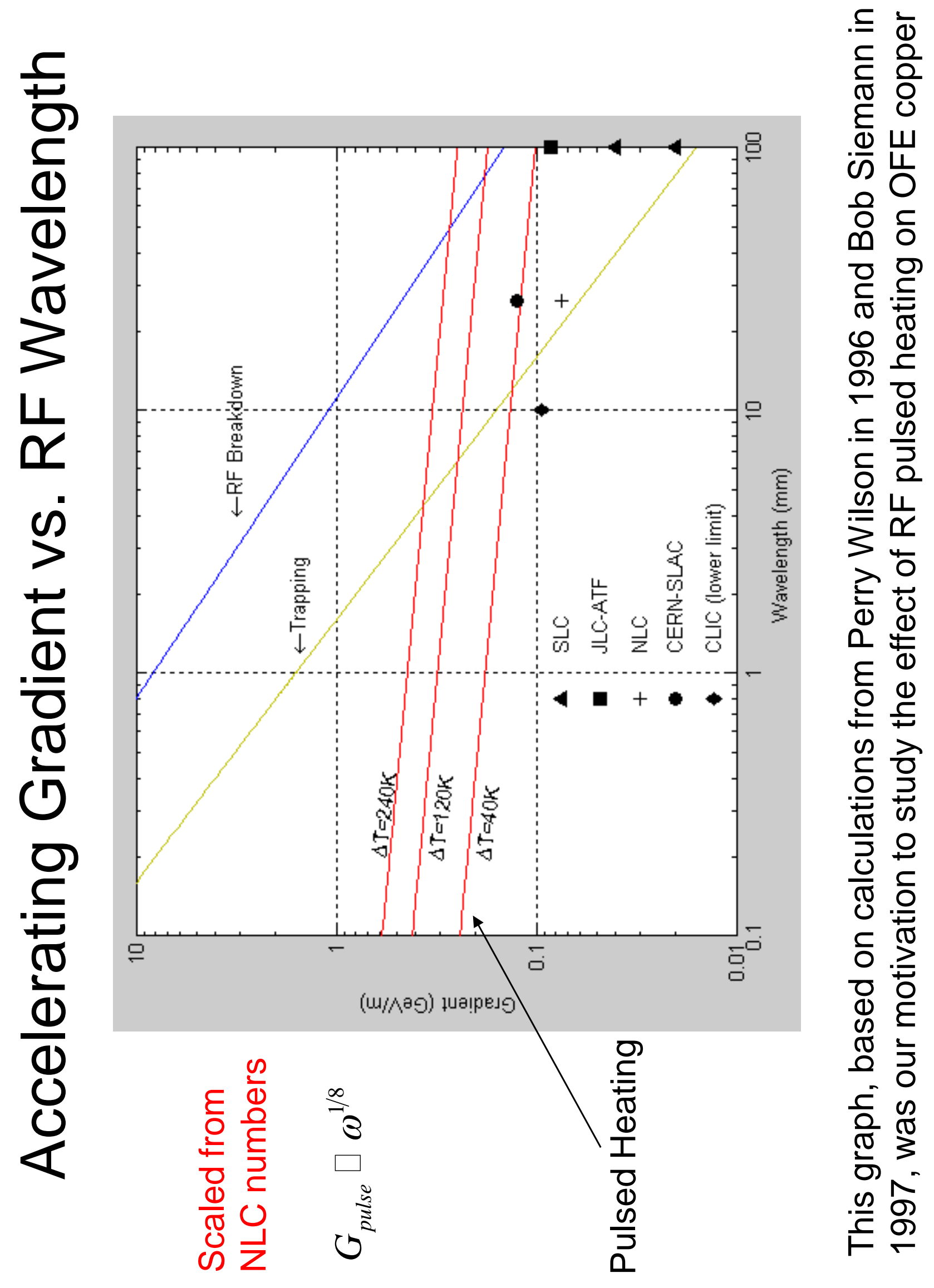




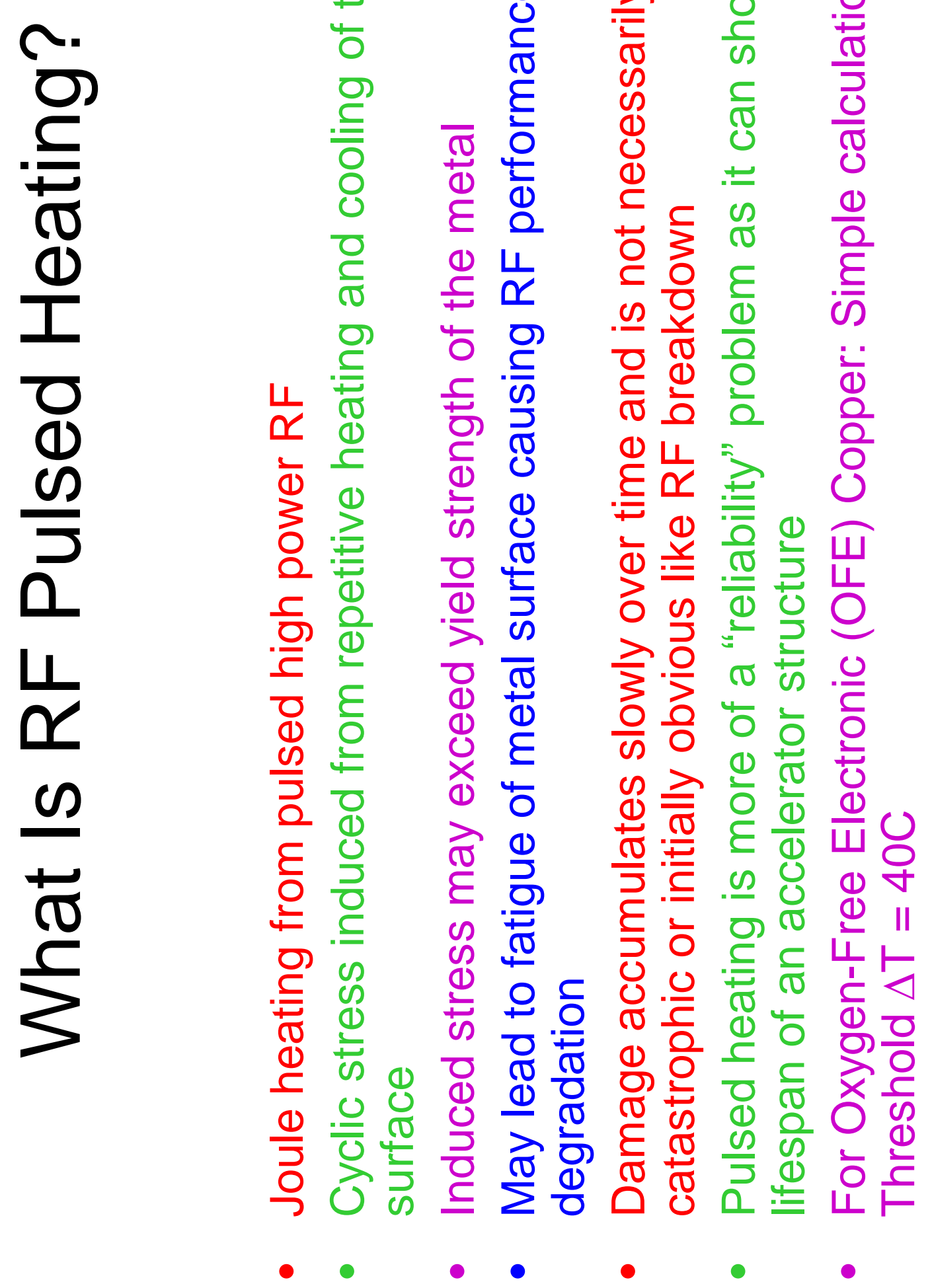



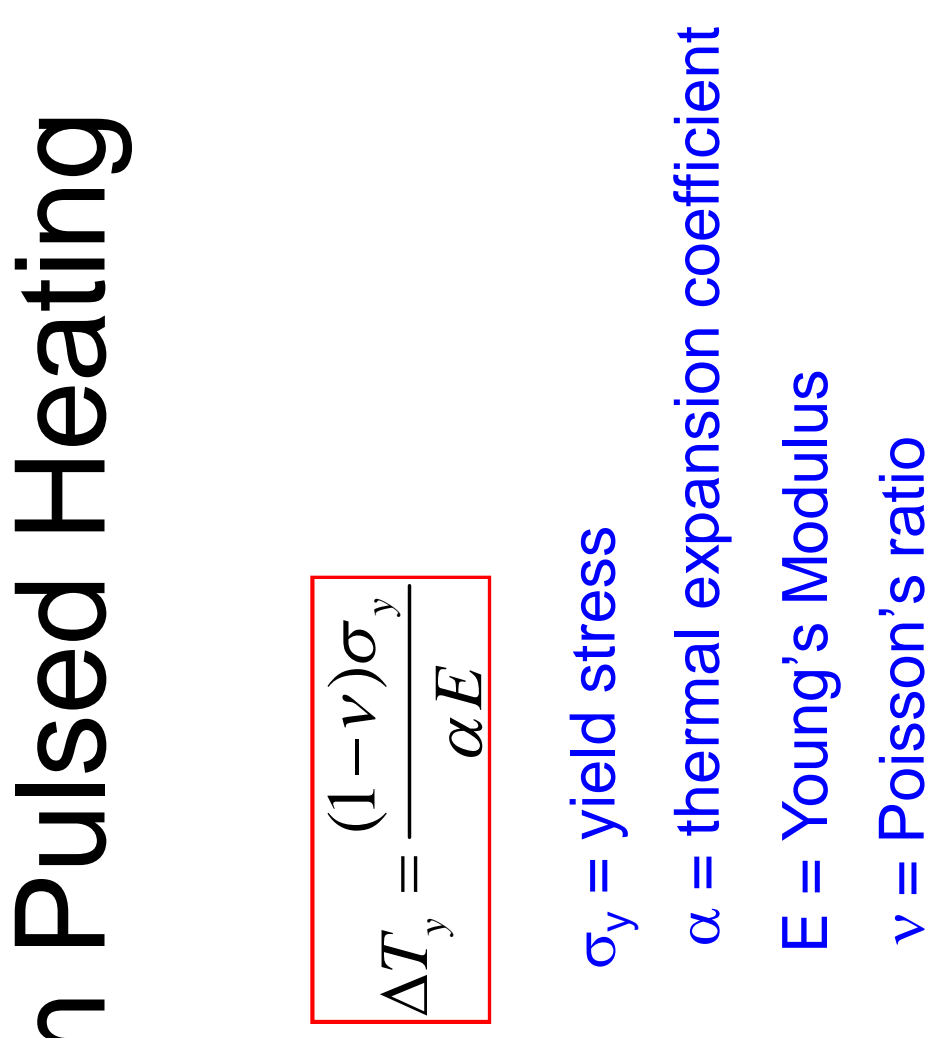

흔

0
0
$\frac{1}{2}$

$\frac{1}{2}$

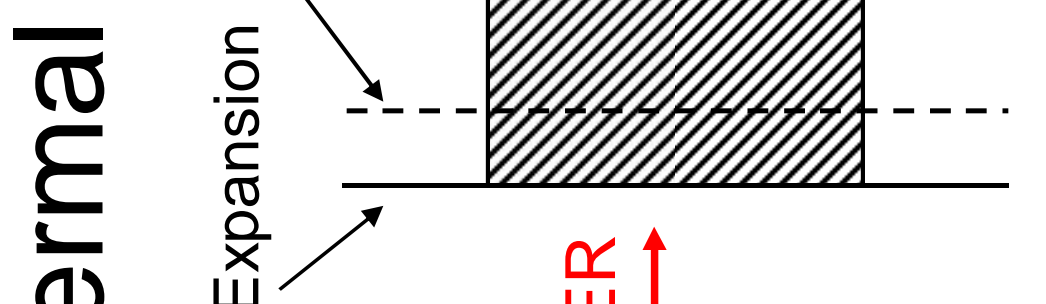

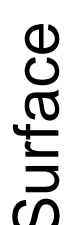

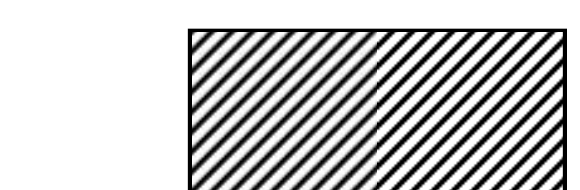

(1)

ع

$\stackrel{\frac{1}{\sigma}}{\frac{D}{d}}$

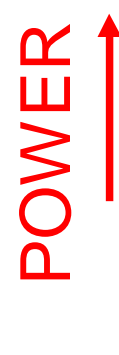

$\overparen{\mho}$

1

气

1

ช్ర

$\frac{\varepsilon}{\sigma}$

0

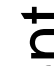

(1)

$\frac{\bar{\sigma}}{\varepsilon}$

हᄐ

으

告

$\frac{\varepsilon}{\varepsilon}$

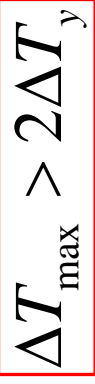

$\pm$ 


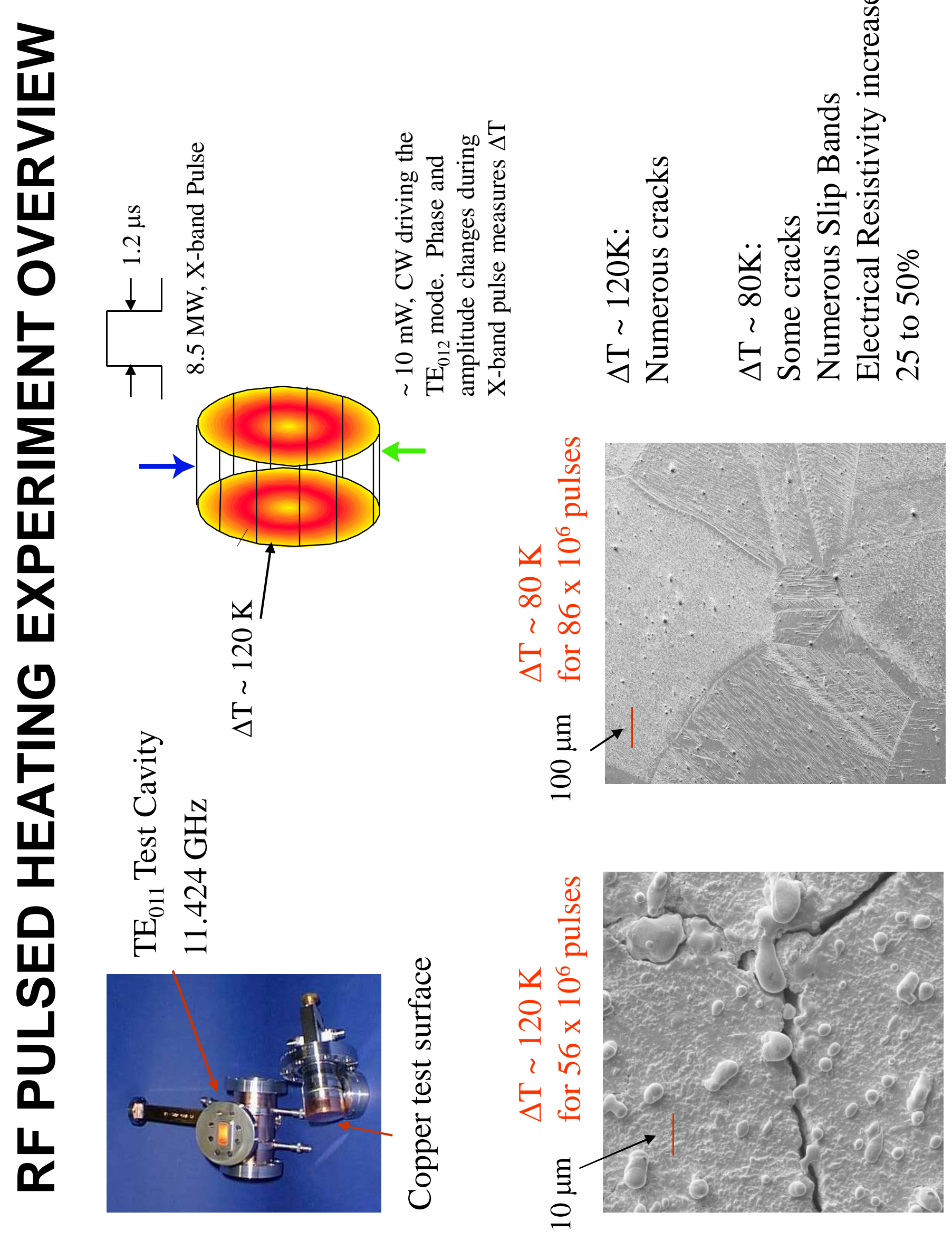



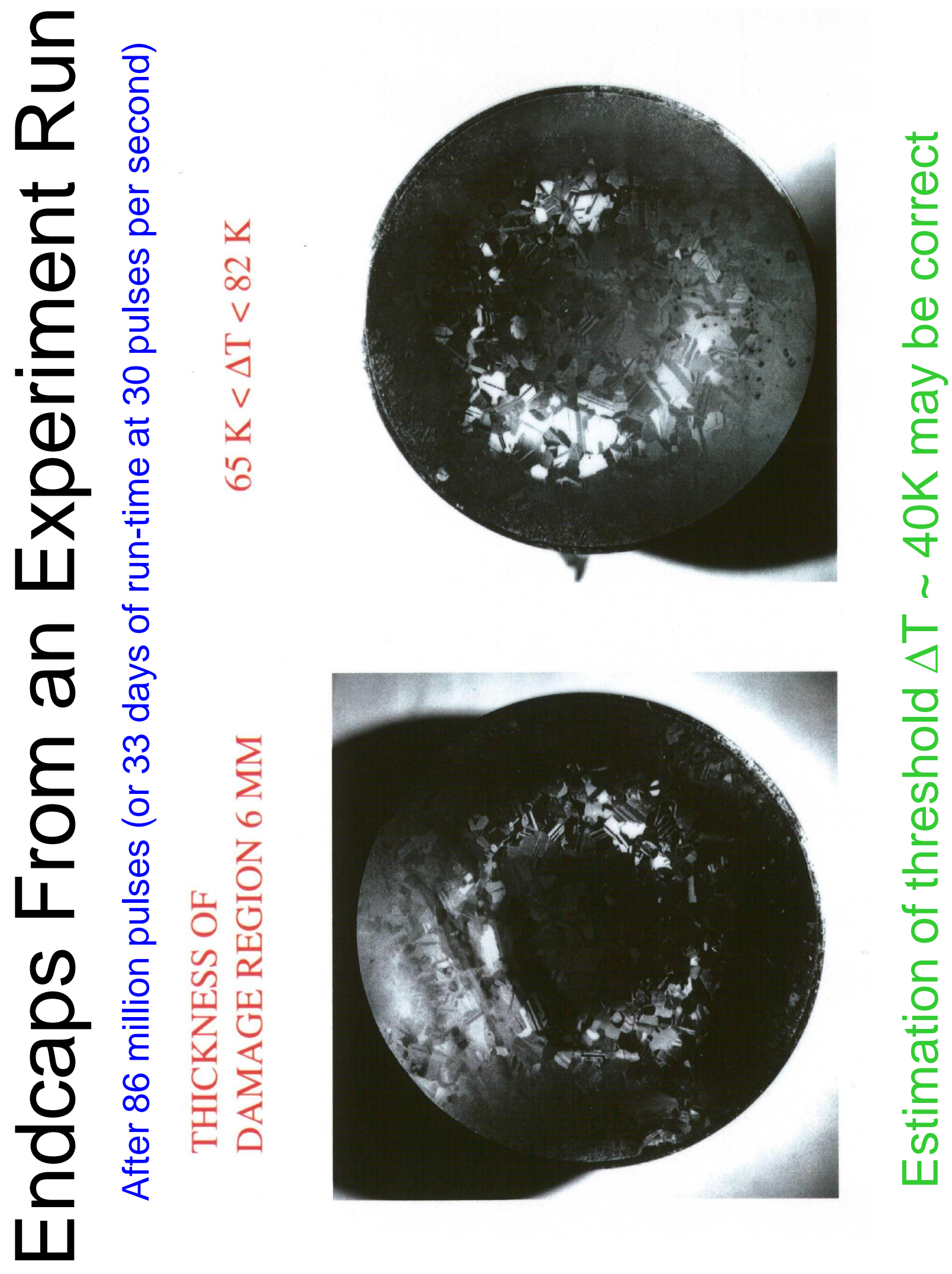


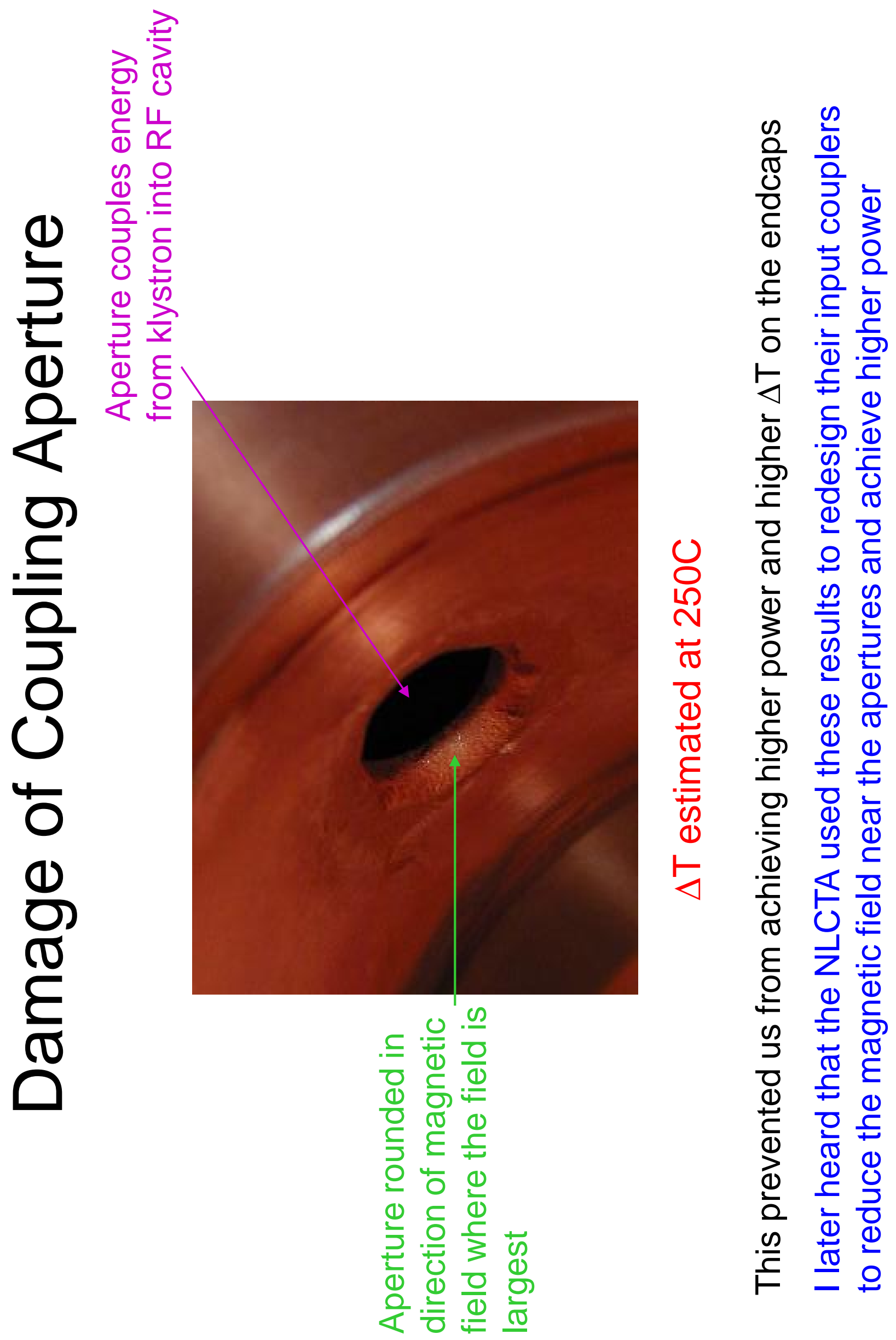




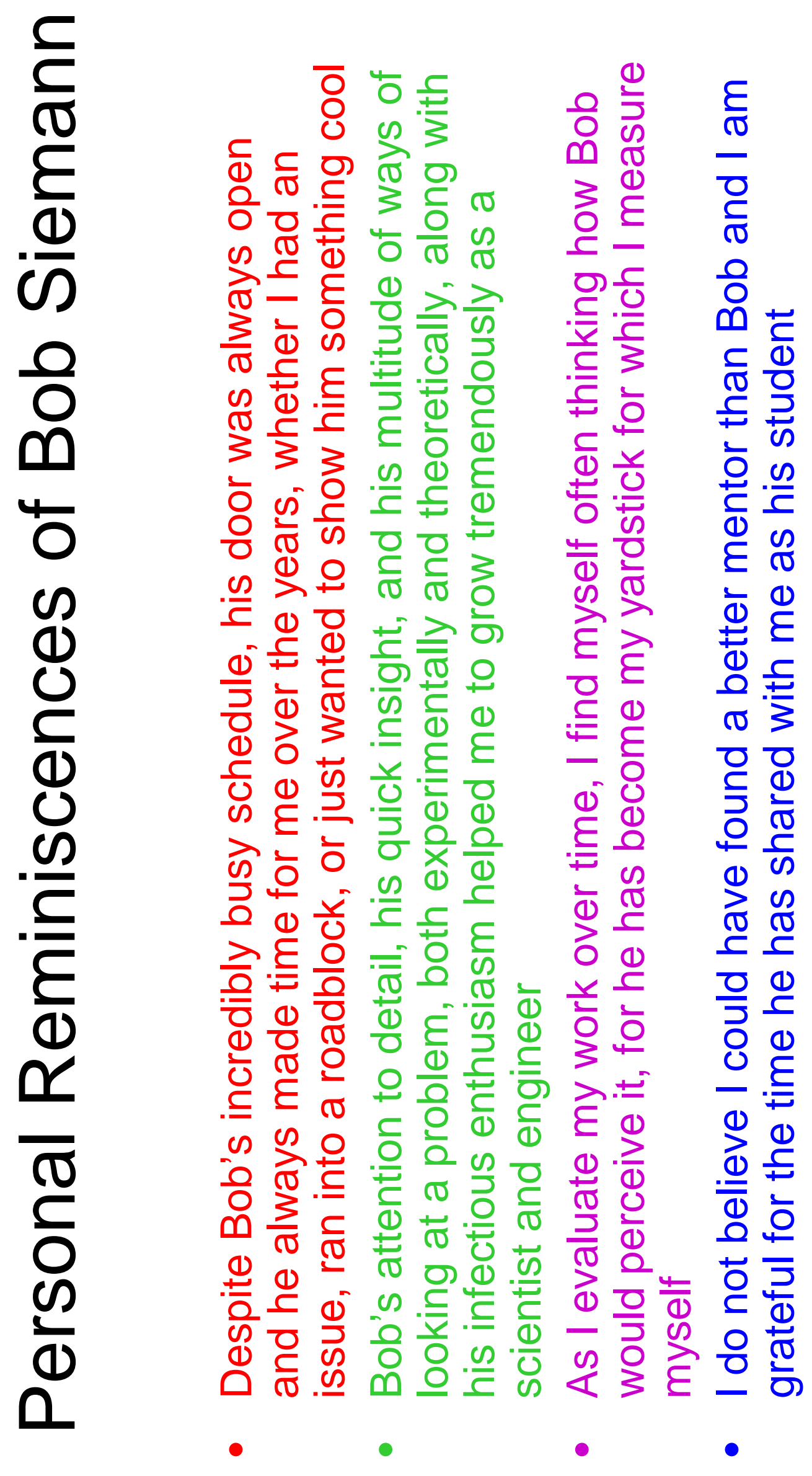



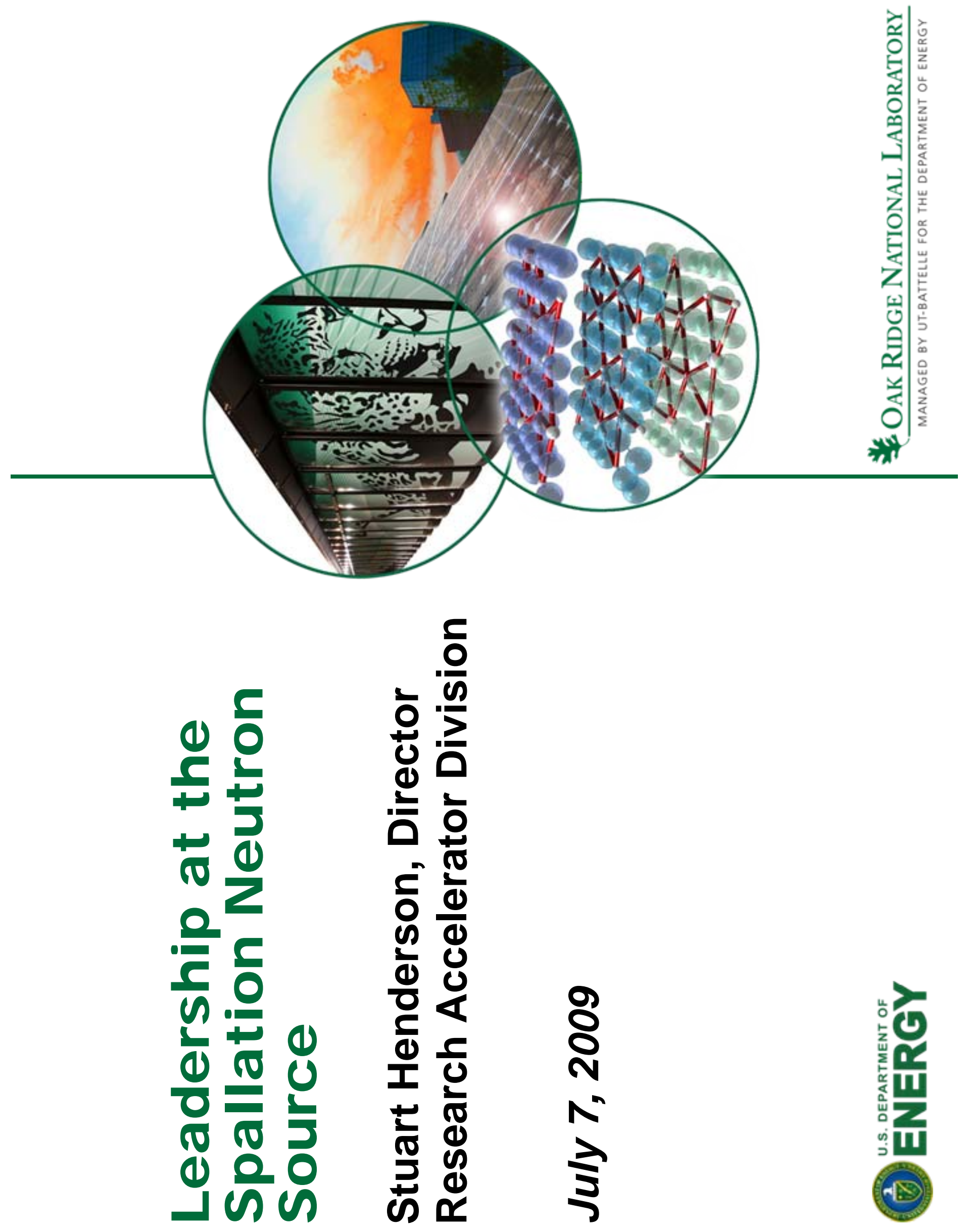

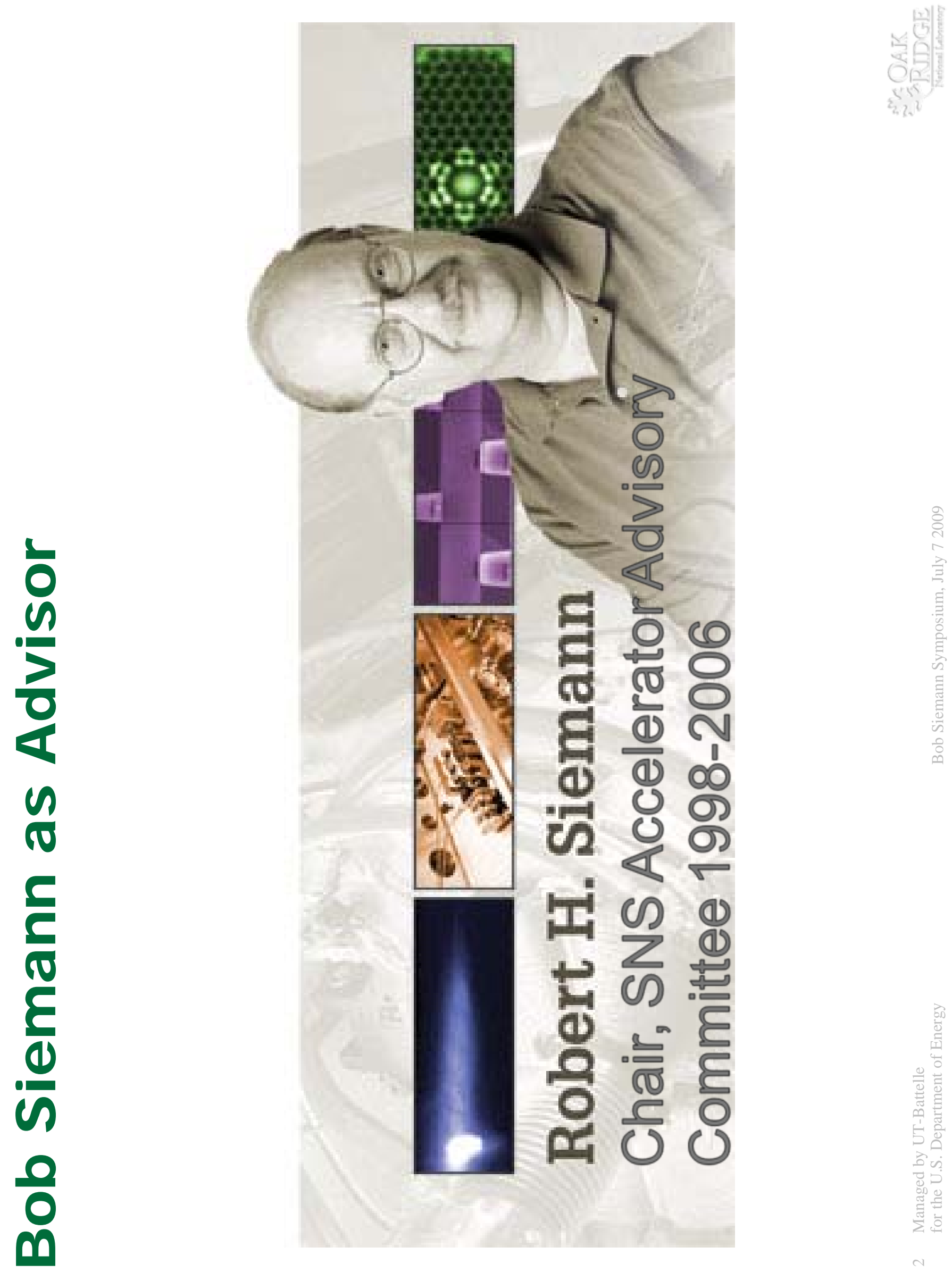


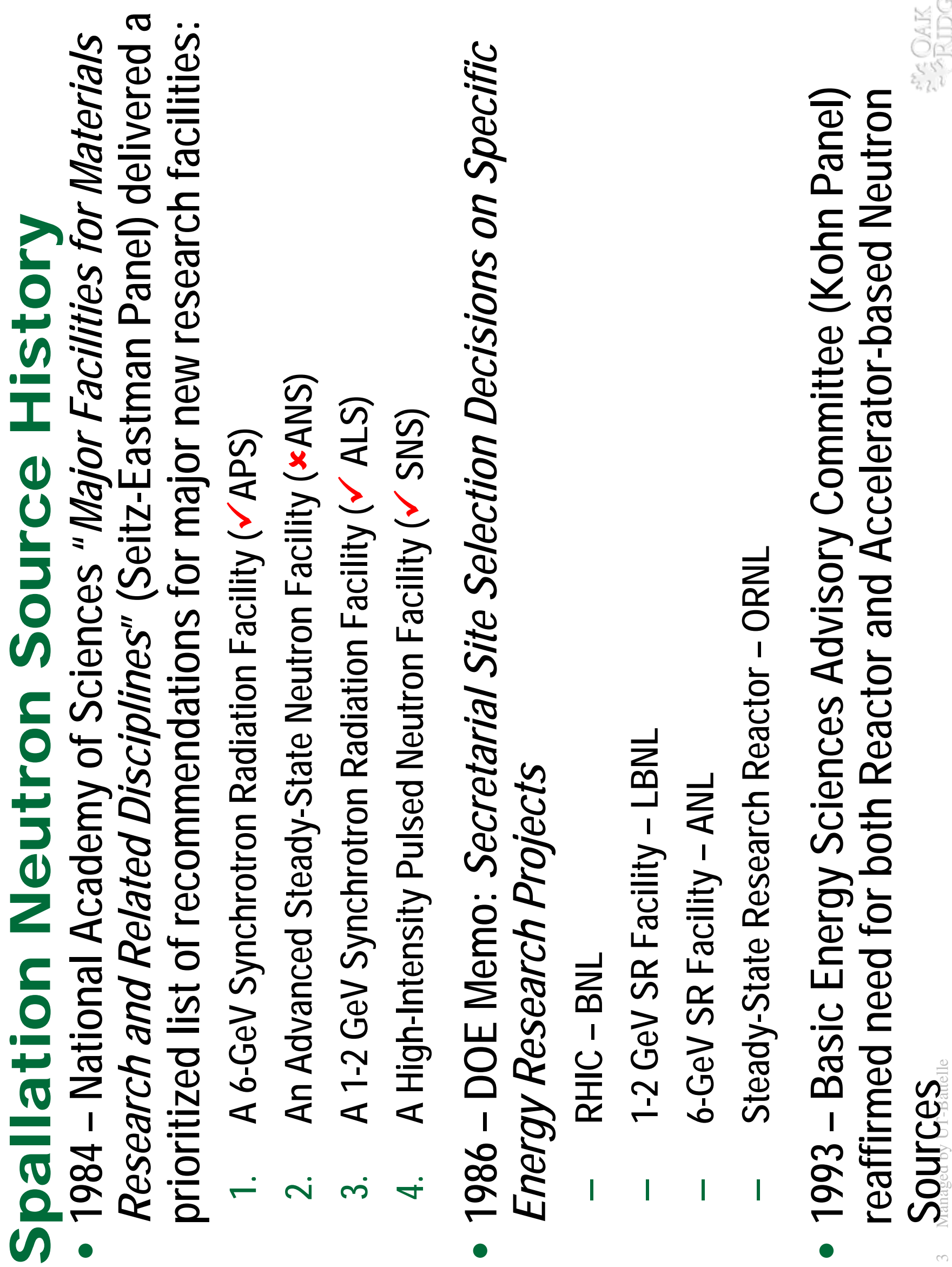




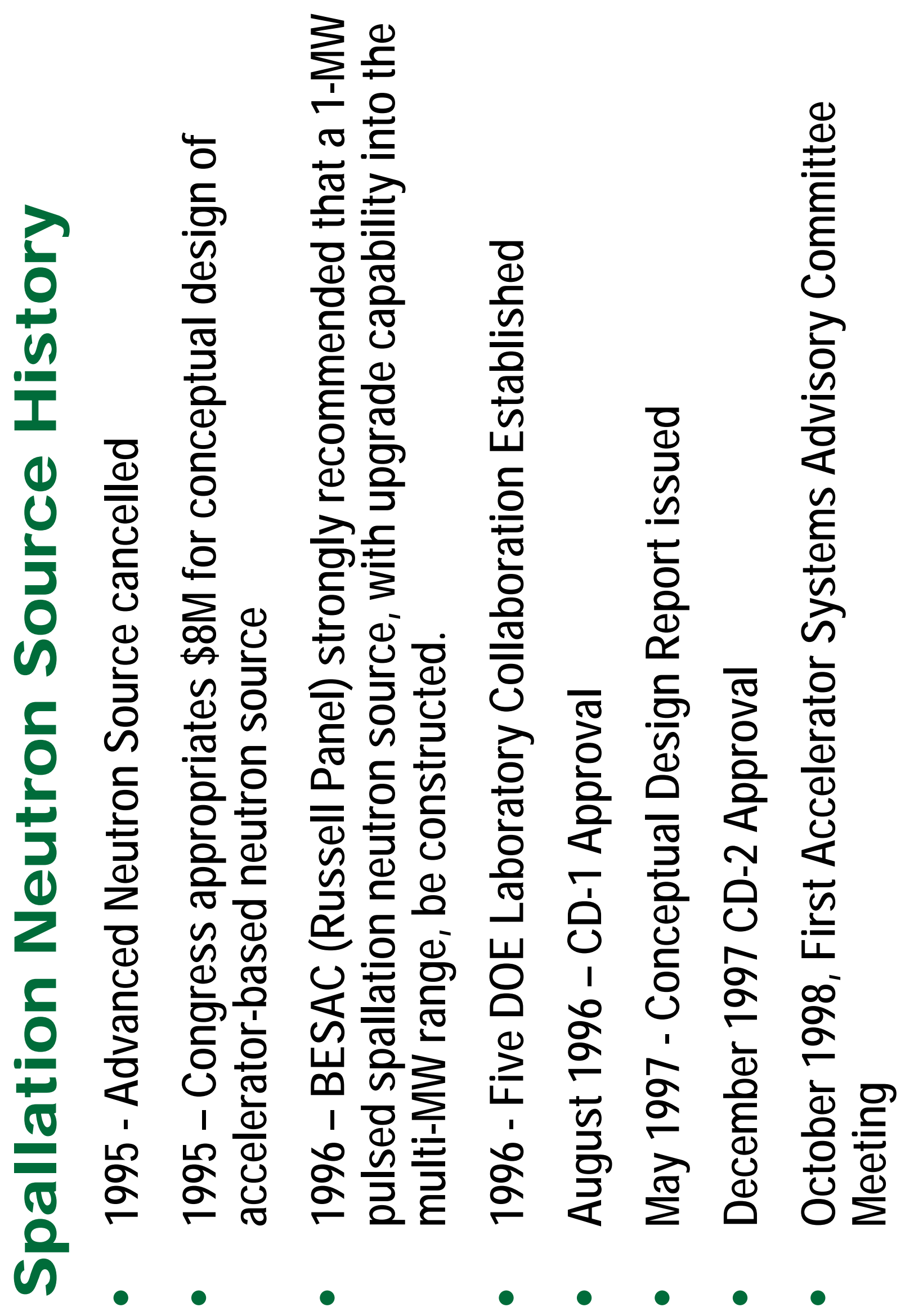




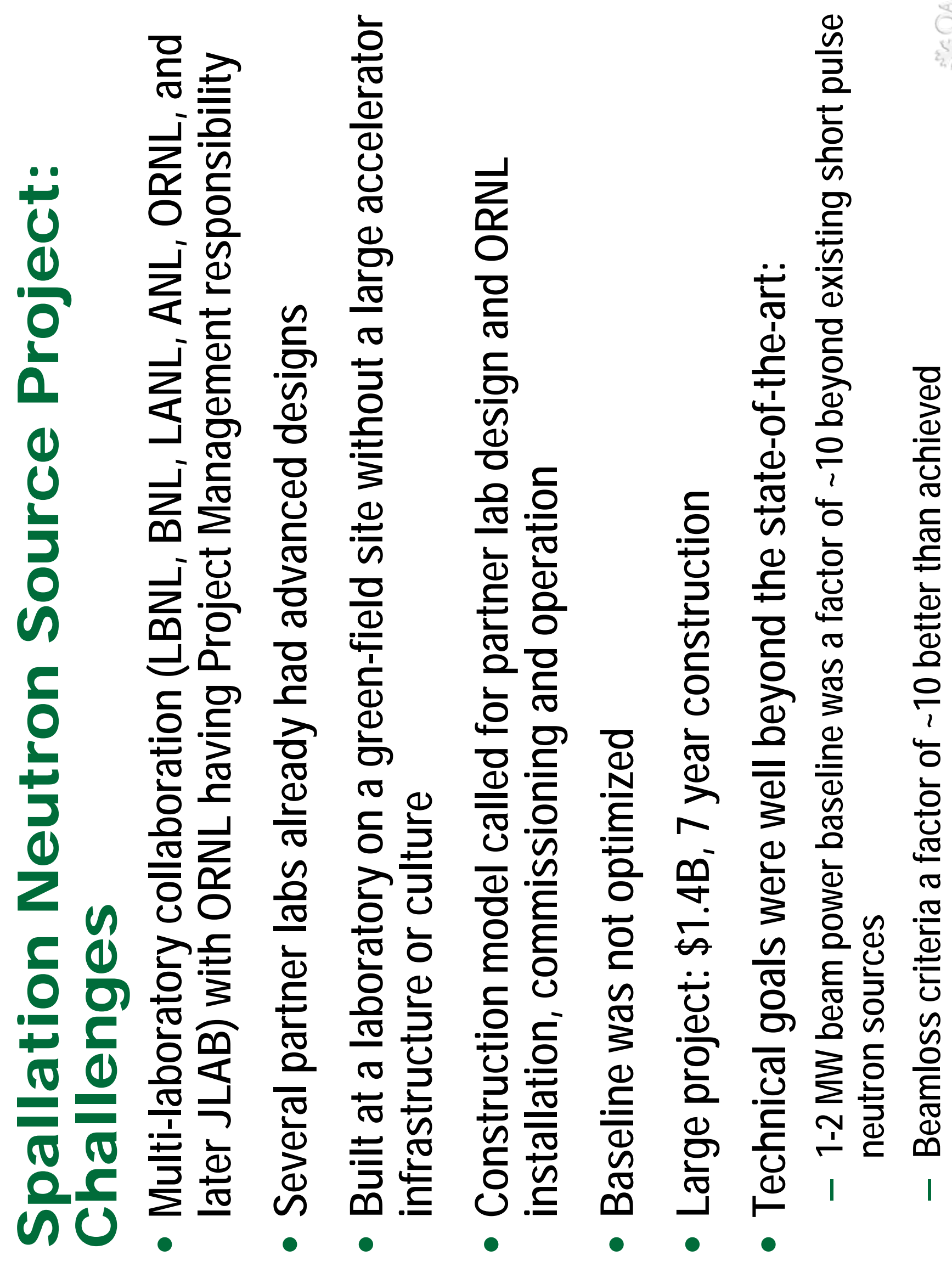




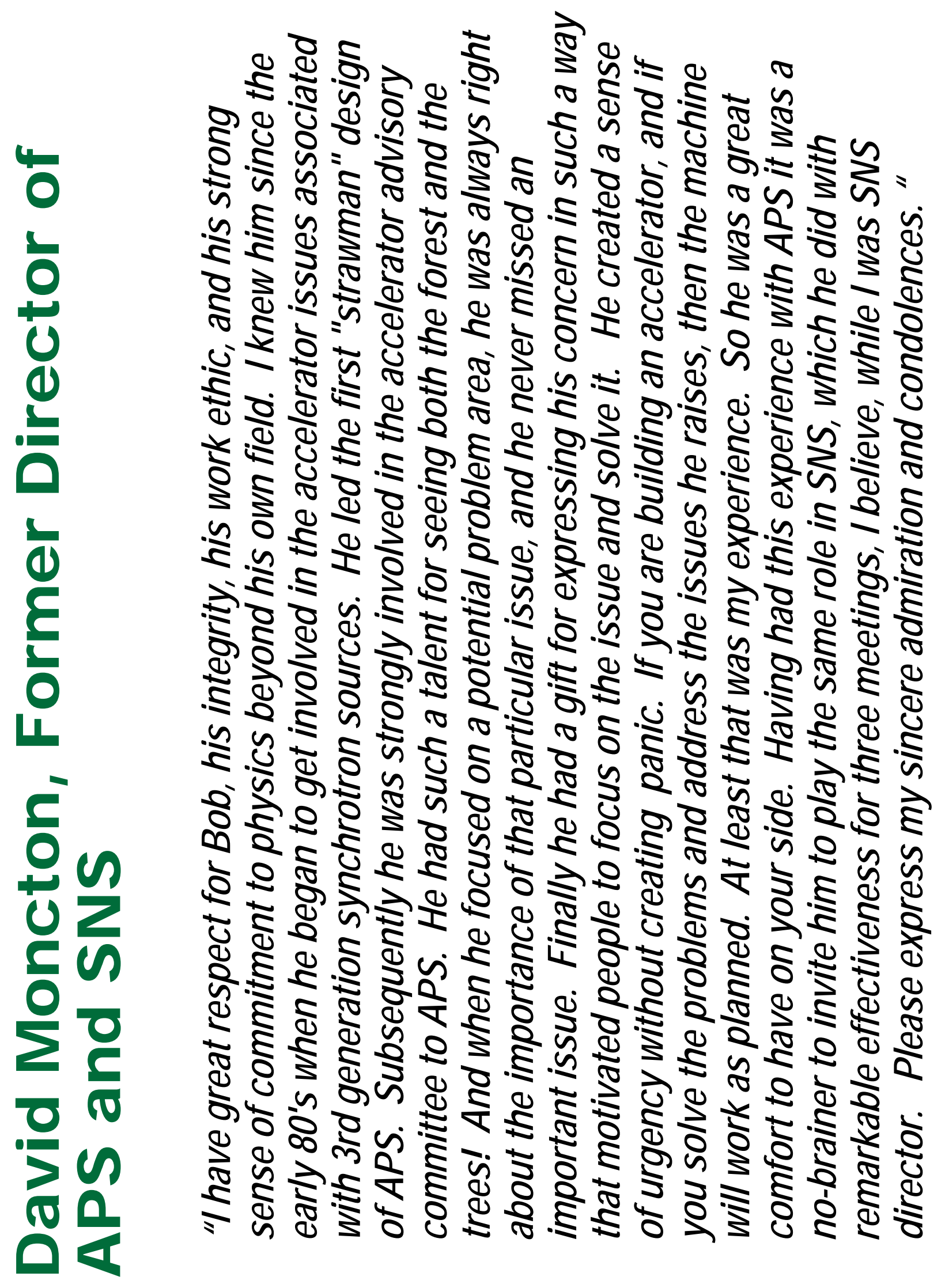




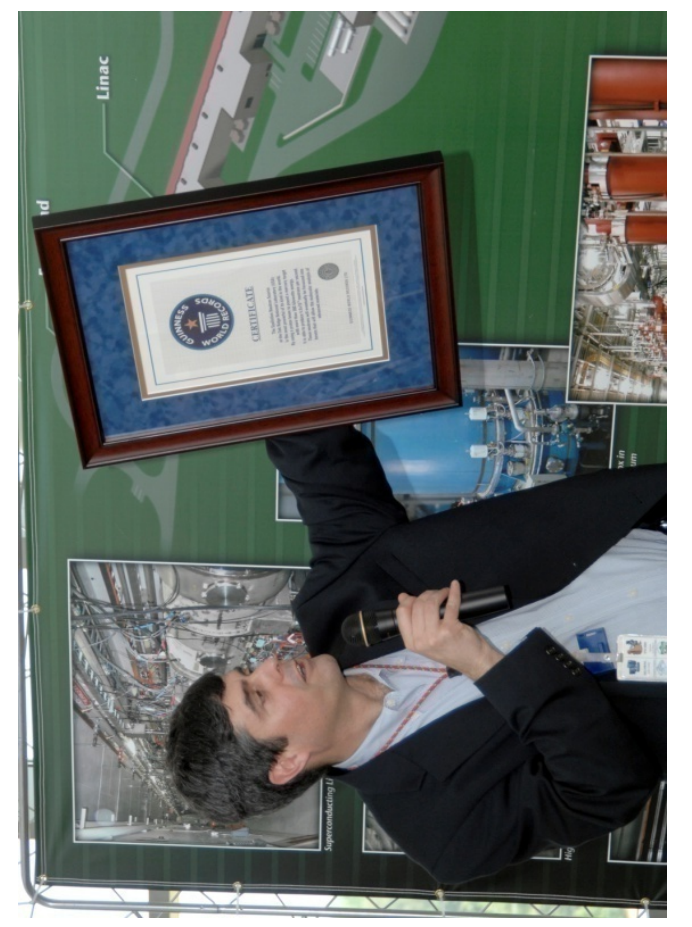

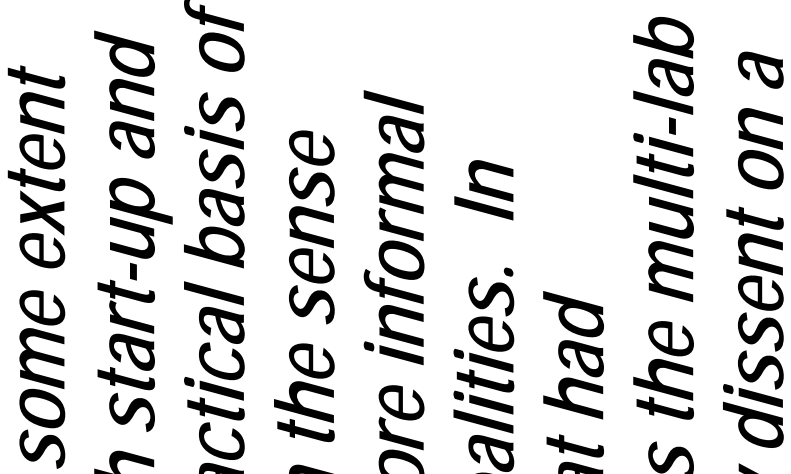

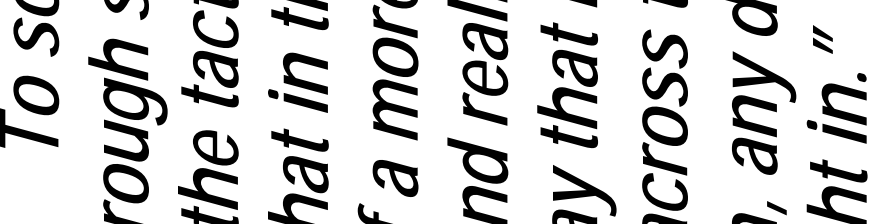

衣方

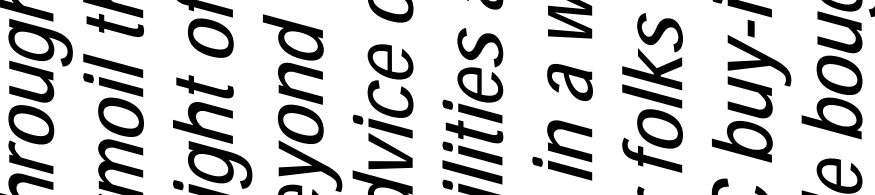

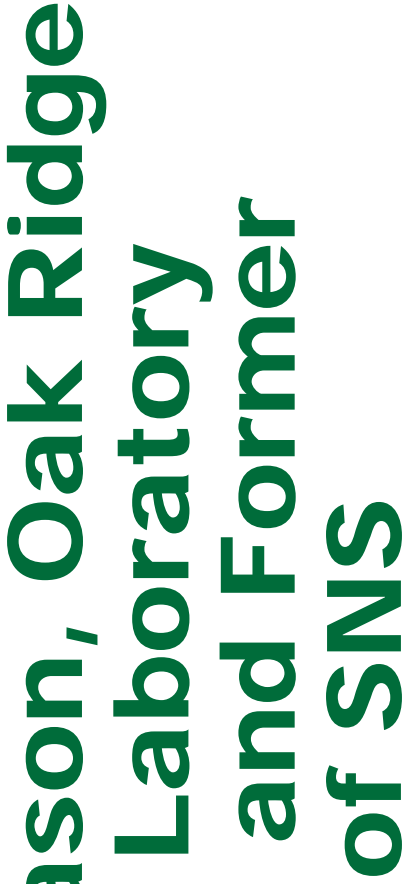

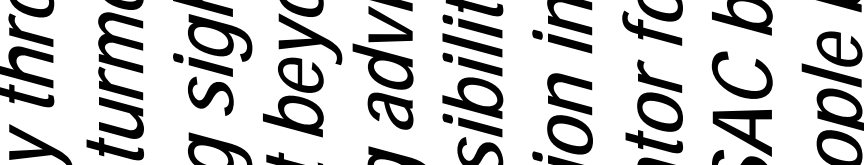

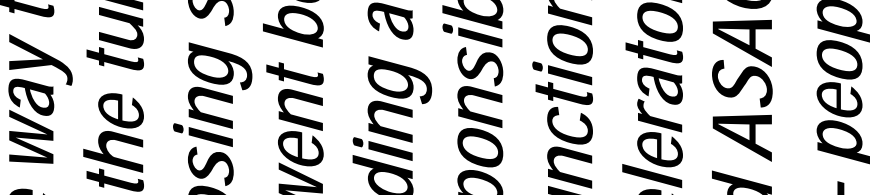

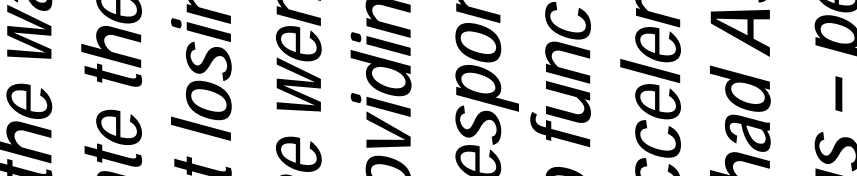

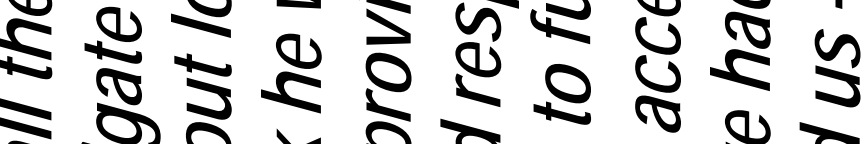

क

पु

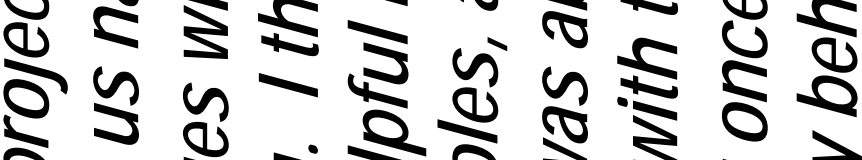

2 व के कर

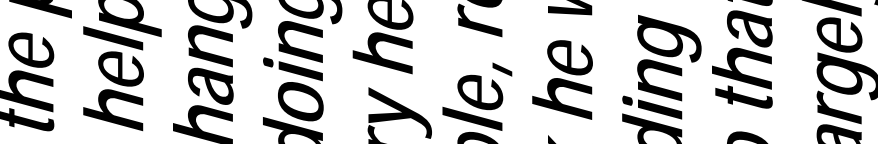

₹ 9 ठ

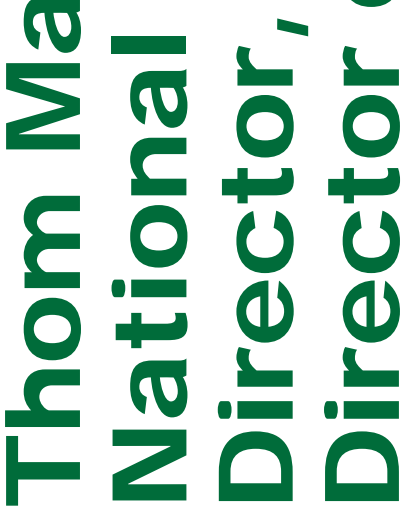

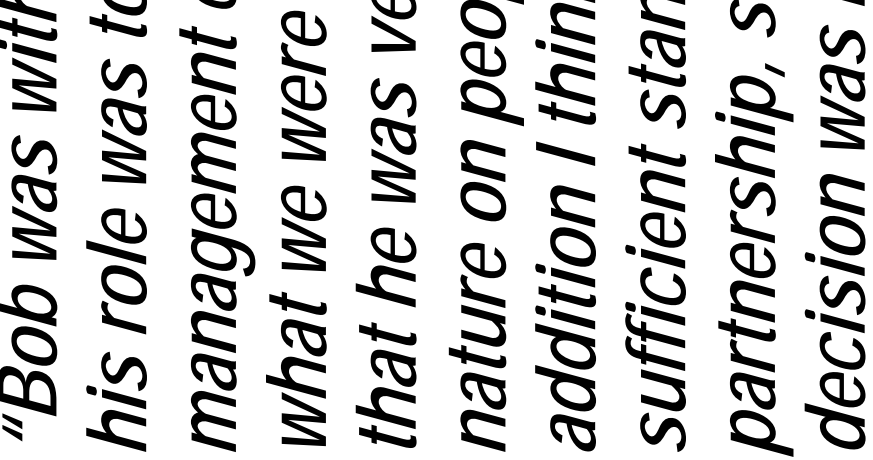




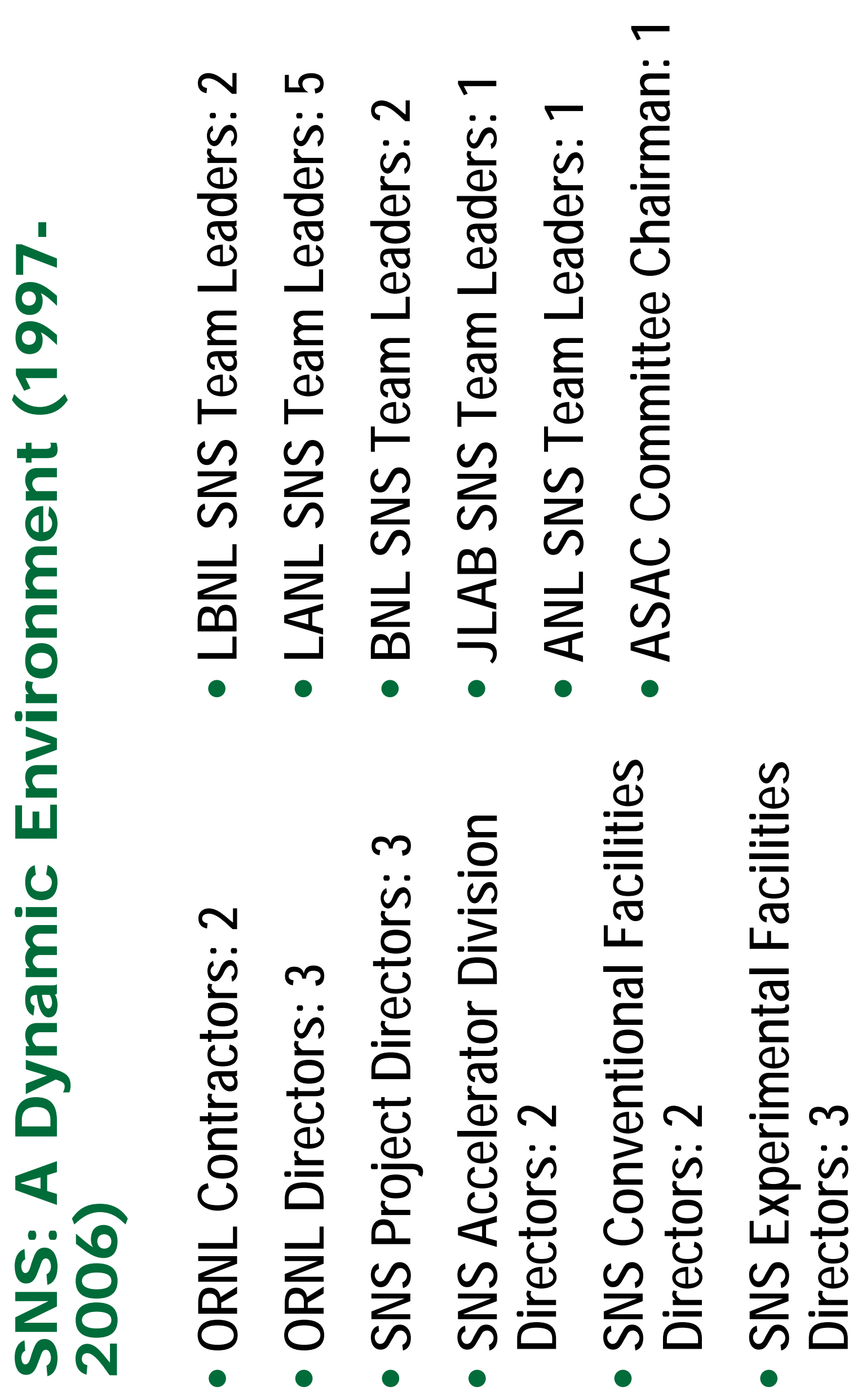




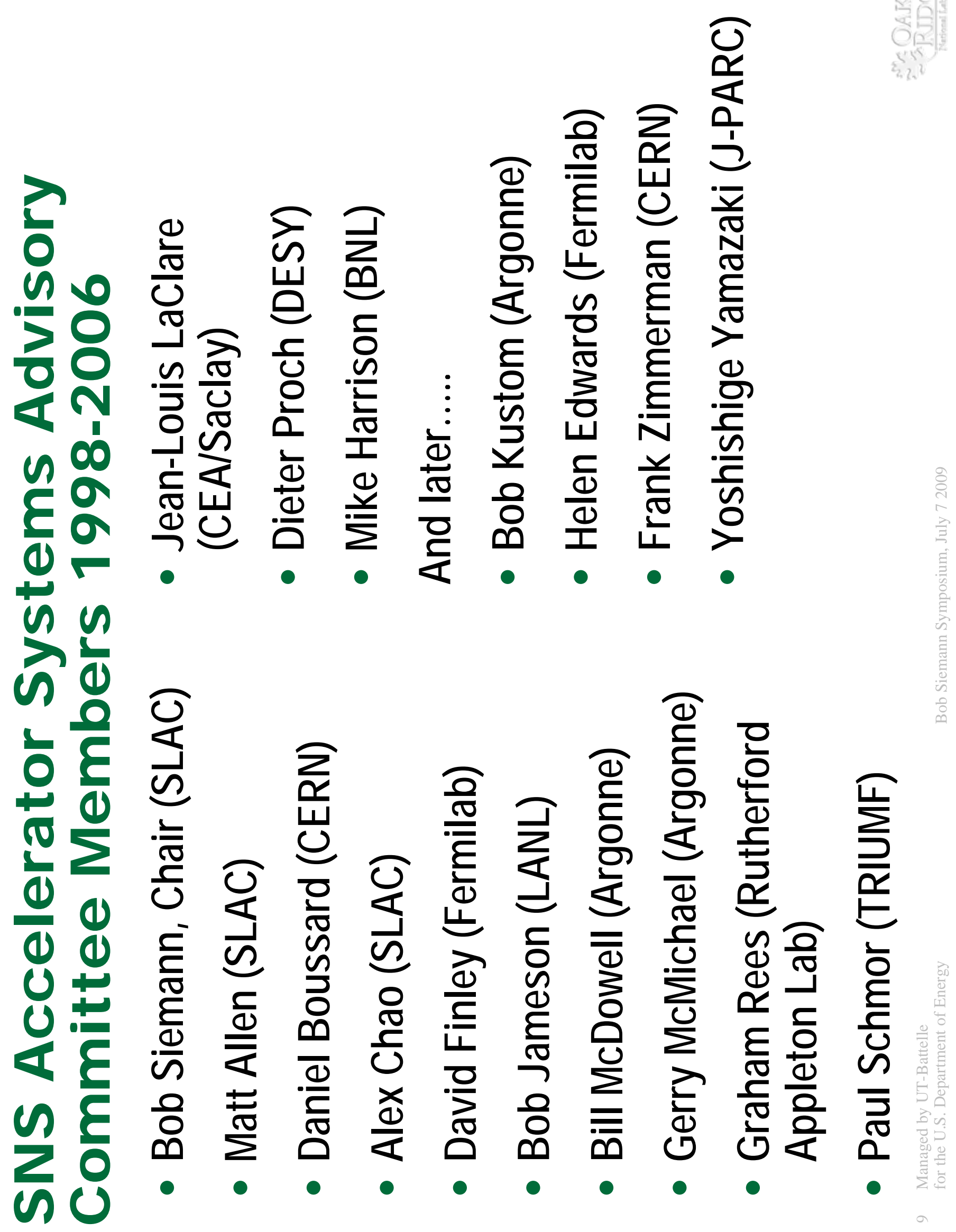




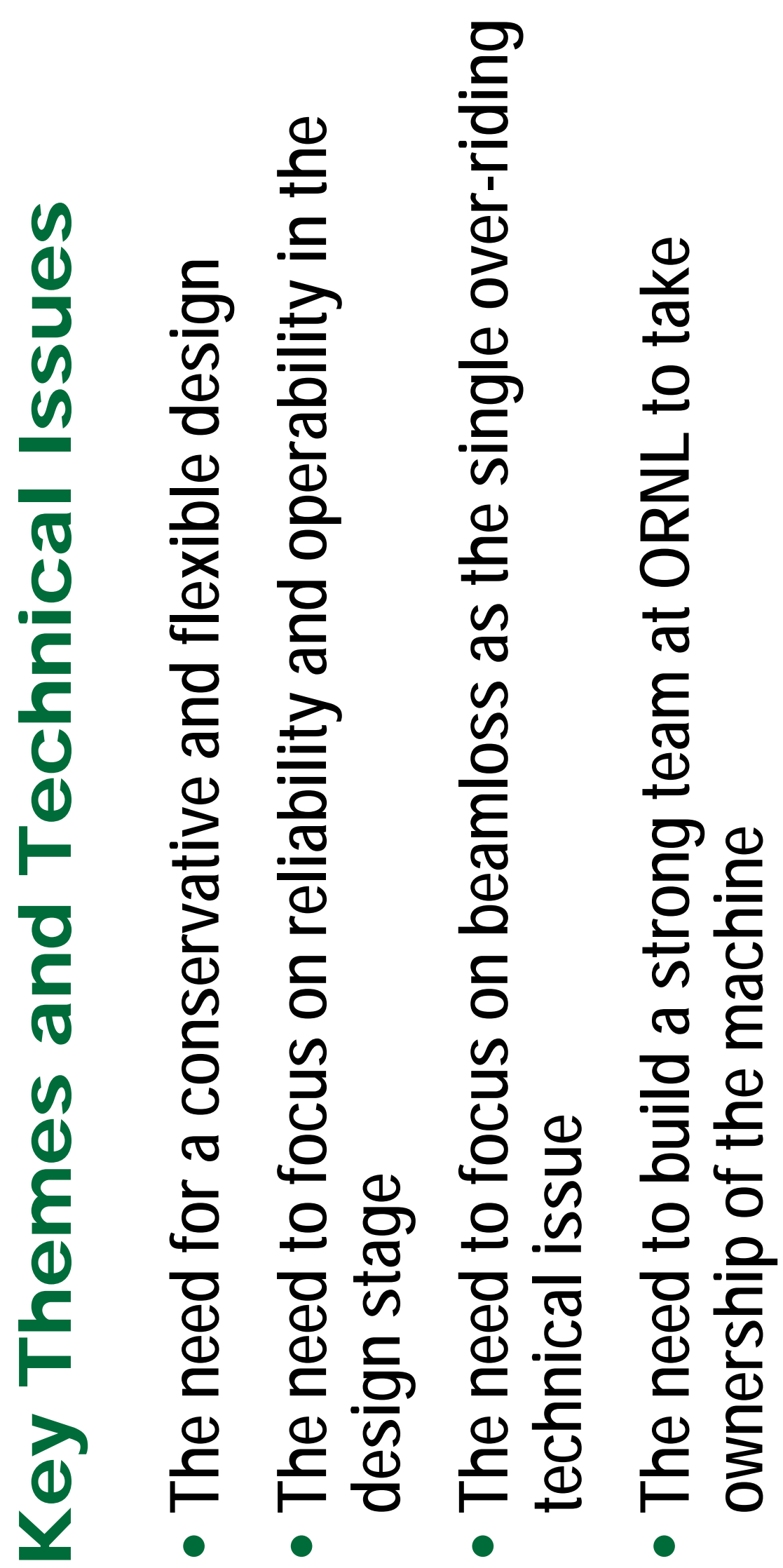




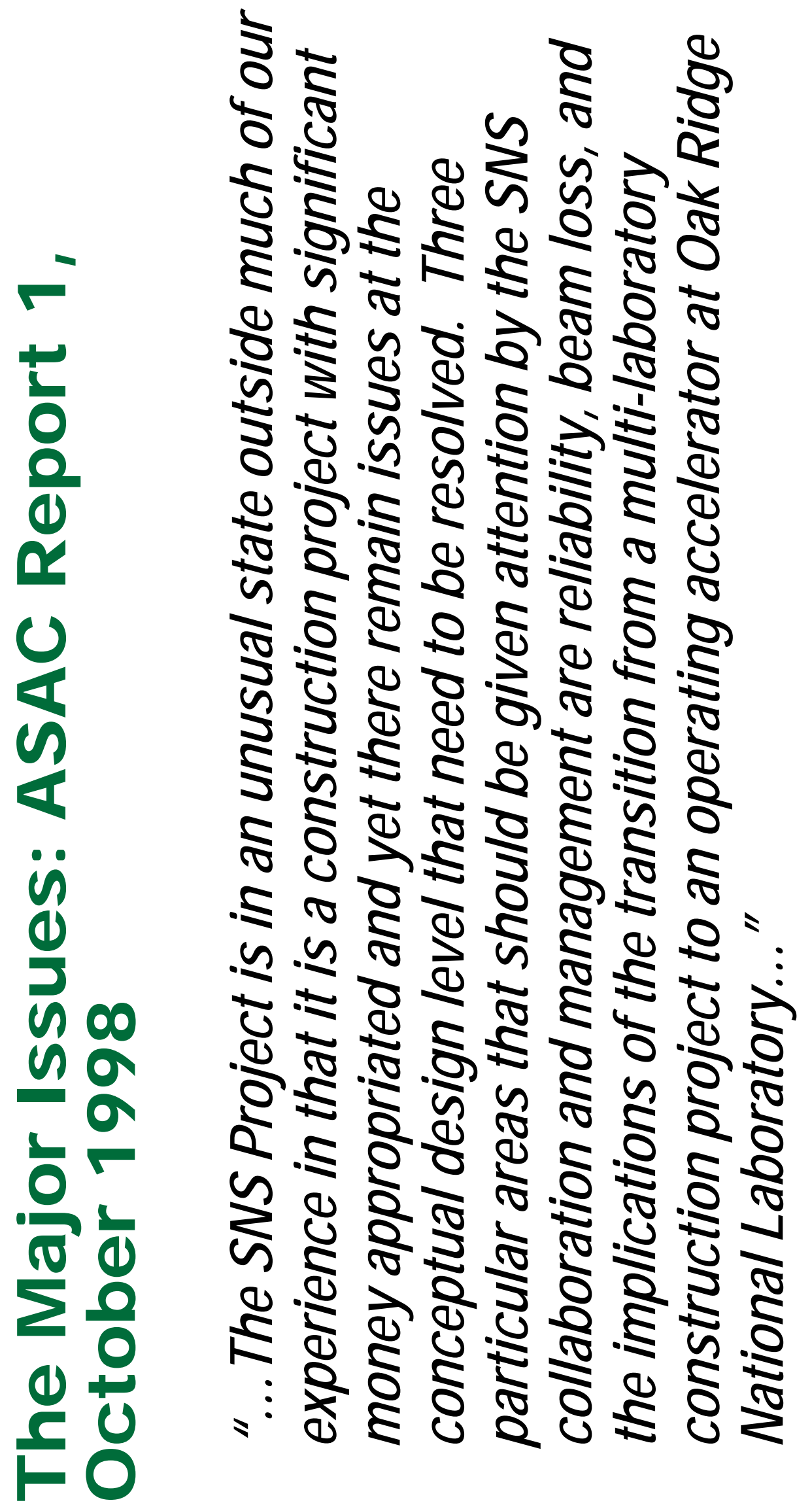




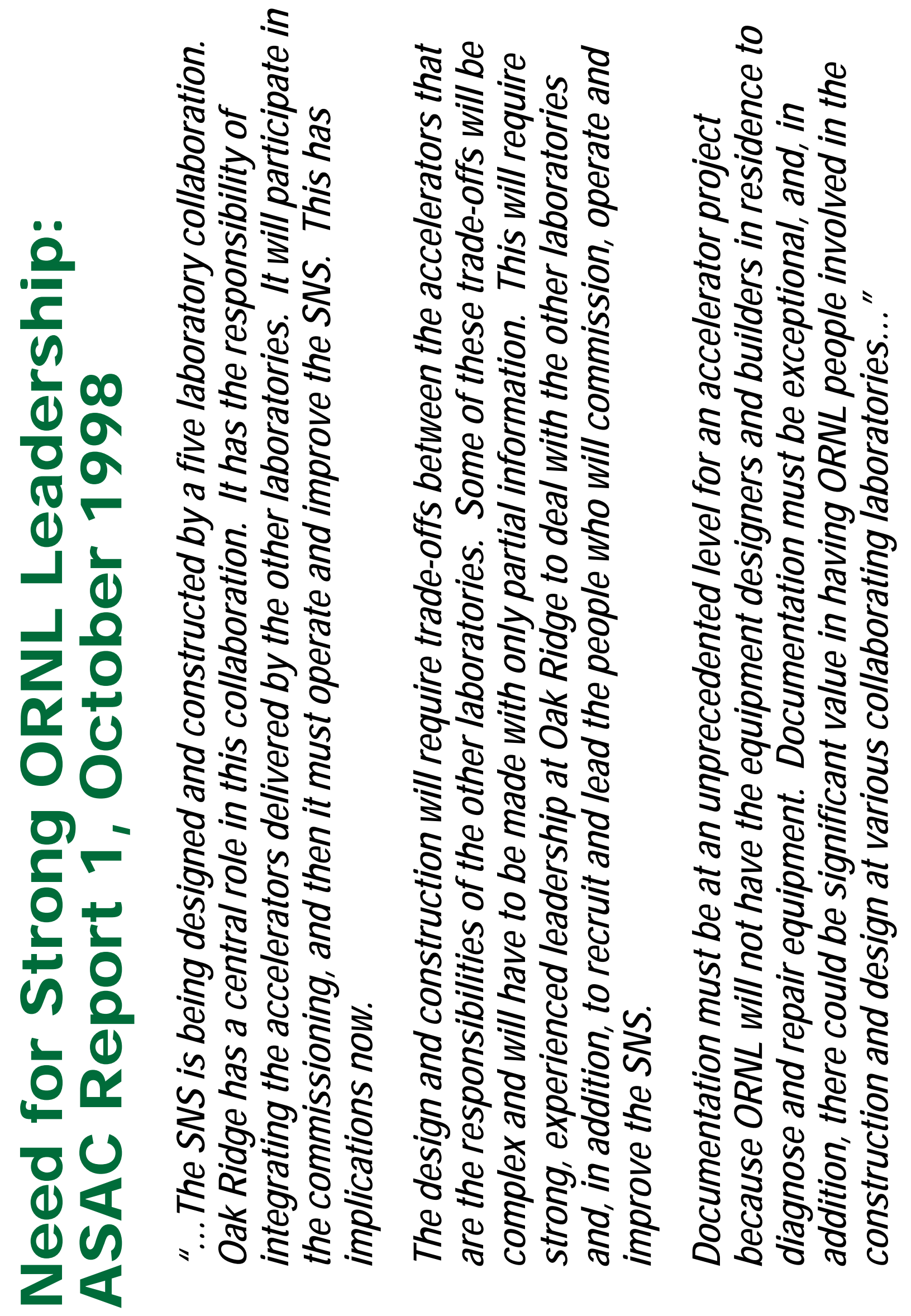


(1)

응

$+$

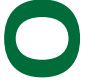

H

능

(1)

$\alpha$

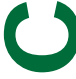

4

今

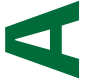

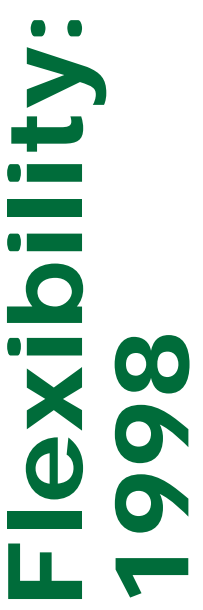

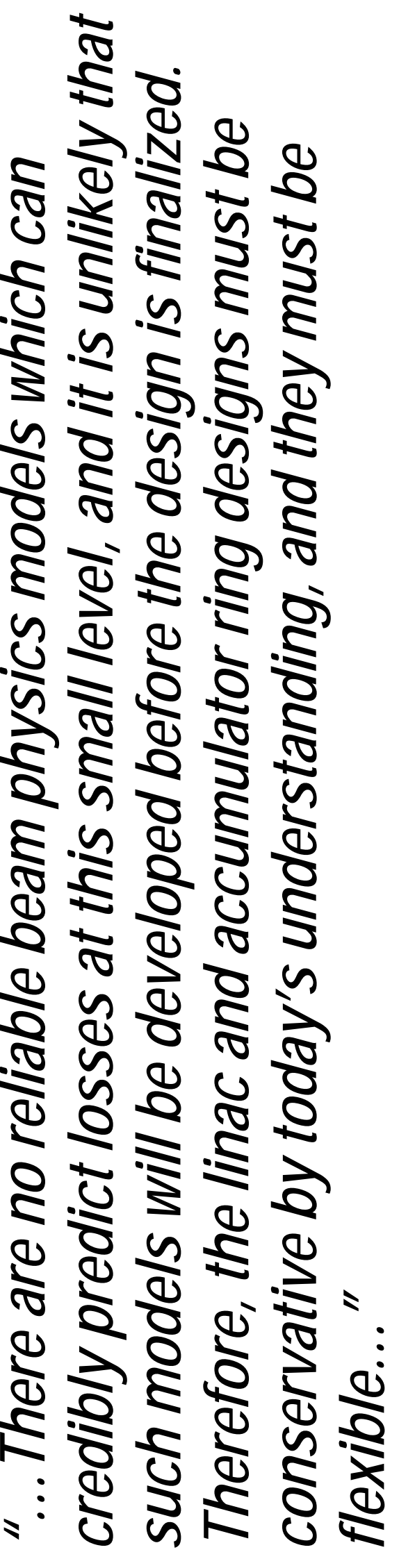




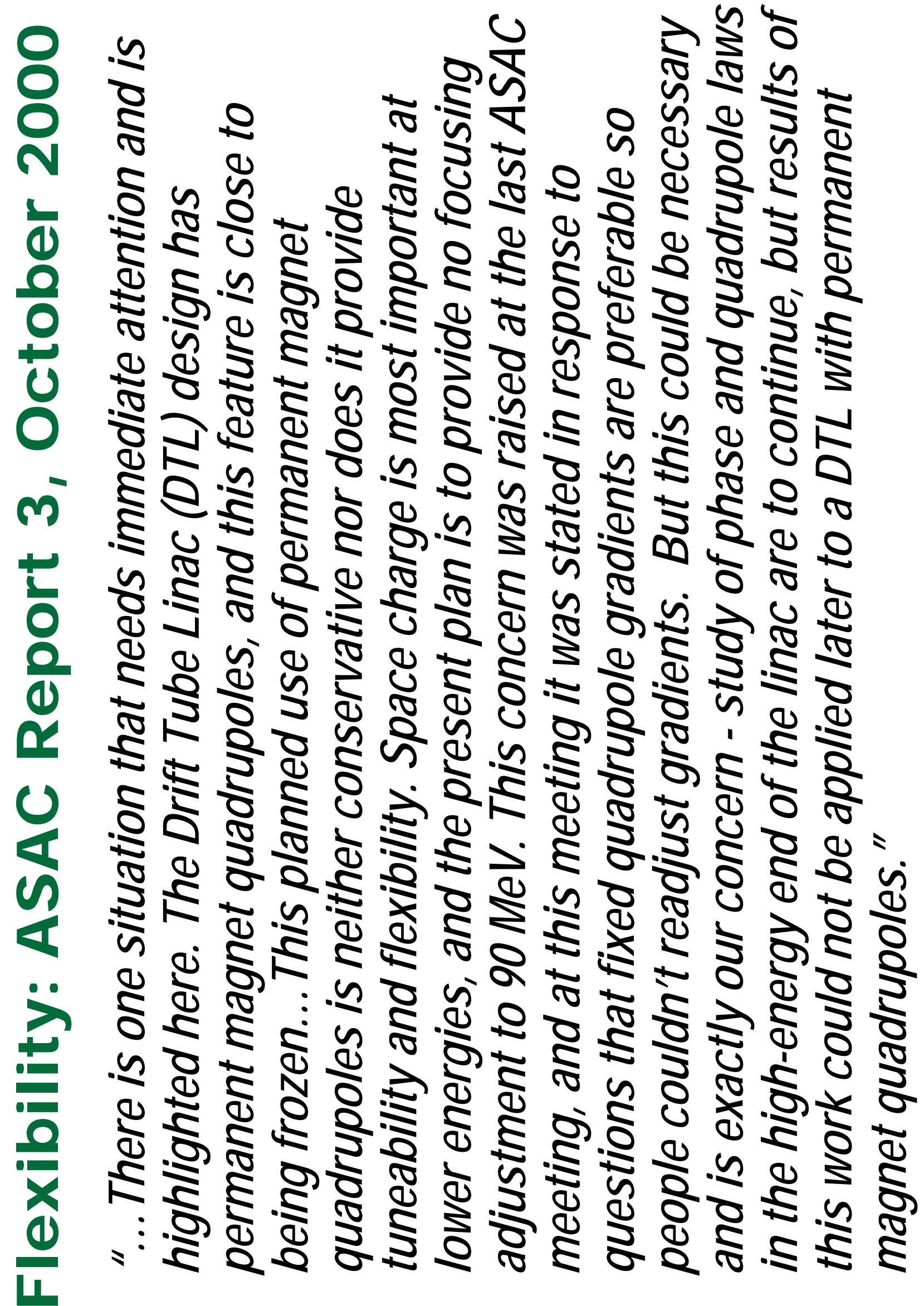




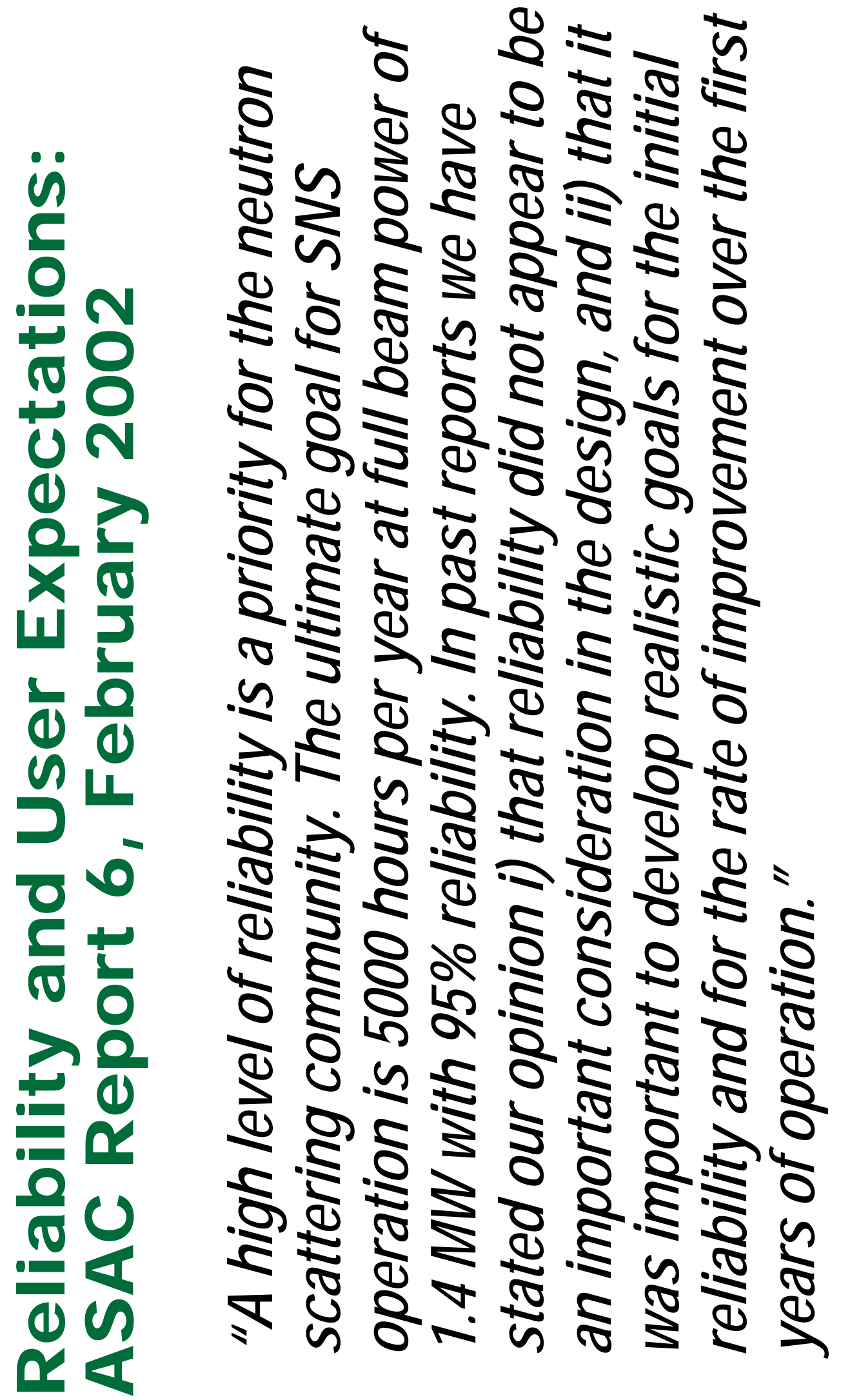




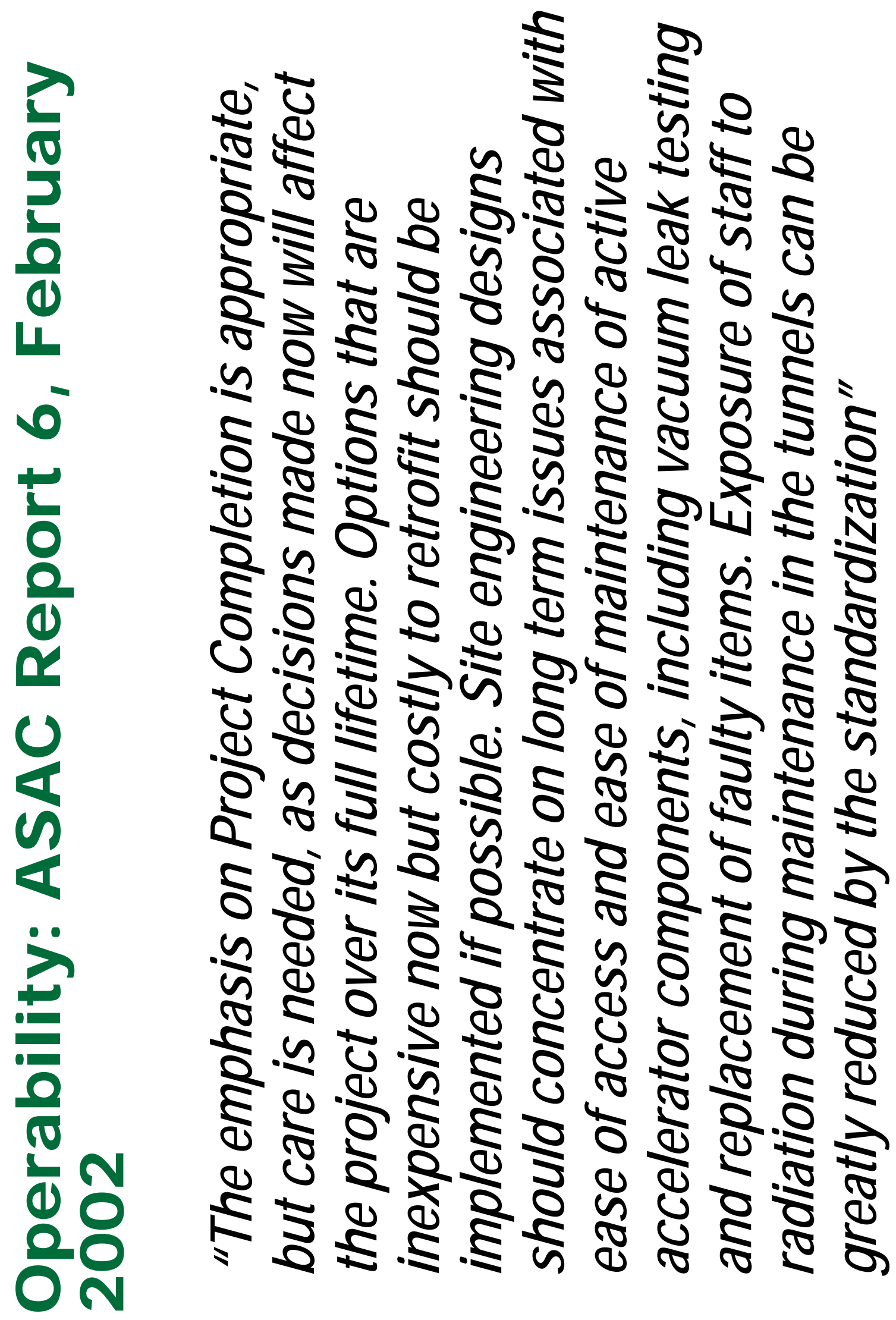


5

\%

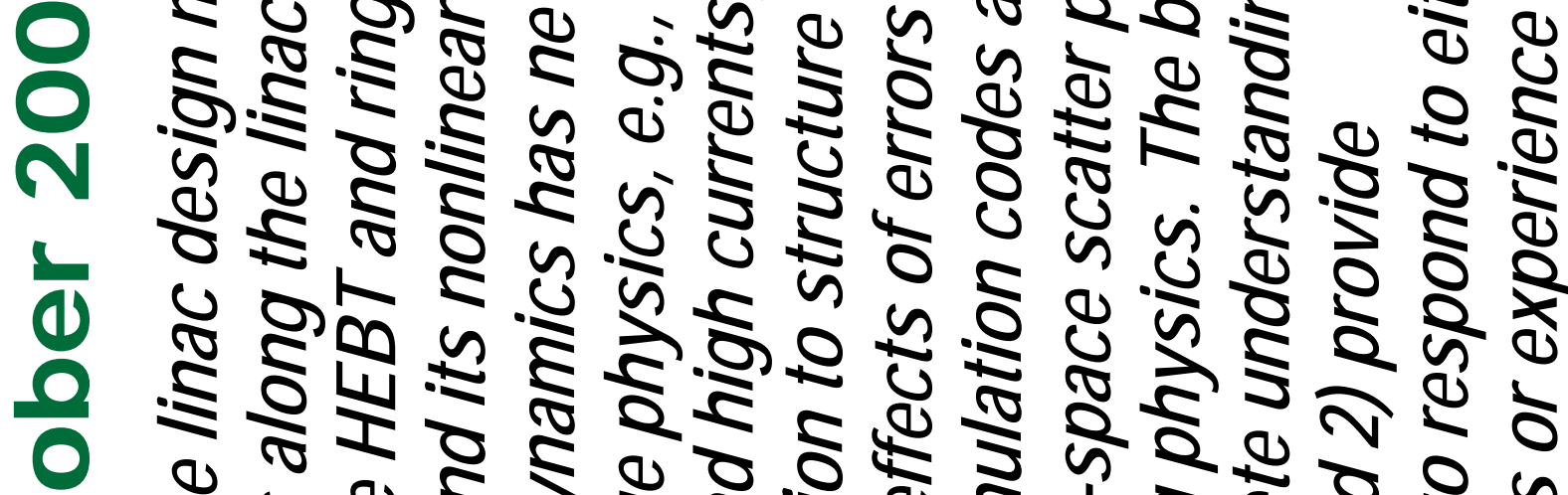

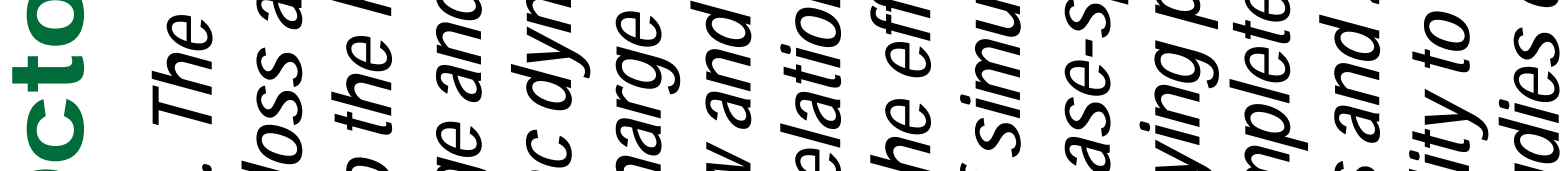

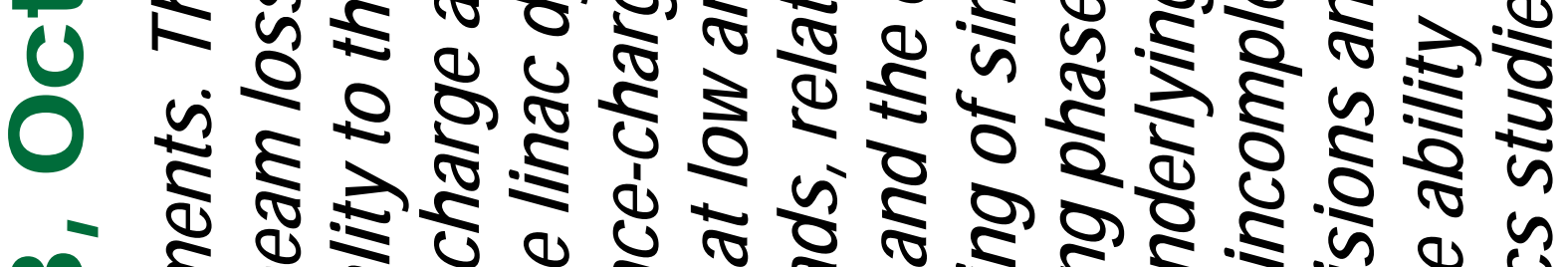

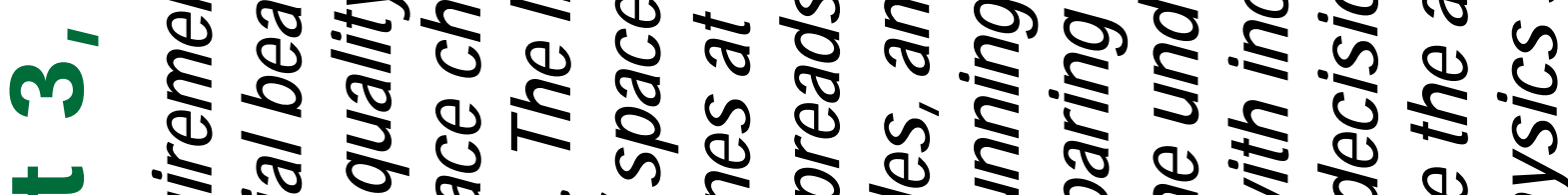

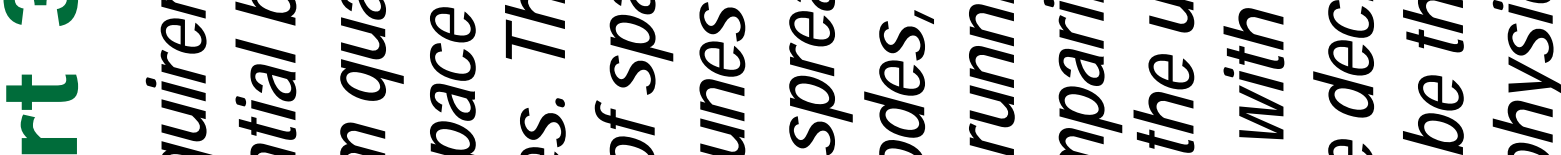

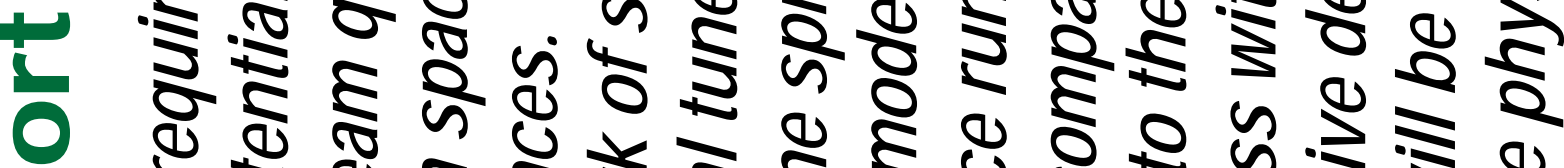

- पे

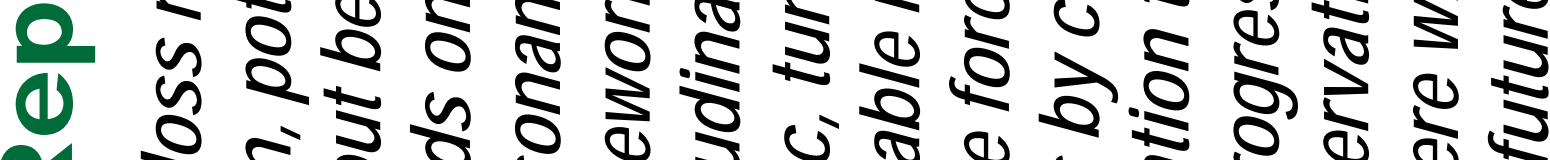

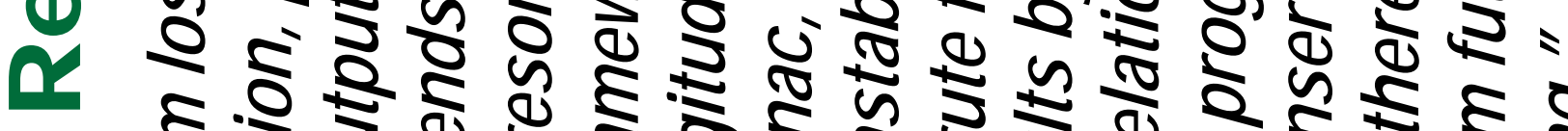

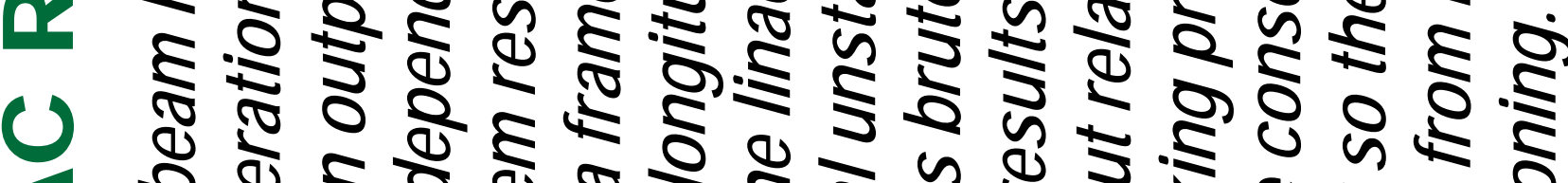

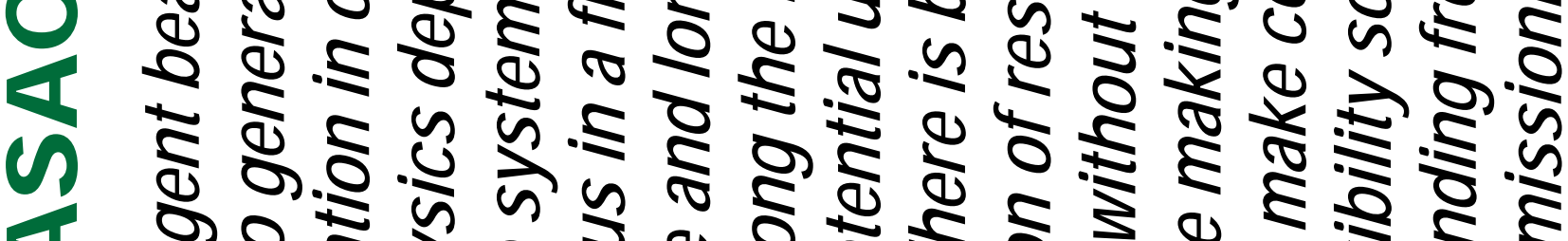

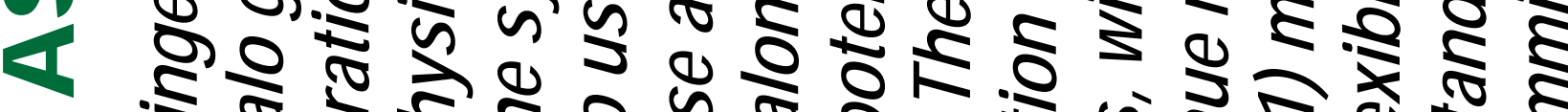

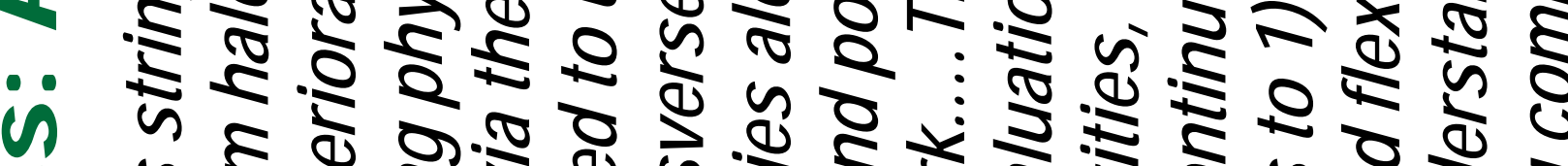

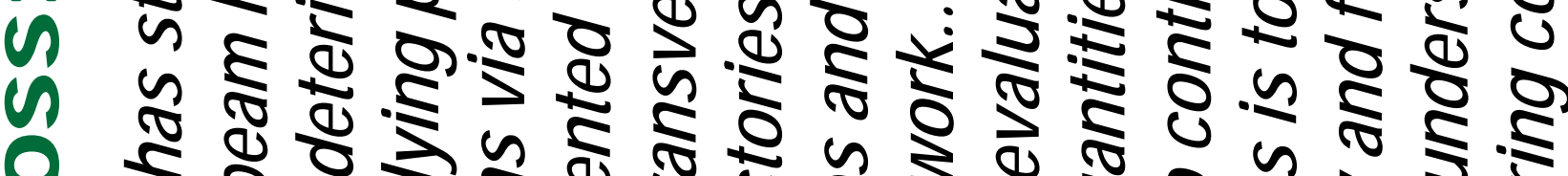

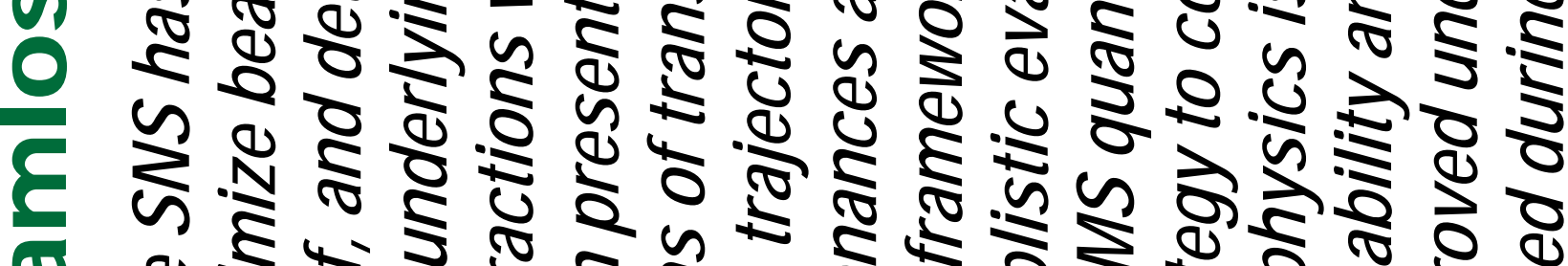

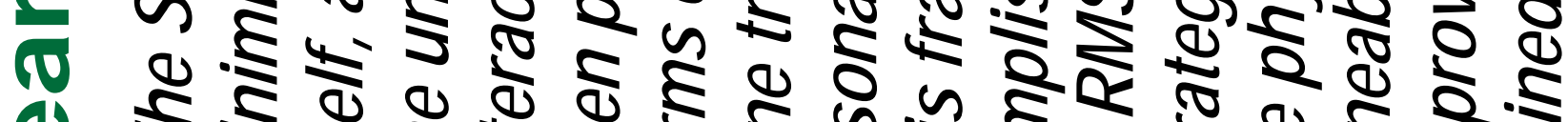

(1) है. 


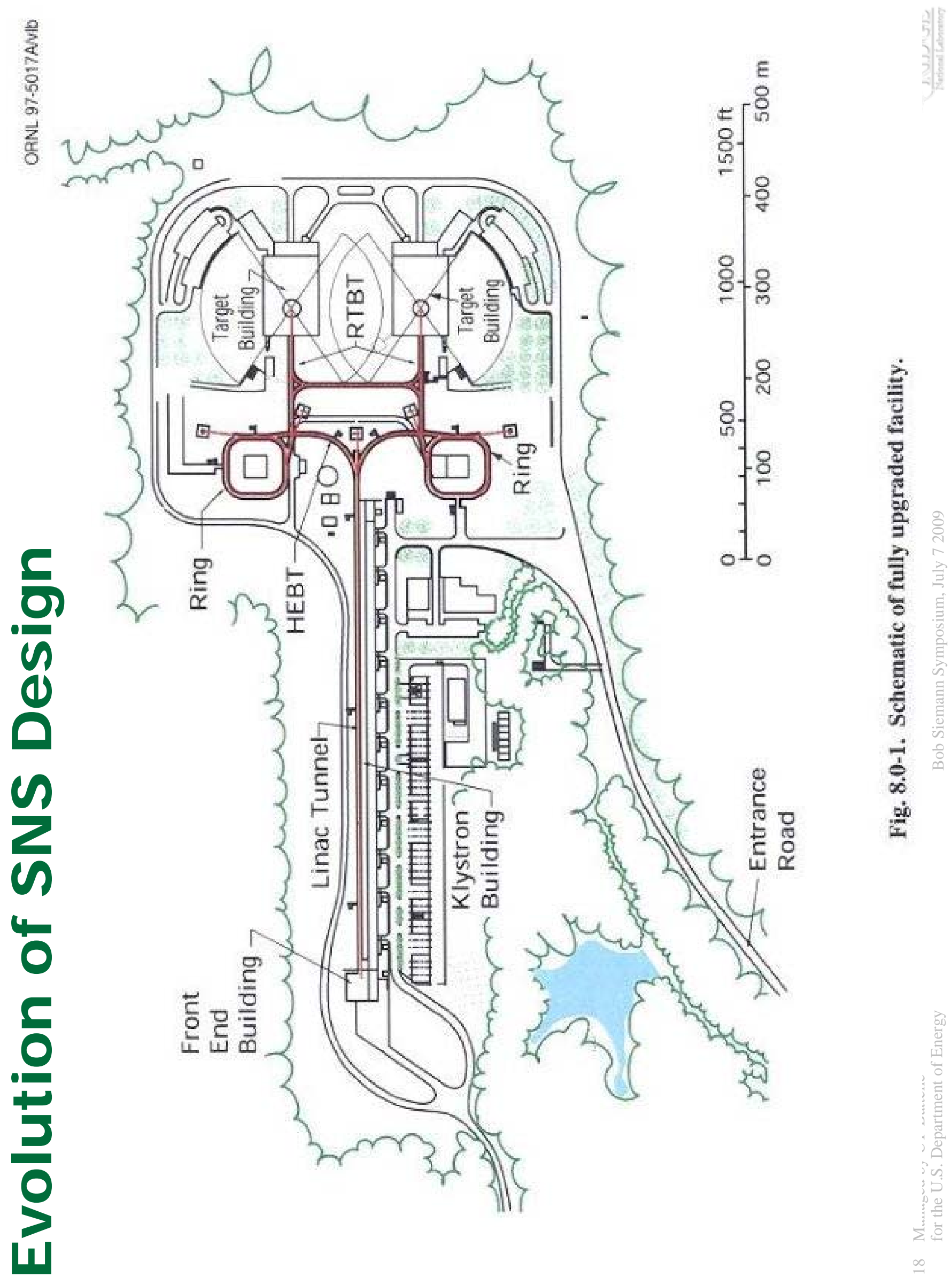




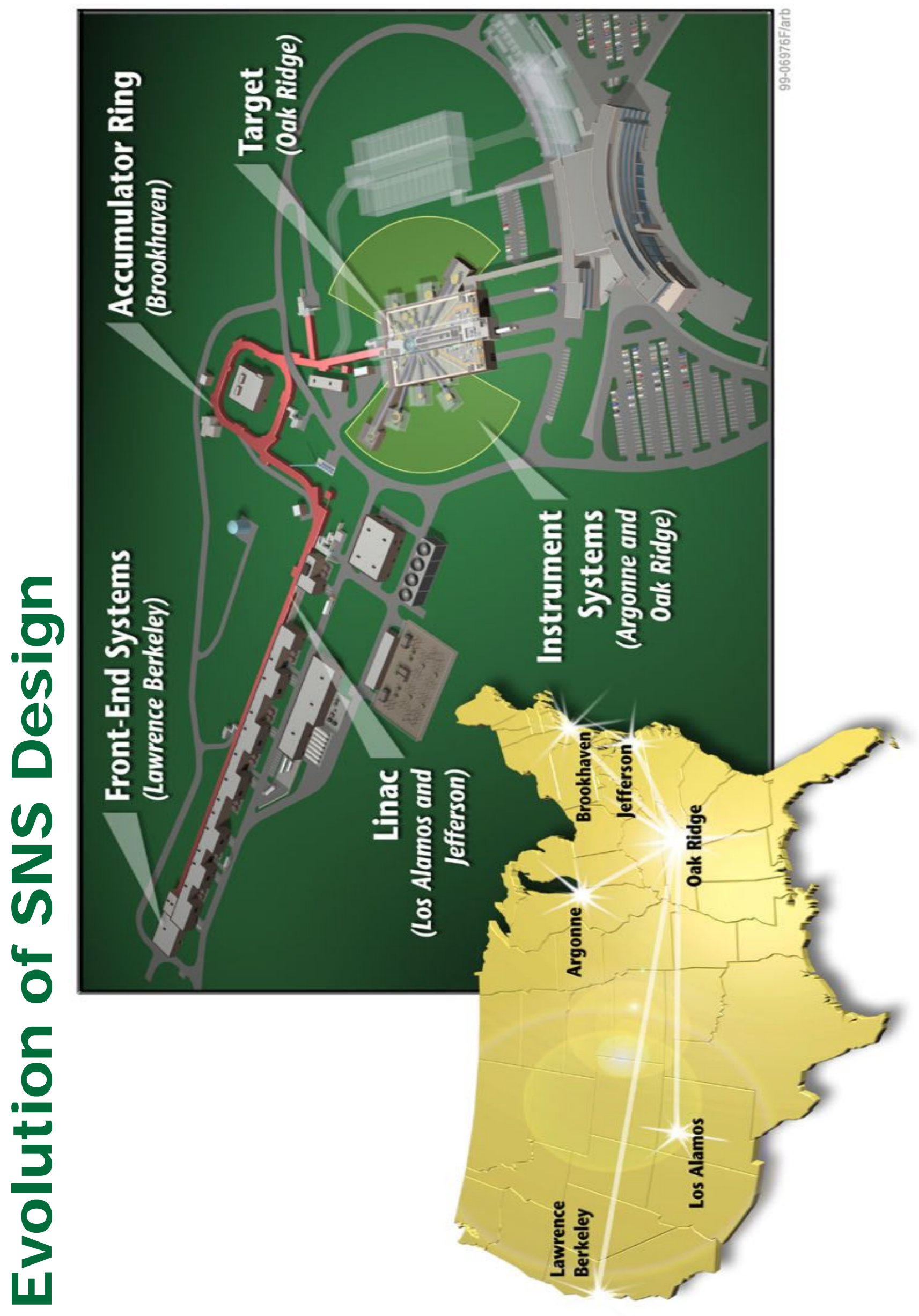




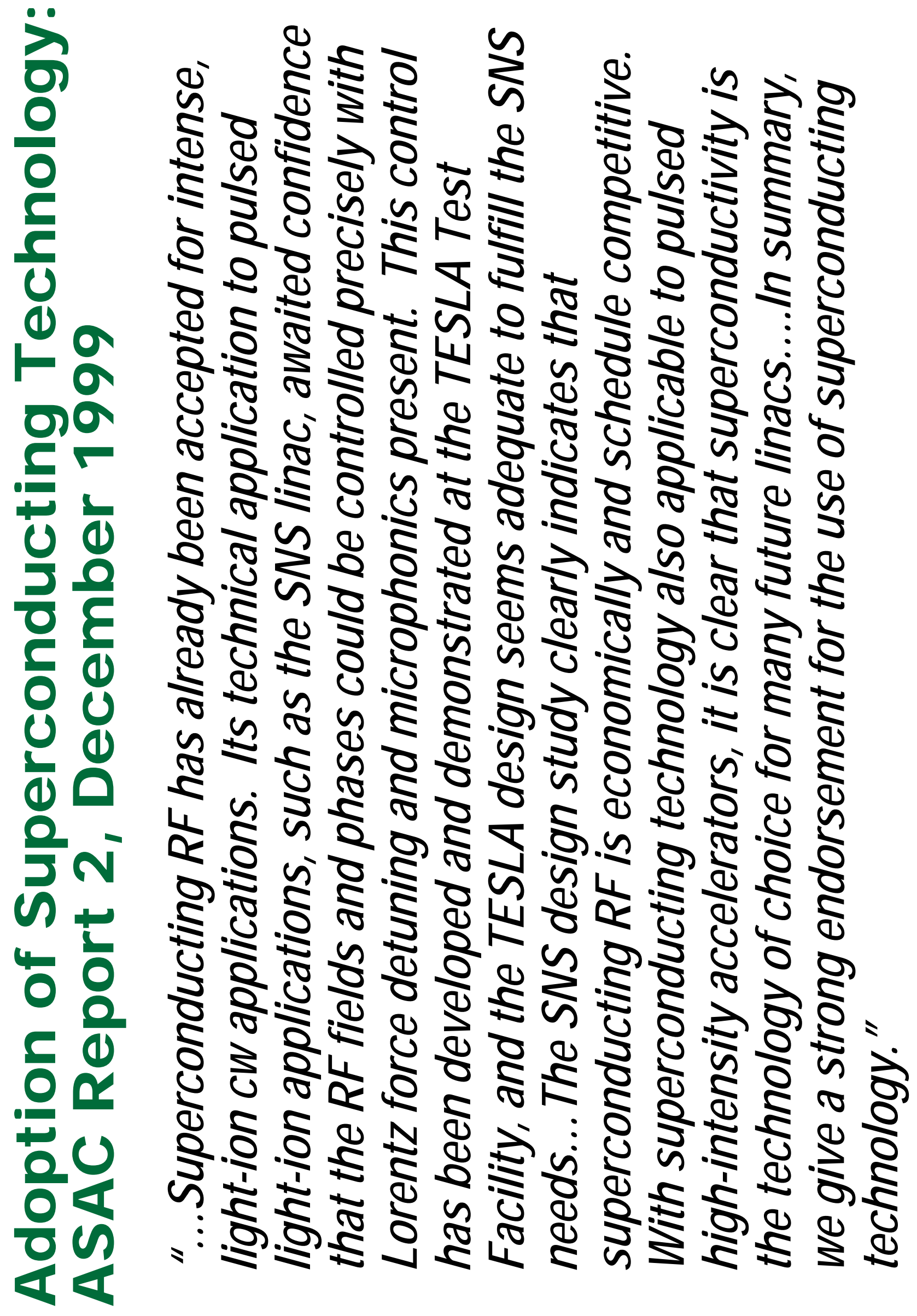



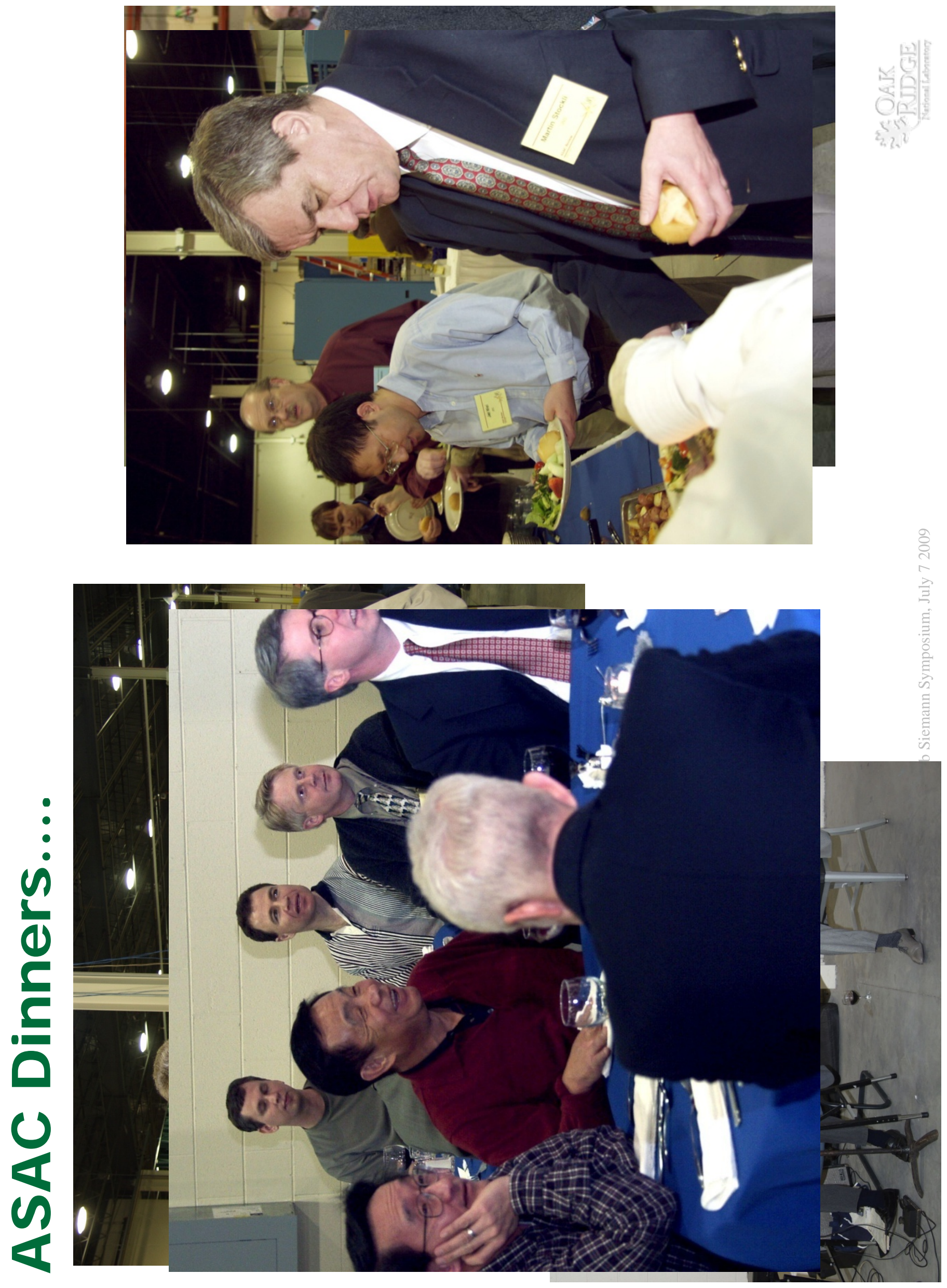

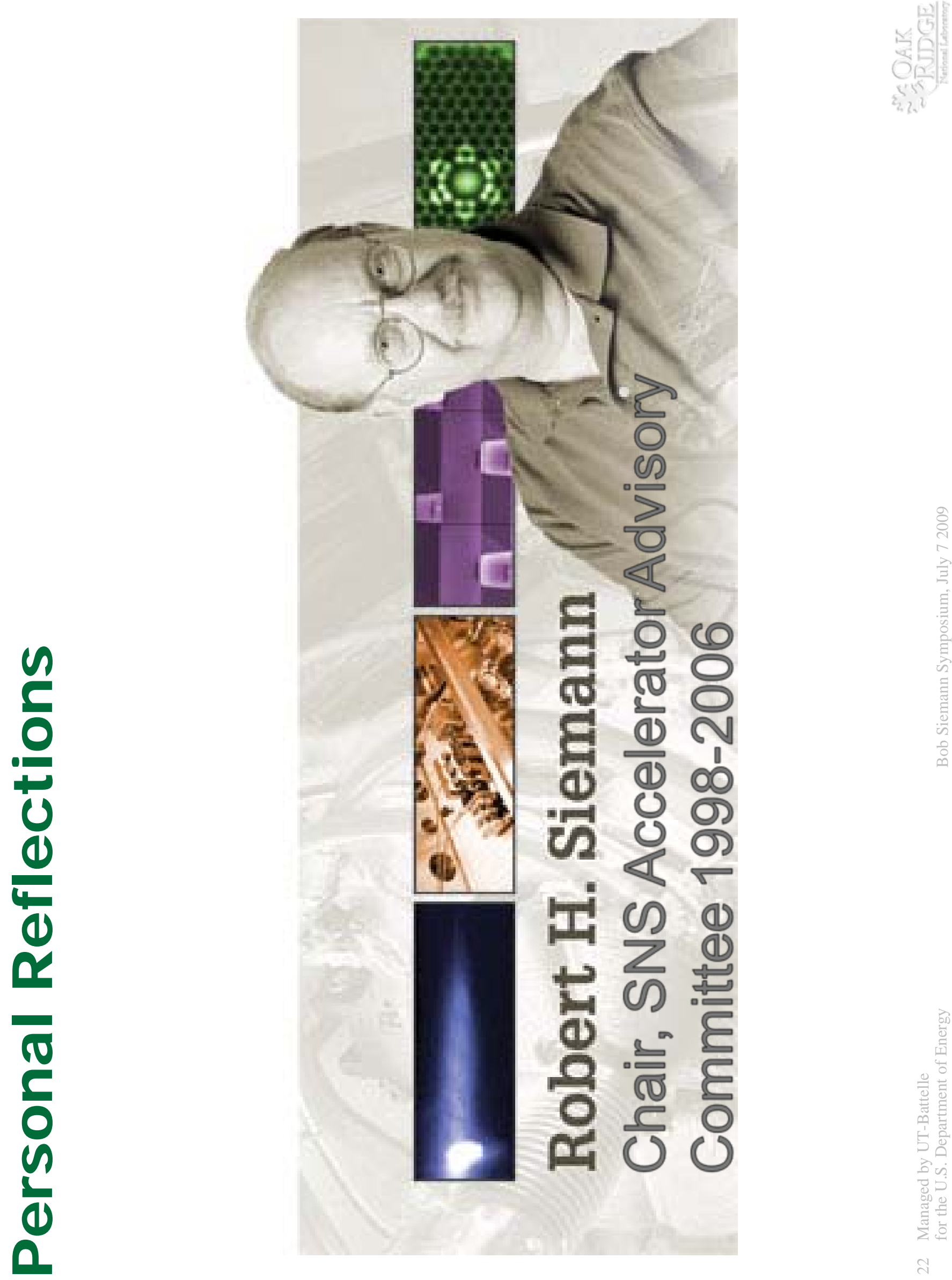


\section{Beam Power on Target (kW)}

号

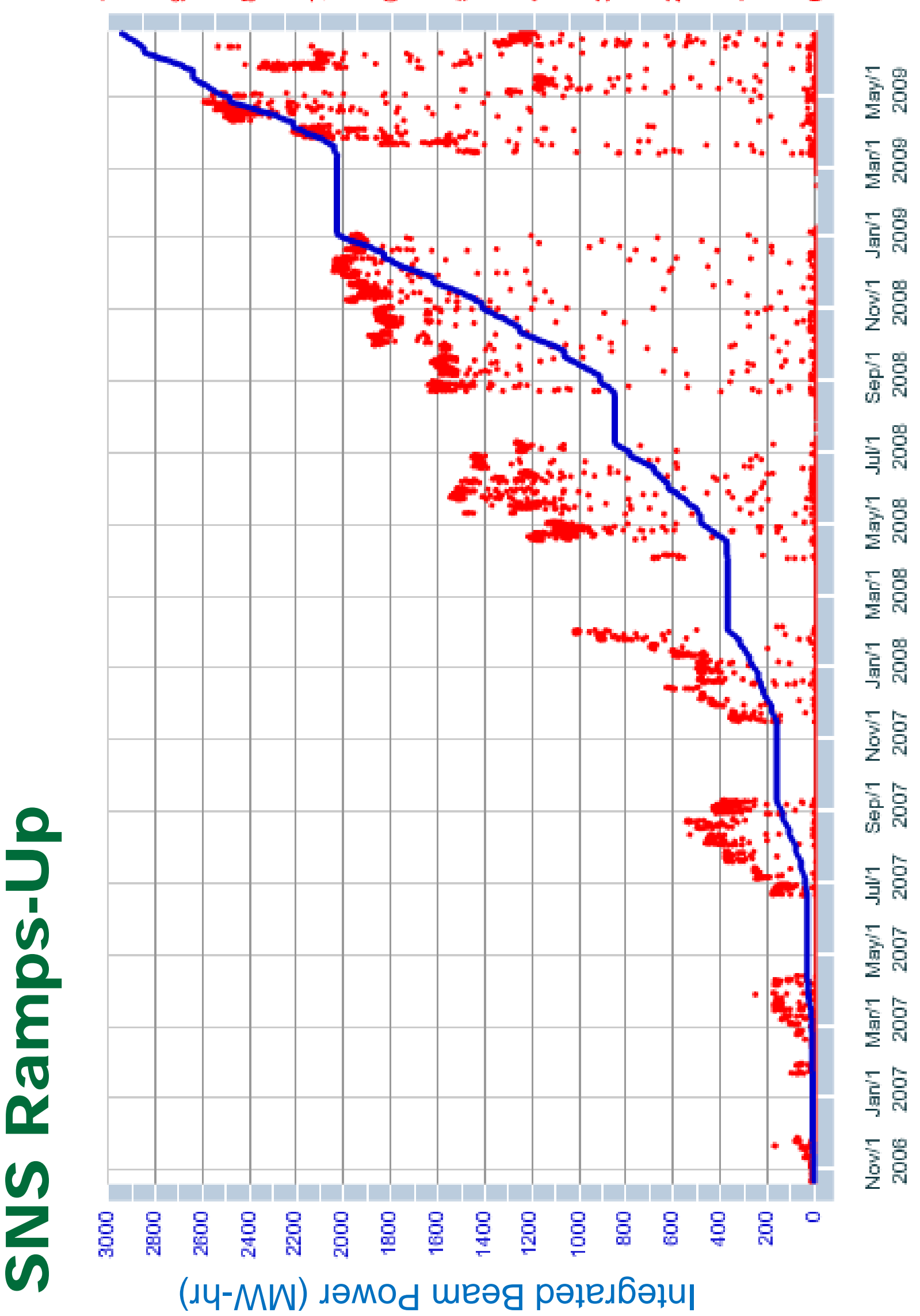




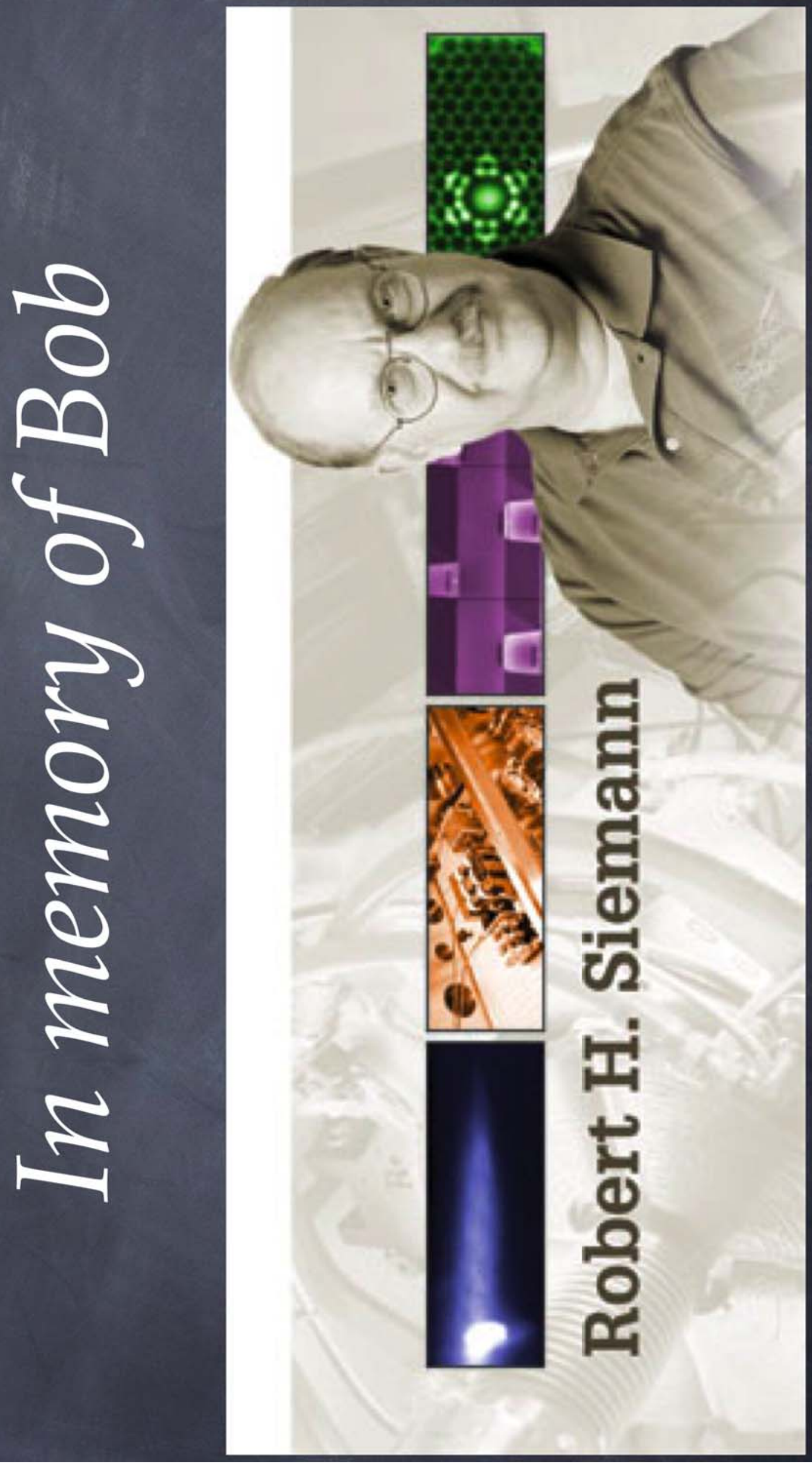




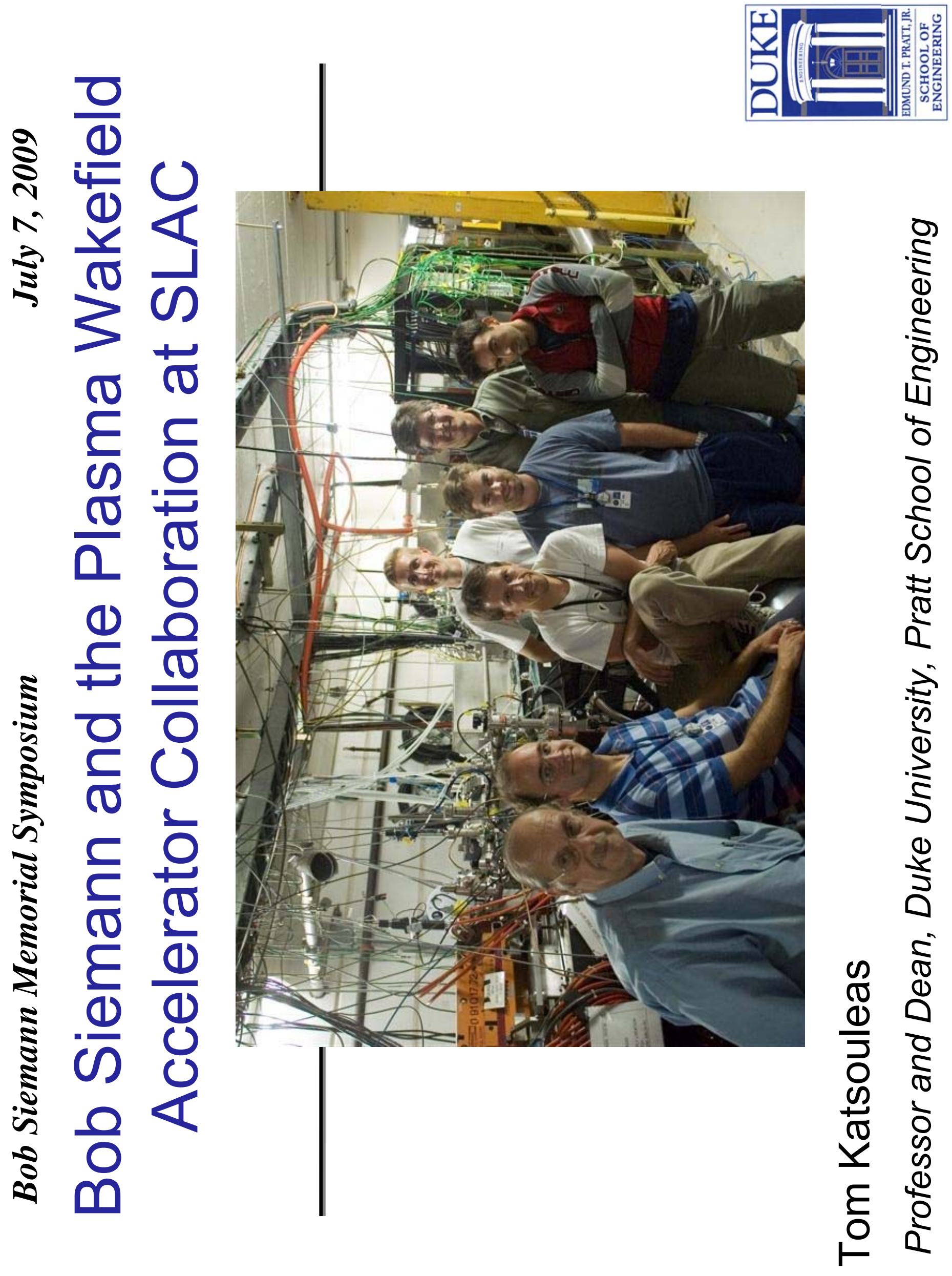




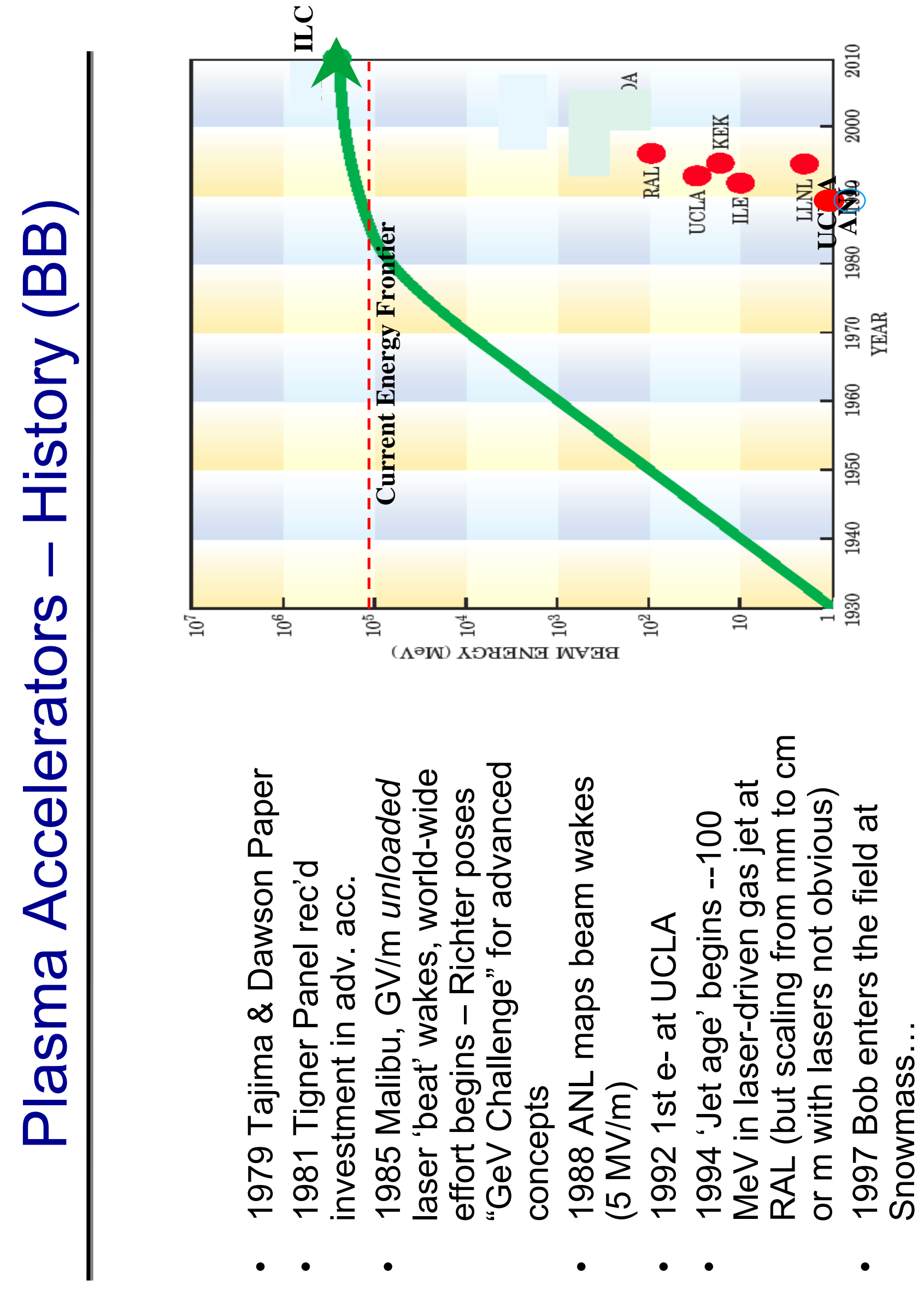




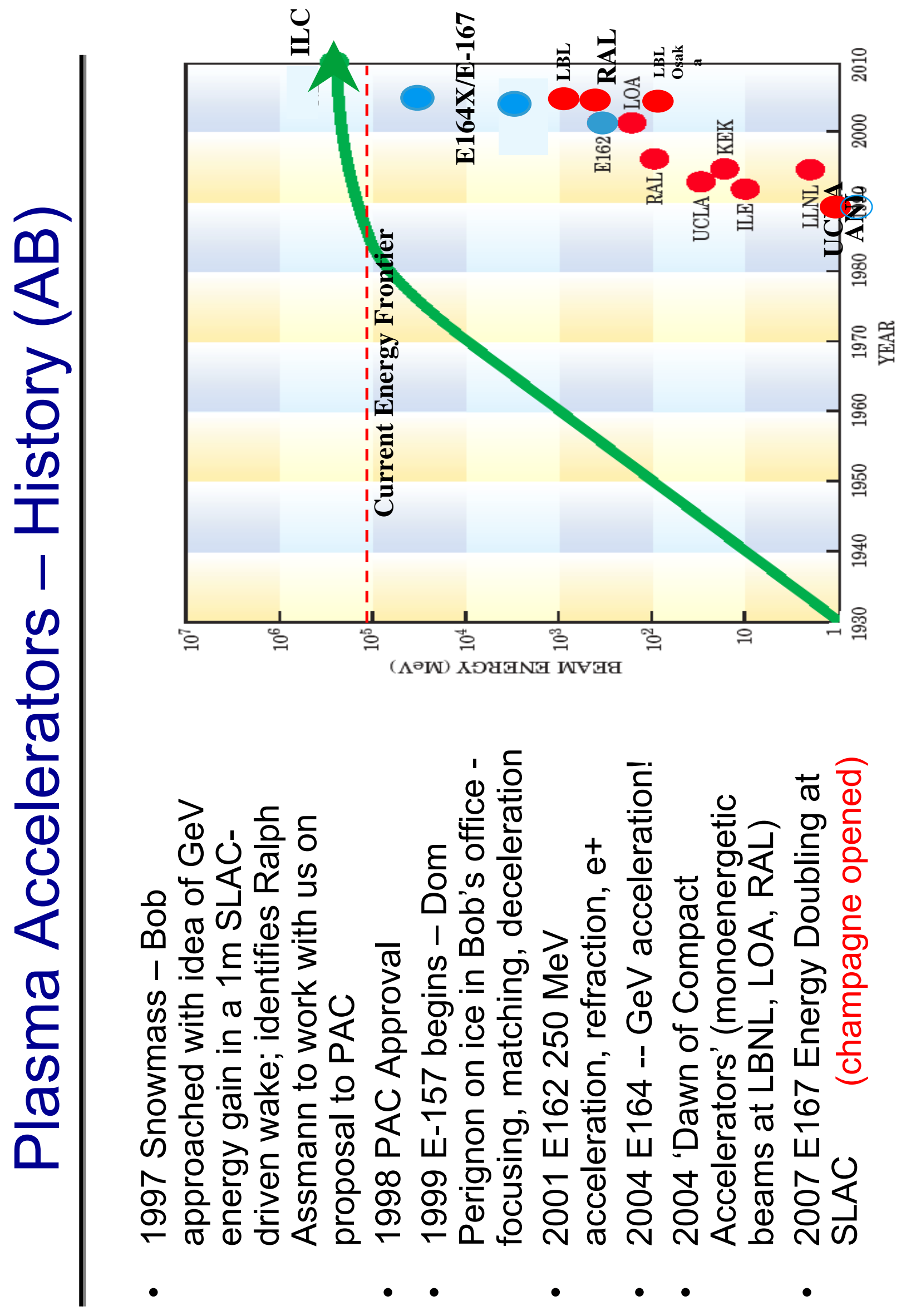



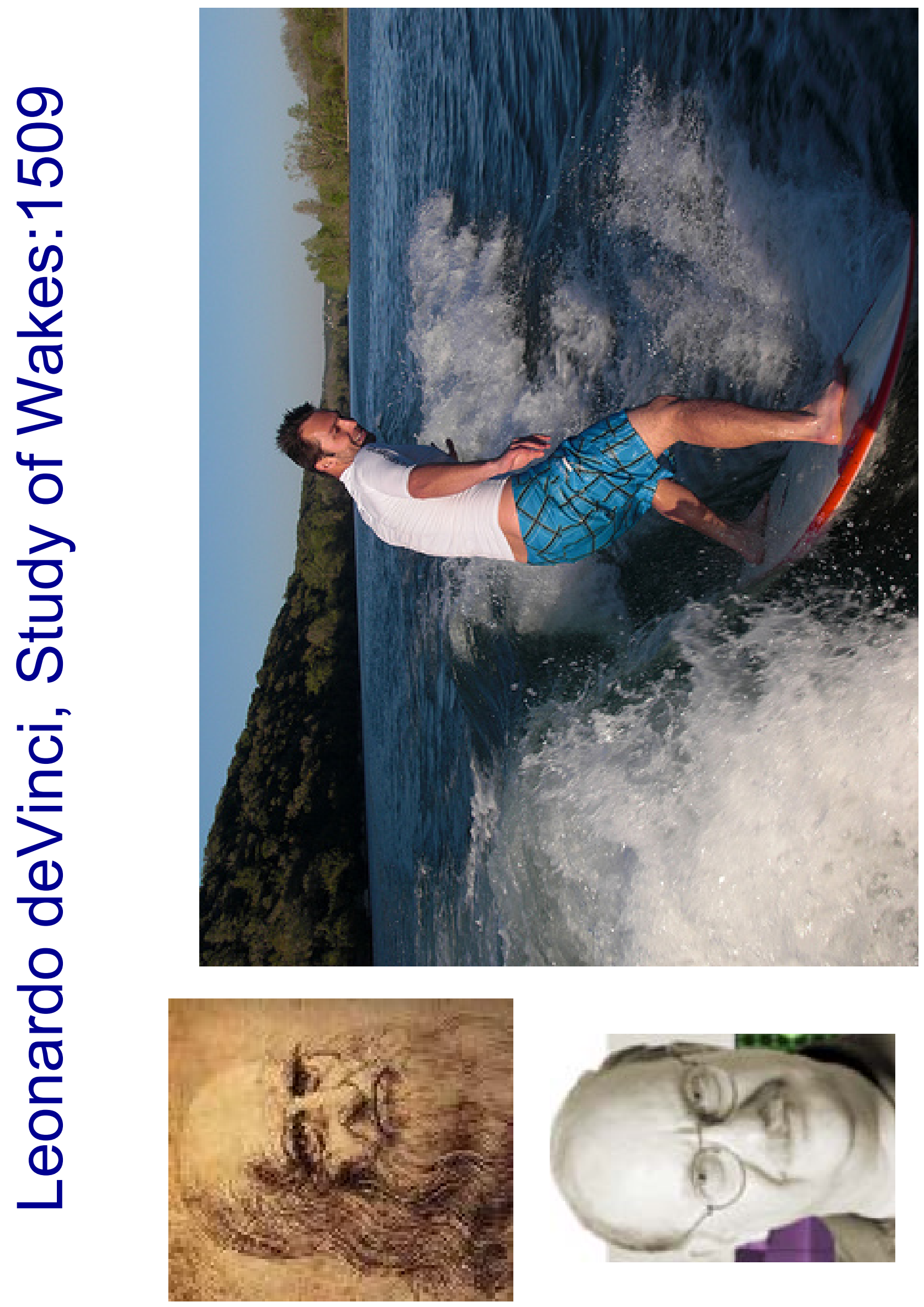


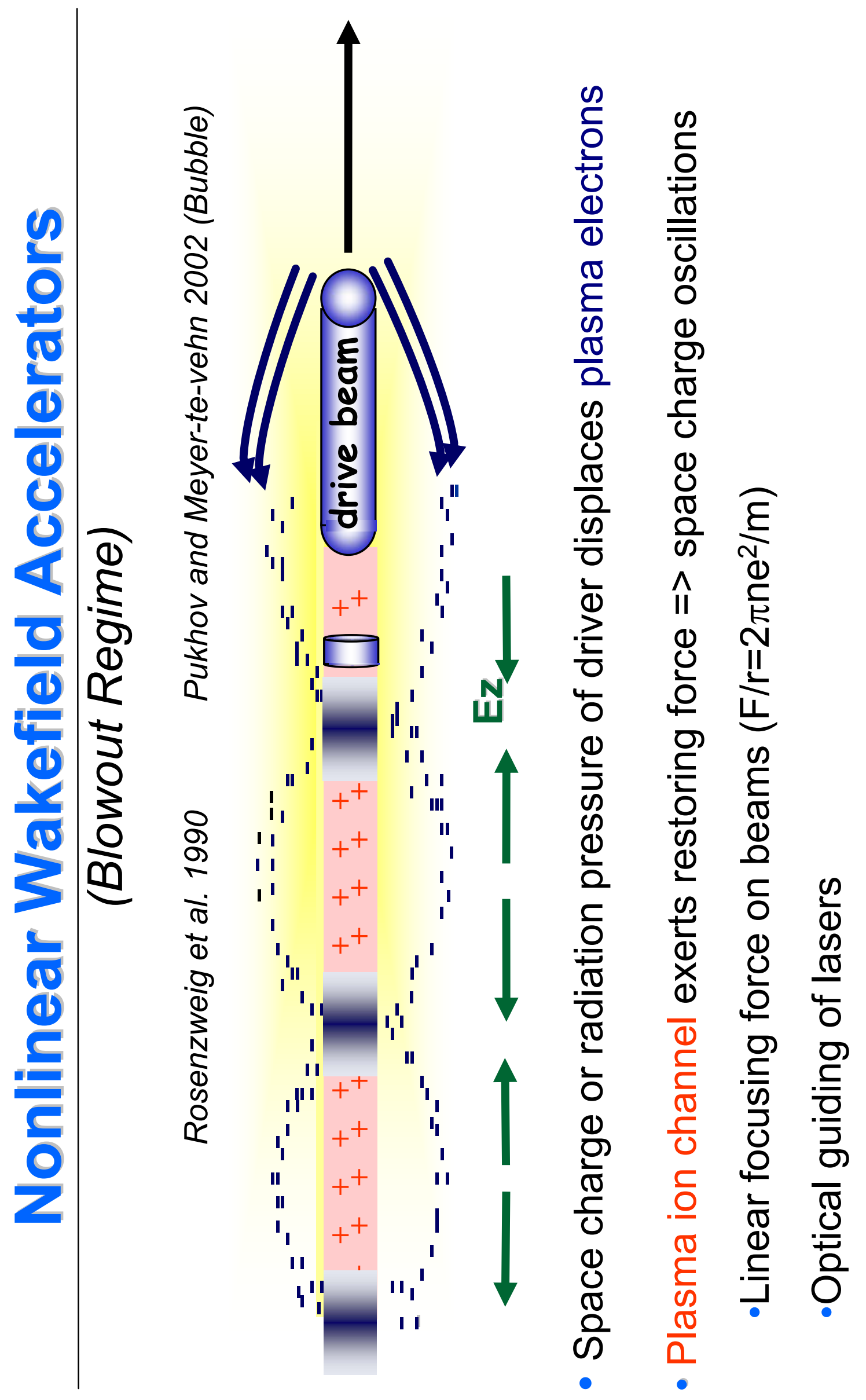




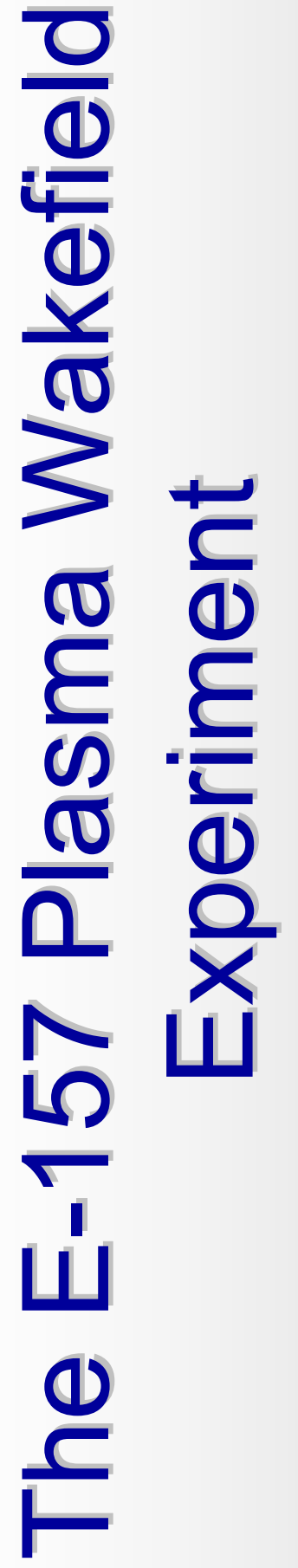

\section{志}

$\frac{0}{\infty}$

11. क क

$\therefore$ -

iㅜ를

$8=$

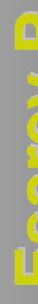

It

든

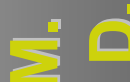

- 1

i.

ด

$\rightarrow 50$

iv

(1) $\frac{2}{11}$

क

두 둘

$=\pi$

के

c 


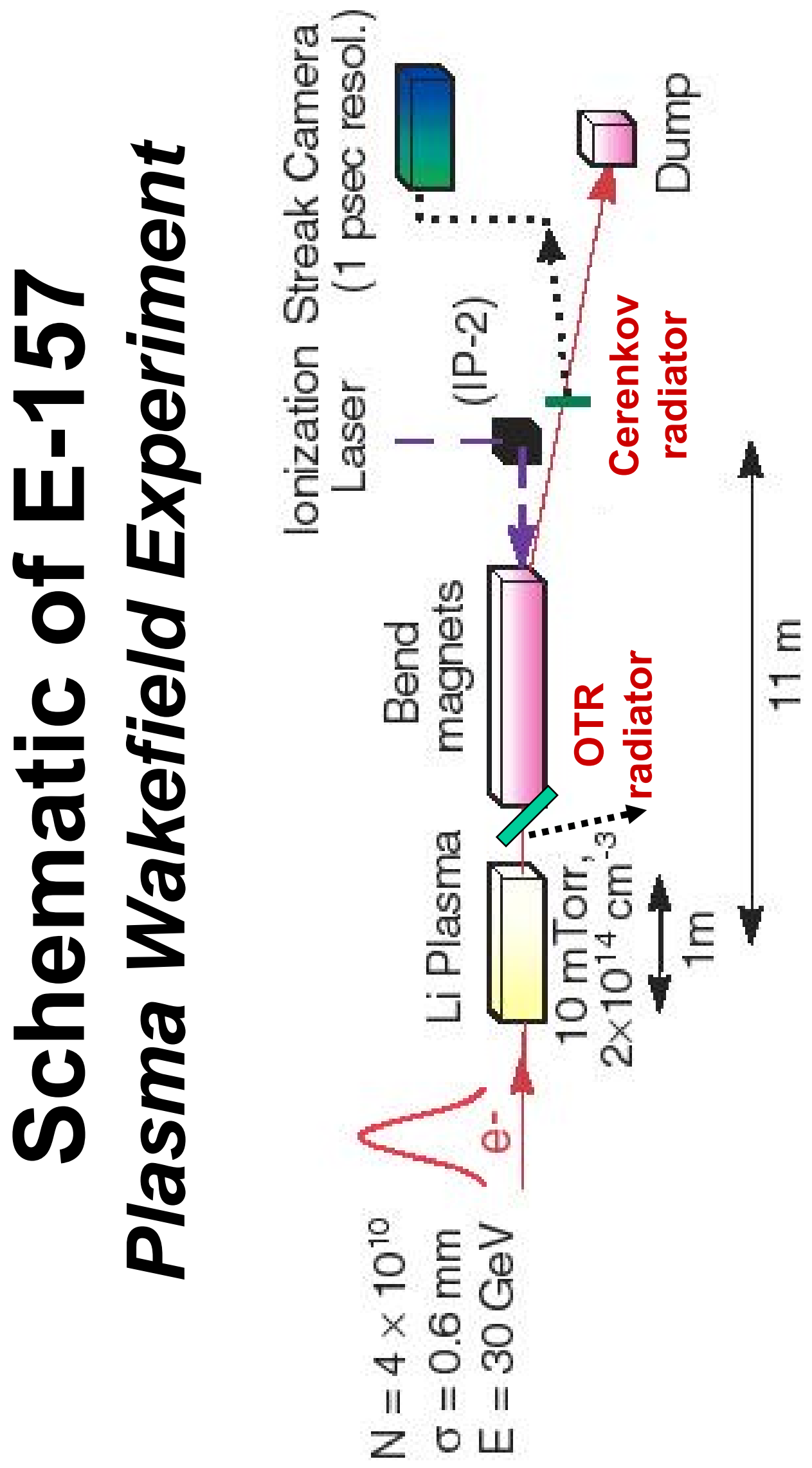



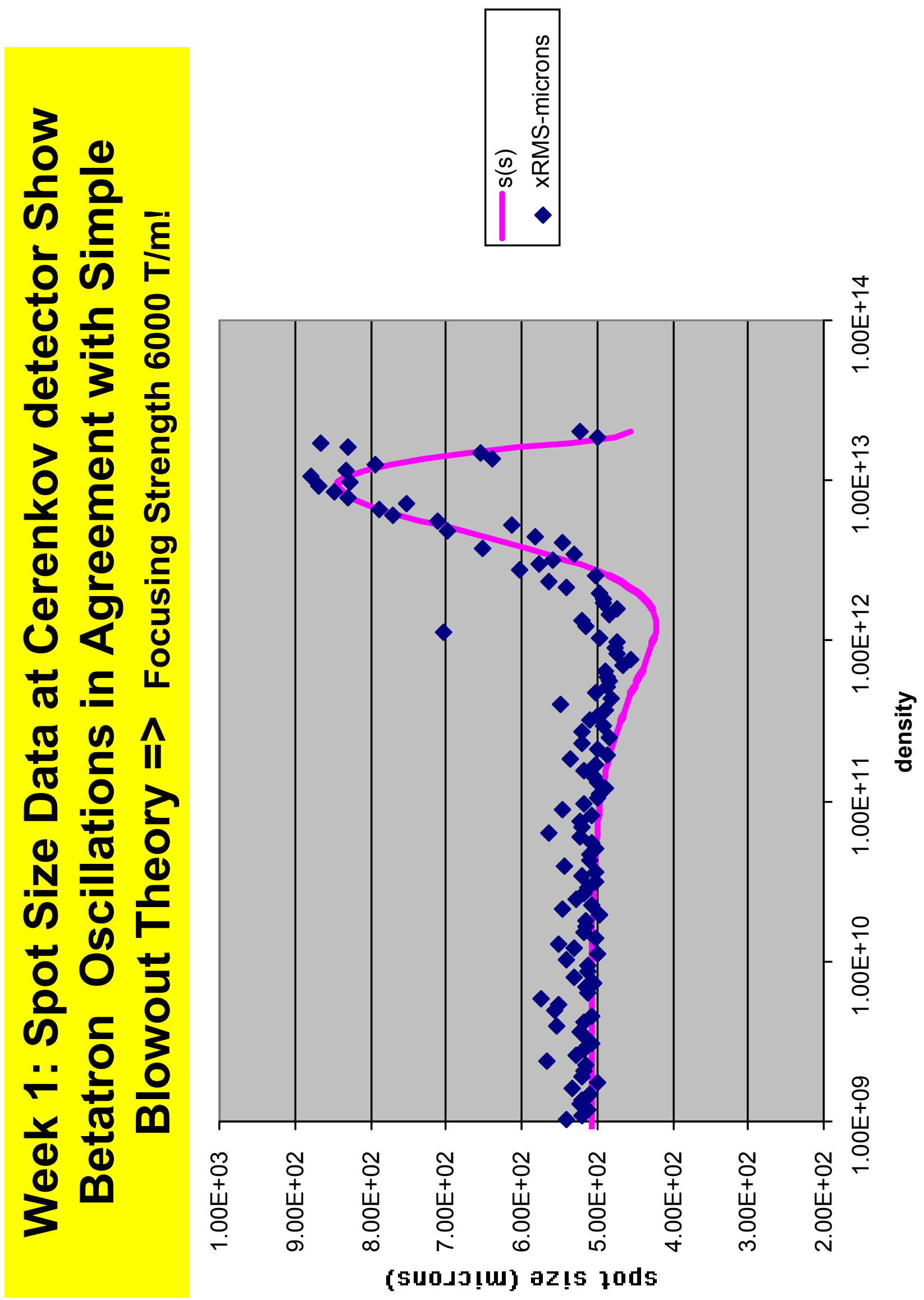

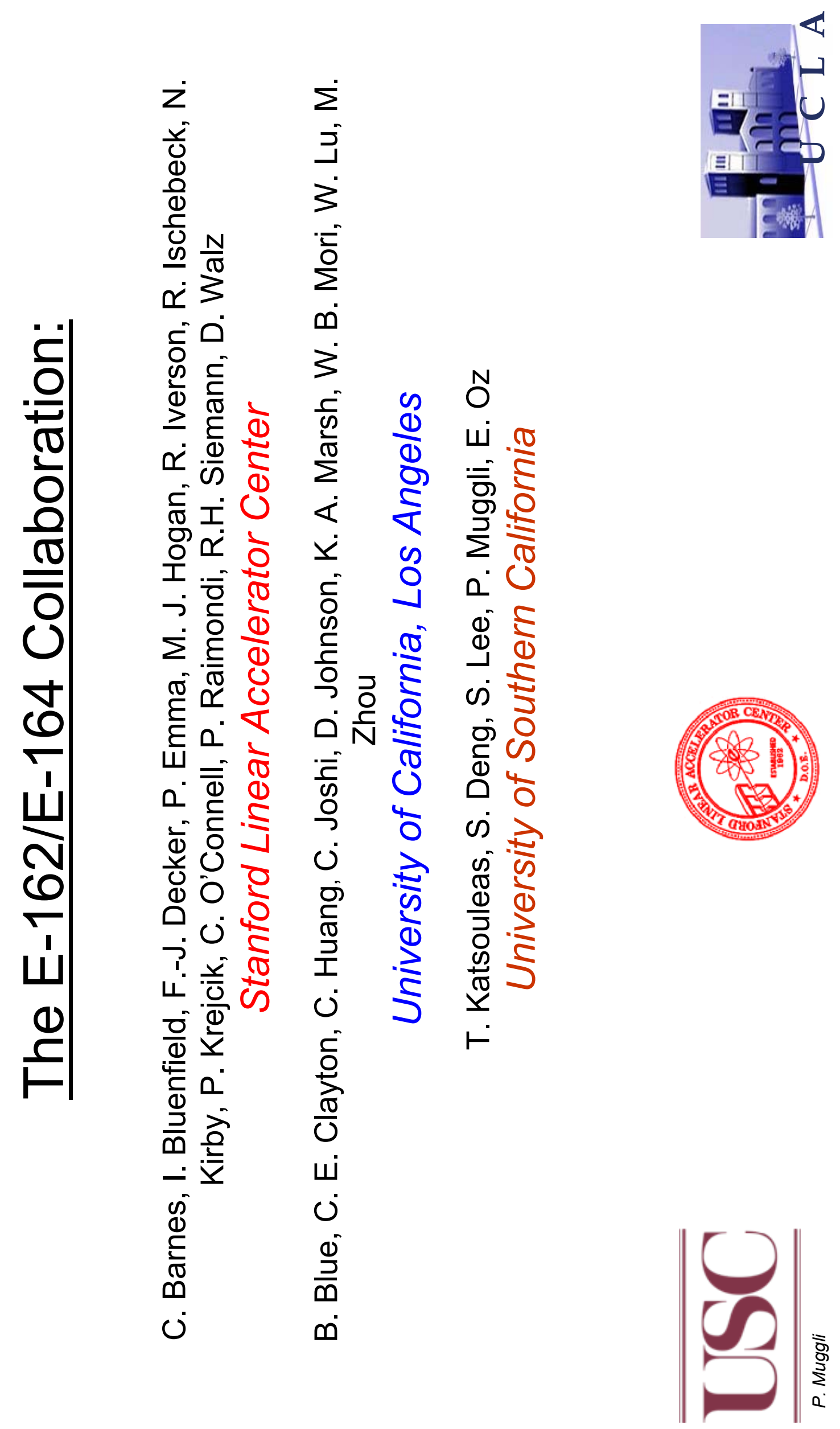


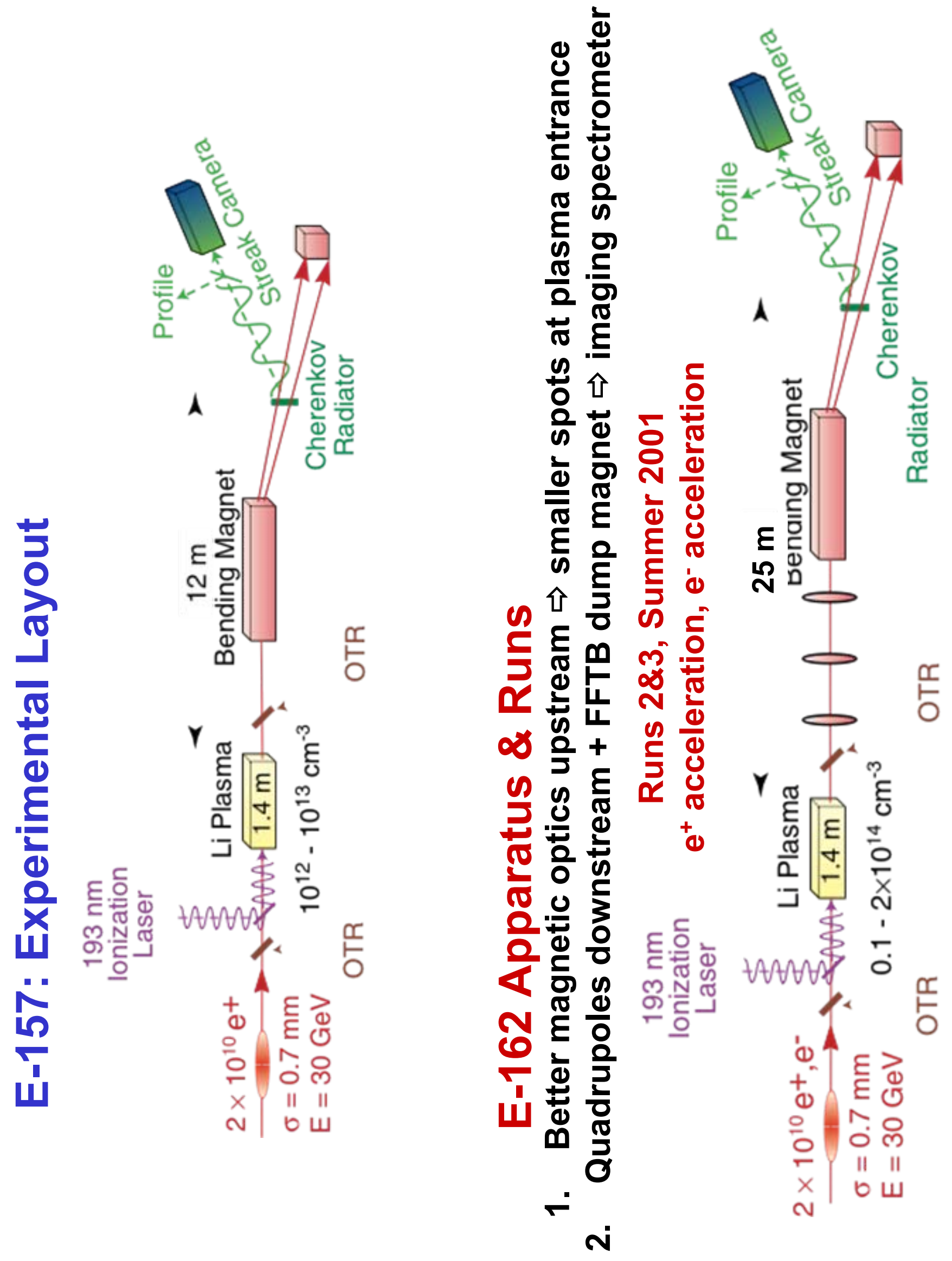




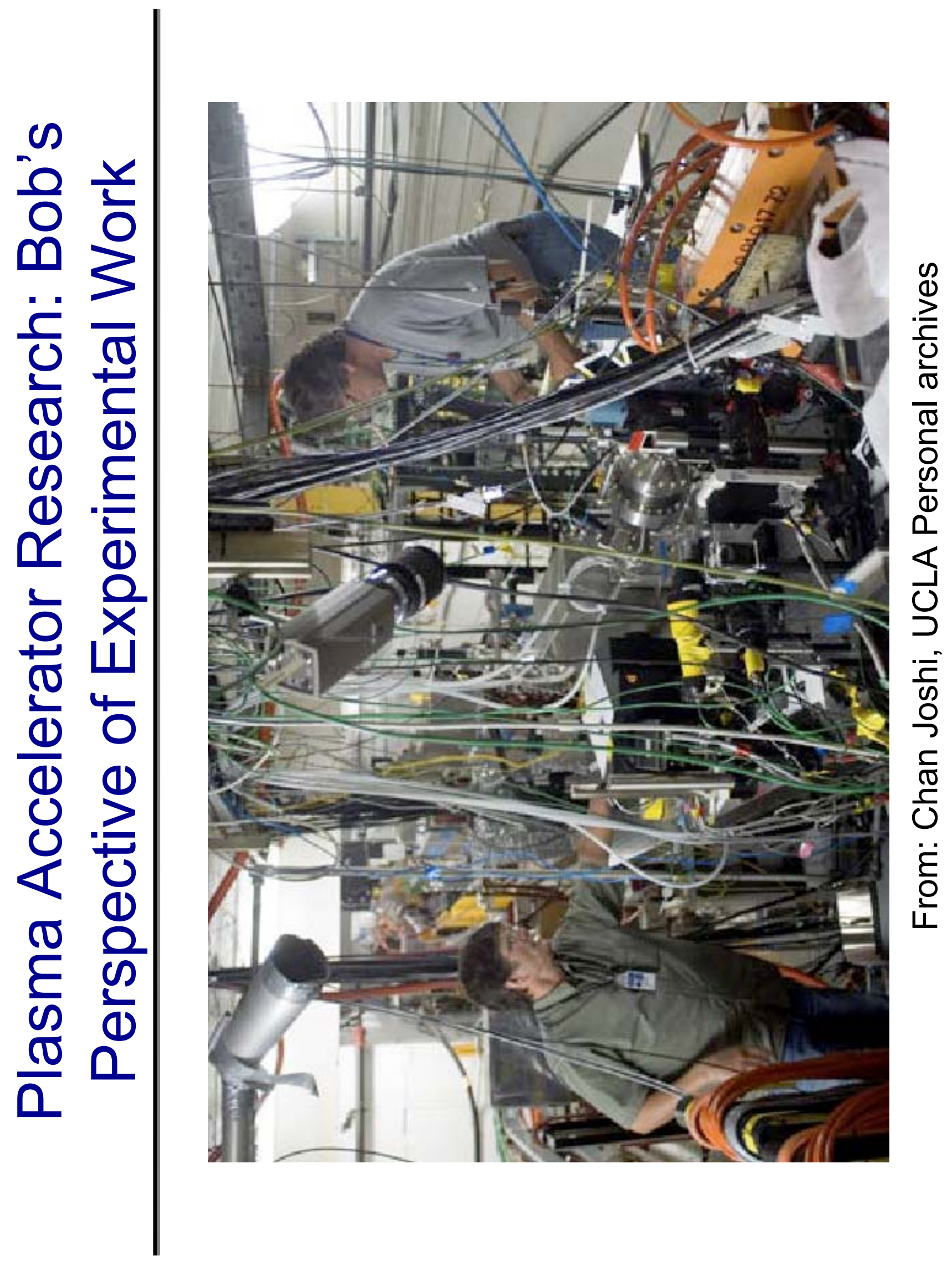



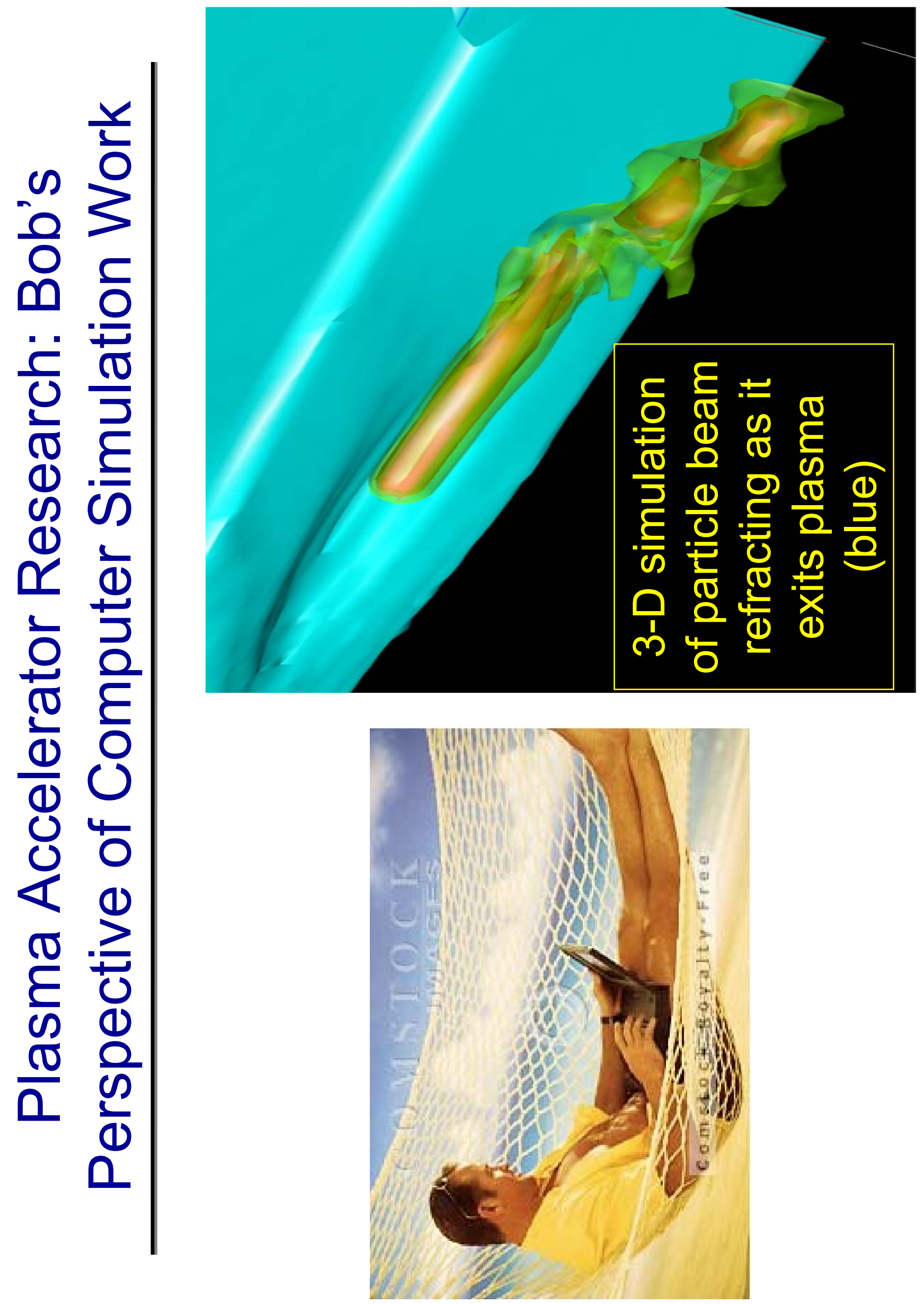


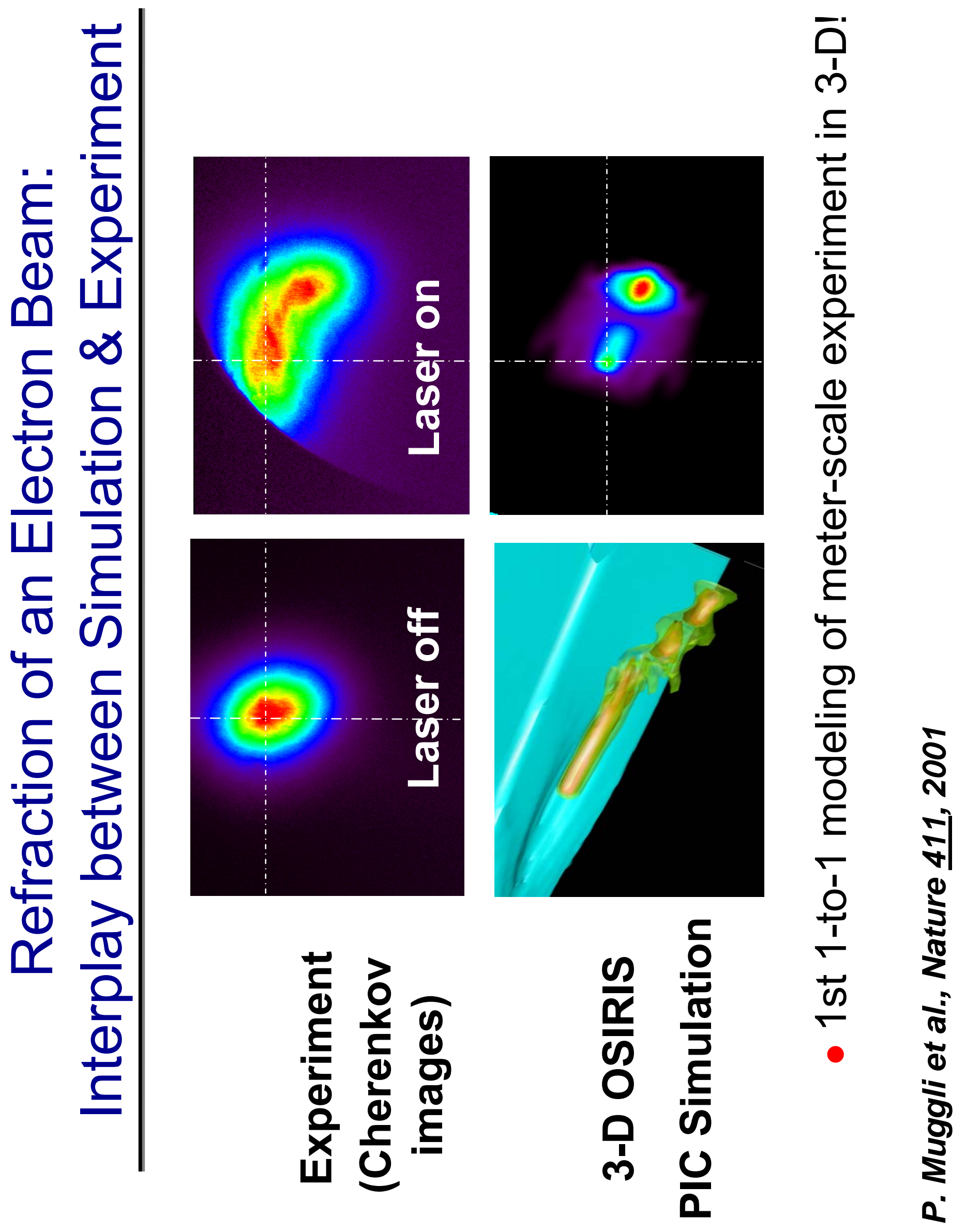




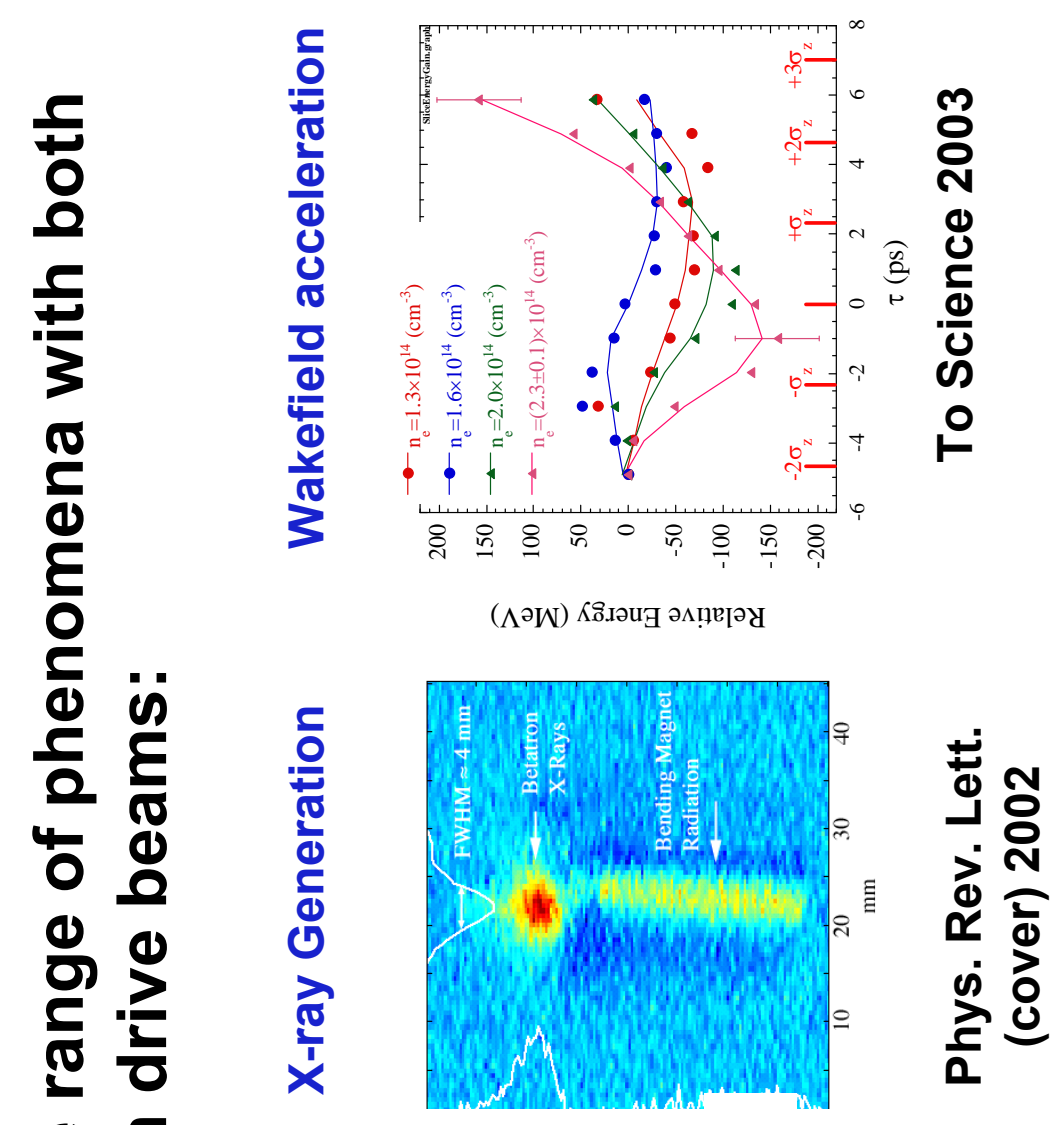

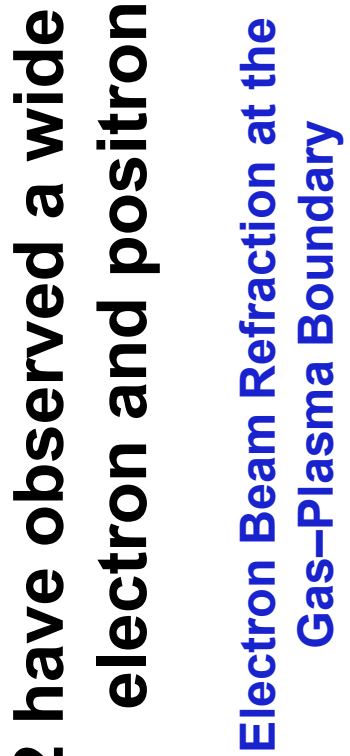

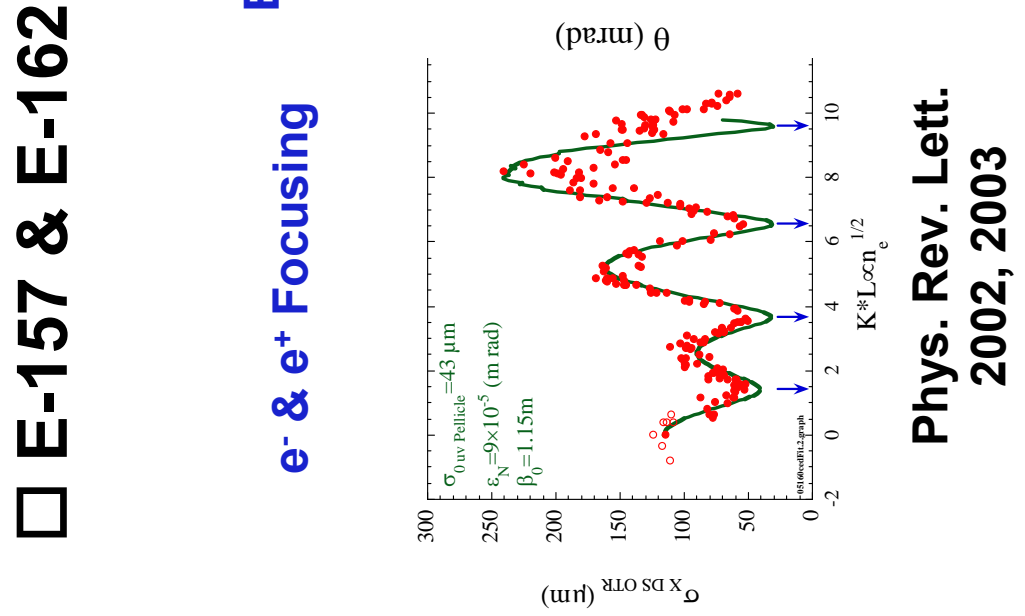



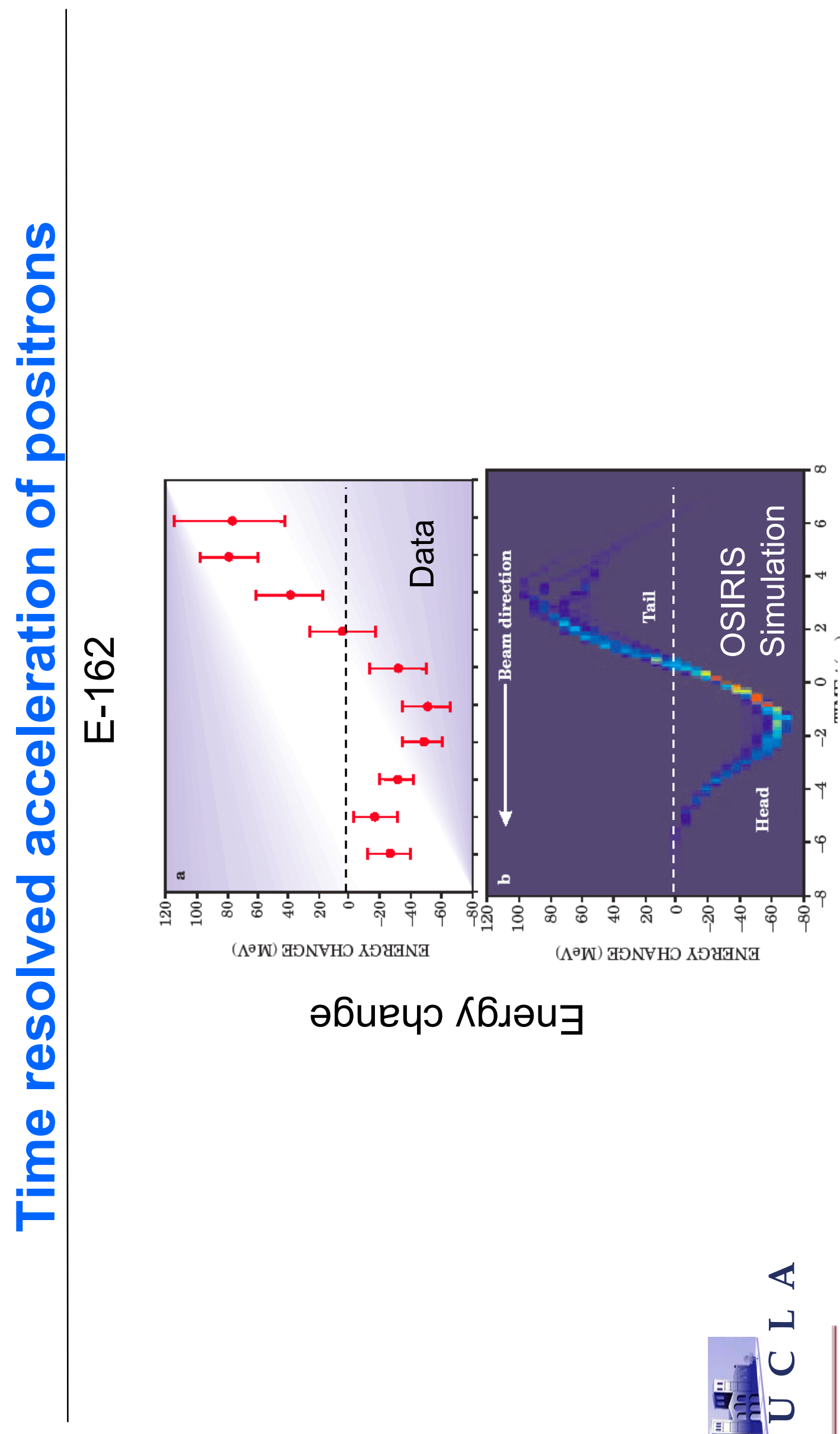

$>2$

(1)

เค 0 क

II के ले

든

()

(1)

0
0
0
10
11
0
0
0
1

\section{әбиечо Кбьәи}

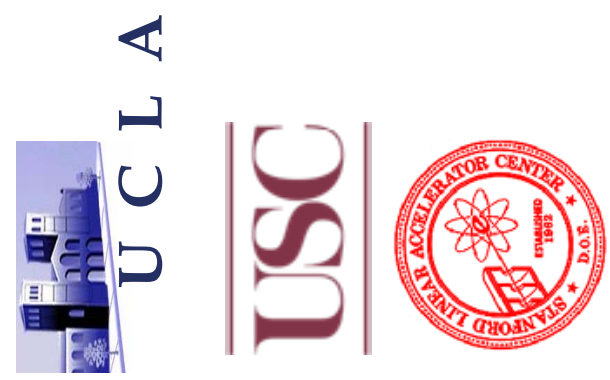



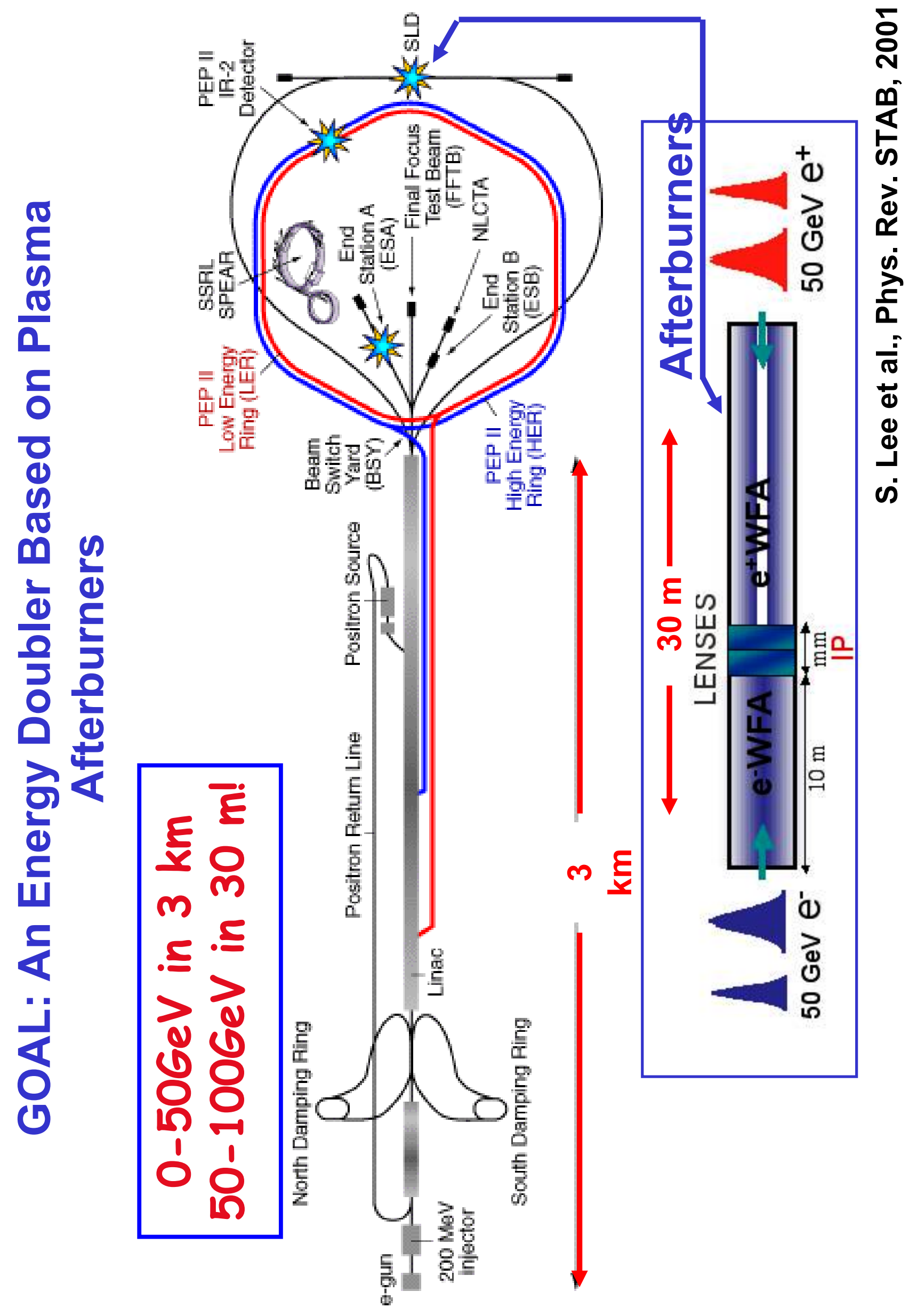


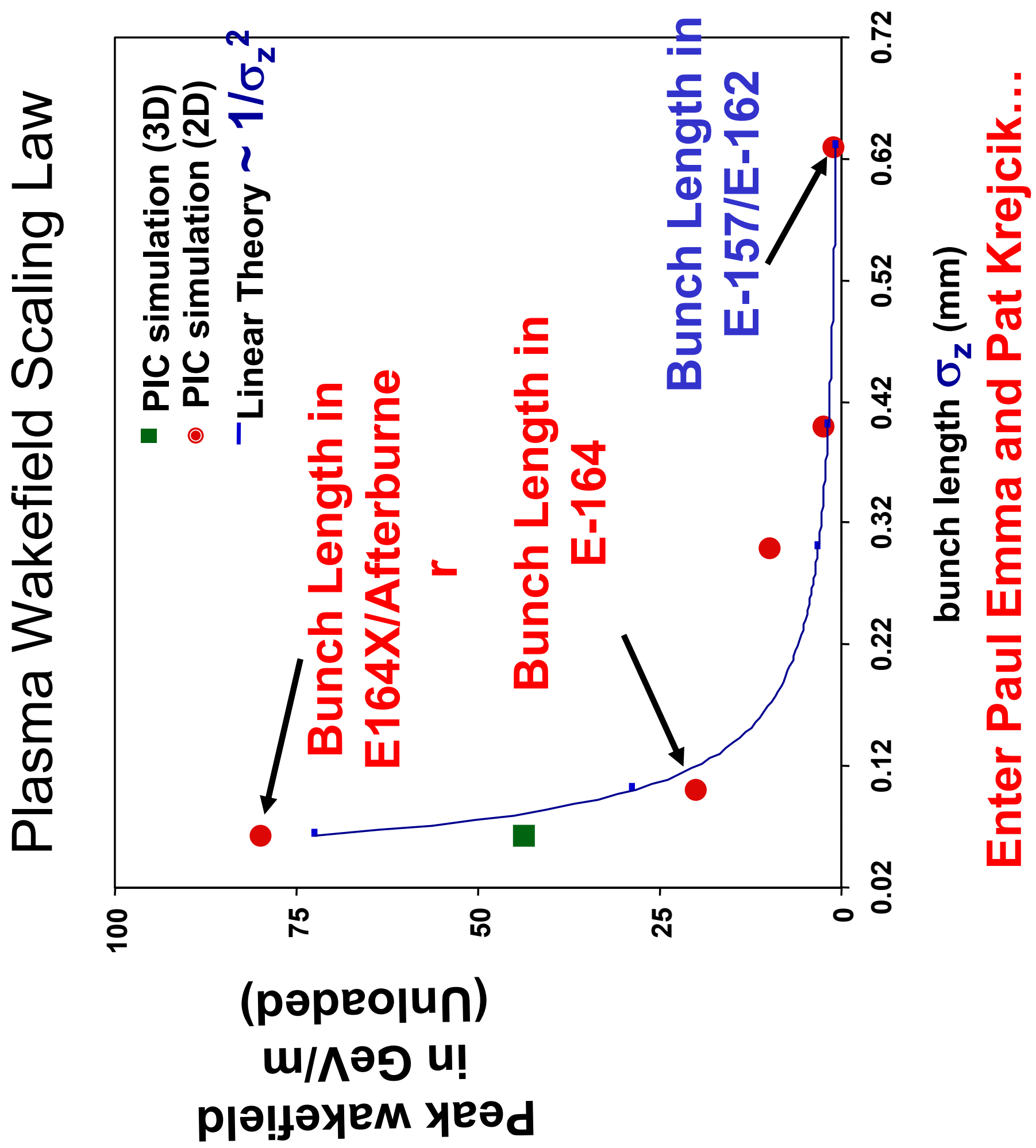



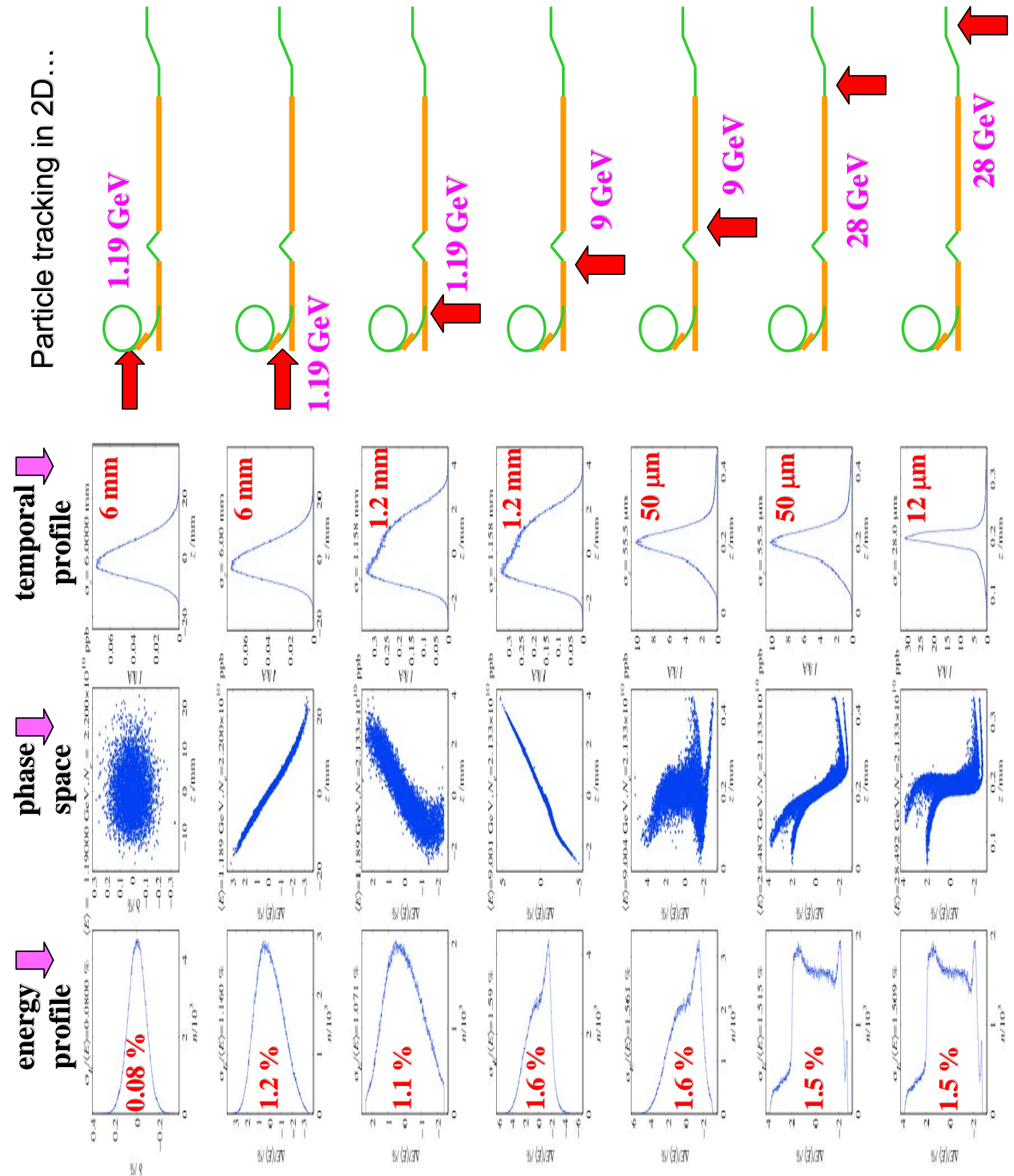

$$
\text { bip }
$$

Bil (j) II
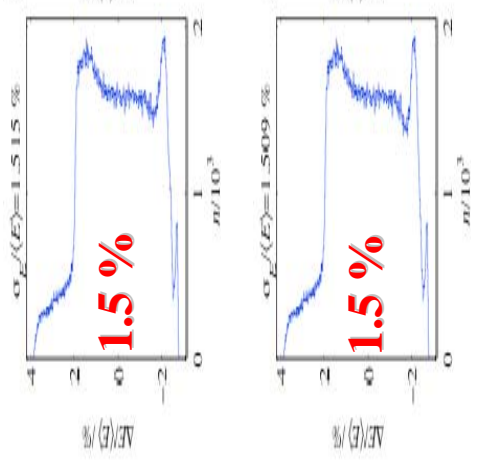


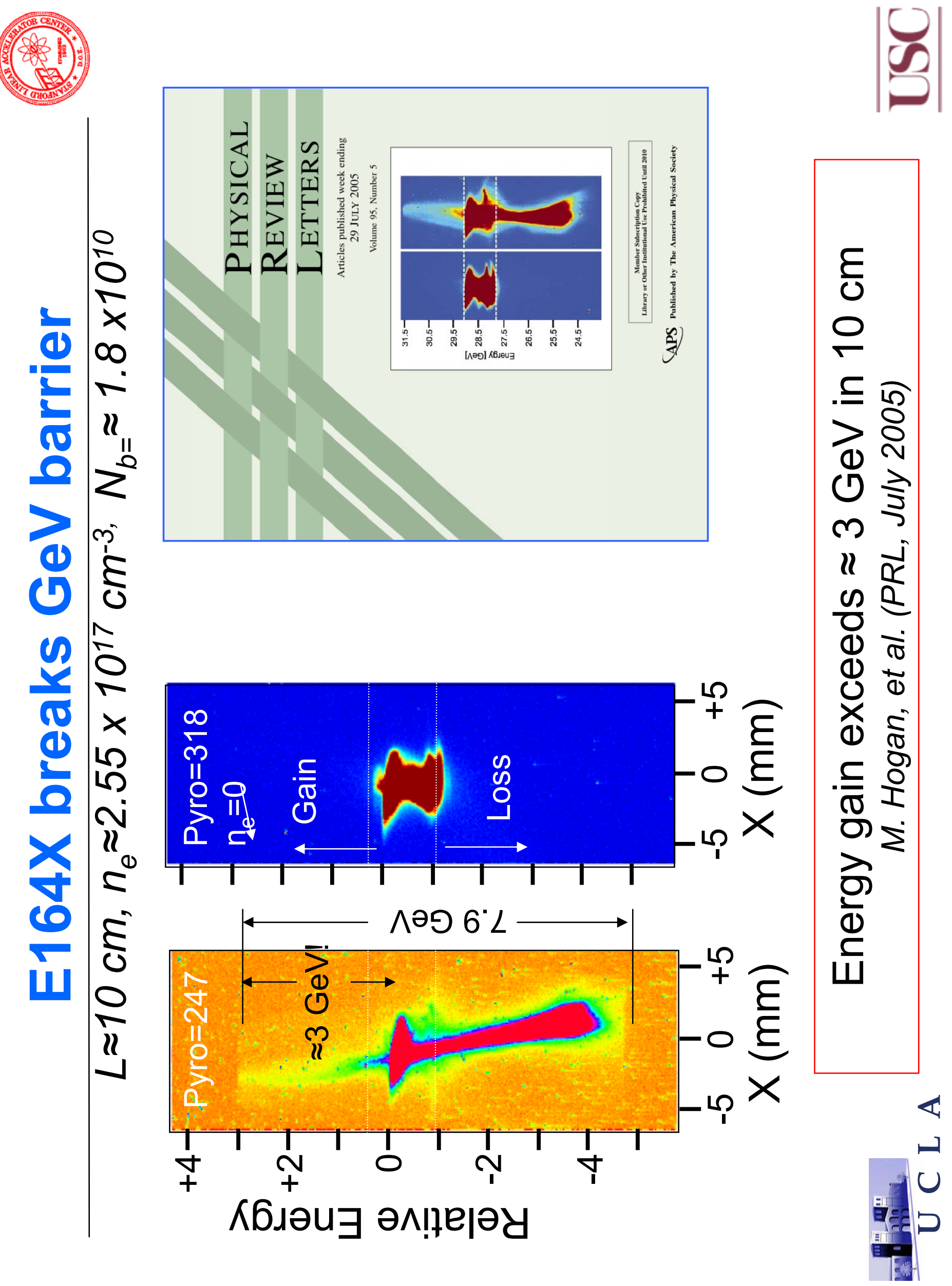



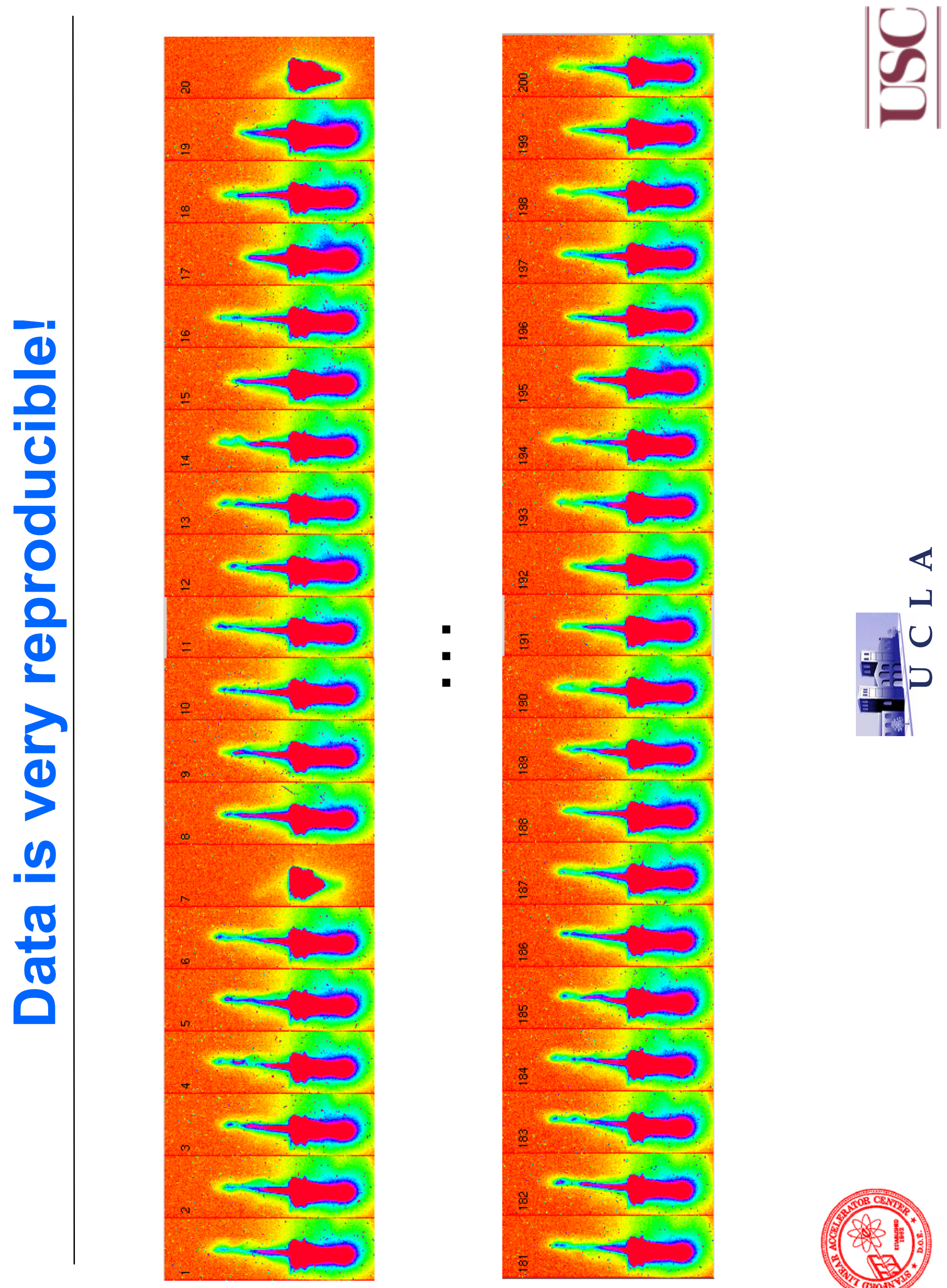


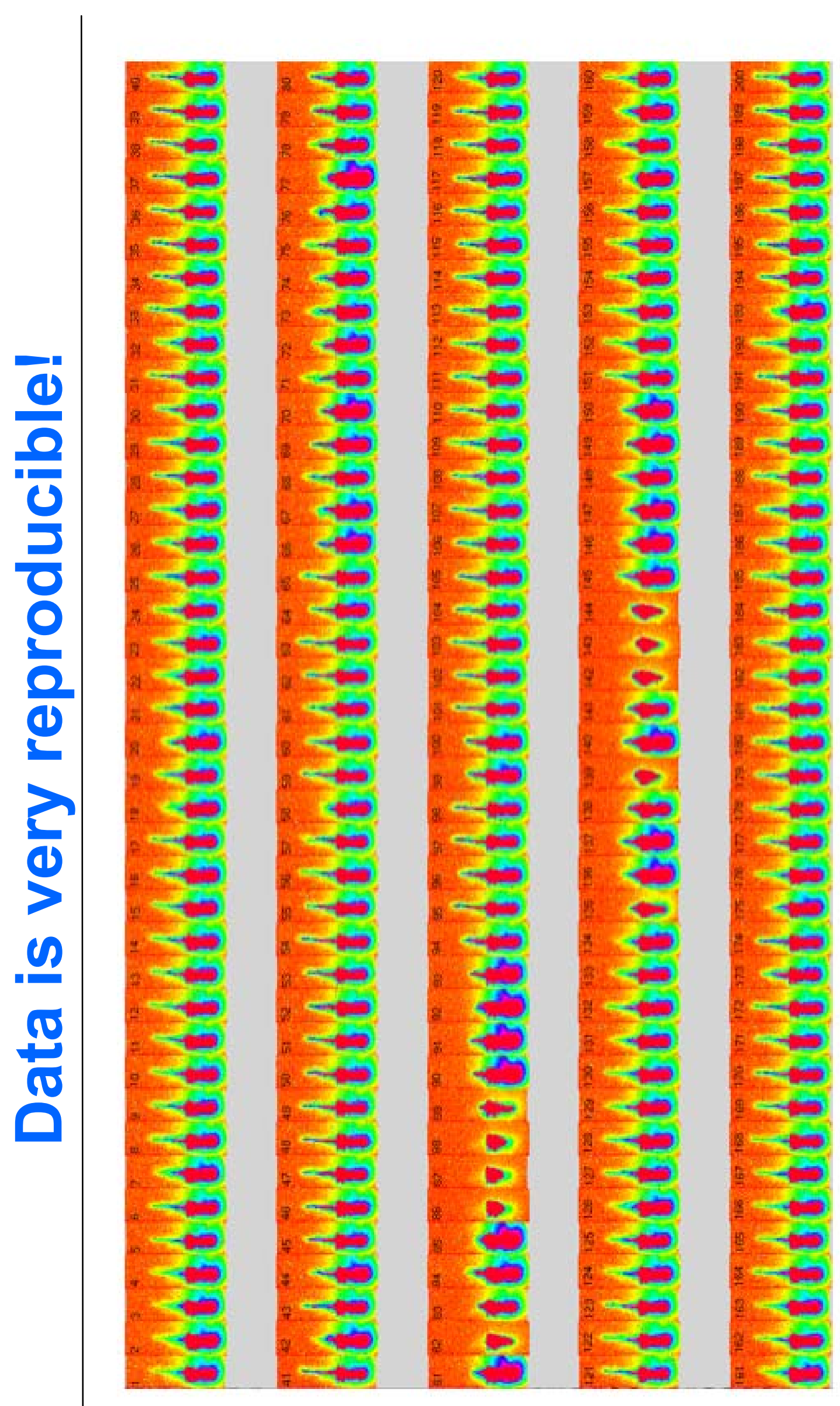




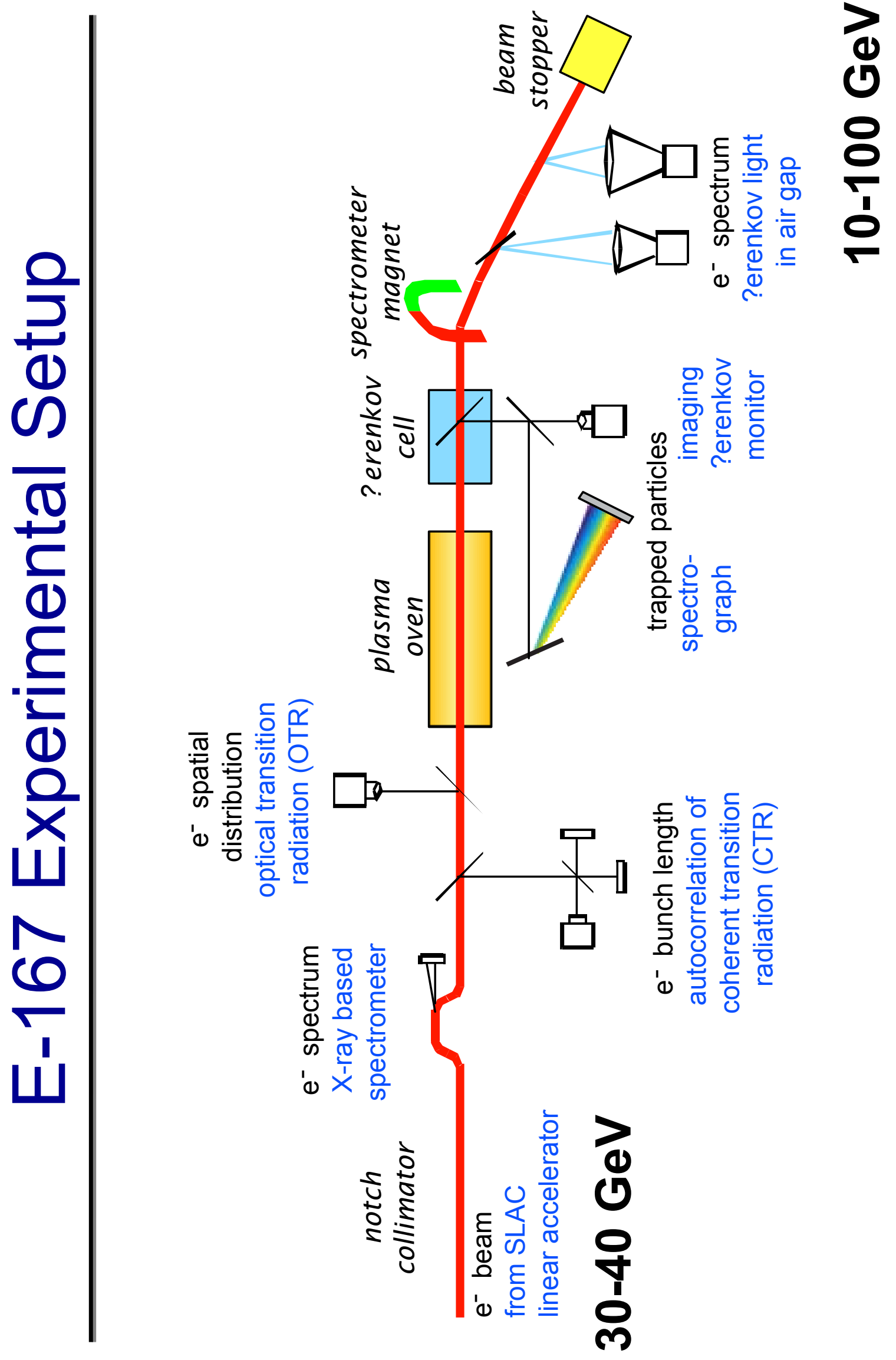




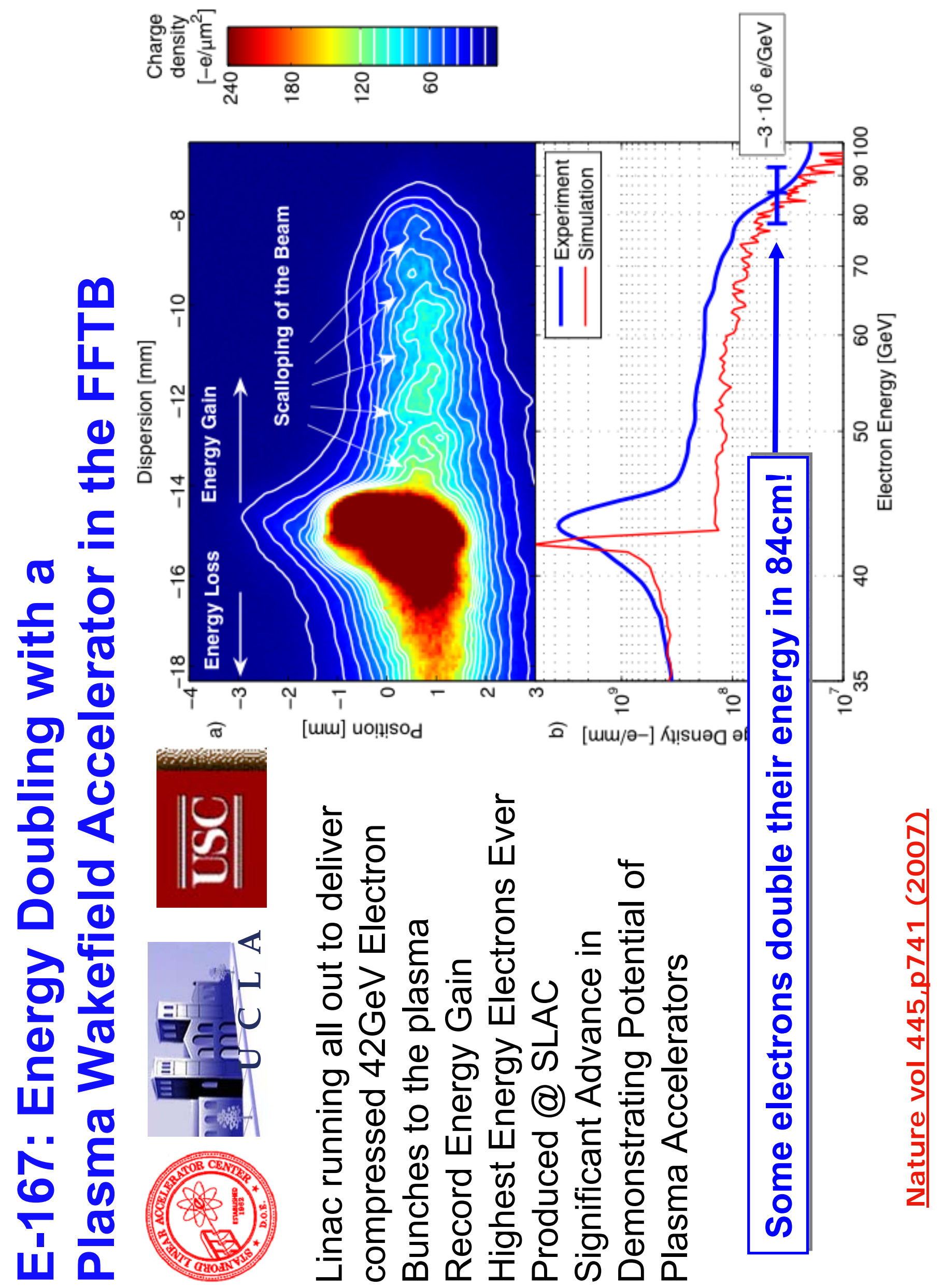




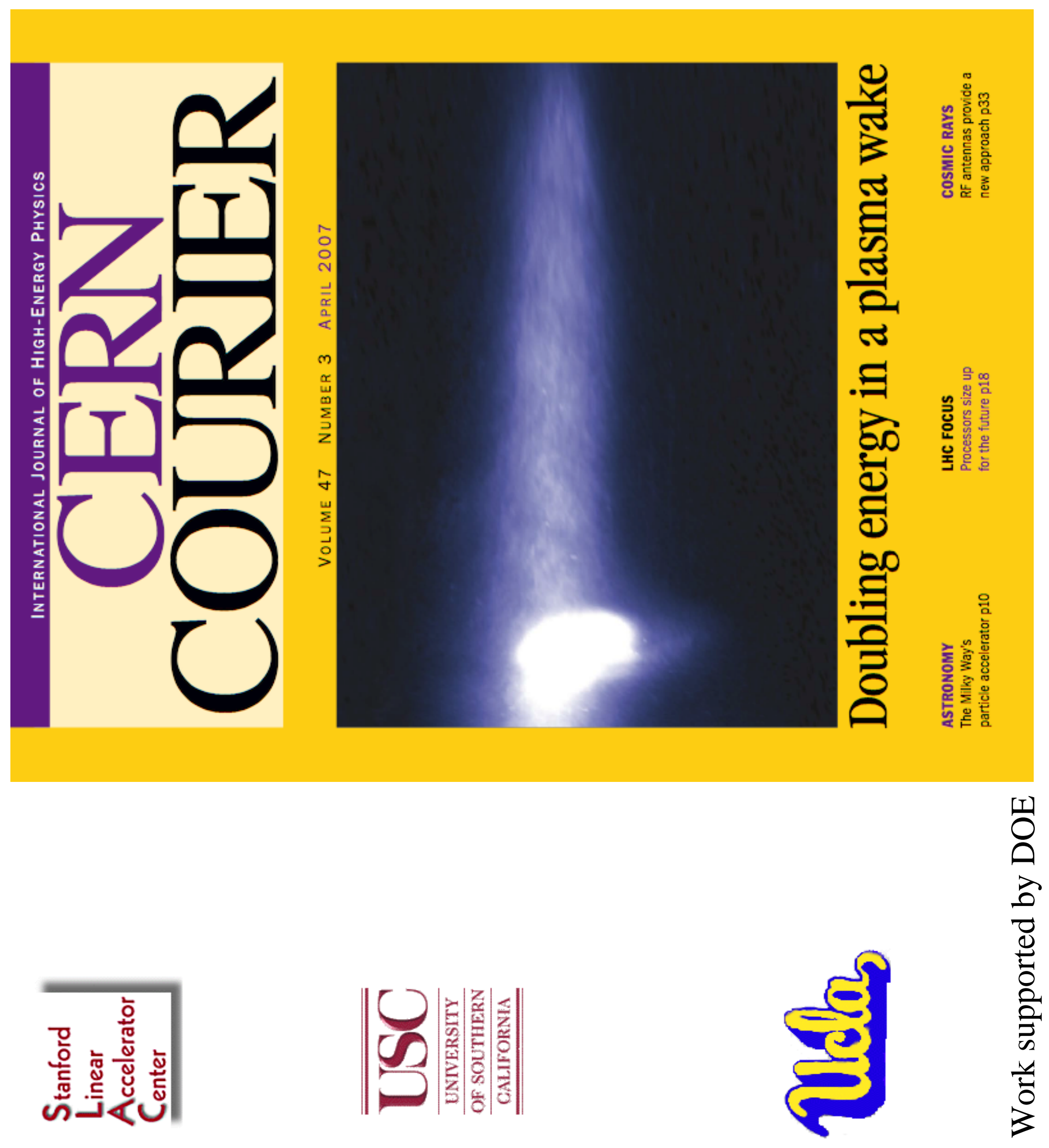




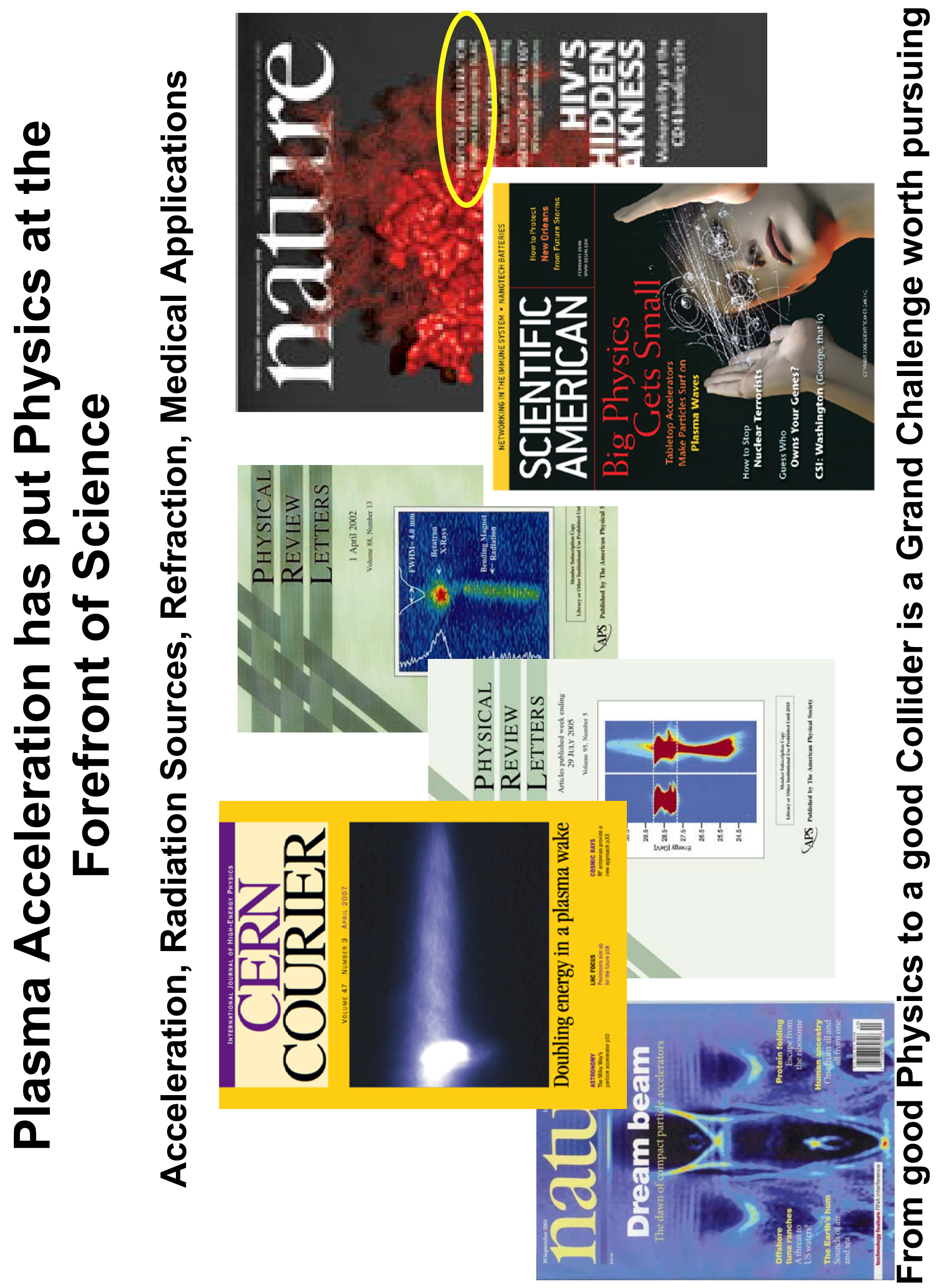




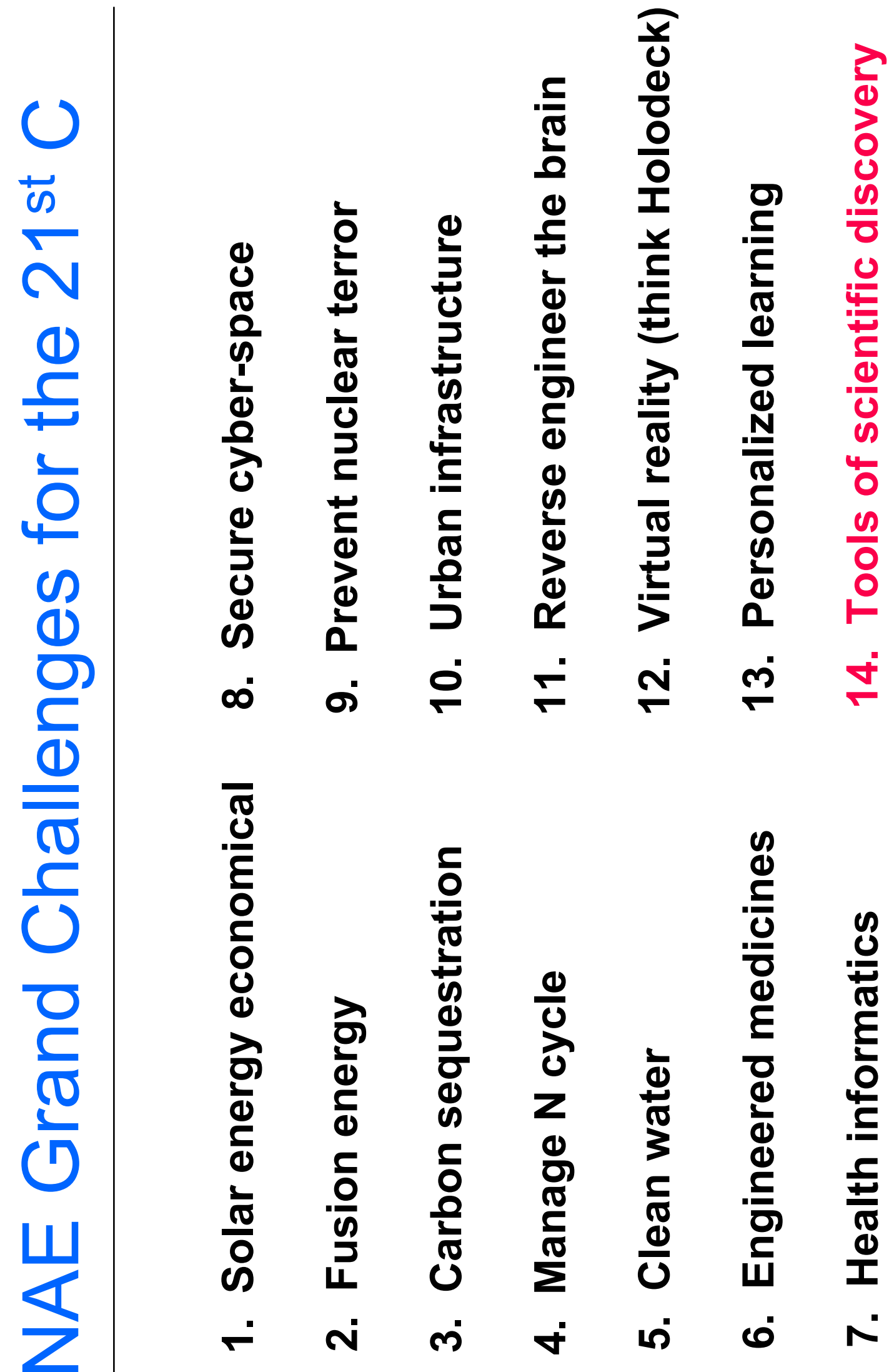




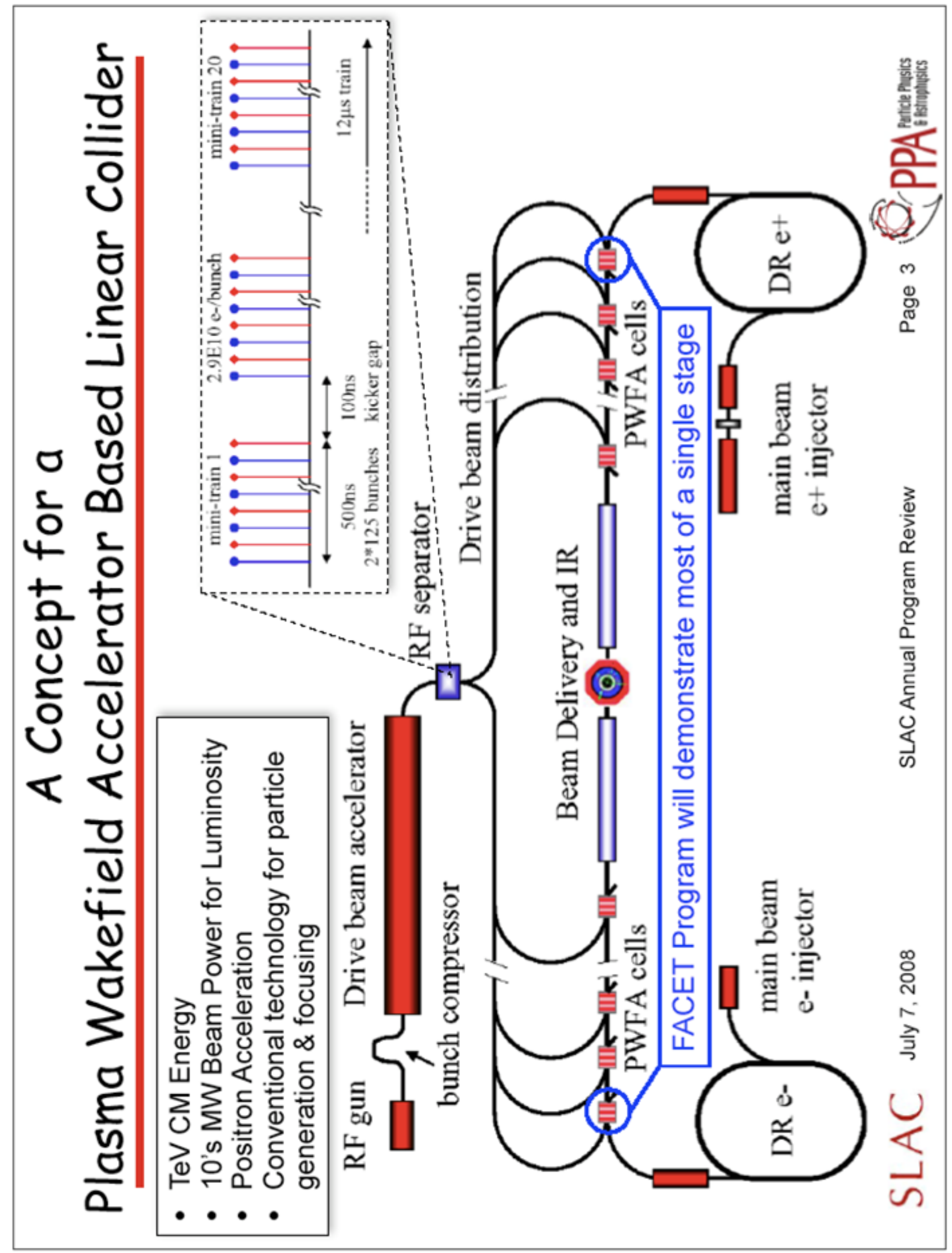




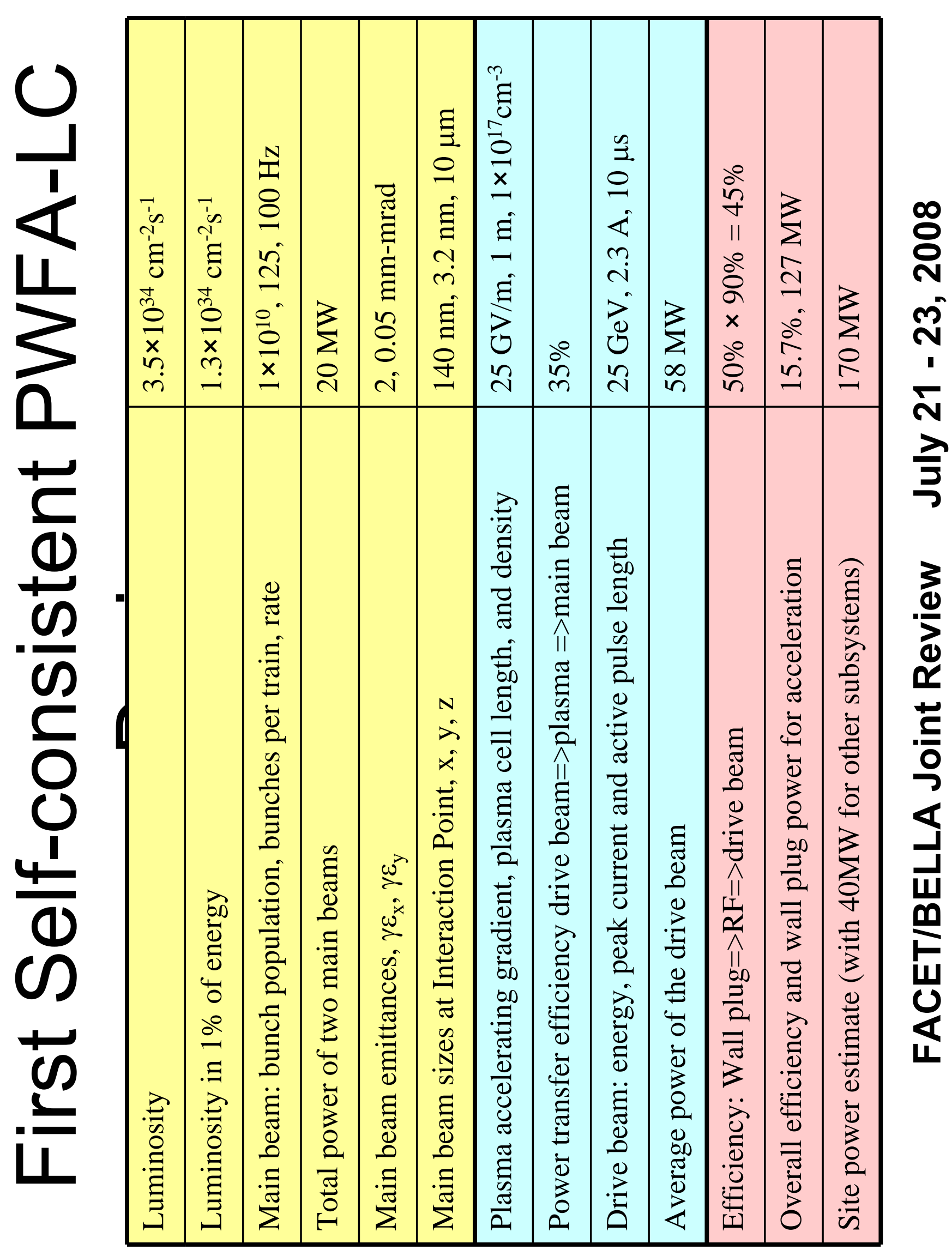




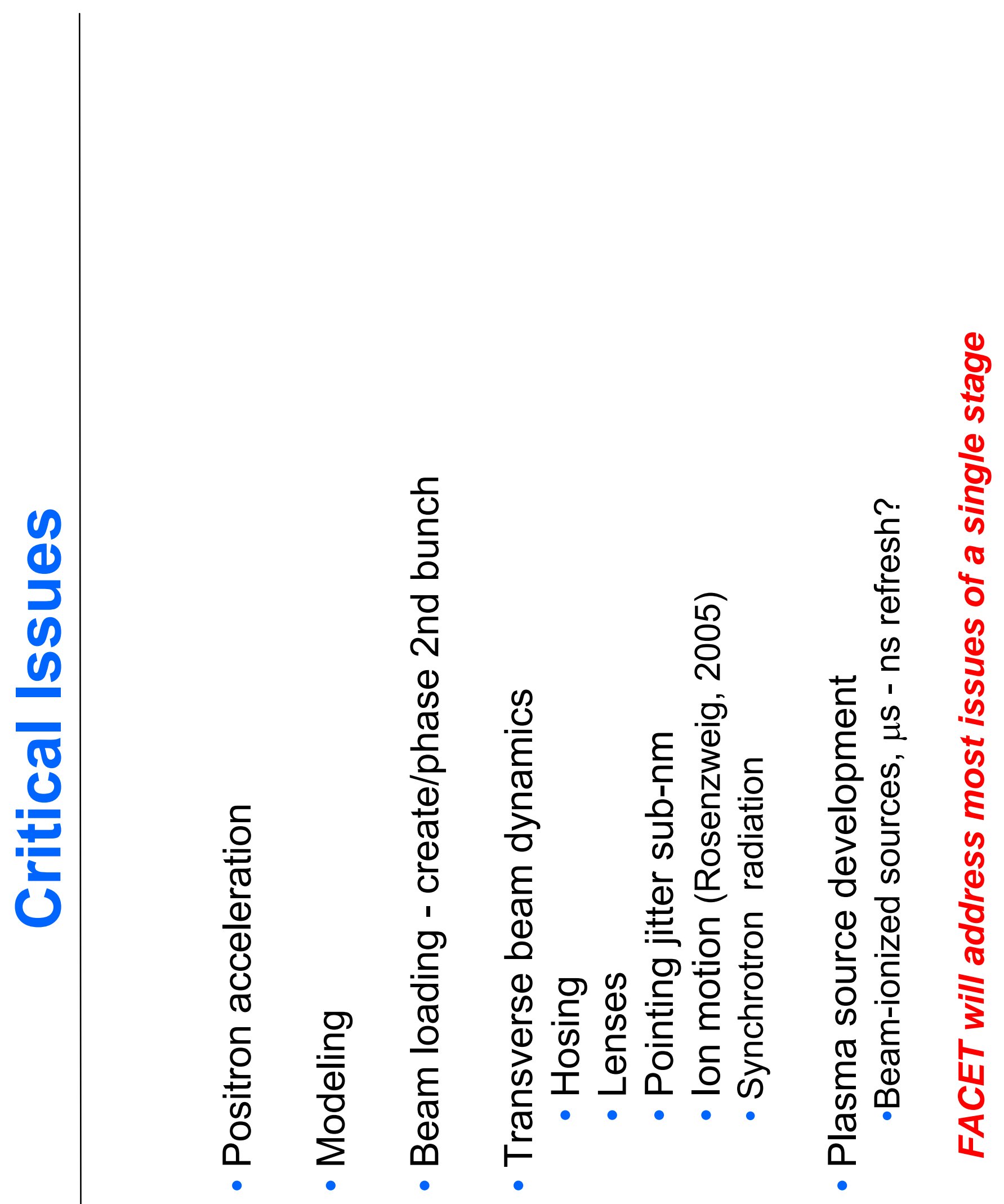




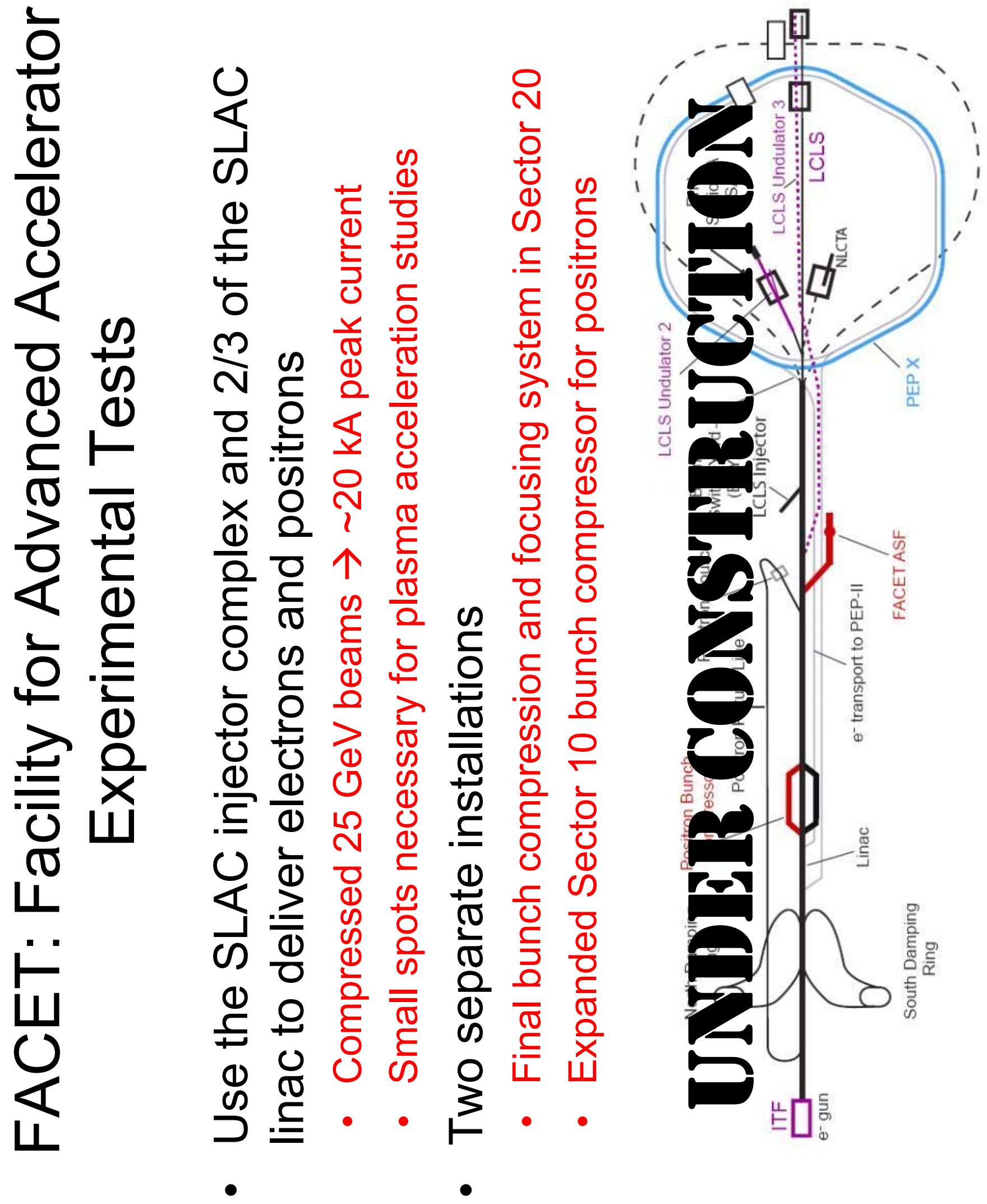




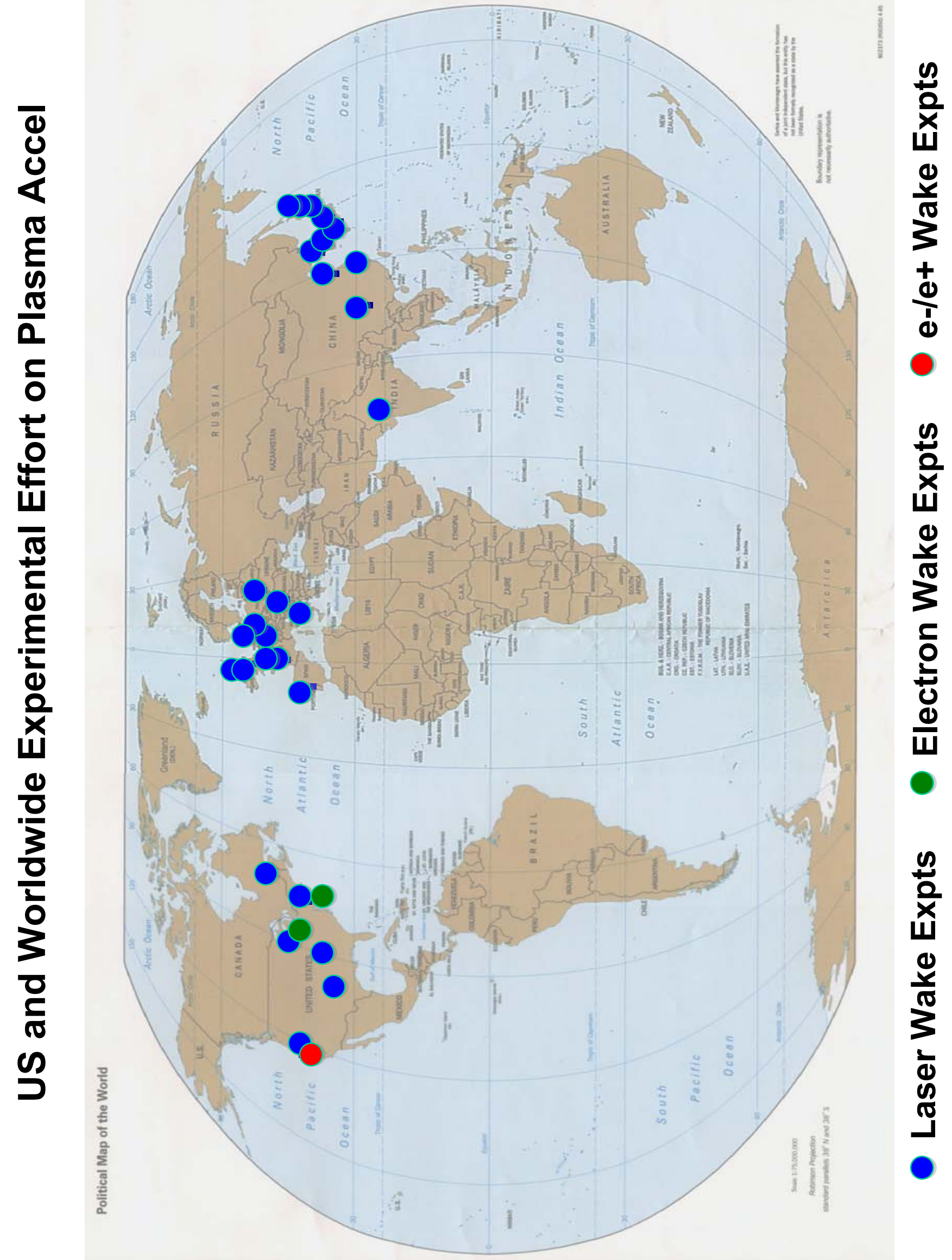




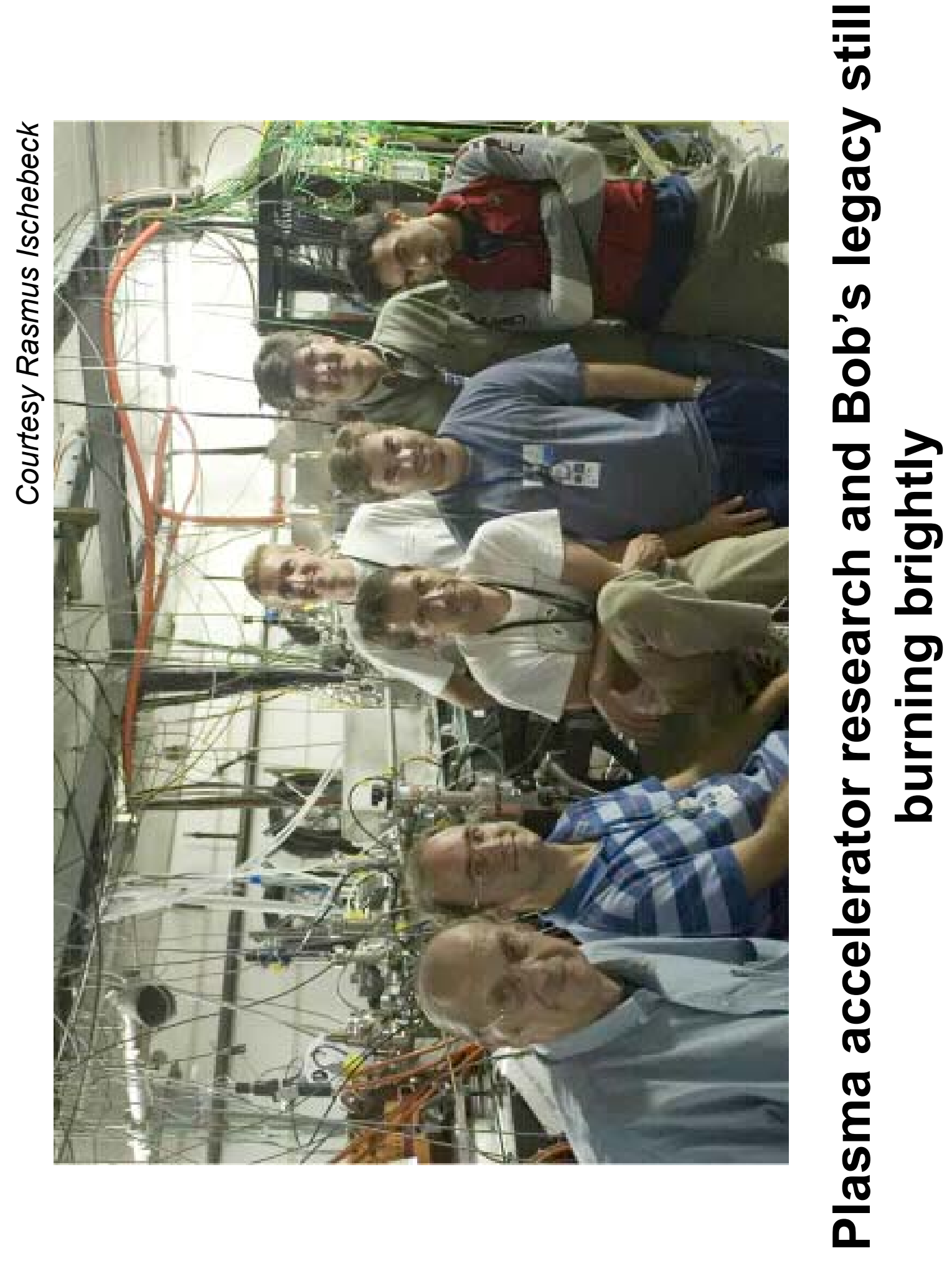




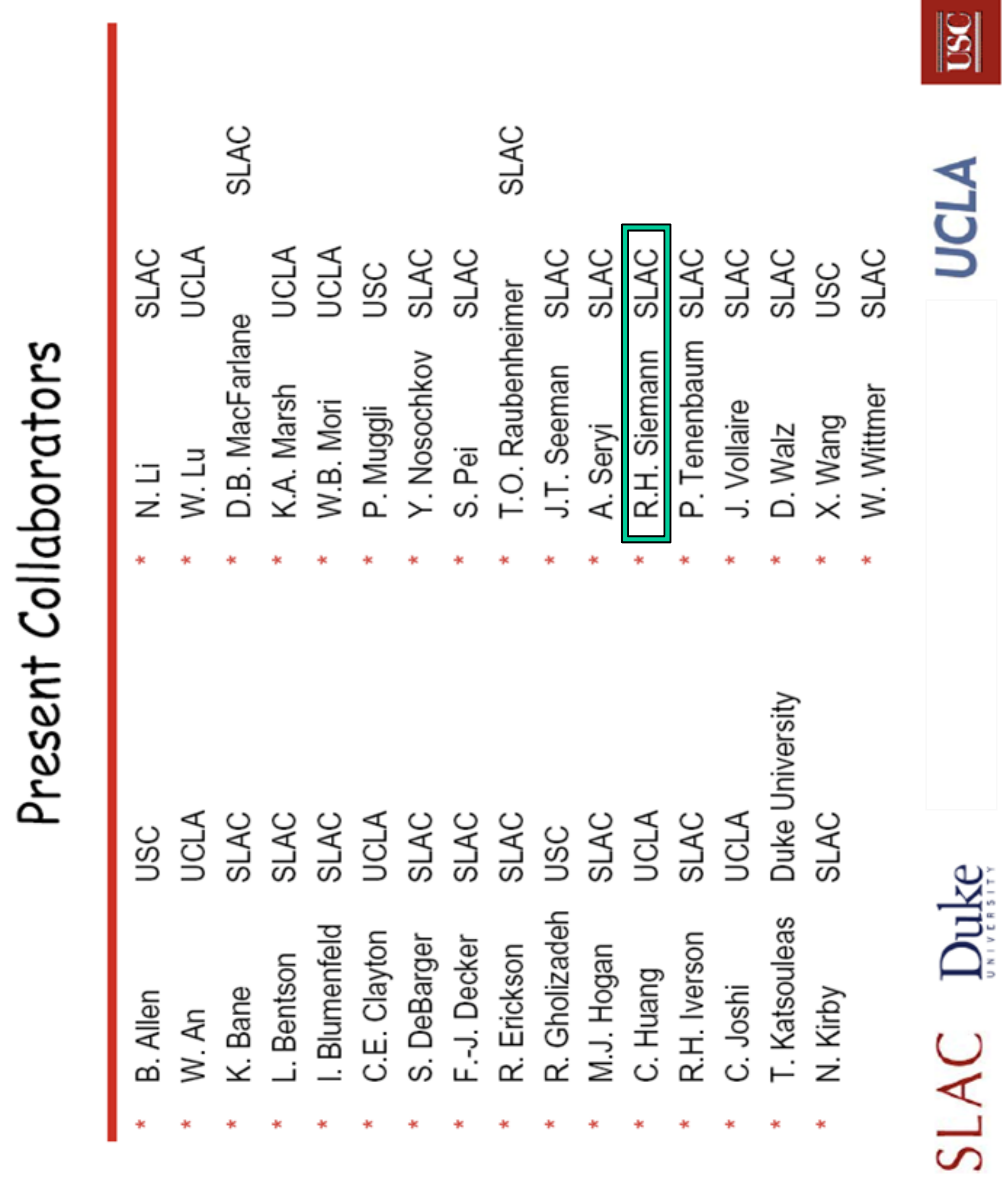




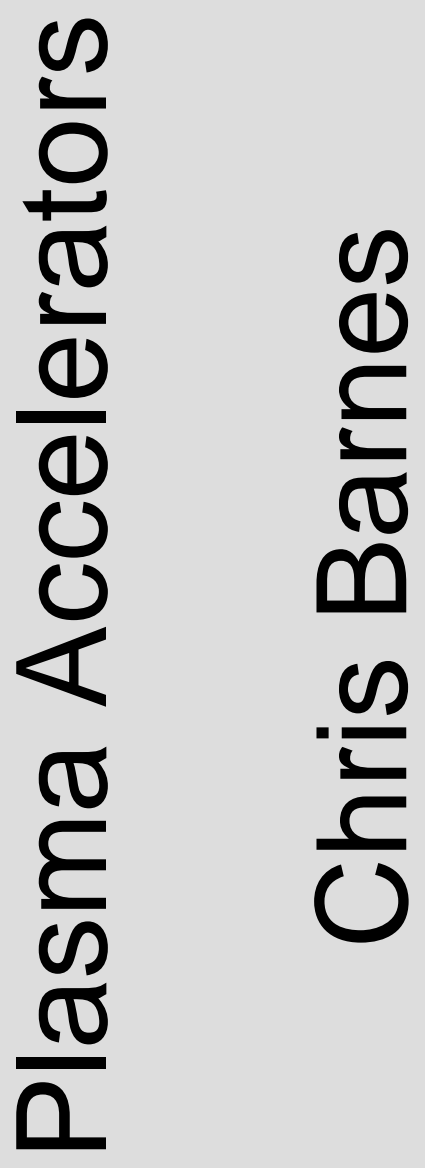




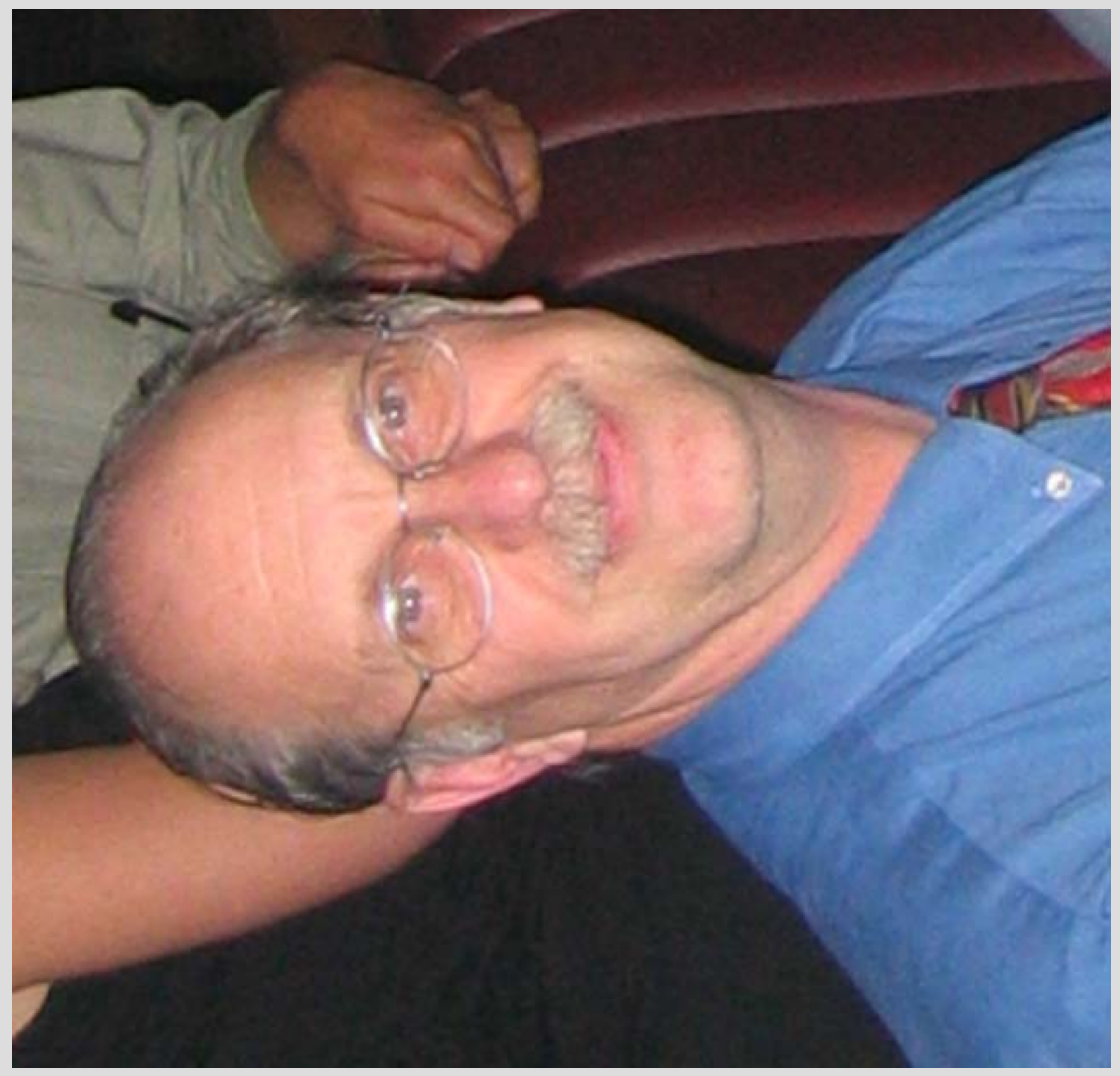




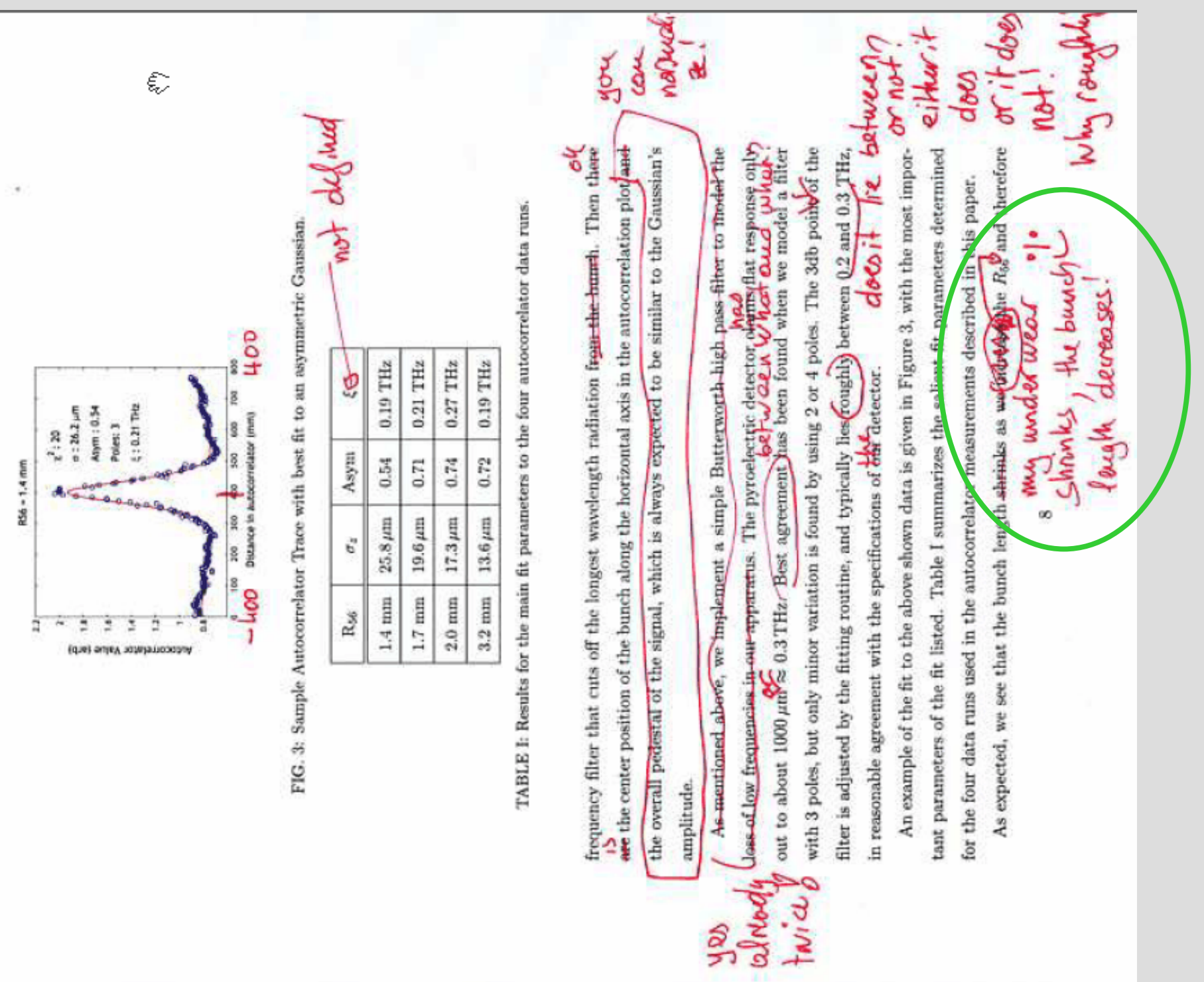




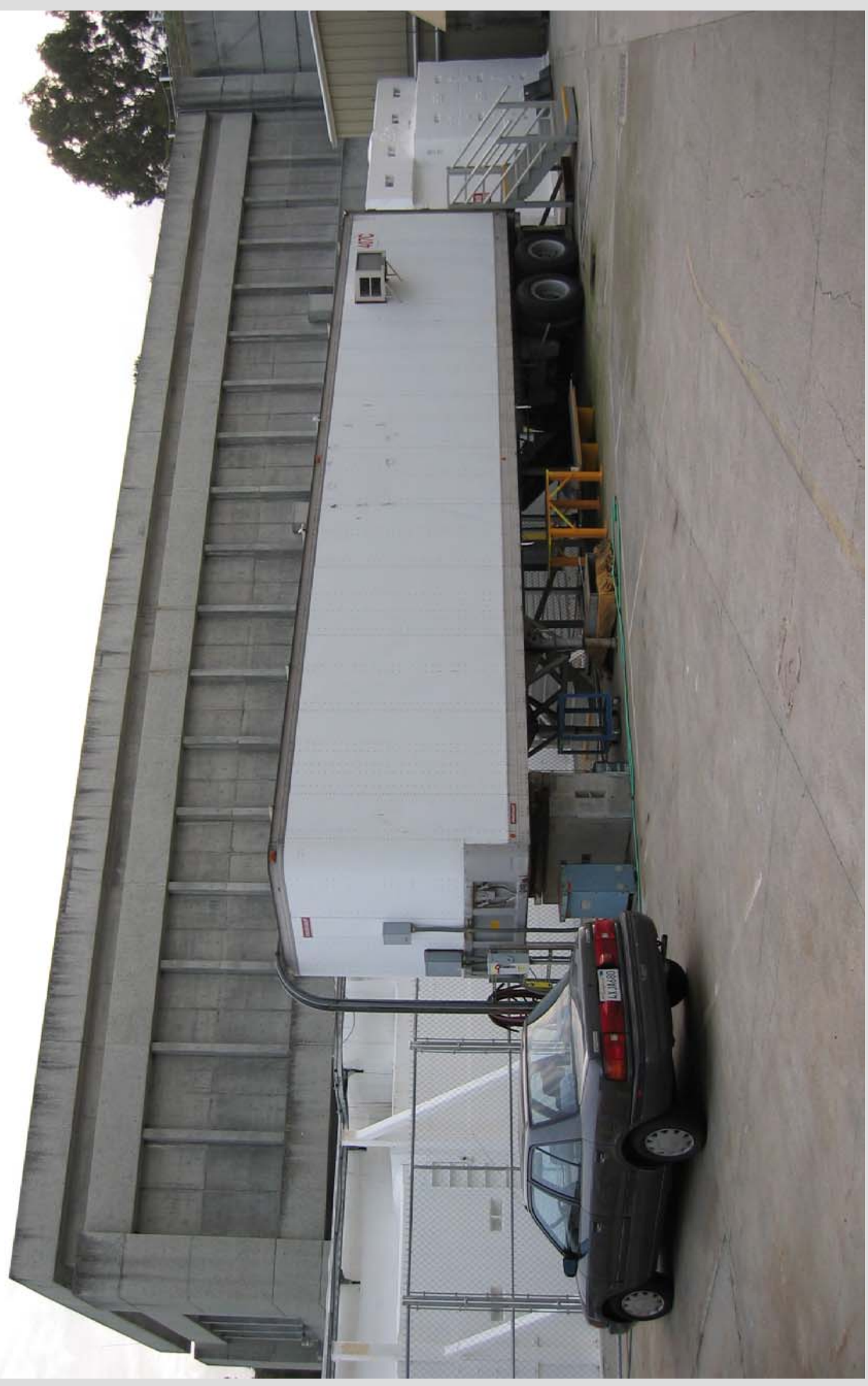




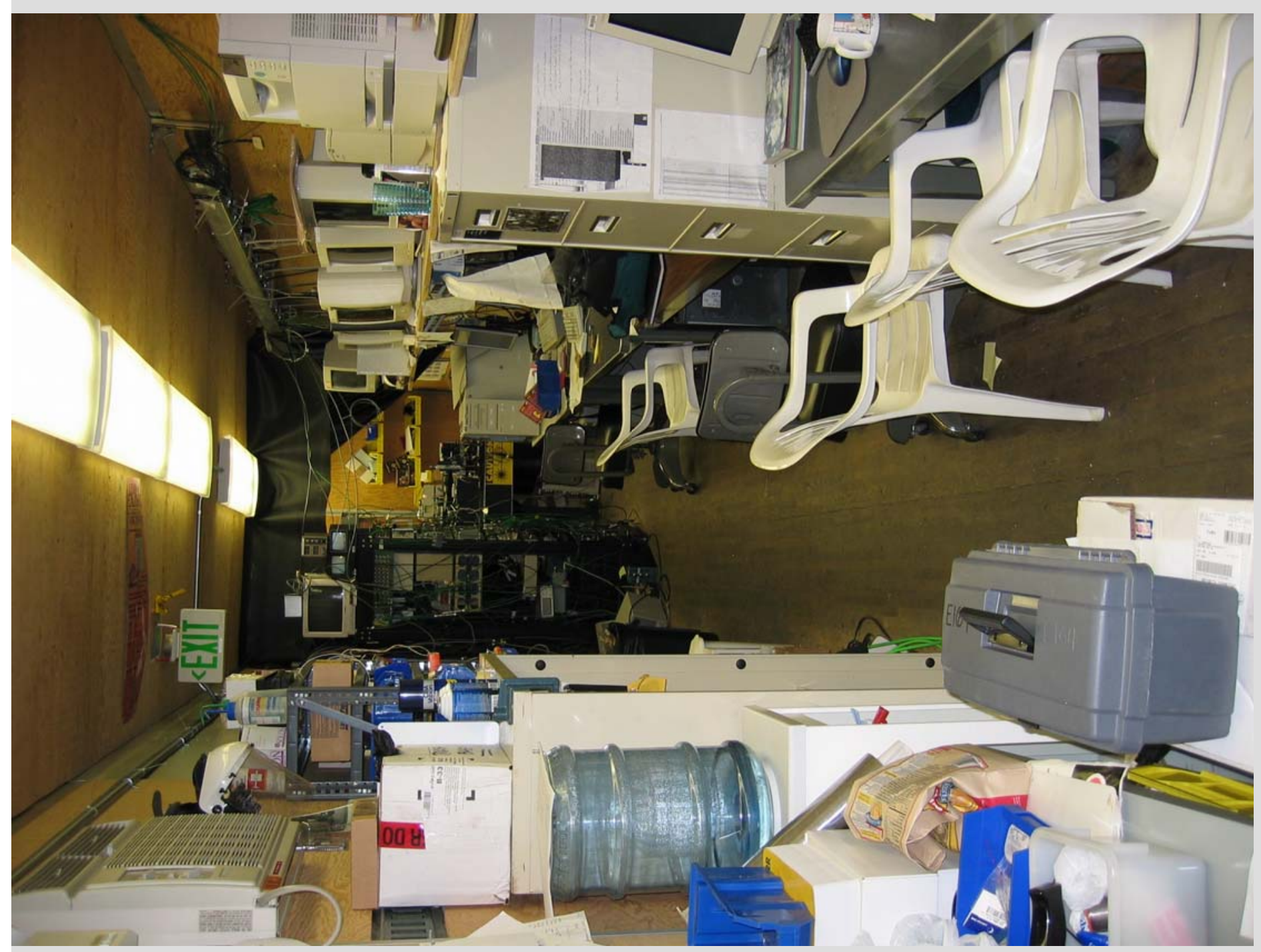




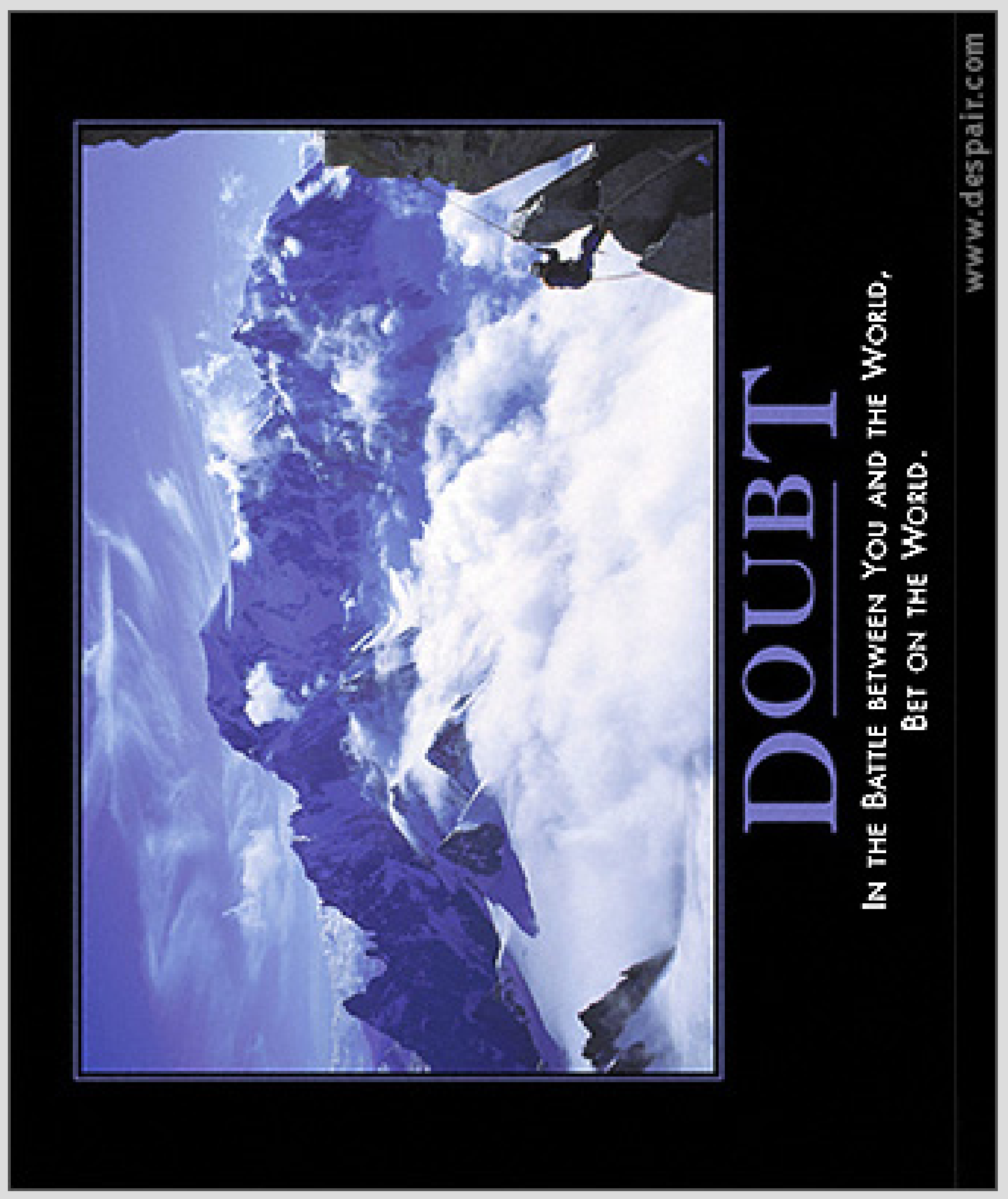




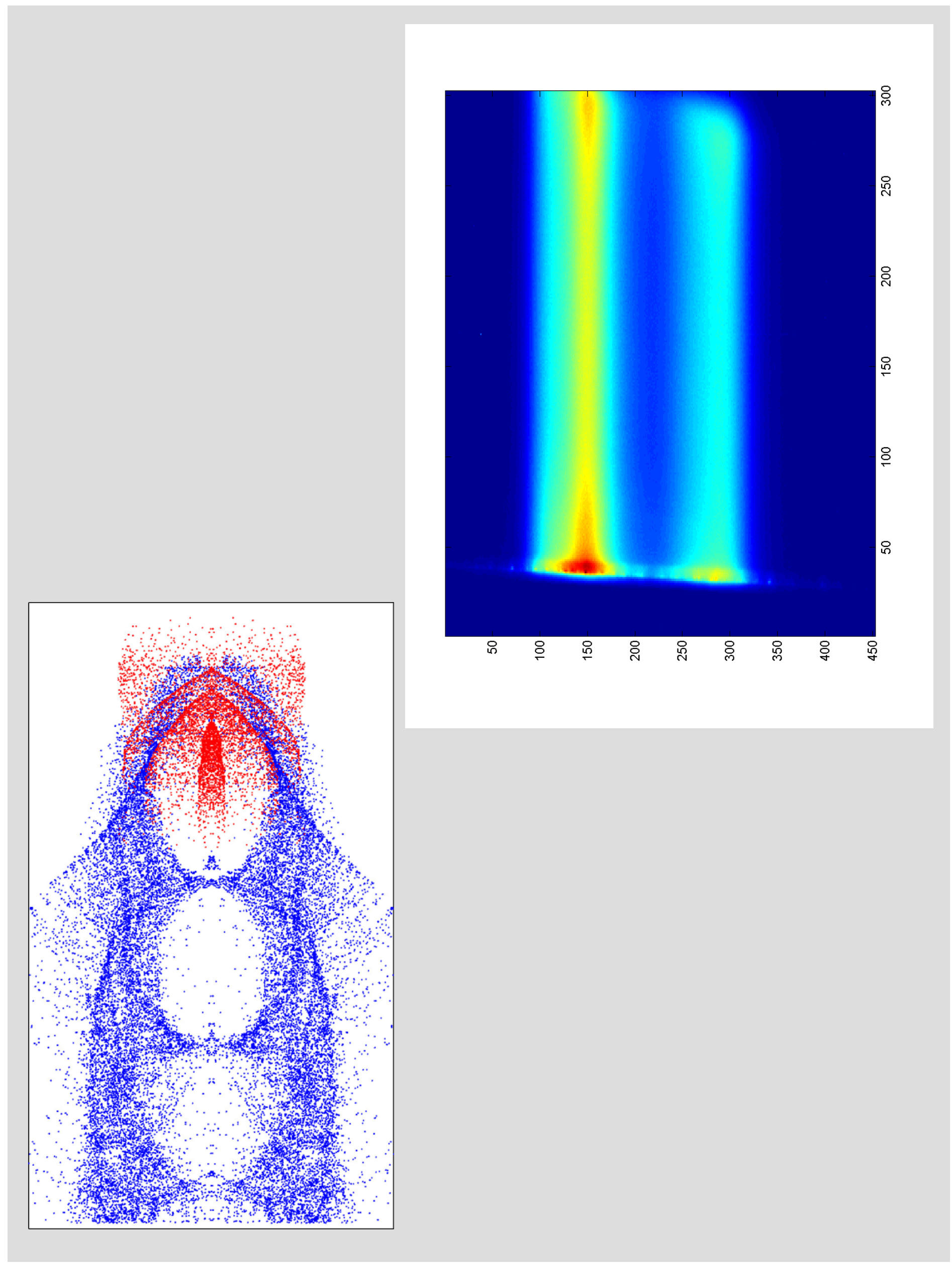




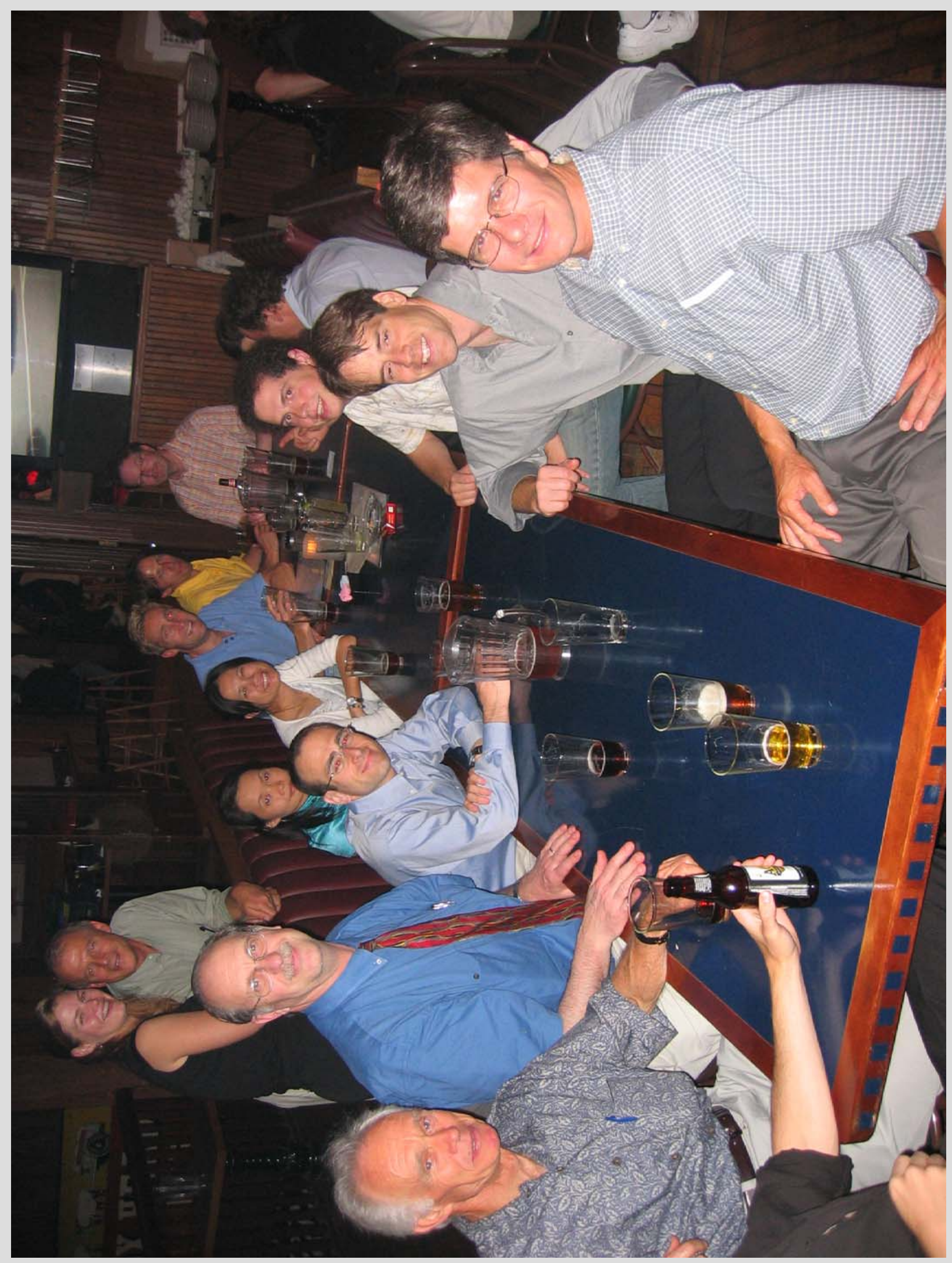



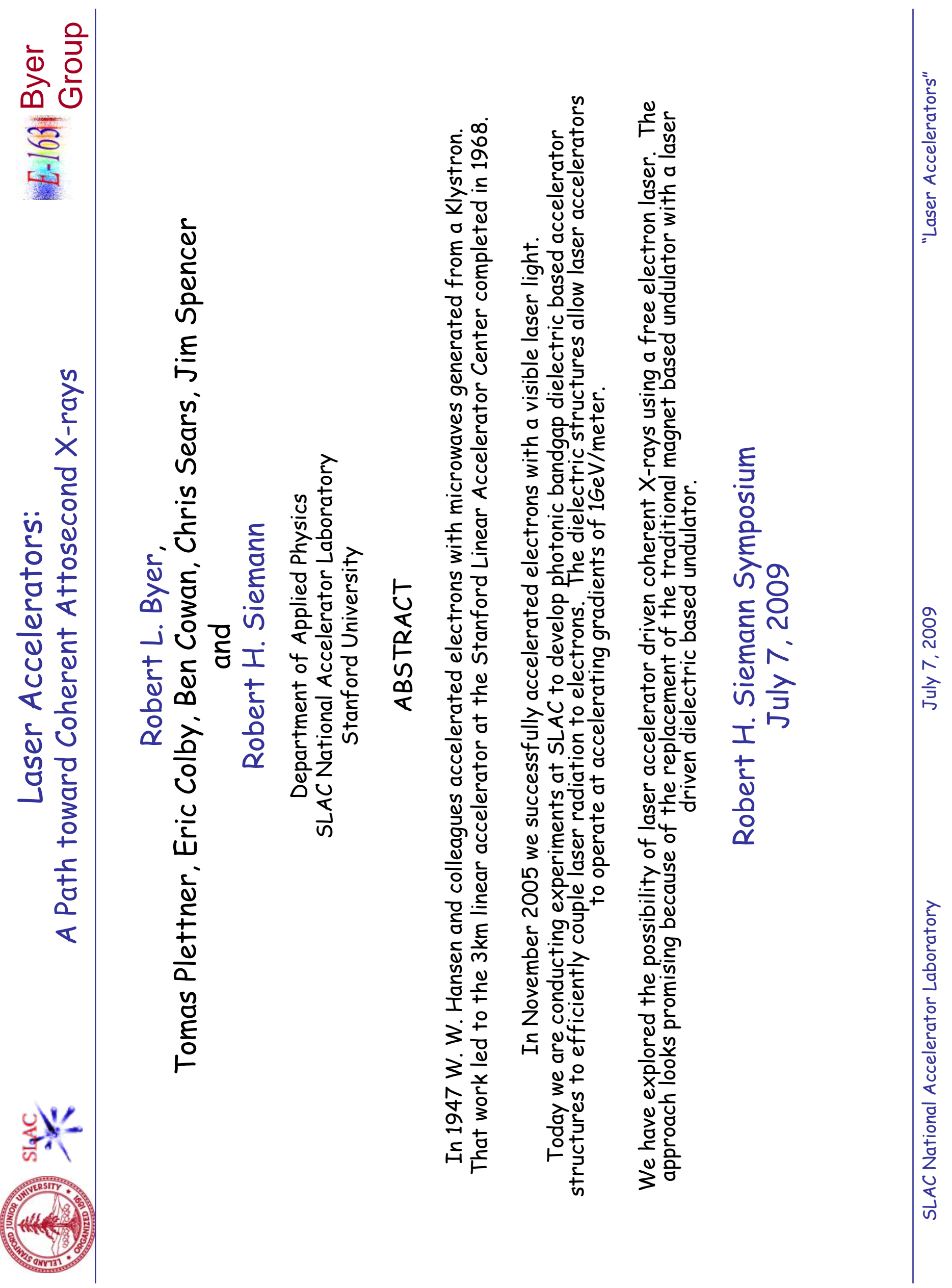


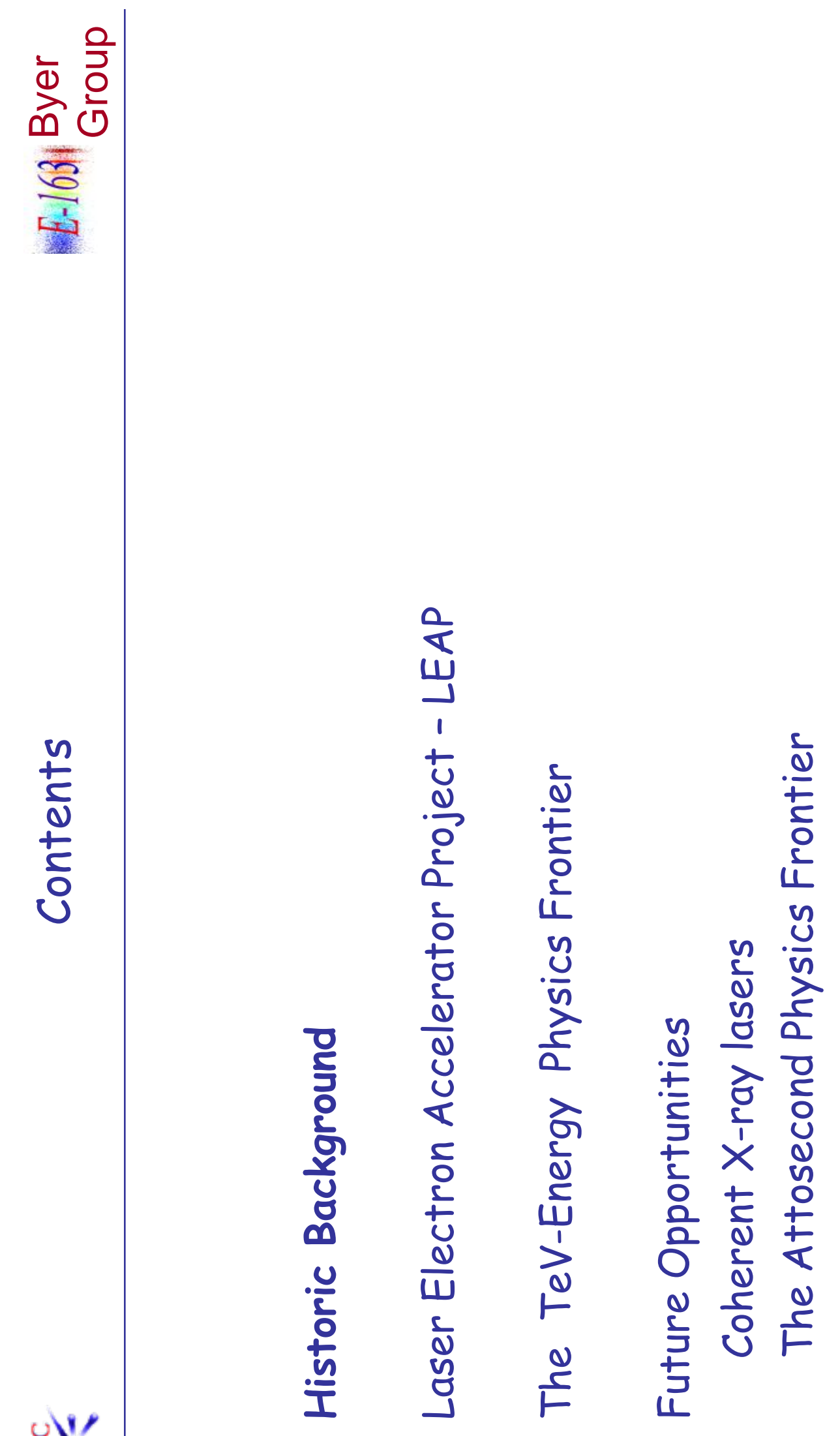




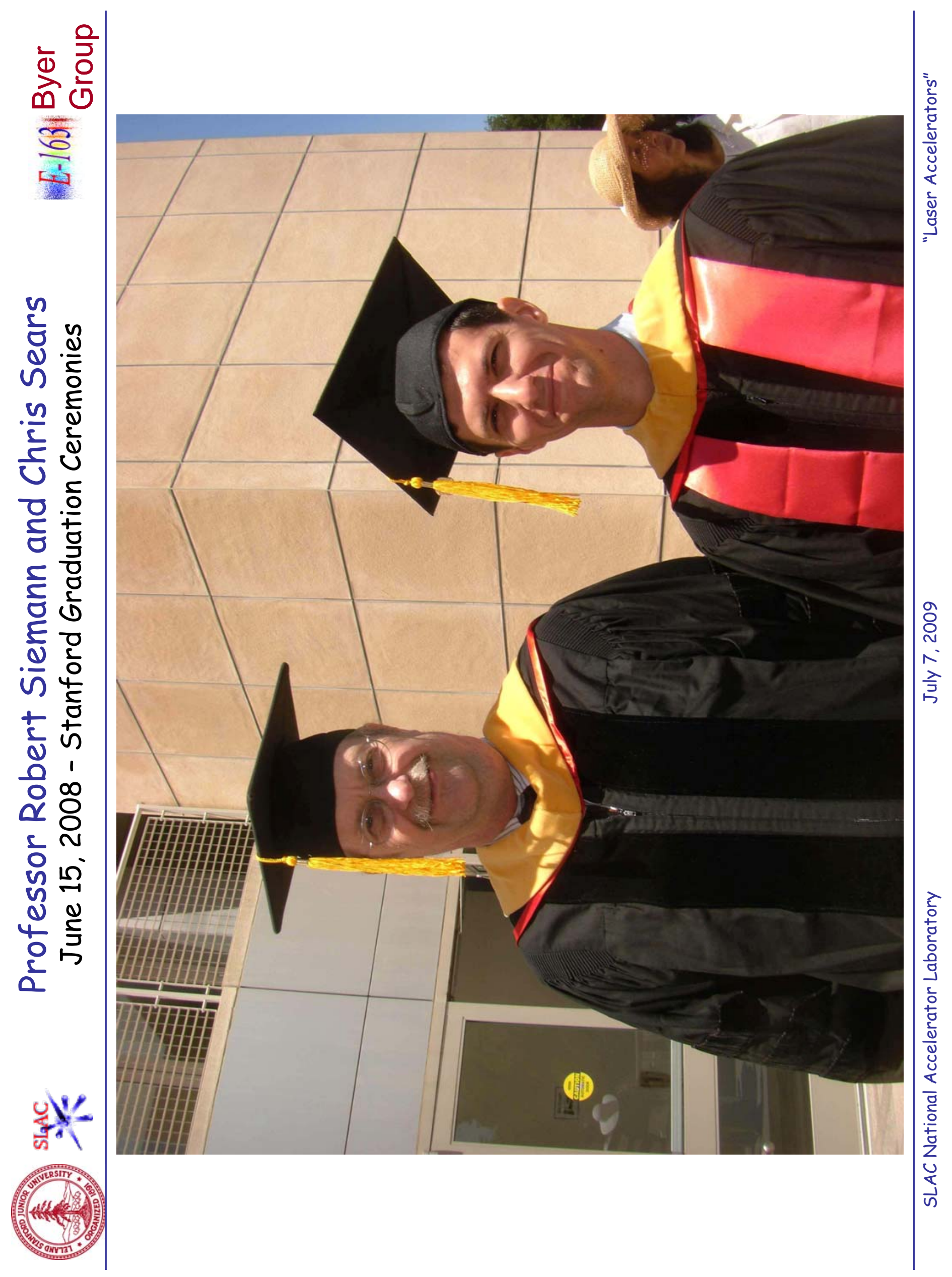




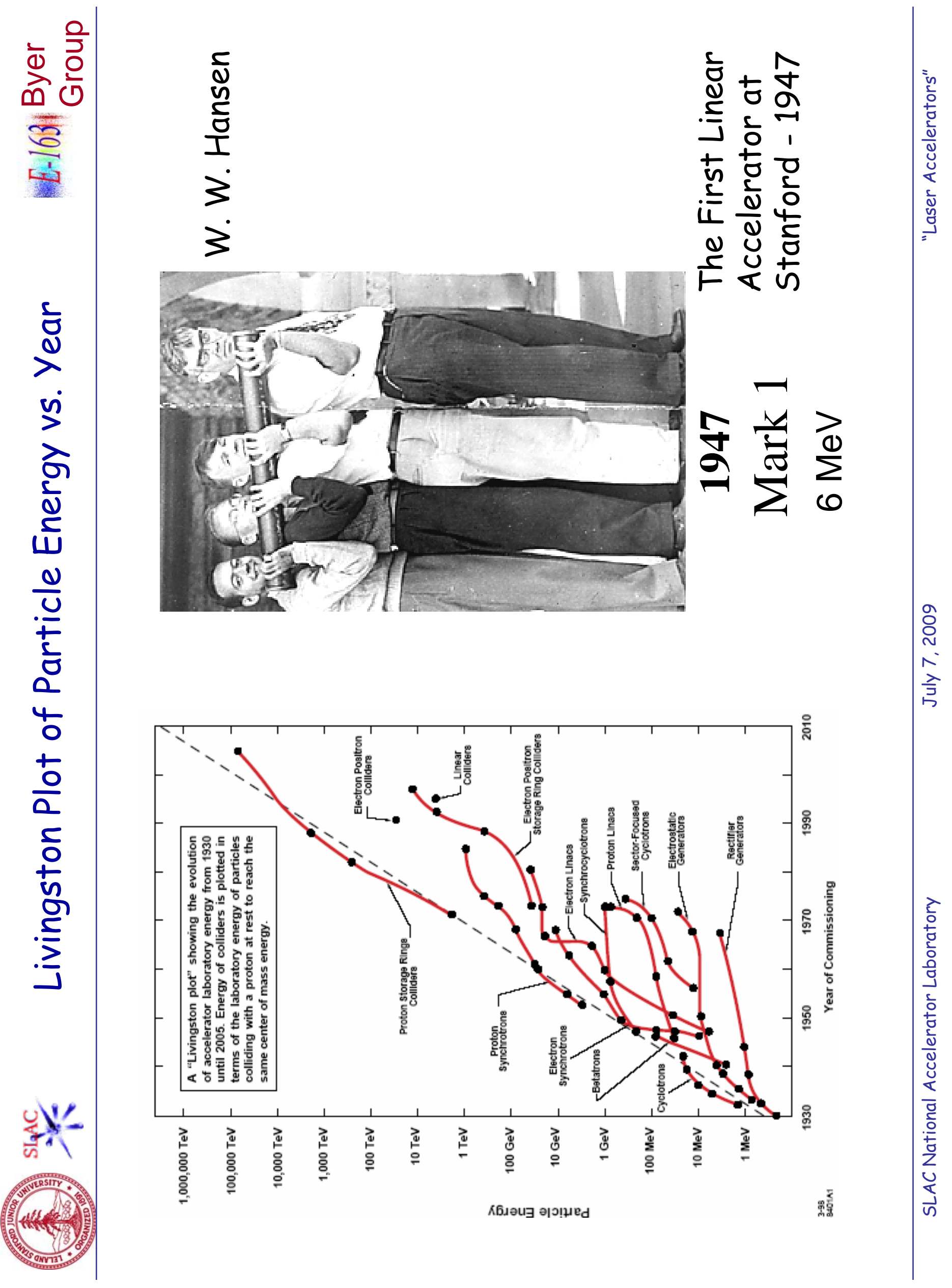




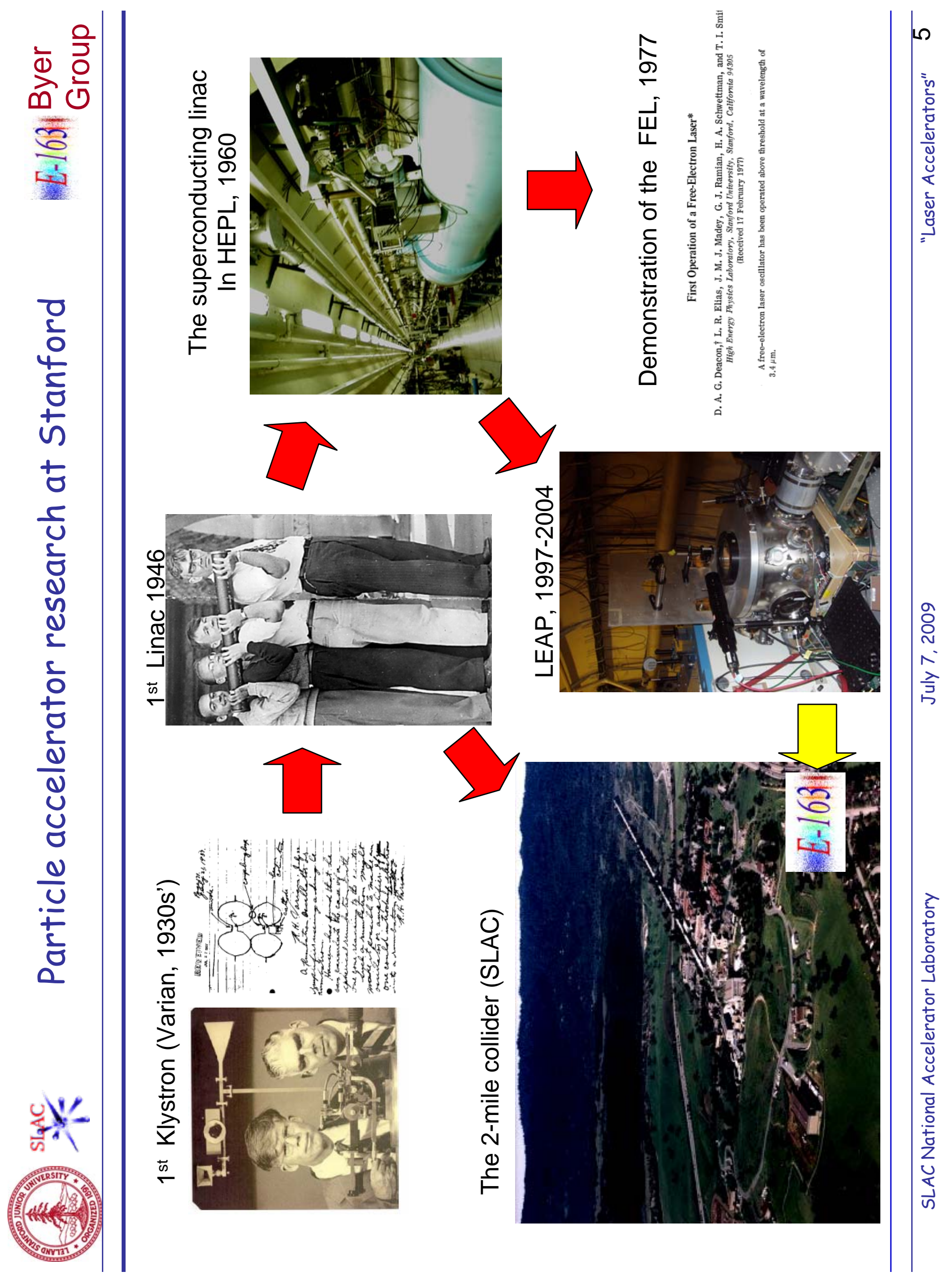




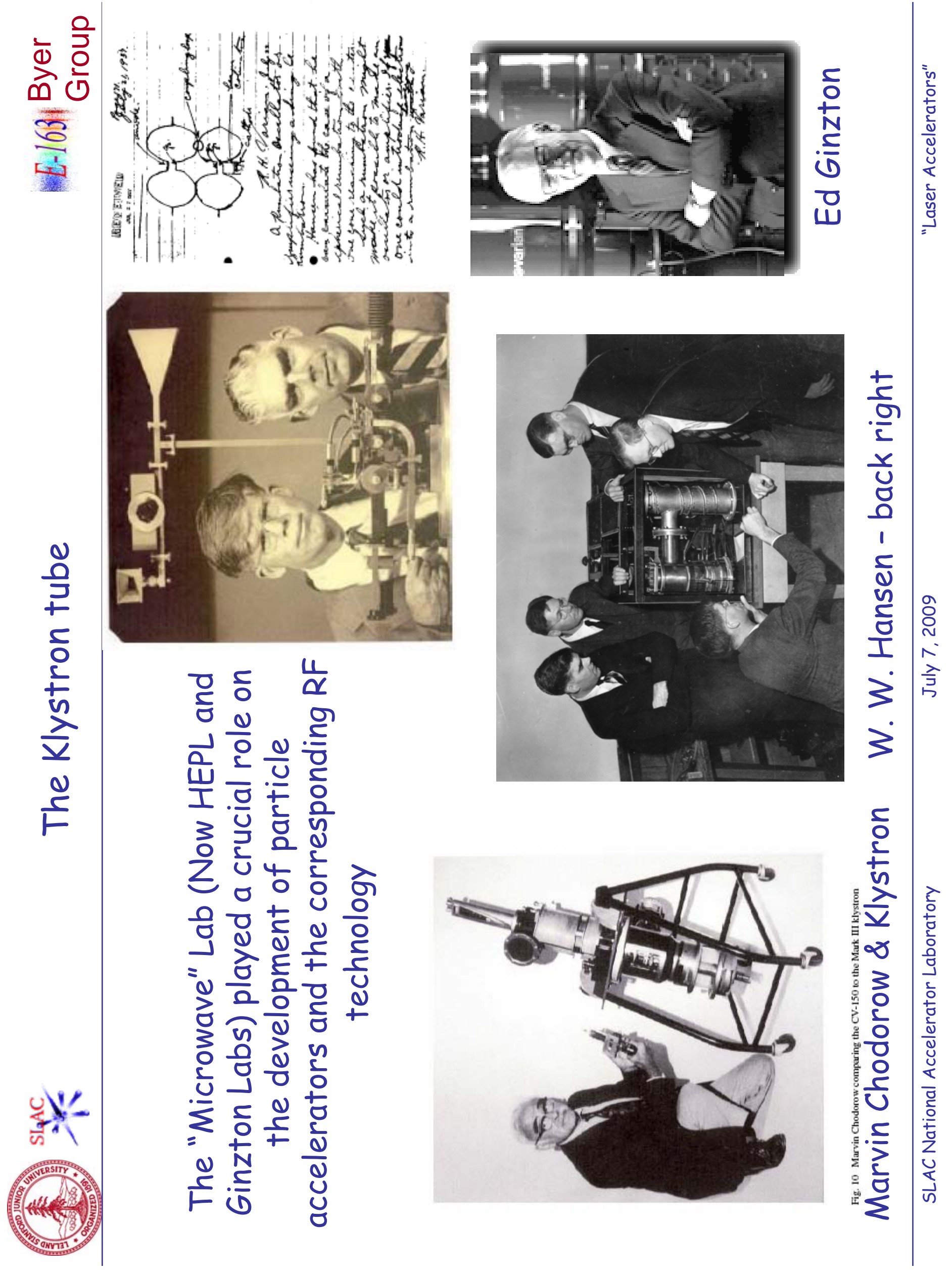




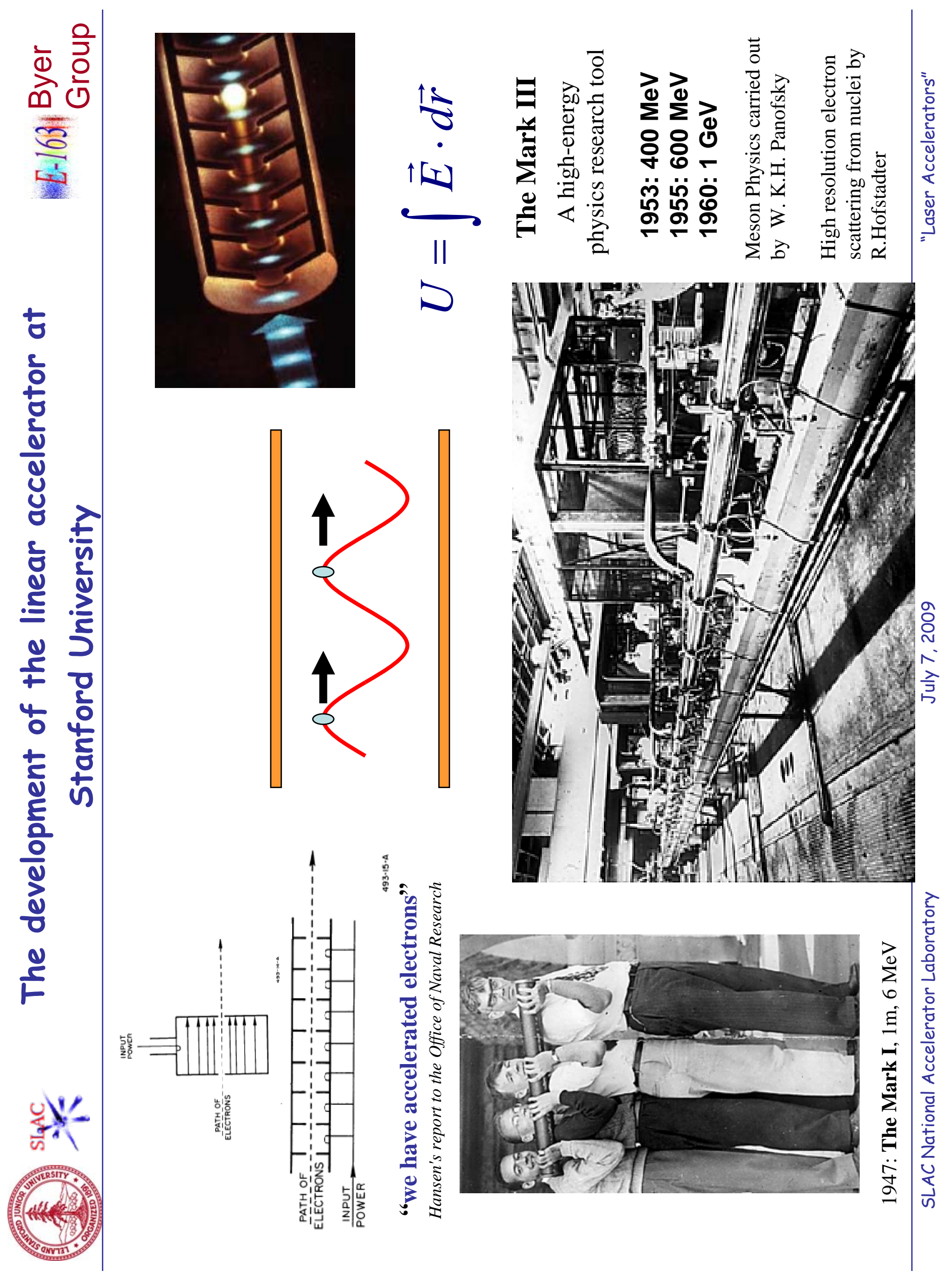




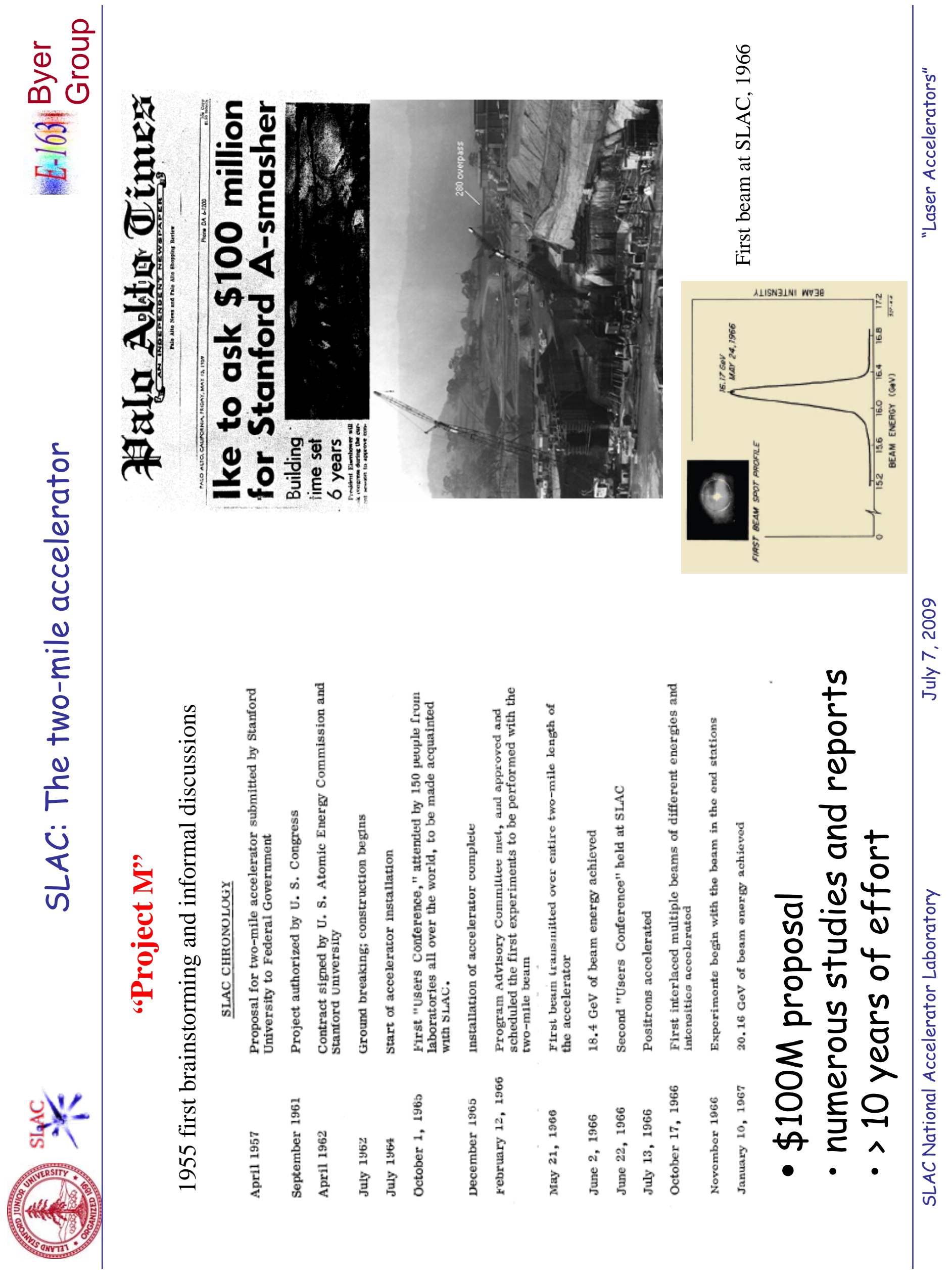




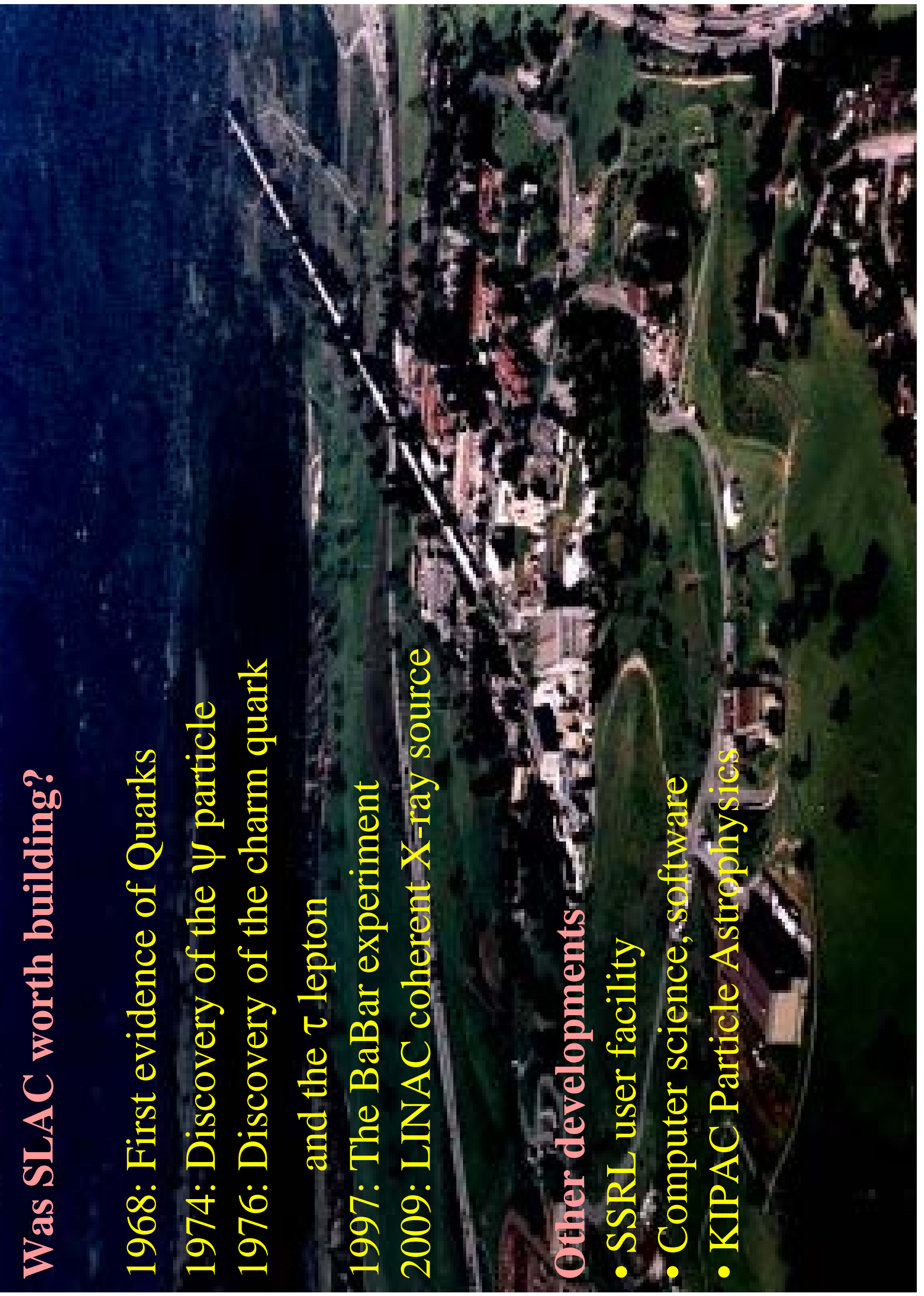




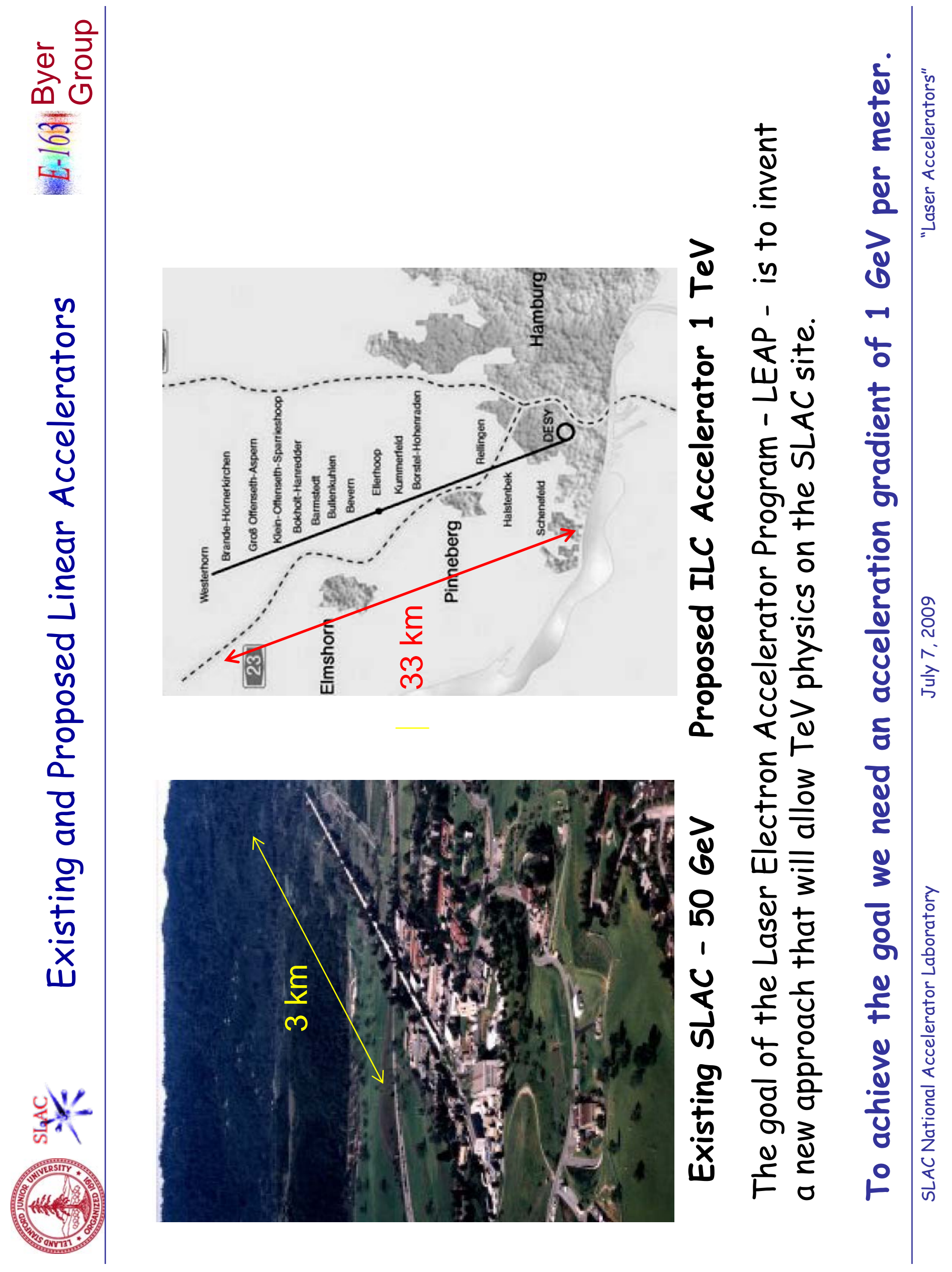




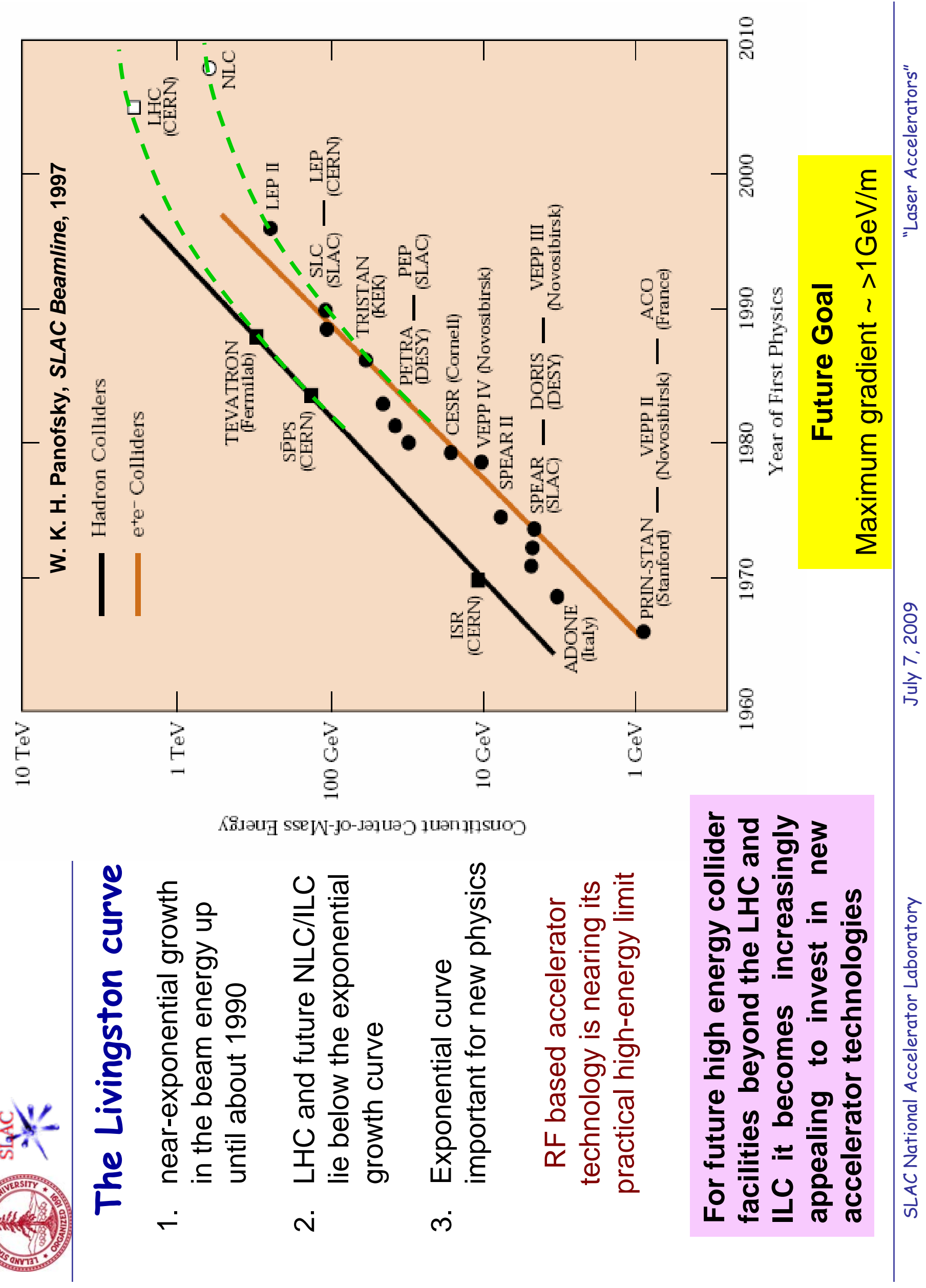




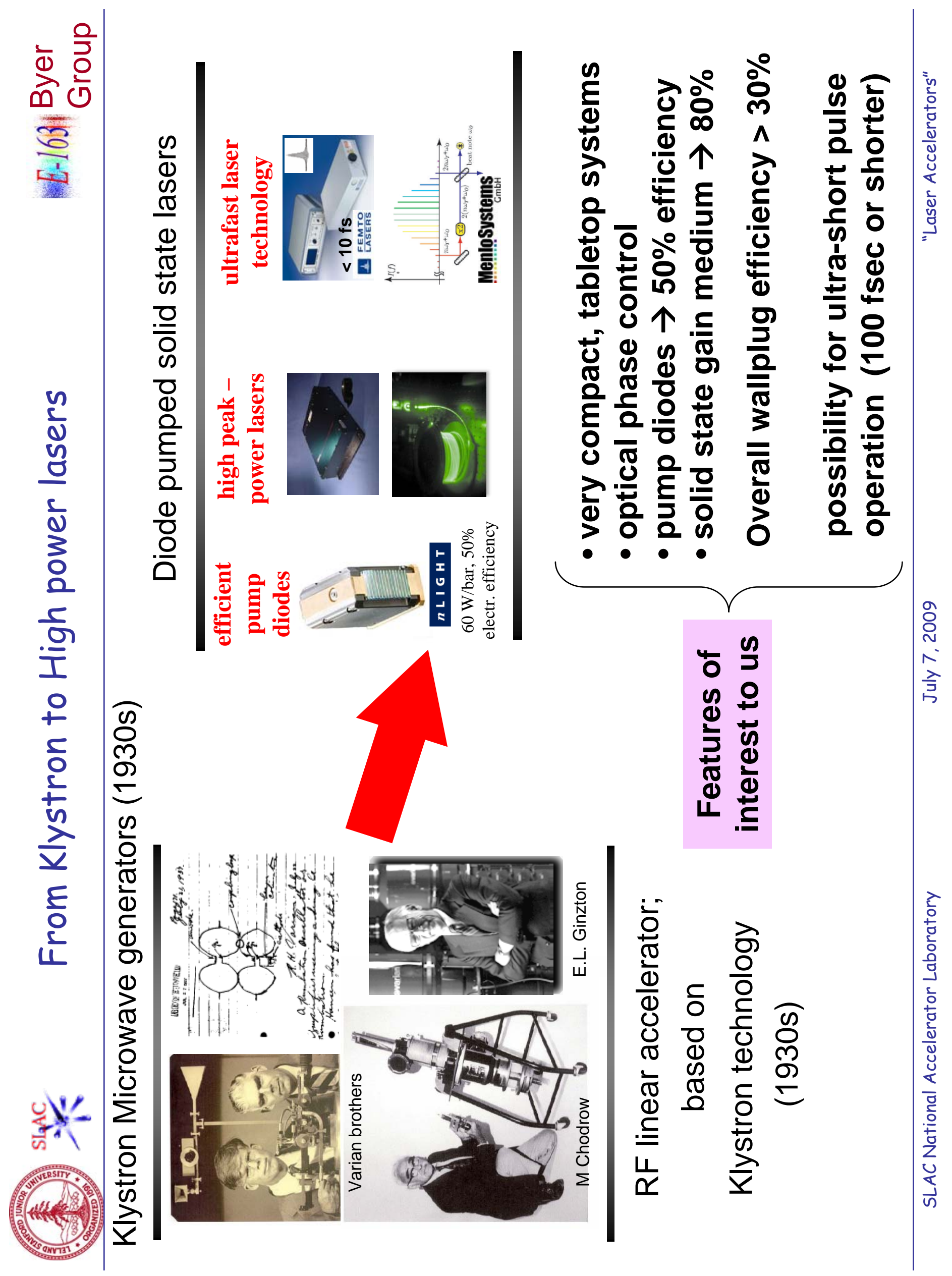




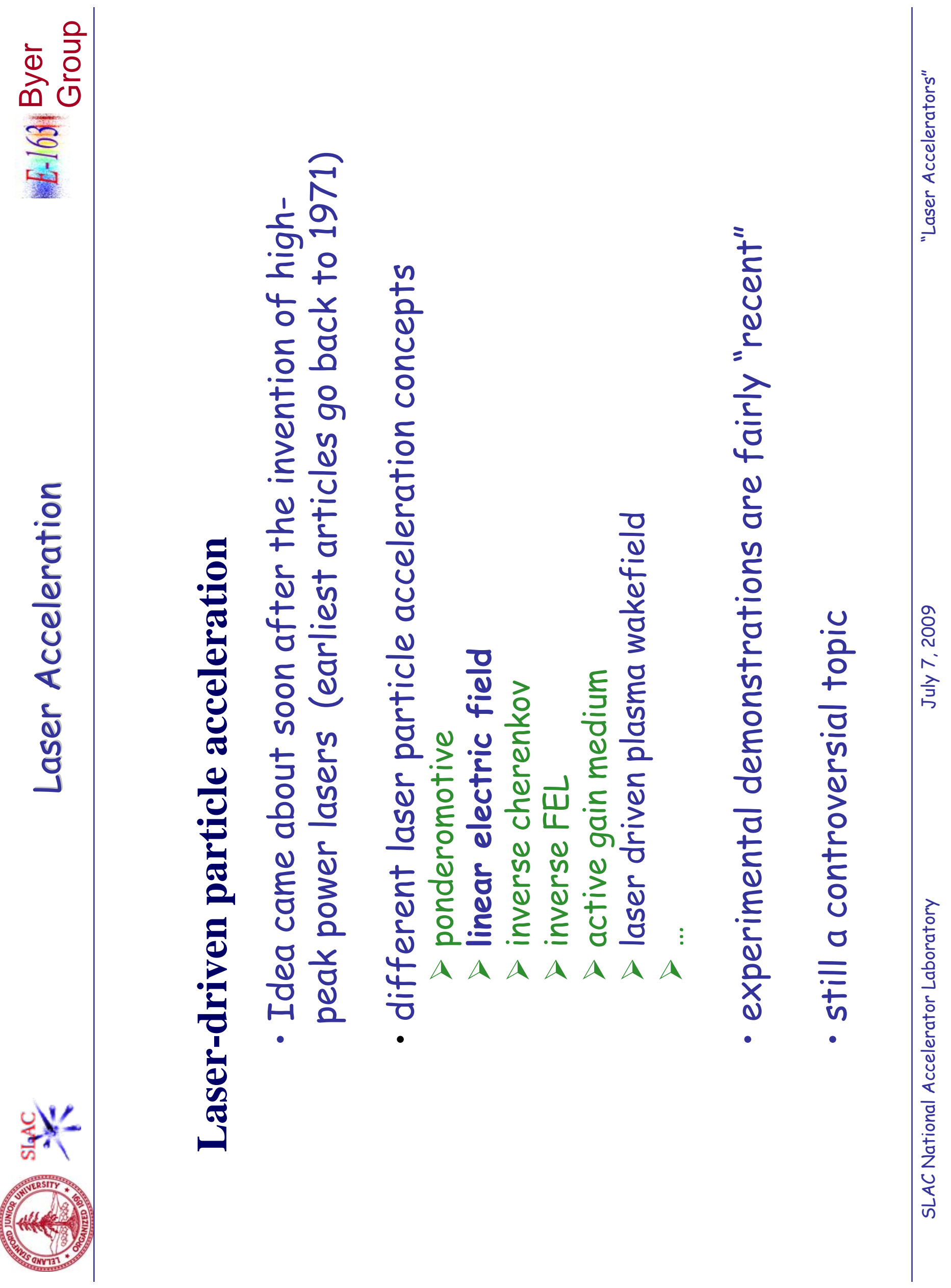




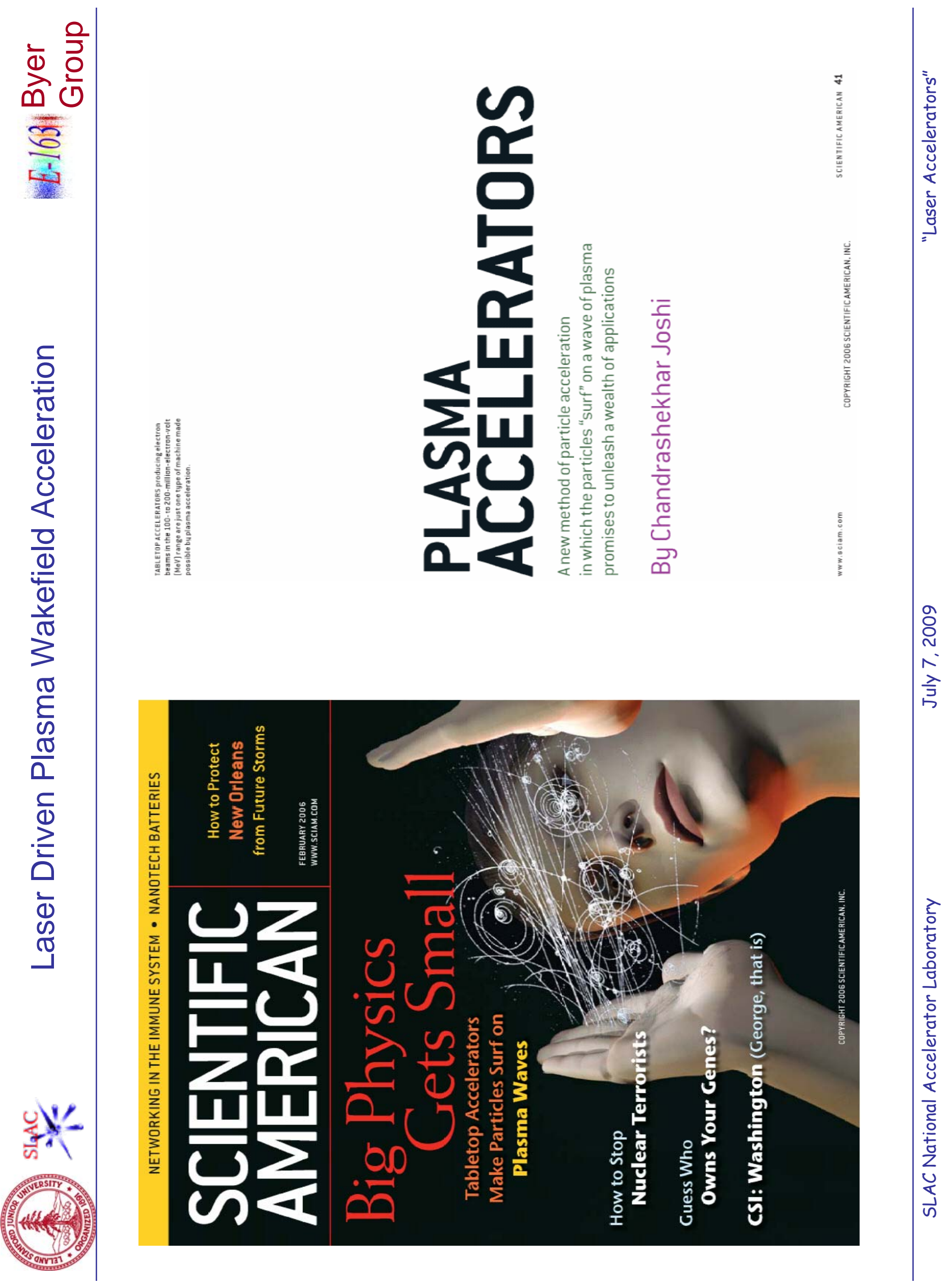




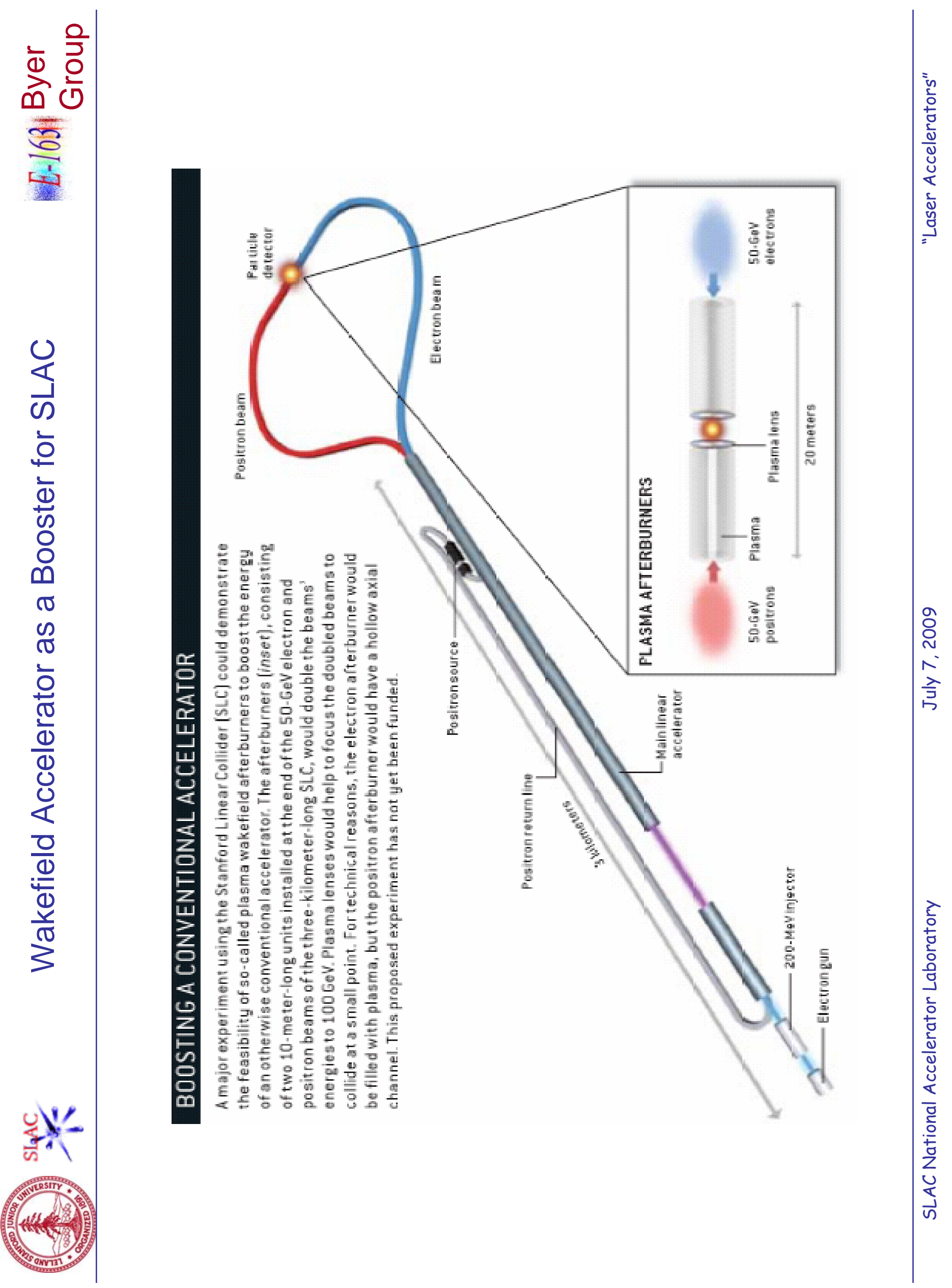




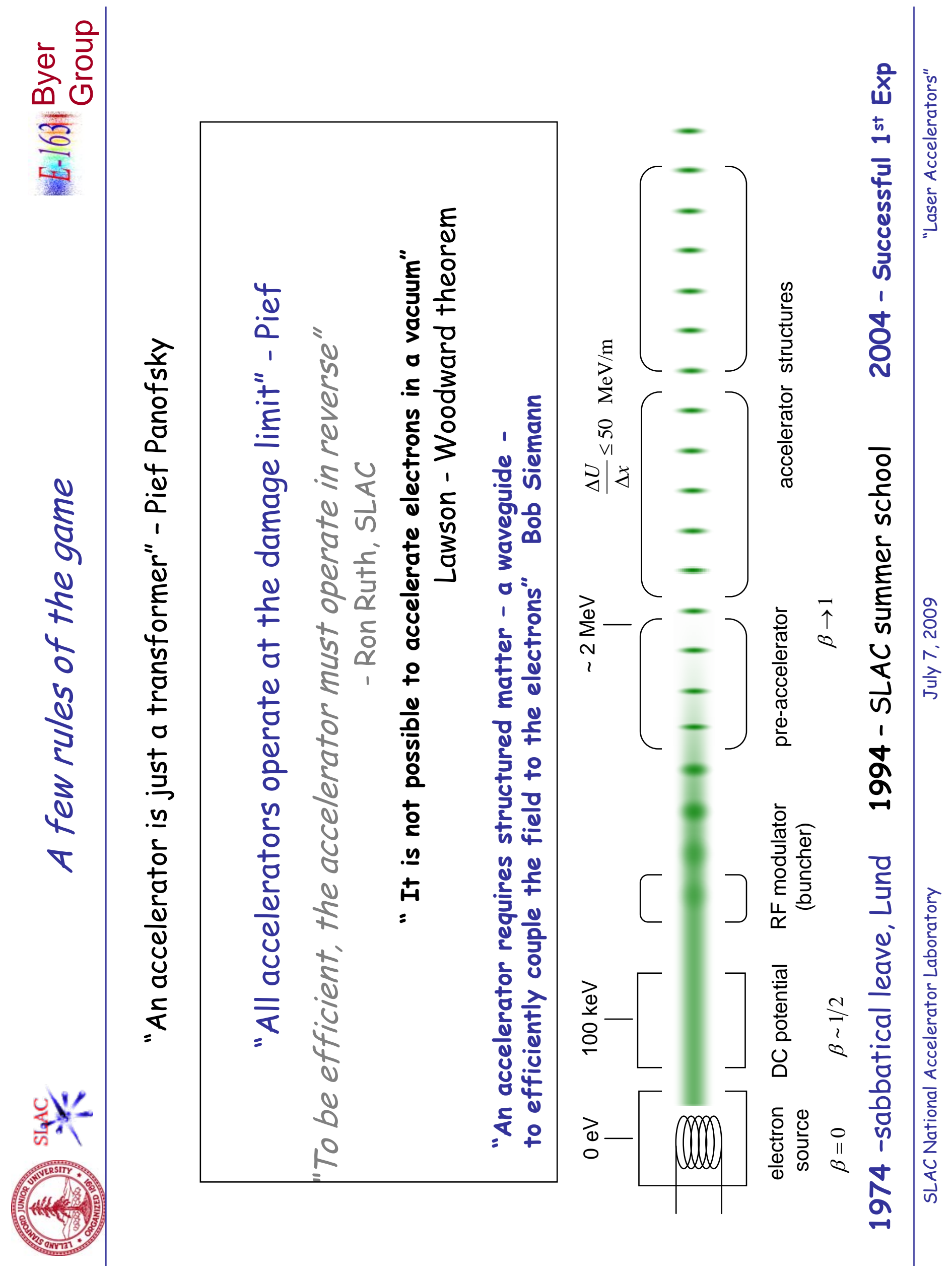



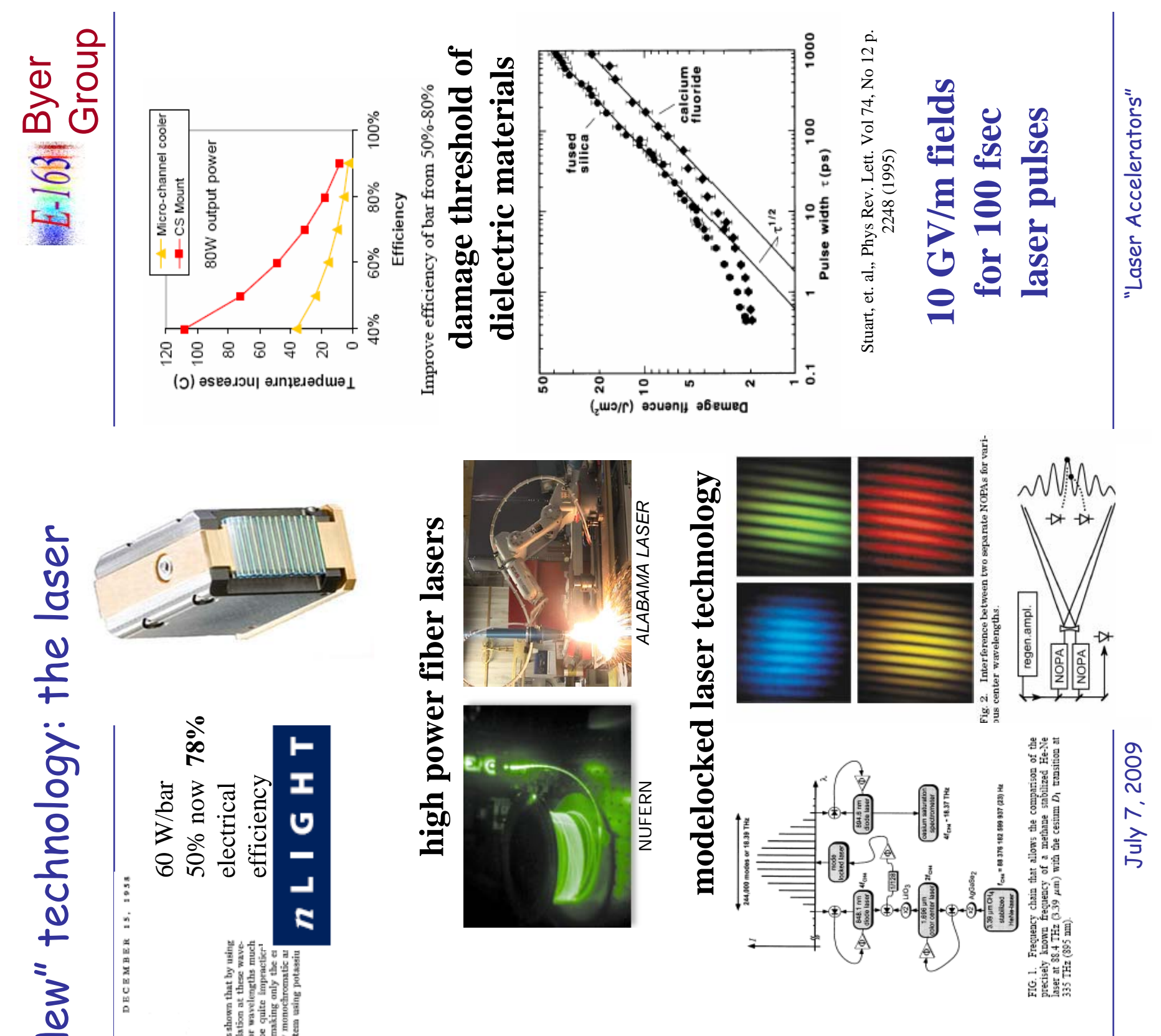

$=$

离
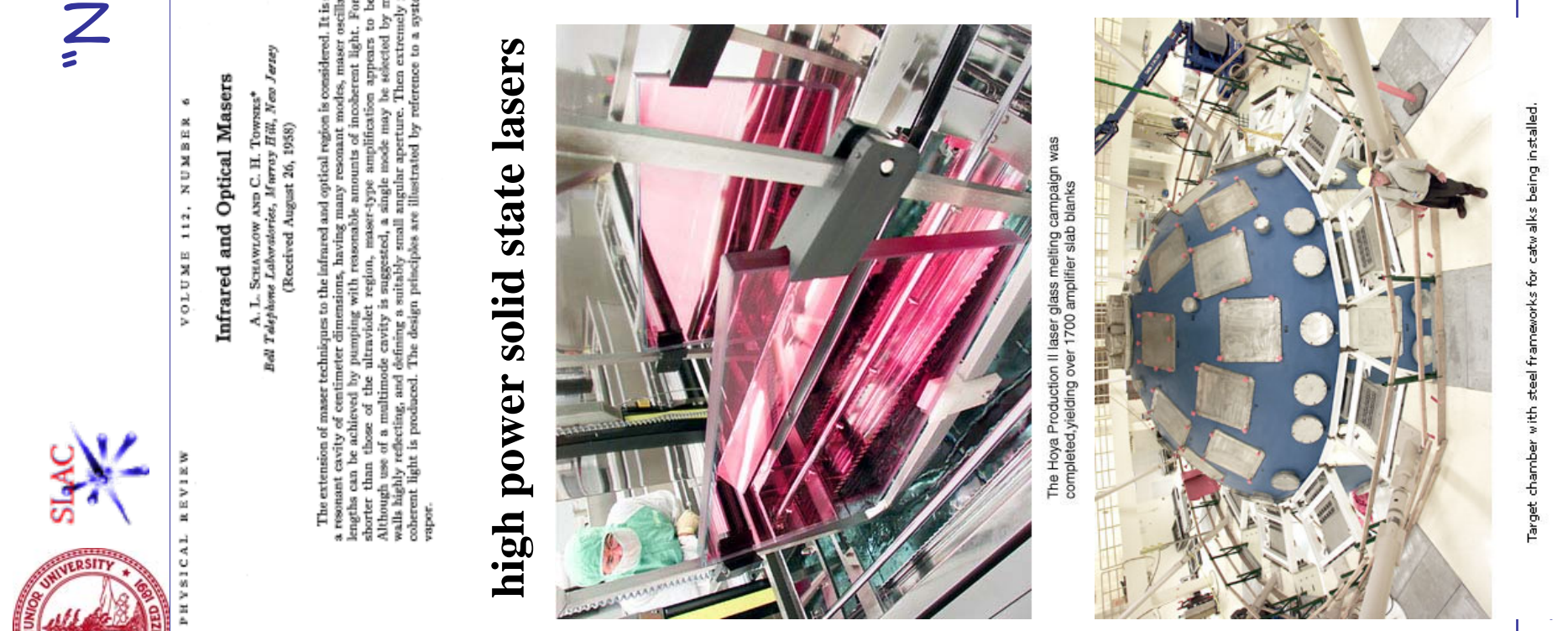


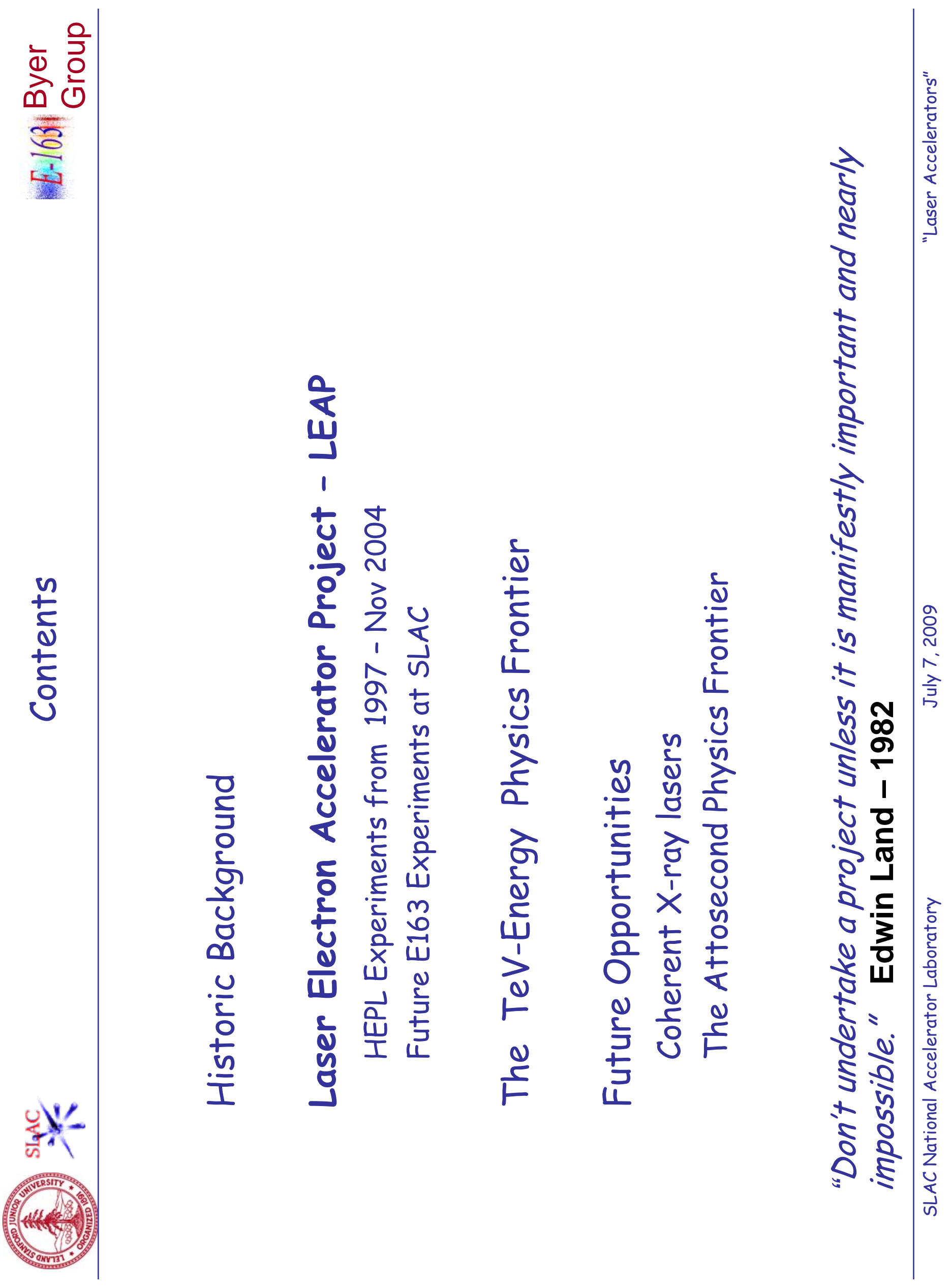




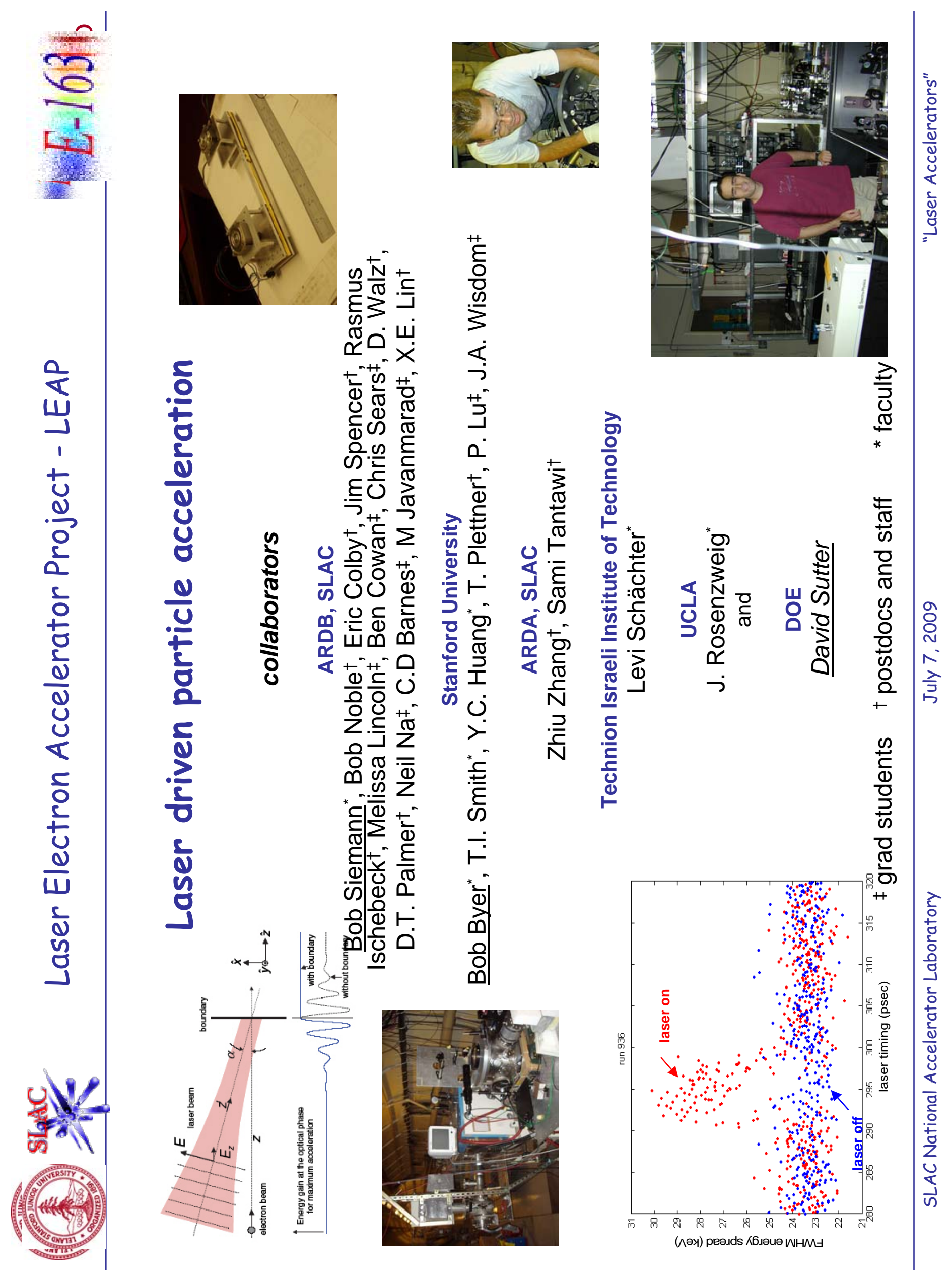




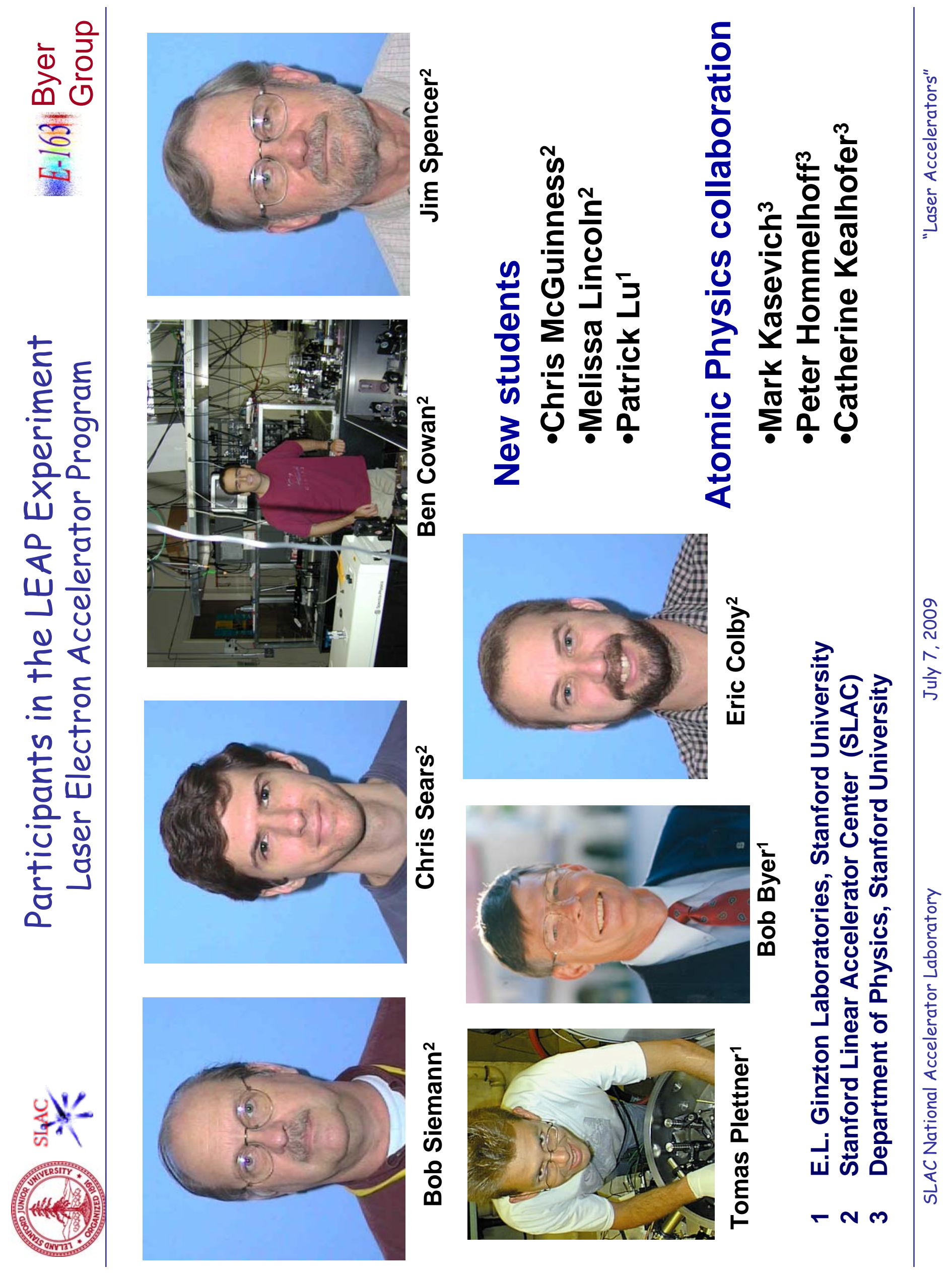




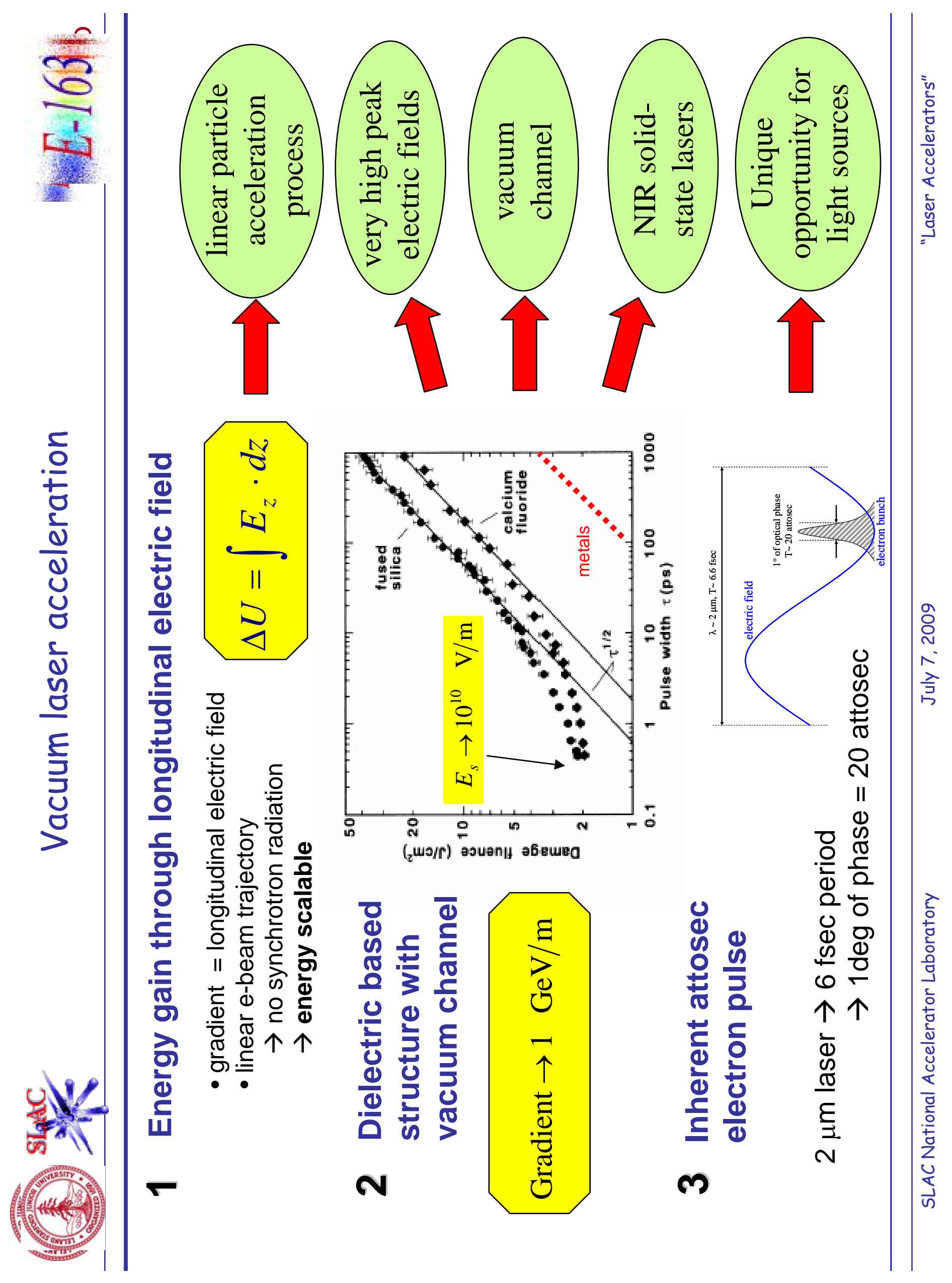



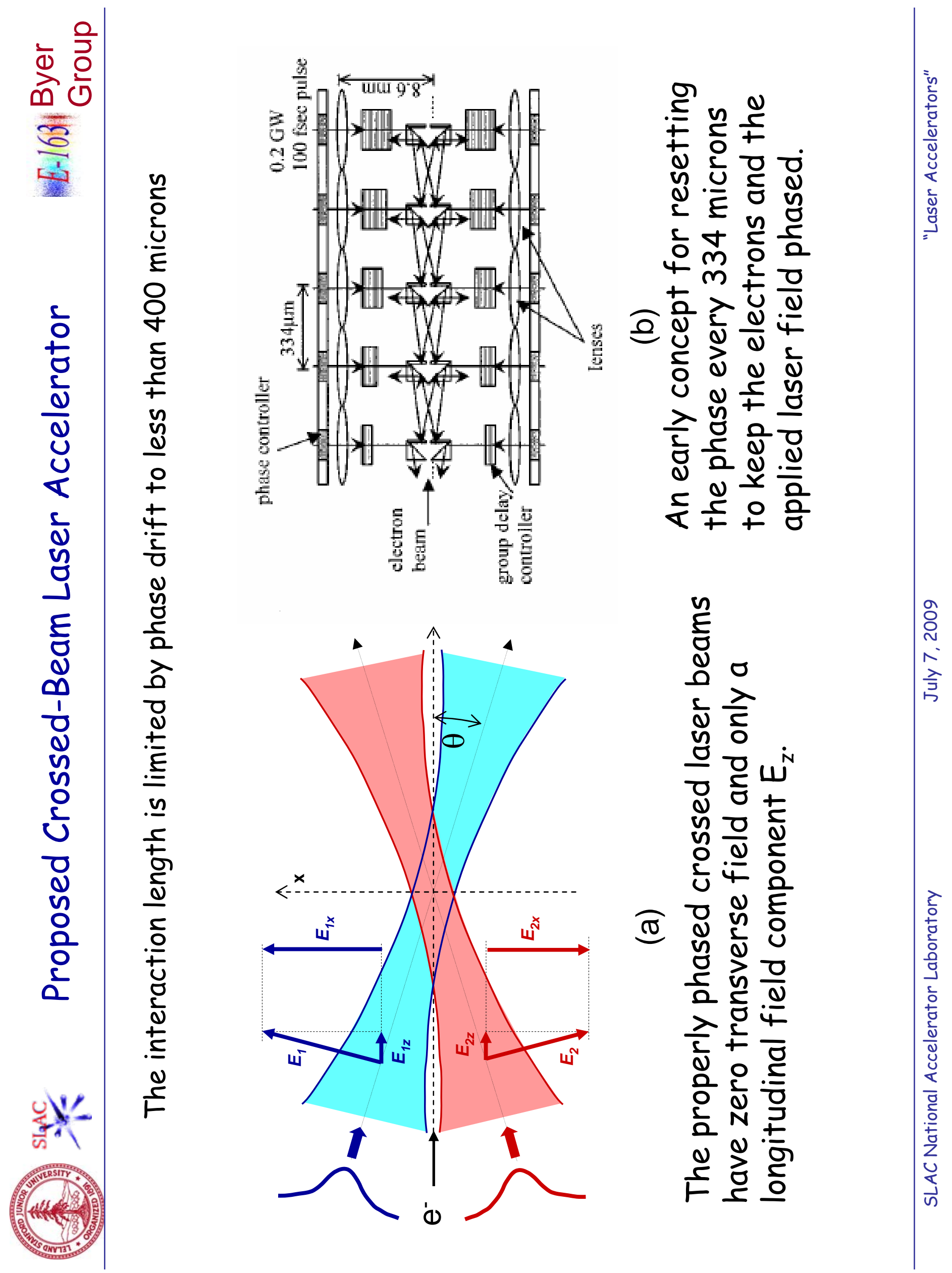


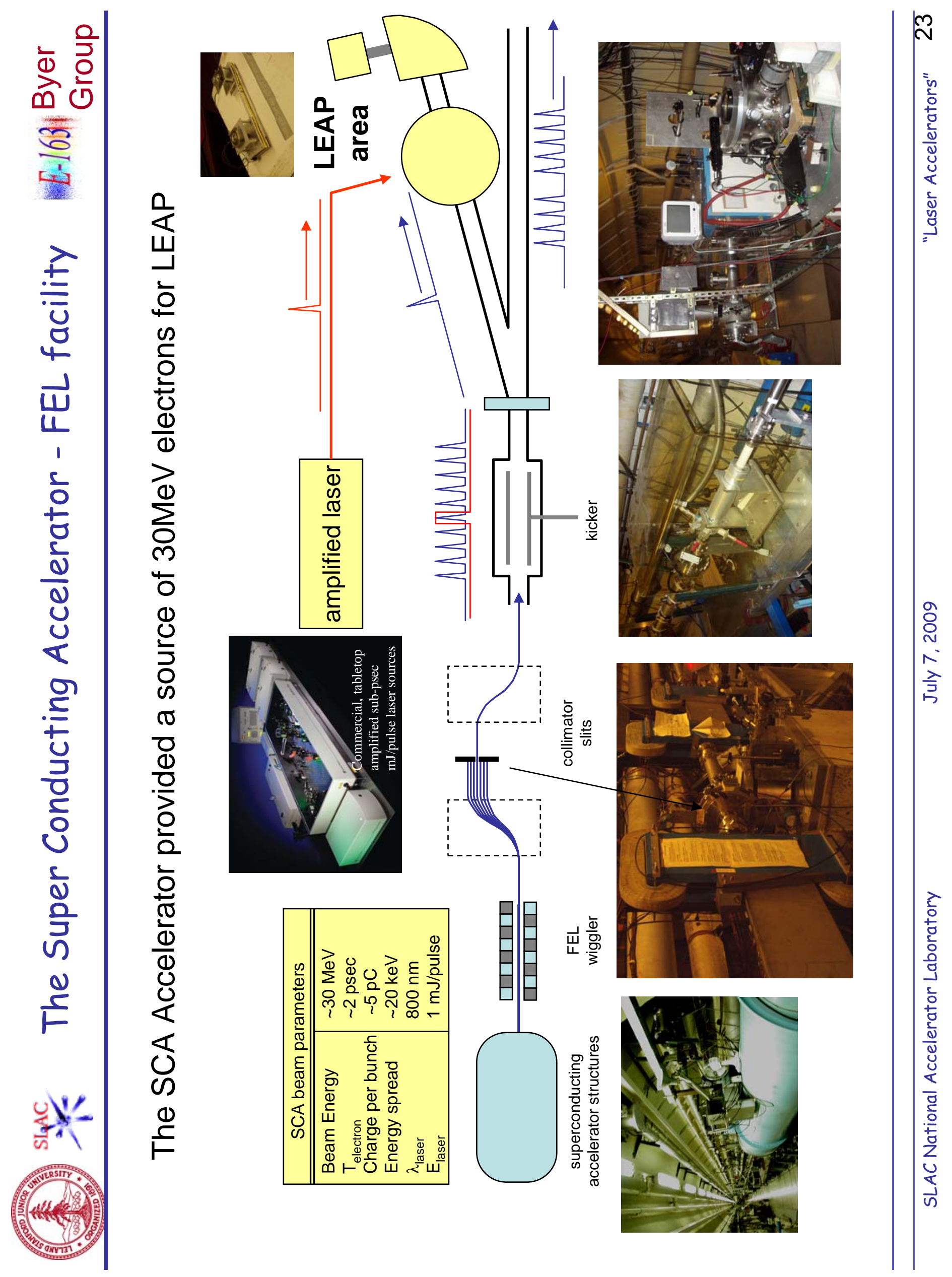




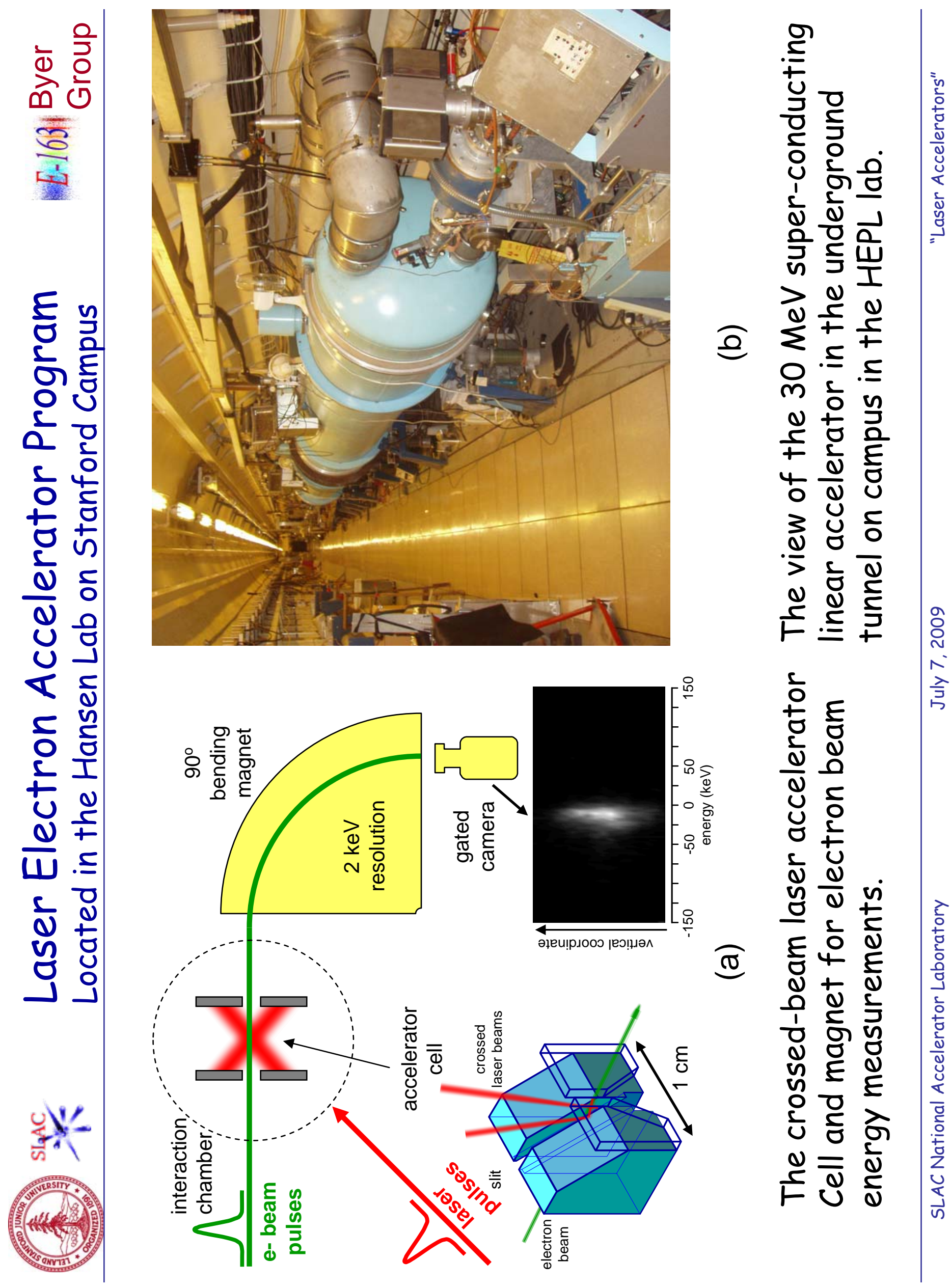




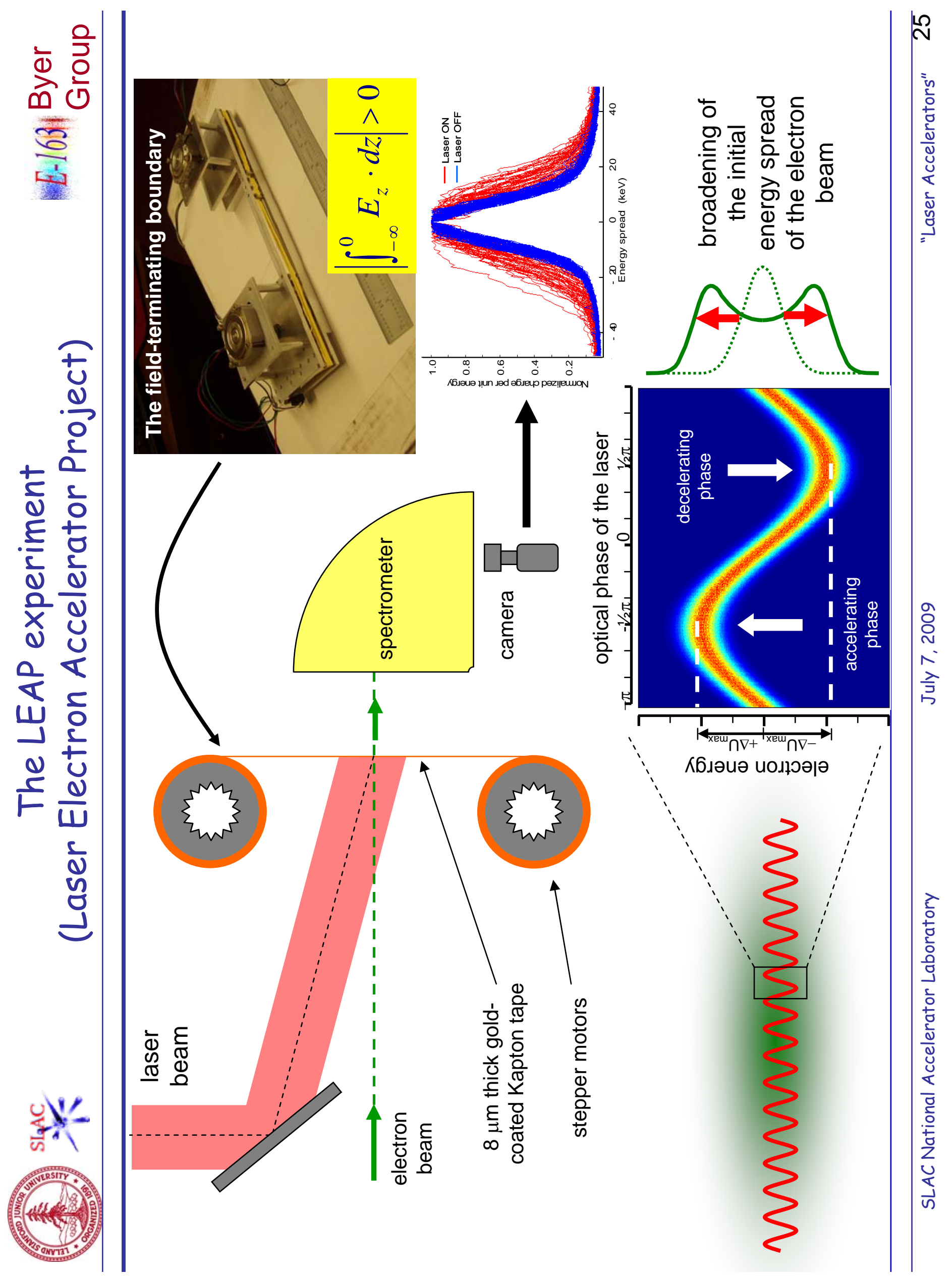




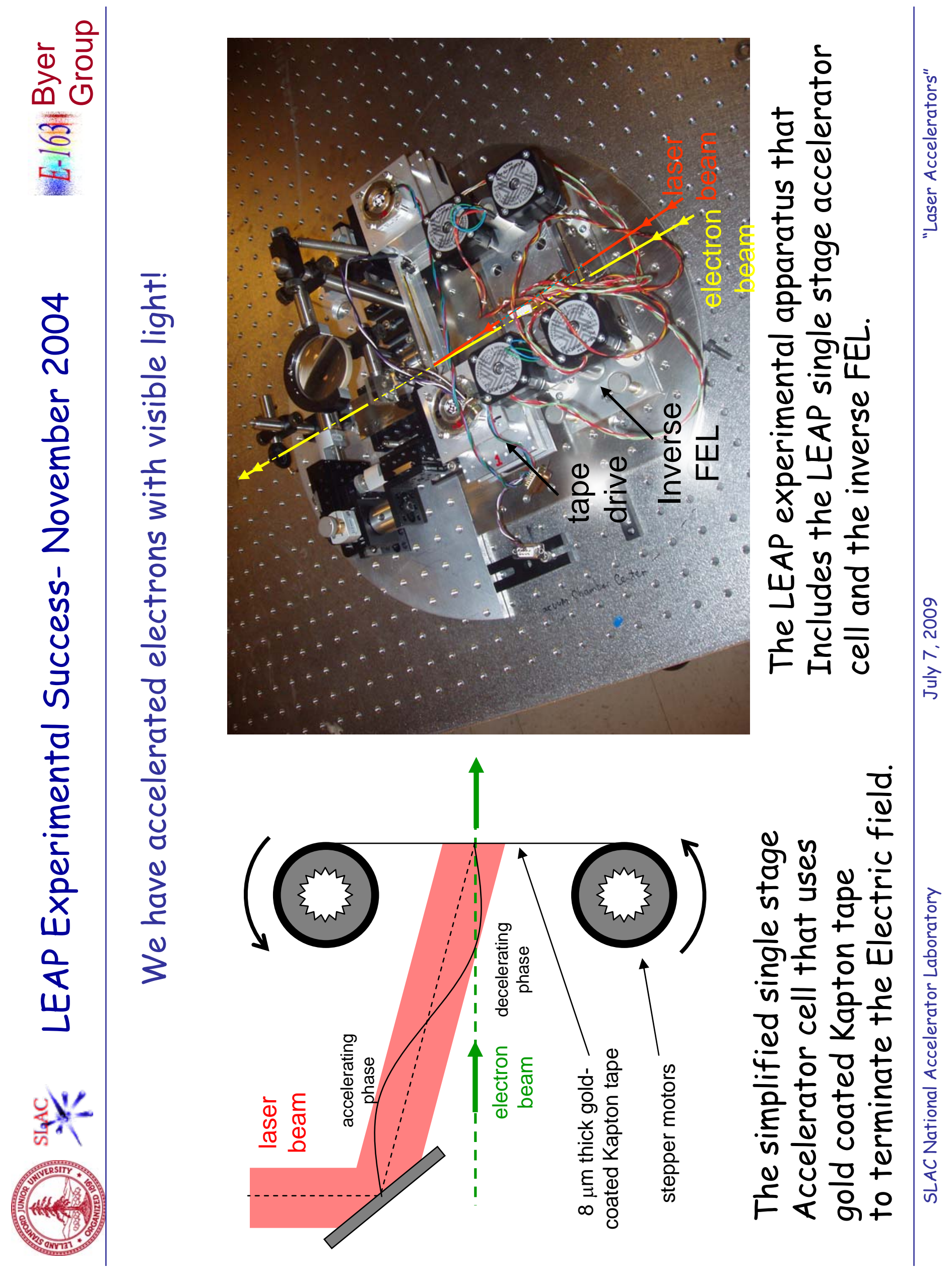




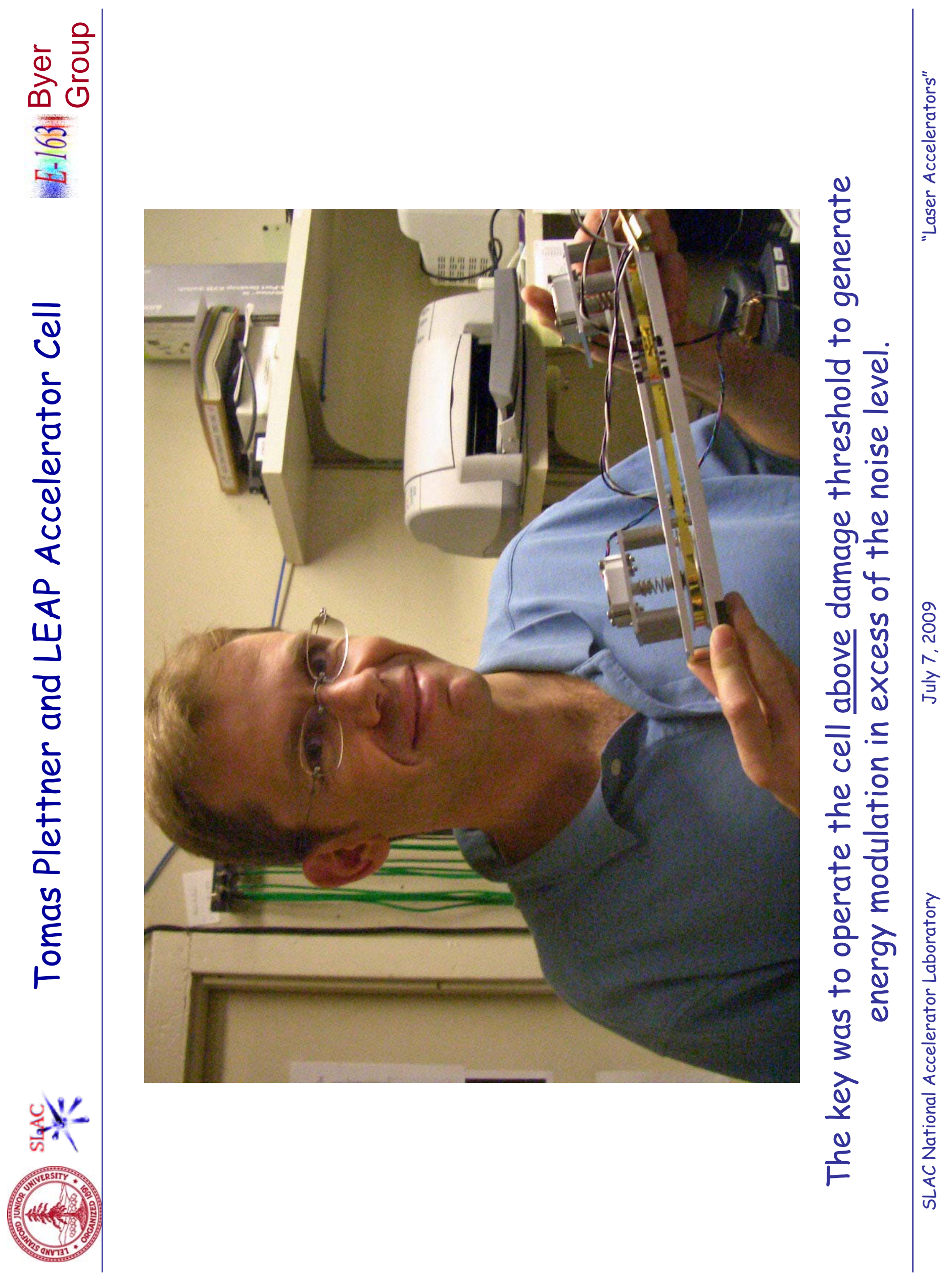




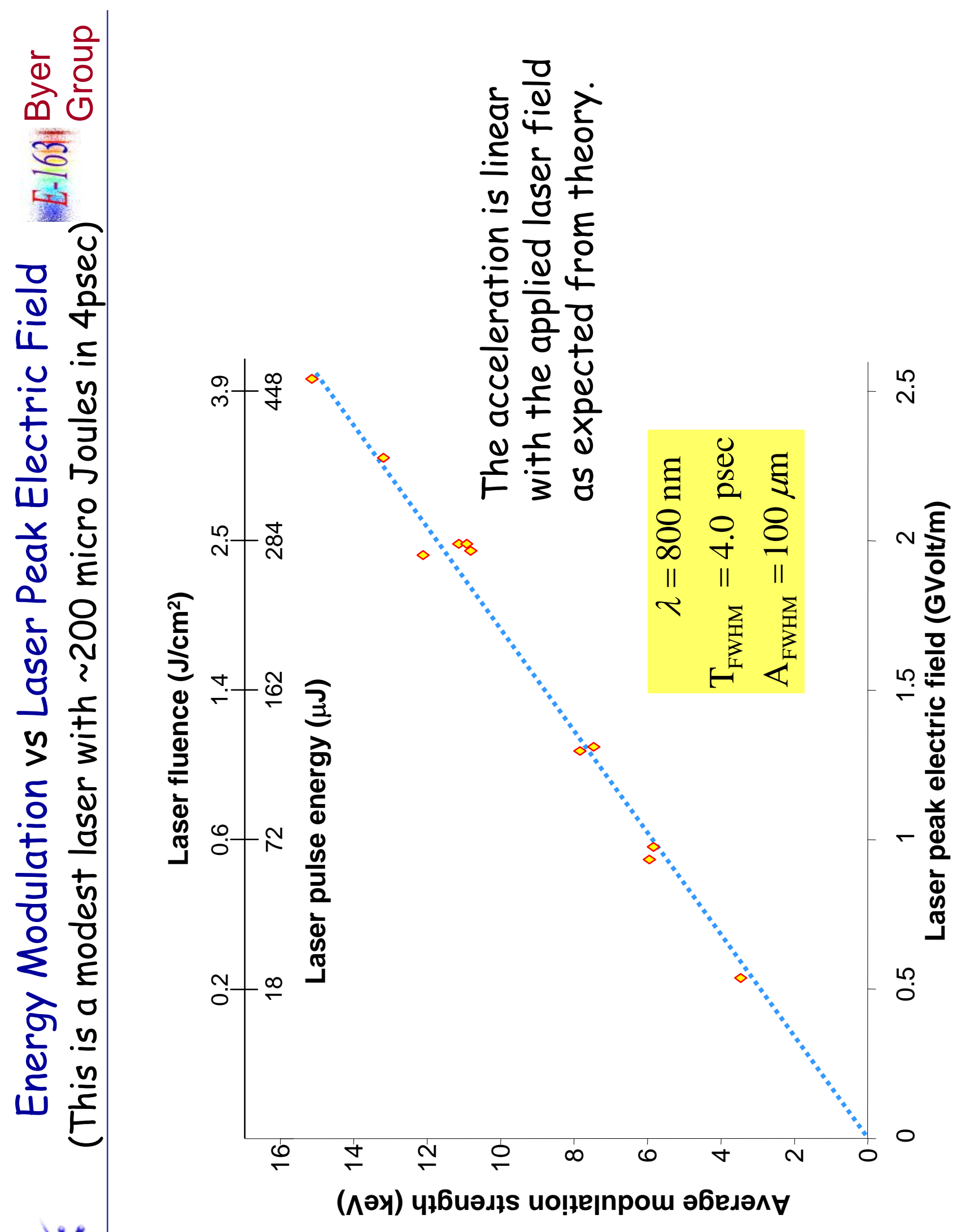




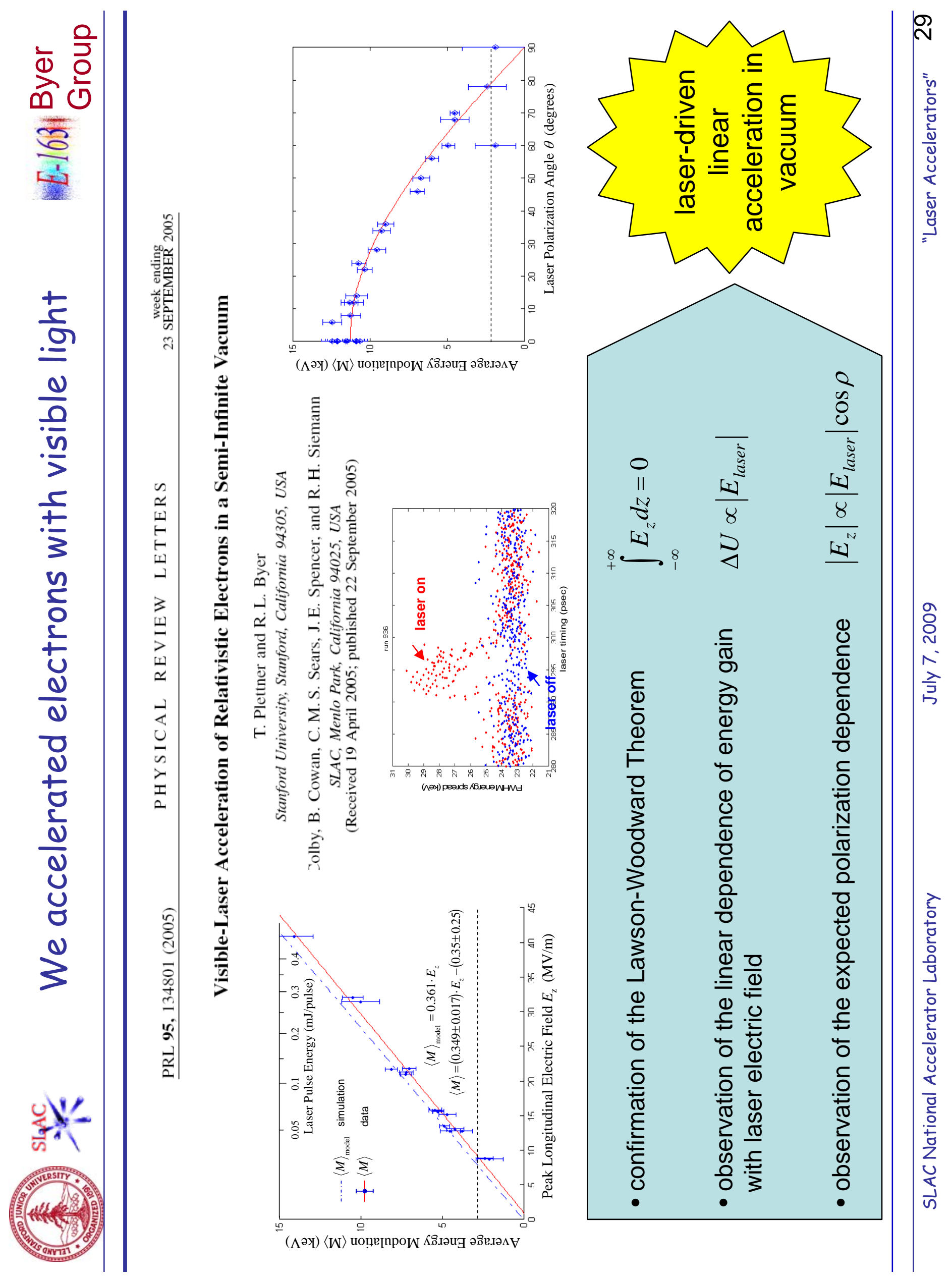




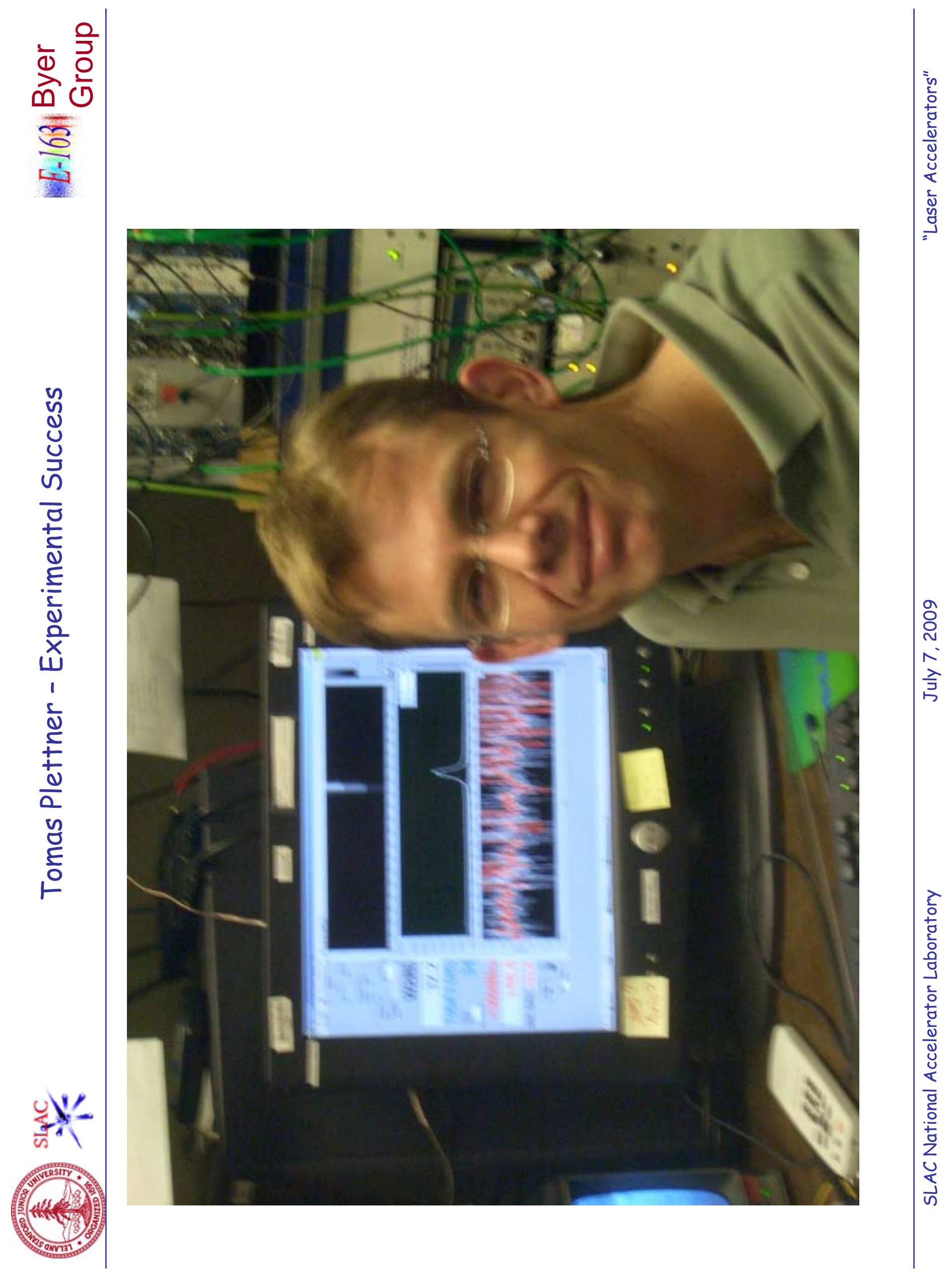



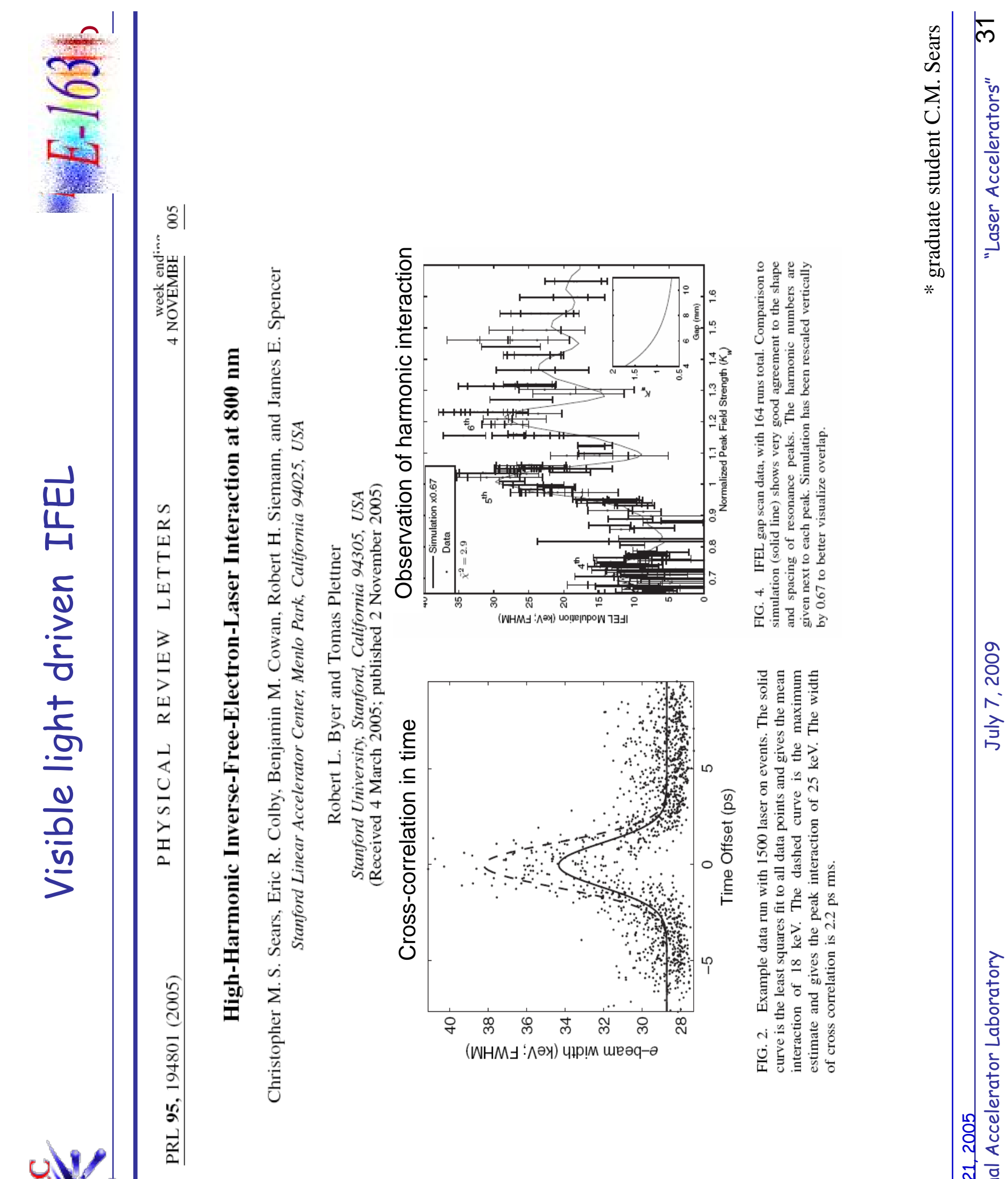


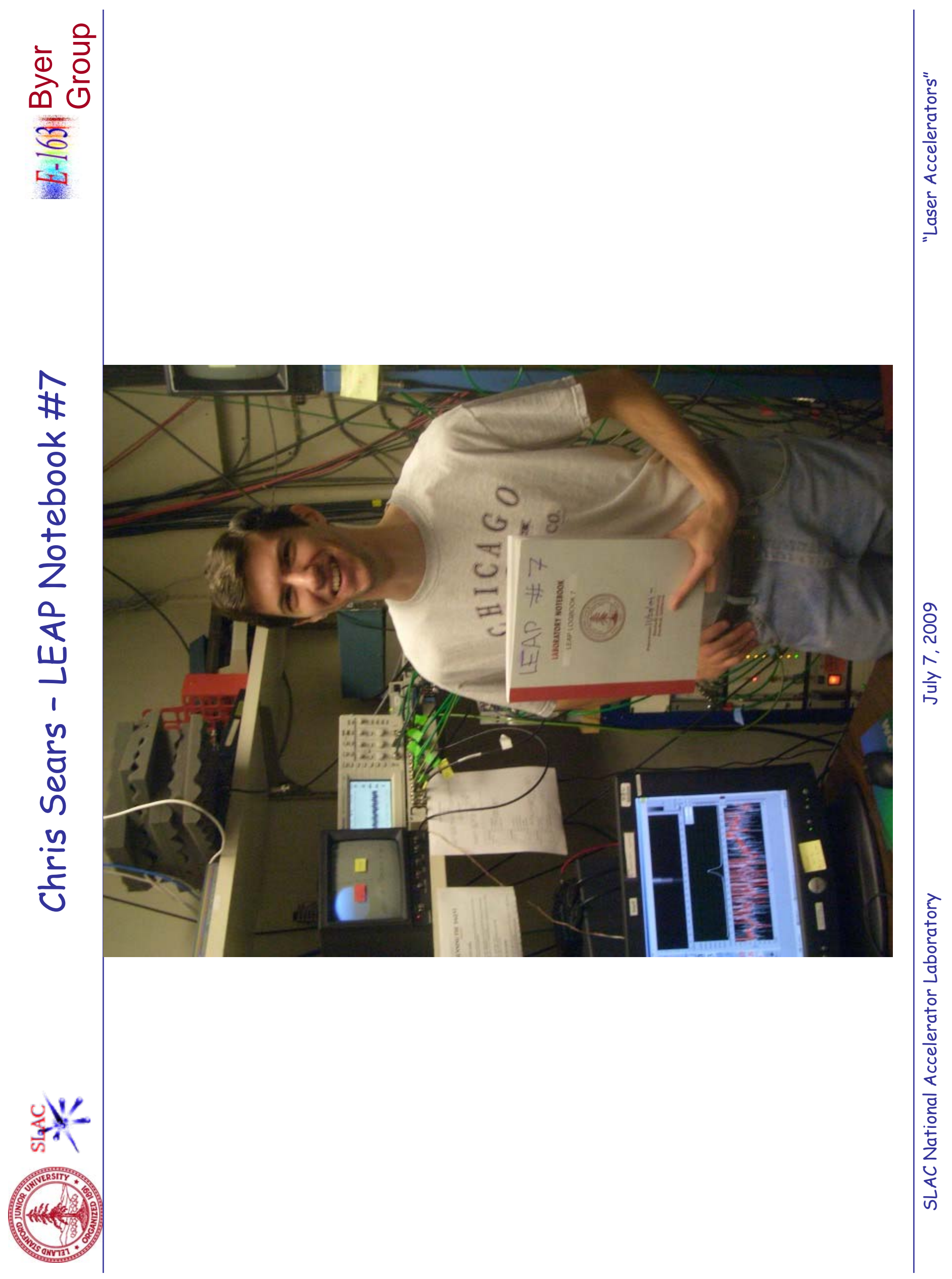




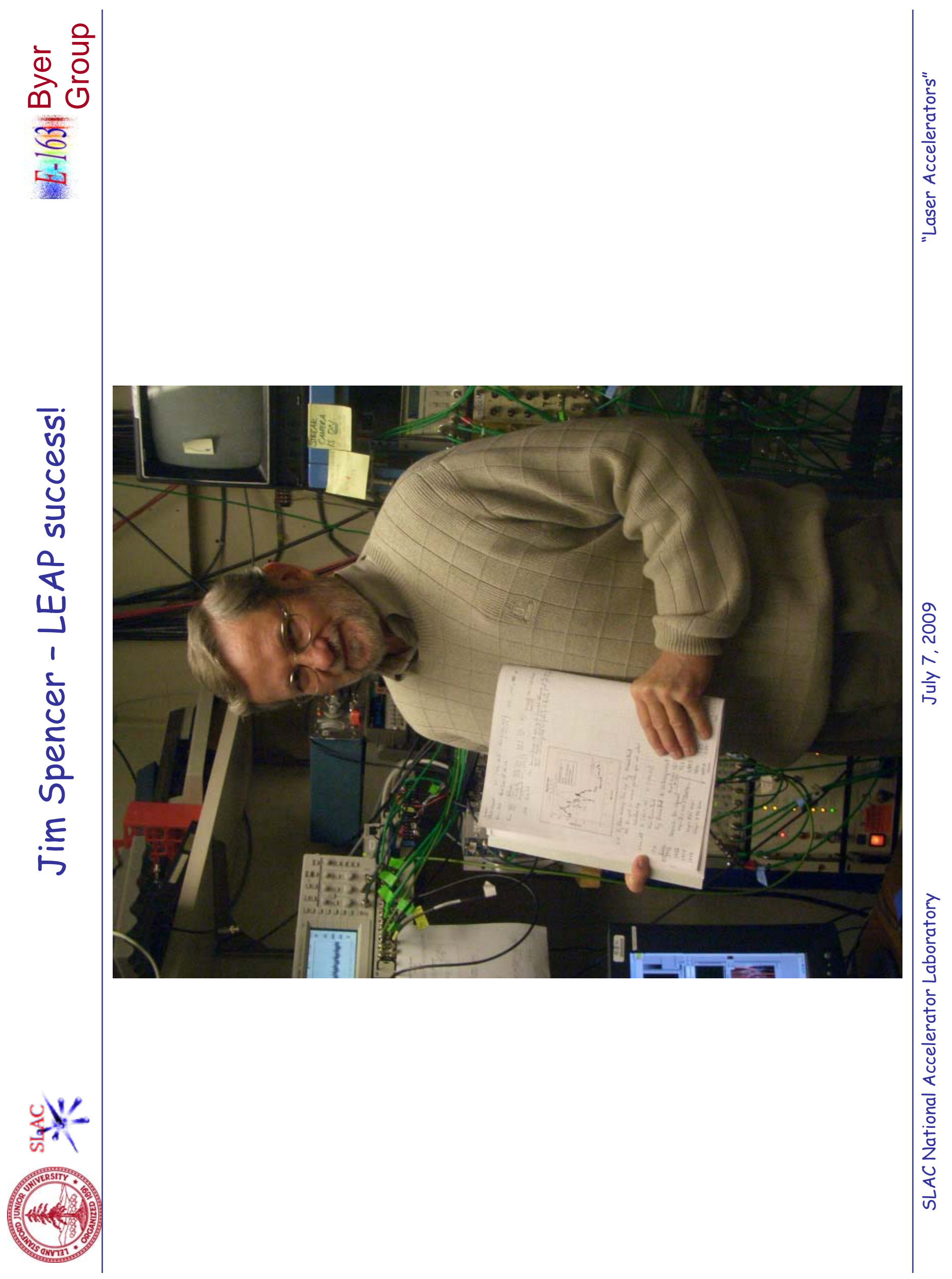




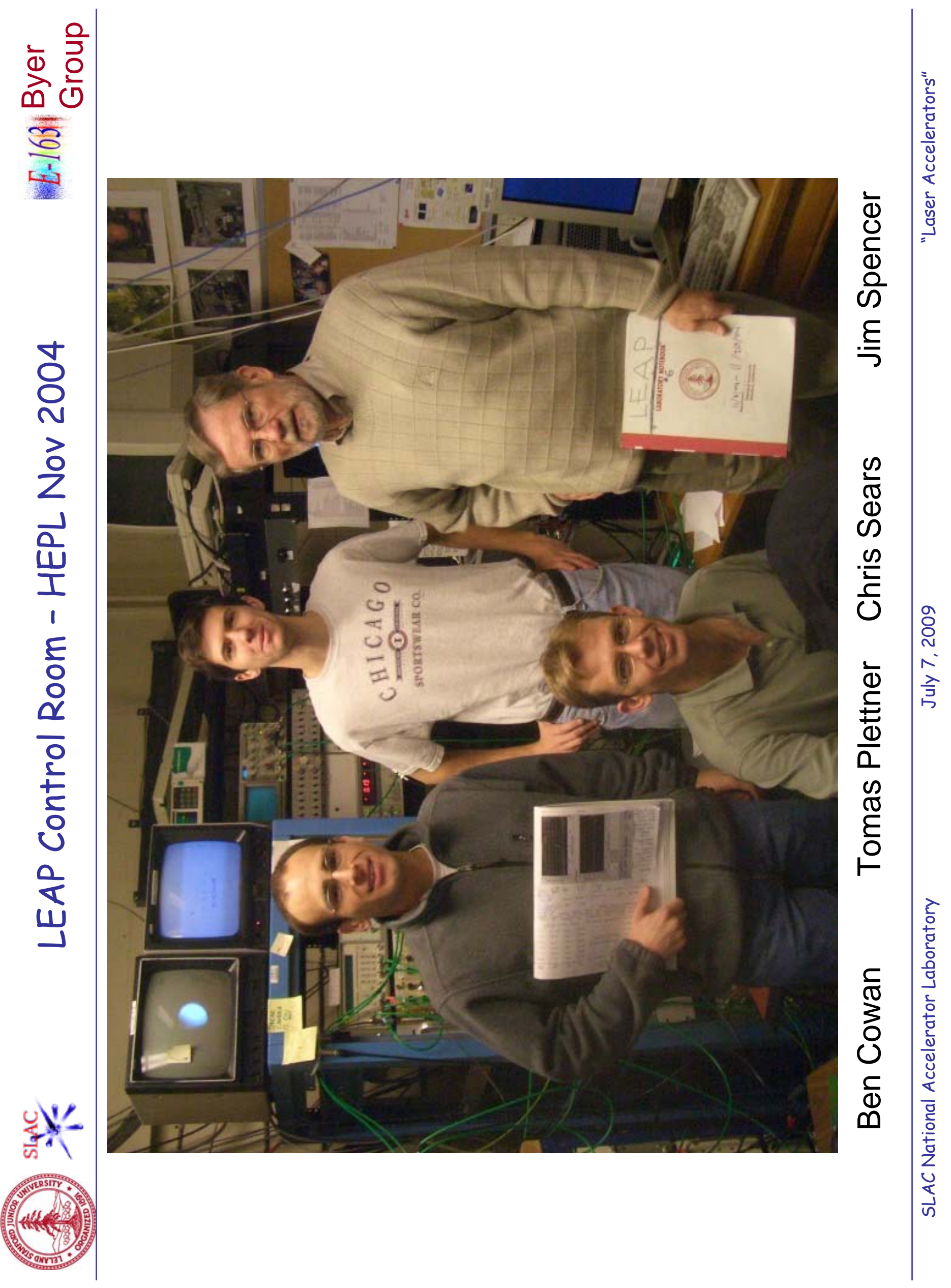




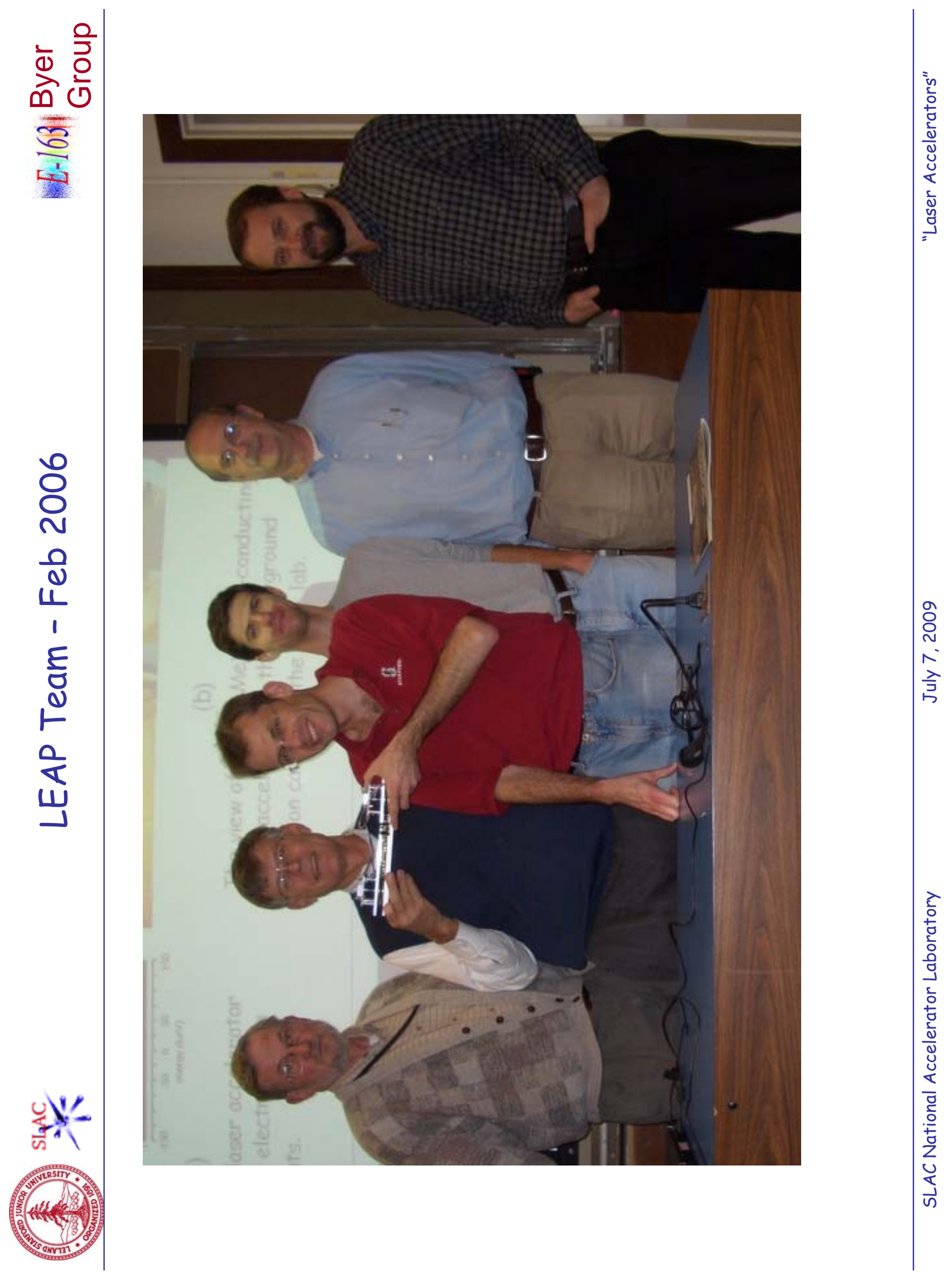




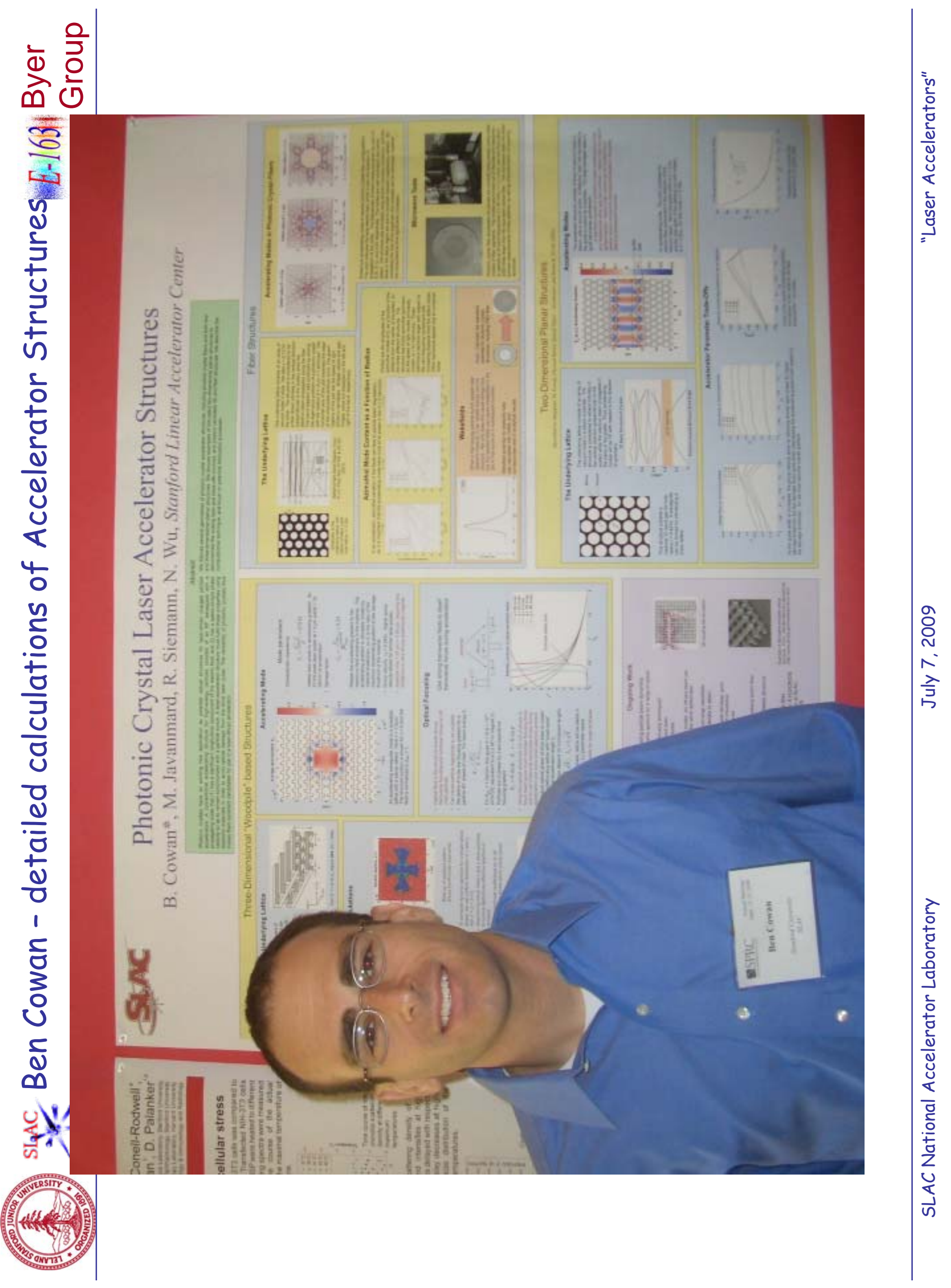




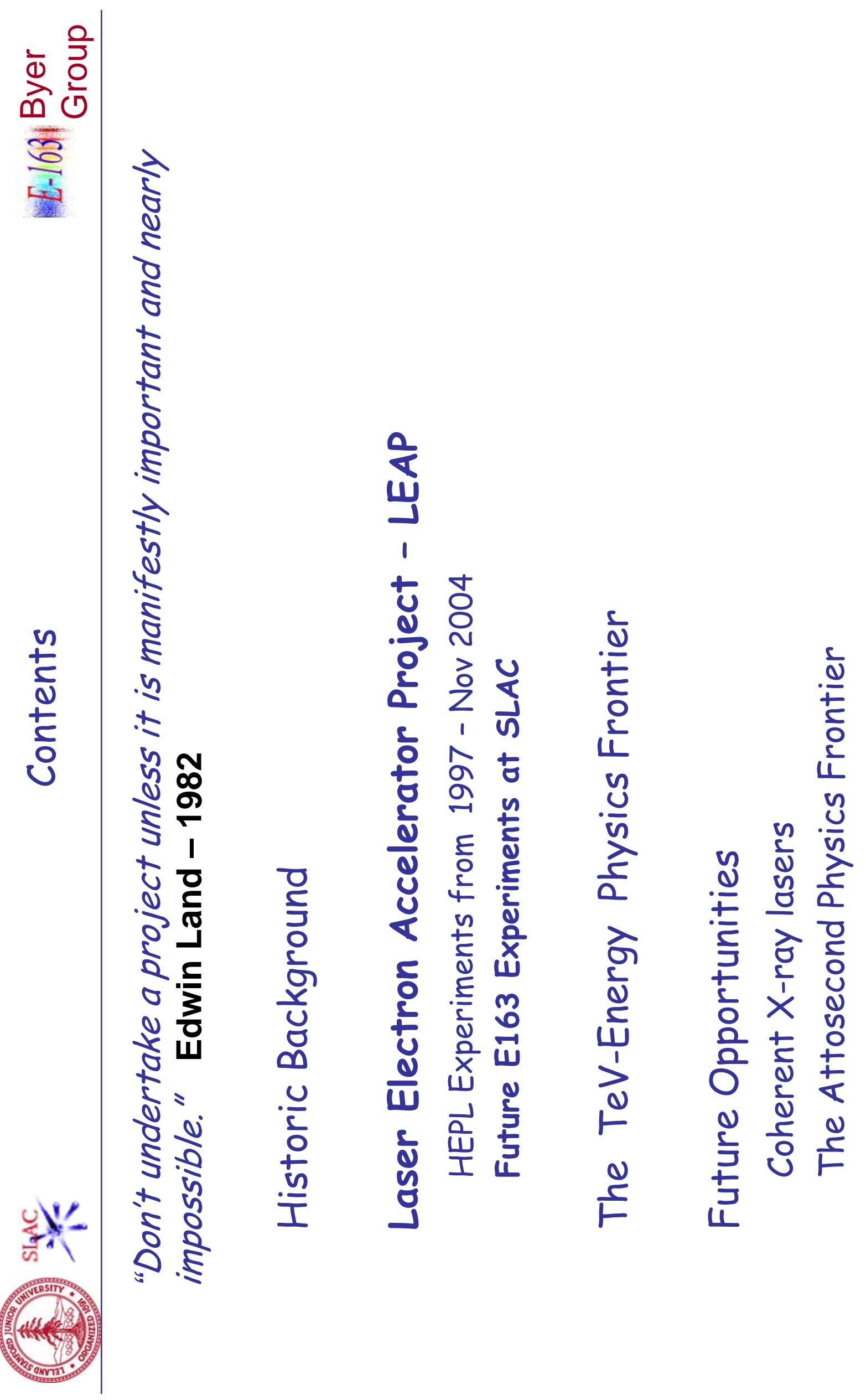




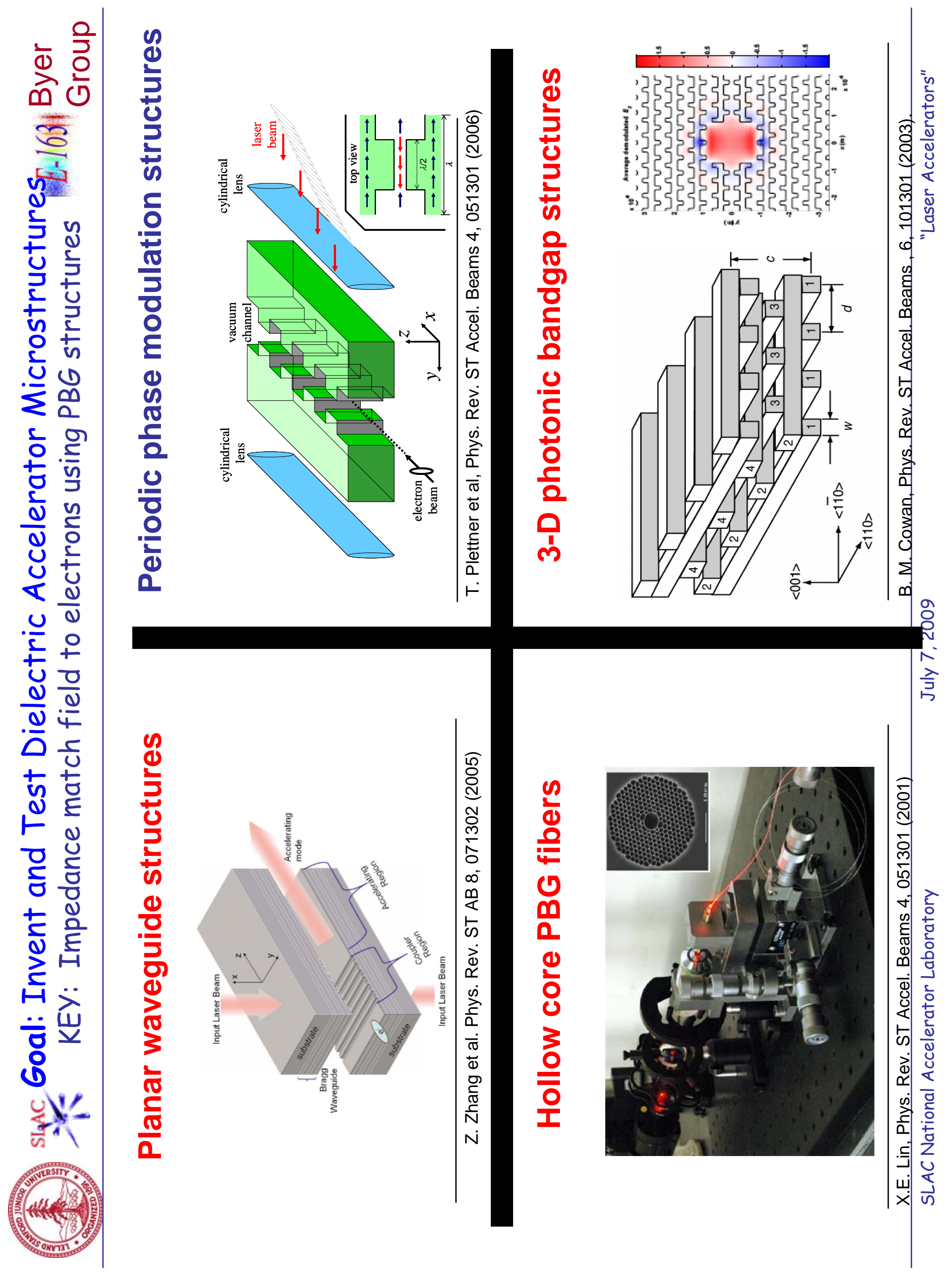




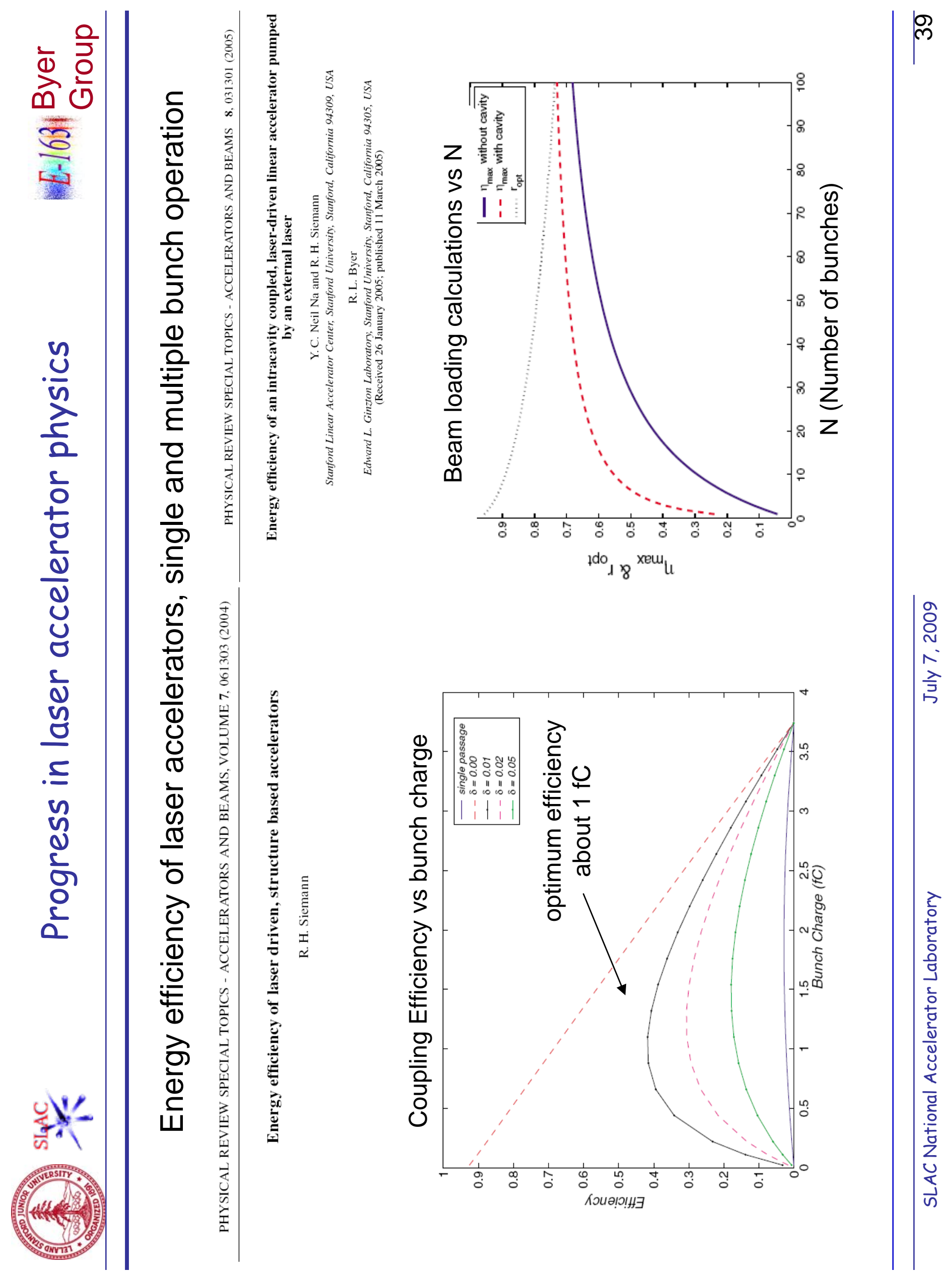




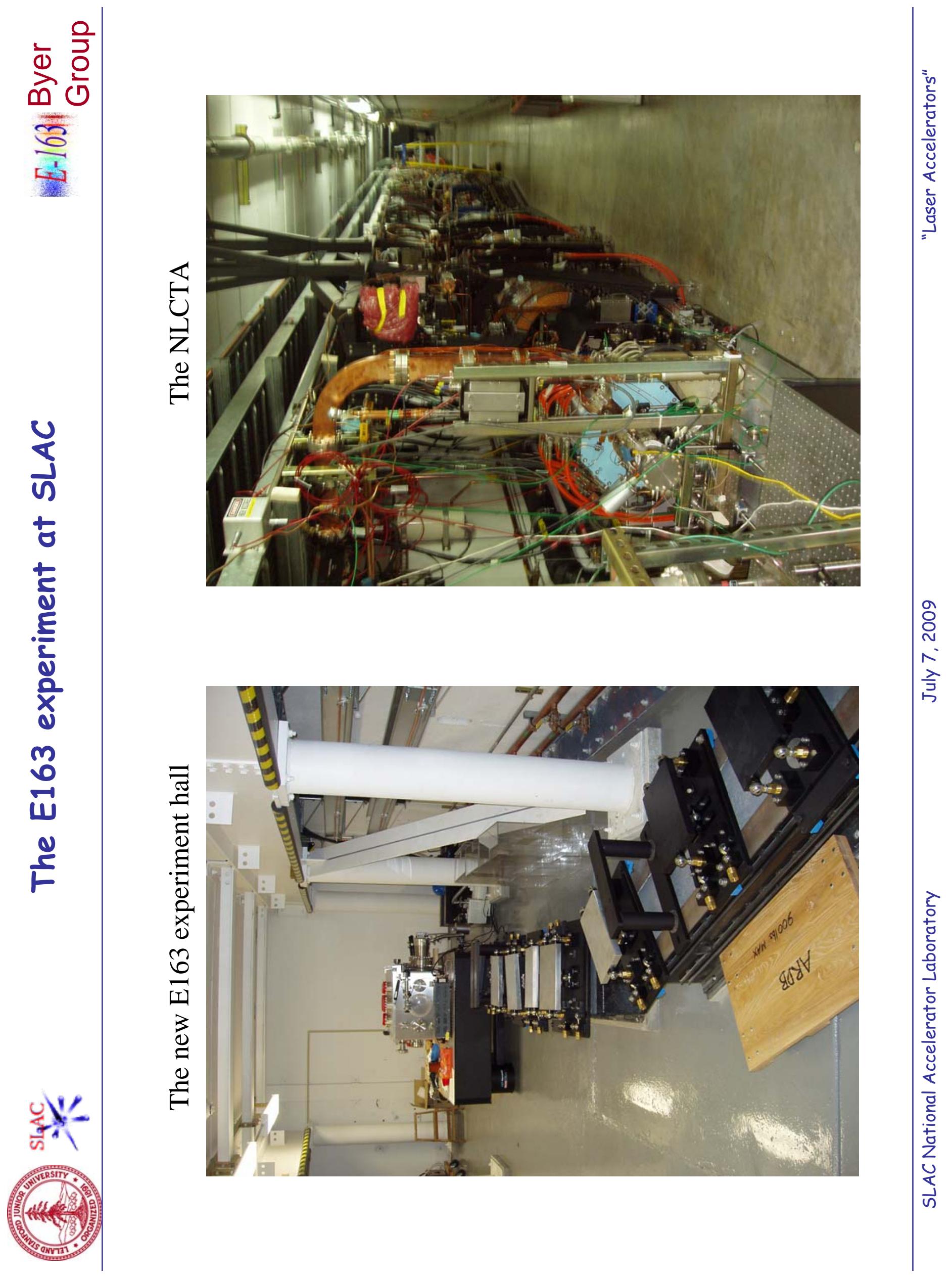




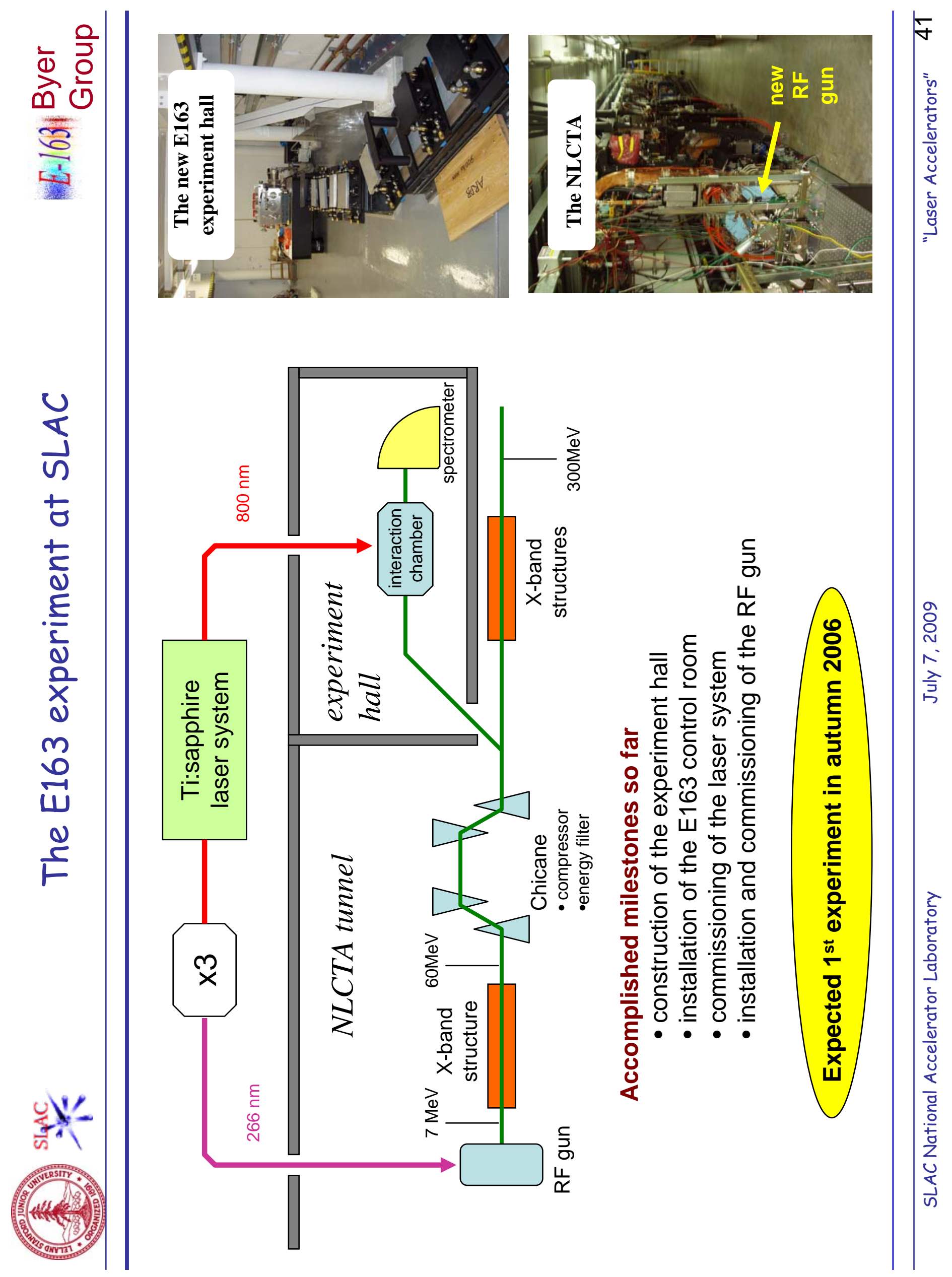




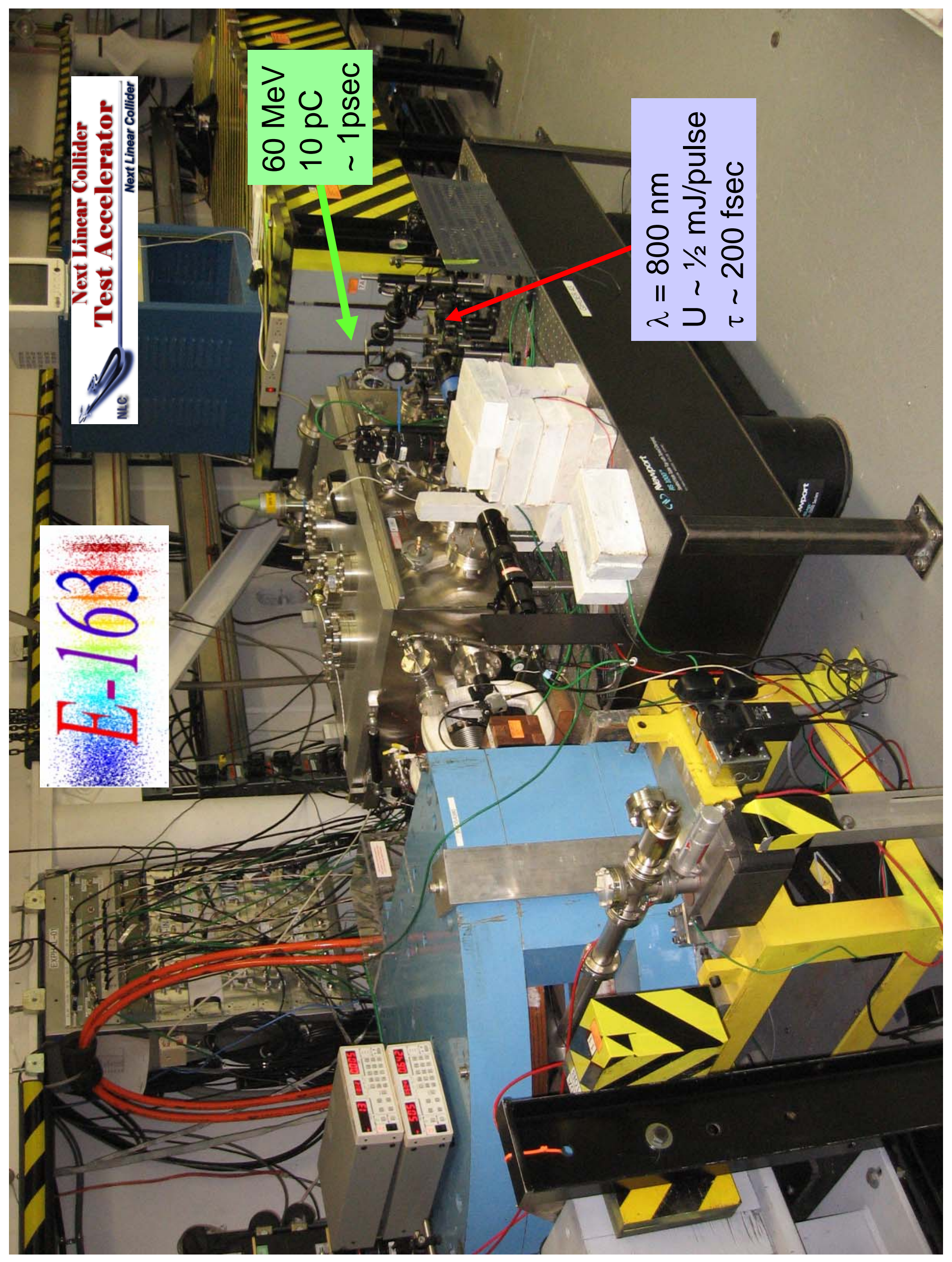




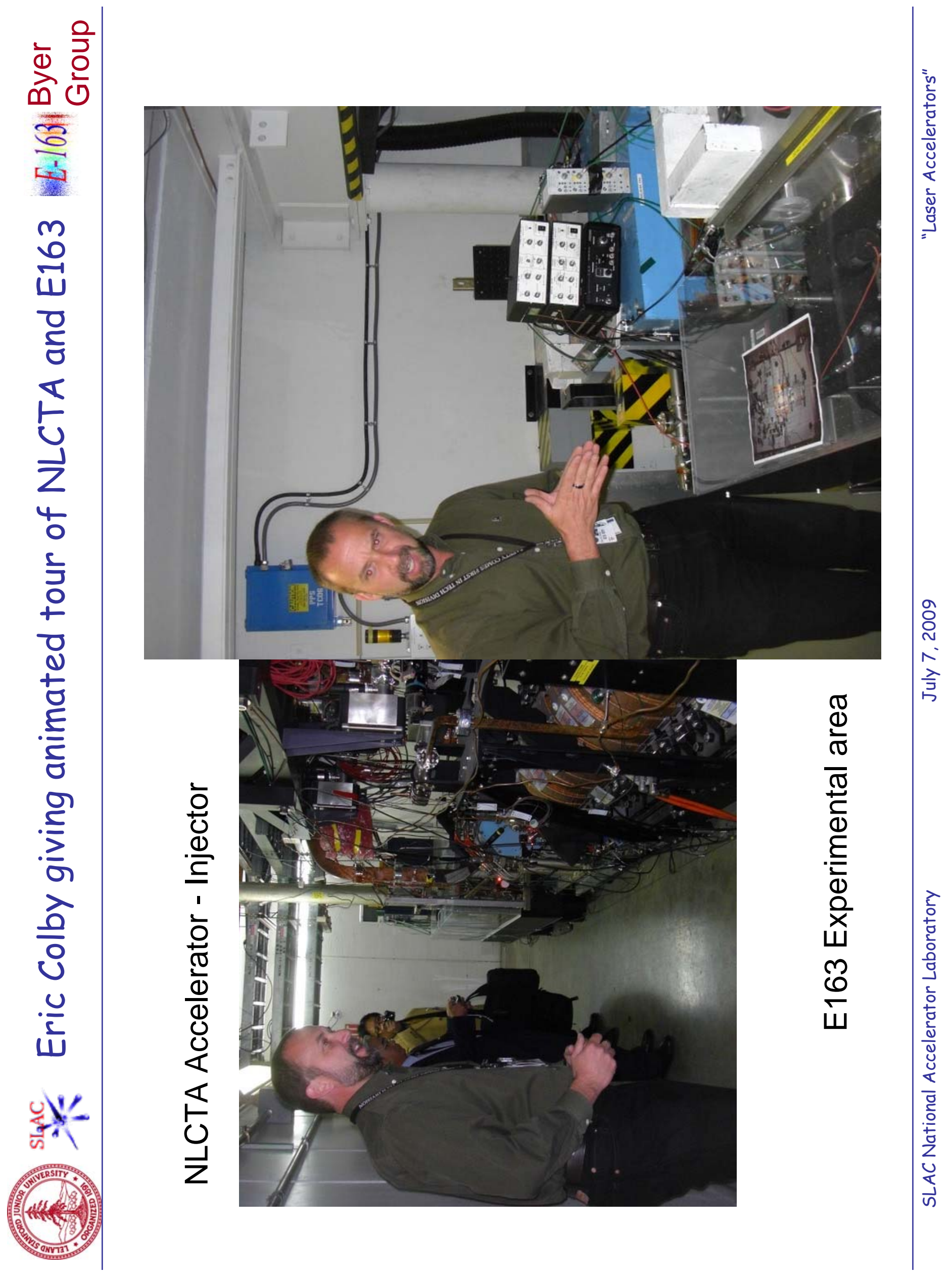




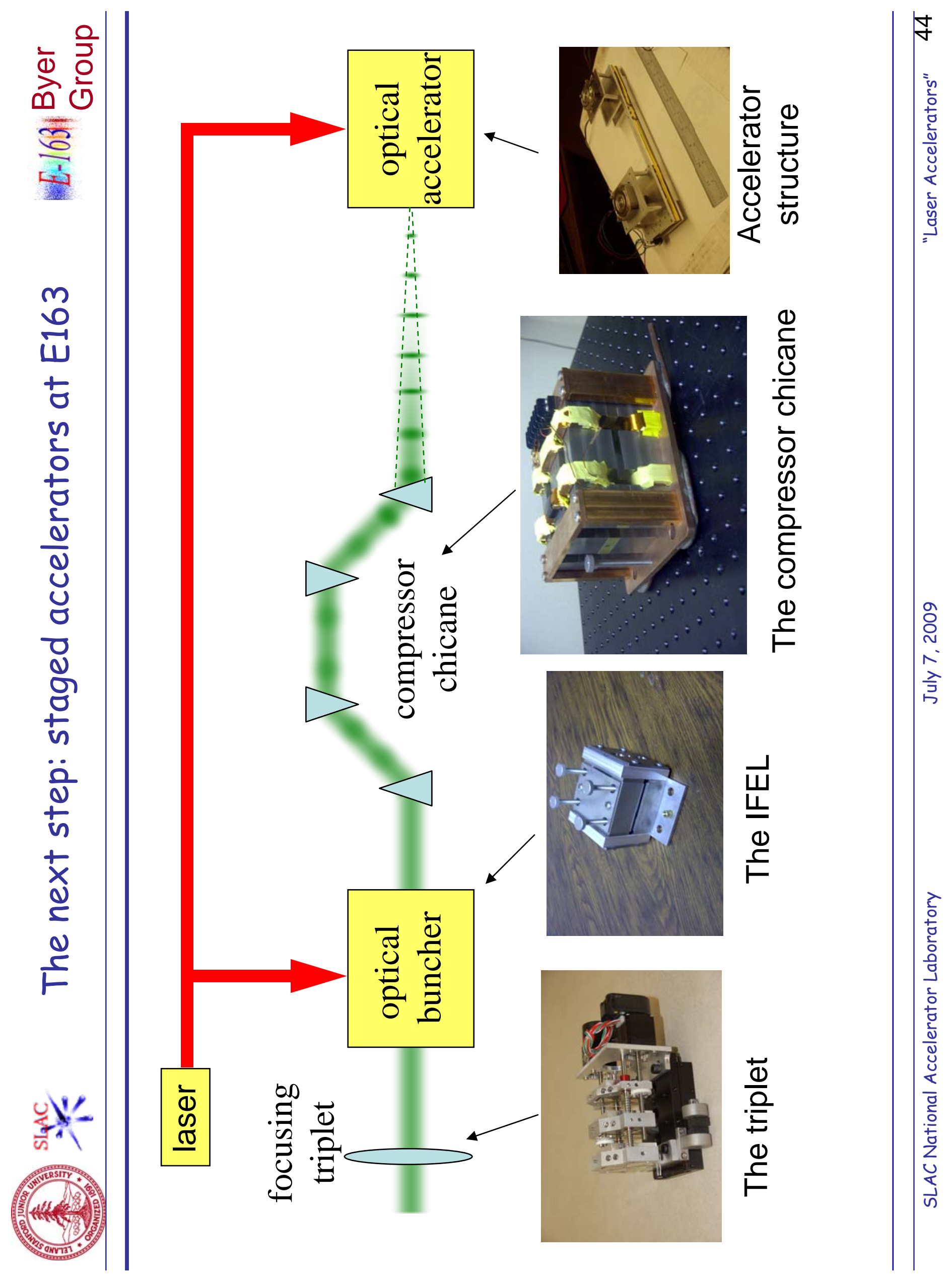




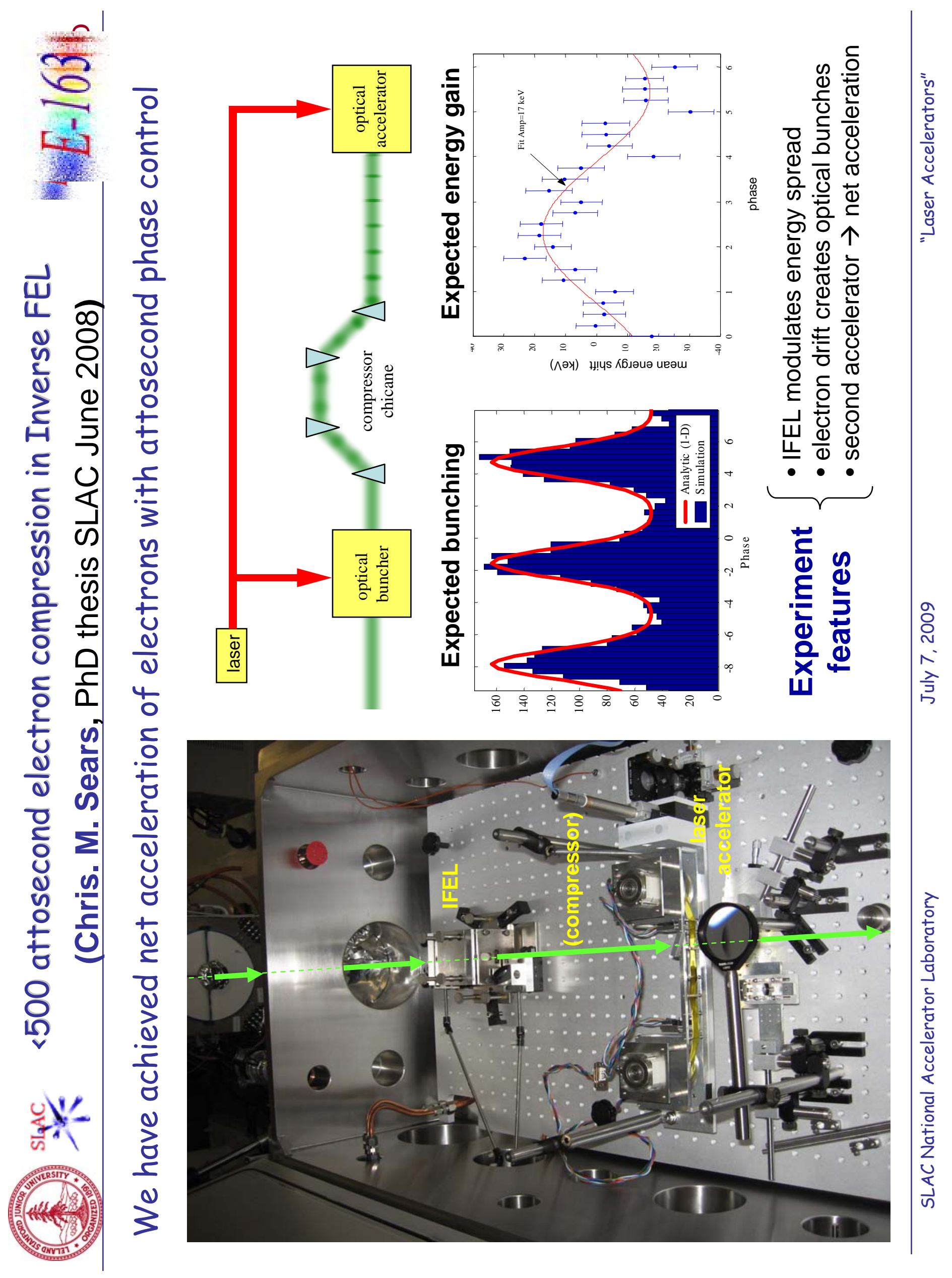




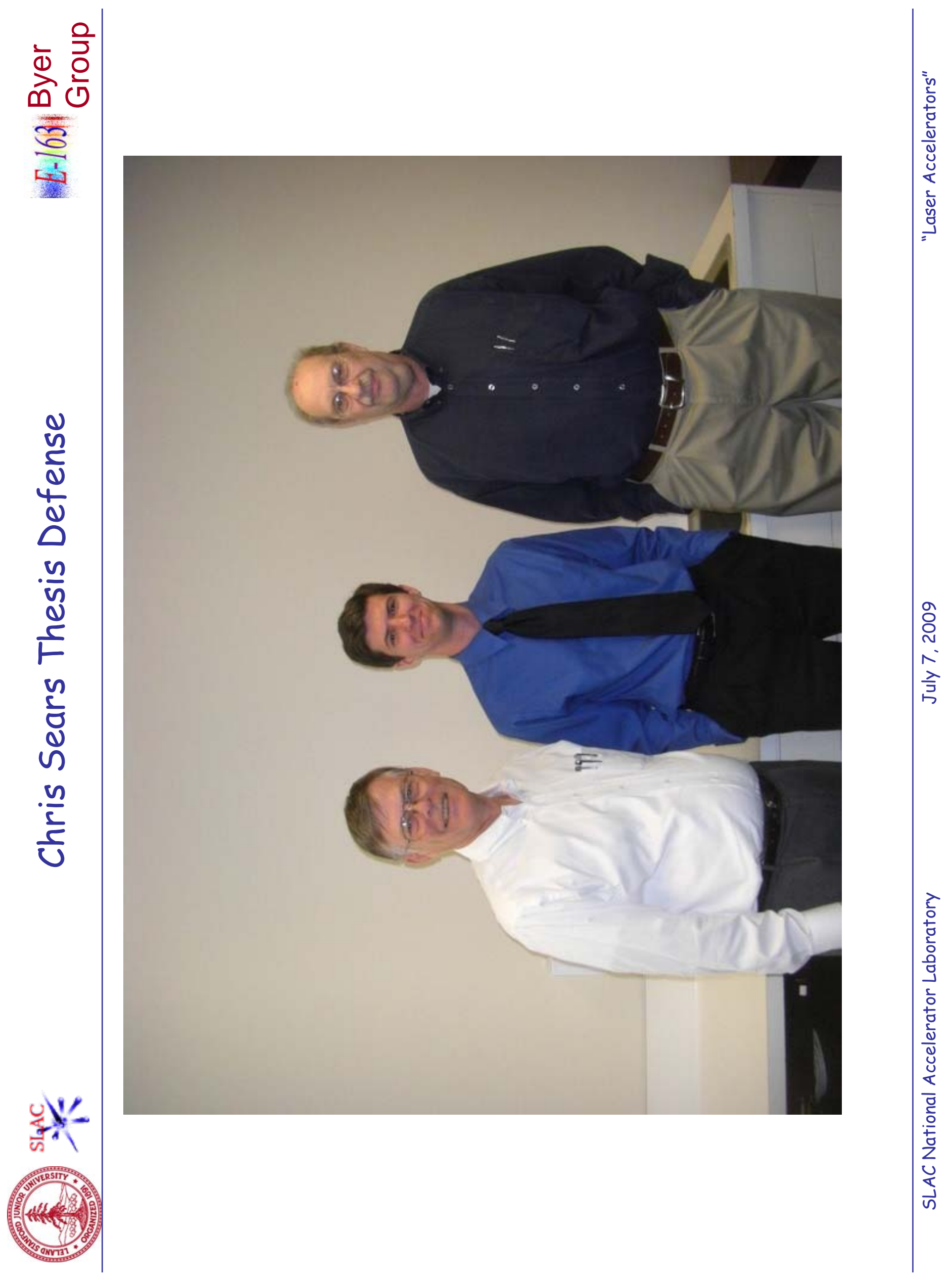



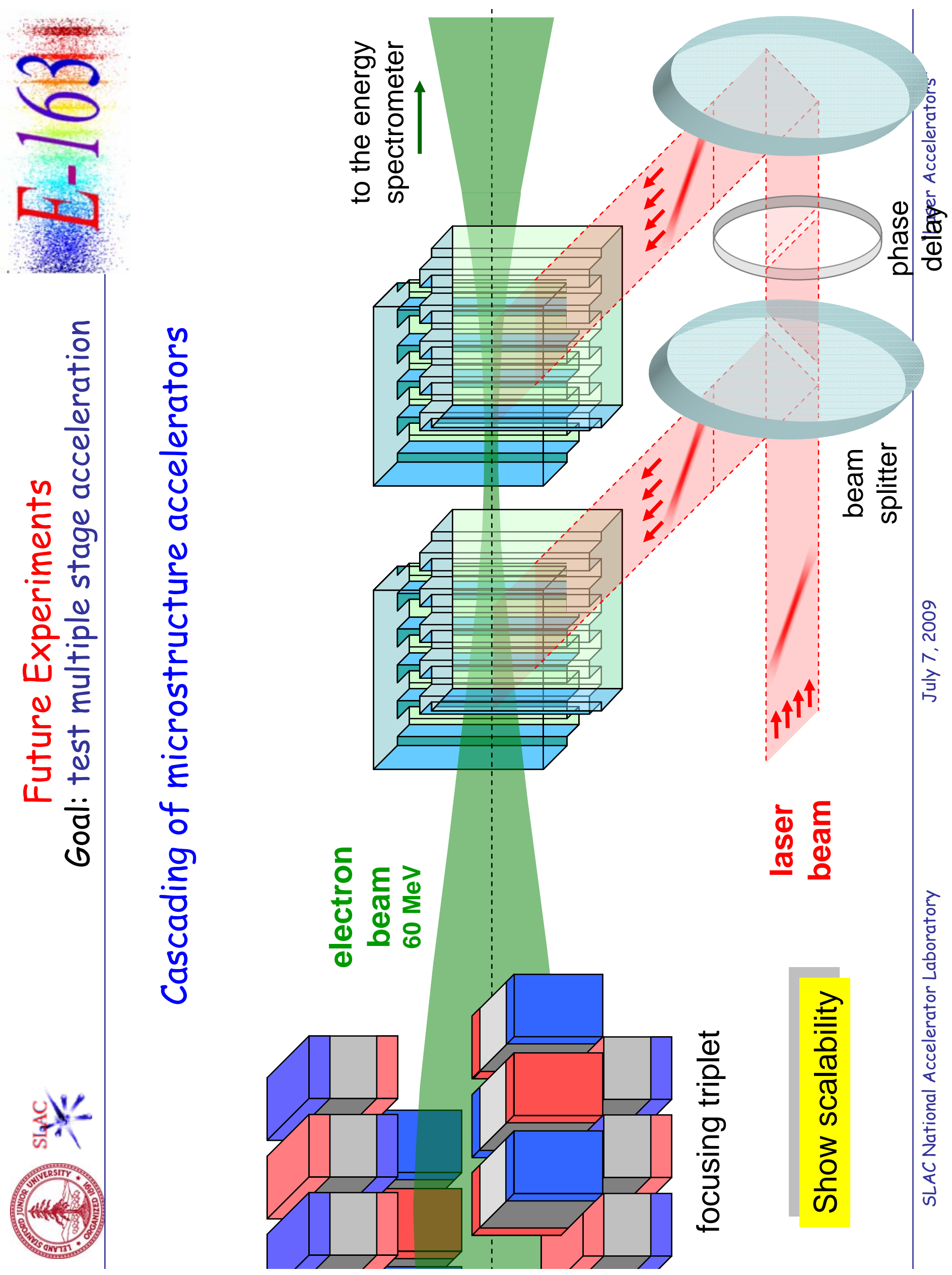


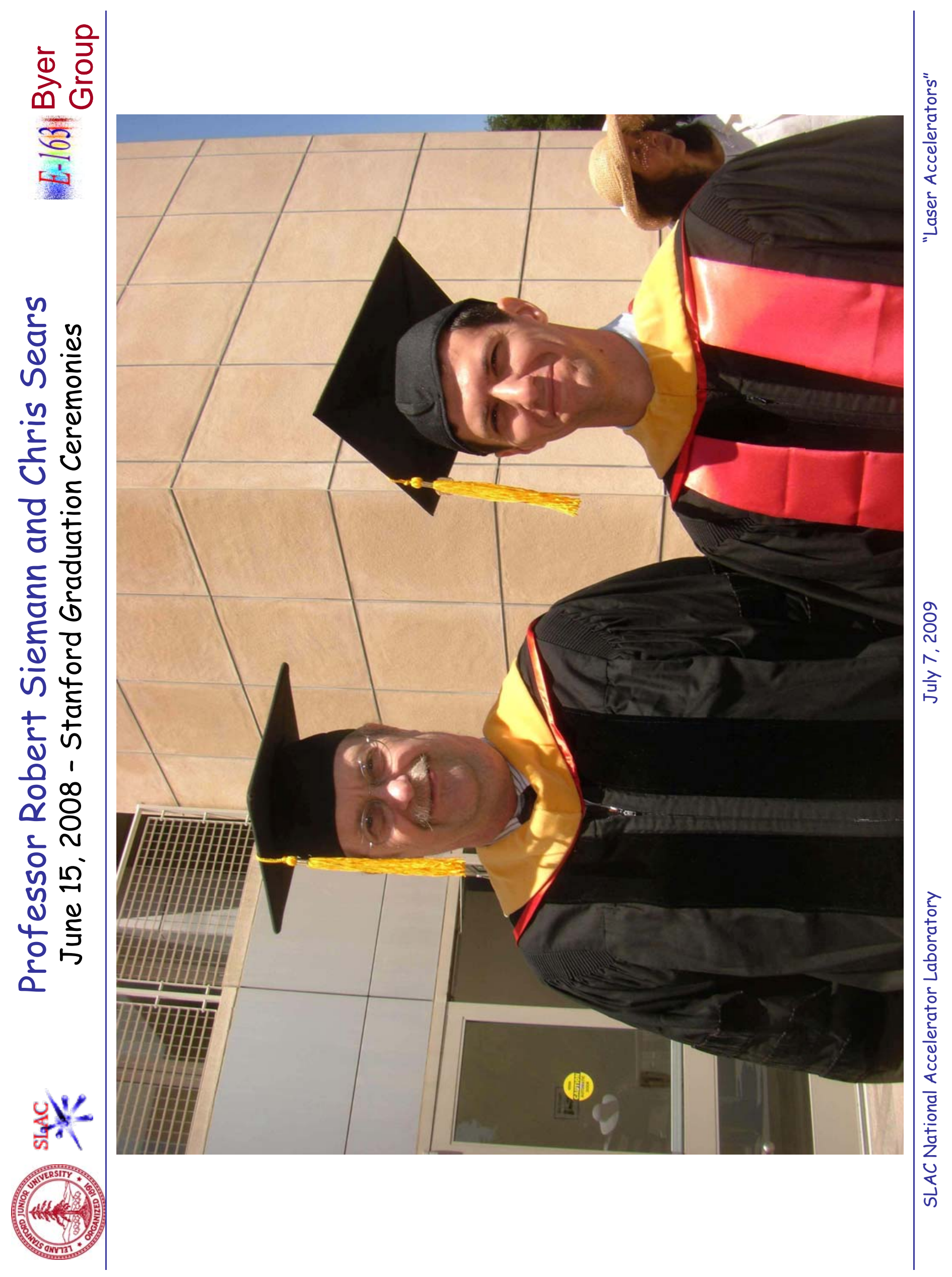




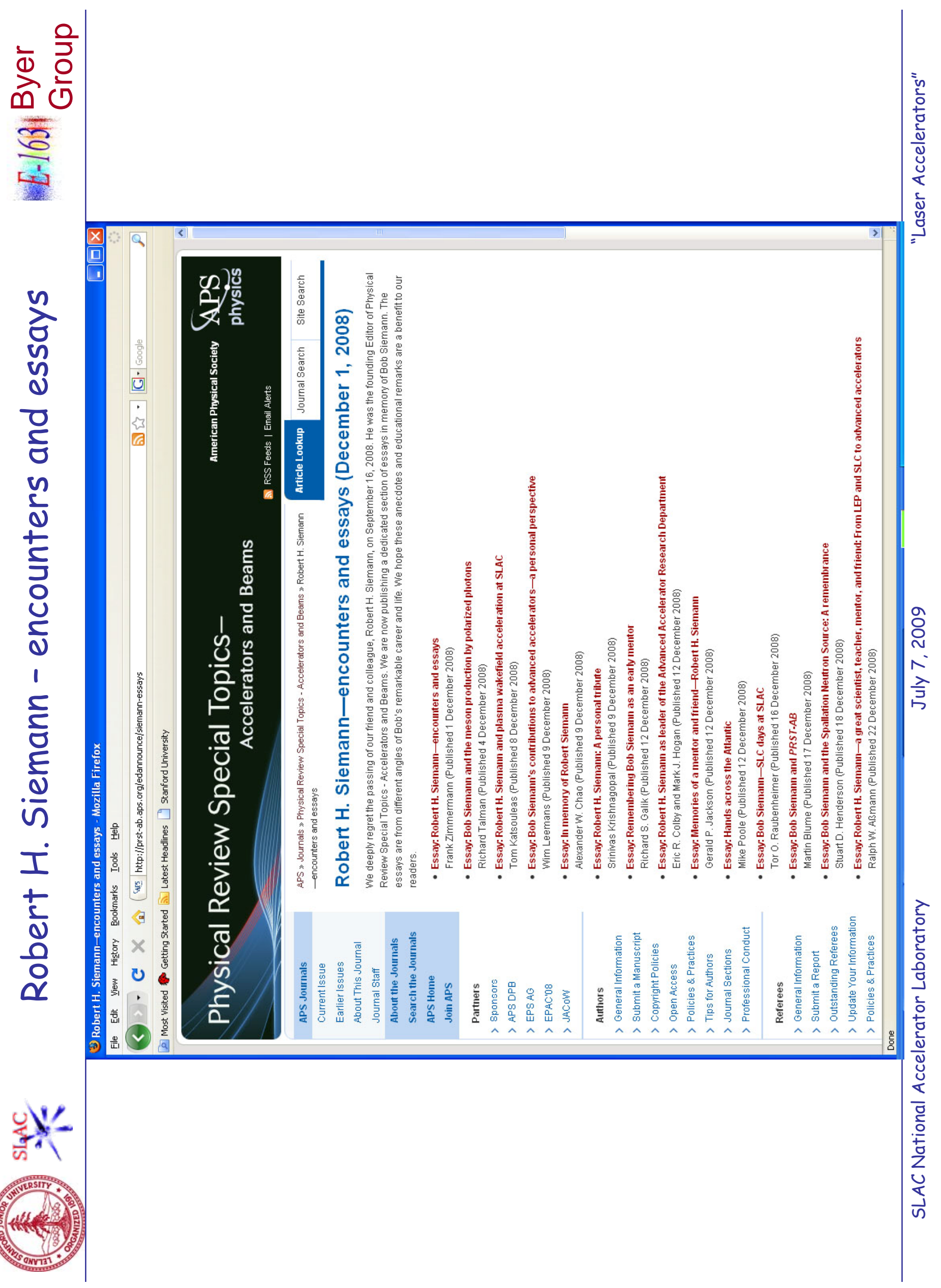




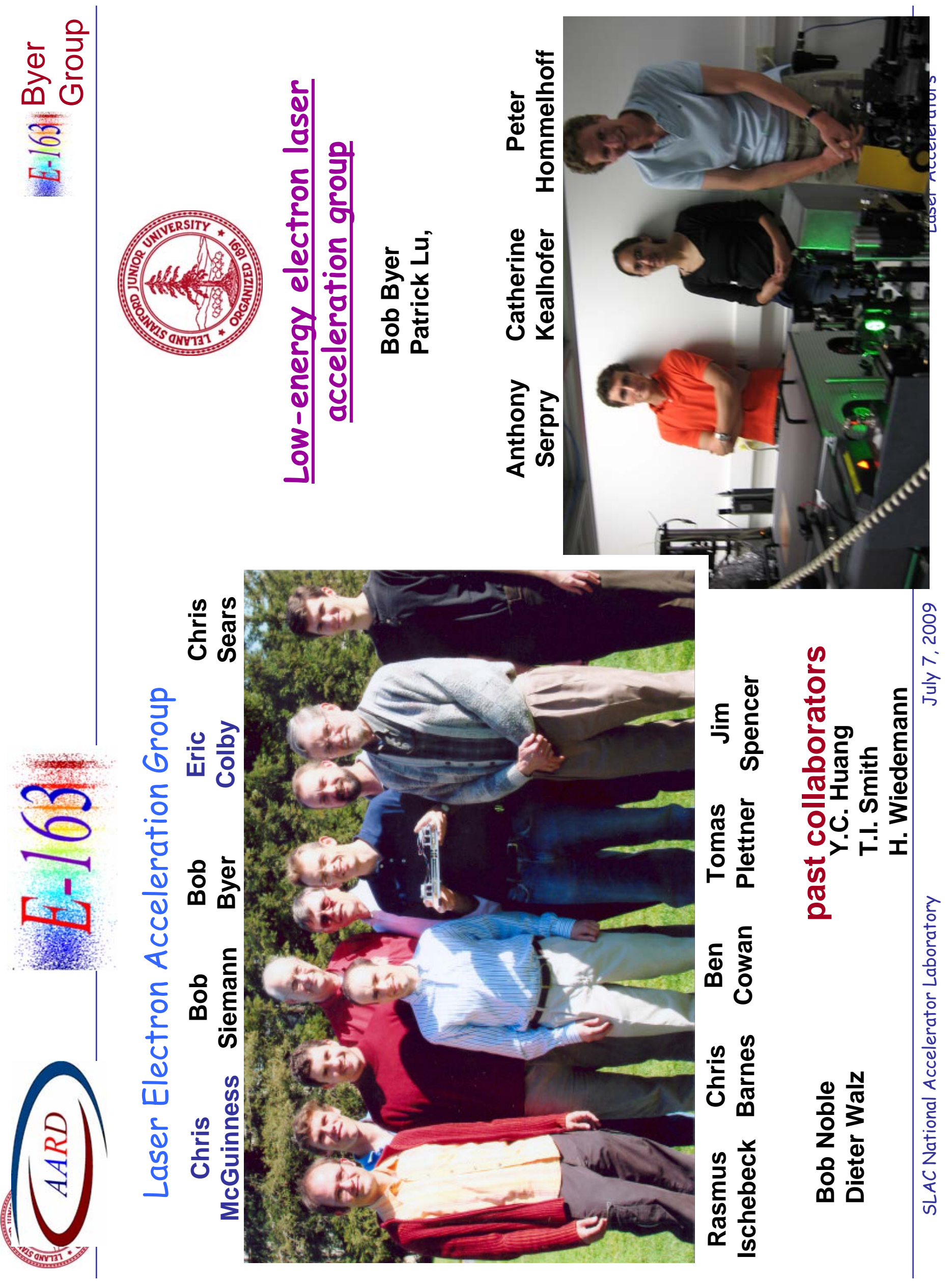




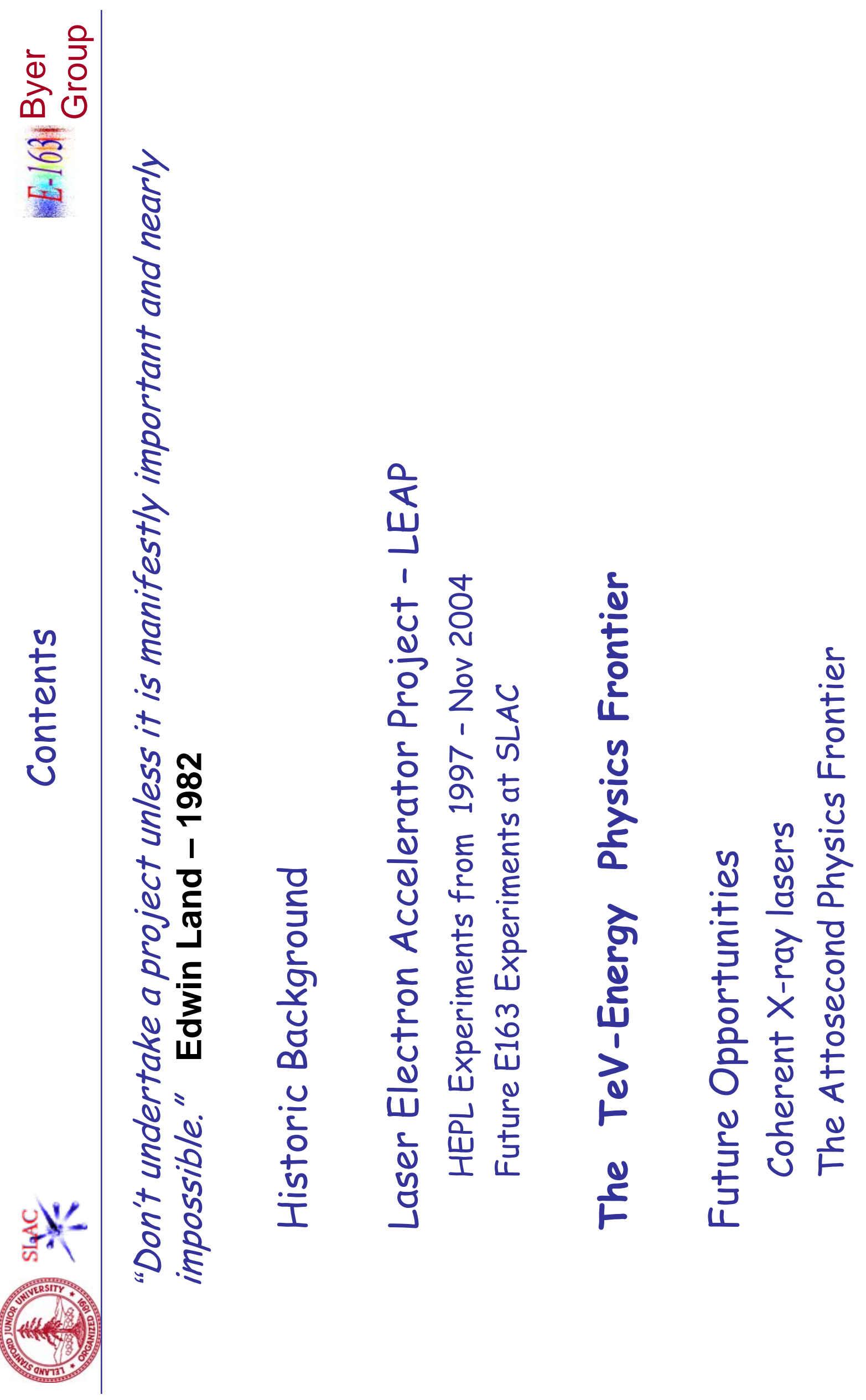




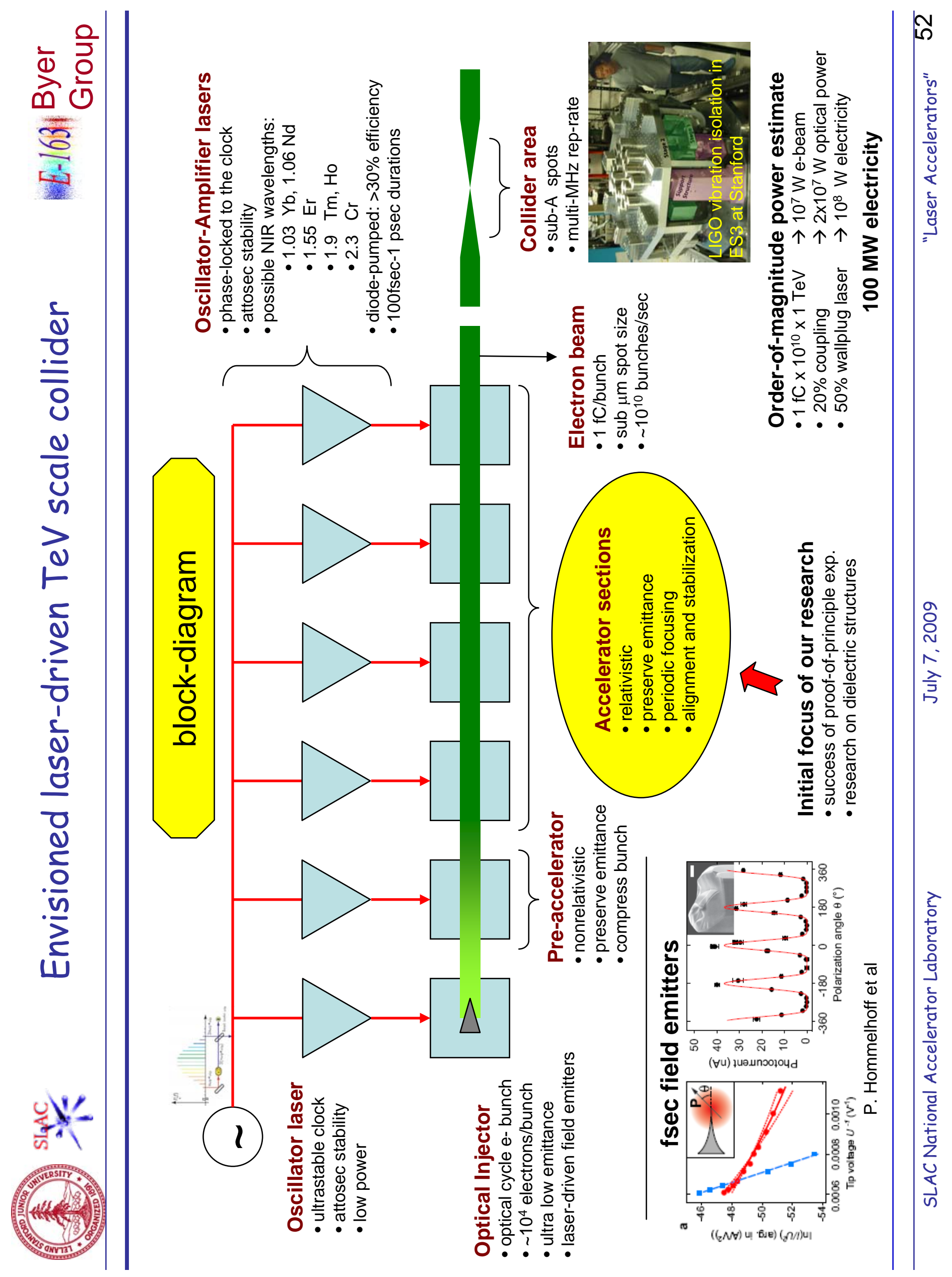




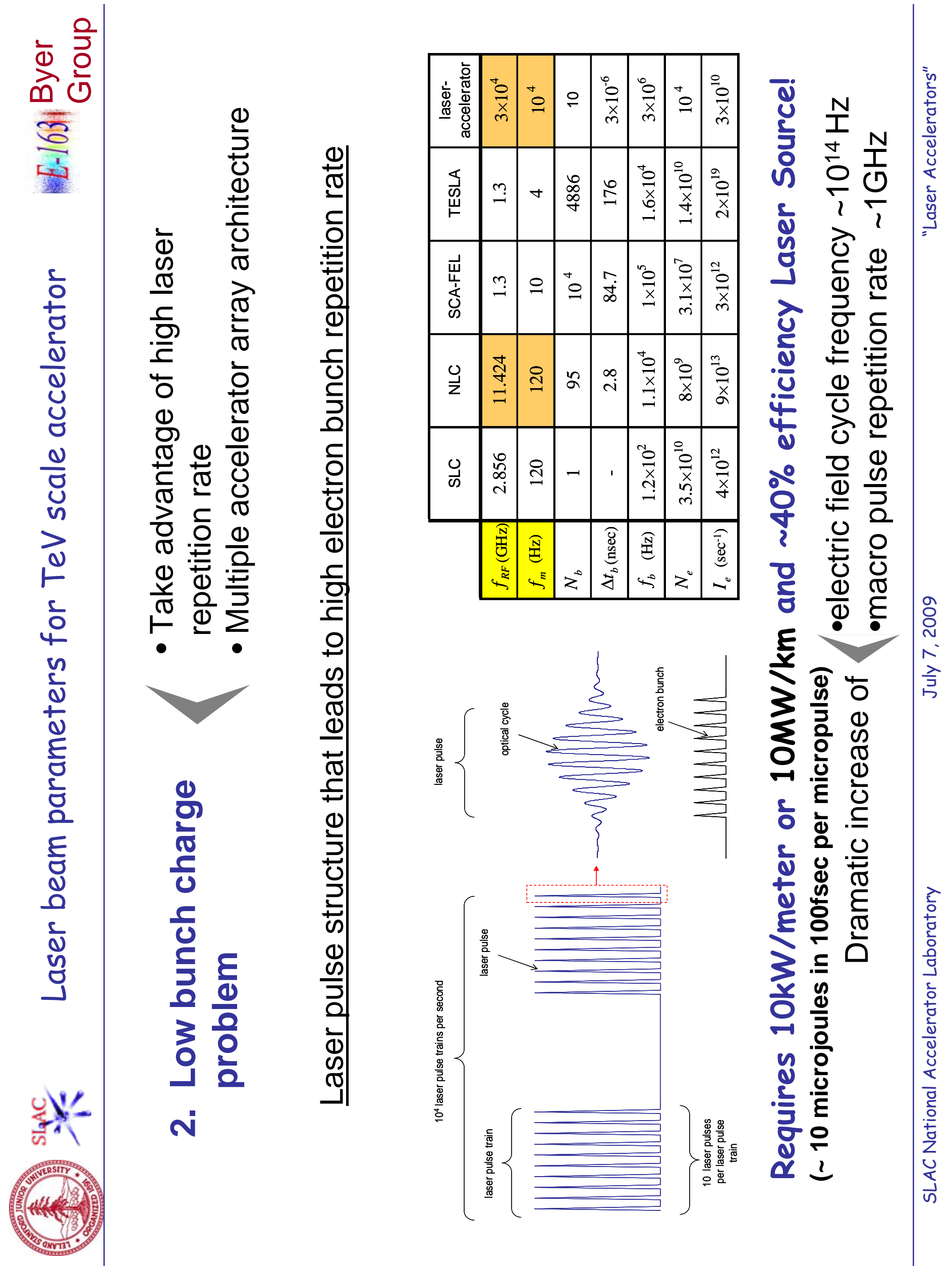




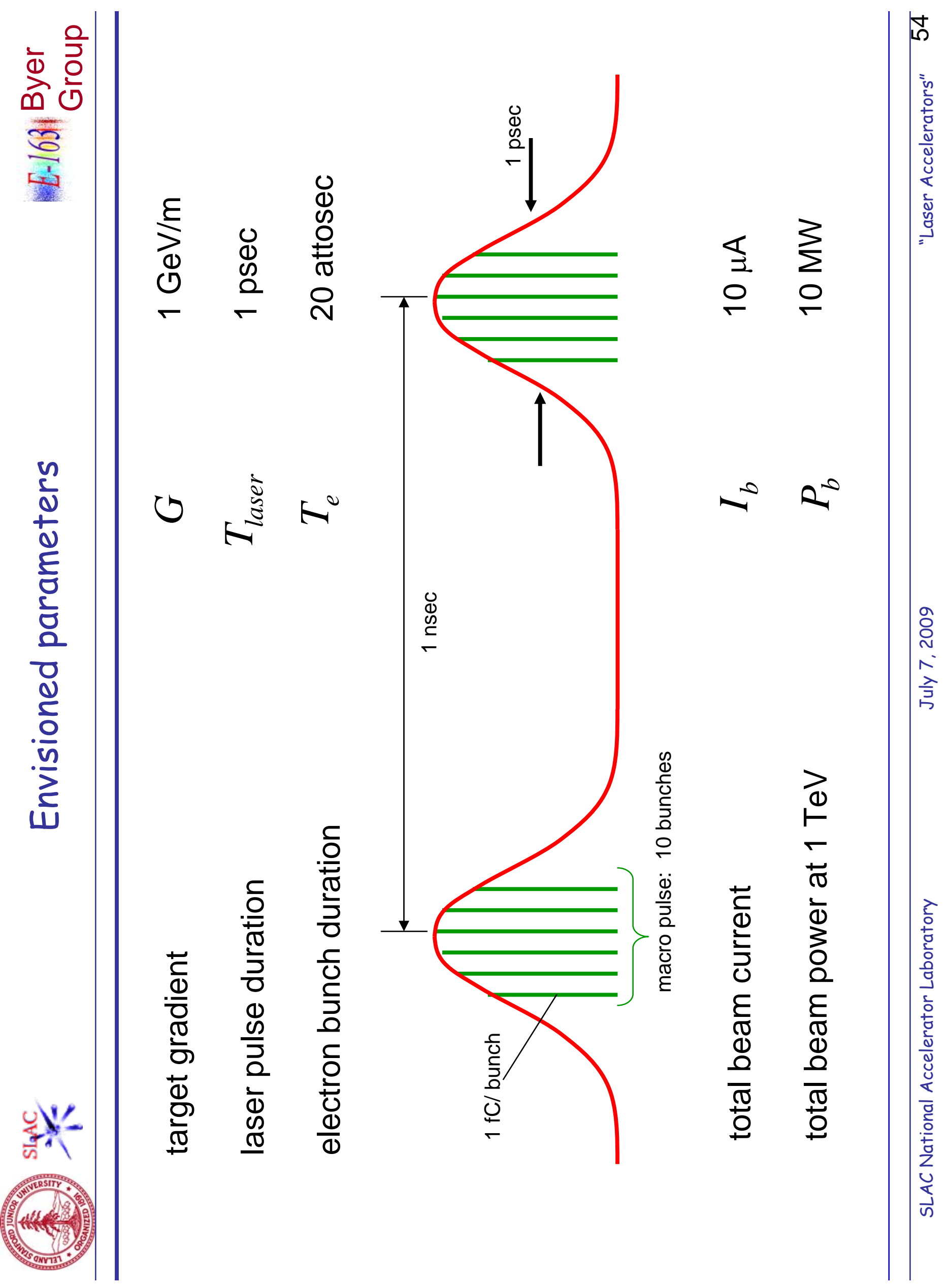



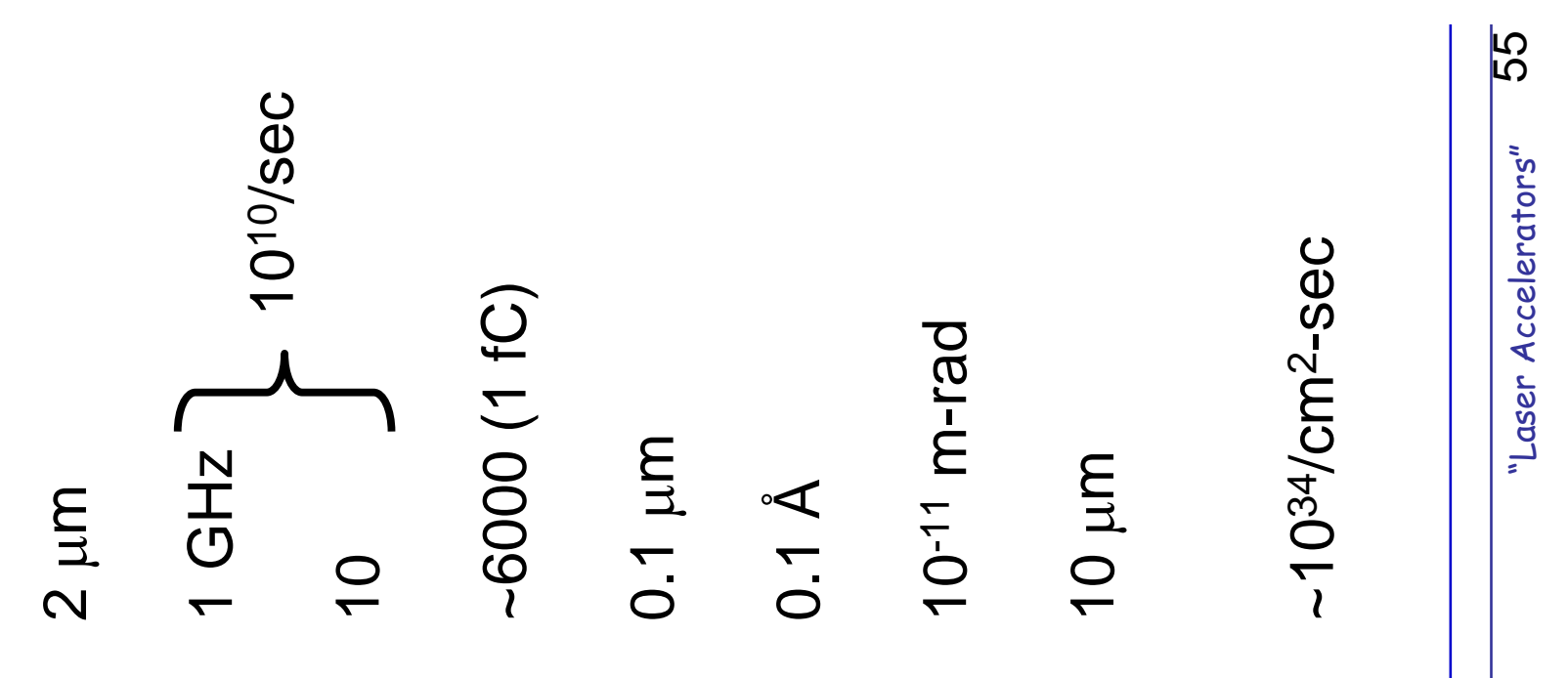

$\frac{v}{U}$
$\frac{v}{\sigma}$
$\frac{\sigma}{\sigma}$
$\frac{\varepsilon}{\sigma}$

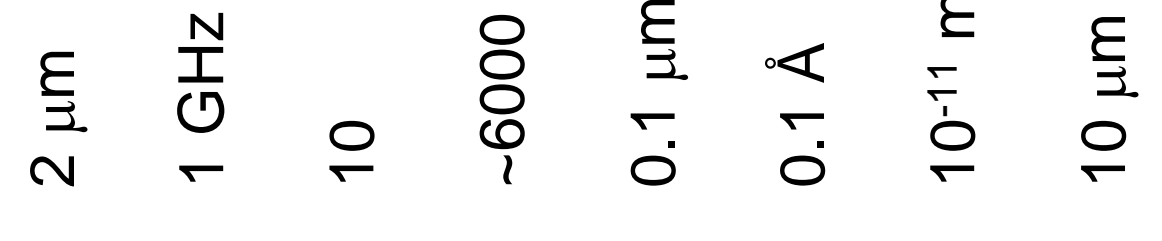

$₹+z \quad b \quad b \quad \omega \quad a^{0}$
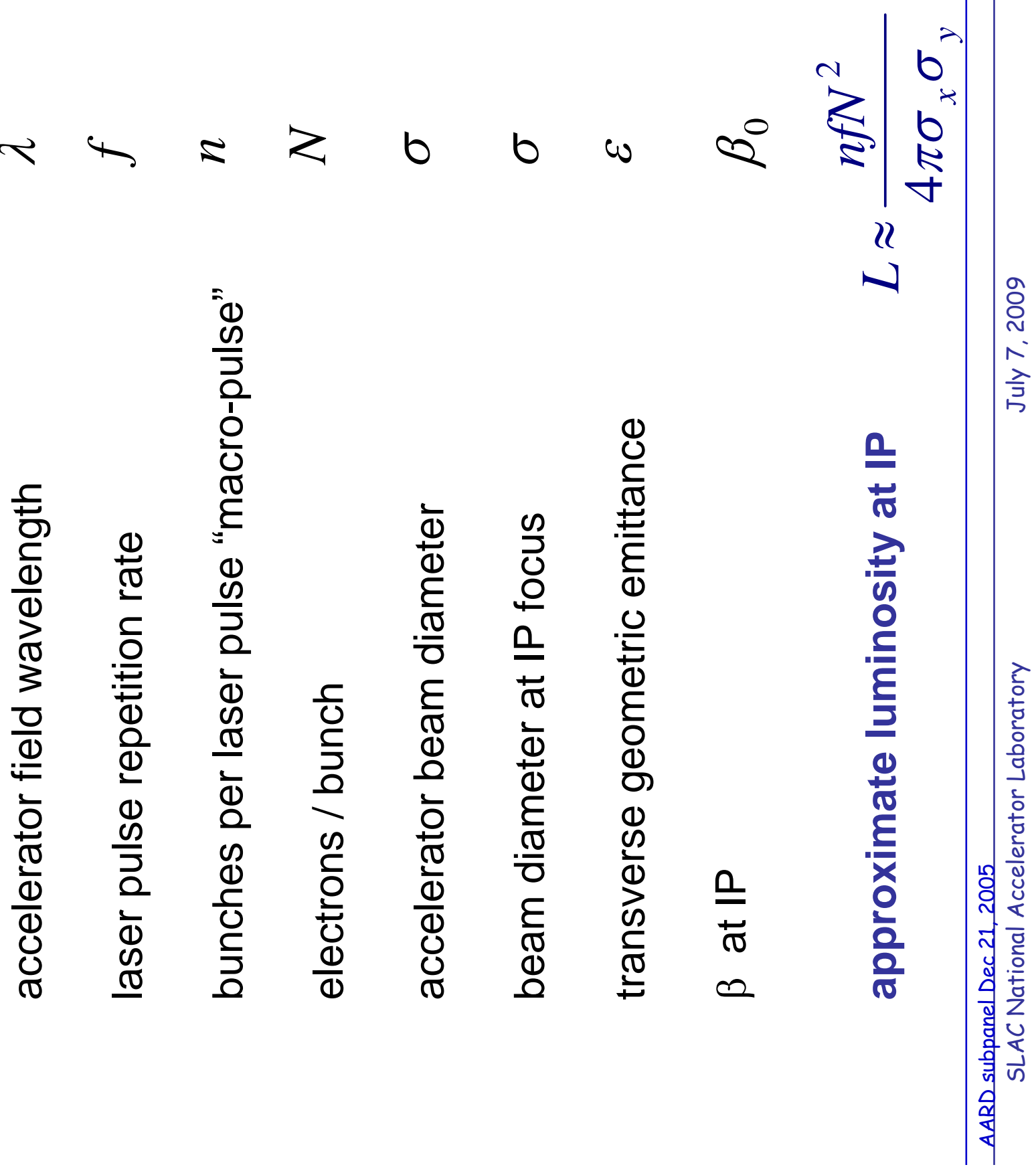


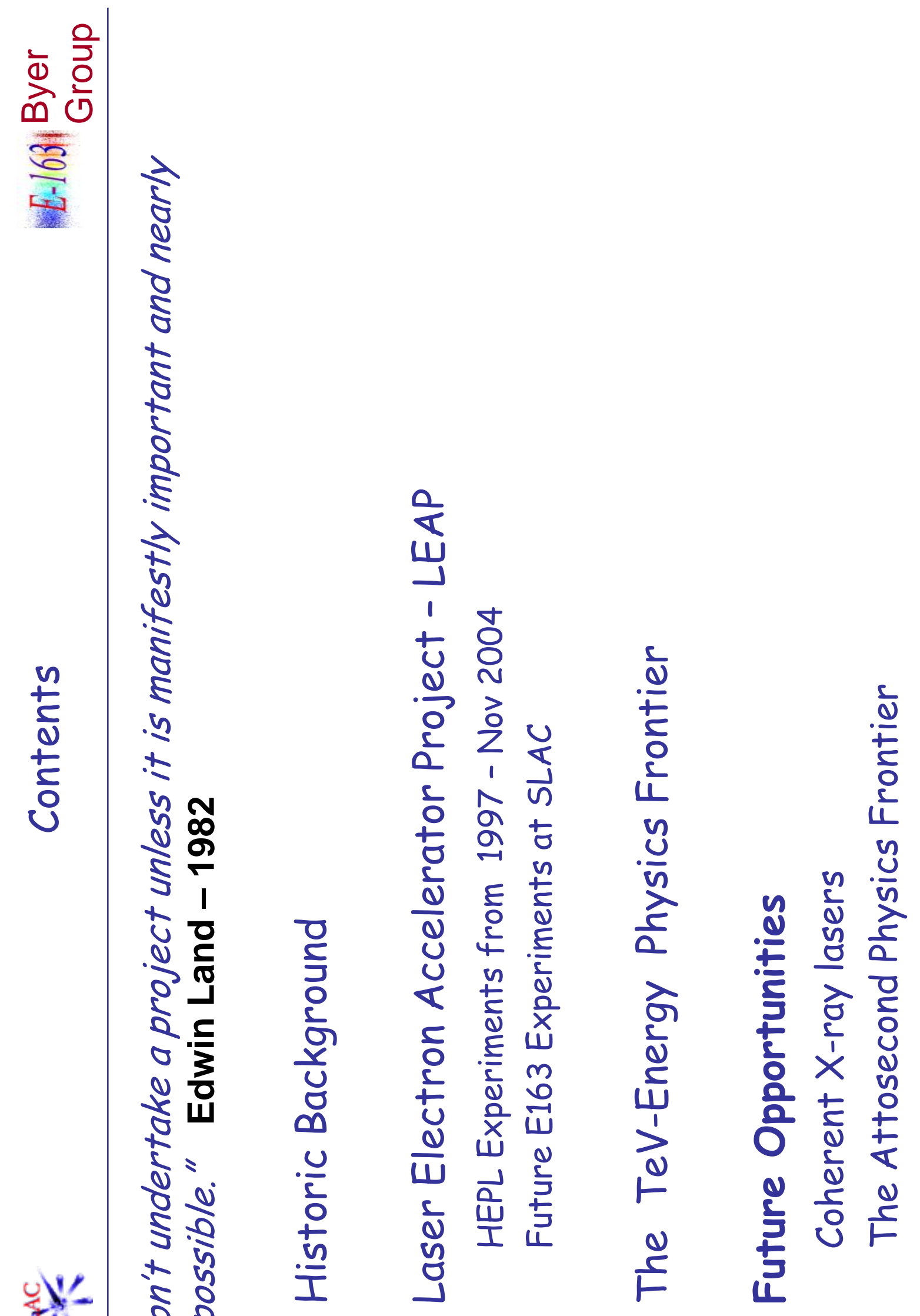




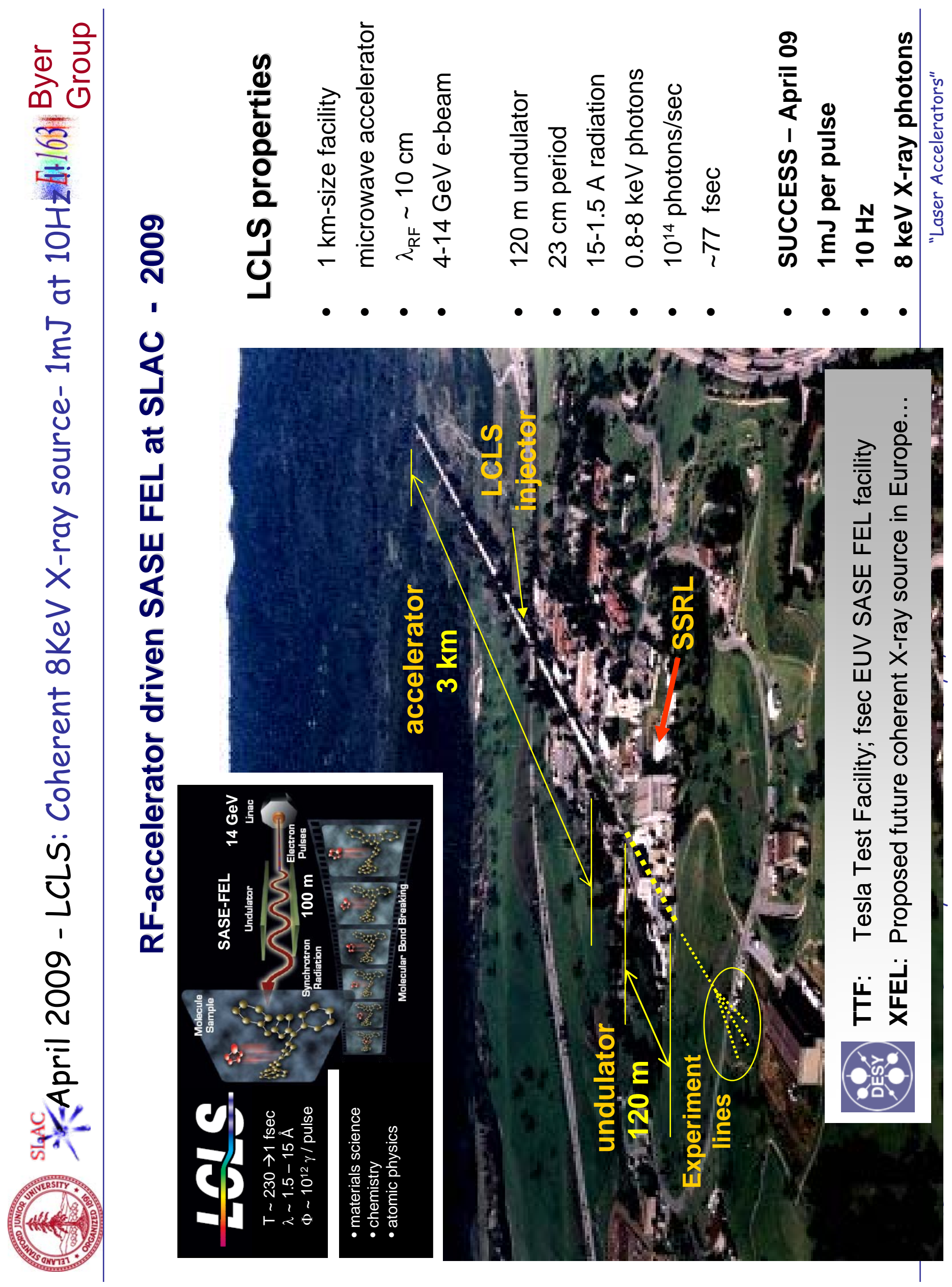




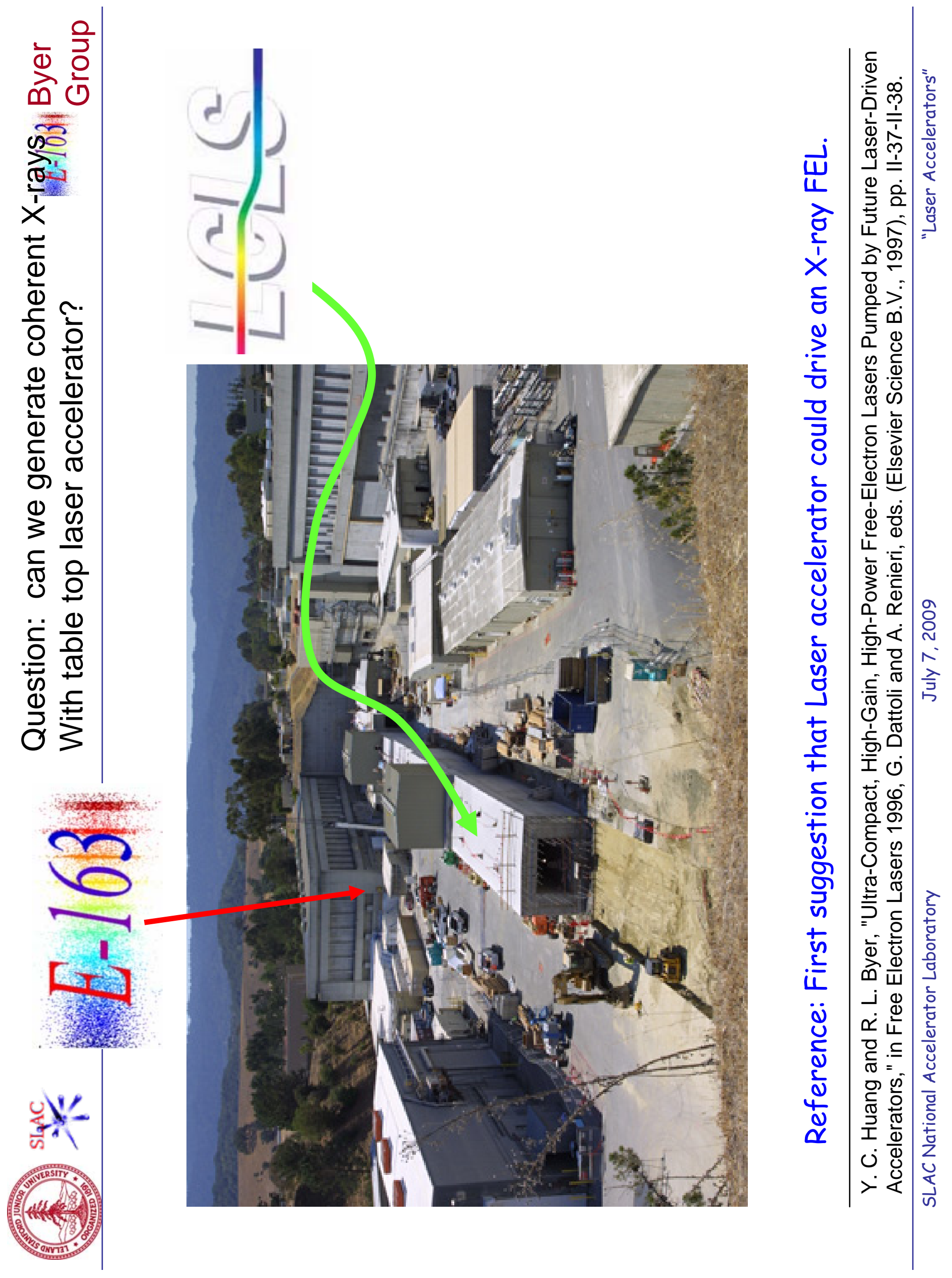




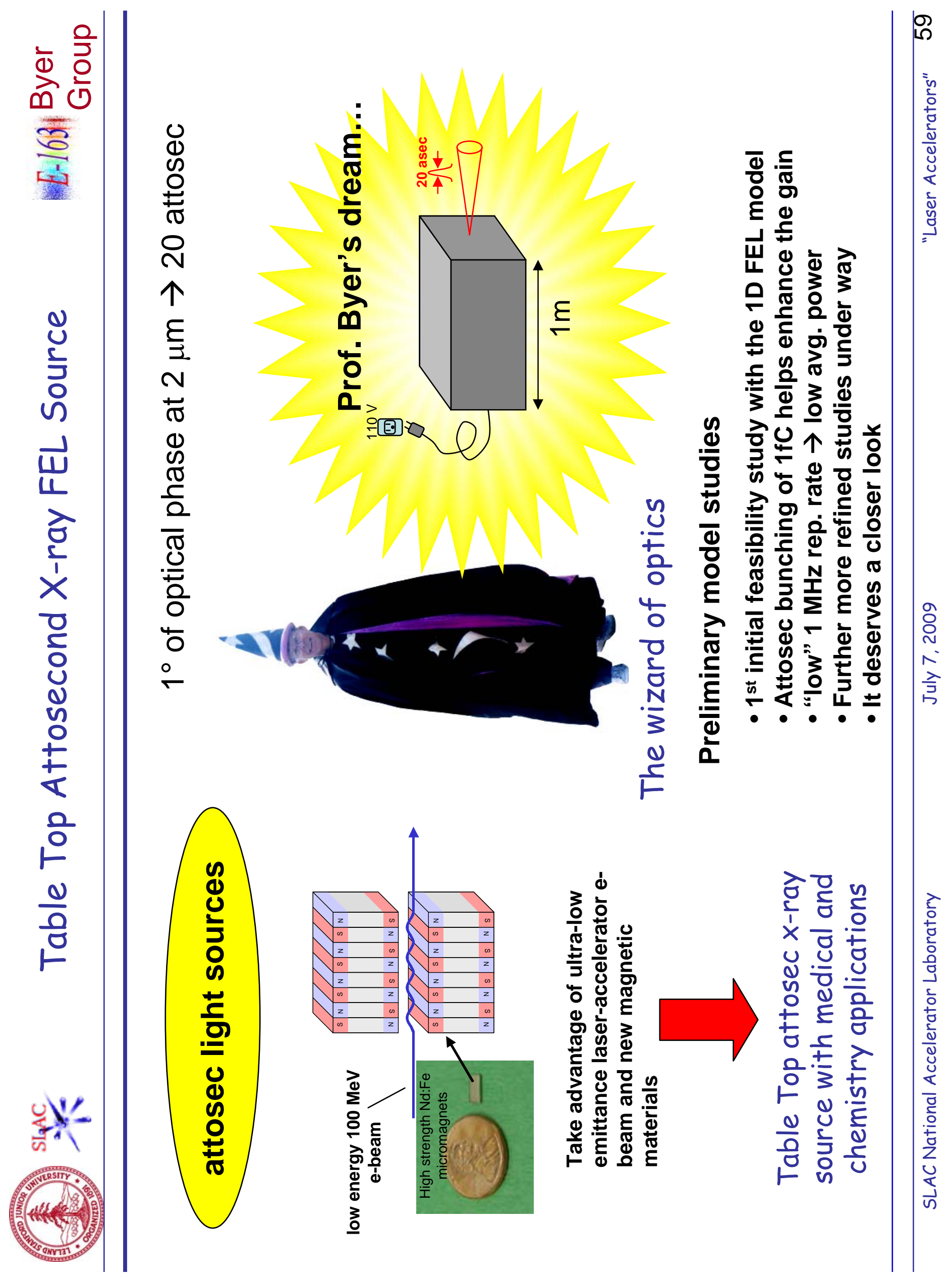




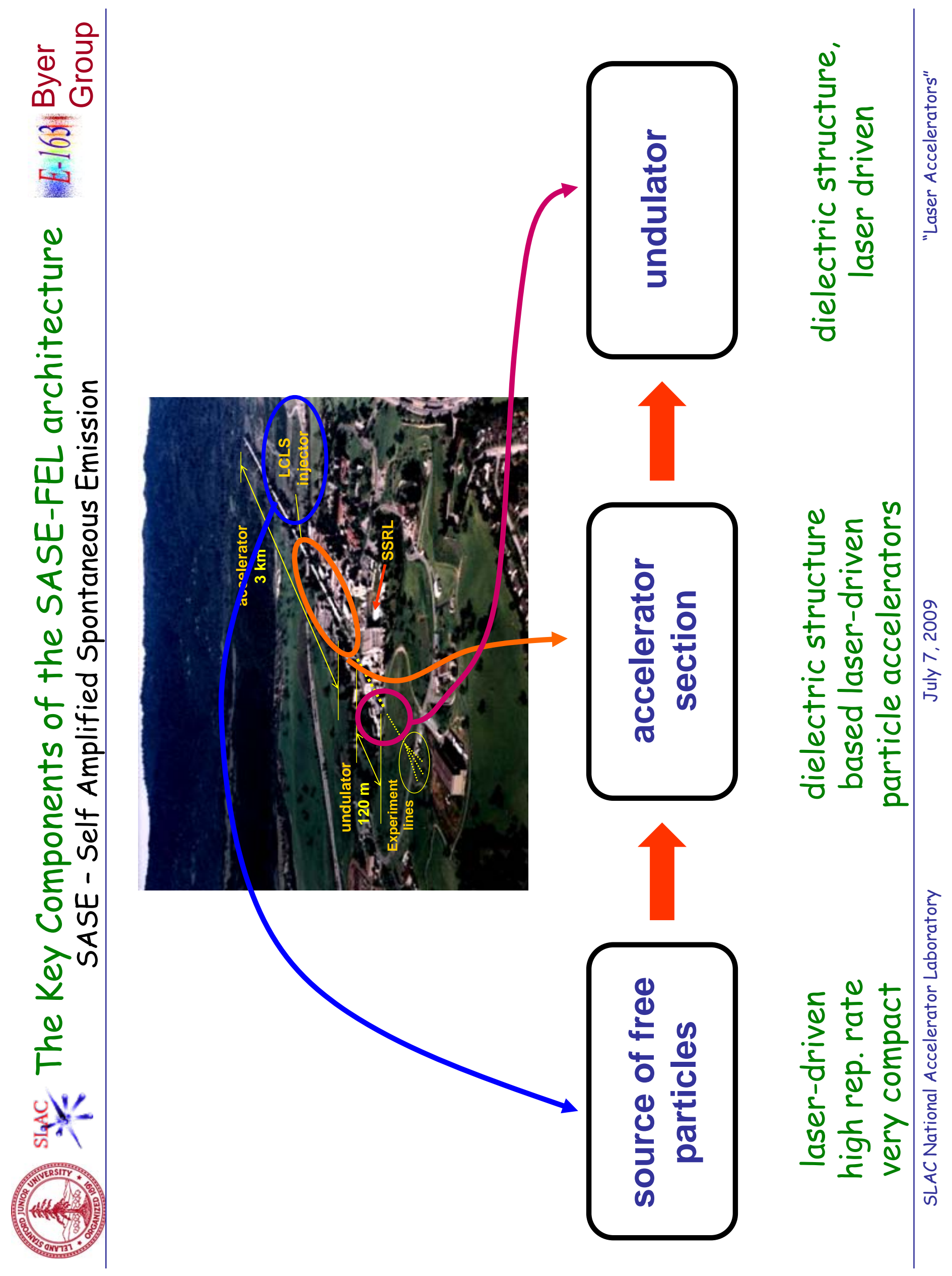




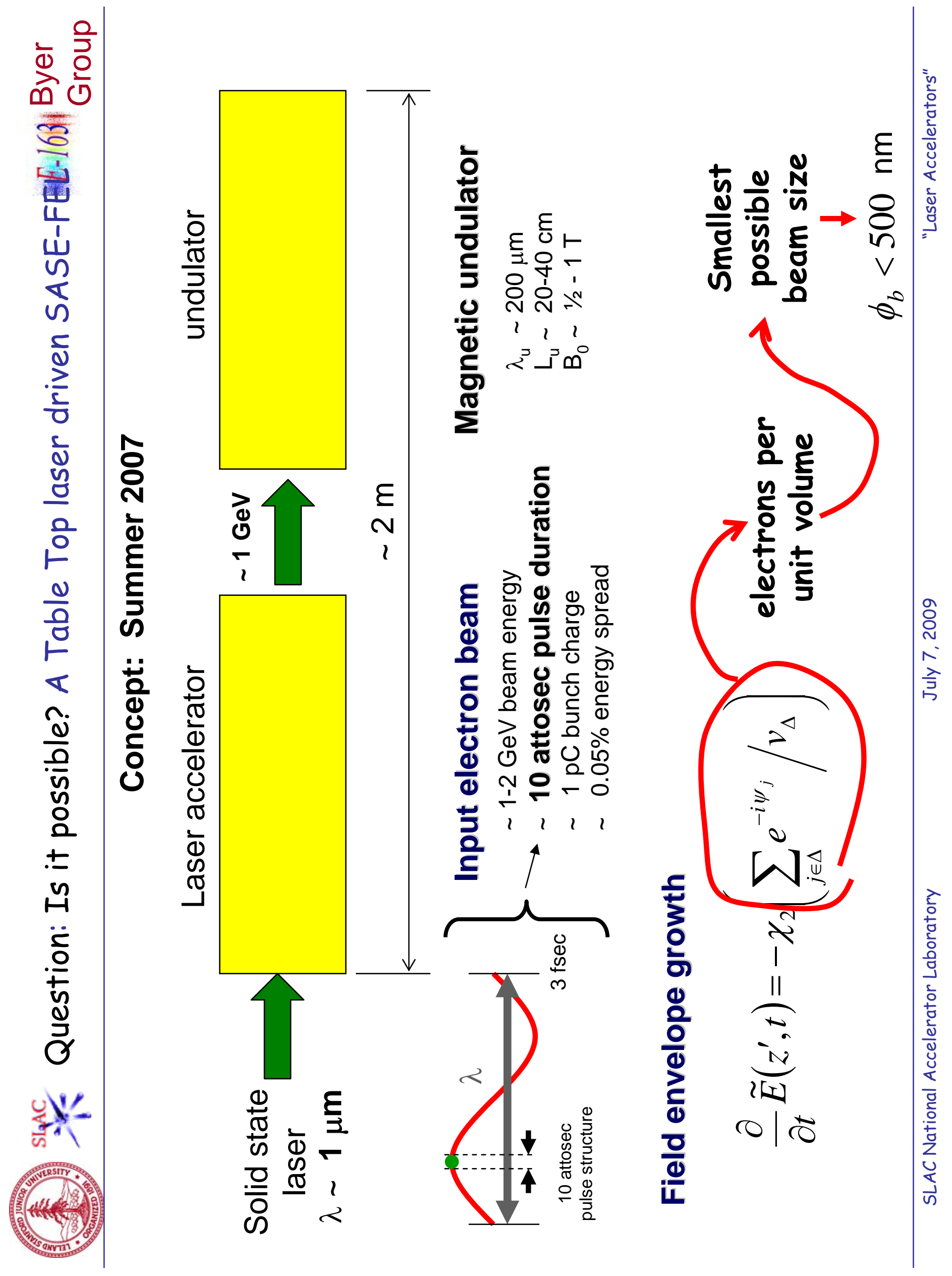




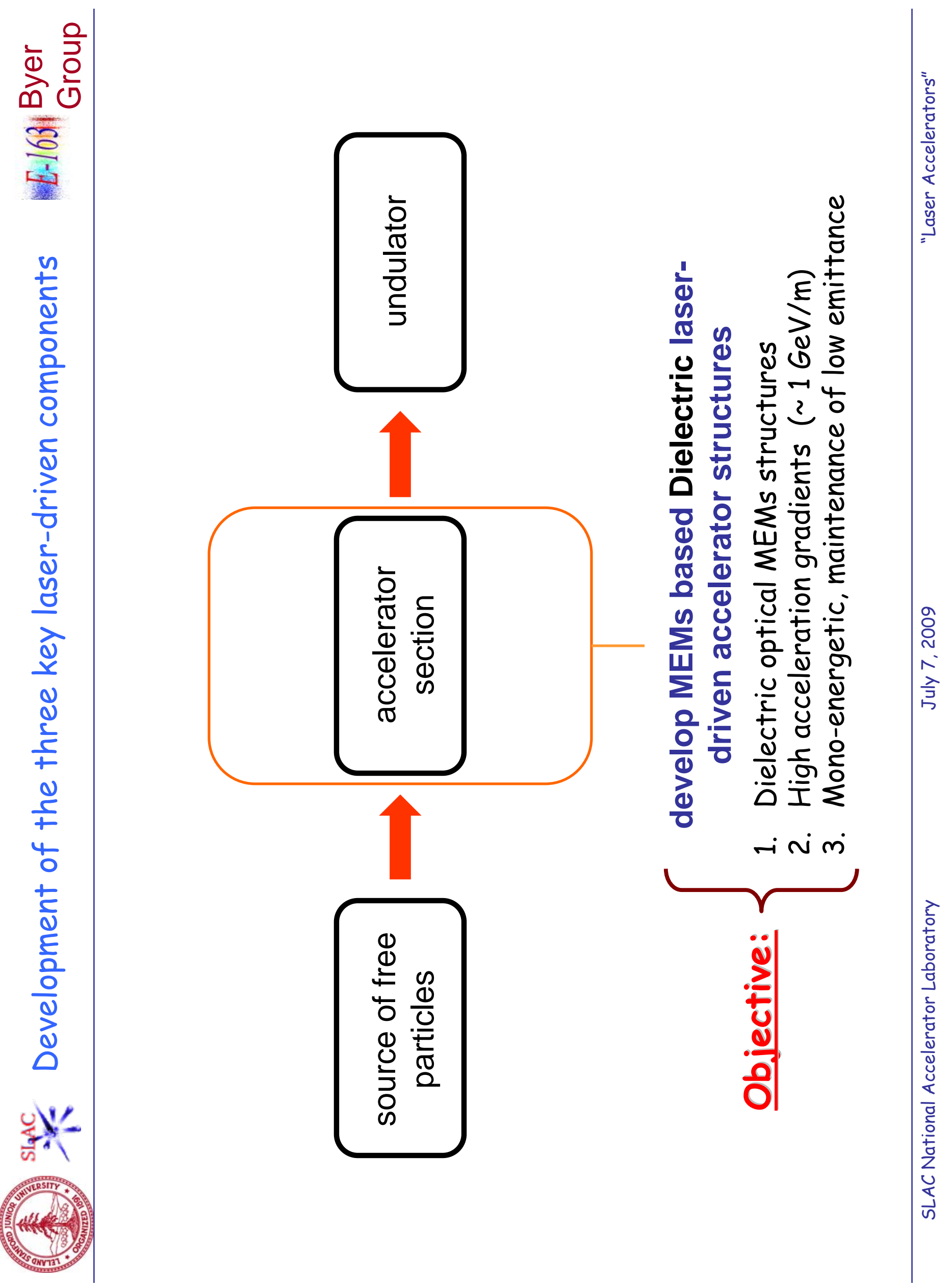




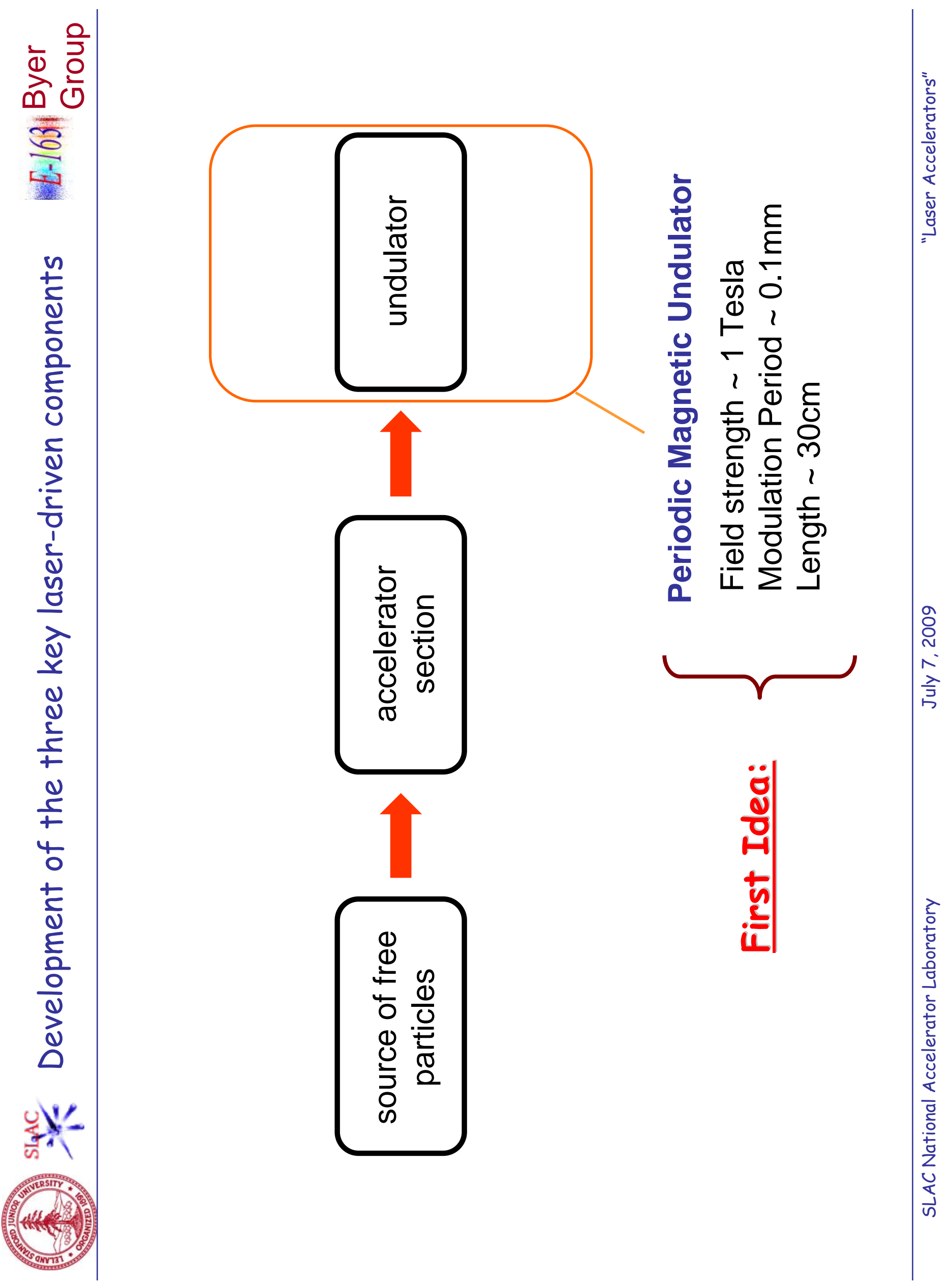




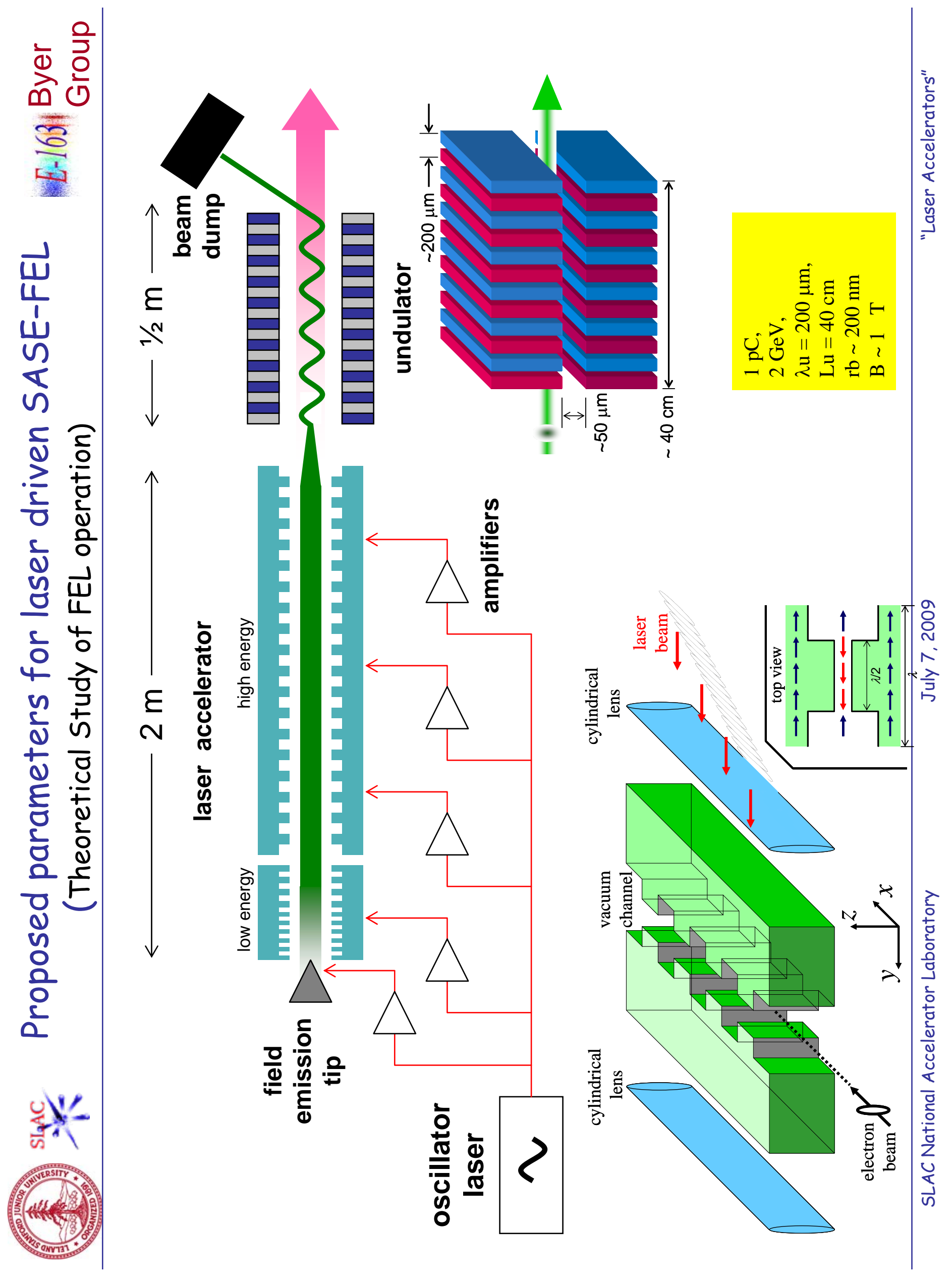




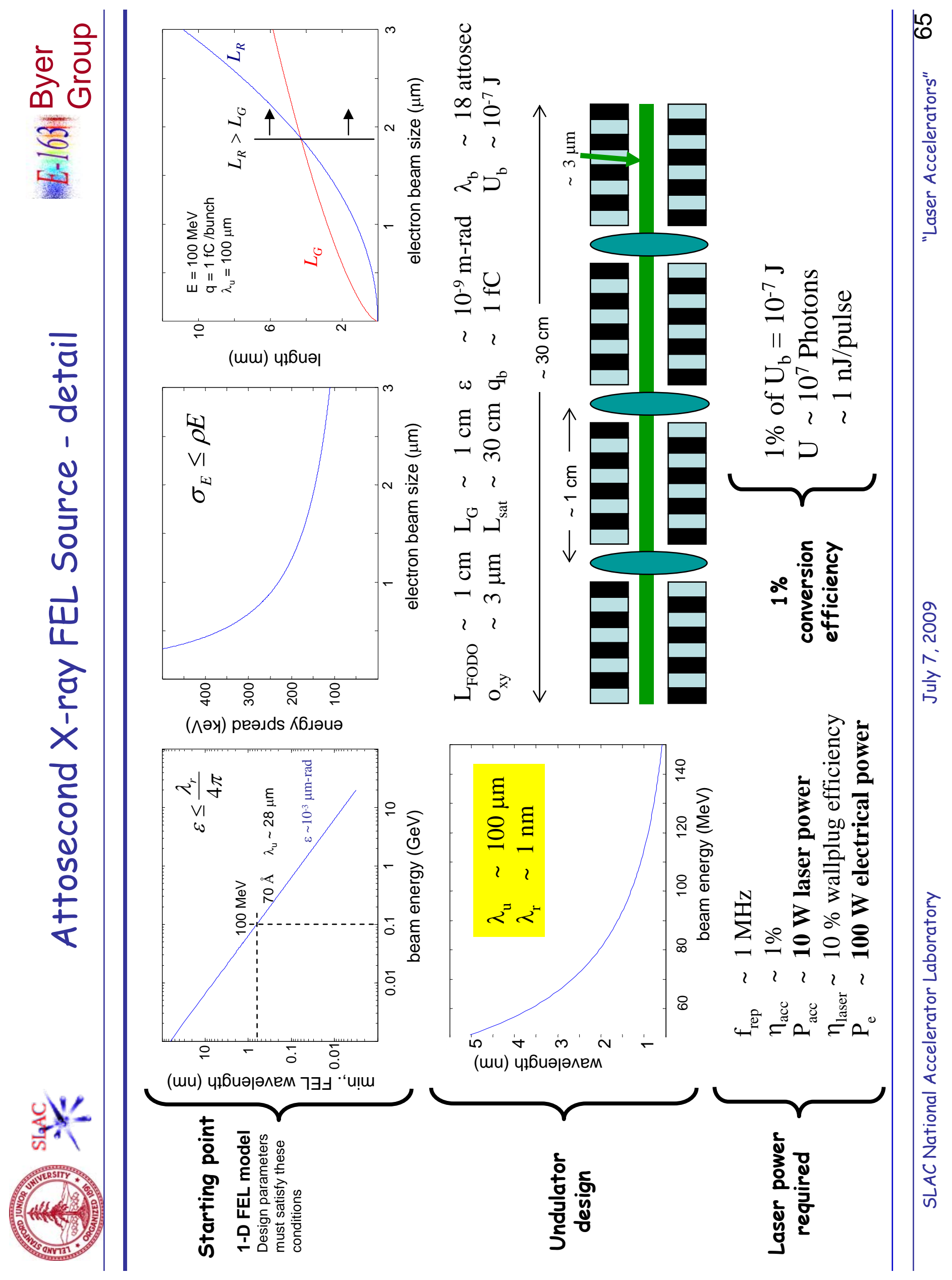




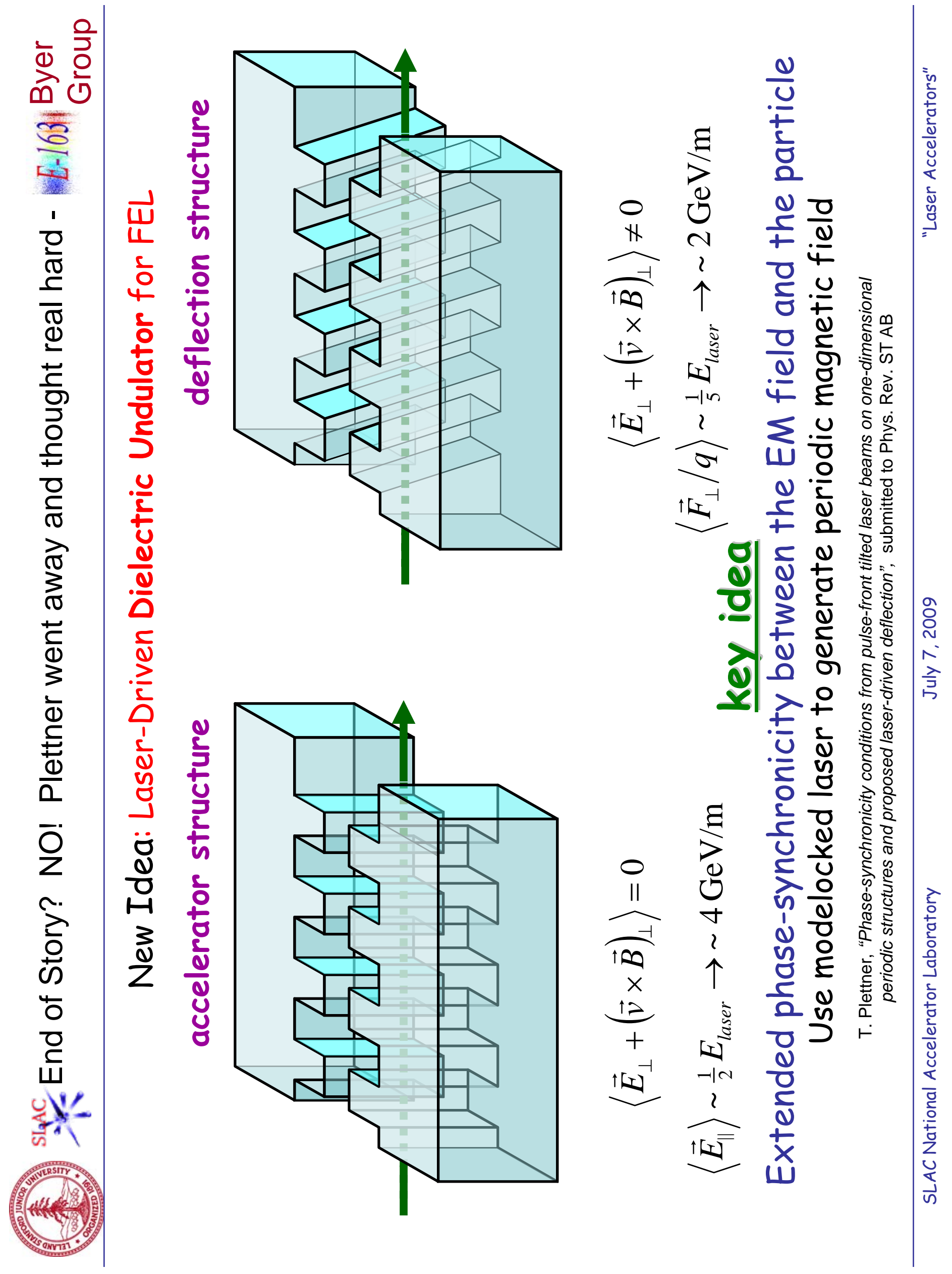




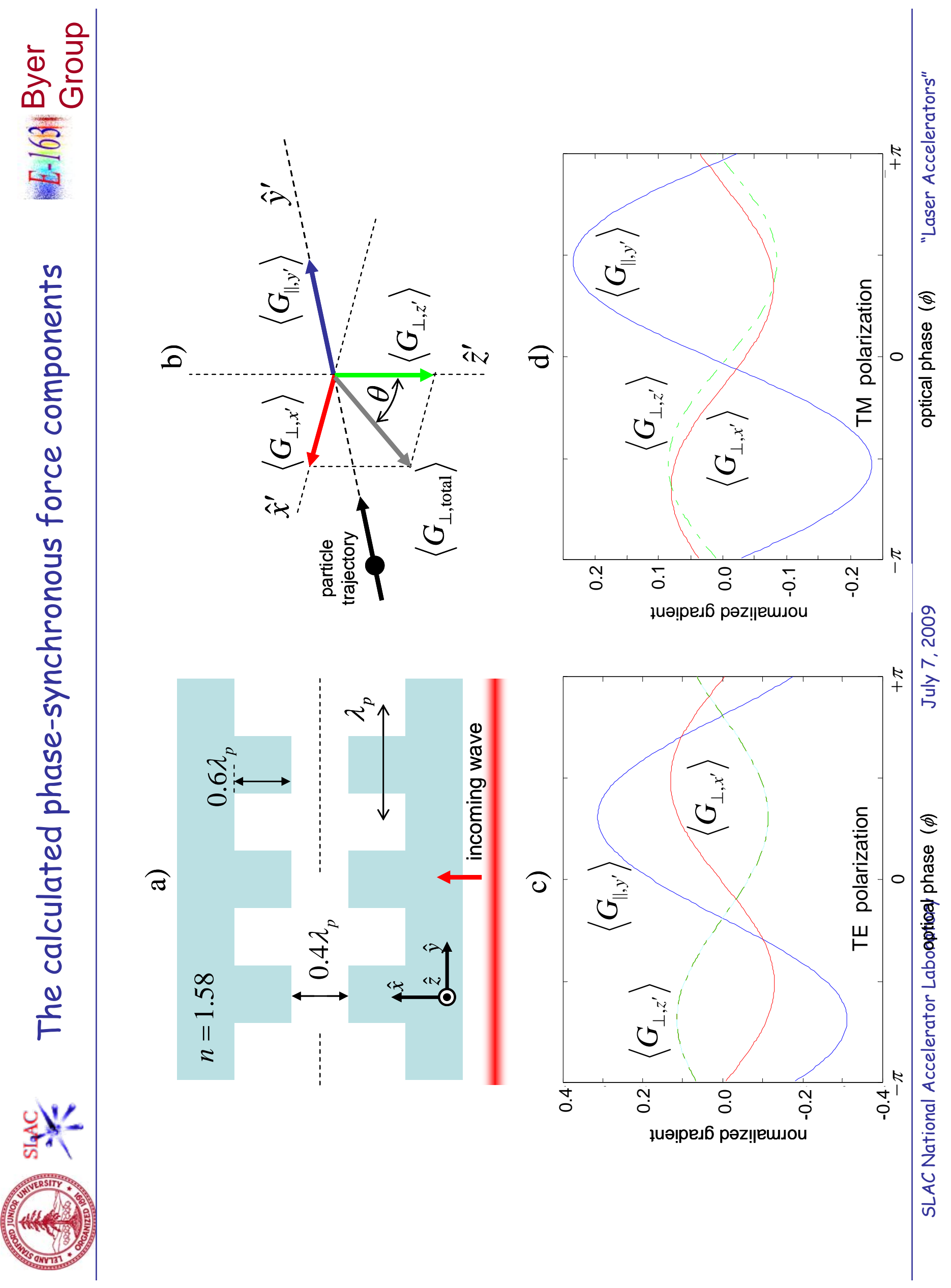




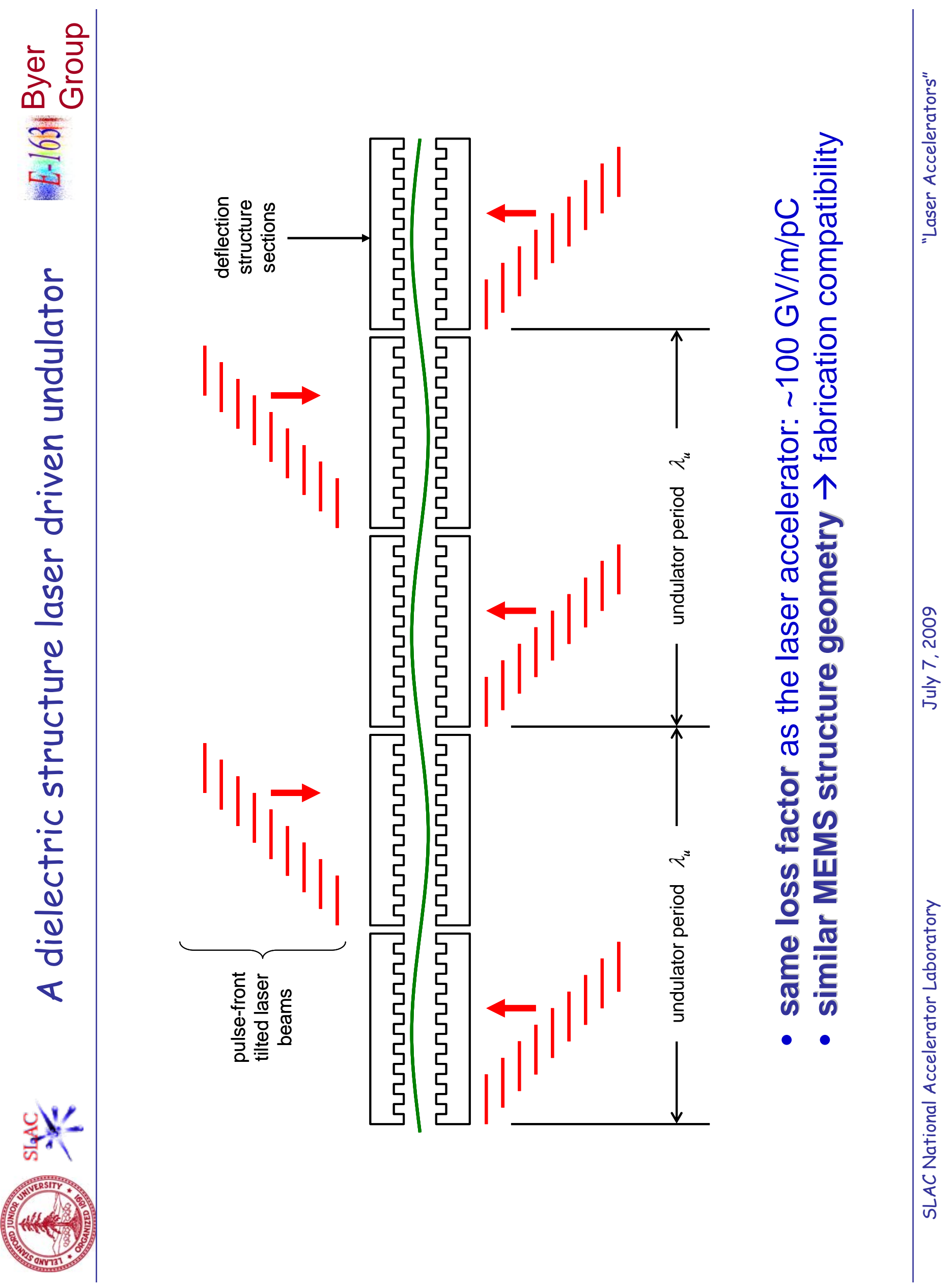




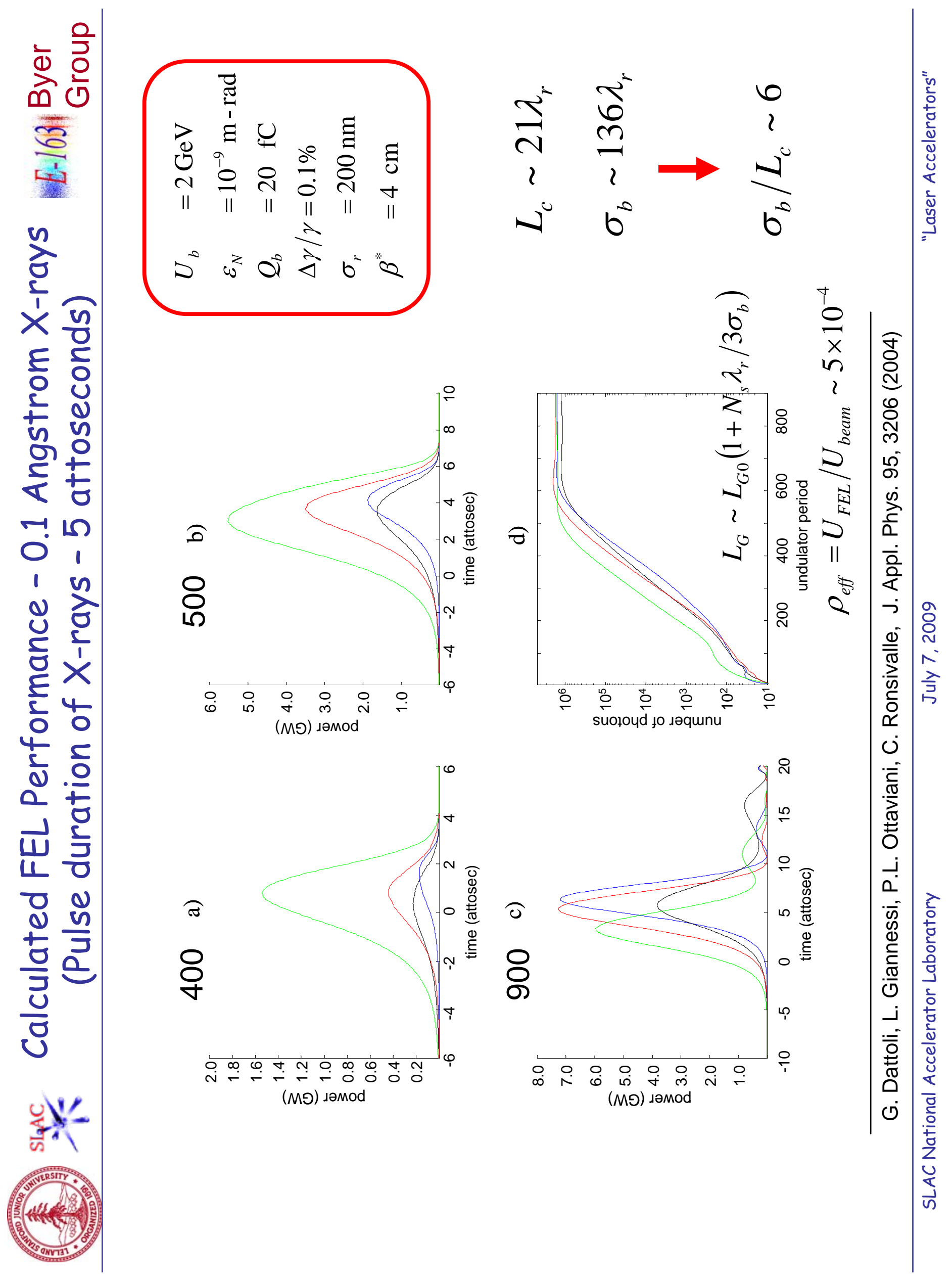



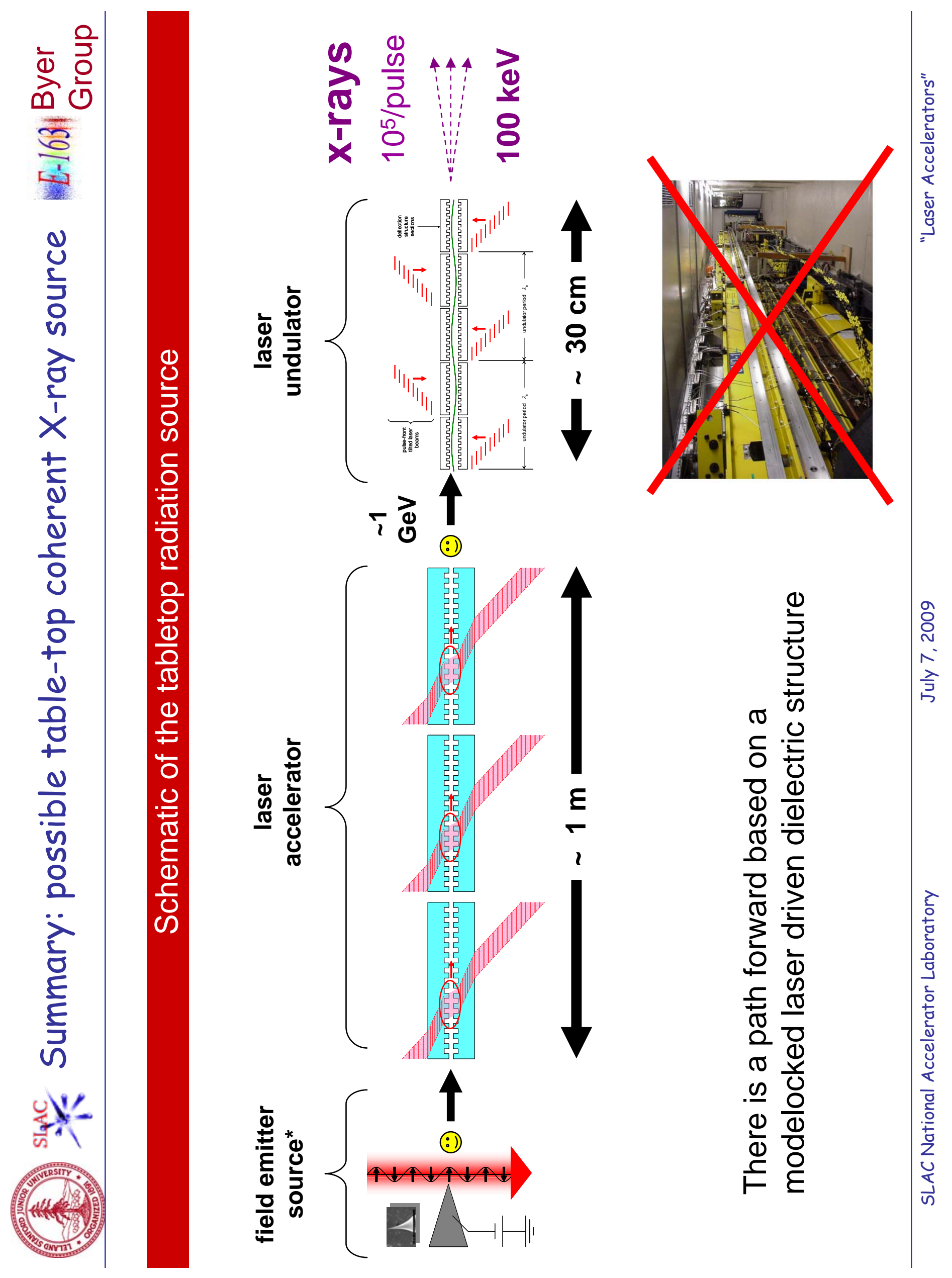


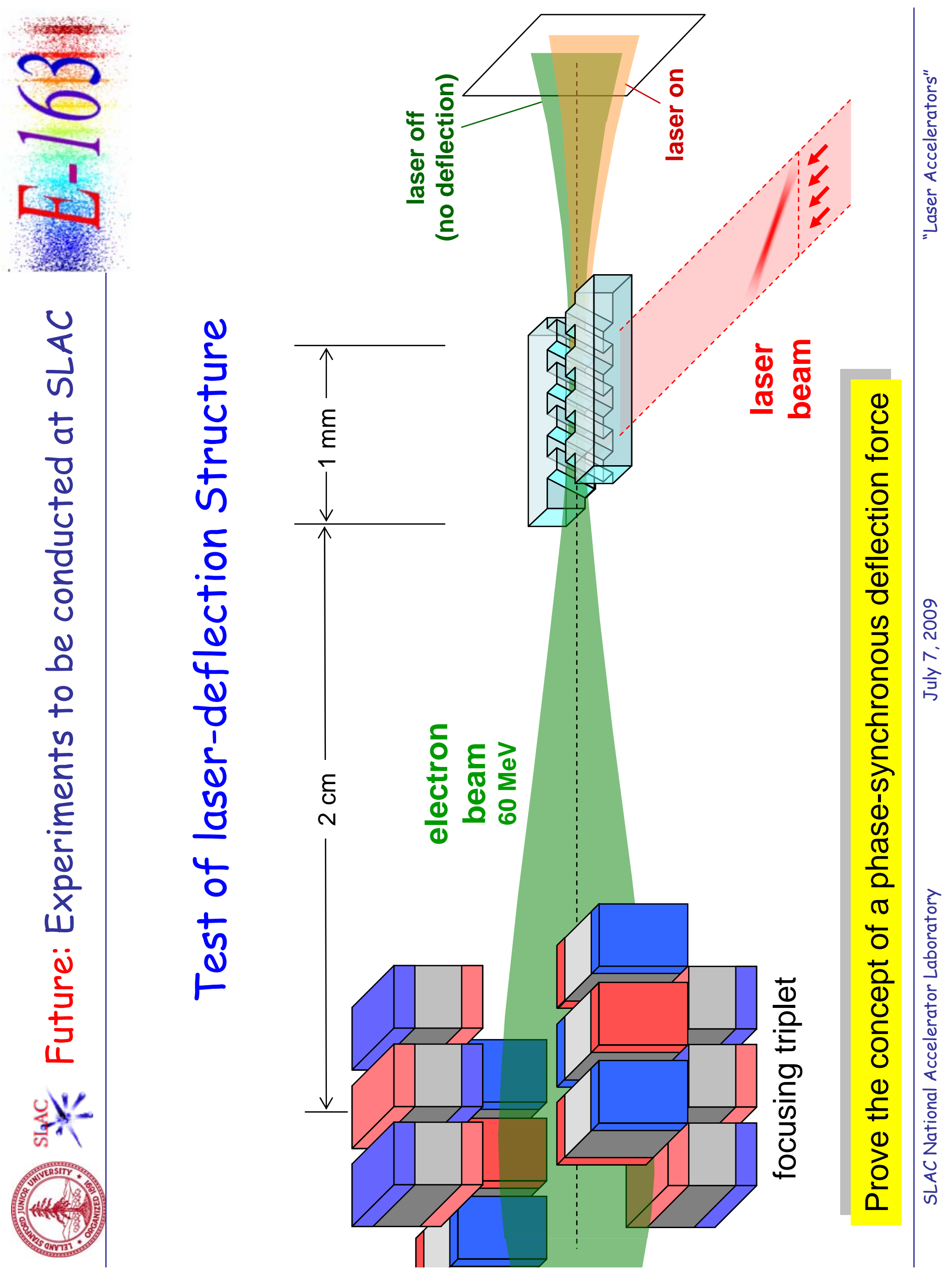




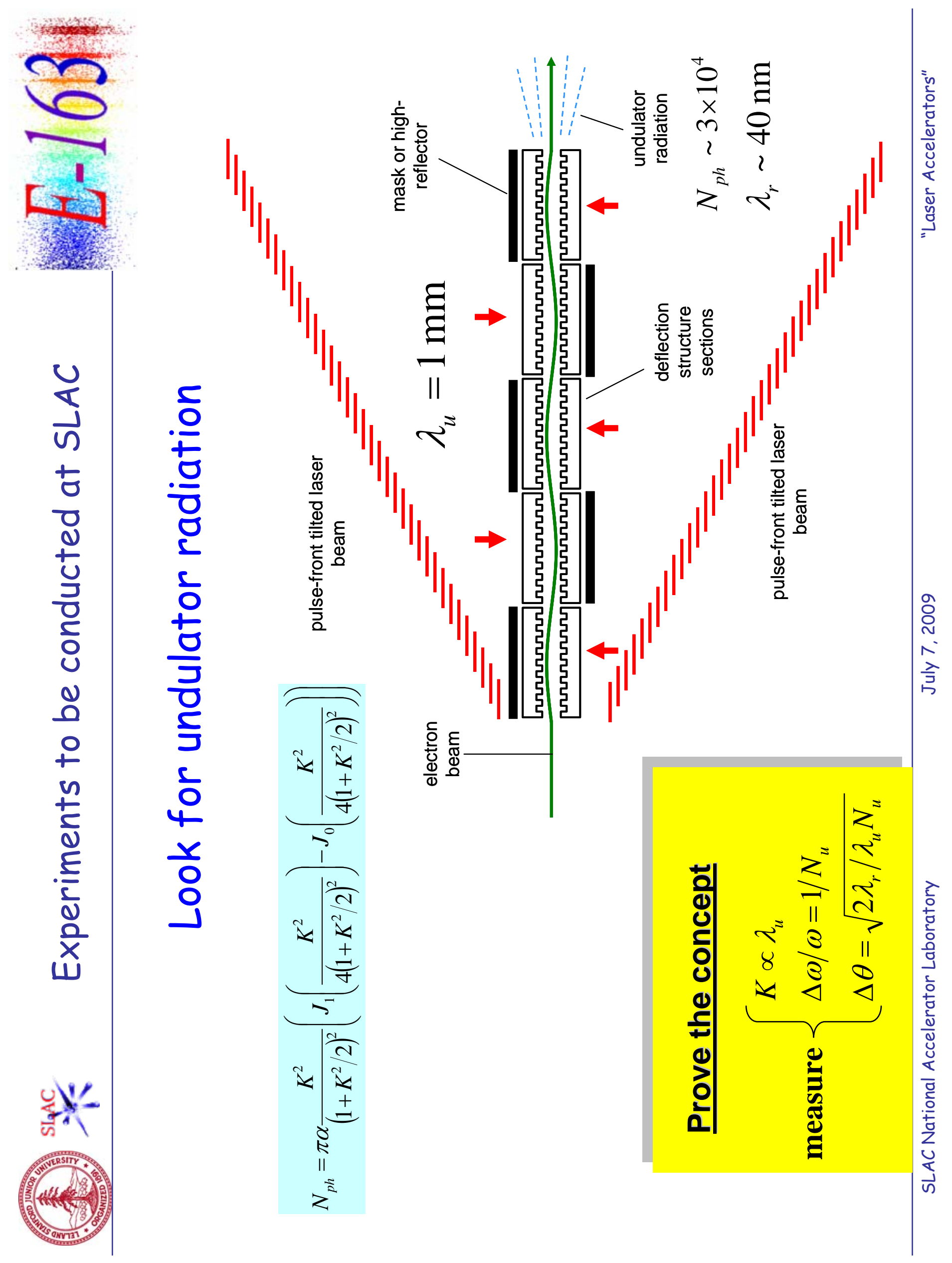




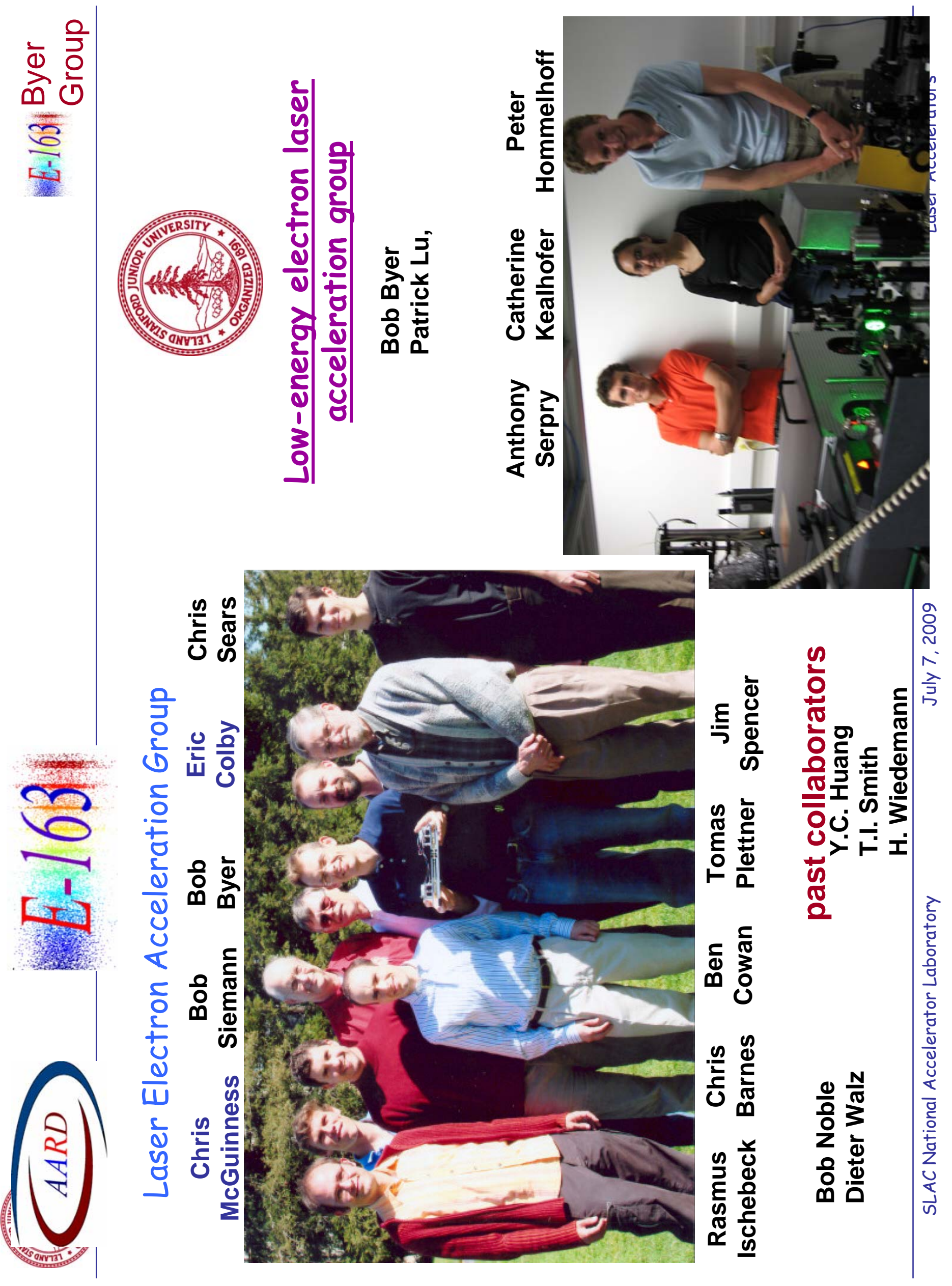




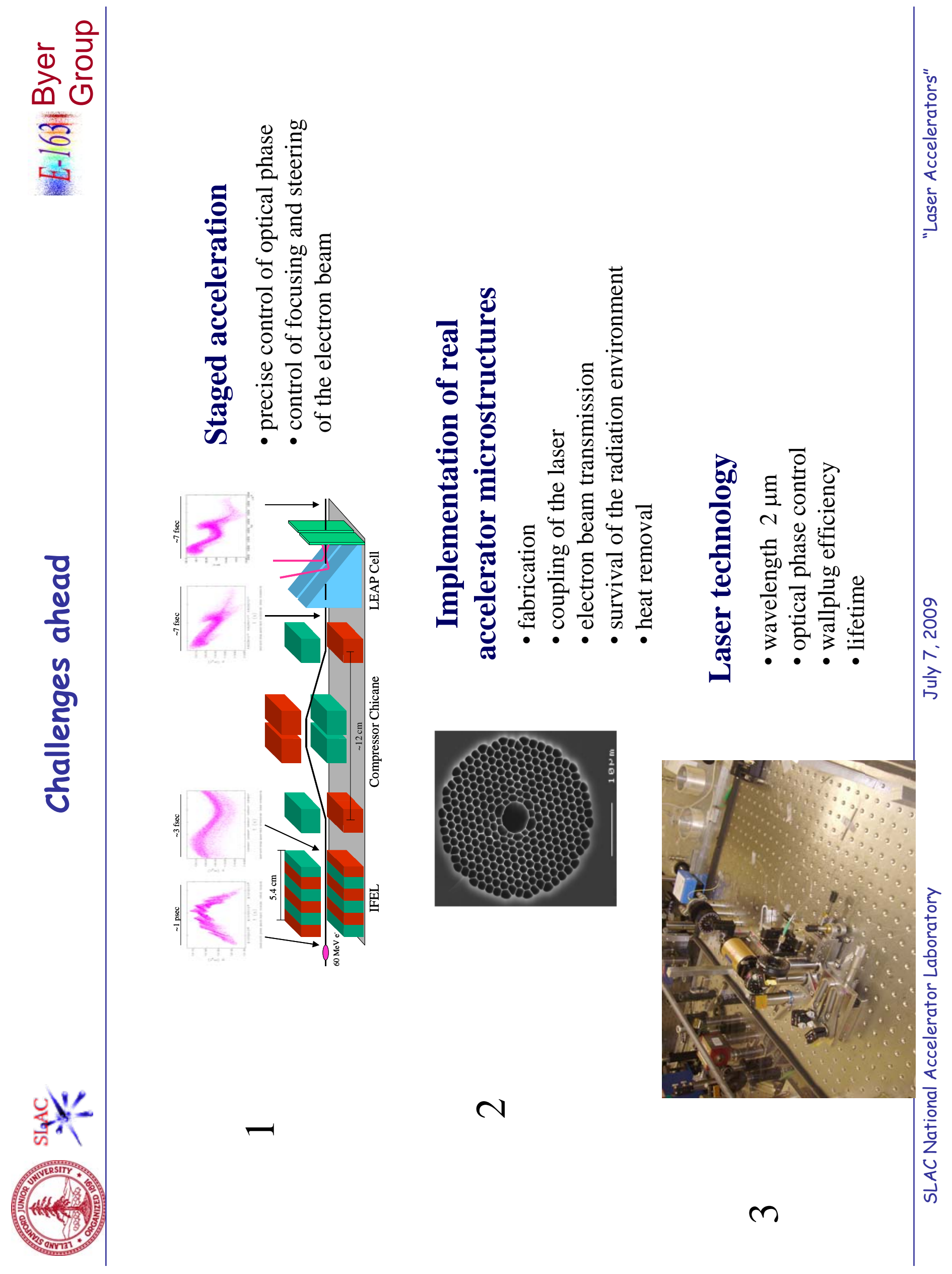




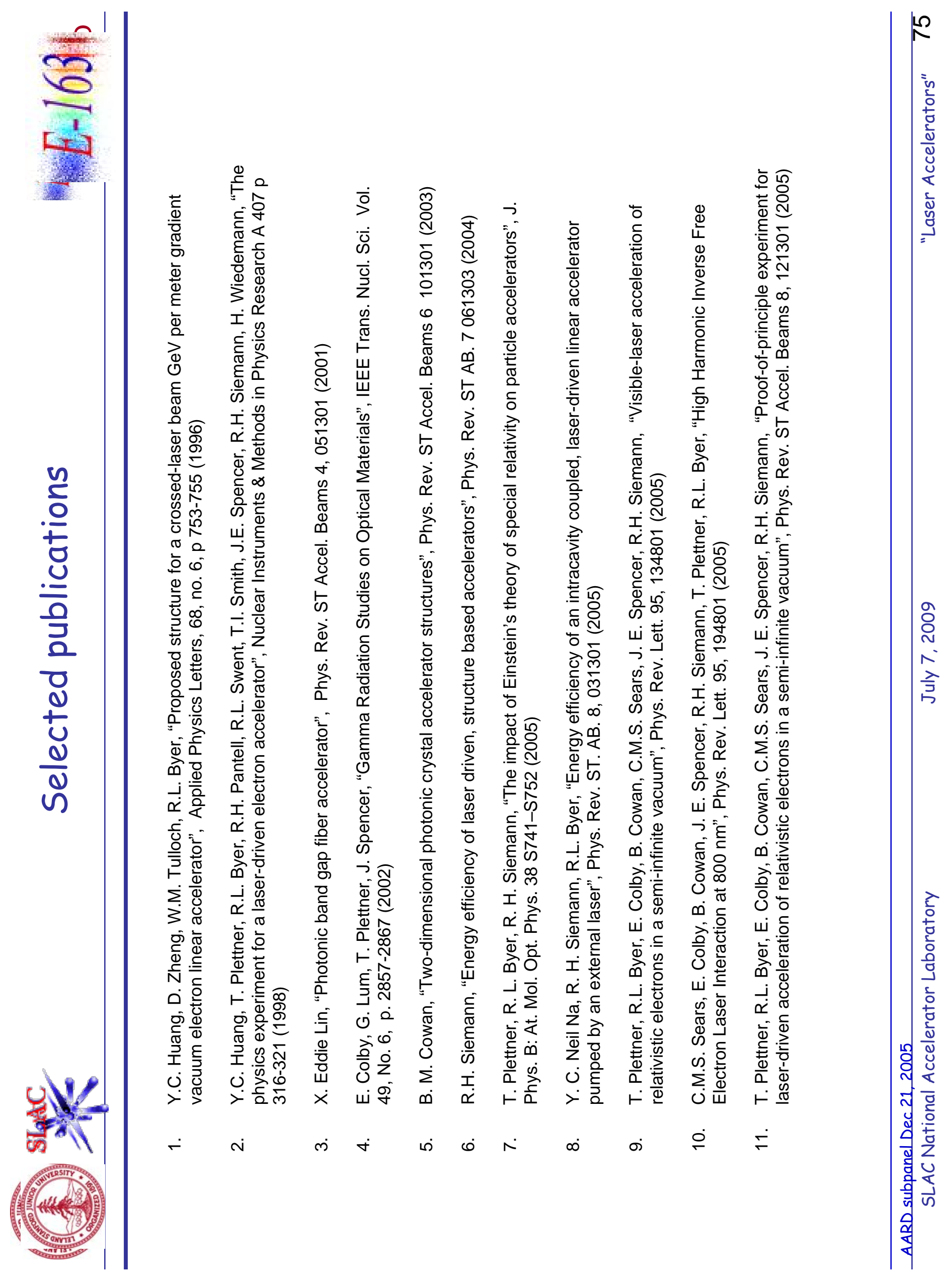



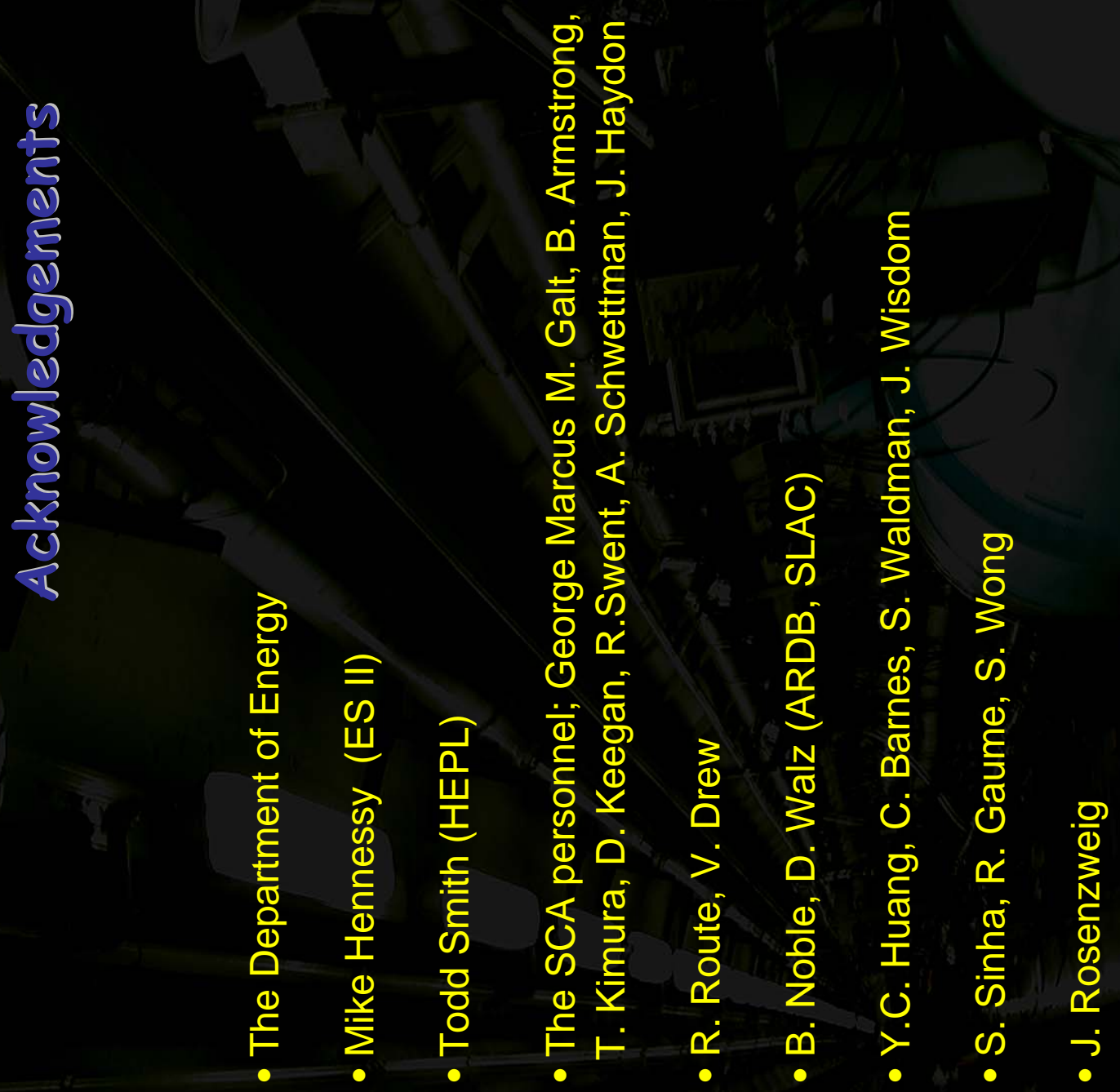


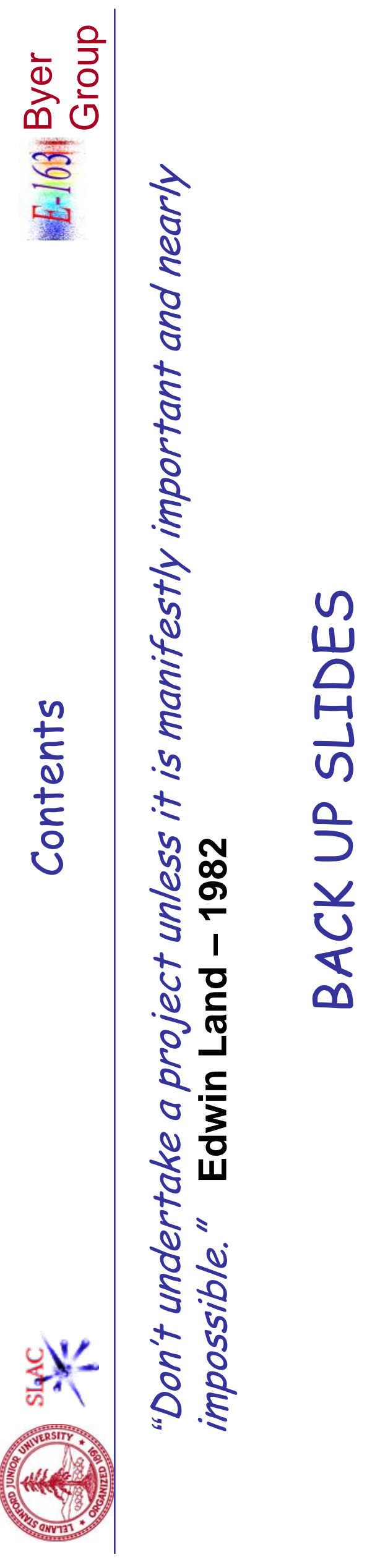



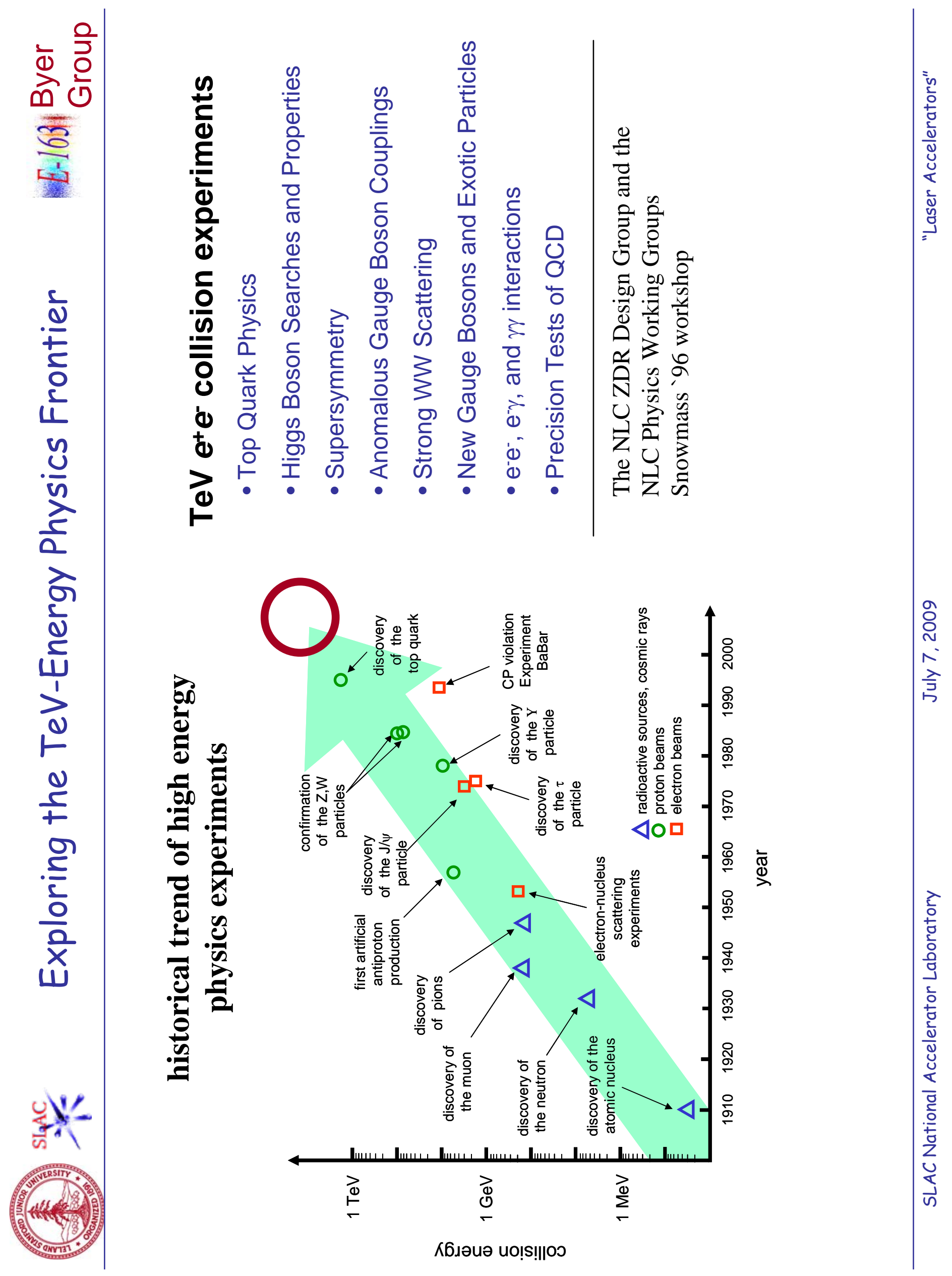


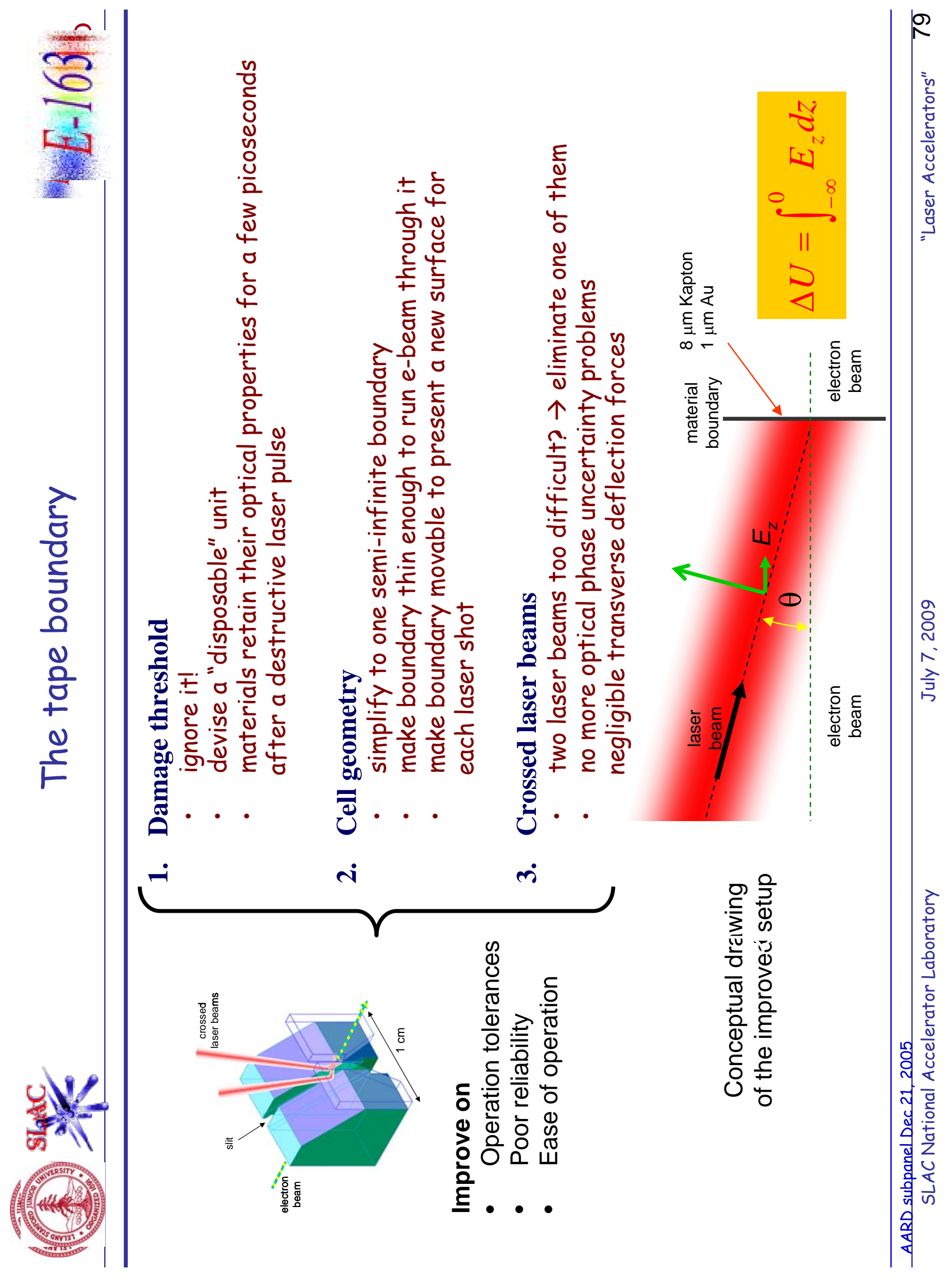




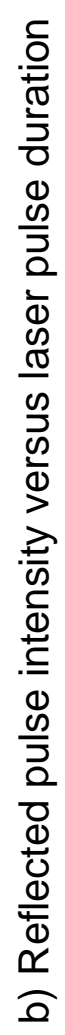
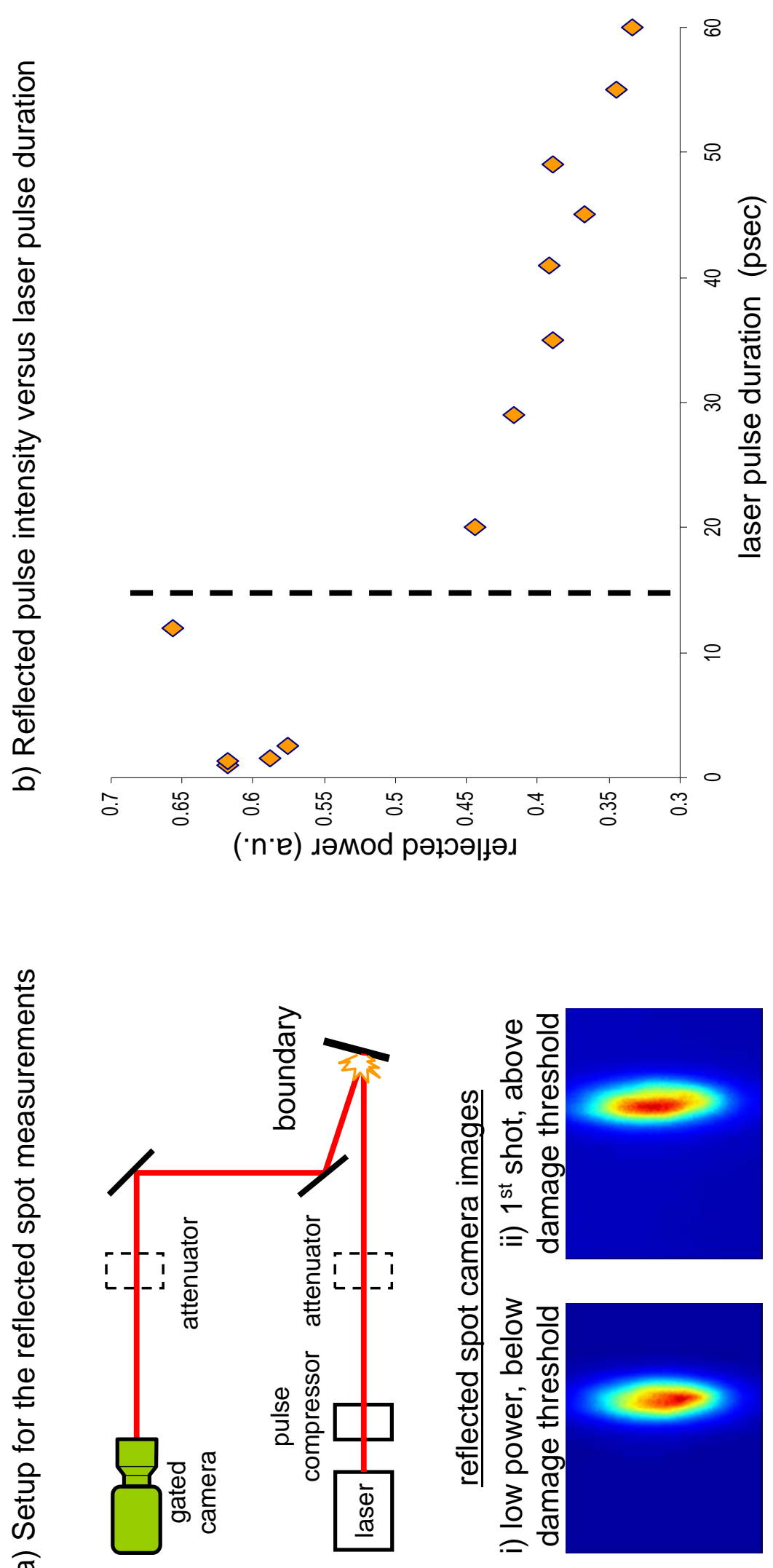


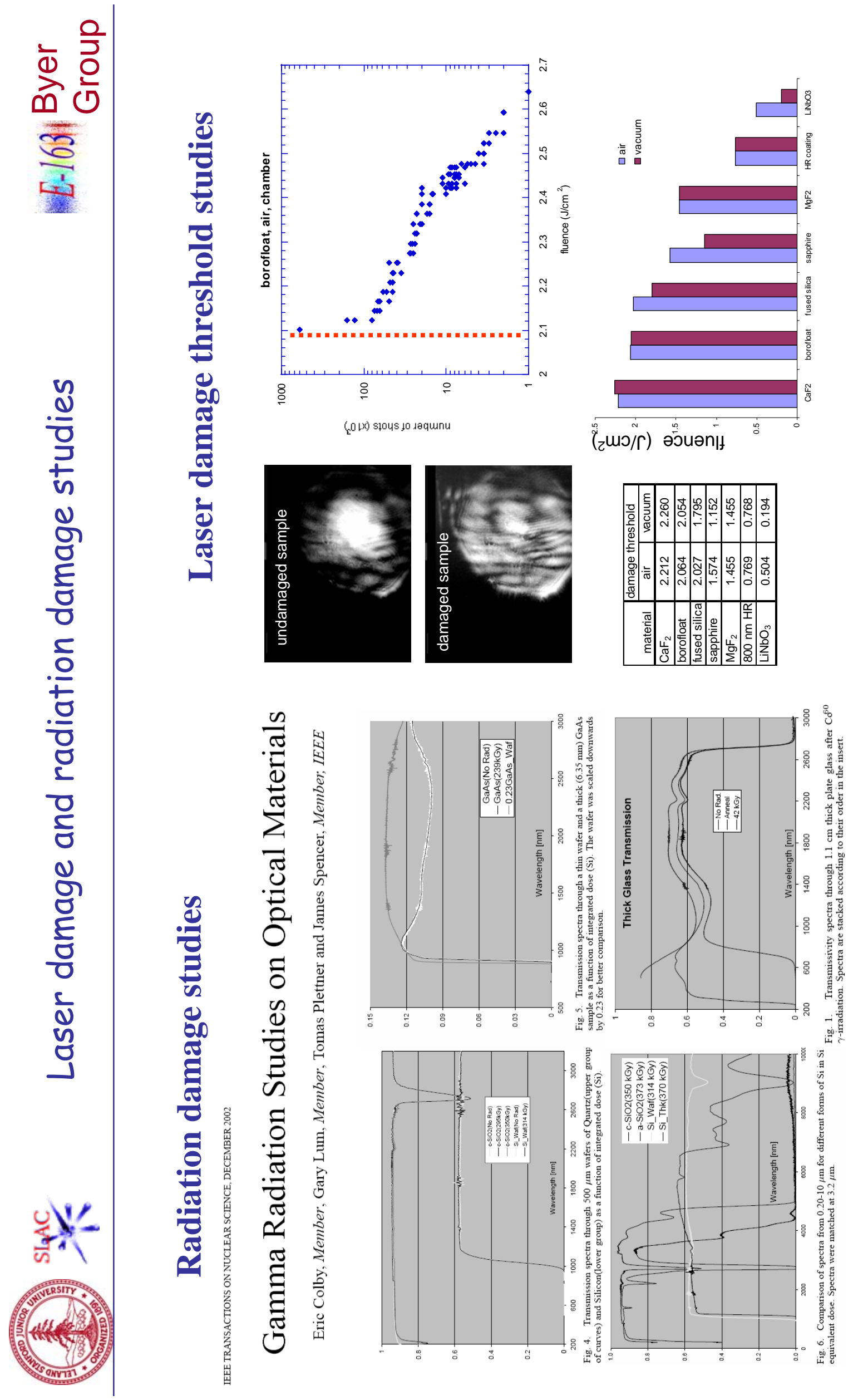

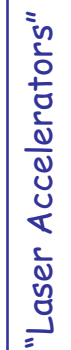

8
8
섬
곤

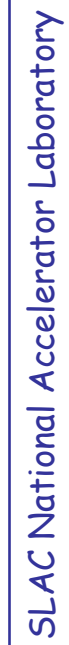




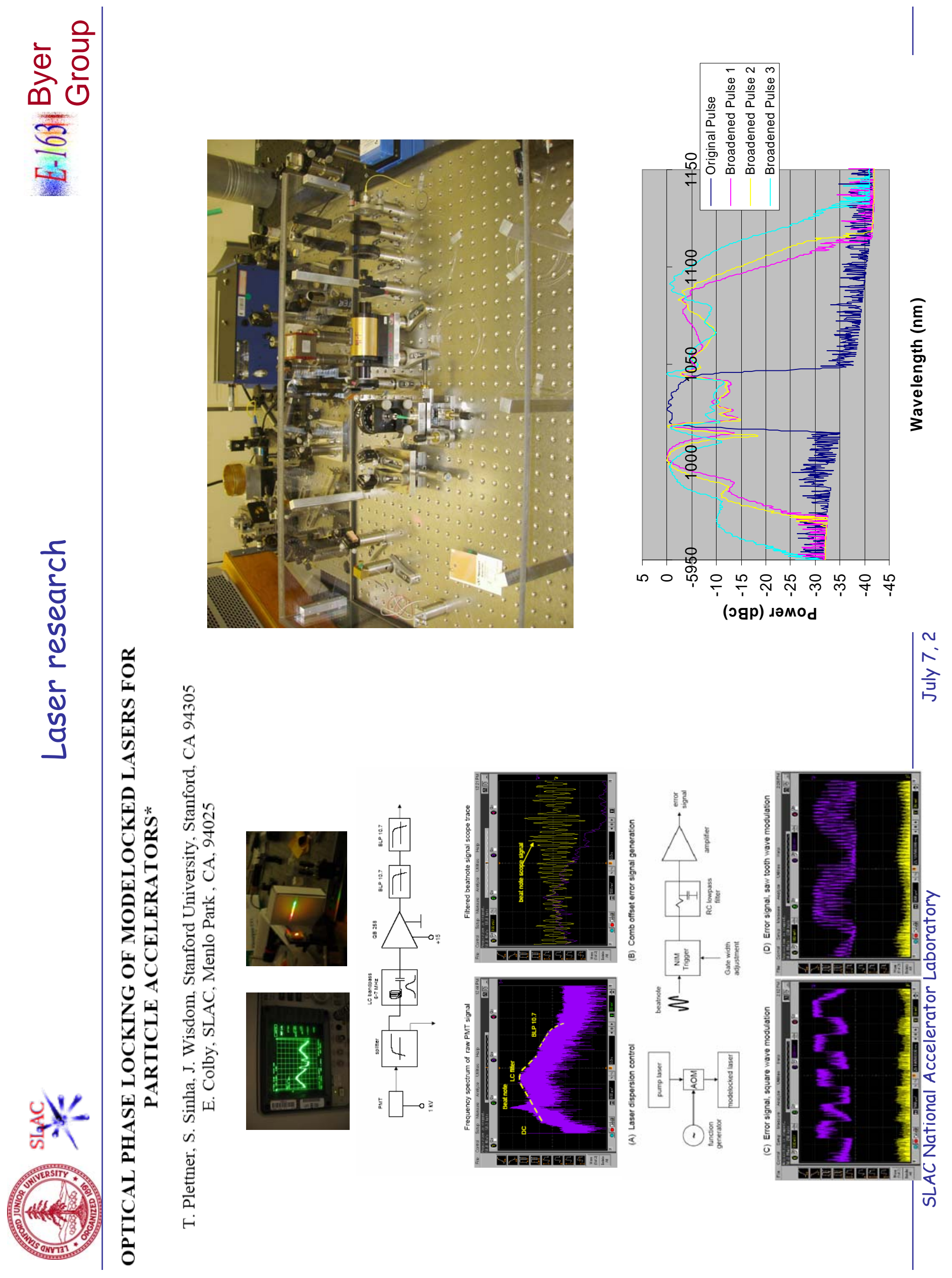




\section{In Memory of Bob Siemann}

\section{Ben Cowan}

Last September, when I heard that Bob had passed away, I was naturally quite saddened - and I was also surprised. Of course, I knew that Bob had been fighting health problems for several years, but through it all he always exuded a great optimism. I remember eating lunch in the courtyard with some other students when Bob returned to SLAC after being away for treatment for a few months, and he came right out to join us. He was excited to return to work, to catch up with us, and with all the advanced accelerator work that was going on - and to give me a gentle push to get more of my thesis written. He really knew that each day would bring new ideas, new discoveries, and new possibilities, and he wanted to be part of every minute of it.

The great thing about this optimism and enthusiasm that Bob had in such great supply was that it was so contagious. Being around him, and working with him, I couldn't help but be confident that success wasn't far off, and that the next modification to the experimental setup, or the simulation process, no matter how small, would yield a breakthrough.

In the laser acceleration project, optimism was really something of a necessity. As you just heard from Bob Byer, it was a very long road from the conception of the experiment, through the instrumentation of the setup, to the results. The experimental work was quite intense. The accelerator we used was superconducting, and quite expensive and time-consuming to cool down. So once it was ready, it was important to squeeze every last ounce of data out of the time we had with it. That meant that there would be months - sometimes years - of preparation work building up to an experimental run of just a few weeks. You really had to believe that the next run would be the one that would yield the great result. And during the run, Bob would be there to inspire confidence in the next shift, and even the next data set.

I'd like to show you a little bit of the environment of this experiment. Of course, we'd run around the clock, in shifts. The action was centered in our control room; here's what it looked like. If it seems to be about as big as a closet in this picture, that's because it was. And this was taken in 2000, and in subsequent years it only got more crowded. Bob would usually be there from 8 in the morning until about 8 at night, every day of the run. It would be amusing to see someone of his size, sitting in this tiny room, hunched over his tiny laptop, methodically analyzing every data set. His size was sometimes a point of contention between us, since he wouldn’t think twice about installing an important piece of equipment on the very highest 
shelves, and then I'd arrive for the night shift and not be able to reach it. But then, I'd install things under tables or tucked away in corners that were hard for him to reach.

Of course Bob wasn't just around for the experimental runs; he'd be actively involved in the preparation as well, often taking the lead on key diagnostics. Here's the first of two streak cameras he set up for the experiment. His depth of electronics knowledge was also invaluable to deciphering some of the systems we worked with. Here's some instructions he wrote down on reviving one of the feedback loops. We all quickly learned that the effort required to read Bob's handwriting was well worth it. And not only would he be there to provide encouragement and advice in difficult times, he'd also be there to celebrate our successes.

As the LEAP experiment at HEPL was reaching its conclusion, an effort was ramping up to develop a new class of optical accelerators, based on photonic crystals and other microstructured geometries. What was amazing to me, working on this topic as my thesis project, was that Bob didn't have any formal training or extensive background in photonics, and yet he'd always be able to provide insight and advice that kept the work moving forward.

This is something that has been remarked upon several times today: Bob’s accessibility to his students. The optimism that Bob engendered didn't come just from kind words; it came from always being available to get advice from him or just bounce ideas off him. We knew that we could always count on Bob in a tough situation. And he made us, his students, a priority, making sure we had the resources we needed. He would often utter the words that a student, especially one working on an experiment, wants to hear (second only, of course, to "your thesis looks good, you can go ahead and defend”): He'd usually say, when we needed to get some equipment, “just write a purchase order.”

Bob made students a priority even over some of the more prestigious activities that come to scientists of Bob’s accomplishments. I remember discussing something with him in his office when he got a phone call related to some administrative responsibility, and he almost gleefully told the caller that he'd have to call back, since he had a student in his office. In fact, Bob had a sanctuary where he could concentrate fully on science. That was building 223, our electronics prep area. And you can tell how important that was to him by the occasional emails he'd send out reminding us gently (most of the time) to keep it clean and well stocked. (The label maker was one of his favorite pieces of equipment. Bob had one bin labeled the "clearly something bin" for miscellaneous mystery items.) 
Speaking of Bob's approachability in the face of high-level responsibilities, I remember when he was chair of the PAC 03 conference. His consolation prize, I guess you could say, for the responsibility of organizing the conference was the presidential suite at the Portland Hilton. So of course, he invited the whole group, including the students, up for a party one evening. The status he had earned just became another opportunity to have a good time, like so many celebrations at the Alpine after a new result or successful thesis defense. In fact we just joked about the setting, especially the presence of a mysterious bright red button in the bathroom of the suite; Bob speculated that it meant "eject."

As another example of Bob's humility, I remember trying to find a shelf I could use to mount some equipment on a rack in that LEAP control room you saw. I couldn't find one anywhere, and the stockroom didn't have any, but a day or two later he managed to find one. When questioned about where he got it, he freely admitted to finding it while dumpster diving in the research yard. Another student recalls Bob setting up BPMs for E163, the successor to the LEAP facility. He always stayed in direct touch - literally - with the experiments.

Bob’s approachability, his down-to-earth character, made him mean much more to me than a mentor and scientist; he was also a friend. I could never have made it through grad school without him. And though I'm no longer at SLAC, I think of Bob often - especially when I'm walking around my neighborhood, since I happen to live just a few hundred feet from his daughter in Longmont, Colorado. I will always be grateful to him. Thank you, Bob. 


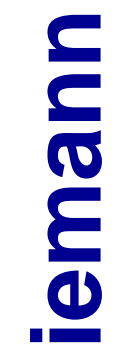

ज

으

ㅇ

$m$

放

?

0

E

(1)

ह

I 


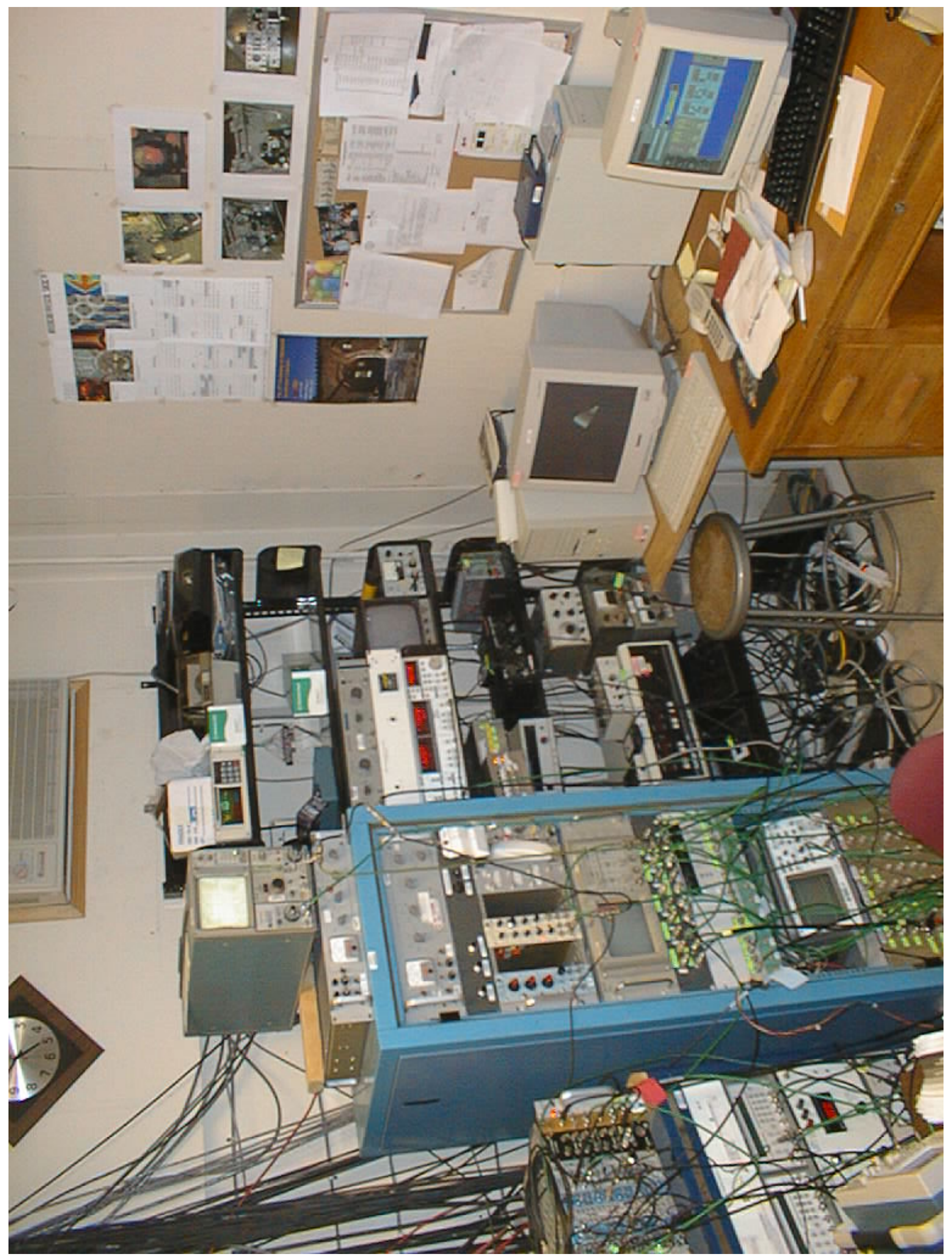




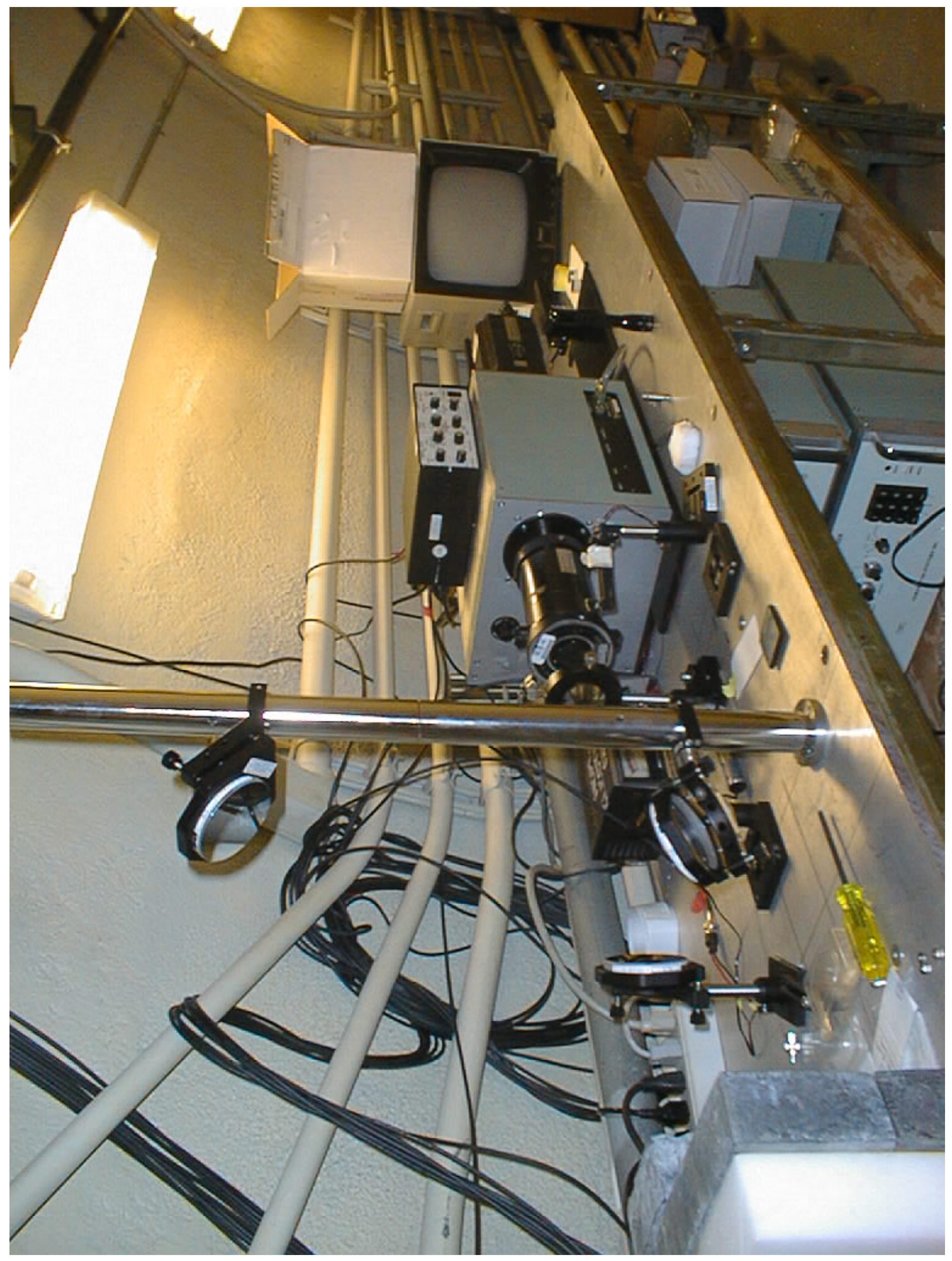




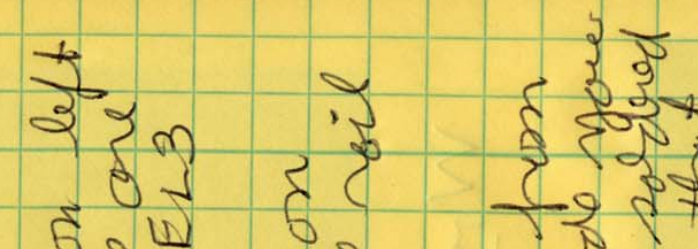

ई इै का दे

इ

$\infty$ ई ई है 1 है

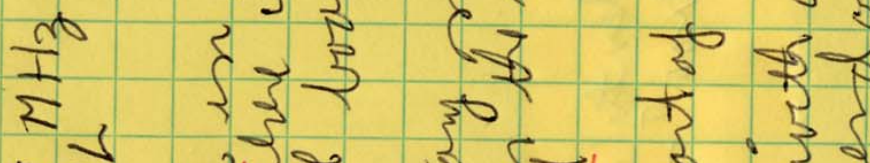

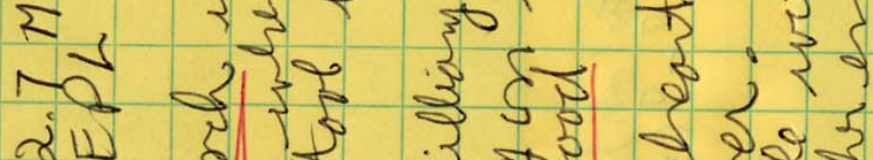

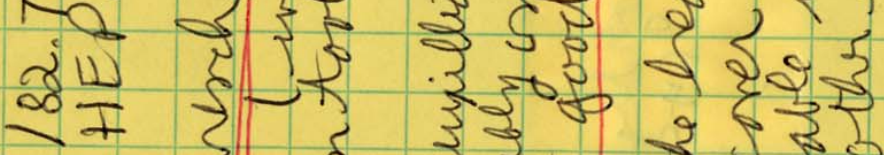

का

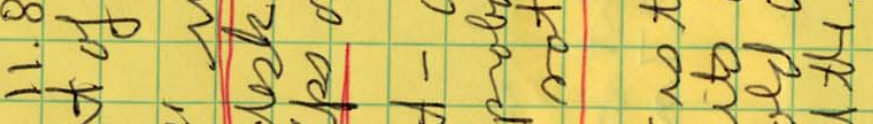

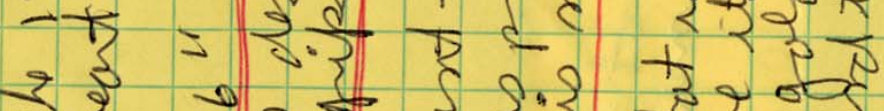

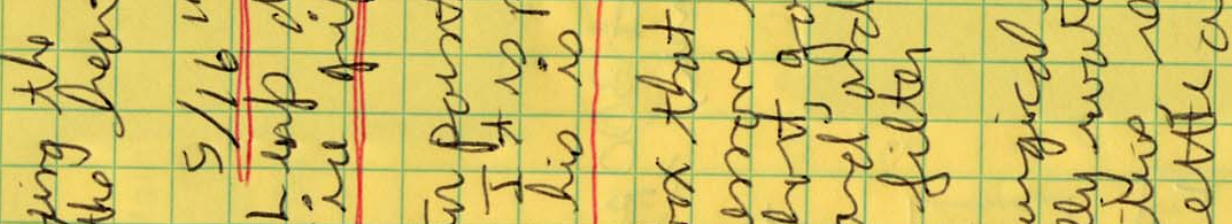

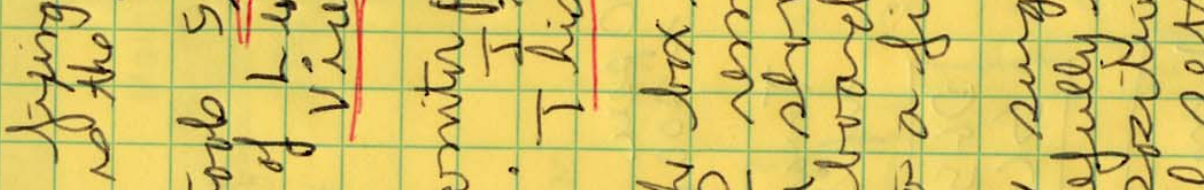

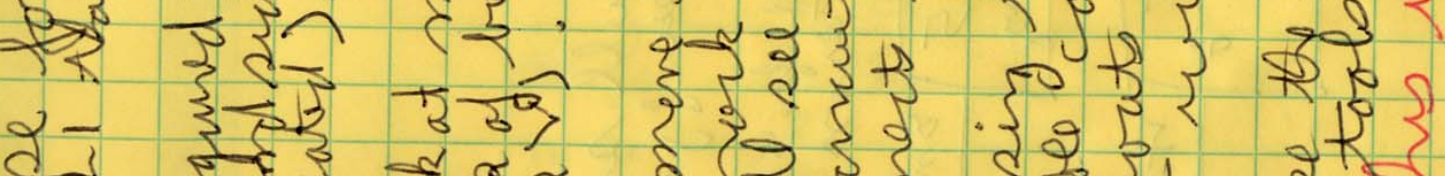

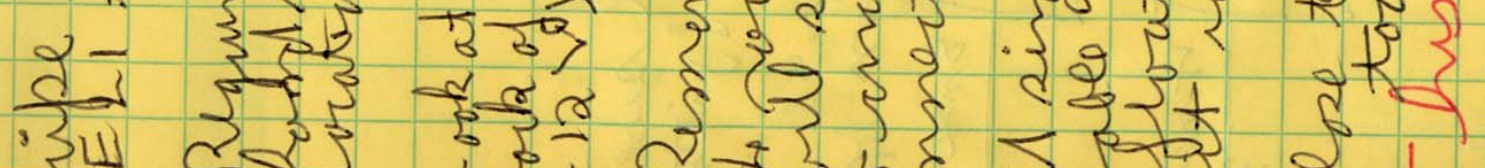

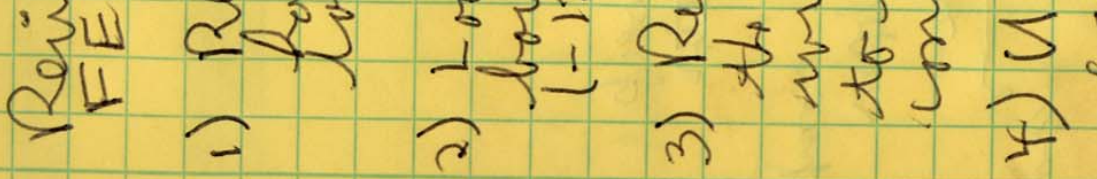

$\mathcal{2}$

in

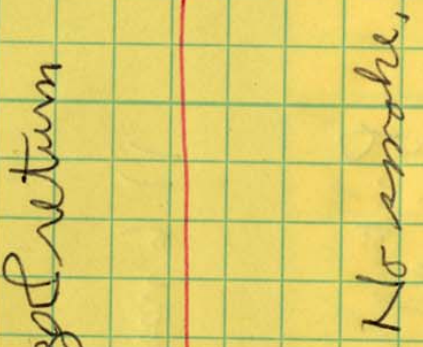

ई

3

$\sum_{2}^{3}$

t

I 3

i) 3 $3 \int_{3}^{\frac{3}{3}} \frac{3}{3}$ \{\}

तु 3 $3+3$ ई $\left.\frac{1}{3}\right\}$ $3_{3}^{5} 5$ 


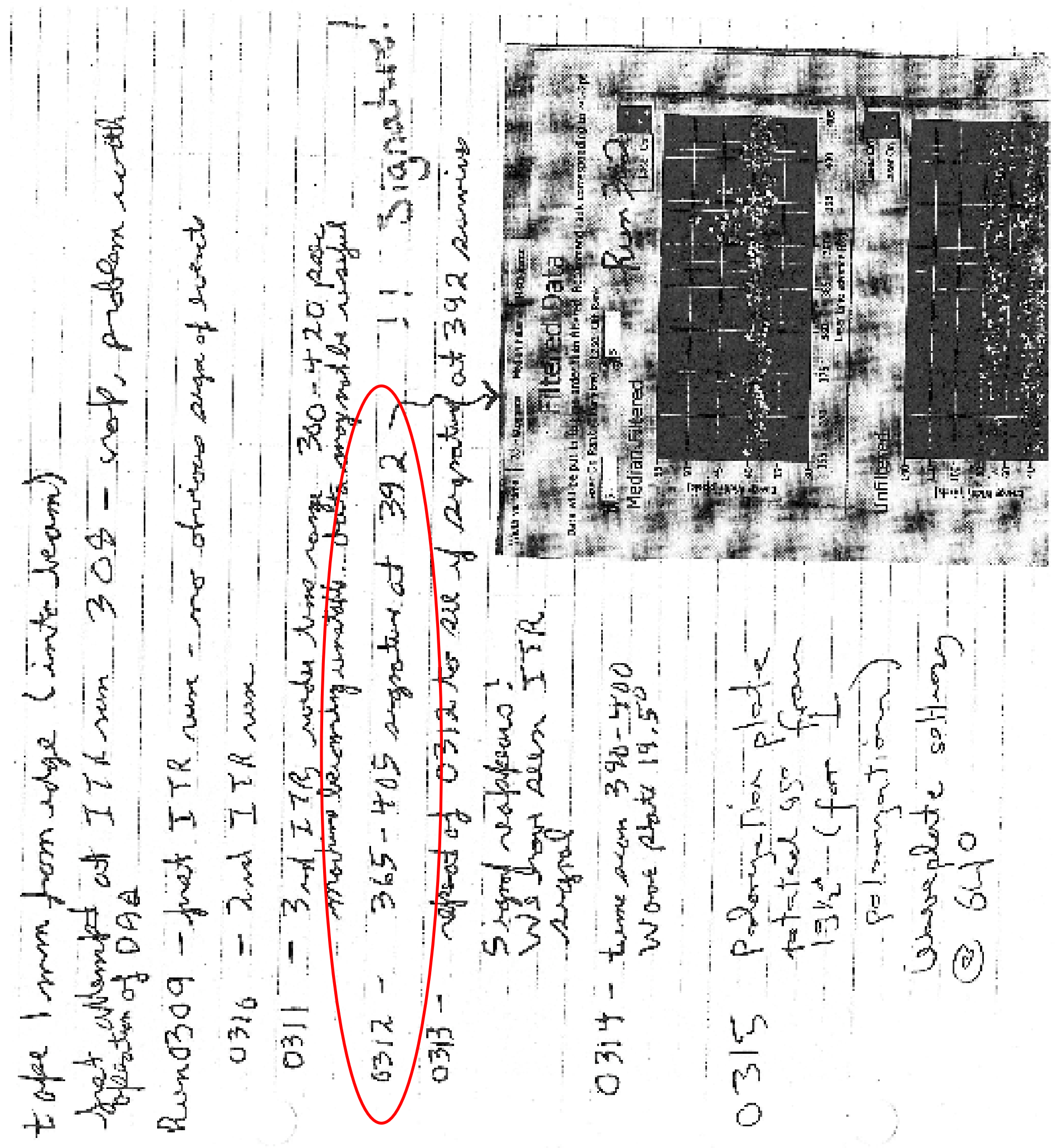




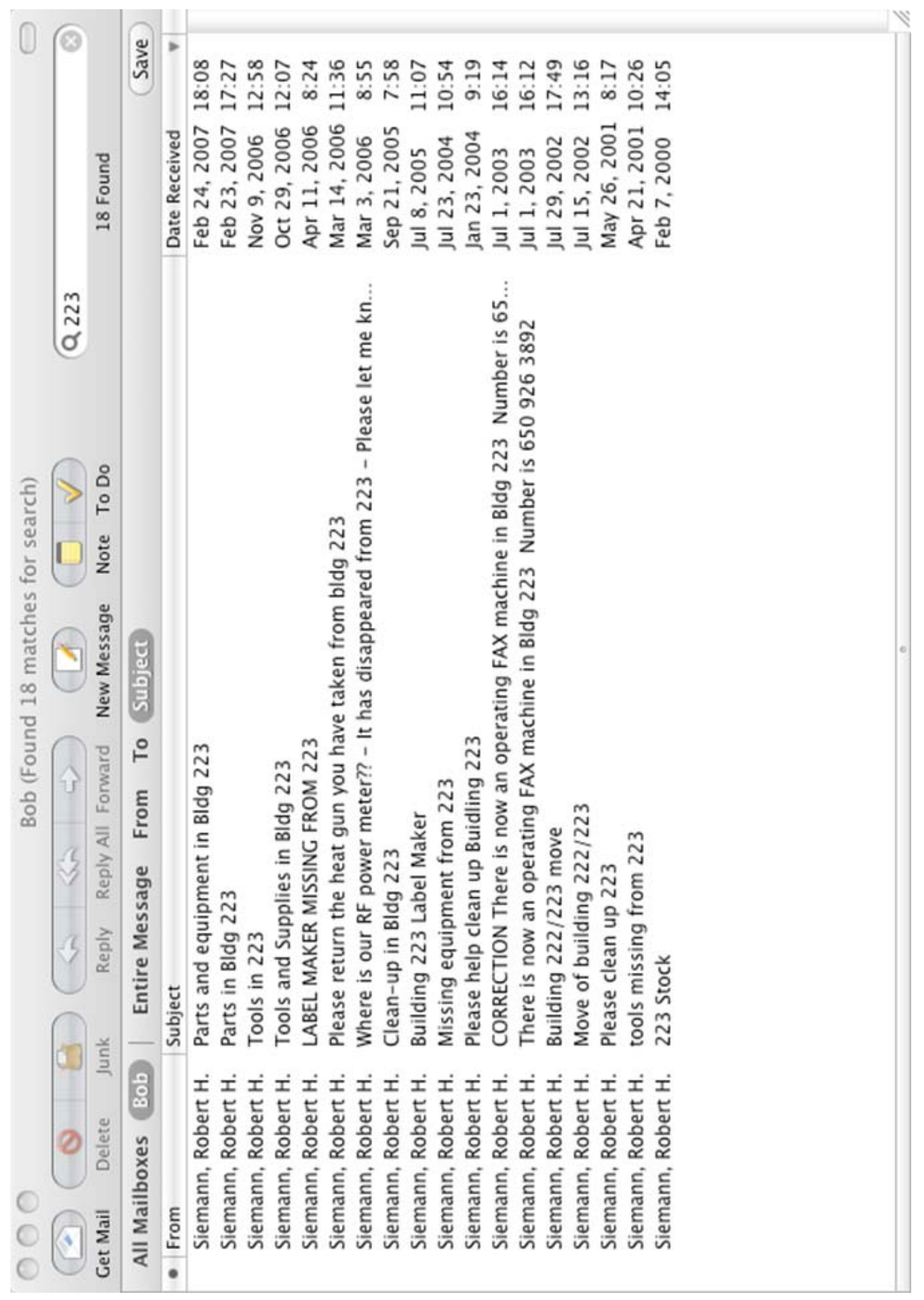




\title{
Bob Siemann and the Community Organizer
}

\author{
Mel Month \\ USPAS Founding Director \\ Longtime DPB Secretary-Treasurer
}

Bob Siemann was a good man. It's not that everything he did was right and proper, or not even that he had a moral edge. No! Like most, sometimes his judgment failed him. Sometimes he did something that hurt someone. So, come to think of it, maybe it's better to see and describe him as a principled man. Before acting, he would instinctively balance the scale of right and wrong, and more often than not, what he did moved our science forward. It's not that the end justified any means he used, but to him it was important, not paramount mind you, but important to leave his chosen field better than he found it. And in that he succeeded.

I met Bob sometime in the eighties. It was probably in 1985 at the Vancouver PAC Conference. There, I ran all over the place with a clipboard trying to accumulate signatures for a petition to form an APS Topical Group (TG) on beams and accelerators. What fun it was cornering people in the effort to persuade them that beams had scholarly merit and so should be represented as a distinct field of study in its own right. In doing this organizing work, as memory recalls, I had a lot of contact with Bob and came to see a mature philosophical side to his nature. I was convinced that work on beams was worthy of scholarly merit, that's why I was there, but he would not easily concede and tended to play devil's advocate. Our talk then was no more than bantering. We both knew he would add his name to the petition. But, as we would discover in a couple of years, this back-and-forth had only been a prelude to discussions about the USPAS method, discussions that would lead to his deep involvement in the accelerator school.

Now, for the record, allow me to take a brief detour and tell you a little story of how the APS Division of Physics of Beams (DPB) got started. As spirited as my Vancouver discussions were with Bob, they were equally so with others the likes of Richter, Panofsky, Lederman, Wilson and Wallenmeyer. And when the dust settled, I found I had over 250 signatures, ten times more than needed according to the APS rules for TG formation. More important than the numbers, the list boasted many of the big boys. Yet, in spite of the size and quality of the petition, permission to form a beams topical group was not a done deal. Most of you probably don't know the inside story of how the petition fared in its travels to find a place for beams in the APS, so let me tell it to you. The APS Executive Committee (EC) met sometime later in 1985 with the matter of the beams topical group on the agenda. There, Havens, the EC Executive Secretary, called for a decision on the petition of the beams community. Almost immediately, Sid Drell, the APS President, objected, saying that beams were an integral part of high-energy and should remain represented within the Division of Particles and Fields (DPF). A topical group on beams would divide the two allies, he said, and, in the end, would be detrimental to both. For a bit after the president spoke, the room was silent. But, just when Havens was about to table the motion, Bob Wilson, the Past President, stepped in. He countered Drell with an eloquent defense of the accelerator field as separate and distinct and claimed that a Beams Topical Group would open the door to the participation of other areas of physics requiring beams and thereby become an even greater asset to high-energy. Well, to make a long story short, Drell backed off, the motion for a beams topical group was passed, membership flourished and, within a year or so, the magic 
1000 mark was reached and the topical group was upgraded to a bona-fide APS division. I'm not sure why Wilson spoke up like that at the last minute, but maybe that hour-long discussion I had with him about a month before the EC meeting played a part.

During the eighties, USPAS was changing and advancing at a fast clip. In 1987, in conjunction with the University of Chicago, the university-style courses were initiated. In 1988, the second such school was held at Cornell. Bob Siemann, then on the Cornell physics faculty, agreed to give a course on "Beam Simulation". He was a tireless teacher and really gave his students a run for their money. During those two hot Ithaca weeks in August of eighty-eight, though he was intensely occupied with the fine-tuning of his lectures, Bob and I found the time to talk and became close friends. There was one issue that clung to us and, in the coming years, we would argue endlessly about the relative merits of a USPAS course as compared to a standard university course. The formal contact hours are much the same, but in USPAS they are concentrated in a 2-week period, while in the university they are spread out over many more weeks. He argued that the time was crucial. For deep and difficult concepts, he would say, the mind needs the time to absorb new ideas so that it can work its miracle of learning. I, on the other hand, argued that immersion provided the mind with the raw material, stimulating it to build a base of knowledge that in time would make for learning of an equal or even greater depth. I further argued that concentration, isolation and nighttime study with teacher contact, all characteristic of USPAS courses, is more conducive to learning than the dilute lecture schedule, competing courses and interfering life activities that prevail in the university environment.

During the years that followed, Bob and I took every opportunity we got to see each other and I came to know his varied talents. Early on, I realized that he was a perfect candidate for an administrative role in the School. After a few conversations about this, he was finally persuaded to take on the job of Program Committee Chairman on a quasi-permanent basis. Understand that Bob was a man with gravitas. He could access the top and the men there would listen to him. Many a time, he would intercede in a disagreement among USPAS Board members as to whether USPAS should or should not do more. In such instances, he and I were a formidable growthpromoting duo. On program matters, he would inevitably come down on the side of curriculum expansion. This turned out to be a key factor in permitting USPAS, in 1992, to move from annual to semi-annual programs. Not only was he a welcome administrative ally, he was also a hands-on type of guy. For the Harvard school in the summer of 1993, I asked him to introduce an innovative and difficult program - a course teaching experimental methods. He grabbed the challenge. Recruiting Gerry Jackson, they dived right in and the result was our Microwave Laboratory course, still a USPAS hallmark.

Our talks on the scholarly value of beams continued into the nineties and, as the decade progressed, I kept pushing for accelerator articles to be published in Physical Review, using my long-term Secretary-Treasurer position in the DPB as base. At the time, beam physics was essentially limited to the two journals, Particle Accelerators and Nuclear Instruments and Methods. But something kept gnawing at me. Since we were part of the APS, why shouldn't beam physics have a place in Physical Review, the society's flagship journal? My efforts had a small effect and articles on beams did begin to appear here and there, but they were spread thin in the various sectors of the journal. We just did not have a place that we could call our own. To accomplish that took Bob’s ingenuity and know-how. He discovered the path that gave Beams 
and Accelerators a true publication home in Physical Review. The story of how Bob, with Marty Blume's visionary thinking and collaboration, created the electronic journal, Physical Review Special Topics - Accelerators and Beams, is truly a fascinating one. But that happened long after I was out of the picture and I'll leave that story to Marty.

Since I heard of his impending passing, many a time thoughts of him would appear spontaneously in my consciousness. I recall our breakfast dialogues at hotels during conferences and meetings all over. And how could I forget the private strategy sessions we engaged in! There were instances that we found ourselves in a moral dilemma. I remember, in the late nineties when I was on the verge of retiring, TD Lee asked me to organize a USPAS School in Beijing. To me, it seemed a good thing to do, for USPAS and for US-China relations. But when I asked Bob to teach one of his patented experimental courses in microwaves, he surprised me. Not only did he refuse but he voiced concern about teaching the communist Chinese advanced technology. I disagreed with him and went on with the program. However, I must say that as time passed, I began to wonder whether perhaps his moral instinct might have been superior to mine.

Yes, Bob was a great friend and the loss of this strong-willed yet thoughtful man will be felt not only by those he was close to but also by the accelerator community as a whole. What a great force he was. He lifted up accelerator education at Cornell, Stanford and at USPAS. Also, recognizing the scholarly value of accelerators and beams, he promoted its publication and was the key figure in giving the field an independent journal within the prestigious Physical Review. There is no doubt that both professionally and personally he will be sorely missed. 


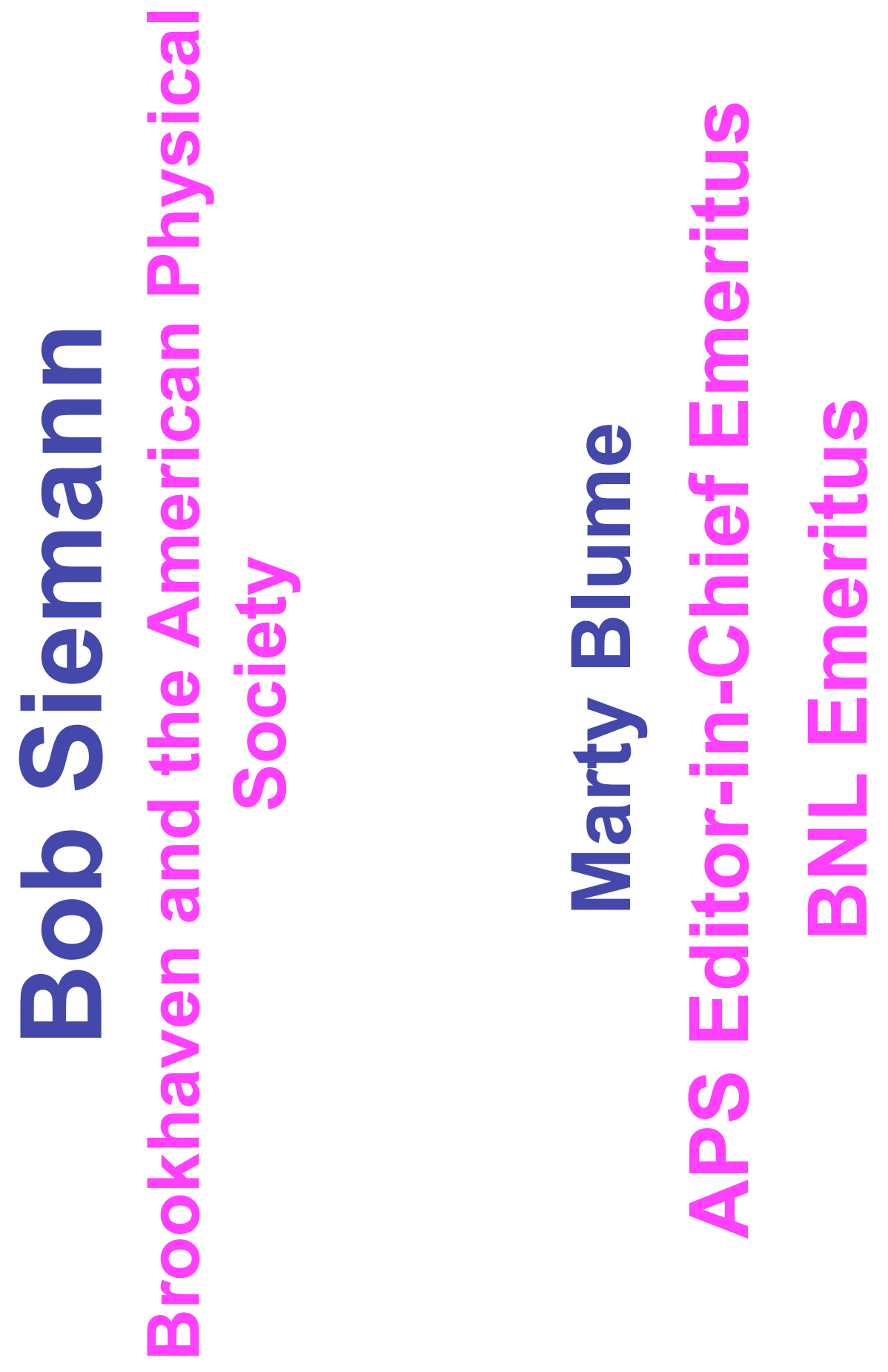




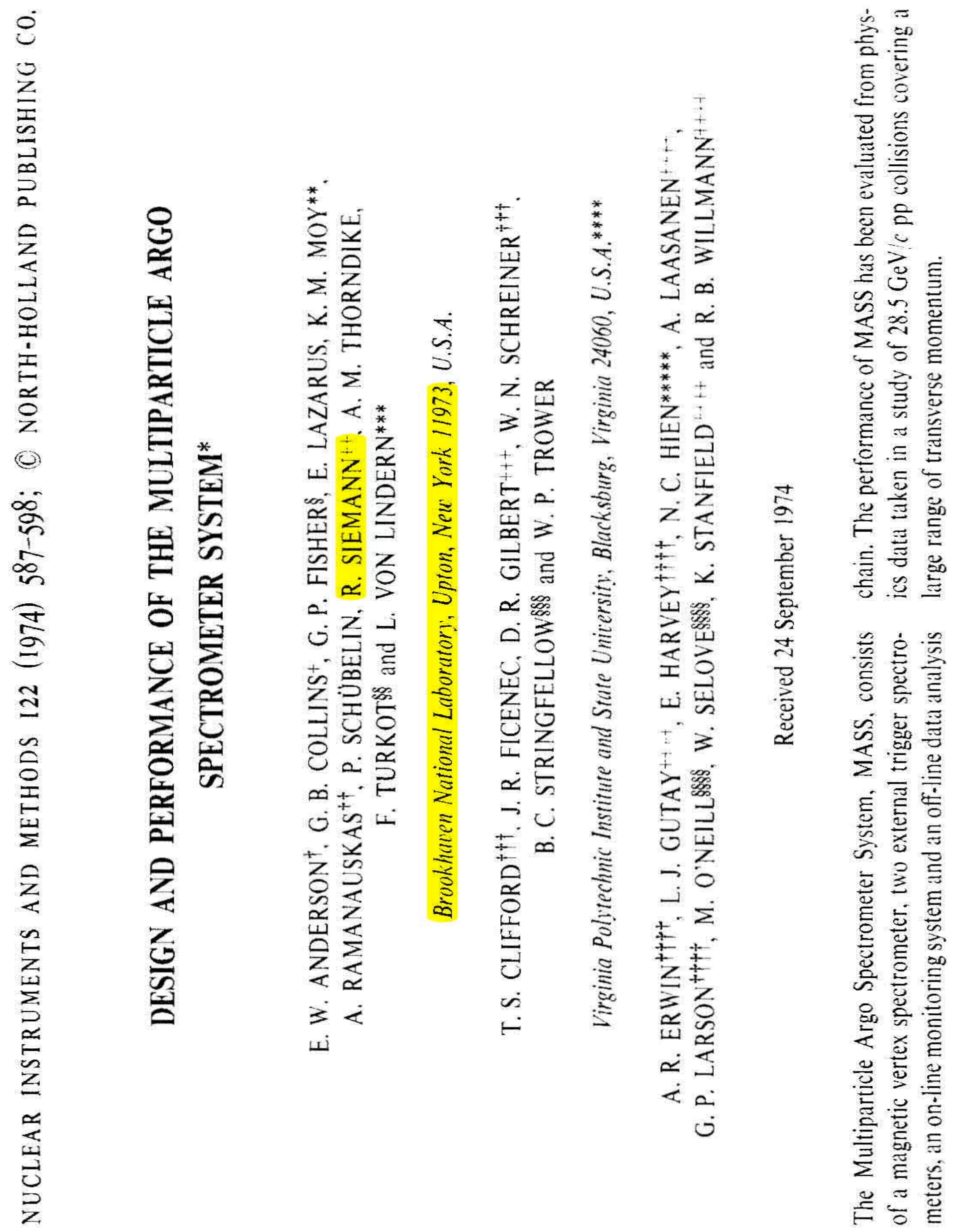




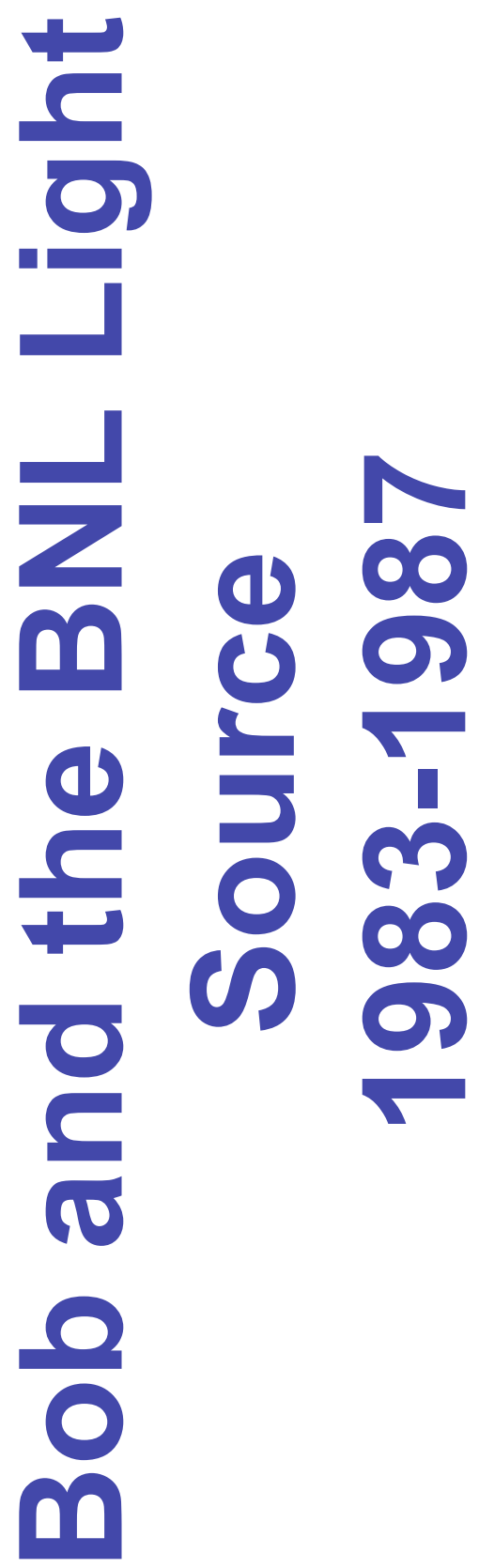



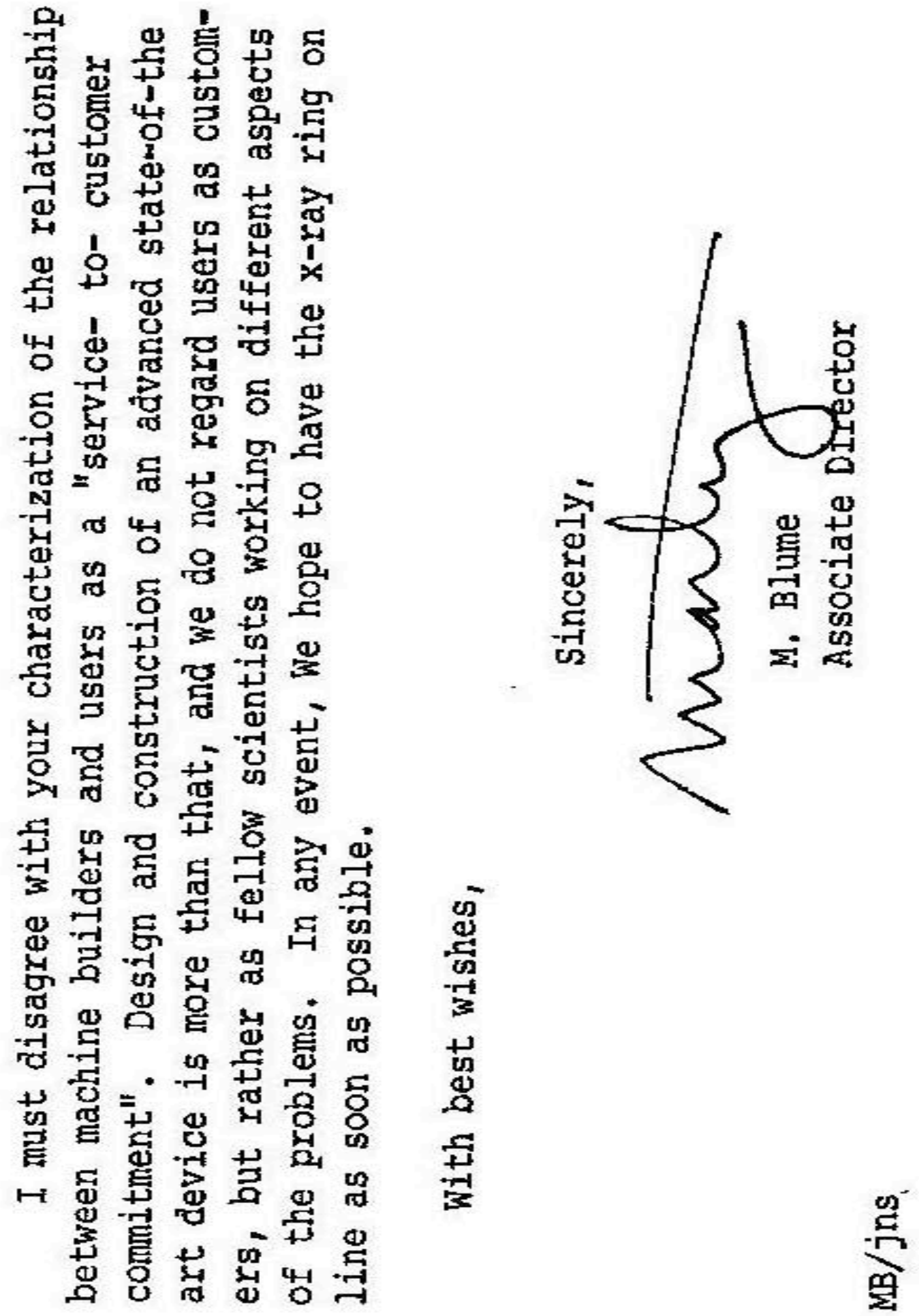


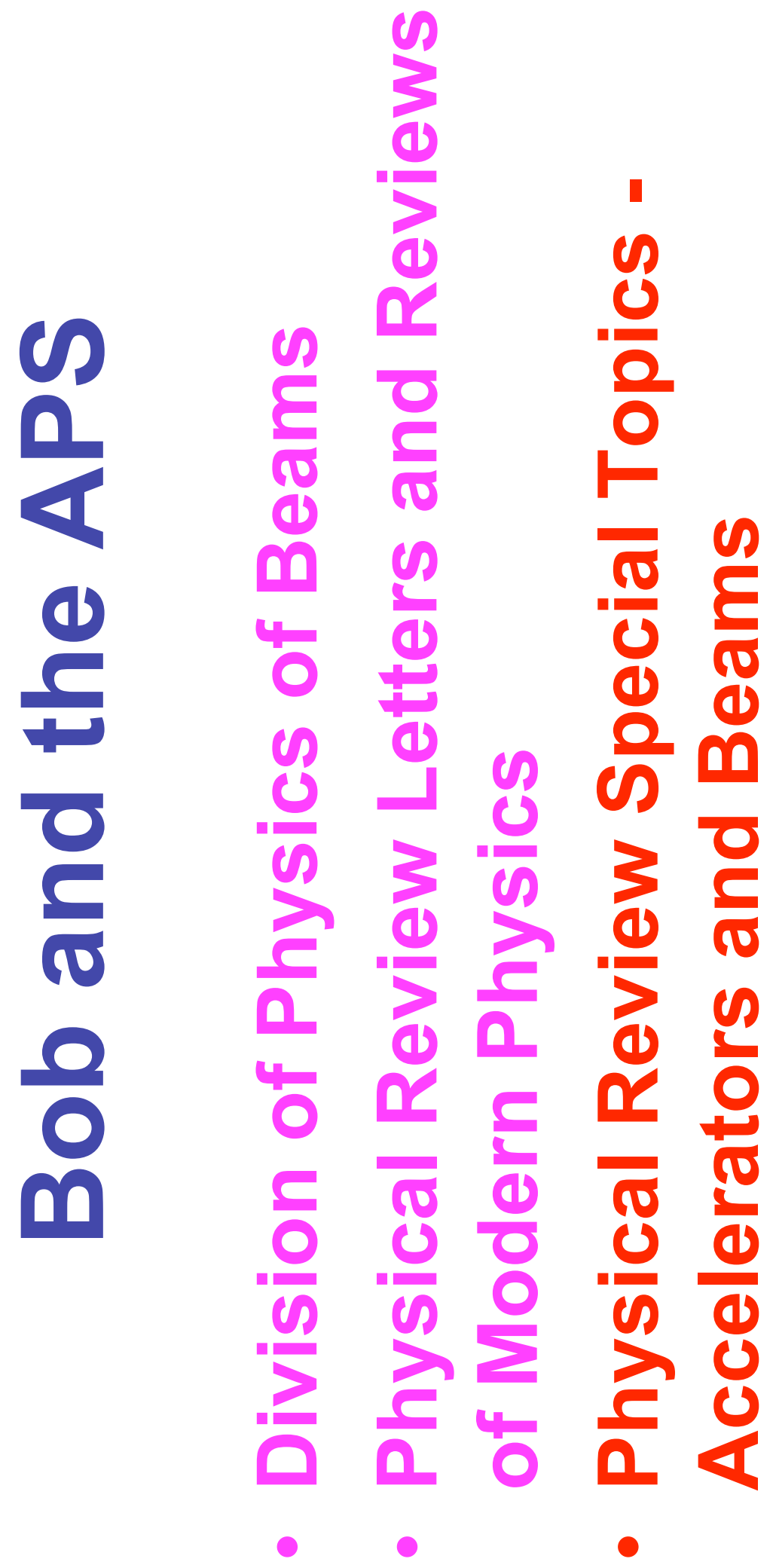




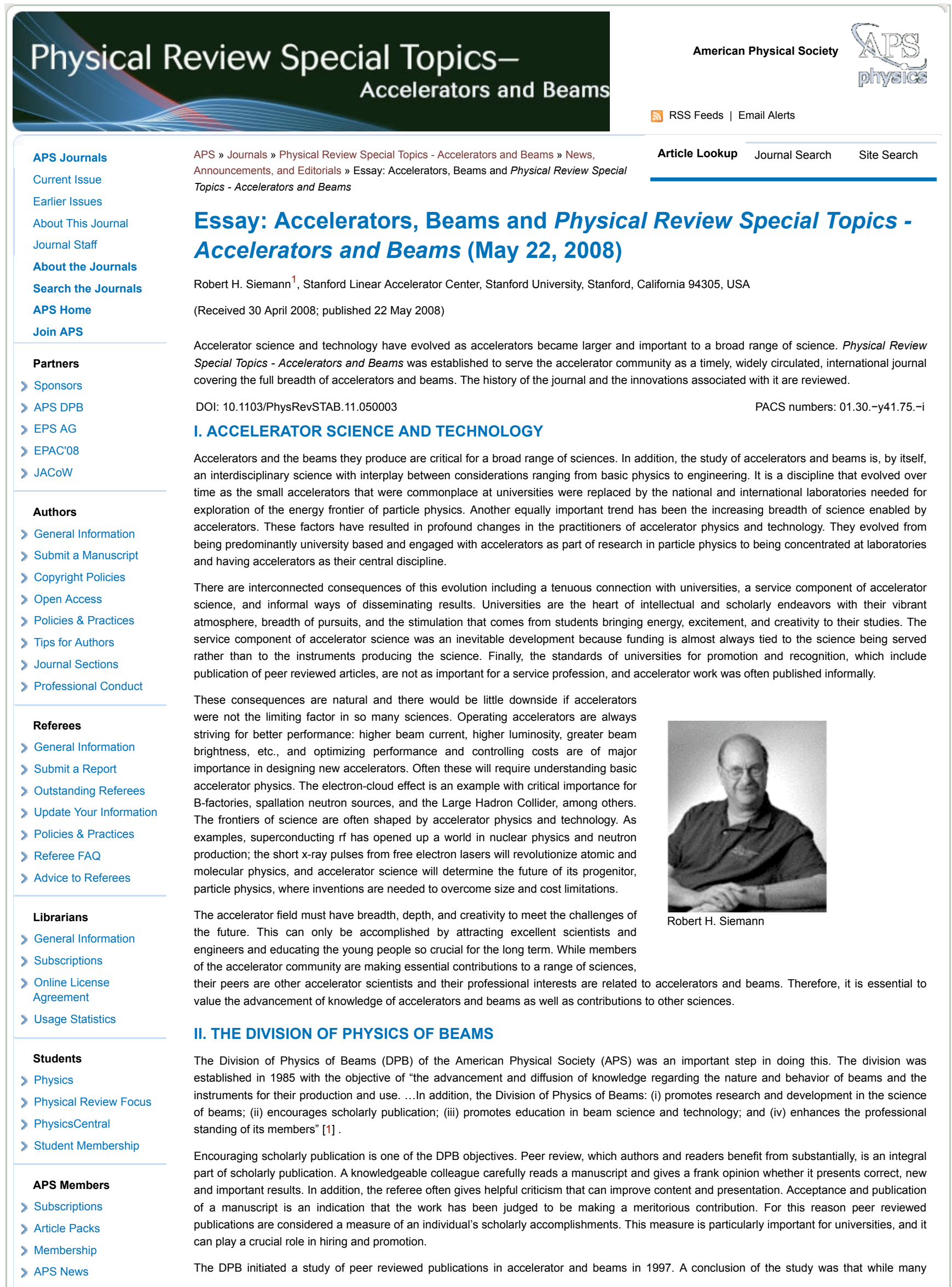


journals published such articles there was no single, widely circulated journal with this as the focus. As a result the literature was fragmented and not effective as a means of communication within the accelerator community. The division recommended establishing a scholarly, peer reviewed journal devoted to the science and technology of accelerators and beams that would (1) cover the full breadth of accelerators and beams, (2) be timely, (3) be inexpensive to promote wide circulation, and (4) be international with an international editorial board and pool of referees. This recommendation led to the establishment of Physical Review Special Topics - Accelerators and Beams (PRST-AB) [2] .

\section{THE EARLY DAYS}

The recommendation reached the American Physical Society at a most opportune time. Marty Blume was the APS Editor-in-Chief. He had previously served as Chair of the National Synchrotron Light Source Department at Brookhaven, and this experience gave him appreciation for accelerator science itself and for the contributions accelerators were making to a wide range of science. In addition, he understood the intimate connection between accelerator science and accelerator technology, and he was willing to champion an editorial policy of publishing both science and technology. This was critical for covering the full breadth of accelerators and beams.

The other factor that made the timing opportune was that the APS recognized that scholarly publication was moving rapidly from hardcopy to electronic format. A small journal like PRST-AB would be an ideal initial effort where lessons could be learned before embarking on electronic versions of the much larger, established Physical Review journals.

Electronic publication satisfied the objectives of timely publication and the potential of wide circulation. However, it had the problem of covering costs, which include, among other things, editorial expenses, composition and production costs, and the costs associated with buying and maintaining servers. Charges to authors or readers would be a significant drawback when establishing a new journal, and it was decided to seek sponsorship from laboratories with large accelerator programs instead. Eight U.S. laboratories agreed to be the initial sponsors, and the APS covered the shortfall in the beginning as a service to the accelerator community and in recognition that PRST-AB was a testing ground for electronic publication. As a result, PRST-AB was available to authors and readers at no cost and without a subscription.

Sponsorship has grown steadily, and PRST-AB now has twenty sponsors: (i) Argonne National Laboratory, (ii) Brookhaven National Laboratory, (iii) The Cockcroft Institute, (iv) Cornell University Laboratory for Elementary-Particle Physics, (v) Deutsches Elektronen-Synchrotron (DESY), (vi) 11th European Particle Accelerator Conference (EPAC '08), (vii) European Organization for Nuclear Research (CERN), (viii) Fermi National Accelerator Laboratory, (ix) Gesellschaft für Schwerionenforschung mbH (GSI), (x) INFN-Laboratori Nazionali di Frascati, (xi) INFN-Laboratori Nazionali di Legnaro, (xii) INFN-Laboratori Nazionali del Sud, Catania, (xiii) Lawrence Berkeley National Laboratory, (xiv) Los Alamos National Laboratory, (xv) National Superconducting Cyclotron Laboratory at Michigan State University, (xvi) Oak Ridge National Laboratory, (xvii) Princeton Plasma Physics Laboratory, (xviii) Stanford Linear Accelerator Center, (xix) Thomas Jefferson National Accelerator Facility, and (xx) TRIUMF-Canada's National Laboratory for Particle and Nuclear Physics. Because of their generous support, PRST-AB continues to be provided without charge to authors or readers.

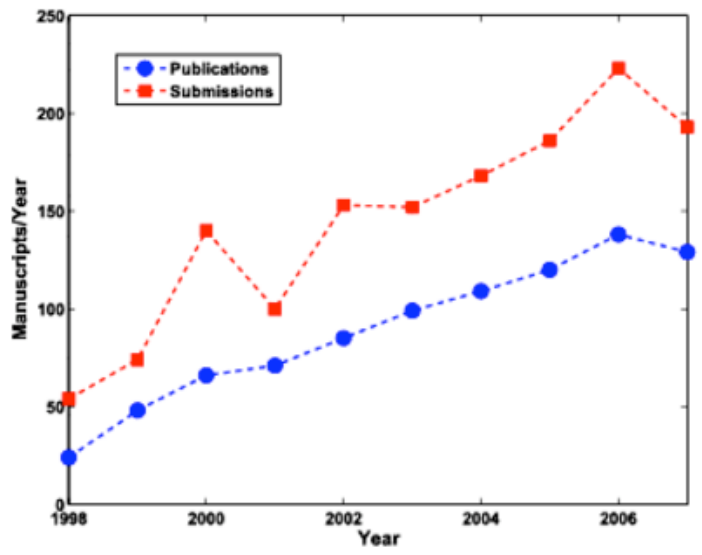

FIG. 1. PRST-AB submissions and publications.

Publication of PRST-AB was approved by the APS Council in November 1997, and I was appointed the Editor shortly afterward. A critical meeting was held at the APS Editorial Office in early 1998. It was decided to move forward on an aggressive schedule with the target of being prepared to accept the first submissions in March. This was accomplished. The first PRST-AB submission [3] was received on March 2 and published on May 12. PRST-AB has grown steadily since that first submission (Fig. ). In 2007193 papers were submitted, and 129 papers were published.

\section{INITIATIVES AND INNOVATIONS}

PRST-AB is a small, electronic journal in a specialized field with a publisher who is interested in and encouraging of innovation. Sponsorship was one of the most profound and significant ones. As a consequence of sponsorship PRST-AB is freely available, making it one of the first open access journals and a harbinger of the developments at the forefront of scientific publication today.

It was a goal from the beginning to make PRST-AB a truly international journal. Initially this was done informally through the selection of members of the Editorial Board and by using referees from all over the world. These practices have continued to this day. A more formal arrangement was made in 2002 with the European Physical Society Accelerator Group (EPS-AG) [4] when they joined with the APS-DPB to form the Affiliated Professional Groups of PRST-AB. Together they share the responsibility for the health and vitality of the journal by providing advice and encouraging scholarly publication in accelerator science and technology. The EPS-AG has been instrumental in securing sponsorship from laboratories in Europe, and as part of this agreement either the Editor or an Associate Editor is from Europe.

There were a number of innovations related to the electronic only format. These included the following

All electronic editorial process.-All communications with authors, referees, and editors were electronic and provided the first APS experience with an all electronic editorial process. PRST-AB is now integrated with the "paperless" office used for all the APS journals.

Special editions, conference editions, and special collections.-The all-electronic nature allows editions of peer reviewed articles that are 
associated with conferences or that are about a closely related subject. These articles are published in a regular monthly issue as well as being added to the "special" Table of Contents that is updated when the article is published.

"Virtual Journal" of accelerator related articles published by the APS.-Links to beam physics articles in Physical Review Letters and Physical Review $E$ are available on the PRST-AB Table of Contents so readers can find links to all of the accelerator related articles published by the APS in one location.

Single column PDF format.-The standard, double-column APS format is not easy to read on a computer screen, and PRST-AB articles are published in single- and double-column formats.

Movies.-Some things can best be visualized with a movie rather than a static figure, and movies can be included in articles. This has not been used by many authors to date, but looking at Optics Express [5] gives one an appreciation for the potential of this manner of presentation.

Readers requested other features that have been implemented.

Review articles.-Reviews of topics of particular interest to accelerator scientists and engineers are published.

Monthly Email notification.-The Table of Contents of each monthly issue is Emailed to interested parties. More recently RSS feeds have been made available for all the Physical Review journals.

\section{PRST-AB, ACCELERATORS AND PUBLISHING}

PRST-AB is a timely, widely circulated, freely available, international journal devoted to accelerator science and technology. It has become the premier scholarly, peer reviewed journal in this field. It is part of what is needed to advance the knowledge of accelerators and to attract excellent scientists and engineers. By doing this it will have a significant impact both within this field and in the broader range of sciences that depend on accelerators.

In addition, PRST-AB had the good fortune of opportune timing combined with a publisher interested in serving the physics community and exploring new methods of publication. As a result PRST-AB was an early, successful experiment in electronic, open access scientific publication.

PRST-AB would not have been possible without Marty Blume. Debbie Brodbar is the PRST-AB Senior Assistant Editor. She began her crucial role of providing assistance with and insight into PRST-AB editorial matters from the very beginning. Chris Wesselborg was the Project Manager who oversaw the implementation in early 1998 that took the journal from an idea to realization. I wish to thank Judy Franz, the APS Executive Officer, and Tom Mcllrath, the APS Treasurer and Physical Review Publisher, for their support of PRST-AB and the accelerator community. PRST-AB started as an initiative of the Division of Physics of Beams. Martin Reiser was the DPB Chair who commissioned the study of accelerator publication and was a strong backer of establishing PRST-AB. Mel Month was the DPB Secretary/Treasurer for many years. I learned much from him about accelerator science and scholarship. Many people at the APS Editorial Office in Ridge, NY, made important contributions. In particular, I want to acknowledge Stanley Brown, Bob Kelly, Bill Rushka, Mark Doyle, Arthur Smith, Margaret Malloy, Joanna Popadiuk, and Evelyn Flores. Angie Seymour and Stephanie Santo provided invaluable editorial support for me at SLAC. Mike Poole, Francesco Ruggiero, Brant Johnson, and Chris Wesselborg were Associate Editors during my tenure, and I wish to thank them for their contributions and hard work. Thanks also to the members of the Editorial Board. They were an excellent sounding board and always willing to help with difficult papers. Frank Zimmermann is the present Editor, and Georg Hoffstaetter and Brant Johnson are the Associate Editors. They took over PRST-AB about a year ago, and I am delighted to see it thrive under their leadership. I hope they find the satisfaction that I did.

${ }^{1}$ Robert Siemann is a Professor of Particle Physics and Astrophysics at the Stanford Linear Accelerator Center. He earned a Sc.B. degree in physics from Brown University in 1964 and a Ph.D. in physics from Cornell University in 1969. He returned to Cornell in 1973 as a faculty member in the Physics Department after a postdoctoral appointment at SLAC and a staff position at Brookhaven National Laboratory. He rose through the faculty ranks at Cornell, and his professional interest shifted from experimental particle physics to accelerator physics. He joined the SLAC faculty in 1991 and is also a Professor of Applied Physics, by Courtesy, and the head of the Advanced Accelerator Research Department. His present research is in the areas of plasma and laser-driven acceleration. He was the Editor of Physical Review Special Topics - Accelerators and Beams from its founding in 1998 through 2007.

1. Abstracted from the DPB homepage http://units.aps.org/units/dpb/index.cfm.

2. http://prst-ab.aps.org/

3. F. Clapier et al., Phys. Rev. ST Accel. Beams 1, 013501 (1998).

4. The EPS-AG homepage is http://epac.web.cern.ch/EPAC/EPS-AG/Welcome.html.

5. Optics Express published by The Optical Society of America and available at http://www.opticsexpress.org.

APS | Journals | Contact Information | Phys. Rev. ST - Accel. Beams | Join APS | Feedback

ISSN 1098-4402 @ 2009 by The American Physical Society All rights reserved. Physical Review Special Topics - Accelerators and Beams ${ }^{\mathrm{TM}}$ is a trademark of The American Physical Society. Use of APS online journals implies that the user has read and agrees to the Terms and

Conditions in the Subscription Agreement. 


\section{COURIER}

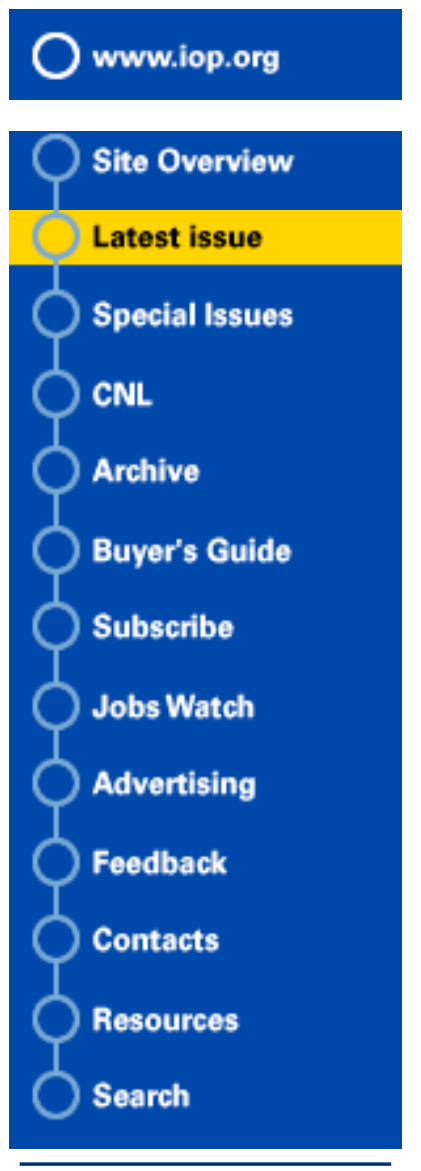

Author:

Ken Peach

\section{This Issue | Back Issues | Editorial Staff}

\section{Viewpoint}

\section{Join the open-access revolution}

\section{Ken Peach argues that particle physicists can lead the way in a paradigm shift in scientific publishing to give everyone free access to research results.}

There is a quiet revolution under way in academic publishing that will change how we publish and access scientific knowledge. "Open access", made possible by new electronic tools, will give enormous benefits to all readers by providing free access to research results.

The scientific articles published in journals under the traditional publishing paradigm are paid for through subscriptions by libraries and individuals, creating barriers for those unable to pay. The ever-increasing cost of the traditional publishing methods means that many libraries in Europe and the US - even the CERN

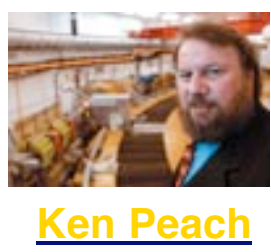
Library, which is supposed to serve international researchers at a centre of excellence - are unable to offer complete coverage of their core subjects.

In 2003 the Berlin Declaration on open access to knowledge in the sciences and the humanities was launched at a meeting organized by the Max Planck Society. Six months later, the first practical actions towards implementing the recommendations of the declaration on an international level were formulated at a meeting held at CERN in May 2004. So far the declaration has been signed by 61 organizations throughout the world, which are now taking concrete measures for its implementation.

An obvious prerequisite for open access is that institutions implement a policy requiring their researchers to deposit a copy of all their published works in an open-access repository. The Council for the Central Laboratory of the Research Councils' library committee in the UK sponsored such a project, ePubs, with the aim of achieving an archive of the scientific output of CCLRC in the form of journal articles, conference papers, technical reports, e-prints, theses and books, containing the full text where possible (CERN Courier May 2005 p44).

The feasibility study, carried out from January to March 2003, demonstrated the business need for this service within the organization. The data, going back to the mid-1960s, can be retrieved using the search interface or the many browse indices, which include year, author and journal title. In addition the ePubs system is today indexed by Google and Google Scholar. The scientific content of the system has further led Thomson ISI (the provider of information resources including Web of Knowledge and Science Citation Index) to classify ePubs as a high-quality resource.

The next step is to encourage the researchers - while of course fully respecting their academic freedom - to publish their research articles in 
open-access journals where a suitable journal exists. In recent years new journals applying alternative publishing models have appeared in the arena. The problem so far is that none of these journals have a long-term business model. They are sponsored either by a research organization or by other titles in the publisher's portfolio, or enjoy sponsorship that will not last forever.

Scientific publishing has a price and will continue to have a price, currently mainly covered by academic libraries through subscriptions. Moving to an open-access publishing model should dramatically reduce the global cost for the whole of the academic community. The publication costs should be considered a part of the research cost and the research administrators should budget for these when the research budgets are allocated. However, a change must not take place without safeguarding the peer-review system, which is the guarantor of scientific quality and integrity.

Outside biology and medicine, few journals that support open access are given the same academic credits as the traditional journals. This situation is further reinforced if there is a direct coupling between research funding and the "impact factors" of journals where results are published. However, by taking the risk and publishing important work in new journals that implement the open-access paradigm, the impact factor will automatically be enhanced.

The example of the Journal of High Energy Physics (JHEP) is striking. This relatively new journal was launched by the International School for Advanced Studies (SISSA) in Trieste in 1997. Today some studies give it an impact factor close to that of Physical Review Letters in publishing papers on high-energy physics. JHEP was launched ahead of its time and was forced, because of the lack of financial support, to become a subscription journal. However, with the support of the main physics laboratories, it would be possible in the present climate for this successful journal to enter the open-access arena once again.

If a change is wanted, it is up to us. Particle physics cannot change the world alone, but a clear position among our authors and our members of editorial boards will have a strong synergy with our colleagues pulling in the same direction in other fields.

- For more about the Berlin Declaration see www.zim.mpg.de/openaccess-berlin/berlindeclaration.html.

\section{Author:}

Ken Peach, director of particle physics, CCLRC Rutherford Appleton Laboratory.

Copyright $($ IOP Publishing Ltd 1998-2005. All rights reserved

\section{Previous article I Next article}




\section{Open access in accelerator physics}

It pleases me that the CERN Courier, itself an open-access journal, has put the debate of scientific publishing on the agenda. Ken Peach's "Viewpoint" (CERN Courier June 2005 p50) is an excellent contribution to the discussion and should be carefully read by everyone in the publication chain.

In the field of accelerator physics a major fraction of our literature is nowadays made available through open-access publications. Our main conference series, i.e. PAC, EPAC, and APAC, are all published as open-access through JACoW (http://accelconf.web.cern.ch/AccelConf/). Our scientific articles are to a large extent submitted to Physical Review Special Topics Accelerators and Beams (PRST-AB), a peer-reviewed, all-electronic journal published by the American Physical Society. The journal is available to everyone without subscription or pay-per-view fees and even without author charges, all thanks to the support of sponsors.

PRST-AB has quickly become the primary means of communicating new results in accelerator physics, but so far, unfortunately, no European institutions are among its sponsors. I hope that this situation will soon change to ensure the continuity of open-access publishing in accelerator physics.

Frank Zimmermann, CERN.

\section{A question of sponsorship}

Ken Peach's article on open access was met, on my part, with a combination of a grain of salt and wry amusement. The American Physical Society started an all-electronic open-access journal called Physical Review Special Topics - Accelerators and Beams that has been successfully published since 1998 . The journal has been endorsed by the Division of the Physics of Beams of the APS and the Accelerators Group of the European Physical Society. It has been supported by sponsorship contributions from large accelerator laboratories in the US and Canada, with no charges either to authors or to readers. This only partially covers its costs, and the shortfall has to be made up by the APS. This is sustainable in the short term, but becomes an increasing burden as more articles than the current 10 per month are published.

Periodically I hear of endorsement by one or another part of CERN for open-access journals, but in spite of occasional requests CERN 
has declined to become a sponsor of PRST-AB. To date 52 articles have been published with one or more CERN authors, and more have been based on results from CERN accelerators. Isn't it time that CERN - one of, if not the, largest accelerator laboratories in the world - came forward?

Martin Blume, editor-in-chief, APS.

\section{Sponsoring open access: more than just wry amusement} (November 2005)

I am delighted to witness the high interest in open access that exists and has been demonstrated by the series of letters on the topic recently published in these pages. In addition, a well-attended debate on "The changing publishing model" held at CERN in September shows that physicists are concerned with this issue.

Following the endorsement of the new CERN publishing policy in March, the library has taken certain steps to support open access where it can. It is therefore my pleasure to announce that CERN will be, as of 2006, the first European financial sponsor of Physical Review Special Topics - Accelerators and Beams. I hope publishers and editors of journals in particle physics will also consider a similar model: in the current climate a publishing model based on sponsorship shows significant potential, at least for the megasciences.

For the future, I would like to propose that we do not mix peerreview and long-term archiving into the debate. Both are of obvious importance, but neither is relevant to the difference between openaccess publishing and the traditional model. The open-access model can of course incorporate peer-review, and with regards to archiving, even traditional subscription journals will most likely be electronic-only in a few years. The real debate is how to finance publishing activities and at the same time ensure equal access for everyone to results from publicly funded research.

A change to full open-access publishing cannot be made by one of the actors in the publishing chain alone. CERN is therefore hosting, in December, a tripartite meeting, which will include funding agencies, research organizations and publishers who already implement some form of open-access publishing. At the workshop we will hope to use our years of open-access experience to work 
out a common strategy for the transition to the new publishing paradigm.

Corrado Pettenati, CERN Library. 


\section{Remarks from the APS Chair \\ Swapan Chattopadhyay}

Well, this has been a wonderful day of reminiscing.... and celebrating Bob and his multifaceted contributions - as an outstanding scientist, teacher, mentor, severe critic of mediocrity at all times and most notably, an untiring servant of the professional society that we call the APS (American Physical Society). On behalf of the entire community of scientists that hails APS as its professional home, in particular on behalf of the scientists and engineers who call APS's Division of Physics of Beams as their home, it is my distinct privilege, honor, and humility to say 'thank you' to the entire Siemann family for sharing this extra-ordinary family member with us in all its glory and grief...!

I have shared a meal or two with Hannah while on invitation from Bob at Cornell or SLAC as symposium/colloquium speaker. I have known about Hannah's love of horses. I have heard about his children, though they do not know me. I have known Bob's frustration while spending time away from Cornell at Fermilab in transition to SLAC; I have known the brilliance of his students and post-docs who he shared with me over time; I have known his impatience as a collaborator in plasma-acceleration experiments at SLAC.

But it is his total dedication to the society APS and all it entails-DPB, Particle Accelerator Conferences, the APS journal in our field PRST-AB, ... many of which he has founded and created himself-that I must uphold, celebrate and bring to our collective acknowledgement from my platform. And it is with this sense of gratitude that I will close my remarks today-reinforcing Persis Drell's announcement this morningthat APS, and its Division of Physics of Beams, is motivated more that ever to contribute to Siemann-fellowship and I pledge to work with our Executive Committee to make it happen.

To "Bob"

Swapan Chattopadhyay

Chair, APS-DPB 


\section{Robert H. Siemann Symposium Closing}

E. R. Colby, 7/7/09

Thank you, Swapan, for sharing remarks from the APS.

I have the privilege of closing the Symposium today by offering a few final comments and words of thanks to those who helped make this event possible.

I met Bob at an annual review of Fermilab in 1996. I was an impressionable grad student presenting a poster; he was the extremely tall committee member who questioned me at length. He asked pointed questions about the simulations and the hardware I had developed. He warmed toward the end of the exchange, leaving me feeling relieved that I had not damaged the program. What I'd not appreciated was that I had been interviewed.

This combination of technical insight and, to use one of Bob's favorite words:

'craftiness', were two of his essential attributes. These traits, together with the conviction that accelerator science is an intellectual pursuit worthy of a university that has given rise to a tremendous legacy.

Bob's contributions to accelerator science span more than 200 publications, covering such diverse topics as wakefield theory, pulsed heating, acceleration of electrons with light, and energy doubling of the SLAC linac, among others.

Bob's technical judgment was prized by the community. Through service on more than 100 national and international committees, Bob advised governments, lab directors, corporations, accelerator schools, machine designers, journal editors, and others. He served on panels that established the long-term directions of High Energy Physics, Light Sources, Fusion and Nuclear Research. He gave advice on such diverse machines as the Tevatron, SSC, Advanced Photon Source, Laser Interferometer Gravitational-wave Observatory, and Spallation Neutron Source.

Trained with one of the last generations to span both the accelerator and experimental sides of high energy physics, Bob willingly rolled up his sleeves and engaged in theory, computer simulation, experiment, and wire wrapping. [explain wire wrapping]. He was as excited about discovering physical connections between the subtleties of complex analysis and spectral features of wakefields as he was seeing a new BPM circuit work flawlessly. Bob often relaxed on Saturday mornings in the electronics lab developing new circuitry for his experiments.

Bob appreciated that progress in accelerator design required more than technical prowess. Advancements in the performance of accelerators — of the scale and magnitude depicted in the Livingston plot-require deep understand of electromagnetism, material science, and an insight that penetrates faster than simulations can provide. This portion of the craft - the science of accelerators — demands the mathematical rigor and clear-headed application of the scientific method to uncover subtleties that are not evident from a 
"simple application of Maxwell's equations". This portion of the craft is clearly worthy of the University.

Bob's conviction in this idea contributed to the expansion of Stanford's academic charter to admit accelerator science, and accelerator scientists as full professors. It led to the founding of a department - with the character of a university research group—dedicated to accelerator science. Through this department—as earlier at Cornell—Bob trained many young students in accelerator science. He was widely respected as a teacher, giving his time willingly when asked, and even when the frowning face of the little statue on his desk was facing the door. (This was Bob’s “do not disturb” sign).

Bob developed courses in accelerator physics and in experimental techniques, teaching at the USPAS, NATO ASI, and US-CERN joint accelerator school in addition to Cornell and Stanford. In several cases, the courses were the first of their kind-for example the USPAS Microwave Laboratory developed with Gerry Jackson—and are still a valued part of the curriculum today.

Bob contributed to the founding of a new unit of the American Physical Society to provide a new forum and voice for beam physicists, and served as an early chair of the division.

He contributed to the establishment of a new segment of the prestigious Physical Review journal dedicated specifically to accelerator science, where he served for a decade as Editor-in-Chief. Where there once was diffused documentation of accelerator science through the conference proceedings and journals, there now is a central archival journal for recording this body of work. The journal is innovative both in content and in form: it's both free and all-electronic.

I hope you will join me in a round of applause in recognition of all that Bob has done for our field.

Bob trained more than 30 young scientists and touched the lives of hundreds more. These individuals today contribute, as Bob did, to a wealth of human endeavors ranging from computer chips, to medicine, to solar power, to — of course—accelerator physics.

I am grateful for opportunity to have known and worked with him.

[thanks to organizers] 

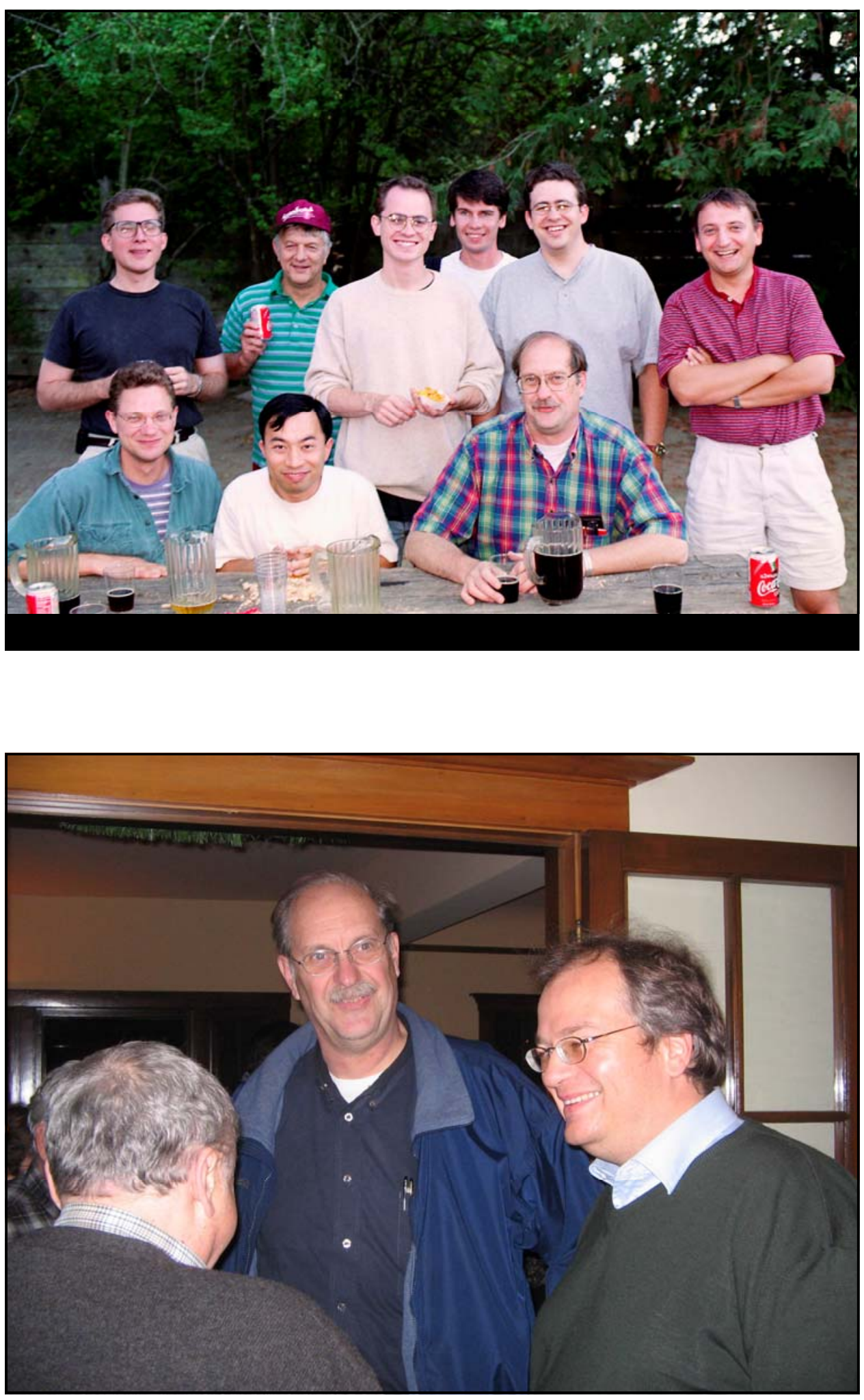

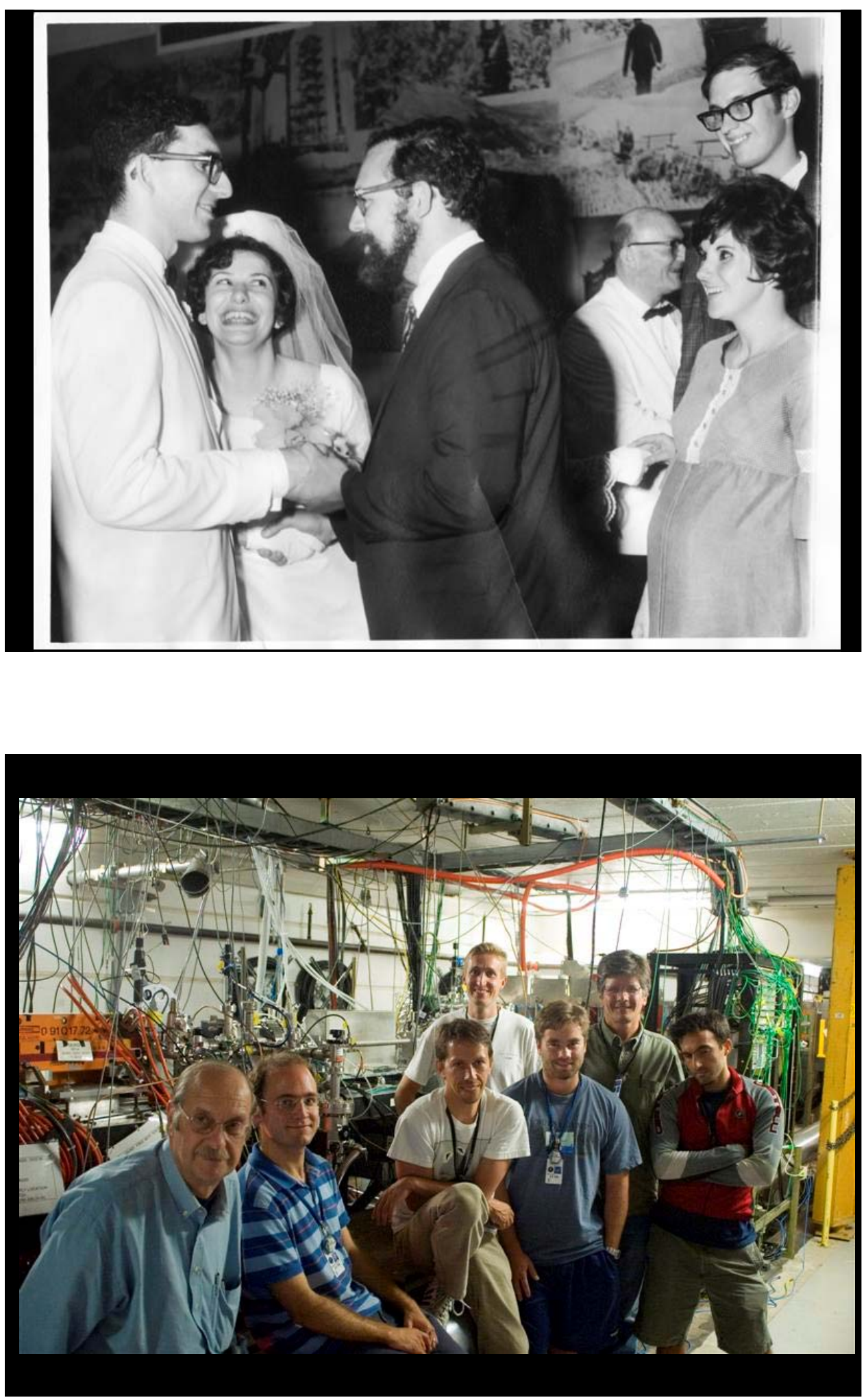

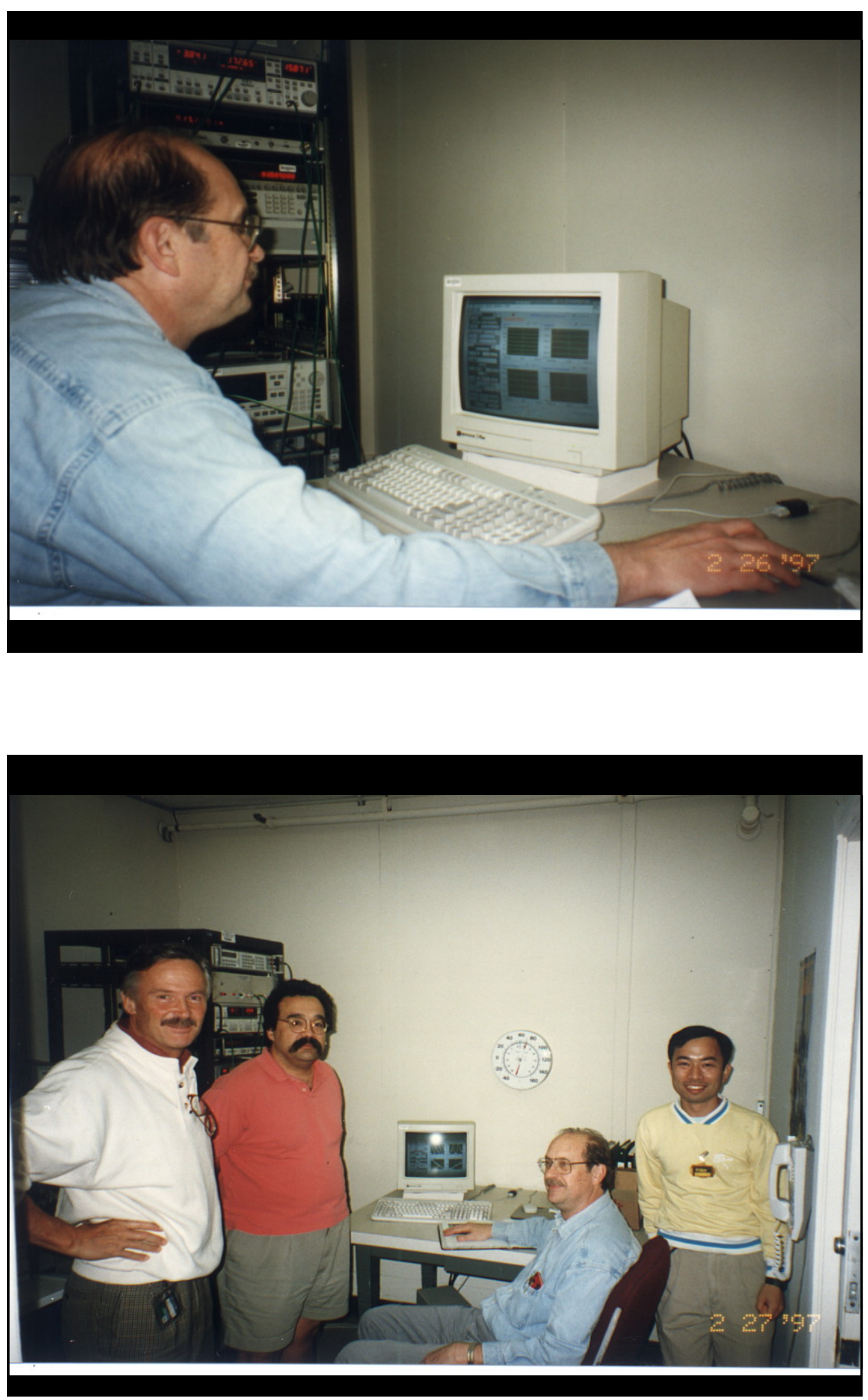

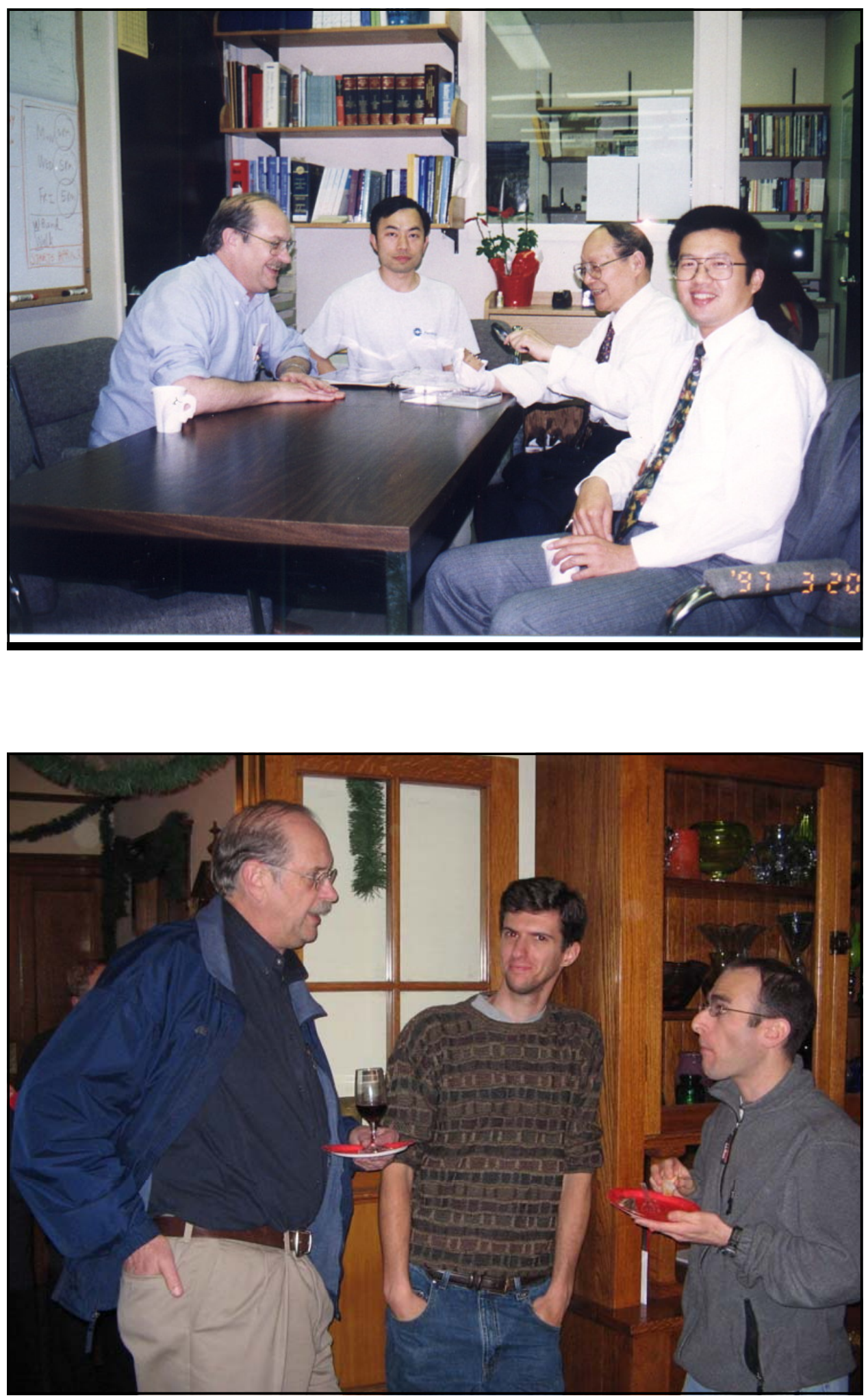

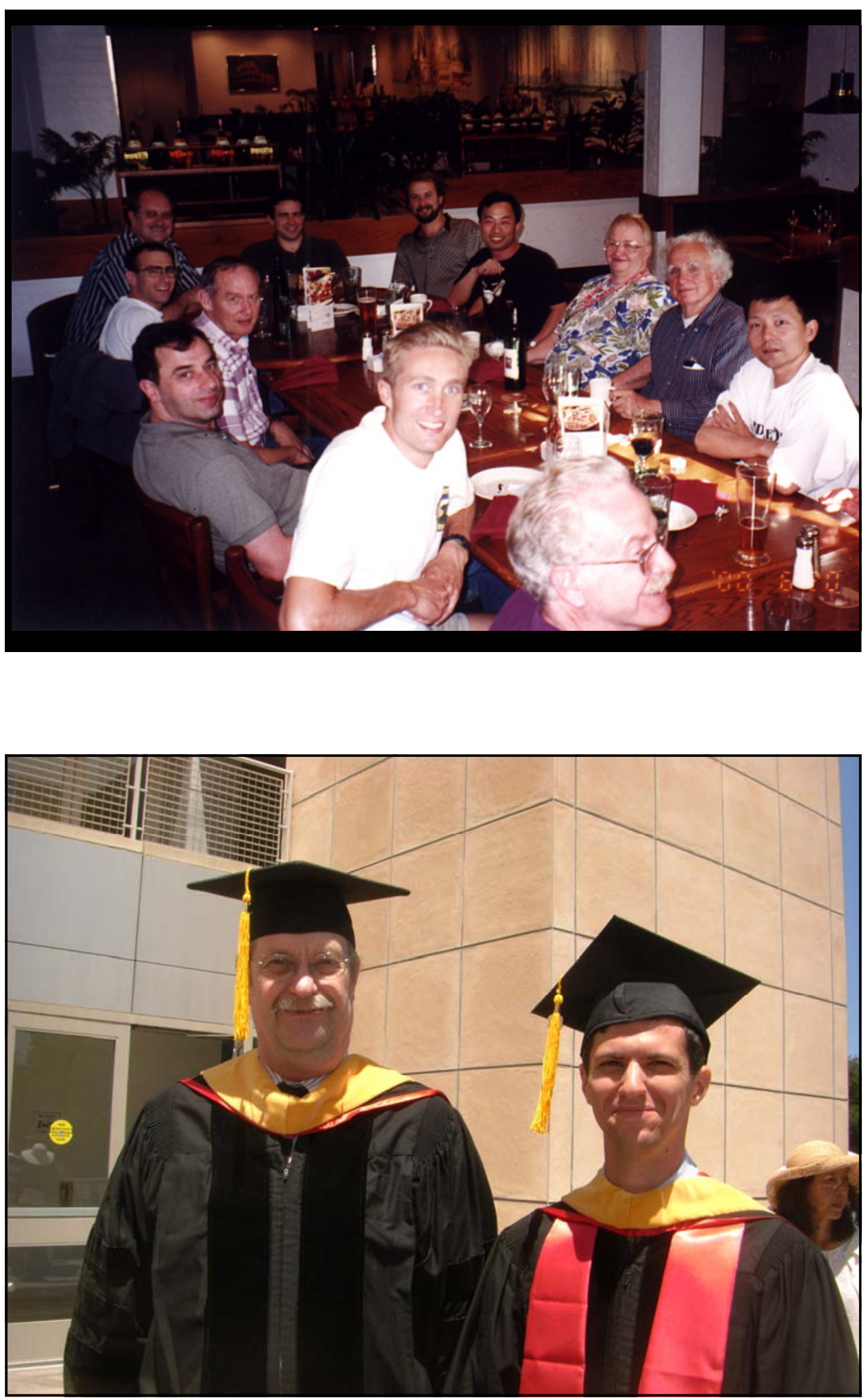

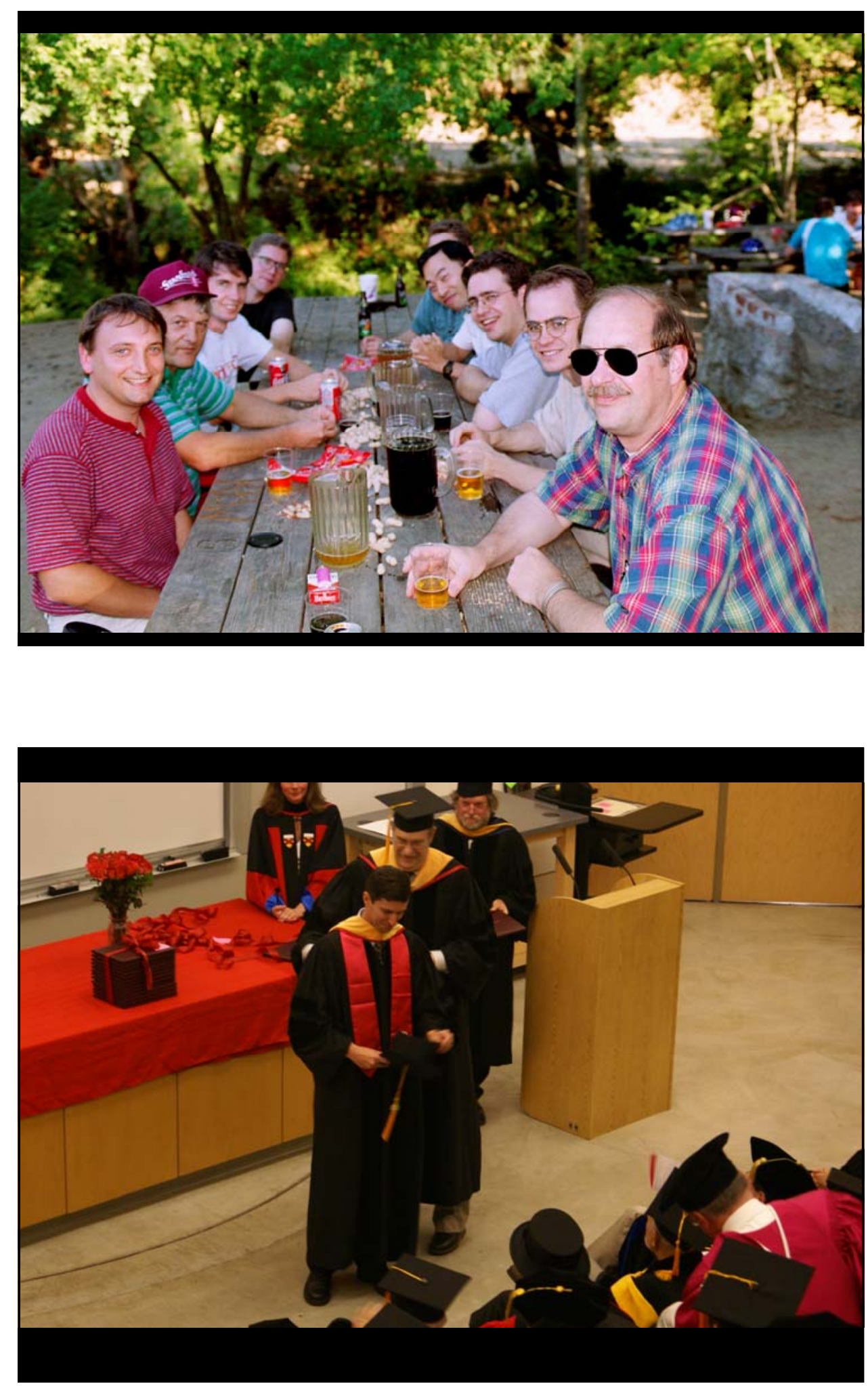

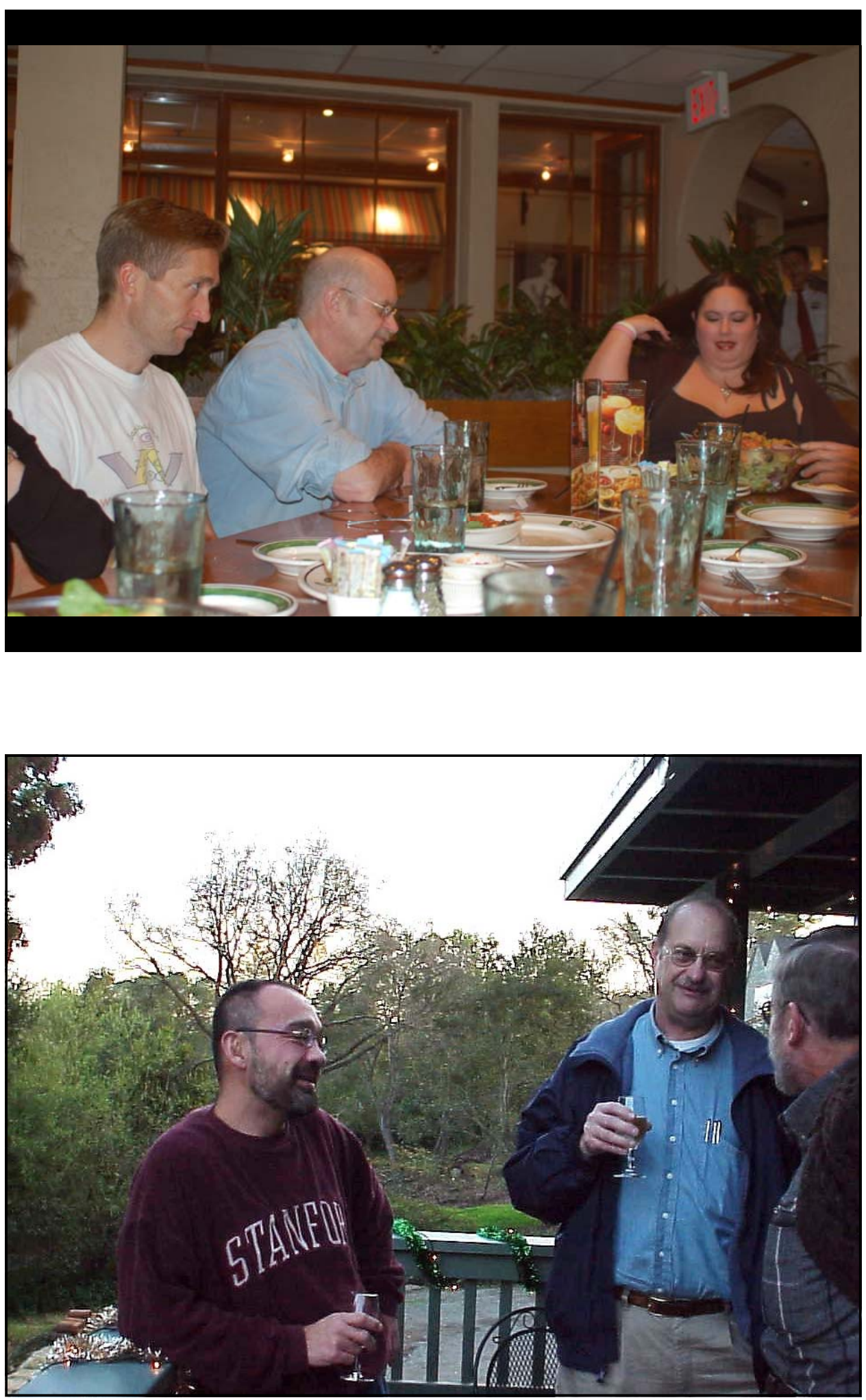

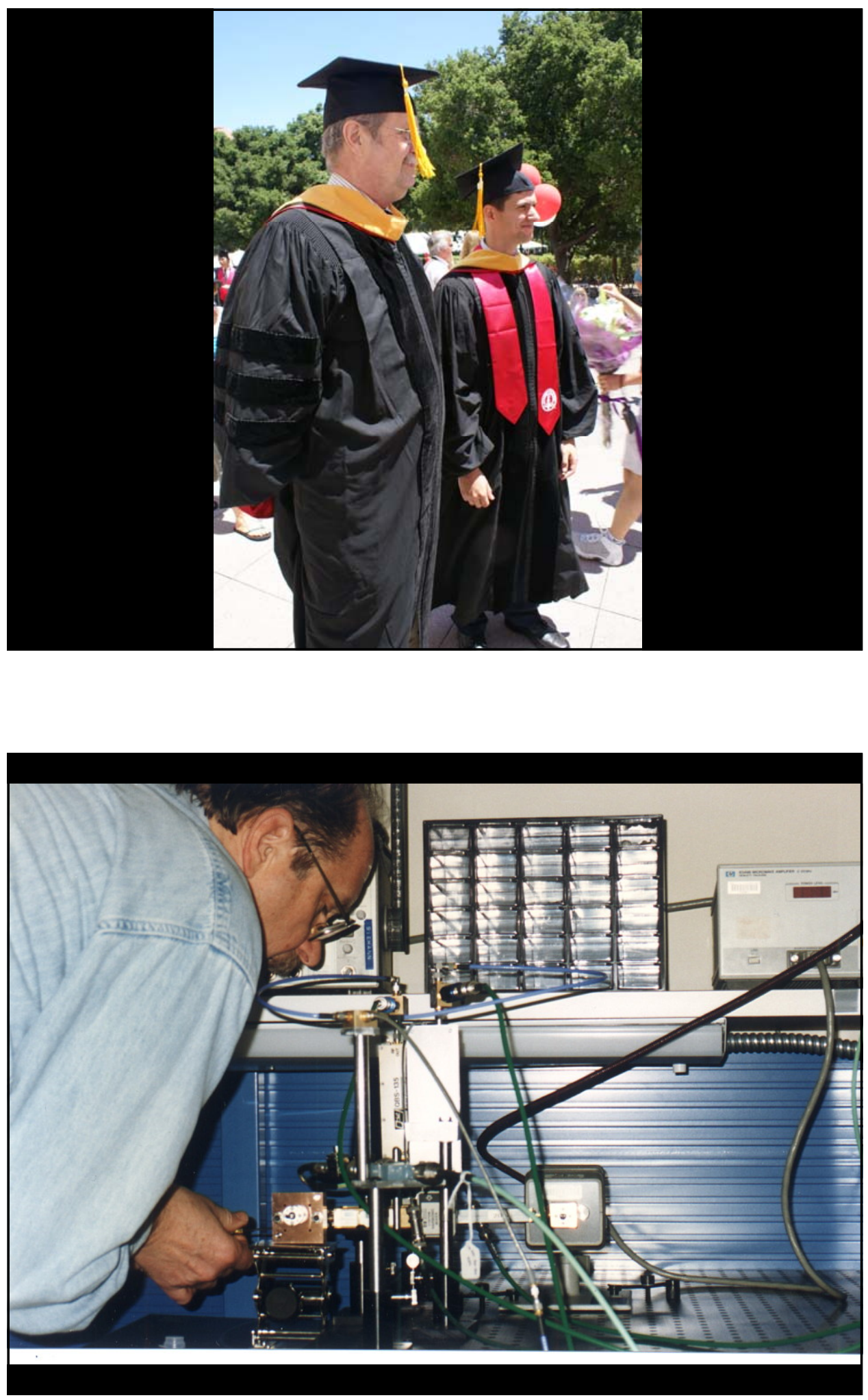

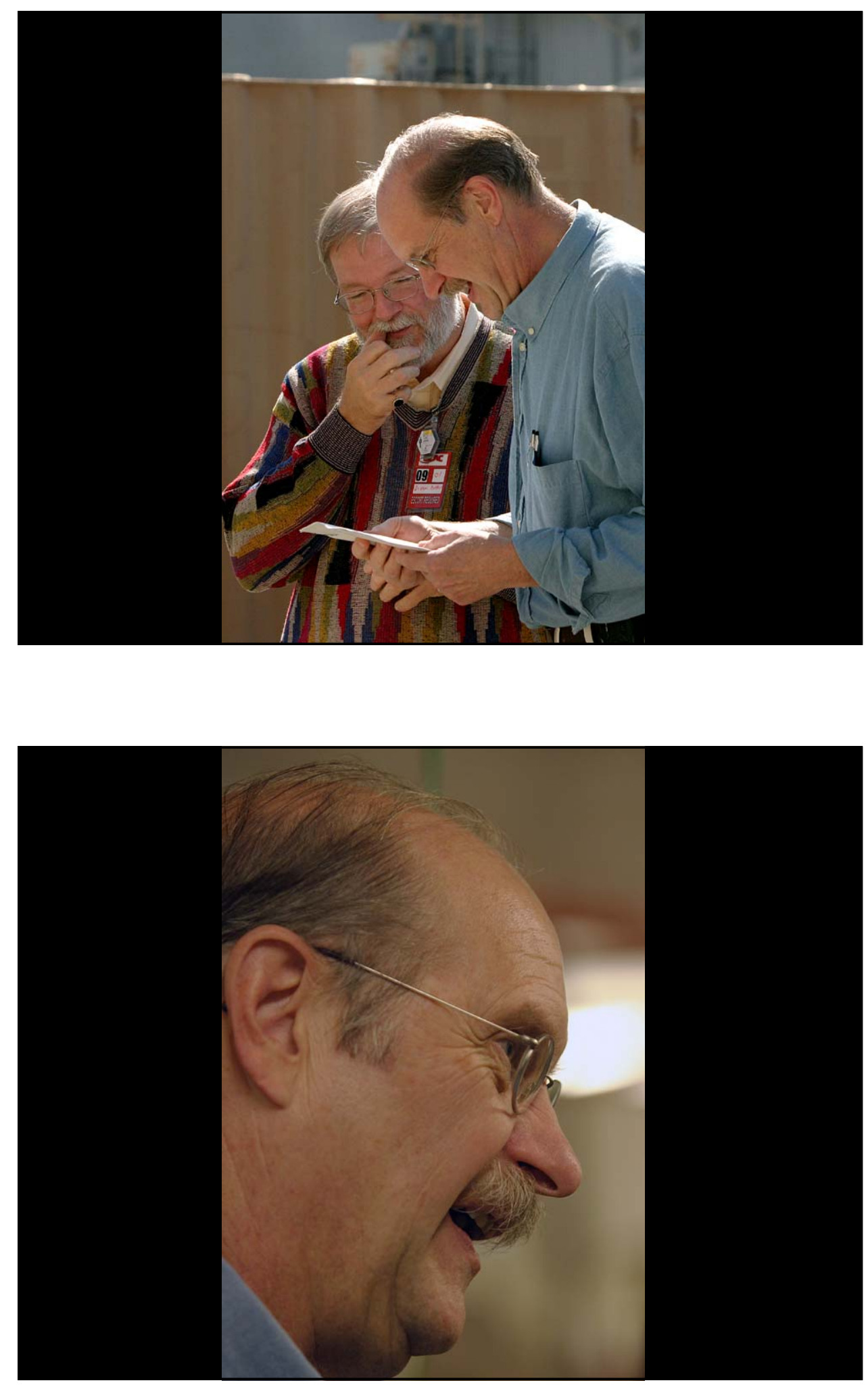


\title{
Thank You
}

\author{
Joan Valine \\ Naomi Nagahashi
}

\section{Stephanie Santo “Nixx" Arias}

Jimmy Pham, Terry Anderson, Chip Dalby, Stephanie West

Rod Reape \& the Communications Group

\section{Members of AARD}

\section{Thank You}

\section{Symposium Speakers}

Persis Drell, Roger Erickson, Dick Talman, Gerry Jackson, Burt Richter, Tor Raubenheimer, Boris Podobedov, Ping-Jung Chou, David Pritzkau, Stuart Henderson, Tom Katsouleas, Chris Barnes, Bob Byer, Ben Cowan, Dave Sutter, (Mel Month), Marty Blume, and Swapan Chattopadhyay

\section{Symposium Committee}

Marty Blume, Bob Byer, Alex Chao, Mark Hogan,

Gerry Jackson, Chan Joshi, Maury Tigner 

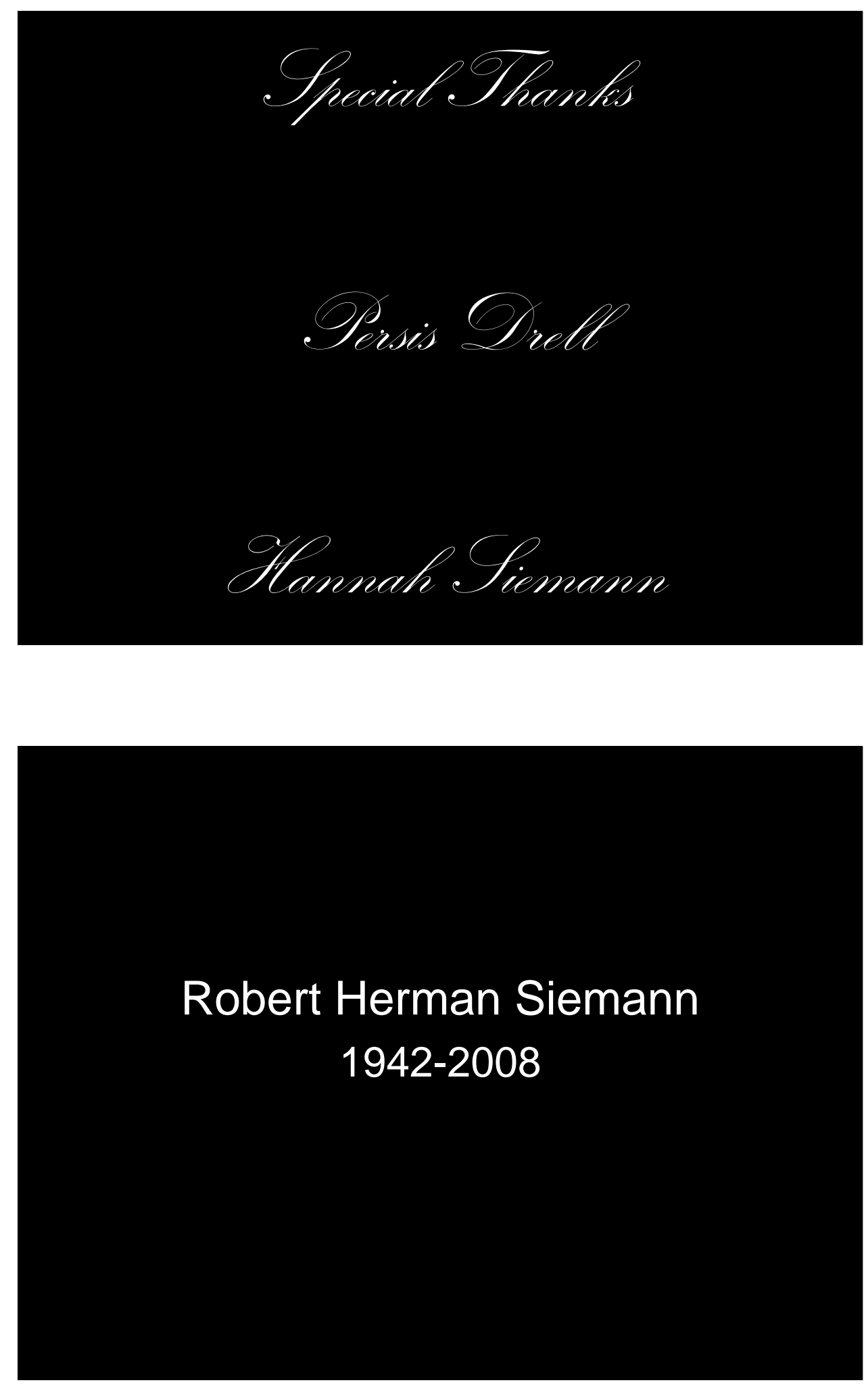


\title{
Tree Dedication
}

E. R. Colby, 7/7/09, 5pm

Good afternoon. We're gathered here to dedicate this tree in Bob’s memory.

This is a Coastal Redwood tree, a native of the northern coasts of California, where it draws moisture from the fog and the streams. It routinely grows to heights exceeding 300 feet, making it the tallest living thing on earth. Its wood is highly prized for its extraordinary resistance to termites and rot. It is also an endangered species, having been logged aggressively in the early $20^{\text {th }}$ century to supply railroad ties and structural members for the railroads of the Pacific Northwest.

It is appropriate, then that this adaptable, hardy, and extraordinarily tall tree is planted in memory of a man who shared these qualities. Bob nurtured the professional growth of many of us who are here today, and cast a long shadow over his scientific field. In a few short years this redwood, like Bob, will both provide shelter and cast its long shadow at SLAC.

A dedicatory plaque has been placed in Bob's memory; mounted to a stone donated by Hannah Siemann from their estate, providing a "piece of home" upon which to display it.

[Hannah Siemann uncovers the plaque]

The inscription reads:

\author{
"In Memory of \\ Robert H. Siemann \\ Pioneering accelerator physicist, \\ respected leader and mentor
}

1942-2008."

This tribute was the idea of Joan Valine, who ably brought the idea to reality. Thank you Joan, for making this fitting memorial to Bob happen.

[Group photo]

[Reception] 


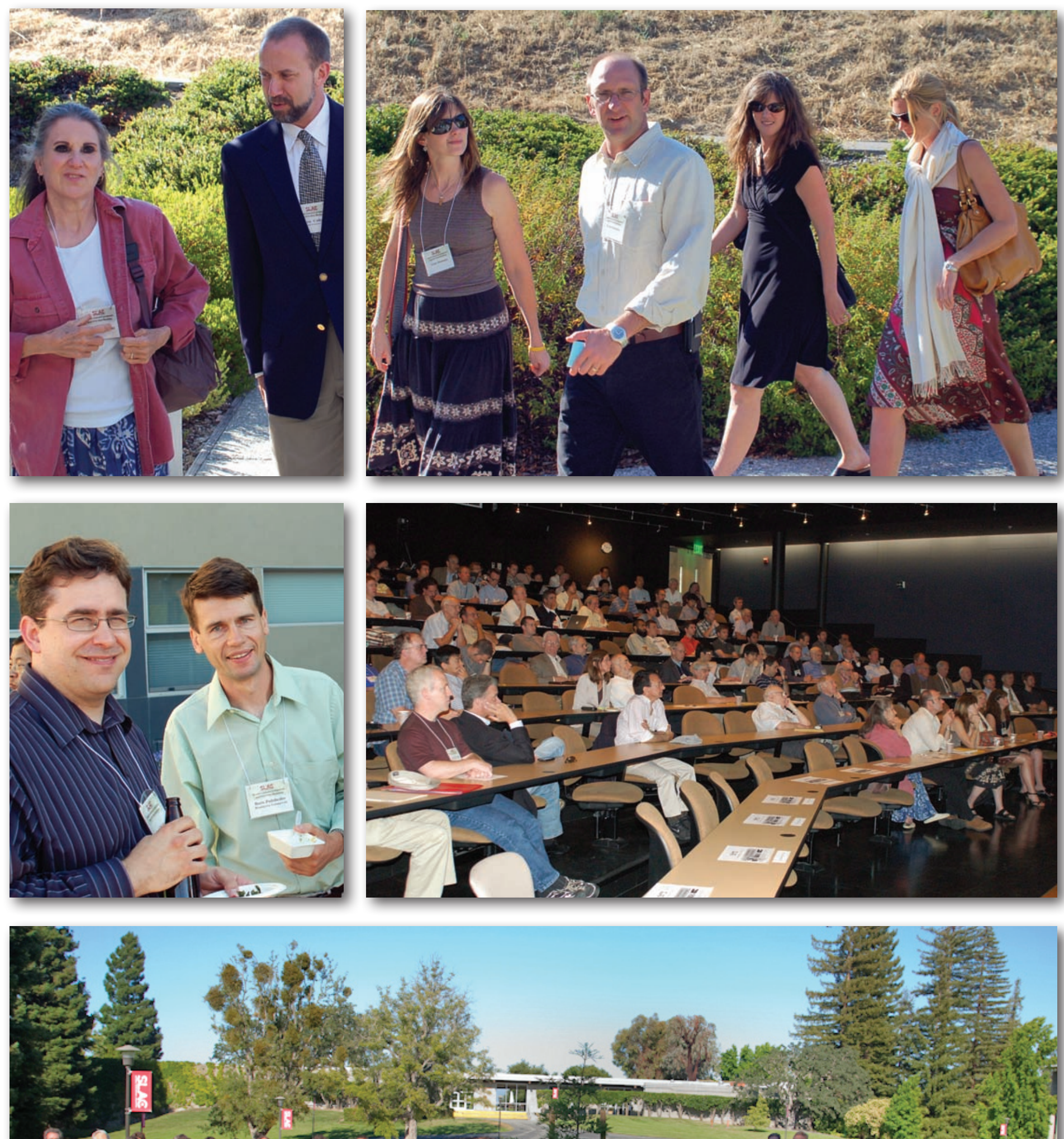

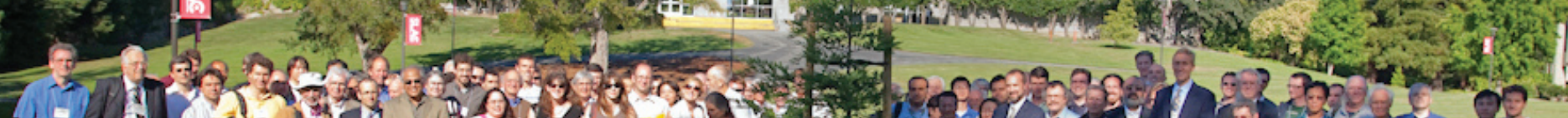
I.

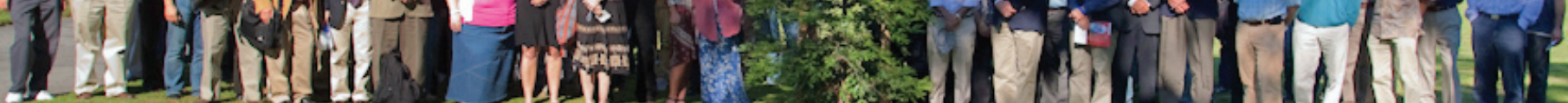

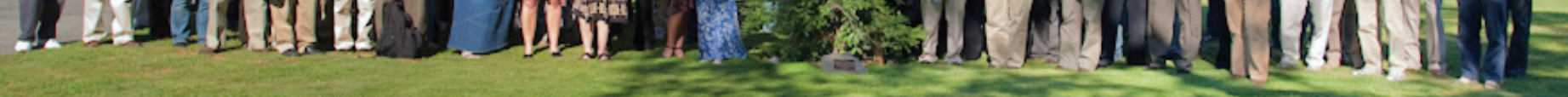


